

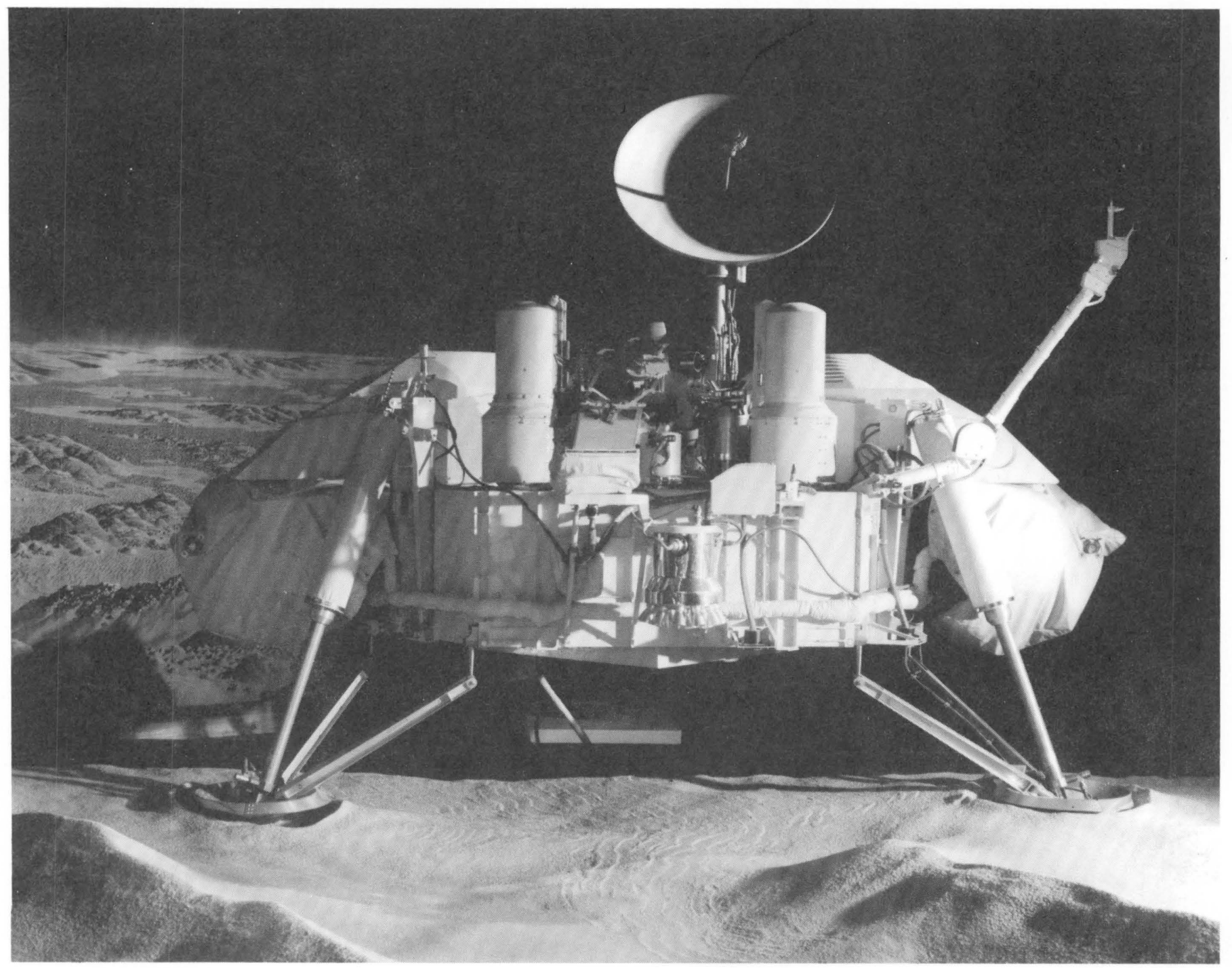

VIKING SCIENCE TEST LANDER

This full-scale model with fully operational lander cameras and surface-sampler subsystem was installed adjacent to a large sand box representing the area in reach of the surface sampler. The Science Test Lander was used during the mission to develop and verify surface-sampler commands and to perform some experiments for the Physical Properties Investigation. Circular S-band radio antenna of lander is $0.76 \mathrm{~m}$ wide. Locations of spacecraft parts, cameras, and surface-sampler subsystem are shown in figures 1 and 3. 
PHYSICAL PROPERTIES OF THE SURFACE MATERIALS AT THE VIKING LANDING SITES ON MARS 


\section{Physical Properties of the Surface Materials at the Viking Landing Sites on Mars}

By H.J. MOORE, R.E. HUTTON, G.D. CLOW, and C.R. SPITZER

U.S. GEOLOGICAL SURVEY PROFESSIONAL PAPER 1389

Prepared on behalf of the

National Aeronautics and Space Administration

A summary of the results of

the Physical Properties Investigation

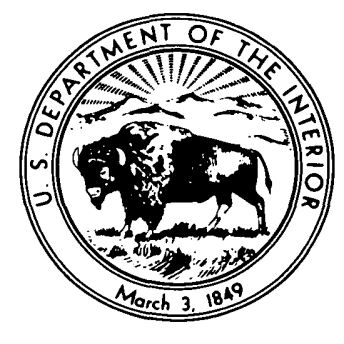

of Viking Landers 1 and 2 during

the Viking 1975 project 


\section{DEPARTMENT OF THE INTERIOR}

DONALD PAUL HODEL, Secretary

\section{U.S. GEOLOGICAL SURVEY}

Dallas L. Peck, Director

\section{Library of Congress Cataloging-in.Publication Data}

Physical properties of the surface materials at the Viking landing sites on Mars.

(U.S. Geological Survey professional paper 1389)

"A summary of the results of the Physical Properties Investigation of Viking Lander 1 and 2 during the Viking 1975 project." "Prepared on behalf of the National Aeronautics and Space Administration."

Bibliography

Supt. of Docs. no.: I 19.16:1389

1. Mars (Planet)-Geology. 2. Mars (Planet)—Surface. 3. Mars (Planet)-Exploration. I. Moore, H.J. (Henry J.), 1928-

II. United States National Aeronautics and Space Administration. III. Series: Geological Survey Professional Paper 1389. QB641.P47 1987 $559.9^{\prime} 23$ 87-600380 


\section{CONTENTS}

Abstract _. . . . . . . . . . . . . . .

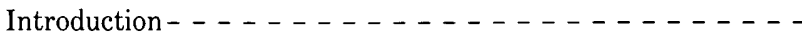

Viking missions - - - - - - - - - - - - -

Supplemental sections - - - - - - - - - - -

Acknowledgments - - . - . - - - . - . - - -

Mission description and conventions _ _ . . . . . -

Viking lander - . - . - . - . - . - . - -

Spacecraft parts _.................

Surface-sampler subsystem - - - _ - - . - .

Mission operations _ - - - - - - - - . - -

Other Viking experiments and investigations - . - . -

Coordinate systems and measurements - - - - - - - -

Time _. . . . . . . . . . . . . . . .

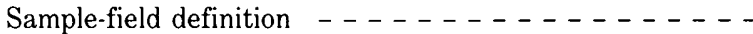

The landing sites $\ldots \ldots \ldots \ldots$

Orbiter views - . . . . - . . . . . . -

Lander panoramas _................

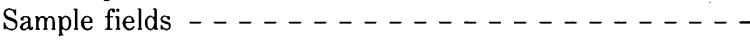

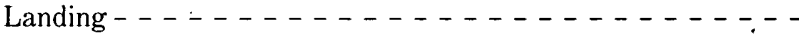

Descent - - - - . - - - - - - - - -

Touchdown - . . . . . . . . . . .

Lander 1 . . . . . . . . . . . .

Lander $2 \ldots \ldots . . . \ldots-\ldots$

Discussion - . - . . . . . . . . . .

Engine-exhaust erosion - . . . . . . . . . - -

Surface-sampler data - . . . . . . . . . . .

Activities - . . - . . . . . . . . . -

Sample trenches _...............

Drift material _. . . . . . . . . . . .

Blocky material -.....- - - - - - - -

Crusty to cloddy material .............

Discussion - . . . . . . . . . . . .

Surface-bearing tests $\ldots \ldots \ldots \ldots \ldots$

Drift material $\ldots \ldots \ldots \ldots$

Blocky material - - . - . - . - . . . -

Crusty to cloddy material $\ldots \ldots \ldots \ldots$

Backhoe touchdowns _...............

Surface impacts - - . - . - . - . - . - -

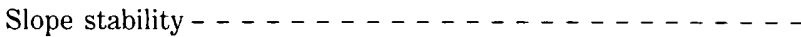

Trench walls $\ldots \ldots \ldots \ldots$

XRFS 1 trench $\ldots \ldots \ldots$

Physical Properties 1 trench

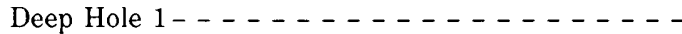

Inferred cohesions - . . . . . . . . . . . .

Natural slopes - . . . . . . . . . . . . -

Conical piles and tailings

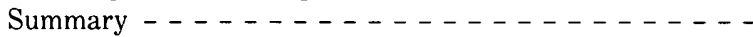

The quest for rocks - . . . . . . . . . . . .

Sample acquisitions - . . . . . . . . . .

Purges and rock piles - - - - - - - - - - -

Comminution _. . . . . . . . . . . . . . .

Rock pushing - - - - - - - - - - - - -

Summary

Temperatures - . . . . . . . . . . . . . . .

Surface-sampler diurnal temperatures - . . - . . . -

Lander 1 . . . . . . . . . . . . . .

Lander $2 \ldots \ldots \ldots \ldots$

Interim period _...................

Discussion - . . . . . . . . . . . . .

\begin{tabular}{|c|c|c|}
\hline & & \\
\hline 1 & Visual observations of changes $-\cdots-\cdots$ & \\
\hline 2 & Conical piles- ----------------- & 10 \\
\hline 2 & Materials in the footpads $\ldots-\cdots$ & \\
\hline 5 & Trenches ------------------- & \\
\hline 5 & al surfaces ---------------- & \\
\hline 6 & ditraviolet sensitive paints $-\cdots-\cdots$ & \\
\hline 6 & ovement ----------------- & \\
\hline 7 & ng experiments and investigations $\ldots \ldots$ & \\
\hline 8 & ------------------ & \\
\hline 11 & ------------------ & \\
\hline 1 & --------------- & \\
\hline 15 & ation or pyrolytic release $-\cdots----$ & \\
\hline 17 & ----------------- & \\
\hline 18 & lysis ------------- & \\
\hline 18 & ; - - - - - - - - - - - - - - - & \\
\hline 20 & $-\cdots----------\cdots---$ & \\
\hline 25 & ------------------- & \\
\hline 26 & ------------------ & \\
\hline 27 & Ing-- - - - - - n & \\
\hline 27 & --------------- & \\
\hline 29 & $-\cdots-----\cdots,-\cdots$ & \\
\hline 29 & ----------------- & \\
\hline 3 & ---------------- & \\
\hline $3 t$ & iction --------------- & \\
\hline 3 & ------------------- & \\
\hline 44 & ------------------- & \\
\hline 4 & -------------------- & \\
\hline 48 & $--\cdots-\cdots,-\cdots \cdots,-\cdots$ & \\
\hline 48 & - $\cdots \cdots-\cdots-\cdots-\cdots$ & \\
\hline 5 & - - - - - - & \\
\hline 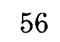 & $\cdots-\cdots-\cdots \cdots-\cdots-\cdots$ & \\
\hline 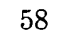 & $--\cdots-\cdots-\cdots-\cdots--\cdots$ & \\
\hline 6 & erial $------\cdots------$ & \\
\hline 6 & - - - - & \\
\hline 6 & $--\cdots----------$ & \\
\hline & ---------------- & \\
\hline & $-\cdots \cdots \cdots \cdots$ & \\
\hline & nates ------------ & \\
\hline 8 & $----\cdot---$ & \\
\hline & $---\cdots---\cdots---$ & \\
\hline & $----\cdots---$ & \\
\hline 8 & $--\cdot$ & \\
\hline 8 & es ------ & \\
\hline 8 & $-\cdots-$ & \\
\hline & - & \\
\hline & - & \\
\hline 8 & - & \\
\hline 8 & - & \\
\hline & - & \\
\hline 9 & - & \\
\hline 9 & - & \\
\hline 9 & - & \\
\hline 101 & & \\
\hline 10 & _. & \\
\hline & ing tests - - & \\
\hline 0 & 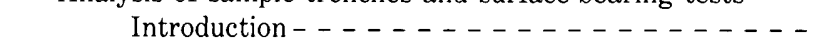 & \\
\hline 0 & & \\
\hline 0 & $s \ldots$ & \\
\hline 1 & he sample fields and rocks $\ldots \ldots$ & \\
\hline
\end{tabular}




\title{
ILLUSTRATIONS
}

\author{
[Plates are in pocket]
}

Frontispiece. Photograph of the Viking Science Test Lander.

Plate 1. Generalized maps of the Viking Lander 1 sample field.

2. Generalized maps of the Viking Lander 2 sample field.

FIGURE 1-4. Sketch of:

1. Viking lander showing locations of spacecraft parts $\ldots \ldots \ldots \ldots \ldots \ldots$

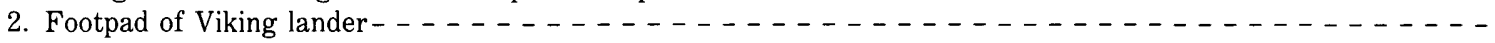

3. Viking lander showing locations of parts for surface-sampler subsystem and science experiments - - - - -

4. Surface-sampler collector head $\ldots \ldots \ldots \ldots \ldots$

5. Diagrams of coordinate systems used in this report $\ldots \ldots \ldots$

6. Viking Orbiter pictures of Lander 1 site $-\ldots \ldots \ldots \ldots$

7. Viking Orbiter pictures of Lander 2 site $\ldots \ldots \ldots \ldots$

8. Sketch of Lander 1 site $\ldots \ldots \ldots \ldots$

9. Graph showing cumulative frequency of craters at the Lander 1 site $\ldots \ldots \ldots$

10. Geologic sketch map showing relation between Lander 2 site and crater Mie $\ldots \ldots \ldots$

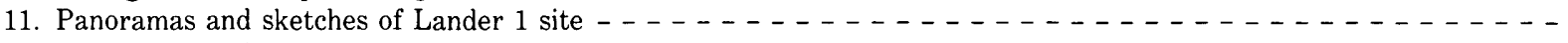

12. Panoramas and sketches of Lander 2 site $\ldots \ldots \ldots-\ldots, \ldots \ldots$

13. Graph showing fraction of area of sample field of both landers covered by rocks with intermediate diameters larger than $3.5 \mathrm{~cm} \ldots \ldots \ldots \ldots \ldots$

14. Graph showing cumulative and incremental frequencies of rocks with intermediate diameters larger than $3.5 \mathrm{~cm}$ for

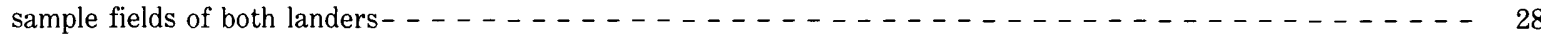

15. Sketches of approximate traces of flight paths of both landers $\ldots \ldots \ldots$

16. Photographs showing penetration of footpads 3 and 2 of Lander $1 \ldots \ldots$

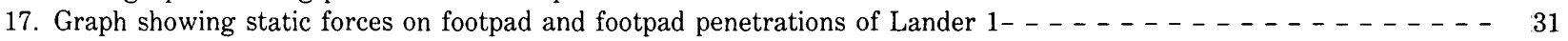

18. Graph showing density-penetration curves of prototype footpads on Earth and footpads 3 and 2 of Lander 1 on

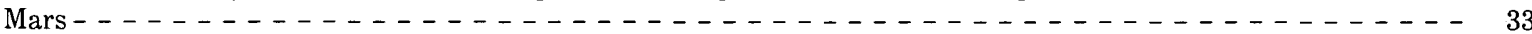

19. Photographs showing footpads 3 and 2 of Lander $2 \ldots-\ldots \ldots$

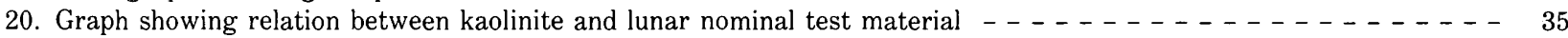

21. First pictures of martian surface taken by Viking landers $-\ldots \ldots \ldots$

22. Photographs showing engine-exhaust erosion by Lander 1- $\ldots \ldots \ldots$

23. Sketch map showing the effects of erosion by engine exhausts of Lander $1 \ldots \ldots \ldots$

24. Mosaics of pictures to right of engine 2, Landers 1 and $2 \ldots-\ldots$

25. Photographs showing engine-exhaust erosion by Lander $2 \ldots \ldots \ldots \ldots$

26-28. Graphs showing:

26. Centerline-impingement pressures related to nozzle heights for Viking lander terminal-descent engine- - - - 27. Impingement pressure related to distance from centerline-impingement point for Viking lander terminal-descent

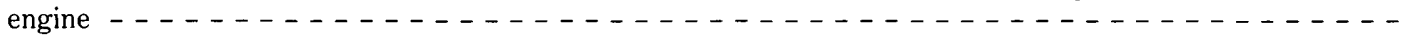

28. Depths of craters produced by engine-exhaust erosion from tests on Earth and Landers 1 and 2

29. Diagrammatic plan views of sample field of Lander $1 \ldots \ldots \ldots \ldots$

30. Diagrammatic plan views of sample field of Lander $2 \ldots \ldots \ldots \ldots \ldots \ldots$ 31-33. Photographs showing:

31. Physical Properties 1 trench excavated by Lander 1 on Sol 41

32. Drift material of Sandy Flats, first sample trench (Biology 1), and first trench for Inorganic Chemical Analysis

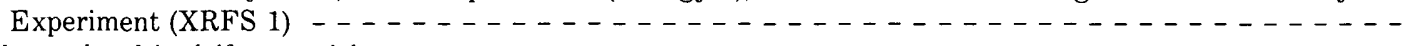

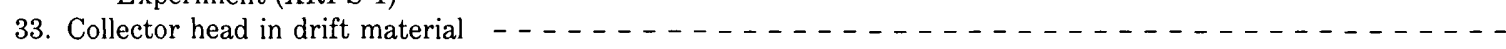

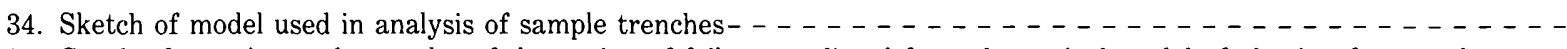

35. Graph of exterior wedge angles of the region of failure predicted from theoretical model of plowing for sample trenches in drift and blocky material of Lander $1 \ldots \ldots \ldots \ldots \ldots$

36. Graph showing comparison between apparent cohesions and probable cohesions for sample trenches in drift, blocky,

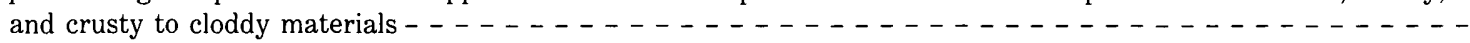

37-41. Photographs showing:

37. Rocky Flats, first, second, and third trench excavated in blocky material (GCMS 3, XRFS 2, XRFS 3),

Lander $1 \ldots \ldots \ldots \ldots \ldots$

38. Rocky Flats, at the end of Sol 350, Lander $1 \ldots \ldots \ldots$

39. Bonneville Salt Flats and trenches excavated by Lander 2 $\ldots \ldots \ldots$

40. Trench in cloddy material (XRFS 3) $\ldots \ldots \ldots \ldots \ldots \ldots \ldots$

41. Sample trenches in Beta site, Lander $2 \ldots \ldots \ldots \ldots \ldots \ldots$

34
9
1


Figure 42. Graph showing exterior wedge angles of the region of failure predicted from theoretical model of plowing for sample

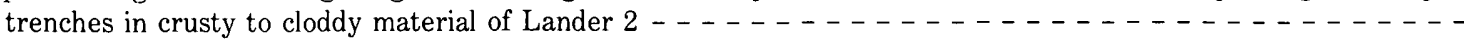

43. Graph showing cohesion as a function of angle of internal friction for drift, blocky, and crusty to cloddy

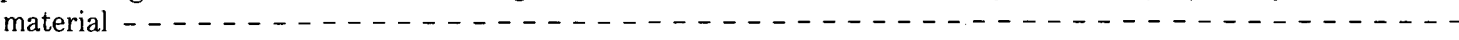

44. Photograph showing surface-bearing test on drift material, Lander 1

45. Map and profile of surface-bearing test on drift material, Lander 1

46. Graph showing exterior wedge angles of region of failure predicted from theoretical model of plowing for the

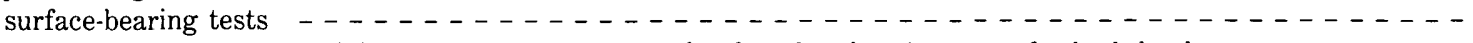

47. Graph showing forces inferred from motor-current records of surface-bearing tests for both landers _ - . . - .

48. Photographs showing surface-bearing test on blocky material of Lander 1- $\ldots \ldots$

49. Map and profile of surface-bearing test on blocky material, Lander $1 \ldots$

50. Photograph showing surface-bearing test on crusty to cloddy material of Lander 2 - - - - - . - . - -

51. Map and profile of surface-bearing test on crusty to cloddy material of Lander $2 \ldots$

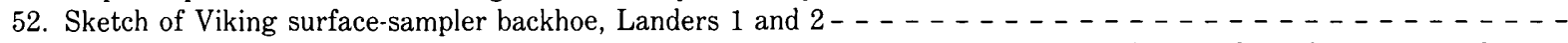

53. Graph and sketch showing comparison between backhoe penetration and shear-vane strength, and two types of

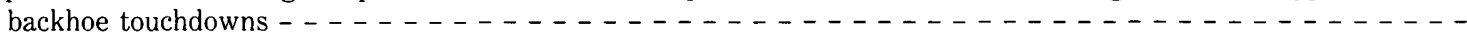

54. Photographs of selected backhoe-touchdown tests of Lander 1- $\ldots$

55. Photographs of selected backhoe-touchdown tests of Lander 2- $\ldots \ldots \ldots \ldots \ldots \ldots$

56. Graph showing comparison between cohesions of lunar nominal material from triaxial tests and shear-vane

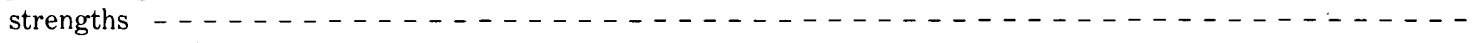

57-63. Photographs showing:

57. Impact craters and pits in drift material produced by fragments propelled by engine-exhaust gases,

Lander 1 . . . . . . . . . . . . . . . . . . . . . . . . . . . . .

58. Impact crater in blocky material produced by ejection of shroud unit on Lander $1 \ldots \ldots$

59. Crater produced by restraint or latch pin, Lander $1 \ldots \ldots$

60. Droppings on drift material from surface sampler, Lander 1

61. Impact crater produced by purging collector head of and on drift material, Lander 1

62. Results of Sol 280 impact experiment on Mars, Lander $1 \ldots$

63. Results of Sol 350 impact experiment on Mars, Lander $1 \ldots$

64. Profiles of three trenches in drift material, Lander 1 _ $\ldots \ldots \ldots$

65. Photographs showing deep holes excavated on Mars, both Landers _ _ _ $\ldots \ldots \ldots$

66. Graph showing slope stability factors as a function of slope angle $\ldots \ldots \ldots \ldots$

67-72. Photographs showing:

67. Open fractures in drift material, Lander $1 \ldots \ldots \ldots \ldots \ldots$

68. Natural slope failures of drift material on leeward sides of large rocks, Lander $1 \ldots \ldots$

69. Drift material $10 \mathrm{~m}$ behind Lander $1 \ldots \ldots \ldots \ldots$

70. Funnel above chamber of the X-ray fluorescence spectrometer showing fragments collected by end of Sol $586 \ldots \ldots \ldots \ldots \ldots \ldots \ldots$

71. Nominal pungè site in stereoscopic field of view of Lander 1 $\ldots \ldots \ldots$

72. Nominal purge site in stereoscopic field of view of Lander 2 $\ldots \ldots \ldots$ 73-78. Graphs showing:

73. Relation between grain-size and comminutor motor currents on Earth and on Mars - - - - - - . - .

74. Relation between fragments of porous and dense materials and increase in comminutor motor currents on

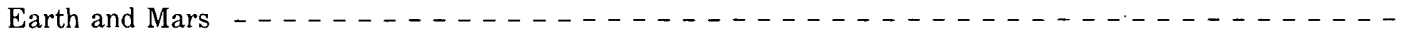

75. Format 5 comminutor motor-current records for Science Test Lander $\ldots \ldots \ldots$

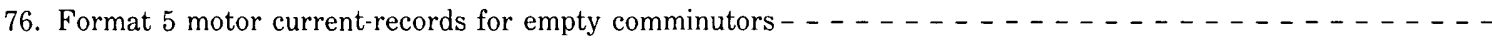

77. Format 5 motor current-records for loaded comminutors on Mars $\ldots \ldots \ldots$

78. Surface-sampler diurnal and footpad temperatures, both Landers $\ldots \ldots \ldots \ldots \ldots \ldots$ 79-90. Photographs showing:

79. Collector head of Lander 2 inserted in crusty to cloddy material $\ldots \ldots \ldots \ldots$

80. Conical pile 1 of Lander 1 _ $\ldots \ldots \ldots \ldots$

81. Conical piles 2, 3, and 5 of Lander $1-\ldots \ldots-\ldots-\ldots$

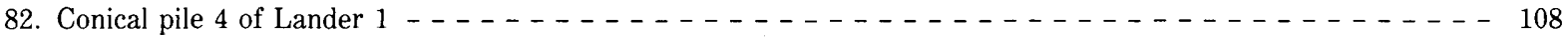

83. Conical piles 1 and 3 of Lander $2 \ldots \ldots \ldots$

84. Conical pile 2 of Lander $2 \ldots \ldots \ldots-\ldots \ldots \ldots$

85. Conical pile emplaced on grid of Lander 1 body $\ldots \ldots \ldots \ldots$

86. Changes in footpad 3 of Lander $1 \ldots \ldots-\ldots$

87. Changes in footpads 3 and 2 of Lander $2 \ldots \ldots \ldots \ldots$

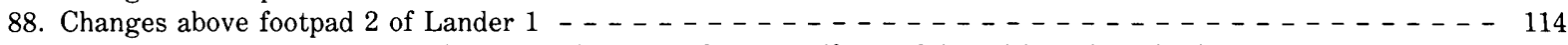

89. Two backhoe-touchdown trenches in Rocky Flats showing effects of deposition of bright fine-grained

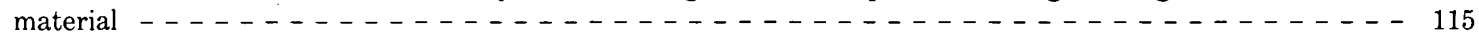

90. Trenches of Lander 2 showing effects of deposition of bright fine-grained material and "snow fall" - - - - - 116

91. Graph showing thermal inertias of surfaces with one or more components

92. Explanation for maps and profiles (figs. 93-150) $\ldots \ldots \ldots \ldots \ldots \ldots \ldots$ 
FiguREs 93-120. Map, profile, and, where shown, Format 5 data for Lander 1 trench sites:

93. First trench excavated in drift material on Sol 8 $\ldots \ldots \ldots \ldots \ldots$

94. In drift material on Sol $8 \ldots \ldots \ldots \ldots \ldots$

95. In drift material on Sol $14 \ldots \ldots \ldots \ldots$

96. In blocky material on Sol $34 \ldots \ldots \ldots \ldots \ldots$

97. In blocky material on Sol $40 \ldots \ldots \ldots \ldots \ldots \ldots$

98. In drift material on Sol $41 \ldots \ldots \ldots \ldots$

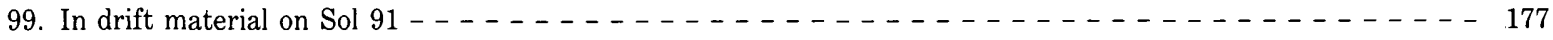

100. In blocky material on Sols $177,178,179$, and $180 \ldots \ldots \ldots \ldots$

101. In Deep Hole 1 in drift material on Sols 202, 204, 218, and $219 \ldots \ldots \ldots$

102. In drift material on Sol 270 during magnetic mineral abundance experiment- - - - - - - - - - - - 179

103. In blocky material on Sol $280 \ldots \ldots \ldots \ldots \ldots$

104. In drift material on Sol $280 \ldots \ldots \ldots$

105. In wind tail produced by skimming on Sols 285 and $286 \ldots \ldots \ldots$

106. In drift material on Sol $296 \ldots \ldots \ldots \ldots$

107. In blocky material on Sols 311 and $312 \ldots \ldots \ldots \ldots$

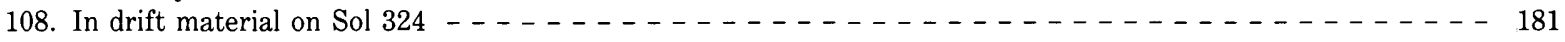

109. In drift material used for diurnal temperatures on Sols 343 and $344 \ldots \ldots \ldots \ldots$

110. In blocky material on Sol $350 \ldots \ldots \ldots \ldots \ldots-\ldots \ldots$

111. Near rock 5 (Bashful) on Sol 378 and later on Sol $527 \ldots \ldots \ldots \ldots$

112. Near right edge of sample field on Sols 430 and $431-\ldots-\ldots-\ldots-\ldots-\ldots$

113. Region of sample trench in Deep Hole 2 on Sol $456 \ldots \ldots \ldots$

114. In Deep Hole 2 on Sol $502,-\ldots \ldots-\ldots-\ldots-\ldots-\ldots-\ldots-\ldots$

115. In drift material on Sol $520 \ldots \ldots \ldots$

116. Produced in tailings of Deep Hole 1 on Sol $550 \ldots \ldots \ldots$

117. In tailings of Deep Hole 2 on Sol $586 \ldots$

118. Surface-bearing test in drift material on Sol $612 \ldots \ldots \ldots \ldots \ldots \ldots$

119. Surface-bearing test in blocky material on Sol $612-\ldots-\ldots-\ldots-\ldots-\ldots-\ldots$

120. In Rocky Flats in blocky material on Sol 639 _ $\ldots \ldots \ldots$

121-150. Map, profile, and, where shown, Format 5 data for Lander 2 trench sites:

121. First trench excavated in Beta site on Sol $8 \ldots \ldots \ldots \ldots$

122. In Bonneville Salt Flats in crusty material on Sol 21_ $\ldots \ldots \ldots \ldots$

123. In Beta site in crusty to cloddy material on Sol $28 \ldots \ldots \ldots$

124. In Bonneville Salt Flats in crusty to cloddy material on Sols 29 and $30 \ldots \ldots$

125. In area originally under rock 3 (Badger) on Sol $37 \ldots \ldots \ldots \ldots$

126. In Beta site in crusty to cloddy material on Sols 46 and $47 \ldots$

127. In area originally under rock 7 (Notch) on Sol $151 \ldots \ldots-\ldots$

128. In Alpha site on Sol 56 _ $\ldots \ldots \ldots \ldots$

129. In Alpha site in crusty to cloddy material on Sol $57 \ldots \ldots \ldots \ldots$

130. In Alpha site in crusty to cloddy material on Sol $58 \ldots \ldots \ldots \ldots$

131. In area originally under rock 7 (Notch) on Sol $131, \ldots \ldots \ldots$

132. In Beta site in tailings of earlier trench on Sol $145 \ldots \ldots \ldots \ldots$

133. In Spalling Valley (near rock 15) for pile of coarse fraction on Sol 145- $\ldots$

134. In Spalling Valley (near rock 15) on Sol $161 \ldots \ldots \ldots \ldots$

135. In Spalling Valley (near rock 15) for pile of coarse fraction on Sol 172- $-\ldots$

136. In area originally under rock 3 (Badger) on Sols 185 and $186 \ldots$

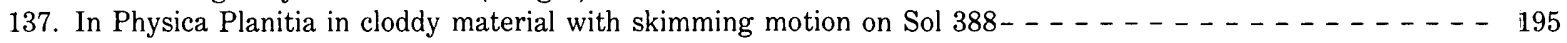

138. Diurnal-temperature trench on Sols 405 and $406 \ldots \ldots \ldots$

139. In Deep Hole 1 on Sol $417 \ldots \ldots \ldots \ldots \ldots$

140. In Deep Hole 1 on Sols 442 (and 417) $\ldots \ldots \ldots \ldots$

141. In Deep Hole 1 on Sol $442 \ldots \ldots \ldots \ldots$

142. In Physica Planitia in crusty to cloddy material on Sol $479 \ldots \ldots \ldots \ldots$

143. In front of rock 11 (Snow White) on Sol 483 $\ldots \ldots \ldots \ldots \ldots$

144. In Bonneville Salt Flats in crusty to cloddy material on Sol $506 \ldots \ldots \ldots$

145. In Deep Hole 1 tailings, base of tailings, and Deep Hole 1 on Sols 522, 523, 535, and 545 $\ldots$

146. Retraction trenches on Sol $559 \ldots \ldots \ldots \ldots$

147. In Physica Planitia in crusty to cloddy material on Sol $595 \ldots \ldots \ldots \ldots$

148. Adhesion test in crusty to cloddy material on Sol $595 \ldots \ldots \ldots$

149. Surface-bearing test in crusty to cloddy material on Sol $595 \ldots \ldots$

150. In Bonneville Salt Flats in crusty to cloddy material on Sols 595 and $596 \ldots$

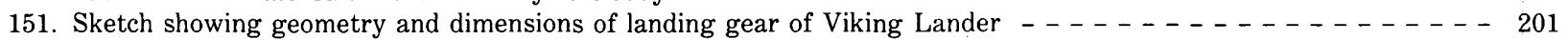

152. Graph showing relation between static axial force and leg stroke for Viking Lander main strut $---_{-}--_{-} 202$

153. Sketch showing relation between forces on the primary strut $\ldots \ldots$ 
FIGUREs 154-157. Graphs showing:

154. Relation between force on footpad and leg stroke for six coefficients of friction _............

155. Relation between lateral force on footpad and lateral translation of footpads for six coefficients of friction - - 210

156. Total work performed on primary-strut shock absorbers, Lander $1 \ldots \ldots$

157. Total work performed on primary-strut shock absorbers, Lander $2 \ldots \ldots \ldots$

158. Sketch showing geometry of failure region in front of plow blade $\ldots \ldots \ldots \ldots$

159. Graph showing relation of exterior wedge angle to dimensionless plowing factor

160. Graph showing procedure for estimating angles of internal friction of martian surface material $-\ldots$

161. Diagram of two-dimensional model used to estimate horizontal force exerted by interface of leading edge of

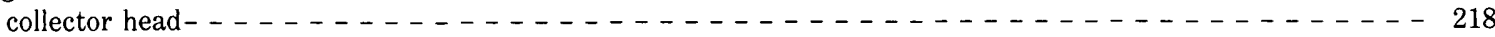

162. Graph showing ratio of forces on collector head and surface sampler as a function of sliding friction $\ldots \ldots$

163. Diagram of plowing model used for empirical analyses $\ldots \ldots \ldots \ldots \ldots$

\section{TABLES}

TABLE 1. The principal sources of data for the Physical Properties Investigation-

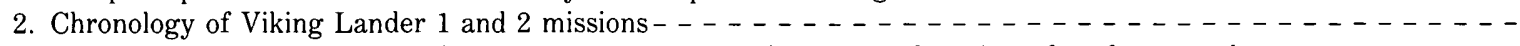

3. Overlap of the planning cycle for mission operations and design and testing of surface-sampler sequences- - - -

4. Detailed surface-sampler sequence designed to collect the coarse fraction of surface materials at the Viking

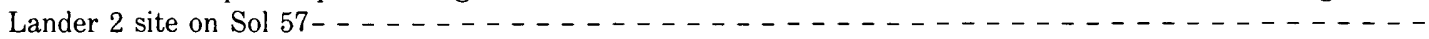

5. Locations and relevant parameters at the Viking landing sites $-\ldots-\ldots-\ldots-\ldots-\ldots$

6. Touchdown conditions and landing data for Landers 1 and $2 \ldots \ldots \ldots$

7. Mechanical properties of surface-material simulants used in footpad tests on Earth $\ldots$

8. Generalized conditions for footpad penetrations during tests on Earth and Mars - . . . . . . . . . .

9. Estimated angles of internal friction of drift material from data on sample trenches- . . . . . . . . . .

10. Cohesion of drift material as a function of angle of internal friction, using the model of McKyes and Ali (1977) - -

11. Apparent and probable cohesions of drift material and expected horizontal forces for sand - _ - . - . - .

12. Estimated angles of internal friction of blocky material from data on sample trenches- - . - . - . - . -

13. Cohesion of blocky material as a function of angle of internal friction, using the model of McKyes and Ali (1977) - - - - - - - - - - - - - - - - - - - - - - - - - - - - - - - -

14. Apparent and probable cohesions of blocky material and expected horizontal forces for sand _. . - . . . -

15. Estimated angles of internal friction of cloddy material from data on sample trenches, Lander 2

16. Cohesion of crusty to cloddy material as a function of angle of internal friction, using the model of McKyes and Ali (1977), Lander $2 \ldots \ldots \ldots \ldots \ldots \ldots$

17. Apparent and probable cohesions of crusty to cloddy material and expected horizontal forces for sand,

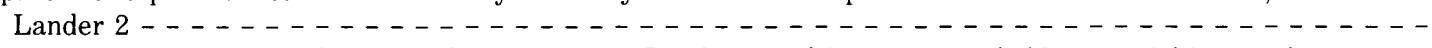

18. Surface-bearing tests in drift and blocky material by Lander 1 and in crusty to cloddy material by Lander 2 - - -

19. Commanded positions, surface-sampler data, and inferred positions from stereometric measurements and mapping for the surface-bearing test in drift material (Lander 1) $\ldots \ldots \ldots \ldots$

20. Cohesions from surface-bearing tests as functions of angles of internal friction, using the model of McKyes and Ali (1977) - - - - - - - - - - - - - - - - - - - - - - - - - - - - - - - - -

21. Commanded positions, surface-sampler data, and inferred positions from stereometric measurements and mapping

for the surface-bearing test in blocky material (Lander 1)
22. Commanded positions, surface-sampler data, and inferred positions from stereometric measurements and mapping for the surface-bearing test in crusty to cloddy material (Lander 2)

23. Backhoe touchdowns and data on locations, times, surface-sampler positions, photographs, and remarks- - . - -

24. Backhoe-touchdown tests conducted with the Science Test Lander $\ldots \ldots \ldots$

25. Data from impact experiments on Mars and Earth and penetration parameters . . . . . . . . . . . .

26. Slopes, reliefs, slope-stability factors, and limits for cohesions, assuming three angles of internal friction for

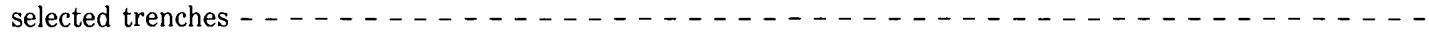

27. Acquisitions for coarse samples by Lander 1 and Lander $2 \ldots \ldots \ldots \ldots$

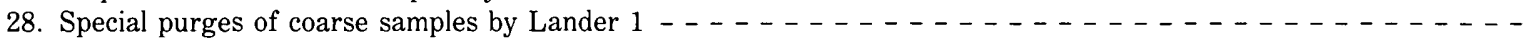

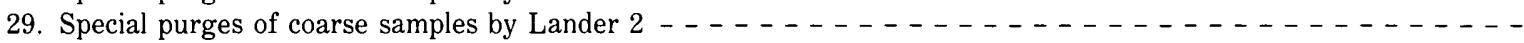

30. Comminution data for Viking Landers 1 and $2 \ldots \ldots \ldots \ldots$

31. Maximum and mininum temperatures measured by the surface sampler in the surface materials, and predicted maximum and minimum surface temperatures $\ldots \ldots \ldots \ldots \ldots$

32. Conical piles of Viking Landers 1 and $2 \ldots \ldots \ldots$

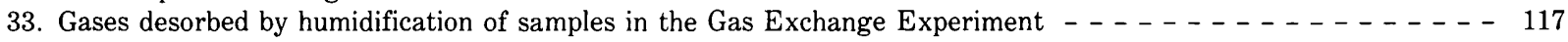


TABLE 34. Estimates of water and carbon dioxide in samples analyzed in the gas chromatograph-mass spectrometer for the

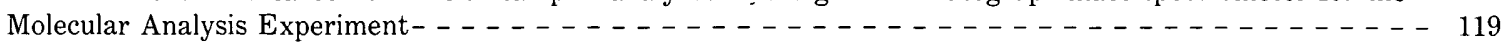

35. Compositions of selected and representative samples from Landers 1 and $2 \ldots \ldots-\ldots$

36. Bulk densities of disturbed materials in the full-analytical chamber of the X-ray-fluorescence spectrometer and inferred fragment (or mineral) densities for two assumed porosities, and derived porosity of sample or fragments for a mineral density of $2,600 \mathrm{~kg} / \mathrm{m}^{3} \ldots \ldots \ldots$

37. Summary of physical properties of the surface materials at the Viking Landing sites $\ldots$

38. Surface-sampler activities related to the Physical Properties Investigation during the Primary Mission of Viking

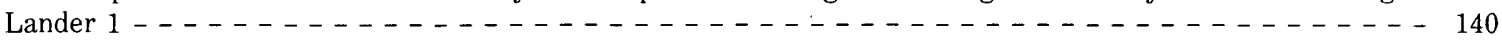

39. Surface-sampler activities related to the Physical Properties Investigation during the Extended Mission of Viking Lander 1 . . . . . . . . . . . . . . . . . . . . . . . . . . . . . . . . 144

40. Surface-sampler activities related to the Physical Properties Investigation during the Primary Mission of Viking Lander $2 \ldots \ldots \ldots \ldots \ldots \ldots$

41. Surface-sampler activities related to the Physical Properties Investigation during the Extended Mission of Viking Lander $2 \ldots \ldots \ldots \ldots \ldots$

42. Calculated static-bearing forces for Viking footpad from static-bearing-capacity equation for footings - . - . - 203

43. Touchdown conditions and landing data for Landers 1 and $2 \ldots \ldots$

44. Leg strokes, footpad travels, footpad penetrations, and work performed on lander shock absorbers by the engines and footpads for Lander $1 \ldots \ldots \ldots$

45. Leg strokes, footpad travels, footpad penetrations, and work performed on lander shock absorbers by the engines and footpads for Lander $2 \ldots \ldots \ldots \ldots \ldots$

46. Data and derived quantities used to calculate the cohesion from the surface-bearing test in blocky material of Lander $1 \ldots \ldots \ldots$

47. Data and derived quantities used to calculate the cohesion from the surface-bearing test of Lander $2 \ldots \ldots$ 


\section{GLOSSARY AND ACRONYMS}

Acquisition assembly. Part of the surface-sampler subsystem that was used to acquire samples. Assembly consists of the collector-head unit, boom, boom housing, and related drive motors.

Areocentric longitude of the Sun $\left(L_{s}\right)$. The longitude of the Sun on the celestial sphere centered on Mars and measured westward in the orbital plane of Mars from its vernal equinox, in degrees. Zero is the vernal or spring equinox $\left(L_{s}=0\right)$; ninety degrees is the northern summer solstice $\left(L_{s}=90^{\circ}\right)$; one hundred eighty degrees is the fall equinox $\left(L_{s}=180^{\circ}\right)$; and two hundred seventy degrees is the northern winter solstice $\left(L_{s}=270^{\circ}\right)$.

Azimuth. An angle measured clockwise, in degrees, as viewed from above the lander on Mars. Camera and surface-sampler azimuths were measured clockwise in a plane parallel to the upper surface of the lander body. Azimuths on Mars were measured clockwise from north in a horizontal plane.

Backhoe. A metal blade with a serrated tip on the surface-sampler collector head used to excavate trenches by pulling the collector head toward the lander. The backhoe has two circular magnet arrays and wire brushes mounted on it.

Biology Experiment. An experiment designed to detect living organisms on Mars assuming that life on Mars would be similar to life on Earth. The experiment was composed of three separate experiments using three instruments or experimental techniques: Gas Exchange, Carbon Assimilation or Pyrolytic Release, and Labeled Release.

Biology or BIO. An abbreviation and acronym used to indicate the Biology Experiment. They are used on maps and figures and in tables to indicate sample trenches excavated and surface-sampler activities for the Biology Experiment.

Blocky material. A soillike surface material at the Lander 1 site that formed cohesive blocks when disrupted by the surface sampler.

Boom. A tube that has the surface-sampler collector head mounted on its tip. The hollow tube is made of two metal strips, joined by welded seams that collapse to form a ribbon when wound around a drum. The collector head was extended from the lander by unwinding the metal strips from the drum and retracted toward the lander by winding onto the drum.

Boom housing. Part of the surface-sampler acquisition assembly that contains the boom, drum, and drive motors.

Camera aligned coordinate system (CACS). A coordinate system with the origin at the center of the rotation axis of a camera mirror. Azimuths are measured clockwise in a reference plane that is parallel to the upper surface of the lander body. The zero azimuth for camera 1 is a line nominally $9.5^{\circ}$ clockwise of the direction of camera 2; the zero azimuth for camera 2 is a line nominally $5.5^{\circ}$ clockwise of the direction of camera 1 . Elevations are measured in degrees above or below the reference plane.

Carbon Assimilation Experiment. One of three biology experiments designed to detect the synthesis of organic substances by constituents in the surface materials resulting from abiologic processes or biologic processes such as photosynthesis or respiration of $\mathrm{CO}_{2}$. Pyrolytic Release (PR) is another name for this experiment.

Clockwise. A rotation or azimuth direction that is the same as that of the hands of an ordinary clock when viewed from above the lander and, separately, as viewed by a lander camera. For the collector- head rotation about the boom axis, the collector head is viewed from its tip toward the boom housing.

Collector head. A device designed to collect and deliver samples of surface materials to the Viking analytical experiments and to perform other scientific tasks. The collector head, which is part of the surfacesampler acquisition assembly, is attached to the end of the boom. A ball joint allows it to rotate $10^{\circ}$ upward. It has a movable upper jaw actuated by a solenoid, a fixed or lower jaw with a serrated tip, and a hinge-mounted backhoe at its base.

Comminutor. A device in the gas chromatograph-mass spectrometer (GCMS) processor that was used to reduce the sizes of grains of surface materials to less than $300 \mu \mathrm{m}$ for delivery to the GCMS ovens for molecular analyses.

Continuation Mission. The scientific exploration of Mars by the Viking landers and orbiters from 25 May 1978 through 26 February 1979.

Counterclockwise. A rotation or azimuth that is opposite to that of the hands of an ordinary clock when viewed from above the lander and, separately, as viewed by a lander camera. For collector-head rotation about the boom axis, the collector head is viewed from its tip toward the boom housing.

Crusty to cloddy material. A surface material at the Lander 2 site that broke into crusty slabs and clods when disrupted by the surface sampler.

Drift material. A very fine grained surface material at the Lander 1 site that is cross laminated and forms dunelike features. Minor amounts of drift material are present at the Lander 2 site.

Elevation angle. The angle of a vector measured in degrees above or below a plane parallel to the upper surface of the lander body. For the surface sampler, the plane passes through sampler-elevation axis, and the vector is parallel to the axis of the boom (assumed to be rigid). For the cameras, the plane passes through the axes of rotation of the camera mirrors; the vector is the line between the axis of a camera mirror and an object in the field of view.

Extended Mission. The scientific exploration of Mars by the Viking landers and orbiters during the period from 15 November 1976 to 31 May 1978.

Extension. An increase in length of the boom measured in inches or meters from the original position of the collector head upon landing. The term is also used loosely to indicate any increase in length of boom from one position to another.

Fixed jaw. See Lower jaw.

Flight path. The line described by the center of gravity of the lander, as the lander descended to the martian surface during landing.

Flight-path trace. The line described by the vertical projection of the flight path on a plane parallel to the local horizontal.

Footpad. A basal part attached to a leg of the landed spacecraft. The footpad was in contact with the surface. Each lander has three footpads which resemble inverted sailor hats.

Footpad travel. The apparent motion of a footpad from its original position before landing to its final position after landing, as viewed by the lander cameras.

Format 5. A data-retrieval system that obtains currents and voltages from the lander electrical distribution system (bus). Currents come from a variety of sources but those related to the surface-sampler motors could be identified and isolated from other currents. Voltages apply to all motors. 
Furlable boom. See Boom.

Gas chromatograph-mass spectrometer (GCMS). An instrument designed to detect, identify, and measure organic molecules and some inorganic molecules, such as water and carbon dioxide, in samples of surface materials and the atmosphere. The GCMS was the instrument used in the Molecular Analysis Experiment.

Gas Exchange Experiment. One of three biology experiments designed to detect and measure gases. Gases evolved by reactions between constituents in the surface materials and nutrients in an incubation chamber were detected and measured by using a gas chromatograph; gases in the atmosphere were also detected and measured.

GCMS. An acronym used to indicate the Molecular Analysis Experiment and the gas-chromatograph mass-spectrometer instrument. It is used on maps and figures and in tables to indicate sample trenches excavated and surface-sampler activities for the Molecular Analysis Experiment.

Greenwich Mean Time (GMT). Mean solar time at the meridian of Greenwich, England (longitude zero degrees); equivalent to Universal Time. GMT appears in data blocks of lander camera pictures.

Guidance control and sequencing computer (GCSC). Computer that controlled and sequenced all lander operations for separation, deorbit, entry, parachute descent, terminal descent, and landing; computer performed navigation, guidance, and steering of lander from separation to landing; computer controlled and sequenced all lander operations on the surface and received frequent updates of sequences from Earth.

Honeycomb tube-core cartridge. A porous cylinder in each primary strut of the spacecraft that absorbed energy by crushing during landing.

Inertial reference unit (IRU). An instrument on the lander with four gyroscopes and four accelerometers that supplied attitude, velocity, and acceleration information to the guidance control and sequencing computer.

Inorganic Chemical Analysis Experiment. An experiment designed to detect and measure common rock-forming inorganic elements, such as silicon, aluminum, iron, magnesium, calcium, and titanium in the martian surface materials by using an X-ray fluorescence spectrometer (XRFS).

Interim period. The period of time between 26 February 1979 and 19 July 1979 during which both landers and one orbiter continued the scientific exploration of Mars.

Labeled Release Experiment. One of three biology experiments designed to detect biogenic (or abiogenic) reactions between constituents of a surface material and a nutrient by measuring the release of carbon dioxide or carbon monoxide originally in the nutrient.

Lander. A term used to refer to a Viking spacecraft that landed on Mars or to a mockup of the spacecraft.

Lander 1. The Viking spacecraft that landed on Mars 20 July 1976 at Chryse Planitia.

Lander 2. The Viking spacecraft that landed on Mars 3 September 1976 at Utopia Planitia.

Lander aligned coordinate system (LACS). Similar to lander science coordinate system except that the datum plane is $1.1 \mathrm{~m}$ below the upper surface of the lander body and parallel to it.

Lander science coordinate system (LSCS). A coordinate system with an origin at the geometrical center of the prismatic outline of the upper surface of the lander body. Two of three orthogonal axes lie in the plane of the upper surface of the lander body; one axis passes midway between the two cameras $\left(Z_{L}\right)$ and is positive in that direction; the other axis $\left(Y_{L}\right)$ is positive in the direction of footpad 2 . The third axis $\left(X_{L}\right)$, at right angles to the plane of the upper surface, is positive when measured downward.

Leg. One of three structures that support the landed spacecraft. Each leg has one primary strut attached to the lander at the main sup- port fitting and two secondary struts attached to load limiters at the base of the prismatic body of the lander. The three struts converge to support the footpad. The primary strut contains the shock absorber.

Lid. See Movable jaw.

Load limiters. Metal rods between the prismatic base and secondary struts of each lander that absorb shocks in horizontal directions.

Local Lander Time (LLT). The time of a lander event determined from the day of landing ( $\mathrm{Sol} 0$ ) in sols, hours, minutes, and seconds. The hours, minutes, and seconds are reckoned from local martian midnight. Lander 2 Sols are numerically about 44.5 sols smaller than Lander 1 Sols at the same Universal or Greenwich Mean Time.

Lower jaw. The lower part of the collector head. The lower part, with its teeth, forms an integral, immovable base for the upper jaw.

Magnet cleaning brush. A fork with wire brushes on two tines that is attached to the front side of the lander body on the corner opposite the surface sampler. The brush was used to clean the magnets on the backhoe of the collector head.

Main support fitting. A structural member of the lander to which the primary strut is attached.

Microwave atmospheric water detector (MAWD). An absorption spectrometer used to measure the amount of water vapor in the martian atmosphere. A microwave atmospheric water detector was carried aboard each orbiter.

Molecular Analysis Experiment. An experiment designed to measure, detect, and identify hydrocarbon compounds in the surface materials and the isotopic composition of gases in the atmosphere of Mars using a gas chromatograph-mass spectrometer.

Movable jaw. The upper jaw of the surface-sampler collector head that moves and vibrates in response to the collector-head solenoid and a spring.

Orbiter. A term used to designate a Viking spacecraft that remained in orbit after the lander separated from it and landed.

Orbiter 1. The first Viking orbiter to arrive at and orbit Mars. Lander 1 and Orbiter 1 were mated from launch until separation of Lander 1 while orbiting Mars.

Orbiter 2. The second Viking orbiter to arrive at and orbit Mars. Lander 2 and Orbiter 2 were mated from launch until separation of Lander 2 while orbiting Mars.

Physical Properties or PP. Abbreviation and acronym used to indicate the Physical Properties Investigation. They are used on maps and figures and in tables to indicate sample trenches excavated and surface-sampler activities for the Physical Properties Investigation.

Pitch. The angular rotation of the descending spacecraft about its $Y_{L}$ axis; the angle in the $Z_{L}-X_{L}$ plane between the $Z_{L}$ axis and the local horizontal plane.

Pixel or picture element. The pictures or images taken by the lander cameras are composed of arrays of squares. Each square is a pixel or picture element. Each column or vertical scan line has 512 squares or pixels. A series of adjacent scan lines, each one pixel wide, forms the picture. Each pixel contains scene brightness information.

Precipitable micron $(\mathbf{p r} \mu \mathrm{m})$. The amount of water vapor in a vertical column of the atmosphere that would produce a layer of water at the surface 1 micron thick. Ten precipitable microns $(10 \mathrm{pr} \mu \mathrm{m})$ would produce a layer of water at the surface that is ten microns thick.

Primary Mission. The scientific exploration of Mars by the Viking landers and orbiters during the period from arrival of the spacecraft at Mars on 19 June 1976 and ending 15 November 1976.

Primary strut. A structural member of a leg of the lander between the footpad and main support fitting. A crushable honeycomb tube-core cartridge shock absorber is part of the primary strut.

Processor. Part of the surface-sampler subsystem that received and delivered samples to instruments. There are two processors: one for the biology instruments and one for the molecular analysis (GCMS) instrument. 
Propellant tank. Hollow sphere that contained fuel for terminal-descent engines. Each lander has two tanks.

Pyrolytic Release Experiment. Another name for the Carbon Assimilation Experiment. See Carbon Assimilation Experiment.

Radar altimeter. A device that provides altitude data to the guidance control and sequencing computer during descent of the lander to a point $41 \mathrm{~m}$ above the surface.

Radio Science Investigations. Investigations that used radiowaves transmitted from the Earth, landers, and orbiters in a variety of experiments.

Radioisotope thermoelectric generator (RTG). A device that generates electrical power used to charge the lander batteries. Each lander has two RTG's housed beneath two covers.

Reference test chart (RTC). A chart used to calibrate the lander cameras and to detect ultraviolet radiation. Each chart has eleven gray-scale patches, three color patches, three tri-bar patterns, and two patches with paints that darken when exposed to ultraviolet radiation. Each lander has three reference test charts.

Retraction. A decrease in length of the boom from a given position or length.

Retro engine. See Terminal-descent engine.

Rocks. Objects in and on the martian soillike materials that appeared to be strong, hard, and consolidated; they were much stronger than the soillike surface materials. Most of the martian rocks near the landers had diameters from 0.05 to $2.0 \mathrm{~m}$; but, rocks and rock fragments may be smaller than $0.05 \mathrm{~m}$.

Roll. The angular rotation of the descending spacecraft about its $Z_{L}$ axis; the angle in the $Y_{L}-X_{L}$ plane between the $Y_{L}$ axis and the local horizontal plane.

S-band antenna. Radio antenna that receives command data from Earth and transmits lander data to Earth. Radio frequency is about 2.3 $\mathrm{GHz}$ (13-cm wavelength). Each lander has two S-band antennas: the large dish-shaped antenna is high gain and the small cross-shaped antenna is low gain.

Sample field. The area in front of the lander that was defined to be safely accessible to the collector head of the surface sampler. Relaxation of the criteria for safety permitted access to areas adjacent to the sample field.

Sampler aligned coordinate system (SACS). A coordinate system of the surface sampler or acquisition assembly. The azimuths of the boom axis are measured clockwise in a reference plane that is parallel to the upper surface of the lander body from a line that is $10^{\circ}$ clockwise from the (-) $Z_{L}$ direction. The elevation angles of a line parallel to the boom axis are measured in degrees above or below a horizontal plane passing through the elevation axis, which is offset from the azimuth axis. The extension is the increase in length of the boom from its original position upon landing on Mars.

Science Test Lander (STL). A full-scale model of the Viking lander with lander camera and surface-sampler subsystems that was installed adjacent to a large sandbox at the Jet Propulsion Laboratory, Pasadena, Calif., and used during the Viking missions for operational and science testing.

Secondary strut. Part of the lander leg between the primary strut at the footpad and the base of the lander at the load limiters. Each leg had two secondary struts.

Shock absorber. The structure in the primary strut of the lander leg that absorbed energy upon touchdown. Energy was absorbed by honeycomb tube-core cartridges in the primary strut and by load limiters attached to the secondary strut and lander body.

Shroud. A hollow cannister that covered the stowed surface-sampler collector head during launch, cruise, descent to the surface, and landing. Shroud protected the collector head during landing and helped fulfill planetary cleanliness requirements.

Sol. A sol is a mean martian solar day (24.66 hours), but, when capitalized, Sol refers to the Local Lander Time of an event.
Standard temperature and pressure (STP). A standard condition of temperature $\left(0^{\circ} \mathrm{C}\right)$ and pressure $(1$ bar $)$ used to express volumes or amounts of gases.

Stroke. The decrease in length of the primary strut caused by compressing the shock absorber during landing.

Stroke gauge. A rod with quarter-inch bands in the primary strut that moved when the leg stroked against the shock absorber. It was used to measure the stroke of the primary strut.

Surface sampler. Surface sampler is almost equivalent to the acquisition assembly.

Surface-sampler control assembly (SSCA). Controls and handles data for the acquisition assembly and processors.

Surface-sampler subsystem. The components of the lander used to acquire and deliver samples to the instruments of the analytical experiments and to perform other tasks.

Survey Mission I. The scientific exploration of Mars by Viking Orbiter 1 and both landers during the period 19 July 1979 to 5 November 1979.

Survey Mission II. The scientific exploration of Mars by Viking Orbiter 1 and both landers during the period of 5 November 1979 to 7 August 1980.

Survival Automatic Mission. The scientific exploration of Mars by the Viking landers while operating in an automatic mode during the martian winter of the Extended Mission.

Telemetry. The combined processes of measurements, recordings of measurements, and transmission of measurements to Earth.

Terminal-descent engine. A rocket engine used to decelerate descending lander from an altitude of $1.45 \mathrm{~km}$ to touchdown. Each lander has three engines and each engine has 18 small nozzles. Engines were fueled with hydrazine and were permanently shut down at touchdown. Also called retro engine.

Terminal-descent landing radar (TDLR). A four-channel continuous wave doppler radar that measured velocity along the centerline of each of four antenna beams. Provided velocity data to guidance control and sequencing computer during landing.

Touchdown. The moment when, or conditions where, any one of the three footpads of the landers touched the surface during descent of the lander. A small continued downward motion of the lander caused the footpad to stroke the primary strut, which shut down the terminal-descent engines.

Ultra-high frequency antenna (UHF). Radio antenna that transmits lander science and engineering data to orbiter for subsequent relay to Earth. The UHF antenna was used to estimate dielectric constants of surface materials. Radio frequency is $381 \mathrm{MHz}(79-\mathrm{cm}$ wavelength).

Ultraviolet (UV). A term that specifies the wavelengths of radiation to be between 0.2 to $0.4 \mu \mathrm{m}$.

Universal Time (UTC). The mean solar time on the Greenwich meridian, reckoned in days of 24 mean solar hours beginning with 0 hours at midnight. Universal Time is equivalent to Greenwich Mean Time (GMT) in navigational almanacs published after 1925 .

Upper jaw. See Movable jaw.

X-ray fluorescence spectrometer (XRFS). An instrument carried aboard the lander capable of detecting, identifying, and measuring inorganic elements such as silicon, aluminum, iron, calcium, potassium, and titanium. Instrument used by Inorganic Chemical Analysis Experiment.

XRFS. An acronym used to indicate the Inorganic Chemical Analysis Experiment and the X-ray fluorescence spectrometer. It is used on maps and figures and in tables to indicate sample trenches excavated and surface-sampler activities for the Inorganic Chemical Analysis Experiment.

Yaw. The angular rotation of the descending spacecraft about its $X_{L}$ axis; the angle in the $Z_{L}-Y_{L}$ plane between the $Z_{L}$ axis and the vertical plane that includes the flight path. 



\title{
PHYSICAL PROPERTIES OF THE SURFACE MATERIALS AT THE VIKING LANDING SITES ON MARS
}

\author{
By H. J. Moore, R. E. Hutton, G. D. Clow, and C. R. Spitzer
}

ABSTRACT

This report summarizes the results of the Physical Properties Investigation of the Viking ' 75 Project, activities of the surface samplers, and relevant results from other investigations. The two Viking Landers operated for nearly four martian years after landing on July 20 (Lander 1) and Sept. 3 (Lander 2), 1976; Lander 1 acquired its last pictures on or about Nov. 5, 1982.

Lander 1 rests on a smooth, cratered plain at the west edge of Chryse Planitia $\left(22.5^{\circ} \mathrm{N}, 48.0^{\circ} \mathrm{W}\right.$ ), and Lander 2 rests $200 \mathrm{~km}$ west of the crater Mie in Utopia Planitia $\left(48.0^{\circ} \mathrm{N}, 225.7^{\circ} \mathrm{W}\right)$. Lander 1 views showed that dunelike deposits of drift material were superposed on rock-strewn surfaces. Soillike material from the rock-strewn areas was called blocky material. Lander 2 views also showed a rock-strewn surface. Polygonal to irregular features, etched by the wind, revealed crusty to cloddy material among rocks.

Both landers descended to the surface along nearly vertical trajectories. Velocities at touchdown were about $2 \mathrm{~m} / \mathrm{s}$ for both landers. Footpad 2 of Lander 1 penetrated drift material $0.165 \mathrm{~m}$, and footpad 3 penetrated blocky material $0.036 \mathrm{~m}$. The two visible footpads of Lander 2 struck rocks. Erosion by exhausts from the forward engines produced craters with rims of mixed fine-grained material and platy to equidimensional clods, crusts, and fragments. Comparison of engine-exhaust erosion on Mars with terrestrial data suggested that drift material behaved like a weakly cohesive material with a grain size less than 3-9 $\mu \mathrm{m}$. Although not sand, blocky and crusty to cloddy materials eroded like sand-with grain sizes of 0.01 or $0.2 \mathrm{~cm}$.

The surface samplers accomplished an impressive number of tasks. All experiments that required samples received samples. Deep holes, as much as $0.22 \mathrm{~m}$ deep, were excavated by both landers. Lander 2 successfully pushed rocks and collected samples from areas originally beneath the rocks. Tasks specifically accomplished for the Physical Properties Investigation include: (1) acquiring motor-current data while excavating trenches, (2) performing surface-bearing tests, (3) performing backhoe touchdowns, (4) attempting to chip or scratch rocks, (5) comminuting samples, (6) measuring subsurface diurnal temperatures, and (7) constructing conical piles of materials on and among rocks.

Sample trenches in the three major types of soillike materials were different from one another. Trenches in drift material, which were typically $0.06 \mathrm{~m}$ deep, had steep walls along much of their lengths, lumpy tailings and floors, and smooth domed surfaces with sparse fine fractures around their tips. Trenches in blocky material, which were typically 0.03-0.04 m deep, had steep walls near their tips, and surfaces around their tips were displaced upward and some appeared blocky. Trenches in crusty to cloddy material, which were typically $0.04-0.05 \mathrm{~m}$ deep, had steep and often irregular slopes near their tips, clods and slabs of crust in their tailings, and disrupted areas around their tips composed of mixed fine-grained material and slabs of crust or thick polygonal clods that had been displaced upwards.

Data acquired during landing, trenching, surface-bearing tests, backhoe touchdowns, and from other science experiments were used to determine the mechanical properties of drift, blocky, and crusty to cloddy materials. Drift material appeared to be very fine grained, with local planes of weakness; in general, the drift material was consistent with a material having an angle of internal friction about $18^{\circ}$, a cohesion ranging from 0.7 to $3.0 \mathrm{kPa}$, and a bulk density of $1,200 \mathrm{~kg} / \mathrm{m}^{3}$. Blocky material was consistent with a material having an angle of internal friction about $30^{\circ}$, cohesions from 1.5 to $16 \mathrm{kPa}$, and a bulk density of 1,600 $\mathrm{kg} / \mathrm{m}^{3}$. Crusty to cloddy material had variable properties. For chiefly crusty to cloddy material, angles of internal friction were about $35^{\circ}$, and cohesions were from 0.5 to $5.2 \mathrm{kPa}$. For mixed fines and crusts, angles of internal friction were about $31^{\circ}$, and cohesions were from 0.2 to $2.3 \mathrm{kPa}$. A bulk density of $1,400 \mathrm{~kg} / \mathrm{m}^{3}$ was plausible for Lander 2 soillike materials. Samples of a coarse fraction $(0.2-1.2 \mathrm{~cm})$ were acquired by Lander 1, but not by Lander 2 . Comminutor motor currents indicated that there were no strong coarse fragments in drift or crusty to cloddy materials. Very large comminutor motor currents indicated that significant amounts of millimeter-size fragments of metal oxides, silicates, or strong clodlets must have been present in blocky material. Very little was learned about the rocks. The rocks did not have weak, friable, weathered rinds because none of the rocks were chipped, spalled, or scratched when pushed or scraped by the sampler.

Diurnal temperatures were measured with the sampler collector heads of both landers while immersed in the surface materials during the winter. Lander 2 also made measurements during the late winter. Winter maxima and minima were 215.4 and $187.6^{\circ} \mathrm{K}$ (Lander 1 ) and 170.5 and $146.5^{\circ} \mathrm{K}$ (Lander 2). The maximum late winter curves of Lander 2 were truncated with constant temperatures of $217^{\circ} \mathrm{K}$; the truncation may have been the result of a phase change in eutectic compositions of $\mathrm{H}_{2} \mathrm{O}$ $\mathrm{H}_{2} \mathrm{O}_{2}$ or $\mathrm{CaCl}_{2}-\mathrm{MgCl}_{2}-\mathrm{H}_{2} \mathrm{O}$ brine.

Changes that occurred at the landing sites included small natural slope failures of drift material, deposition of a thin veneer of fine-grained material in the sample fields, wintertime "snowfalls" at the Lander 2 site, darkening of paints that were sensitive to ultraviolet radiation, and movement of martian surface materials on the lander. Some conical piles of surface materials in the sample fields were unchanged after two martian years. They were eroded much later during a duststorm of the third winter. Lander 2 rotated $0.5^{\circ}$ counter-clockwise and settled downward $8 \mathrm{~mm}$ in small increments by the end of 1.76 martian years.

Other Viking experiments provided information on the surface materials. Data from the Gas Exchange Experiment suggested that drift material had grain sizes ranging from 0.1 to $2.0 \mu \mathrm{m}$. Results from the Labeled Release Experiment were equivocal and consistent with (1) life on Mars, (2) the presence of $\mathrm{Fe}$ - or $\mathrm{H}_{2}$-rich montmorillonite-type clays, or (3) the presence of silica sand, $\mathrm{H}_{2} \mathrm{O}_{2}$, and $y-\mathrm{Fe}_{2} \mathrm{O}_{3}$. Results from the Pyrolytic Release Experiment were equivocal, but compatible with Fe-rich montmorillonite-type clays and $\gamma-\mathrm{Fe}_{2} \mathrm{O}_{3}$. Crude estimates of water and $\mathrm{CO}_{2}$ in some samples were determined with the gas chromatograph-mass spectrometer. The compositions measured by the X-ray fluorescence spectrometer were consistent with a mixture dominated by iron-rich clays or their degradation products. Bulk densities of the fine fraction in the X-ray fluorescence spectrometer analysis chamber were about $1,150 \mathrm{~kg} / \mathrm{m}^{3}$; individual fragments of the coarse fraction may have had densities as large as $1,880-2,300 \mathrm{~kg} / \mathrm{m}^{3}$. The magnetic 
fraction may have been thin grain coatings or admixed crystallites of 1-7 percent $\gamma-\mathrm{Fe}_{2} \mathrm{O}_{3}$. According to the Radio Science Investigations, the dielectric constant of the Lander 1 site was $3.3 \pm 0.7$; the low value of the Lander 2 dielectric constant was 2.8 . Thermal inertias from orbital data were $9 \pm 0.5$ and $8 \pm 1.5$ (in $10^{-3} \mathrm{cgs}$ units) for the Landers 1 and 2 sites, respectively. Water vapor was saturated in the atmosphere at the sites, and exchange between the surface and atmosphere appeared to occur seasonally.

\section{INTRODUCTION}

The Physical Properties Investigation and Magnetic Properties Investigation were given the lowest priorities of the Viking ' 75 Project because the goal of the Viking missions was the exploration of Mars with special emphasis on the search for life (Soffen, 1977). Because of this priority, the use of the surface sampler was given first to the Biology Experiment, next to the Molecular Analysis Experiment, then to the Inorganic Chemical Analysis Experiment, and finally to the Physical and Magnetic Properties Investigations. Certain important experiments were conducted near the very end of the surface-sampler activities on Mars.

Sources of data to be used by the Physical Properties Investigation were administratively constrained in the Viking '75 Project Mission Definition (U.S. Natl. Aeron. and Space Adm., 1970, p. 32) which states:

"This investigation is to be performed using available data such as those from the lander imaging system, the surface sampler and engineering sensors. Physical properties will be derived from these data. Only instrumentation for engineering measurements and other scientific investigations will be provided for this investigation. This investigation will be planned to maximize the use of the engineering measurements, the use of the surface sampler, and the use of the imaging system to obtain physical properties of the surface without interfering with the primary objectives of the devices used to accumulate the data."

Because of the Mission Definition, no instruments were specifically designed for or allotted to the investigation, nor were engineering and scientific measurements specifically designed to fulfill the goals of the investigation. Thus, interpretations of the engineering data and results from other experiments are markedly constrained by the nature of the instruments, measurements, and experiments.

Although the Mission Definition was partly restrictive, it was also partly unrestrictive because it required the examination of many sources of information. In fact, data relevant to the investigation were obtained beginning with the landing and the first pictures of the surface taken $25 \mathrm{~s}$ after landing and, then continuously throughout the Primary and Extended Missions. Four items were also placed on the spacecraft for the investigation. Two mirrors were placed on the surface-sampler boom housing to enhance viewing under the number 2 terminal-descent engines (retro engines) and to view the temperature sensors on footpad 2. A magnifying mirror was placed near the main reference test chart. Chips coated with paints that darken when illuminated by ultraviolet radiation were placed on the reference test charts.

Specific goals of the Physical Properties Investigation were briefly to deduce: (1) bearing strengths, (2) cohesions, (3) angles of internal friction, (4) porosities (bulk densities), (5) thermal properties, (6) grain-size distributions, (7) adhesions, and (8) heterogeneities of the surface materials (Shorthill and others, 1972). Experiments, observations, and deduced physical properties, grouped by principal data sources were: (1) spacecraft parts, (2) surface sampler, (3) results of other investigations, and (4) natural features and conditions (see table 1).

\section{VIKING MISSIONS}

Performances of the Viking spacecraft were so remarkable that operations continued 4 Mars' years after the first arrival at Mars and Viking Lander $1^{1}$ continued to acquire pictures and collect additional data until November 19 , 1982. After the Primary Mission, additional missions were defined and accomplished (Snyder, 1979) because of the remarkable performances (table 2). The Primary Mission began with the arrival of the mated Lander 1 and Orbiter 1 pair at Mars. Original plans to land at the preselected landing site on 4 July 1976 were changed because the site appeared to be too hazardous (Soffen, 1977). Viking Orbiter 1 pictures revealed an apparently rough rocky eroded surface that was not visible in preViking pictures taken by Mariner 9 because the site was obscured by atmospheric dust. In addition, echoes from Earth-based radio transmissions were so effectively scattered by the roughness elements of the surface that very weak to nonexistent echoes were received on Earth from the site (Tyler and others, 1976). After considerable search to the northwest, the final site was chosen because broadening of the radio echoes indicated acceptable surface roughness at the scale length of the spacecraft, the reflection coefficient appeared to be average for Mars, and the pictures of the surface from orbit indicated the presence of substantial amounts of fine-grained surface materials. Lander 1 landed on 20 July 1976-7 years after the landing of Apollo 11 on the Moon. Subsequently, on 3 September 1976, Lander 2 landed successfully.

${ }^{1}$ On 7 January 1981, Lander 1 was redesignated as the Thomas A. Mutch Memorial Station in honor of the NASA Administrator, Viking Lander Imaging Team Leader, scientist, and educator. With all due respect, Lander 1 will be used throughout most of this paper. 
For the landers, the Primary Mission was nearly a complete success (Cooley and Lewis, 1977). Excellent pictures were acquired throughout the mission, and these included pictures of spacecraft parts and sample trenches. All experiments that required samples did receive and analyze samples, and even the Physical Properties and Magnetic Properties Investigations had their days on Mars (Lander 1, 31 August 1976; Lander 2, 31 October and 1 November 1976). Surface-sampler activities were highlighted by successful attempts to push rocks and by acquiring samples from areas originally beneath the rocks (Moore and others, 1978). All the science experiments were successfully conducted except for the failure of the seismometer of Lander 1 to uncage.

During the period of solar conjunction beginning in November 1976, the Extended Mission activities of the landers consisted of the automatic acquisition of pictures and science data which were stored on the spacecraft tape recorders. Severely curtailed communications during the conjunction period required automatic operations of the landers. Surface-sampler operations resumed after the conjunction in mid-January 1977. By the end of May 1977, the Biology and Molecular Analysis experiments had terminated, and the samples for inorganic chemical analyses were acquired from January 1977 to 8 May 1978. During the Extended Mission, many experiments were performed by the surface samplers for the Magnetic and Physical Properties Investigations. These included magnetic mineral abundance tests accompanied by cleaning of the collector-head backhoe-magnets, comminuting selected samples, constructing conical piles of surface materials, surface-bearing tests, and other experiments. A major accomplishment of the surface sampler was the excavation of three deep holes. About 6 hours of operating time over periods of 18 to 31 martian days (sols) were required to excavate each hole. Surface-sampler activities were not continuous. The surface-sampler of Lander 2 did not operate in the martian winter for about 5 months during the Survival Automatic Mission. Lander 1 had a similar but shorter period of automatic operation. The

TABLE 1.-The principal sources of data for the Physical Properties Investigation

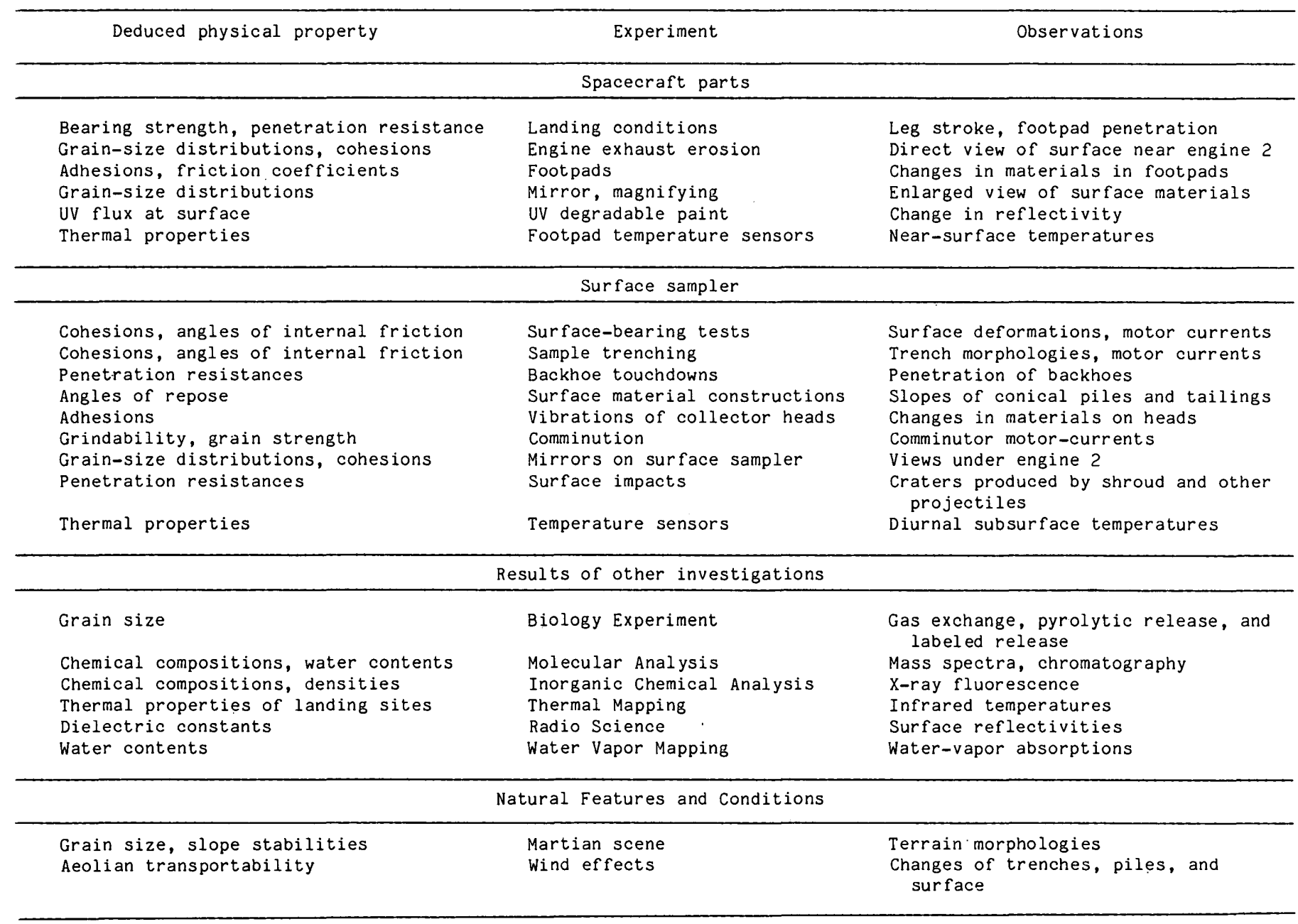


Lander 2 seismometer was turned off at the end of the Extended Mission.

During the Continuation Mission, lander activities were confined to continued acquisition of pictures, meteorology data, and engineering data.
Operation of the surface sampler of Lander 2 in May 1978 was a highlight of the interim period. The sampler executed all the planned sequences, obtained wintertime diurnal temperatures with the collector-head sensor immersed in the surface materials, and positioned its col-

TABLE 2.-Chronology of Viking Lander 1 and 2 missions

[VL means Viking Lander; VO means Viking Orbiter. Sol is the day number of the event (martian days) for each lander; day of landing is Sol 0]

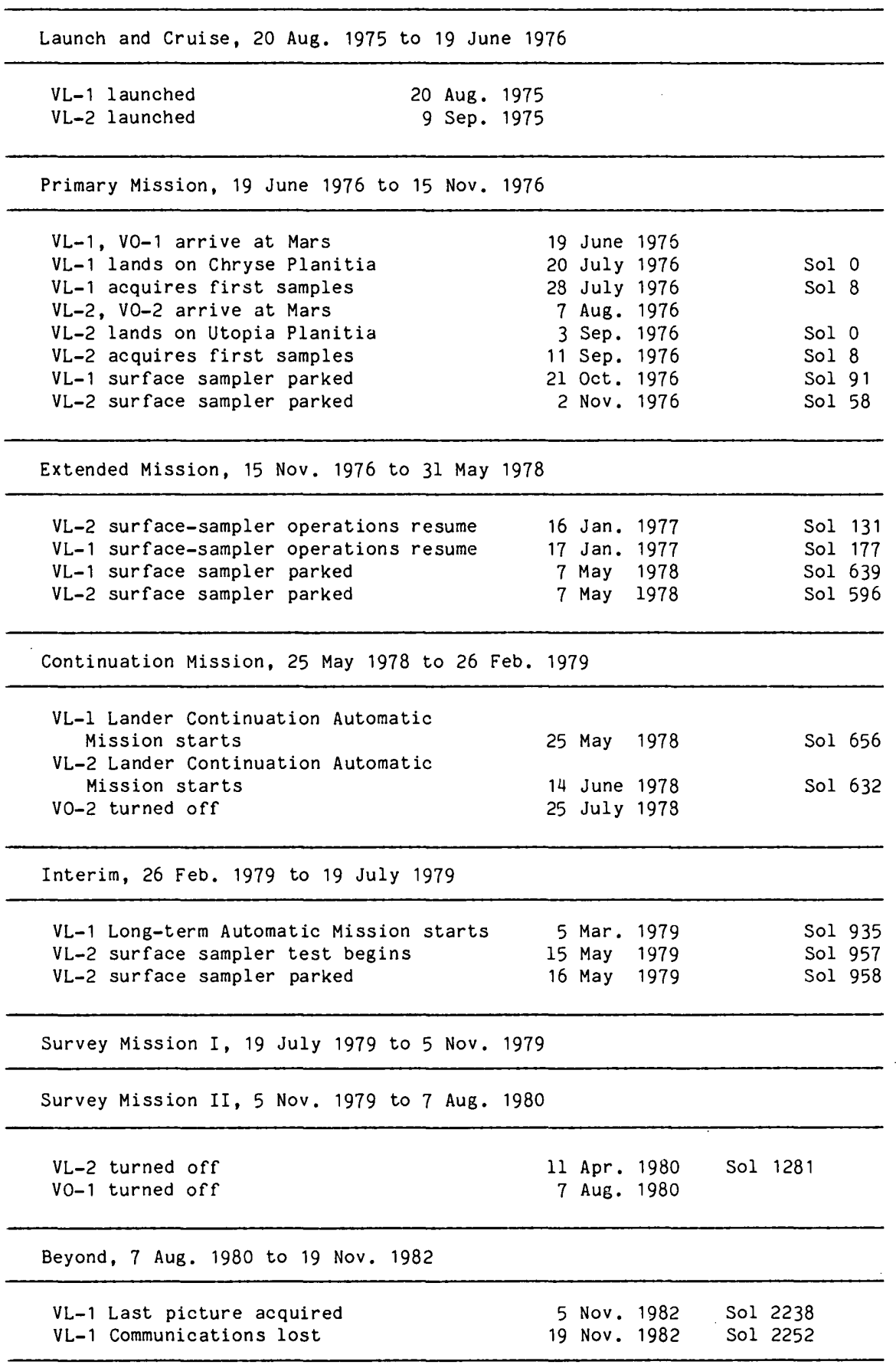


lector head for the very first stereoscopic pictures of the laden magnets on the backhoe.

Both Lander 1 and Lander 2 continued taking pictures and collecting meteorology data during subsequent missions. Orbiter 2 was turned off 25 July 1978, Lander 2 was turned off 11 April 1980, and Orbiter 1 was turned off 7 August 1980. Lander 1 was earlier programmed to automatically acquire pictures, meteorology data, and engineering data until December 1994; but, unfortunately, communications were lost in November 1982. The Mutch Memorial Station (Lander 1) furnished exciting data on a significant windstorm before contact was lost. It was interesting to learn what this lone surviving Viking explorer saw and felt on Mars.

\section{SUPPLEMENTAL SECTIONS}

Six supplemental sections in this paper contain specialized information and data. The first five sections will be of interest to some readers but not to the general reader. The sixth section may be of interest to all readers. These sections, which appear after the references, are: (1) Measurements and coordinates, (2) Summary of surface-sampler activities, (3) Outline maps, profiles, and data on trenches, (4) Analysis of landing data, (5) Analyses of sample trenches and surface-bearing tests, and (6) Names of areas in the sample fields and rocks. The first section provides procedures and equations that may be used by readers who wish to measure Viking lander pictures and use surface-sampler coordinates in order to gain a firsthand knowledge of the sample fields of the landers. The second section contains a chronological summary of the activities of each surface sampler that are relevant to the Physical Properties Investigation; this section contains data on the samples used in analyses, Physical Properties experiments, and other activities, as well as surface-sampler coordinates for each activity. The third section contains outlines and profiles of trenches obtained from measurements of pictures taken with the lander cameras, frame numbers of pictures that include the trenches, and selected surface-sampler motor-current records; this section will interest readers who want to know more about the acquisition of specific samples or Physical Properties experiments. The fourth section contains the details of the analysis of the landing data necessary for the specialized reader but unnecessary for the general reader. The fifth section contains a description of the procedures used in the analyses of trenches and surface-bearing tests in order to estimate angles of internal friction and cohesions of the surface materials of Mars; this section provides the level of detail necessary for interested and specialized readers to understand our methods. The sixth and final section gives the reasons for naming areas in the sample fields and rocks, the rationales for the names, and the names.

\section{ACKNOWLEDGMENTS}

Many individuals contributed to the success of the Viking Missions. James S. Martin (Project Manager), Thomas A. Young (Mission Director), and G.A. Soffen (Project Scientist) of National Aeronatuics and Space Administration (NASA) Langley Research Center (LRC) were largely responsible for the successes of the mission along with the management of the Martin Marietta Corp. (MMC), Denver, Colo. G.C. Broome (LRC, Mission Director, Extended Mission) and C.W. Snyder (Project Scientist, Extended Mission) of the Jet Propulsion Laboratory (JPL), Pasadena, Calif., were responsible for the host of experiments for the Physical Properties Investigation which were admirably carried out despite a substantial reduction of resources. The contributions of D.S. Crouch (MMC), who was in charge of the design of the surface sampler, were necessary to the entire success of the Viking lander missions. Drs. R.W. Shorthill (University of Utah Research Institute) and R.F. Scott (California Institute of Technology) were active members of the Physical Properties Investigation during the planning phases and Primary Mission. Elizabeth M. Dowey, formerly of the U.S. Geological Survey, Menlo Park, Calif., helped prepare maps and profiles of trenches using stereometric measurements on Viking pictures. We also wish to acknowledge the contributions of the Surface Sampler Science Team (J.S. Hubbard, University of Georgia; D.A. Flory, University of Houston; James Gliozzi, MMC; Sid H. Liebes, Lander Imaging Team; J.F. Ellzey, Kennedy Space Center; V.P. Gillespie, LRC; Al Britting, MMC; Don Macumber, MMC; and J. Timbrook, MMC), and the Surface Sampler Team (D.S. Crouch, MMC; K.Z. Bradford, MMC; R.D. Grossart, MMC; L.V. Clark, NASA-LRC; D.D. Pike, MMC; P.M. Coulson, MMC; J.P. Yarbrough, student intern; L.K. Schwab, MMC; and J. Kaehler, MMC). Sid H. Liebes, Jr., Stanford University, kindly furnished topographic data during the missions as well as later; R.B. Hargraves, Princeton University, assisted in planning surface-sampler sequences during the Primary Mission; P.M. Cates (JPL) was the operations representative of the Physical Properties Investigation during the Extended Mission; and D. Pidek (JPL) and C.E. Carlston (MMC) planned picture sequences for us. Dr. Edward McKyes, McGill University, reviewed our procedures for estimating angles of internal friction and cohesions. Joseph C. Pohlen, D.S. Crouch, and K.Z. Bradford of MMC, kindly reviewed parts of this paper. 


\section{MISSION DESCRIPTION AND CONVENTIONS}

\section{VIKING LANDER}

The body of each lander is a hollow hexagonal aluminum prism supported on the surface by three legs with footpads. Within the body are the spacecraft computer, battery power, surface-sampler control, thermal control, science instruments, and data handling systems. Three terminal-descent (retro) engines, two propellant tanks, three legs, the surface sampler, and inertial reference units are mounted on the six vertical sides of the prismatic body (fig. 1). Two facsimile cameras, two covered radioisotope thermoelectric generators (RTGs), sample processors and delivery systems, a magnifying mirror, a seismometer, and a photographic reference test chart (with magnets and two rectangular squares painted with ultraviolet sensitive paints) are mounted on the upper surface of the prismatic body. A rectangular grid is painted on this upper surface and two additional photographic reference test charts are mounted on the RTG covers. A footpad is mounted on each of the three legs, and one of these footpads has temperature sensors mounted on it. Three radio antennas provided communications between the lander and Earth (high- and low-gain S-band) and between the lander and orbiters (UHF). Two radar units (radar altimeter and terminal-descent landing radar) and the inertial reference unit (IRU) provided velocity, attitude, elevation, and control data for the automatic landings on Mars as well as science data. The mass of the entire landed spacecraft is $611 \mathrm{~kg}$.

The various components of the landers of special interest to the Physical Properties Investigation were: (1) spacecraft parts, (2) surface-sampler subsystem, and

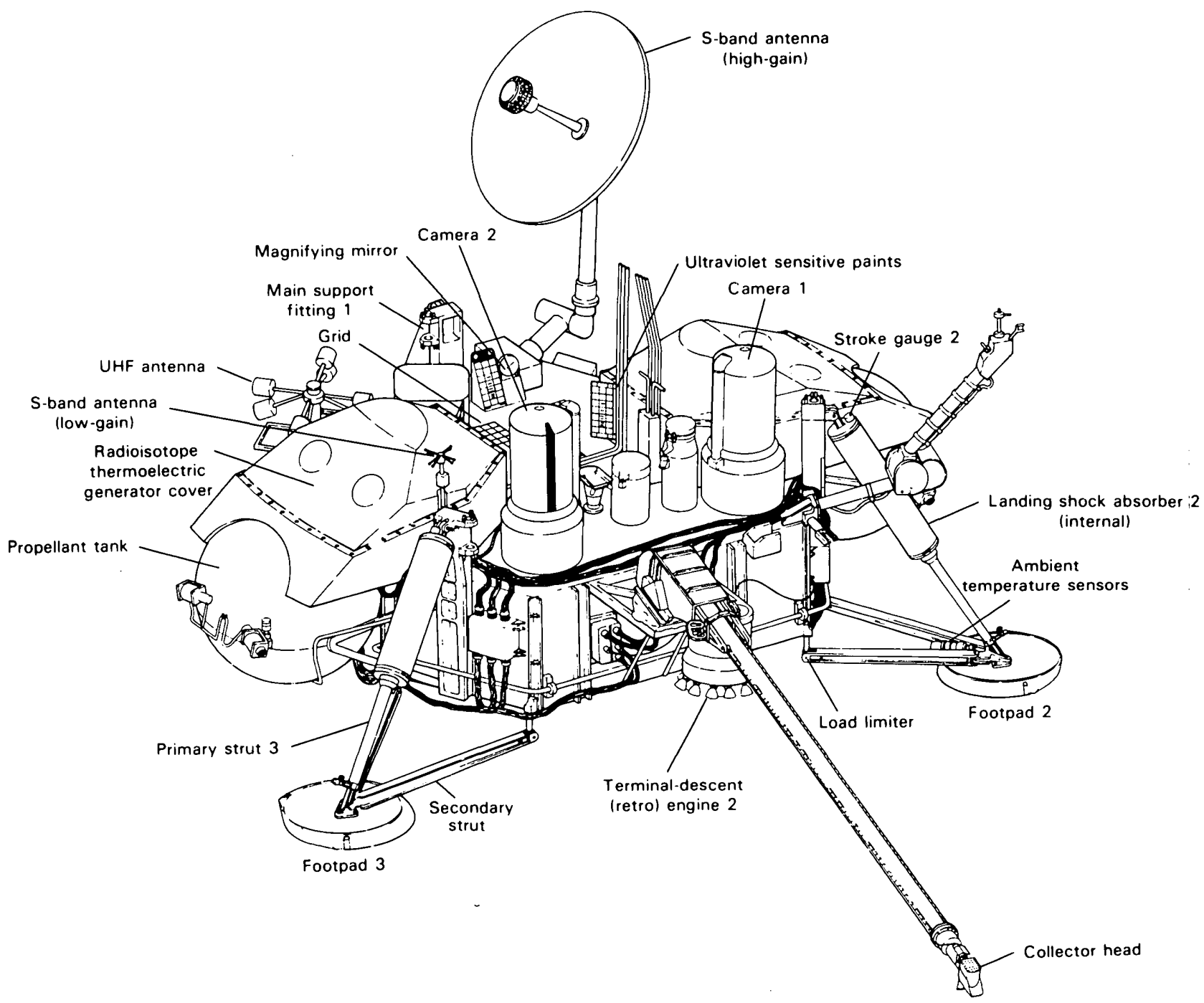

FIgURE 1.-Viking lander showing locations of spacecraft parts important to landing and other selected components. Radar altimeter and terminal-descent landing radar are mounted beneath lander body. 
(3) science instruments (for other investigations). These are briefly described below.

\section{SPACECRAFT PARTS}

The terminal-descent engines (along with pictures of the response of the surface materials to their exhaust gases) and the footpads (along with their penetration into the surface materials and the stroking of the legs against the shock absorbers), provided the first information available on the physical properties of the surface materials (fig. 1). Other spacecraft parts such as mirrors and temperature sensors provided information later.

Firing of the terminal-descent engines began about 1.4 $\mathrm{km}$ above the surface after parachute separation. Constant velocity and vertical descent were achieved about $16.8 \mathrm{~m}$ above the surface. Velocity was maintained at about $2.4 \mathrm{~m} / \mathrm{s}$ with little translation until touchdown. At touchdown, the stroking of any one of the three legs permanently turned off the engines. Each engine developed a peak thrust about $685 \mathrm{~N}$ by expelling gases through 18 individual nozzles that distributed the gas pressures on the surface $\left(2.3 \mathrm{kN} / \mathrm{m}^{2}\right.$ and less). Eighteen nozzles on each engine were required to eliminate the excessive erosion that would have been produced by one large nozzle (Hutton and others, 1980). Only the surface near the forward or number 2 engine could be seen from the landed spacecraft. Parts of the surface in front of engine 2 could be viewed directly with the cameras; camera 1 viewed the left side and camera 2 viewed the right side. The eroded surface beneath and to the right side of engine 2 was also viewed, indirectly, with camera 2 and a suitably positioned mirror (number 2) on the right side of the boom housing of the surface sampler. The response of the martian surface materials to the engine exhausts could be compared with the response of surface material simulants used in terrestrial tests of the engines in an atmosphere like that of Mars.

At landing, forces and energies were absorbed, chiefly, by the spacecraft shock absorbers and penetration of the footpads (fig. 2) into the surface materials. The crushable, honeycomb tube-core cartridges of the shock absorbers collapsed as the primary struts moved upward. The amounts of stroke or movement were measured by gauges, which are simple rods that extend above the shock absorber housing, and also by footpad travel. Each leg has a gauge or rod with alternating black and white bands $0.64 \mathrm{~cm}(0.25 \mathrm{in}$.) wide. Displacements of the rods from their normal pretouchdown positions were measures of leg strokes. Travel or displacements of footpads in the camera pictures were also measures of leg strokes. Forces as a function of stroke are known. Penetration of the two visible footpads (2 and 3 ) were estimated from pictures. Footpad 2 could be seen directly by camera 1 and indirect- ly by a mirror (number 1 ) on the left side of the boom housing of the surface sampler (fig. 1). Footpad 3 could be viewed directly by camera 2 . Uncertainties in the estimates of footpad penetrations depended chiefly on the shape of the footpad and the amount of penetration. Footpad 1 could not be viewed. The penetration of footpads 2 and 3 and the stroke of the legs could be compared with similar data obtained in terrestrial tests.

The remainder of the spacecraft parts (fig. 1) provided information later and continually throughout the mission. The magnifying mirror was used to obtain enlarged views of materials on the tip of the surface-sampler collector head and of magnetic materials attracted to the magnets on the backhoe of the collector head. The grid provided a means of establishing sizes, amounts, and movement of dust and martian surface materials on the upper surface of the lander body. The squares painted with UV sensitive paints were darkened by exposure to UV radiation and were in view of the cameras. The ambient temperature sensors mounted on footpad 2, which were used for science investigations during entry of the spacecraft, were monitored after landing and supplied temperature data throughout the landed phases of the missions. Accuracy
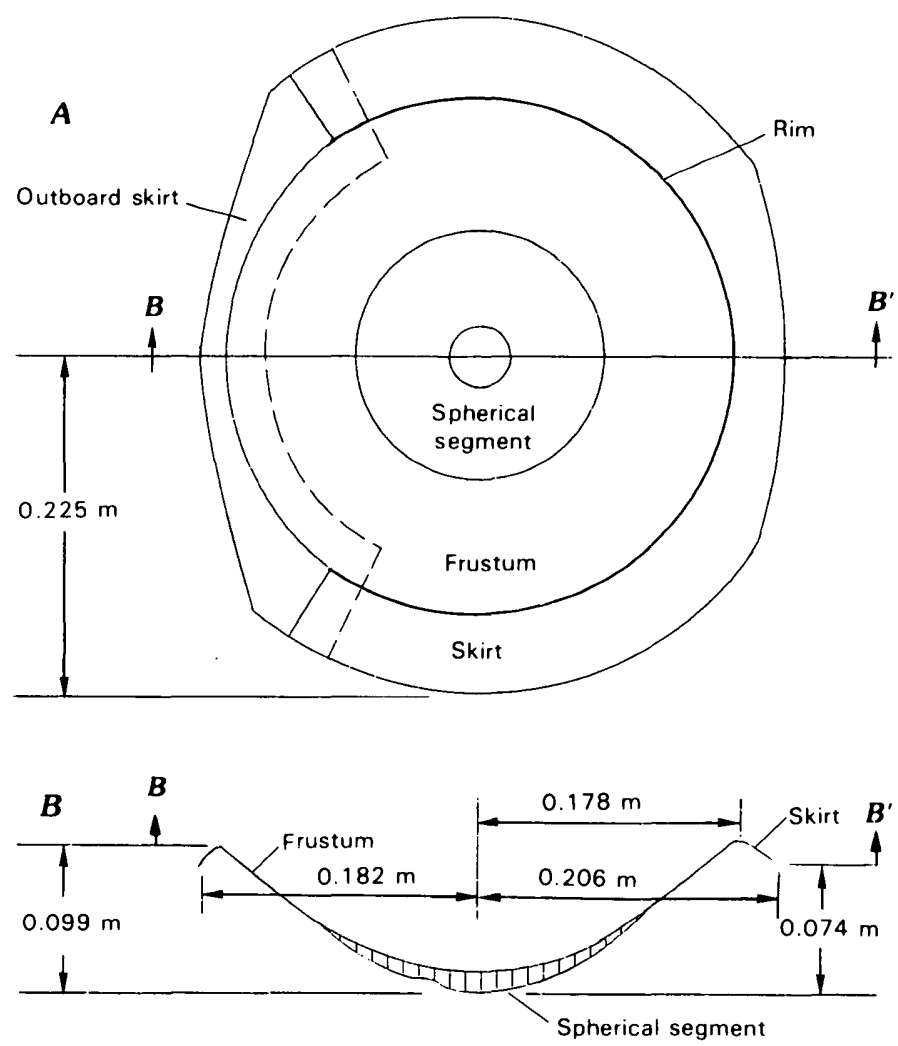

FiguRE 2.-Footpad of Viking lander. Each lander has three footpads, two of which can be seen in frontispiece. Footpad is an inverted frustum of a cone capped by a spherical segment with an irregular skirt around base of frustum. $A$, Top view. $B$, Section B-B'. 
of the thermocouple sensors is $1.3^{\circ} \mathrm{K} \pm 2.1$ percent of the reading over the range from $100^{\circ}$ to $400^{\circ} \mathrm{K}$. Additional information such as adhesions, friction coefficients, and grain sizes were obtained by monitoring pictures of leg struts, footpads, and other spacecraft parts throughout the missions.

\section{SURFACE-SAMPLER SUBSYSTEM}

The surface-sampler subsystem had four major components (fig. 3): (1) acquisition assembly, (2) gas chromato- graph-mass spectrometer (GCMS) sample processor, (3) biology sample processor, and (4) surface-sampler control assembly (SSCA) (Crouch, 1977). These four components are briefly discussed below.

Each acquisition assembly, which is mounted on a bracket located on the right-front side of the lander, is made up of a shroud unit, a boom-housing unit, an azimuth-drive unit, an elevation-drive unit, an extendretract drive unit, a furlable boom, and a collector-head unit. The shroud unit is a canister attached to the boom

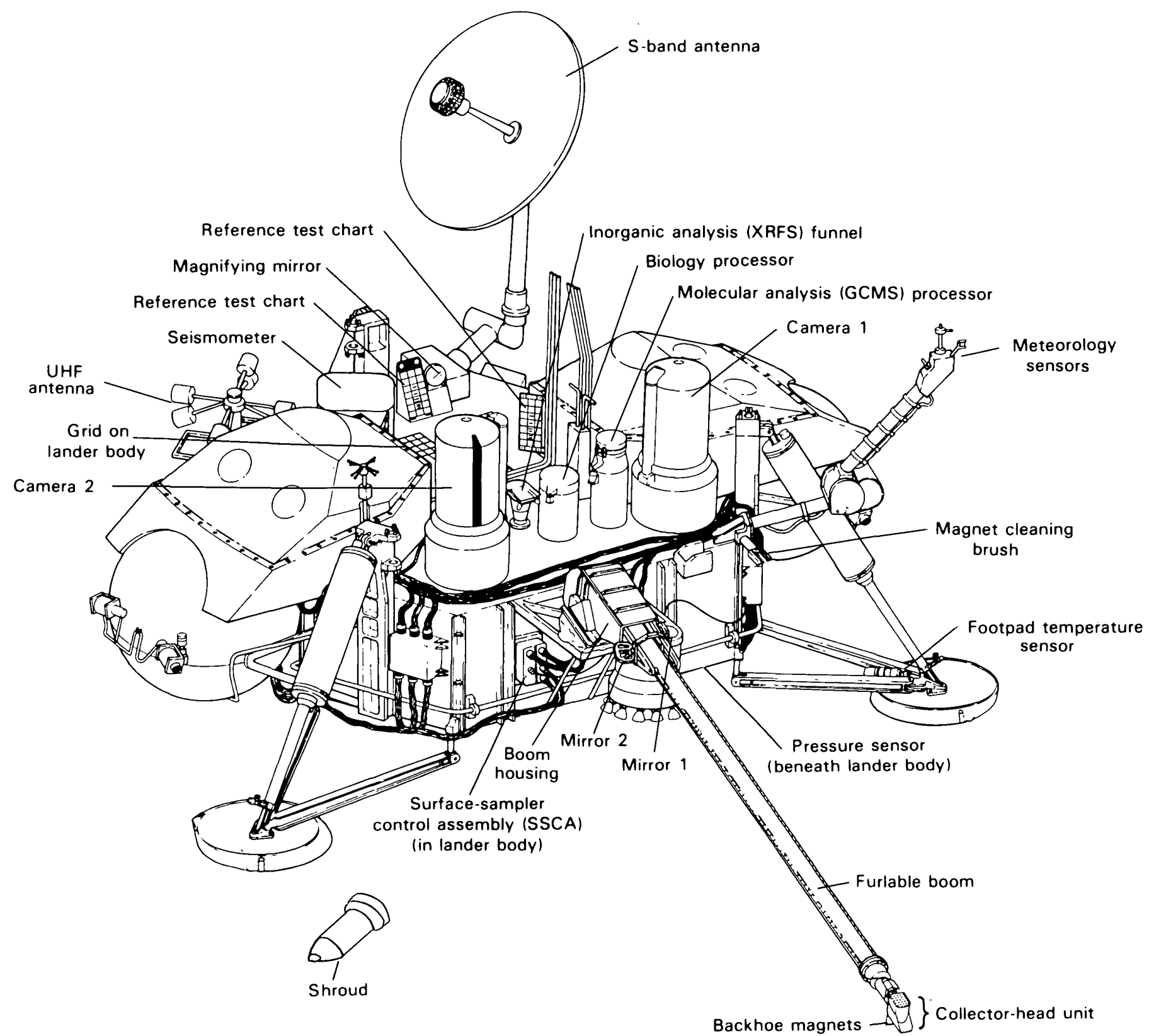

Figure 3.-Viking lander showing locations of visible parts of components of surface-sampler subsystem and science experiments. Analytical instruments are mounted within the prismatic body; sample processors and delivery ports are labeled. All three biology instruments used the

same processor. Pressure sensors and inertial reference unit are not visible. Guidance control and sequencing computer and surface-sampler control assembly are mounted internally. Azimuth, elevation, and extend-retract drive units are located in boom housing. 
housing that covered the collector head through prelaunch check out, launch, cruise to Mars, descent, and landing. The shroud was required to ensure that requirements for cleanliness in interplanetary exploration were met and to protect the collector head during landing. After landing, the 478-g shroud was ejected by springs so as to impact the surface near footpad 3. Tests using high speed photography and flight-type hardware indicated that the ejection velocity was $3.2 \mathrm{~m} / \mathrm{s}$. The boom housing contains the furlable boom and its drum, the elevation-drive unit, and the extend-retract drive unit. Two mirrors (not shown in fig. 3) are mounted on the boom housing so that the surfaces beneath the number 2 engines and the temperature sensor on footpad 2 could be viewed in the mirrors after suitably positioning the boom housing in azimuth and elevation. The azimuth-drive unit is located beneath the boom housing, and its motor drives the housing through an azimuth of $228^{\circ}$ at a rate of $1.8^{\circ} \mathrm{s}$. A separate motor of the elevation-drive unit in the boom housing drives the boom housing in elevation through $74^{\circ}$ at a rate of $1.5^{\circ} \mathrm{s}$. The elevation axis is offset $2.54 \mathrm{~cm}$ from the azimuth axis. Outward (extension) and inward (retraction) motion of the collector-head unit is accomplished by a motor that unwinds and winds the furlable boom, which is wrapped around a drum. When wrapped around the drum, the two metal strips of the boom, joined by welded seams, are flattened against each other. When extended beyond the housing, the two metal strips assume a tubular form in cross section giving the boom strength. The maximum extension, or increase in distance of the collector-head unit from the housing, is $3.45 \mathrm{~m}$, and rates of extension and retraction are $2.54 \mathrm{~cm} / \mathrm{s}$. Displacement of the longitudinal axis of the boom is $0.215 \mathrm{~m}$ above the elevation axis. The collector-head unit (fig. 4) is attached to the end of the boom with a collar. Each collector-head unit consists of a lower fixed jaw with a serrated tip for digging into the surface and a bucket-shaped upper movable jaw. Power to a solenoid rotates the movable jaw upward to open the collector head for sample acquisitions; this rotation acts against springs that close the collector head when power to the solenoid is shut off. The upper part of the movable jaw has $2.0-\mathrm{mm}(2000 \mu \mathrm{m})$ holes in it in order to separate the sample into two size fractions. Separation was accomplished by a motor in the collector-head unit that inverts the collector head by rotation about the longitudinal axis through angles as much as $180^{\circ}$ and then vibrates the bucket-shaped movable jaw at 4.4 or $8.8 \mathrm{~Hz}$ through power variations to the solenoid. A backhoe is attached to the base of the lower fixed jaw by a spring-loaded hinge that keeps the backhoe perpendicular to the lower surface of the fixed jaw. Initially, the backhoe is stored parallel to the lower surface of the fixed jaw but rotation of the collector head releases it from the stowed position. The tip of the backhoe is serrated and the forward sur- face has wire brushes with which to acquire samples from a veneer of loose material on rock outcrops. Two magnets, which could be cleaned by a wire brush attached to the lander body, are mounted in the backhoe to attract the magnetic fraction in the surface materials. Collector-head temperatures were measured with a platinum resistance thermistor mounted in a metal holding plate on the base of the lower fixed jaw. The absolute accuracy of the thermistor temperatures was found by experience to be \pm 3 percent of the temperature measured from $0{ }^{\circ} \mathrm{C}$ and the range was $(-) 129^{\circ} \mathrm{C}$ to $(+) 177^{\circ} \mathrm{C}$. Temperature resolution, constrained by telemetry, was $0.72{ }^{\circ} \mathrm{C}$. Near the collar, the collector head rotates upward. Upon surface contact during sampling, this upward rotation of about $10^{\circ}$ actuates a switch that cuts off the power to the elevation motor in order to stop the downward motion of the collector head and boom. This rotation acts against a spring and requires about $10 \mathrm{~N}$ of force on the backhoe. Surface-sampler positions in azimuth, elevation, and extension were measured with potentiometers so that the achieved positions could be compared with commanded positions. Additionally, motor currents of the acquisition assembly could be measured and these measurements are discussed below.

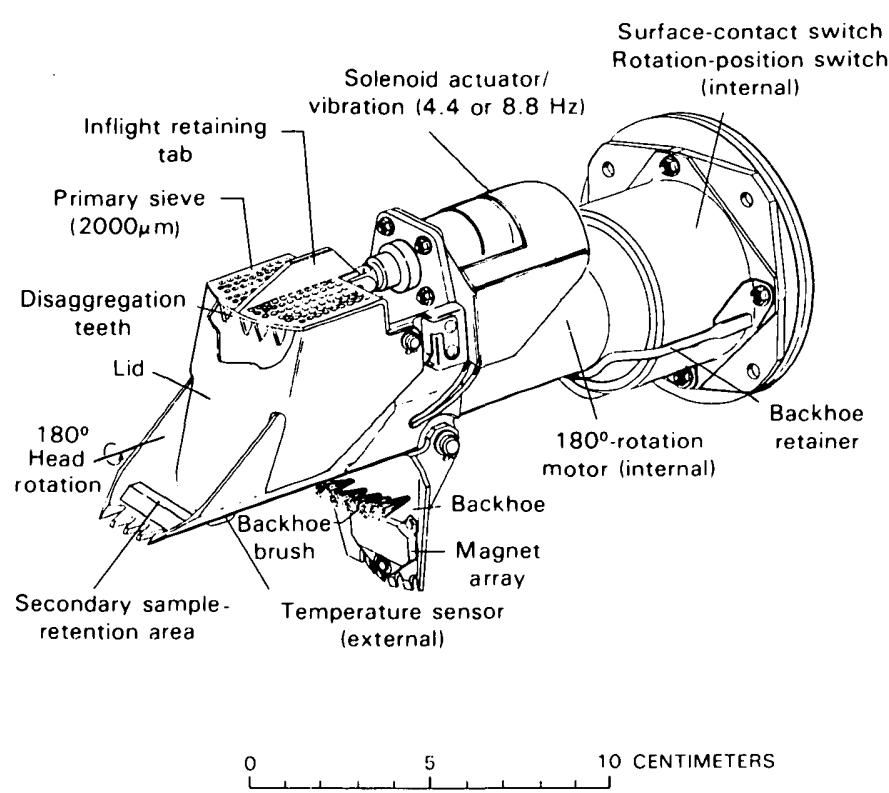

FIGURE 4.-Surface-sampler collector head. Collector head is in upright position. Upright collector-head unit rotates upward through $10^{\circ}$ during touchdown on surface. This rotation actuates a switch which turns off elevation motor upon surface contact and $10^{\circ}$ rotation. Backhoe tip is initially held in retainer but spring deploys it to indicated position when collector head rotates. Solenoid retracts rod and thereby rotates lid to open position for sample acquisition; lid is closed by spring. Upper lid contains 2.0-mm screen for sample processing. Entire collector head can be rotated to inverted position with backhoe upward. 
The GCMS sample processor (fig. 3) received samples from the acquisition assembly, ground the material to pass a $300-\mu \mathrm{m}$ sieve, and delivered the samples to the oven containers for organic chemical analyses in the gas chromatograph-mass spectrometer (GCMS). The processor is located on the upper surface of the lander body. Samples were normally delivered by vibration through the sieve of the movable jaw of the inverted collector head to the processor. Samples fell from the collector head onto and through a $2.0-\mathrm{mm}$ screen on top of the processor (Clark, L.V., and others, 1977). After passing through the screen, the samples fell into an inverted cone that fed the sample to an auger comminutor. The cone-shaped auger was driven by a motor in the processor. Samples passed through the operating comminutor, ensuring that the materials delivered to the oven containers were smaller than $300 \mu \mathrm{m}$. Larger particles were crushed by the comminutor. The crushing required additional power, as discussed below. A small wire loop between the $300-\mu \mathrm{m}$ sieve and comminutor discharge stirred the materials in order to assist them through the sieve. After comminution and sample delivery, the processor was emptied of residual material by lowering the auger cone from the comminution position, rotating it, and allowing the material to fall into a dump cavity.

The biology sample processor, which is located on the upper surface of the lander body, received samples from the acquisition assembly and delivered the samples through a 1.5-mm screen to the three biology instruments. Like the screen on the GCMS processor, the screen is on top of the biology processor.

The surface-sampler control assembly (SSCA) is housed within the lander body. It received digital commands from the spacecraft computer and provided control- and datahandling functions for the components described above. Data handling consisted of commanded and achieved positions in azimuth, elevation, and extension as well as motor currents from filtered circuits. Data resulting from the execution of each command were then stored on the lander tape recorder for transmission to the Viking Orbiters or Earth.

Although not specified as part of the surface-sampler subsystem, the funnel located on top of the lander through which samples were delivered to the X-ray fluorescence spectrometer (XRFS) is covered by a screen with $12.5-\mathrm{mm}$ openings. Samples could be delivered through the $2.0-\mathrm{mm}$ $(2,000-\mu \mathrm{m})$ sieve of the inverted and vibrating movable jaw of the collector head or directly through the opened jaws of the collector head. Thus, either the coarse, fine, or mixed fractions of samples could be delivered for analyses.

Surface-sampler subsystem motor currents were measured in two ways (Clark, L.V., and others, 1977): (1) with the SSCA filtered circuits on the drive motors, and (2) with the lander power system (Format 5).
Voltages, measured at the lander power system bus, ranged between about 31 and $36 \mathrm{~V}$. They were nominally $33.5 \mathrm{~V}$. SSCA motor currents during extension and retraction of the boom and collector head were measured $2 \mathrm{~s}$ and $4 \mathrm{~s}$ after motor start with a resolution of 0.0083 $\mathrm{A} / \mathrm{bit}$. Comminutor motor currents were measured $2 \mathrm{~s}$ and $15 \mathrm{~s}$ after motor start with a resolution of $0.028 \mathrm{~A} / \mathrm{bit}$. In Format 5, currents and voltages were sampled every $192 \mathrm{~ms}$ with resolutions very close to $0.039 \mathrm{~A} / \mathrm{bit}$ and $0.157 \mathrm{~V} / \mathrm{bit}$.

Several factors have to be considered in the interpretation of motor currents. No-load currents had to be established because both types of measurements had bias and base currents. For the SSCA comminutor of Lander 1, no-load currents were about $0.618 \mathrm{~A}$, and for Format 5 currents measured at the lander bus, no-load currents were about $1.38 \mathrm{~A}$. Comminutor motor currents of Lander 2 were irregular because of interference produced by the stirring mechanism. For Format 5 current records, the no-load current was about $1.85 \mathrm{~A}$ and those for the SSCA circuit were about $1.068 \mathrm{~A}$. The primary problem with extend- and retract-motor currents measured by the SSCA was the times at which they were measured. Durations of extensions and retractions were almost always larger than $4 \mathrm{~s}$ so that maximum currents were rarely, if ever, measured. Format 5 motor-current records covered the interval from motor start to motor stop; the interval was nominally from $5 \mathrm{~s}$ to $8 \mathrm{~s}$. These motor current records had a base current of about $0.2 \mathrm{~A}$. Laboratory tests using the Science Test Lander (frontispiece), which is described later, showed that the no-load current measured as the collector head glided over a smooth surface were typically about $0.08 \mathrm{~A}$ larger than the base current (Moore and others, 1977). In addition, cyclic currents unrelated to the extend-retract motor were measured in Format 5. Motor start was often accompanied by a large transient current. These currents were infrequent and easily identified so that they could be omitted from the interpretation of the current record.

Motor currents were experimentally related to force on the collector head (Crouch, 1976). For Format 5 current measurements,

$$
F=0.6362 I,
$$

where

$$
F \text { is the force in newtons, and }
$$

$I$ is the current above the base and no-load current $(280 \mathrm{~mA})$ in milliamperes.

When forces exceeded a certain value, the extend-retract motor decoupled (or clutched). This peak force, judging from Viking spacecraft motor-current records, corresponded to forces ranging from 200 to $225 \mathrm{~N}$ and currents 
above the no-load current were between 314 and $354 \mathrm{~mA}$. Because the resolution of current readings in Format 5 is $0.039 \mathrm{~A}$, the resolution in force was $24.8 \mathrm{~N}$ or, nominally, $25 \mathrm{~N}$.

\section{MISSION OPERATIONS}

Viking mission operations were complicated because: (1) four spacecraft were operating at the same time-two landers on the surface and two orbiters, (2) many experiments were conducted, (3) the distance from Earth to Mars was so great (for the Primary Mission it was about 225 million miles, corresponding to a radio-wave travel time of $20 \mathrm{~min}$.) that all operations had to be controlled remotely, (4) the motions of Mars only allowed a couple of hours for commanding the lander computers each day, (5) data-handling and storage capabilities were limited, and (6) spacecraft-systems limitations in power, operating temperatures, operating times, and other constraining factors could not be violated. Though initial planning for a given set of science experiments began 16 days before commanding (Lee, 1976), 5 or 6 days might elapse after commanding before the data could be analyzed (Crouch, 1977). The duration for each planning cycle and the resulting experiment overlapped the duration of the planning cycle for the following experiment, so that subse-

TABLE 3.-Overlap of the planning cycle for mission operations and design and testing of surface-sampler sequences

[After Lee (1976) and Crouch (1977)]

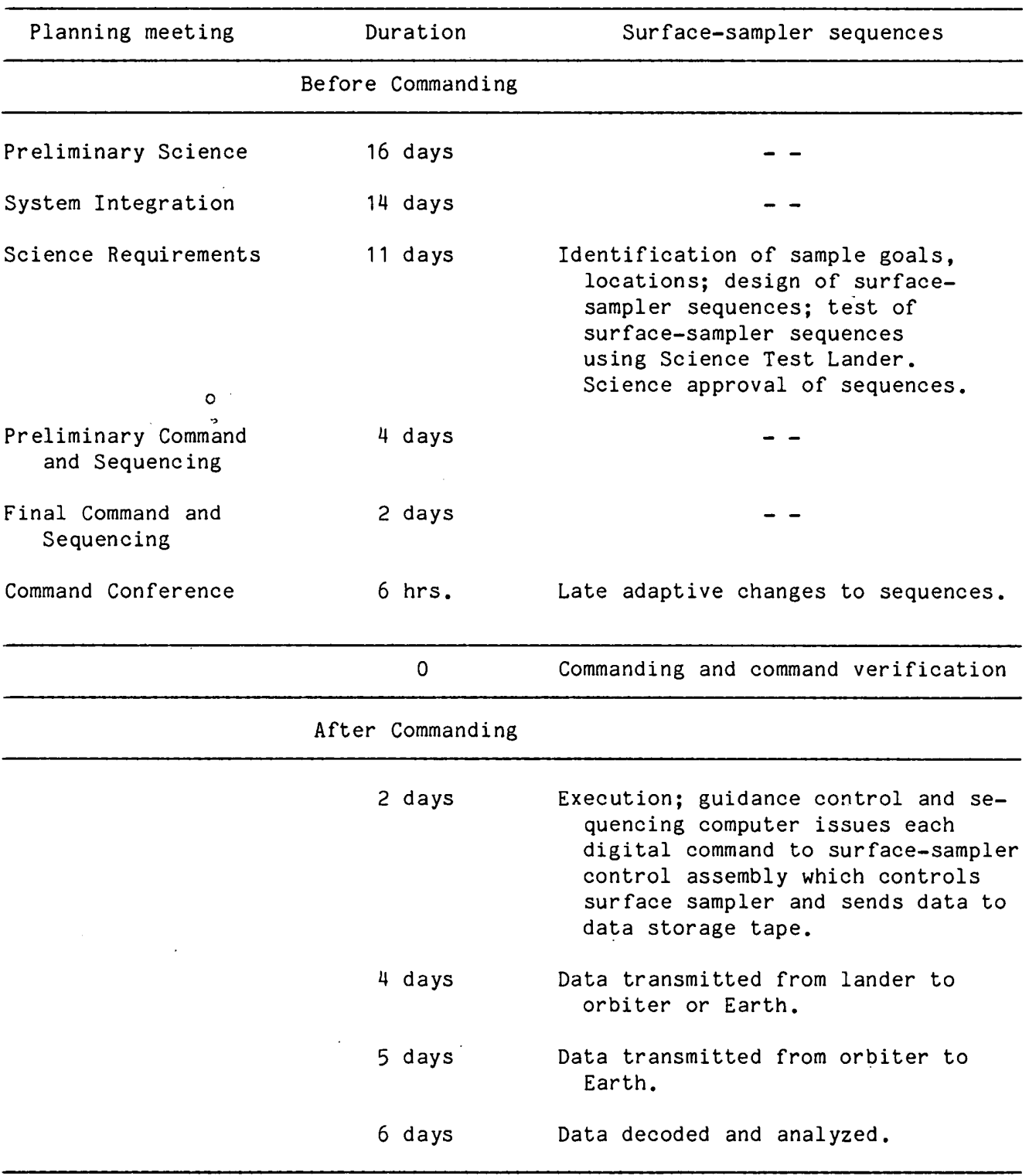


TABLE 4.-Detailed surface-sampler sequence designed to collect the coarse fraction of surface materials at the Viking Lander 2 site on Sol 57 [This sequence corresponds to the first part of item 26, table 40 (see supplemental section on "Summary of Surface-Sampler Activities"). Note that elevations here are taken as ( - ) downward whereas those in table 40 are taken as (+) downward. From Clark, L.V., and others (1977, p. B-107, 106)]

\begin{tabular}{|c|c|c|c|c|c|c|c|}
\hline $\begin{array}{l}\text { La } \\
\text { Sol }\end{array}$ & $\begin{array}{l}\operatorname{der} 1 \\
\text { Hrs }\end{array}$ & $\begin{array}{l}\text { Min } \\
\text { Min }\end{array}$ & Sec & Description & $\begin{array}{l}\text { Position } \\
\text { commanded }\end{array}$ & $\begin{array}{l}\text { Position } \\
\text { achieved }\end{array}$ & Remarks \\
\hline 57 & 8 & 0 & 0 & SSCA POWER ON & & & \\
\hline 57 & 8 & 0 & 10 & START TABL 10 & & & \\
\hline 57 & 8 & 0 & 10 & ELEV UP & 5.3 & 5.3 & \\
\hline 57 & 8 & 0 & 55 & $\mathrm{AZ} \mathrm{CW}$ & 180.3 & 180.3 & Azimuth of sample site. \\
\hline 57 & 8 & 3 & 57 & EXTEND & $76.2^{\prime \prime}$ & $76.2^{\prime \prime}$ & Prevents boom from binding on drum. \\
\hline 57 & 8 & 7 & 7 & RETRACT & $75.2^{\prime \prime}$ & $75.2^{\prime \prime}$ & Extension for sample site. \\
\hline 57 & 8 & 7 & 19 & ELEV DN & -35.1 & -33.2 & Elevation of surface contact. \\
\hline 57 & 8 & 8 & 29 & $\mathrm{CH}$ OPEN & 0.0 & & Collector head opened to collect sample. \\
\hline 57 & 8 & 8 & 46 & NG INHIBIT & 0.0 & & $\begin{array}{l}\text { Allows sequence to continue for allotted } \\
\text { time. }\end{array}$ \\
\hline 57 & 8 & 8 & 51 & EXTEND & $81.7^{\prime \prime}$ & $81.7^{\prime \prime}$ & Collector head extends to acquire sample. \\
\hline 57 & 8 & 9 & 17 & CH CLOSE & 0.0 & & Collector head closed to retain sample. \\
\hline 57 & 8 & 9 & 34 & NG ENABLE & 0.0 & & \\
\hline 57 & 8 & 9 & 39 & RETRACT & $63.3^{\prime \prime}$ & $63.3^{\prime \prime}$ & \\
\hline 57 & 8 & 10 & 20 & ELEV UP & 18.0 & 18.0 & \\
\hline 57 & 8 & 12 & 3 & NG INHIBIT & 0.0 & & \\
\hline 57 & 8 & 12 & 8 & $\mathrm{CH}$ ROT CCW & 0.0 & & Collector head inverted. \\
\hline 57 & 8 & 12 & 18 & $\mathrm{CH}$ CLOSE & 0.0 & & \\
\hline 57 & 8 & 12 & 35 & NG ENABLE & 0.0 & & \\
\hline 57 & 8 & 12 & 40 & $\mathrm{CH} \mathrm{H} / \mathrm{F}$ VIB & 0.0 & & $\begin{array}{l}\text { Collector head vibrates to } 8.8 \mathrm{~Hz} \text { to remove } \\
\text { fines from coarse fraction. }\end{array}$ \\
\hline 57 & 8 & 14 & 10 & $\mathrm{CH}$ CLOSE & 0.0 & & Collector head closed. \\
\hline 57 & 8 & 14 & 27 & EXTEND & $64.4 "$ & $64.4^{\prime \prime}$ & Prevents boom from binding on drum. \\
\hline 57 & 8 & 14 & 40 & CH CLOSE & 0.0 & & \\
\hline 57 & 8 & 14 & 57 & $\mathrm{CH}$ CLOSE & 0.0 & & \\
\hline 57 & 8 & 15 & 14 & NG INHIBIT & 0.0 & & \\
\hline 57 & 8 & 15 & 19 & $\mathrm{CH}$ ROT $\mathrm{CW}$ & 0.0 & & Collector head upright. \\
\hline 57 & 8 & 15 & 24 & $\mathrm{CH}$ CLOSE & 0.0 & & \\
\hline 57 & 8 & 15 & 41 & NG ENABLE & 0.0 & & \\
\hline 57 & 8 & 15 & 46 & RETRACT & $6.0^{\prime \prime}$ & $6.0^{\prime \prime}$ & \\
\hline 57 & 8 & 17 & 35 & $\mathrm{AZ} \mathrm{CCW}$ & 4.2 & 4.2 & $\begin{array}{l}\text { Positioning of collector head for de- } \\
\text { livery of sample to XRFS. }\end{array}$ \\
\hline 57 & 8 & 20 & 35 & EXTEND & $8.4 "$ & $8.4^{\prime \prime}$ & \\
\hline 57 & 8 & 20 & 51 & ELEV DN & -9.2 & -9.2 & \\
\hline 57 & 8 & 21 & 38 & $\mathrm{CH}$ L/F VIB & 0.0 & & $\begin{array}{l}\text { Collector head vibrates at } 4.4 \mathrm{~Hz} \text { for } \\
\text { sample delivery to XRFS. }\end{array}$ \\
\hline 57 & 8 & 22 & 38 & CH CLOSE & 0.0 & & Collector head closed. \\
\hline 57 & 8 & 22 & 55 & ELEV UP & 3.4 & 3.4 & \\
\hline 57 & 8 & 23 & 25 & $\mathrm{CH}$ CLOSE & 0.0 & & \\
\hline 57 & 8 & 25 & 60 & START TBL 9 & & & \\
\hline 57 & 8 & 25 & 60 & ELEV DN & -14.9 & -14.9 & Park. \\
\hline 57 & 8 & 26 & 37 & NG INHIBIT & 0.0 & & \\
\hline 57 & 8 & 26 & 42 & CH ROT CW & 0.0 & & Collector head upright. \\
\hline 57 & 8 & 26 & 47 & $\mathrm{CH}$ CLOSE & 0.0 & & \\
\hline 57 & 8 & 27 & 4 & NG ENABLE & 0.0 & & \\
\hline
\end{tabular}

quent experiments began before the results from the previous cycle were in hand. Indeed, one planning session that dealt with surface operations took place before the spacecraft arrived at Mars. Although inconvenient at times, this overlap was necessary and the planning strategy proved to be successful. A planning cycle is summarized in table 3.

A full-scale mock-up of the Viking lander, dubbed the Science Test Lander, proved to be an important and necessary part of mission operations (Clark, L.V. and others, 1977; Moore and others, 1978; see frontispiece). The Science Test Lander had operational cameras and surface-sampler. A large bed of poorly sorted sand in which sampling sequences were designed and tested was located in front of the Science Test Lander. During the test of sampling sequences, the Science Test Lander was tilted in the same way as the Viking landers on Mars, and the test bed was configured to simulate the sample fields 
on Mars by placing real and synthetic rocks in their correct positions as determined by stereometric measurements of the martian scene (Liebes and Schwartz, 1977; Moore and others, 1978; Wu, 1979).

As a result of the science requirements developed during the planning cycles using martian data, sample locations and goals were identified in Surface Sampler Team meetings. Surface-sampler sequences were then designed, developed, and tested using the Science Test Lander. Normally, developed and tested sampling sequences were completed within 6 or 8 days after they were established, but late adaptive changes were made within a day when the change was required to prevent a deleterious result that was identified after initial sequence design. A typical relation between the development and testing of sampling sequences and a planning cycle is given in table 3 .

A summary of surface-sampler operations during the Viking Missions is given later (see supplemental section on "Summary of Surface-Sampler Activities"). The sequences, which were generalized, provide the minimum amount of information needed to understand the sequence. Where more detail is necessary, it is given in the text. A detailed sequence for one sample acquisition is given in table 4. Noteworthy aspects of this detailed sequence are: (1) each extension command is followed by a retraction command of at least $1 \mathrm{in}$. in order to assure proper operation of the drive mechanism; (2) NG (no-go) inhibit commands allow the sequence to proceed for the allotted time in order to prevent an automatic cessation of the sequence because the commanded extension (or other command) was not achieved; and (3) the nature of the software required that some commands be paired. The detailed sequence in table 4 is condensed in the section on "Summary of Surface-Sampler Activities" to the azimuth $\left(180.3^{\circ}\right)$, extension ( $\left.75.2^{\prime \prime}\right)$ for surface contact, the elevation $\left(33.2^{\circ}\right)$ at surface contact, the achieved extension $\left(81.7^{\prime \prime}\right)$ during sampling, and the final retraction $\left(63.3^{\prime \prime}\right)$ (see item 26 , table 40 ).

\section{OTHER VIKING EXPERIMENTS AND INVESTIGATIONS}

Twelve experiments and investigations were conducted during the Viking Missions, and each of the experiments provided information relevant to the Physical Properties Investigation either directly or indirectly and to greater or lesser degrees. Most of these experiments were conducted by the landers on Mars (fig. 3) but some were conducted as part of the lander entry and descent phases while others were conducted from orbit. The experiments or investigations conducted on the surface were Biology, Molecular Analysis, Inorganic Chemical Analysis, Lander Imaging, Magnetic Properties, Meteorology, and Seismology (Soffen, 1977). Experiments conducted during the descent of the lander from orbit to the surface were grouped as one and called Entry Science. Orbiter-based experiments or investigations were Orbiter Imaging, Thermal Mapping, Water Vapor Mapping, and Radio Science (Snyder, 1977; Soffen, 1977). These experiments or investigations and their relevance to the Physical Properties Investigation are briefly described and discussed below. Results from the experiments are described separately or with other data later in this paper.

The Biology Experiment consisted of three experiments (Klein, 1977): (1) Carbon Assimilation, (2) Labeled Release, and (3) Gas Exchange. The Carbon Assimilation or Pyrolytic Release Experiment was designed to measure the incorporation of atmospheric $\mathrm{CO}_{2}$ and $\mathrm{CO}$ by organisms using photosynthesis or other biologic processes (Horowitz and others, 1972, 1977). In the experiment, a sample of surface material was incubated at $8-26{ }^{\circ} \mathrm{C}$ in a test chamber under an atmosphere containing labeled carbon dioxide and carbon monoxide $\left({ }^{14} \mathrm{CO}_{2}\right.$ and $\left.{ }^{14} \mathrm{CO}\right)$. Incubation was carried out in the dark or under illumination by a xenon lamp filtered to remove ultraviolet radiation and in a dry condition or moist condition by adding water vapor to the atmosphere. The total pressure was about the same as the ambient martian atmosphere. At the end of incubation, the test cells were heated to $120^{\circ} \mathrm{C}$ and the radioactive atmosphere vented. This was followed by pyrolysis of the sample in the chamber at $635^{\circ} \mathrm{C}$ and flushing of the chamber gases into a column operated at $120^{\circ} \mathrm{C}$ where organic molecules larger than methane were retained. Smaller molecules, which included carbon dioxide and carbon monoxide desorbed from the surface materials, passed into a radiation counter where the amount of radioactive carbon was measured. This measurement was designated as peak 1 . The column temperature was then raised to $640^{\circ} \mathrm{C}$; at this temperature the remaining carbon volatized and oxidized to $\mathrm{CO}_{2}$; and the $\mathrm{CO}_{2}$ then flowed to the radiation detector where radioactive carbon was measured. This measurement was designated as peak 2 ; it was a measure of organic matter synthesized by the sample from ${ }^{14} \mathrm{CO}_{2}$ and ${ }^{14} \mathrm{CO}$ during incubation.

In the Labeled Release Experiment (Levin, 1972; Levin and Straat, 1977), radioactive carbon was released from a nutrient by biogenic or abiogenic reactions in the surface materials. The nutrient was composed of water and organic compounds (formate, glycolate, glycene, DLalanine, and DL-lactate). The reactions produced radioactive ${ }^{14} \mathrm{CO}_{2}$ and ${ }^{14} \mathrm{CO}$ gases, which were measured with a radiation counter. The sample of surface material was placed in an incubation chamber that was about $9 \pm 3{ }^{\circ} \mathrm{C}$ and had pressures larger than the ambient pressures on Mars. After both the nutrient and incubation chambers were flushed, the nutrient was injected onto the sample. The evolution of labeled gas was then monitored in the detection chamber. Respiration of biota in a sample would 
produce an exponential increase in evolved gas; but heating to $160^{\circ} \mathrm{C}$ would sterilize the sample and substantially reduce the evolved gases.

In the Gas Exchange Experiment (Oyama, 1972; Oyama and Berdahl, 1977), gases that evolved from biogenic or abiogenic reactions between surface materials and a nutrient containing water and organic compounds were separated, identified, and measured using chromatography. The surface material was placed in an incubation chamber and then purged. At this point there were three choices: nutrient could be added to moisten the surface material, nutrient could be added to humidify the incubation chamber and surface material, or no nutrient could be added to the surface material and chamber so that they would remain dry. Krypton was added as an internal standard. Total pressure was about $200 \mathrm{mbar}$, and incubation temperatures were $8-24.4{ }^{\circ} \mathrm{C}$, although temperatures could be raised to $145^{\circ} \mathrm{C}$. Evolved gases in the chamber headspace were periodically sampled by a valve which removed $100 \mu 1$ of sample and placed it in a helium carrier gas stream. The gases were carried to a chromatograph column where retention time was used to identify the various gaseous components. Amounts of each gas phase were measured using thermistors. Gases measured by this experiment were $\mathrm{H}_{2}, \mathrm{Ne}, \mathrm{N}_{2}, \mathrm{O}_{2}$, Ar-CO, NO, $\mathrm{CH}_{4}, \mathrm{Kr}$ (internal standard), $\mathrm{CO}_{2}, \mathrm{~N}_{2} \mathrm{O}$, and $\mathrm{H}_{2} \mathrm{~S}$. The relevances of the Biology Experiments to the Physical Properties Investigation were: (1) abiogenic chemical reactions could provide information on the mineralogy and chemical properties of the surface materials, and (2) amounts of evolved gases could provide information about the surface areas of the surface materials and hence grain size.

A gas chromatograph-mass spectrometer (GCMS) was used in the Molecular Analysis Experiment to search for organic substances and inorganic volatile compounds in the surface materials of Mars (Biemann and others, 1977). In this experiment about $60-\mathrm{mm}^{3}$ samples of surface materials finer than $300 \mu \mathrm{m}$ were placed in an oven and then sealed. The sample was heated to $50^{\circ}, 200^{\circ}, 350^{\circ}$, or $500{ }^{\circ} \mathrm{C}$ in $1-8 \mathrm{~s}$ and held at each of these temperatures for $30 \mathrm{~s}$. Volatiles were then swept by ${ }^{13} \mathrm{C}$-labeled carbon dioxide (or hydrogen gas) from the opened ovens into the chromatograph columns and carried through the column by hydrogen gas. The various volatile compounds were first separated from each other in the column and next were separated from the hydrogen gas in a separator just before the compounds entered the mass spectrometer where they were identified and amounts were measured. Detection limits of organic molecules ranged from parts per million to fractions of a part per billion, depending on the molecular species. Crude estimates of water and carbon dioxide contents were made at various temperatures, but these measurements were hampered by the complex behavior of the effluent divider that protected the ion pump of the mass spectrometer from being overloaded. The chief relevance of this experiment to the Physical Properties Investigation was the ability to obtain rough measures of the amounts of water and carbon dioxide in the surface materials.

In the Inorganic Chemical Analysis Experiment, samples of surface materials were analysed with a dispersive X-ray fluorescence spectrometer (XRFS) (Toulmin and others, 1973; B.C. Clark and others, 1977). The samples were placed in a cavity $2.5 \times 2.5 \times 3.0 \mathrm{~cm}$ and irradiated by radioisotope sources $\left({ }^{55} \mathrm{Fe}\right.$ and $\left.{ }^{109} \mathrm{Cd}\right)$. Energies of the resulting fluorescent emissions were measured using proportional counter detectors. Elements between $\mathrm{Mg}$ and $\mathrm{U}$ in the periodic table could be measured or detected if they were present in sufficient quantities. The integrated intensity of backscattered primary X-rays was a direct function of the amount and distance of sample material from the radioactive source so that, if the geometry and chemistry of the samples were known, the bulk densities of the samples in the chamber could be estimated. The relevance of this experiment to the Physical Properties Investigation was to determine the chemical composition of the surface materials, to make inferences about the mineralogy of the surface materials, and to estimate bulk densities.

Goals of the Lander Imaging Investigation were generally distinct from those of the Physical Properties Investigation (Mutch and others, 1972; Shorthill and others, 1972), but the lander cameras were essential to both investigations. The lander cameras (Patterson and others, 1977) obtained images by scanning with a mirror in elevation and then rotating the camera in azimuth steps. Light from the scene passed through a window, was reflected by the scanning mirror through a lens, and then struck a detector. There are 12 photodiode detectors masked by a small aperture that defines the field of view. Four of the diodes, which have resolutions of $0.04^{\circ}$, are placed at different distances from the lens to give optimum focuses from the near field outward to the far field. The other eight diodes have resolutions of $0.12^{\circ}$. Six of the low resolution diodes have filters (Huck and others, 1977): blue, green, red, and three near-infrared filters. The eleventh diode, which did not have a filter, was used for rapid area scans. The last diode was used to view the sun directly through a red filter. Of particular importance was the stereometric capability of the two cameras on each lander that permitted accurate measurements of positions and sizes of objects in the sample fields (Liebes and Schwartz, 1977). The relevances of the products of the Lander Imaging Investigation were manyfold. Perhaps the most relevant products were the properly labeled and processed pictures that had to be used in conjunction with the surface-sampler data (Levinthal and others, 1977). 
Geologic observations and interpretations, color estimates of objects and surfaces, and so forth, were also useful to the Physical Properties Investigation.

Magnet arrays on the backhoes of the surface-sampler collector head and main reference test chart (next to the magnifying mirror, fig. 3 ) were the principal tools of the Magnetic Properties Investigation (Hargraves and Petersen, 1972; Hargraves and others, 1977). Effective fields and field gradients are $2,500 \mathrm{G}$ and $10,000 \mathrm{G} / \mathrm{cm}$ and $7,000 \mathrm{G}$ and $3,000 \mathrm{G} / \mathrm{cm}$, respectively, for the strong arrays and weak arrays. Wire brushes are mounted on the left side of each lander in order to clean the magnets on the backhoe (Hargraves and others, 1979). Cleaning was accomplished by rotating the collector head so that the backhoe magnets passed through the brushes. This investigation provided data on the nature of the magnetic materials in the surface materials and the chemistry of the surface materials.

Meteorology temperature and wind sensors are mounted on a boom, which was deployed shortly after landing (Hess and others, 1977). The sensors are about $1.6 \mathrm{~m}$ above the surface. Temperatures were measured with three thermocouples, and wind speeds were measured by two hot film sensors. A quadrant sensor measured the wind direction. The pressure sensor is a stressed diaphragm mounted underneath the lander body. Wind speeds, particularly gusts, were important to the Physical Properties Investigation because changes in the tailings of trenches, piles of surface materials, and morphologies of trenches could be caused by the martian winds.

The seismology instrument has three orthogonal inertial seismometers fitted with velocity transducers (D.L. Anderson and others, 1977). The useful frequency range was $0.1-10 \mathrm{~Hz}$ with a ground amplitude resolution of $2 \mathrm{~nm}$ at $3 \mathrm{~Hz}$ and $10 \mathrm{~nm}$ at $1 \mathrm{~Hz}$. The instrument package is mounted on the upper surface of the lander body near leg 1. Data from the Seismology Investigation was relevant to the Physical Properties Investigation because a seismically active Mars could induce failure of trench walls and lander vibrations could affect the behavior of surface materials deposited on the lander body and in the footpads. Unfortunately, only the seismometer on Lander 2 was operable.

Entry Science consisted of a number of experiments: Use of mass spectrometry to study the upper atmosphere, use of a retarding potential analyser to study the ionosphere, and use of pressure sensors, temperature sensors, and spacecraft motion during descent to study the structure of the lower atmosphere (Nier and others, 1972). Of particular importance here were the measurements of the velocities of the landers at touchdown and the accelerations of gravity at the landing sites using the inertial guidance system and landing radars.
Orbiter Imaging was chiefly a global-scale experiment. The cameras aboard the Viking Orbiters did, however, obtain images of the Viking Landing sites (Carr and others, 1976; Masursky and Crabill, 1976; Snyder, 1977) with identification resolutions about $150 \mathrm{~m}$ and, later, for the Lander 1 site, $25 \mathrm{~m}$ (Dial, 1978). These images provide a broad-scale photogeologic framework on which to base interpretations of origins of surface materials observed at a fine scale by the landers.

Thermal Mapping was accomplished by measuring thermal emissions from the planet's surface from the Viking Orbiters through four telescopes and detectors with responses centered near 7, 9, 11, and $15 \mu \mathrm{m}$ (Kieffer and others, 1976, 1977). Spatial resolution was about $8 \mathrm{~km}$ at periapsis $(1,500-\mathrm{km}$ range). Seven detectors at $0.3-0.9 \mu \mathrm{m}$ responded to reflected sunlight. Thermal behavior of different areas was obtained by repeated observations and after taking into account variations due to diurnal, latitudinal, seasonal, albedo, atmospheric, and elevation factors. Thermal properties, particularly the thermal inertias of the general areas of the landing sites, are relevant to the Physical Properties Investigation.

Water Vapor Mapping was also an experiment conducted from the Viking Orbiter (Farmer, 1976, 1977). The instrument, affectionately known as MAWD, was a (Mars) microwave atmospheric water detector that measured the absorption of reflected solar radiation by the strong lines at the center of the $1.4 \mu \mathrm{m}$ vibration-rotation bands of water. Water ice in the atmosphere was not detected. The instantaneous field of view at periapsis $(1,500-\mathrm{km}$ range) was $3 \times 24 \mathrm{~km}$. Measurements of water vapor in the atmosphere above the landing sites could be relevant to the Physical Properties Investigations because the water vapor could be equilibrated with ice or adsorbed water on mineral surfaces in the surface materials.

Radio Science Investigations were rather varied (Michael and others, 1977). Parts of the investigations that are relevant to this investigation were the locations of the landers on the martian globe (Mayo and others, 1977) and bistatic radar measurements of the electrical properties of the surface at the landing sites (Tang and others, 1977).

\section{COORDINATE SYSTEMS AND MEASUREMENTS}

The lander science coordinate system (LSCS) forms the framework for maps, profiles, and diagrams in this report. In this coordinate system (fig. 5), the upper planar surface of the prismatic lander body is coincident with the $Z_{L}-Y_{L}$ plane and the origin for the three orthogonal axes is at the geometrical center of the polygonal outline of this upper planar surface. $Z_{L}$ passes through the origin and a point halfway between the two cameras. $Z_{L}$ is positive in the direction of the point between the cameras. $Y_{L}$ passes through the origin at right angles to $Z_{L}$ and 
is positive in the direction of footpad 2. $X_{L}$ passes through the origin at right angles to $Z_{L}$ and $Y_{L}$ and is positive in the direction extending downward through the lander body. Positions of objects and features in the sample field
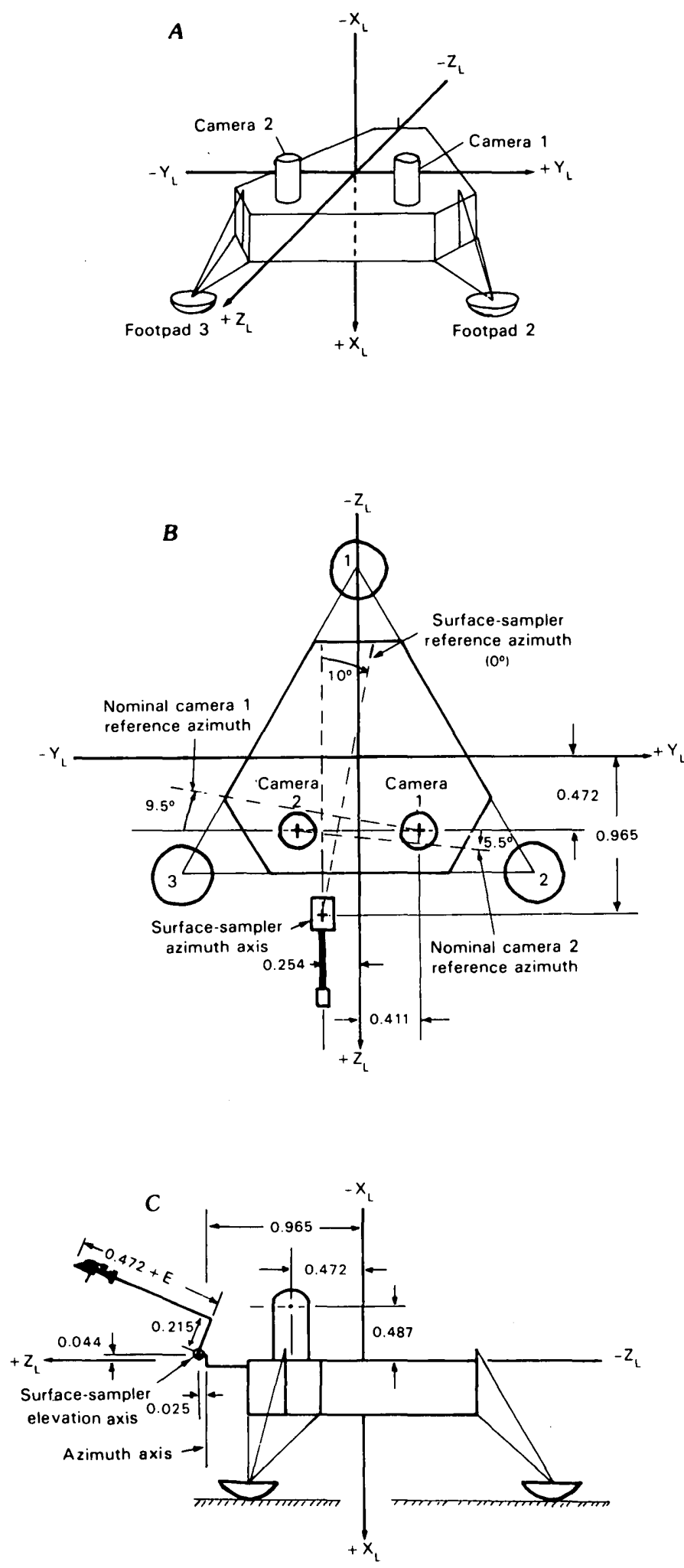

and spacecraft parts are defined as distances, in meters, from the origin in the directions of these axes.

The lander science coordinate system differs from the lander aligned coordinate system (LACS) in that the origin of the LACS is $1.1 \mathrm{~m}$ below the origin of the LSCS; otherwise, the $X$ axes are coincident, the $Y$ and $Z$ axes are parallel, $Y$ and $Z$ positive directions are the same and $X$ is positive upward (see Tucker, 1978).

All elements of the acquisition assembly or surface sampler are offset from the LSCS as well as from each other (fig. 5). The geometry of the surface sampler and three surface-sampler coordinates are used to locate the tip of the collector head in the sampler aligned coordinate system (SACS): (1) azimuth, (2) elevation, and (3) extension. Azimuths are reported in degrees measured clockwise, as viewed from above, from a line passing through the azimuth axis in a plane parallel to the $Z_{L}-Y_{L}$ plane. This line is $10^{\circ}$ clockwise from the $(-) Z_{L}$ direction. Elevation angles are reported in degrees measured from a plane parallel to the $Z_{L}-Y_{L}$ plane and passing through the elevation axis. As used here, elevation angles measured downward were positive. In some conventions, elevation angles measured downward were negative (Clark, L.V., and others, 1977; Pike and others, 1978). Extensions, which represent the increase in distance of the collector head from its original position in the boom housing, were normally reported in inches and subsequently converted to meters. Because the longitudinal axis of the furlable boom and collector-head unit is offset from the elevation axis, equations relating the surface-sampler coordinates to the LSCS had to take these offsets into account.

The camera aligned coordinate system (CACS) was used here in measuring and locating objects in the sample field; the measurements were then translated into the LSCS. The origin of this system is the nominal intersection of the rotation axes of the mirrors of the cameras. For camera 1 , azimuths are measured clockwise, in degrees, from a line passing through the origin in a plane parallel to the $Z_{L}-Y_{L}$ plane. This line is nominally $170.5^{\circ}$ counterclockwise of the $(+) Y_{L}$ direction, but the actual direction had to be corrected for a "bolt-down" error peculiar to camera 1 of each lander. For camera 2, azimuths are

Figure 5.-Coordinate systems used in this report. Distances are in meters. $A$, Perspective view showing relation between lander science coordinate system (LSCS) and lander body. $B$, Plan view of lander showing locations and distances of offsets of camera and surfacesampler azimuth axes from $Z_{L}$ and $Y_{L}$ axes of LSCS. View is along $X_{L}$ axis in positive direction. $C$, Side view of lander showing locations and distances of offsets of camera and surface-sampler elevation axes from the $Z_{L}$ and $X_{L}$ axes of LSCS. View is along $Y_{L}$ axis in negative direction. Note that extension (E) of surface sampler is increase in distance of collector head from its original position in boom housing measured along boom axis. 
measured in degrees clockwise from a line passing through the origin in a plane parallel to the $Z_{L}-Y_{L}$ plane. This line is nominally $5.5^{\circ}$ clockwise of the $(+) Y_{L}$ direction, but the actual direction had to be corrected for a "bolt-down" error peculiar to the camera 2 of each lander. Elevations for both cameras are measured in degrees normal to a plane passing through the origin and parallel to the $Z_{L}-Y_{L}$ plane. In the convention used here, elevation angles measured downward were taken as positive, but it must be realized that they were recorded as negative on standard photographs (Tucker, 1978).

Equations that could be used to obtain lander science coordinates from known surface-sampler coordinates and camera coordinates, as well as surface-sampler coordinates and camera coordinates from lander science coordinates, are given later (see supplemental section on "Measurements and Coordinates").

There were two methods used during the Viking missions to locate features and objects in the fields of view of both cameras of each lander: a sophisticated method and an unsophisticated method. The sophisticated method employed a geometrically calibrated and accurate stereophotogrammetric system (Liebes, 1982; Liebes and Schwartz, 1977; Patterson and others, 1977) and was used chiefly to support surface-sampler operations and the Lander Imaging Investigation. Locations and sizes of many rocks in the sample fields that were established with the sophisticated system were used extensively by the Physical Properties Investigation; but, additional locations and sizes of rocks had to be established and only several trenches were located and measured with this system because of limited resources. Thus, the unsophisticated method, which is mentioned below, was required to obtain the measurements required by the Physical Properties Investigation. These unsophisticated methods yielded accurate measurements of local relief and distances but somewhat less accurate positions in the LSCS. Comparison of the LSCS coordinates derived from the surface sampler and the sophisticated system indicated that errors using the unsophisticated method were small. Thus, whenever possible, measurements made using the sophisticated system were used in preparing maps and diagrams, but the vast majority of measurements were made using the unsophisticated method mentioned below and described later (see supplemental section on "Measurements and Coordinates").

In the unsophisticated method, conjugate images of points in the camera 1 and 2 photographs were identified, and data were obtained from the edges of the photographs that were related to the camera azimuths and elevations (see Tucker, 1978). These data were then used to calculate the LSCS coordinates of the conjugate image points using a small programmable calculator. Programs corrected azimuths for diode displacements and "bolt-down" cor- rections. The final products, such as trench outlines and profiles, were achieved by selecting a multitude of conjugate images, determining the necessary data from the photographs, and calculating their coordinates. This method, though tedious, supplied the only maps showing the locations and dimensions of practically all the trenches in the sample fields and was used sometimes to support surface-sampler operations during the missions.

Not all the sample fields could be viewed by both cameras of each lander. For this reason, some objects in the sample field were located by using a variety of monoscopic techniques. These techniques included gathering as much information as possible about the surrounding area by using stereometric methods, using shadows cast upon the monoscopic area by spacecraft parts, using LSCS coordinates derived from surface-sampler coordinates for trenches in the monoscopic area, and by obtaining estimates of distances from the camera by using camera-elevation angles in conjunction with "best estimates" of the $X_{L}$ coordinate of an object in a monoscopic area. These monoscopic techniques, which are reasonably accurate, were used sometimes to support surfacesampler operations during the missions.

\section{TIME}

Some features of Mars are similar to those on Earth; its axis of rotation is inclined $24.77^{\circ}$ to its orbital plane and martian solar days are nearly the same length as terrestrial days. Mars rotates about its axis every 24.623 hours and revolves around the sun in 687 days. These two motions result in a mean martian day or sol that is 24.66 hours long. The Mars year is equal to 1.88 Earth years 1 (668.6 sols). Lander events were reckoned in Local Lander Time (LLT) and Greenwich Mean Time (GMT) or Universal Time (UTC). In the local time convention, the day of landing of the spacecraft was Sol 0 (Sol is capitalized) and subsequent divisions were measured in sols and hours, minutes, and seconds past martian midnight. Thus, 08070510 represents the eighth sol after landing (Sol 8) at 7 hours, 5 minutes, and 10 seconds past midnight. Because Lander 2 landed approximately $177^{\circ}$ east of and later than Lander 1, local event Sols of Lander 1 are 44.5 sols larger than Lander 2 Sols at the same instant of time. The times of events, which were recorded on pictures and in project documents, can be correlated with one another by GMT or UTC. Seasons, which are unequal in length because of orbital eccentricity, were described in terms of $\mathrm{L}_{\mathrm{S}}$ or the areocentric longitude of the sun: 0 is the spring equinox, $90^{\circ}$ is the northern summer solstice, $180^{\circ}$ is the fall equinox, and $270^{\circ}$ is the northern winter solstice. Perihelion (206.5 Mm, 1.38 AU), or closest approach of Mars to the sun, occurs near $\mathrm{L}_{\mathrm{S}}=250^{\circ}$ (late northern fall), and aphelion (249.1 Mm, 1.67 AU), or 
farthest distance from the sun, occurs near $\mathrm{L}_{\mathrm{S}}=70^{\circ}$ (late northern spring).

\section{SAMPLE FIELD DEFINITION}

The sample fields are the areas in front of the landers that were safely accessible to the surface-sampler collector heads. They are nominally defined as the area between surface-sampler azimuths of $90^{\circ}$ and $250^{\circ}$ that could be reached by the collector head extended to the surface with an elevation angle of $38.1^{\circ}$ (downward) and lowered to the surface with a commanded extension of $110 \mathrm{in}$. Thus, factors that affect the size and shape of the sample field include tilt of the lander on the surface, leg stroke and penetration of the footpads into the surface, and local topography of the surface. Because of these factors, the arcuate traces on the surface that were defined by the collector head deviated from circularity, and widths of the sample fields varied with azimuths. For Lander 1 (pl. 1), at the left edge along an azimuth of $90^{\circ}$, the sample field was $1.54 \mathrm{~m}$ from the azimuth axis of the surface sampler, and the width of the sample field was $1.66 \mathrm{~m}$; at the right edge along the $250^{\circ}$ azimuth, the distance and width were $1.46 \mathrm{~m}$ and $1.80 \mathrm{~m}$. For Lander 2 (pl. 2), at the left edge along an azimuth of $90^{\circ}$, the sample field was $1.42 \mathrm{~m}$ from the azimuth axis of the surface sampler, and the width of the sample field was $1.76 \mathrm{~m}$; at the right edge along the $250^{\circ}$ azimuth, the distance and width were $1.74 \mathrm{~m}$ and $1.28 \mathrm{~m}$. Relaxation of the criteria for safety permitted access to areas adjacent to the defined sample field.

\section{THE LANDING SITES}

The two Viking Landers were separated from one another by approximately $6,500 \mathrm{~km}$. Lander 1 : was situated at the west edge of a basin in Chryse Planitia at an elevation approximately $2 \mathrm{~km}$ below the Mars datum (table 5). Lander 2 was situated about $200 \mathrm{~km}$ west of the rim of the crater Mie in Utopia Planitia at an elevation approximately $3 \mathrm{~km}$ below the Mars datum. Environments at the landing sites differed in several ways. Lander 2 , which was both farther north and lower in elevation than Lander 1, had larger variations in diurnal insolation with the changing seasons, thus, larger variations in average air temperatures than Lander 1. Atmospheric pressures at the Lander 2 site were about 1 mbar higher at a given time than those for Lander 1 because of the elevation difference. For Lander 2, maximum and minimum daily air temperatures were about $242^{\circ}$ and $194^{\circ} \mathrm{K}$ in the summer, but they plunged to about $158^{\circ}$ and $150^{\circ} \mathrm{K}$ in the

TABLE 5.-Locations and relevant parameters at the Viking landing sites

$\begin{array}{lccc}\text { Areographic name } & \text { Viking } 1 & \text { Viking } 2 & \text { References }\end{array}$

\begin{tabular}{|c|c|c|c|}
\hline $\begin{array}{l}\text { Latitude: } \\
\text { areocentric } \\
\text { areographic } \\
\text { Longitude }\end{array}$ & $\begin{array}{l}22.272^{\circ} \\
22.482^{\circ} \\
47.968^{\circ}\end{array}$ & $\begin{array}{r}47.670^{\circ} \\
47.966^{\circ} \\
225.736^{\circ}\end{array}$ & Davies, 1978 . \\
\hline $\begin{array}{l}\text { Acceleration of } \\
\text { gravity }\left(\mathrm{m} / \mathrm{s}^{2}\right)\end{array}$ & 3.7189 & 3.7307 & $\begin{array}{l}\text { Nier and others, } 1976 . \\
\text { Sieff and Kirk, } 1976 .\end{array}$ \\
\hline Elevation $(\mathrm{km})$ & $(-) 2$ & $(-) 3$ & $\begin{array}{l}\text { Sieff and Kirk, } 1976 . \\
\text { Nier and others, } 1976 .\end{array}$ \\
\hline $\begin{array}{l}\text { Atmospheric pressure: } \\
\text { Summer, low (mbars) } \\
\text { Fall-winter, high } \\
\text { (mbars) }\end{array}$ & $\begin{array}{c}6.8 \\
8-5-8.9\end{array}$ & $\begin{array}{l}7.4-7.5 \\
9.0-10.1\end{array}$ & \\
\hline $\begin{array}{cl}\text { Air temperatures: } \\
\text { Late-summer } & \text { high } \\
\left({ }^{\circ} \mathrm{K}\right) & \text { low } \\
\text { Winter } & \text { high } \\
\left({ }^{\circ} \mathrm{K}\right) & \text { low }\end{array}$ & $\begin{array}{l}248 \\
188 \\
186 \\
178\end{array}$ & $\begin{array}{l}242 \\
194 \\
158 \\
154\end{array}$ & Ryan and Henry, 1979. \\
\hline $\begin{array}{l}\text { Viking Orbiter } \\
\text { pictures }\end{array}$ & $\begin{array}{c}452 \mathrm{~B} \\
9,10,11\end{array}$ & $\begin{array}{c}009 B \\
13,14,15,16\end{array}$ & \\
\hline
\end{tabular}




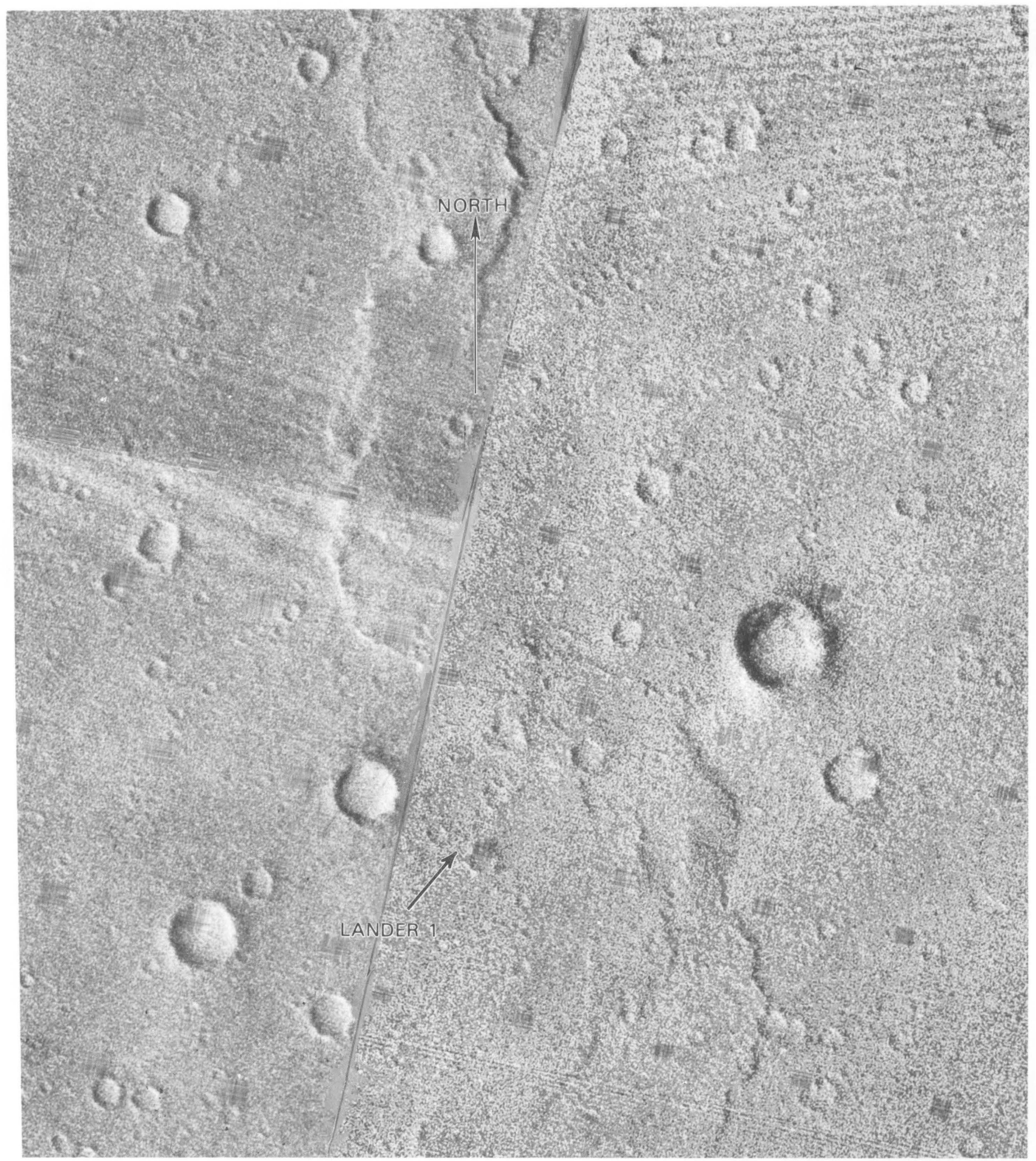

0

2 KILOMETERS

FIGURE 6.-Viking Orbiter pictures of Lander 1 site. Arrow indicates location of Viking Lander 1. Identification resolution of picture is about $25 \mathrm{~m}$. Sun from lower left. Viking Orbiter frames 452B09, 10, and 11. 
winter; for Lander 1, maximum and minimum daily air temperatures were about $248^{\circ}$ and $188^{\circ} \mathrm{K}$ in late summer but dropped to $186^{\circ}$ and $178^{\circ} \mathrm{K}$ in the winter (table 5). Wind speeds at the sites were typically small-several meters per second-but hourly speeds sometimes averaged about $18 \mathrm{~m} / \mathrm{s}$ and gusts as large as $26 \mathrm{~m} / \mathrm{s}$ were reported (Ryan and Henry, 1979). The topography of the two sites also differed.

\section{ORBITER VIEWS}

Perhaps the most significant topographic differences between the two sites as viewed from orbit were the character of the planar surfaces and their relations with craters. The intercrater plains of Chryse Planitia ap- peared smooth except where wide elongate ridges like those of the lunar maria anastomose across the plains (fig. 6). Lander 1 rested on the smooth plains and was surrounded by craters hundreds of meters across. Ejecta from several of these craters were undoubtedly deposited at the site. In contrast, the intercrater plains of Utopia Planitia appeared uneven with broad shallow swales and broad gentle bulges. Lander 2 was very near or on lobes related to ejecta (fig. 7) from the crater Mie, which is $100 \mathrm{~km}$ in diameter. The abundance of craters with diameters less than $1.0 \mathrm{~km}$ was only about $10-15$ percent less than at the Lander 1 site.

Lander 1 was just west of a broad ridge (Morris and Jones, 1980; figs. 6 and 8) and was roughly halfway between two craters. The western crater, which was cir-
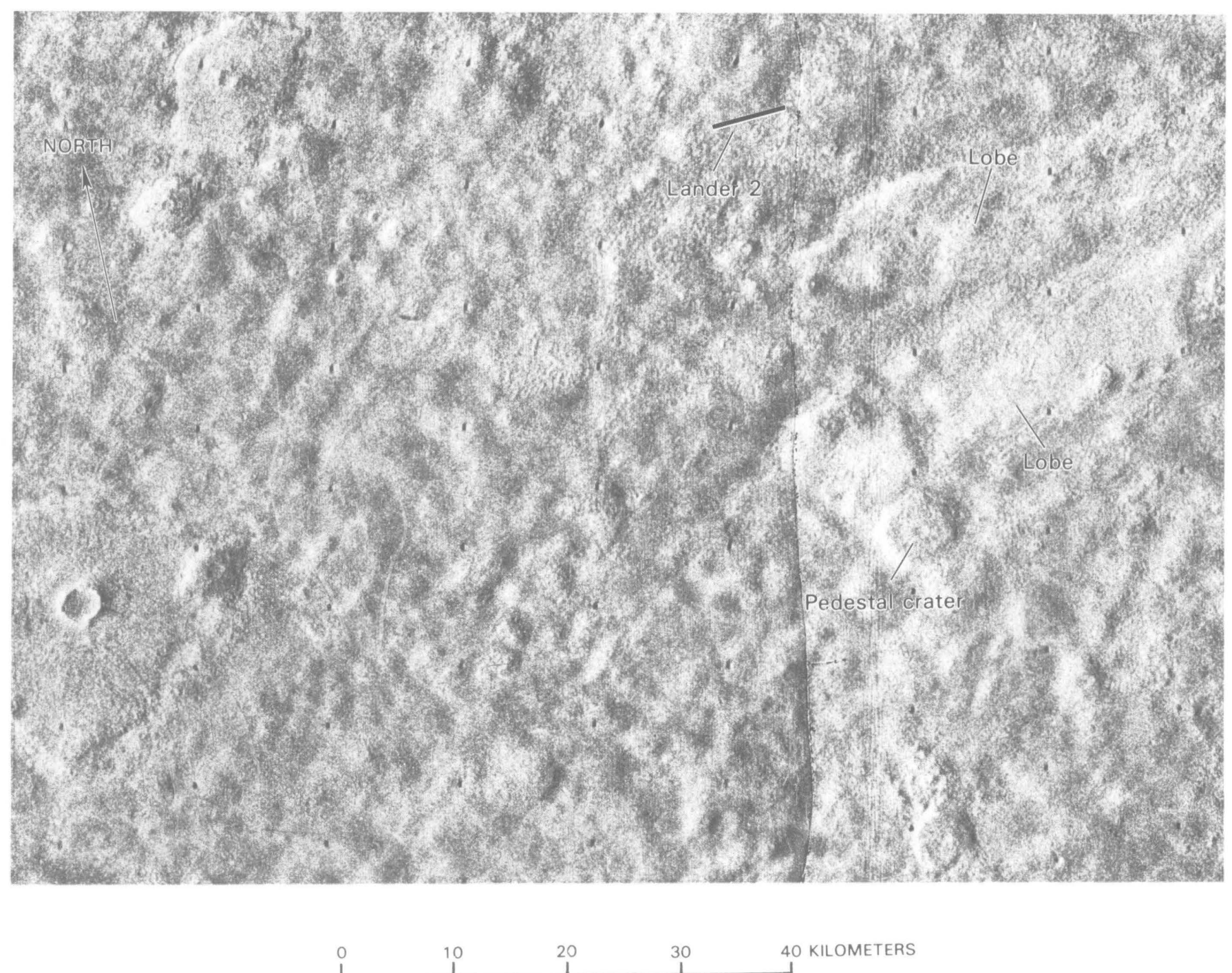

Figure 7.-Viking Orbiter pictures of Lander 2 site. Bar indicates possible locations of Lander 2. Note hummocks and swales. Lobes radial to crater Mie are indicated. Many craters appear to be on pedestals. Sun is from left. Viking Orbiter frames $009 B 14$ and 16. 
cular and $130 \mathrm{~m}$ in diameter, might have been the result of an impact of an object from space (a primary crater) but the eastern crater, $180 \mathrm{~m}$ in diameter, and very irregularly shaped, might have been produced by the impact of ejecta (secondary crater) flung from some distant large primary crater. Because these two craters were so close to Lander 1, it appears probable that ejecta from them was deposited at the site (fig. 8). Two additional craters might also have deposited ejecta at the landing site. One of these, $400 \mathrm{~m}$ to the west, was $160 \mathrm{~m}$ in diameter and appeared to have been modified by erosion and infilling. The other, $390 \mathrm{~m}$ in diameter, was $800 \mathrm{~m}$ to the west-northwest and appeared to be unmodified. A distribution of crater sizes and frequencies for the general Chryse region (Dial, 1978) appeared to have two components (fig. 9). For craters between $100 \mathrm{~m}$ and $1.0 \mathrm{~km}$ in diameter, the cumulative number of craters was inversely proportional to the cube of their diameters, but for craters larger than $1.0 \mathrm{~km}$, the cumulative number of craters was inversely proportional to the 1.5 power of their diameters. Frequencies of craters smaller than $100 \mathrm{~m}$ were invalid because of the resolution of the pic-
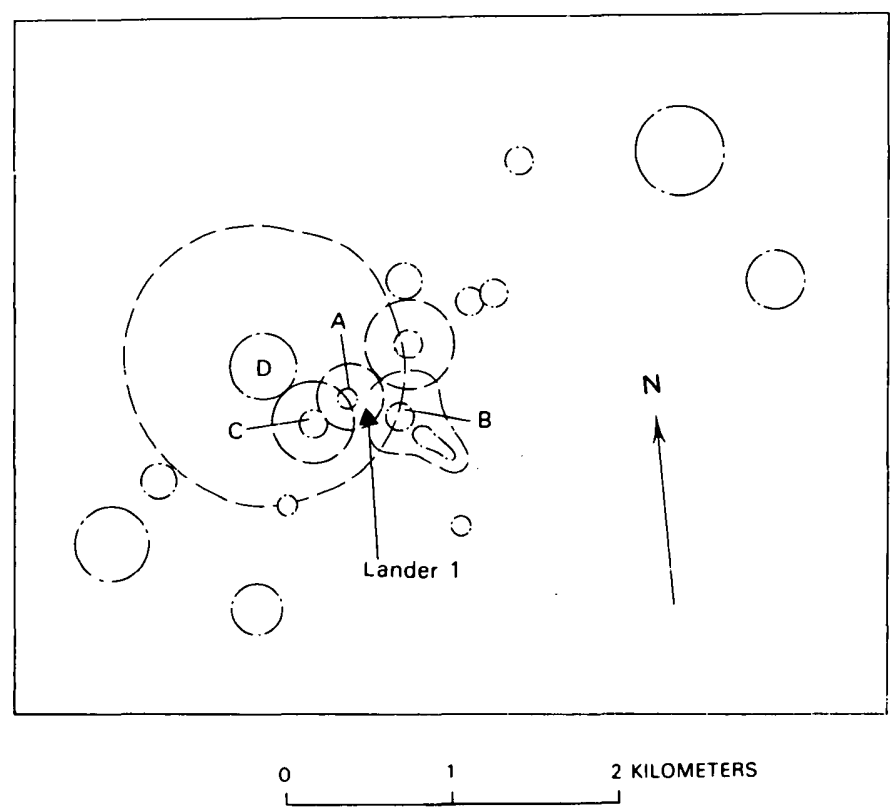

EXPLANATION

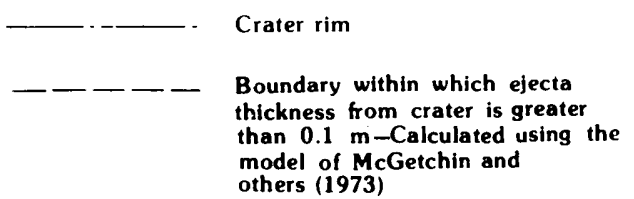

FIGURE 8.-Sketch map of Lander 1 site. Four craters (A-D) may have deposited ejecta at landing site. Diameters of these craters are: $\mathrm{A}$, $130 \mathrm{~m} ; \mathrm{B}, 180 \mathrm{~m}$; C, $160 \mathrm{~m}$; and $\mathrm{D}, 390 \mathrm{~m}$.

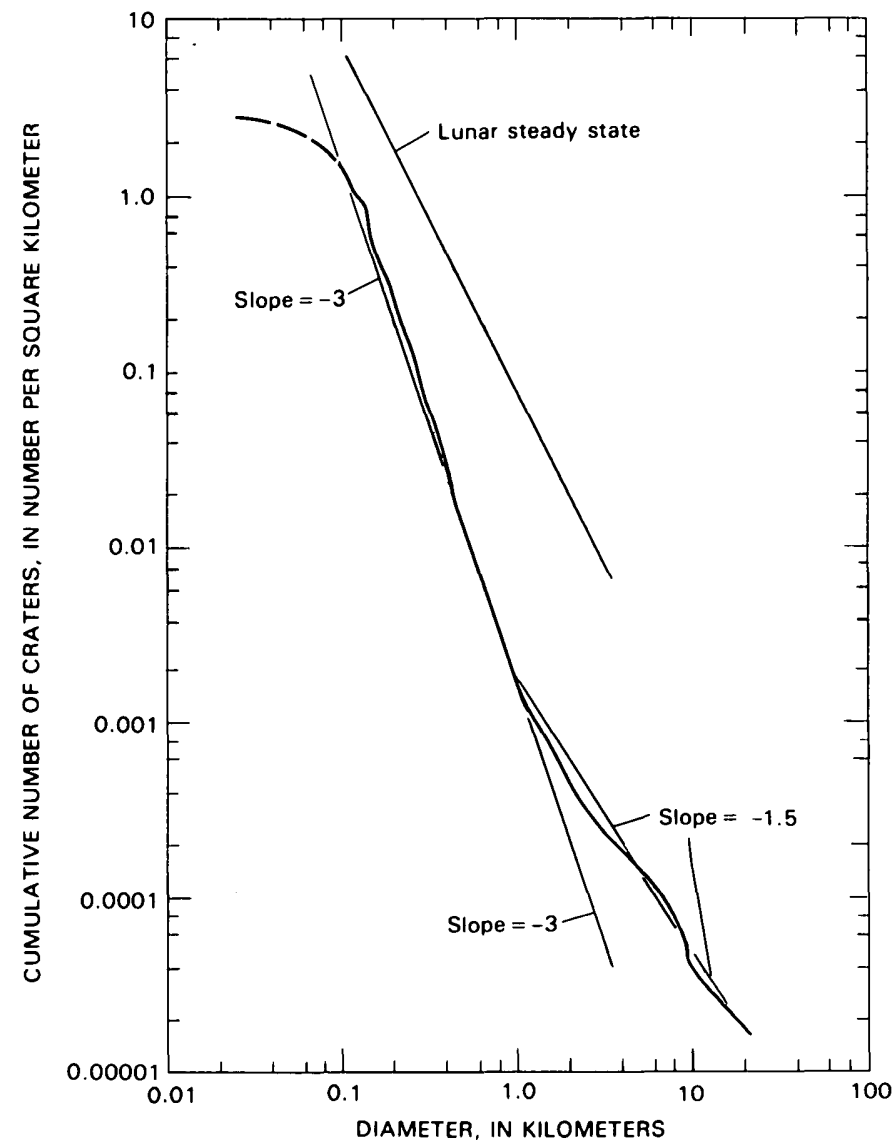

FIGURE 9.-Cumulative frequency distribution of craters at Lander 1 site (from Dial, 1978).
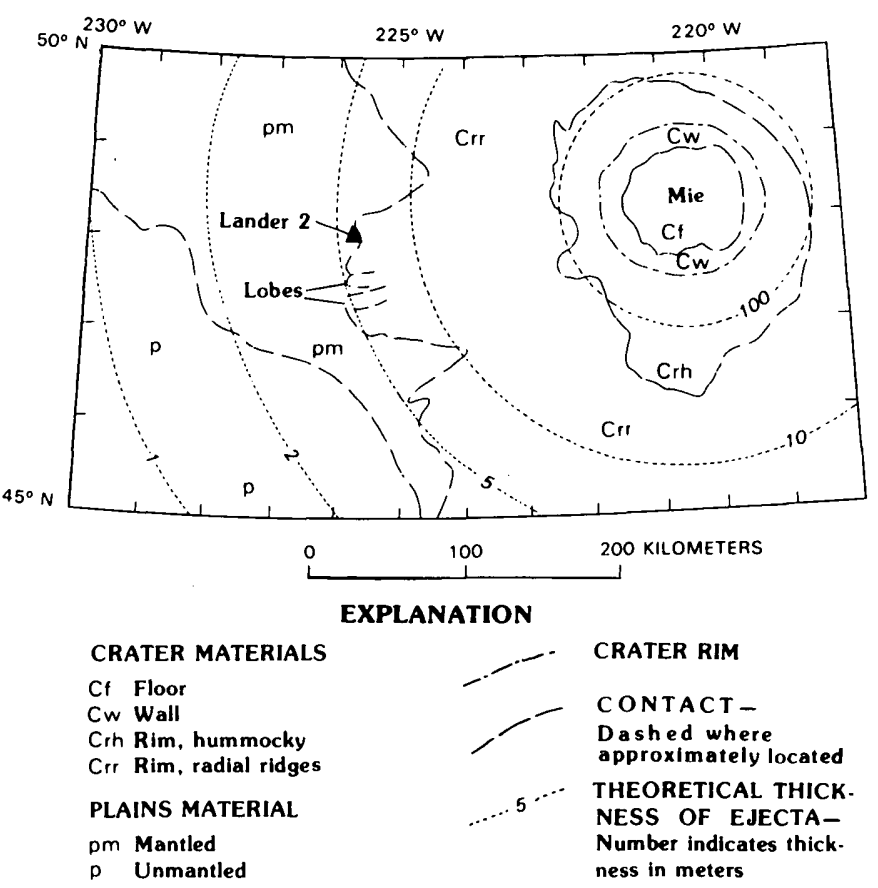

FIGURE 10.-Geologic sketch map showing relation between Lander 2 site and crater Mie. Theoretical thicknesses of ejecta from Mie were calculated with a model for large lunar craters (McGetchin and others, 1973). Geologic units after Mutch and others (1977). 
tures. Craters as small as $25 \mathrm{~m}$ could be identified in the general region of the landing site, but they did not appear to be so abundant as similar size craters on the lunar surface. Although the absence of craters less than $100 \mathrm{~m}$ (relative to the Moon) might be partly related to the resolution of the pictures of the site, the absence might be related to the presence of a martian atmosphere which could produce two effects: (1) burial and destruction of small craters by aeolian processes, and (2) meteorite massloss, break-up, and deceleration in the atmosphere of Mars (Dycus, 1969).

For both components of the crater size-frequency distribution, the cumulative number of craters was $1.5 \times 10^{-3}$ per $\mathrm{km}^{2}$ for diameters greater than $1.0 \mathrm{~km}$. In-
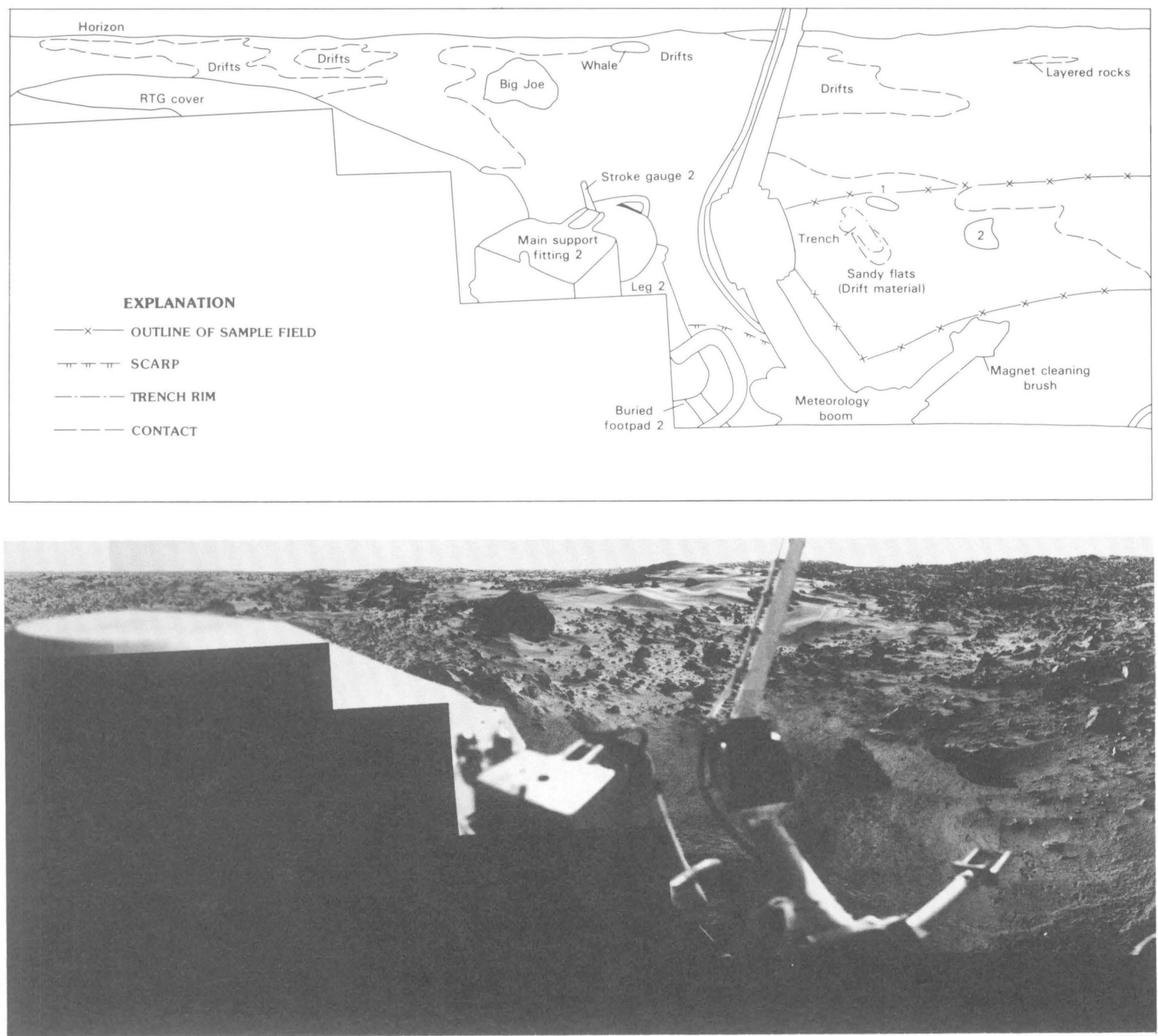

Figure 11.-Panoramas and sketches of Lander 1 site showing abundant rocks and tracts of dunelike drifts. $A$, Mosaic of camera 1 pictures. $B$, Mosaic of camera 2 pictures. Sketches show locations of selected features in panoramas; rocks indicated by number correspond to numbered rocks on plate $1 A$. Panoramas include sample field (see sketches). Trench is result of first sample acquisitions for Biology, Molecular Analysis, and Inorganic Chemical Analysis experiments on
Sol 8 from area informally called Sandy Flats. Second area sampled was informally called Rocky Flats. Rims of distant impact craters rise above local horizon. Large rock, labeled "Big Joe", is $1 \mathrm{~m}$ high and 8-10 $\mathrm{m}$ from Lander 1. Note pitted appearance of surfaces of some rocks. Dune and probable outcrops are indicated. Mosaics were generated by computer from morning pictures (Levinthal and Jones, 1980). 
terpretation of the cumulative number of craters in terms of absolute age was unfeasible because of a complete absence of samples of rocks from Mars for radiometric age determinations in laboratories on Earth and the variety of models used to estimate absolute ages from superposed craters. Direct comparison with lunar crater distributions on surfaces with known ages placed the age of the Chryse region at about 2 b.y., but other models which inferred both greater (about 1.5 times; Soderblom, 1977; see also Soderblom and others, 1974) and smaller (about one-third; Neukum and others, 1978; see also Neukum and Wise, 1976) rates of formation of craters that were the same size as the lunar ones, indicated ages between 1.3 and 3.6 b.y. In any case, the age of Chryse recorded by craters seems to be very old by terrestrial standards.

In detail, the location of Lander 2 was not so well established as that of Lander 1 because there were no clearly identifiable features in both the orbiter and lander pictures. As noted earlier, the cratered surface appeared
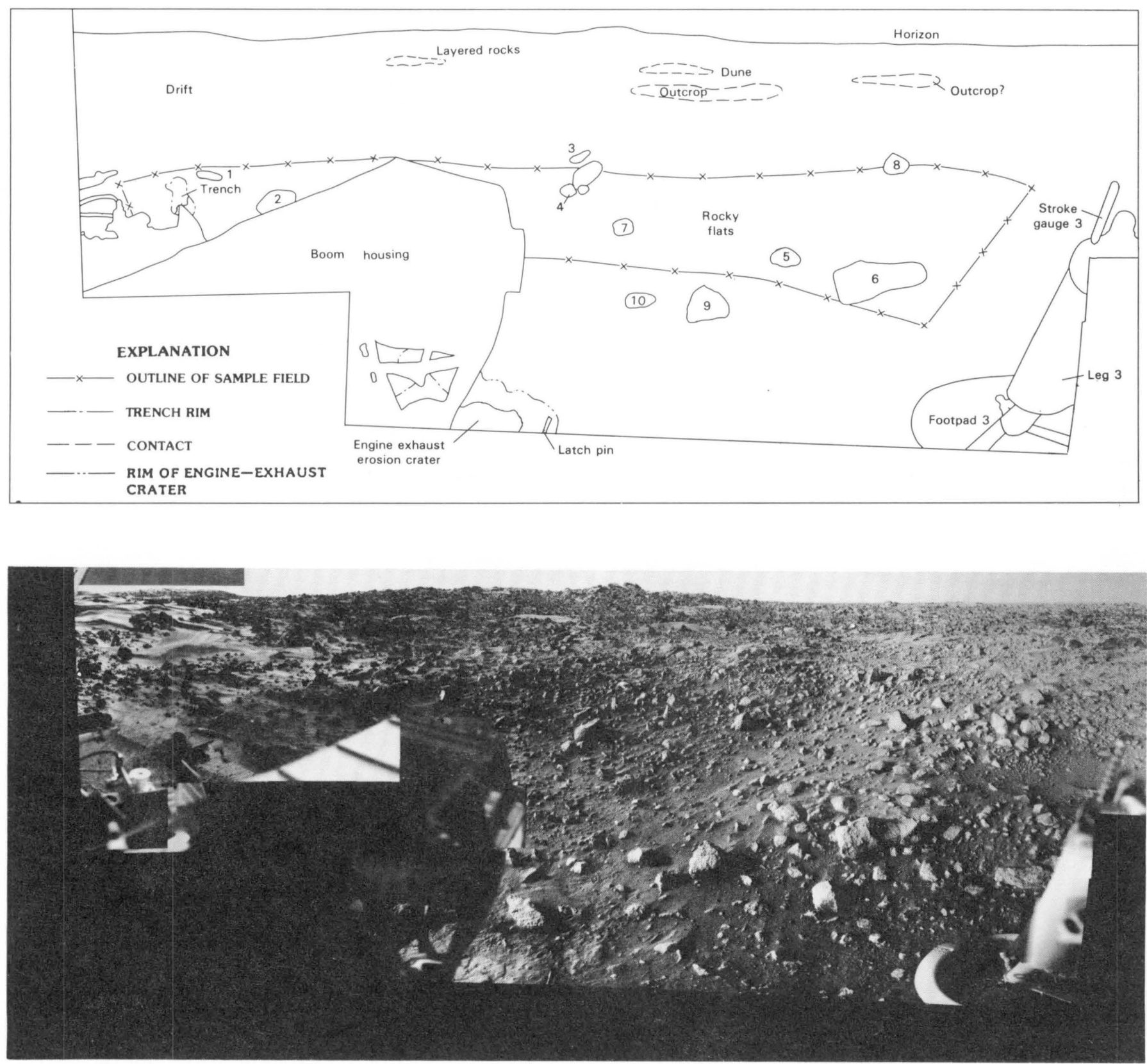

$B$

Figure 11.-Continued. 
uneven with shallow swales about 3-4 km wide and less and with broad, gentle bulges about the same size as the swales. These swales and bulges might be related to the dynamic deposition of ejecta from the crater Mie, the rim of which lay about $180 \mathrm{~km}$ to the east (fig. 10). Indeed, the mapped contact of the radial facies of ejecta from Mie crossed the probable locations of Lander 2 (Mutch and others, 1977), and theoretical calculations indicated that the ejecta reaching the site would be $3.4 \mathrm{~m}$ thick (McGetchin and others, 1973) (fig. 10). To the south of Lander 2, broad lobes with an intervening valley were radial to Mie. Westward, the surface again appeared to have swales and bulges, and some craters there appeared to be filled with some sort of a deposit. Farther west and
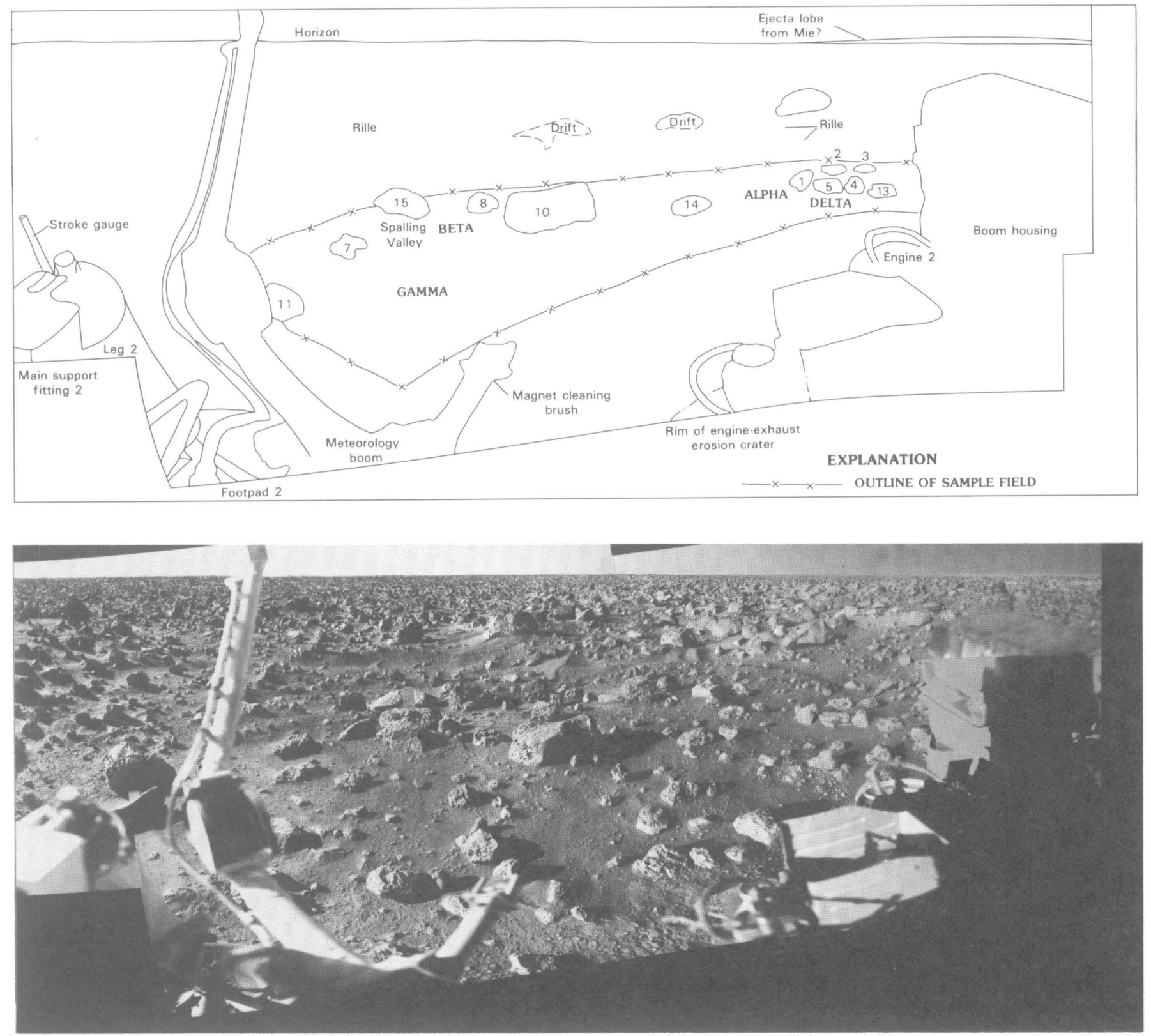

$\boldsymbol{A}$

Figure 12.-Panoramas and sketches of Lander 2 site showing abundant rocks. Rocks are generally larger than those at Lander 1 site. $A$, Mosaic of camera 1 pictures. $B$, Mosaic of camera 2 pictures. Sketches show locations of selected features in panoramas; rocks indicated by number correspond to numbered rocks shown on plate 2 ; names of sample site areas (Alpha, Beta, and so on) also correspond to those on plate 2. Panoramas include sample field (see sketches). Light strand on horizon may be mapped lobes of ejecta from crater Mie (see figs. 7 and 10). Note pitted surface of most rocks. Small rille in foreground, which has small drifts in it, may be surface expression of ice polygon. Mosaics were generated by computer from afternoon pictures (Levinthal and Jones, 1980). 
to the southwest, swales and bulges gradually disappeared, and giant polygonal forms, outlined by shallow depressions, appeared. Small craters about $400 \mathrm{~m}$ in diameter were present near Lander 2, but distances to them were not known.

\section{LANDER PANORAMAS}

The most striking aspects of the panoramic views of Mars obtained by both landers are the myriad of rocks strewn across the surface and the absence of many small craters. The martian surface, unlike the lunar surface, does not have the myriad of small craters (figs. 11 and 12). Additionally, at the Lander 1 site, large tracts of dunelike drifts appear to be superposed on a rocky substrate (fig. 11). Closer inspection of the Lander 1 pictures reveals other characteristics of the rocky surfaces and drifts (Binder and others, 1977). On the local horizon, rims of distant and near impact craters rise above the surroundings; large blocks of ejecta can be resolved on the rims of the closer ones. In other sectors, dunelike forms with crossbeds etched by wind erosion (Mutch and others, 1976a,b) lend a harsh, eerie aspect to the scene. Drifts
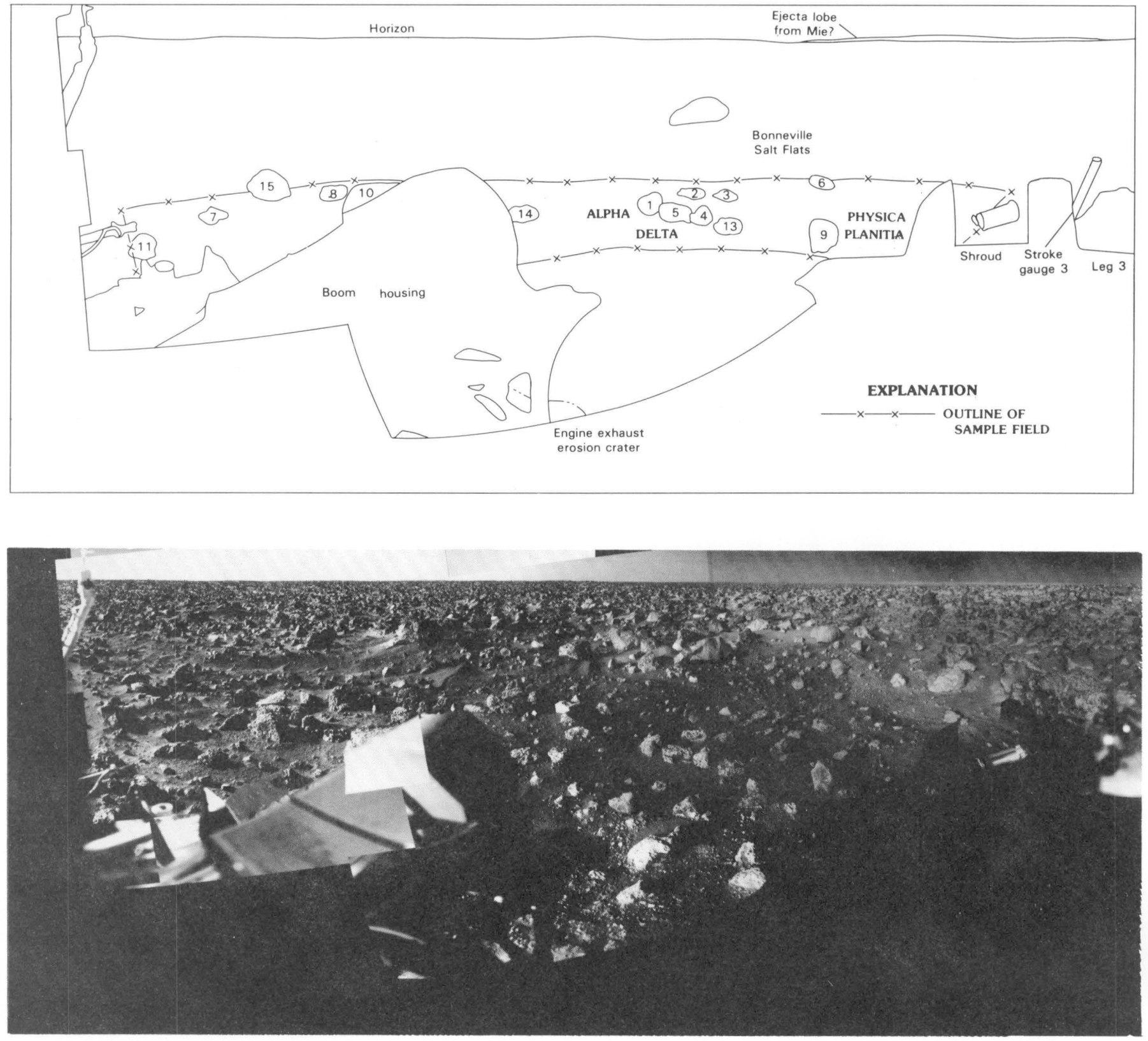

$B$

Figure 12.-Continued. 
are present as large-scale $(\approx 10 \mathrm{~m})$ complexes, individual dunes $(\approx 1-3 \mathrm{~m})$, isolated patches $(<1 \mathrm{~m})$, and wind tails on the lee sides of rocks.

There are a variety of rock sizes, shapes, and morphologies at the Lander 1 site. Sizes of rocks range from a few centimeters to as large as $3.0 \mathrm{~m}$. One of the larger rocks (informally called Big Joe), which is about $2 \mathrm{~m}$ wide, is 8-10 $\mathrm{m}$ to the left (northeast) of Lander 1 . Most of these rocks are angular and have coarsely pitted surfaces, but some rocks have smooth surfaces. Rock structures range from massive and homogeneous to layered. Several ventifacts, common rock forms in terrestrial deserts, are present. Some rocks are perched on the surface; other rocks are set in a matrix of surface materials. Local areas appear to be underlain by bedrock. Evidence for this includes the large area of exposure of units with continuous fracture patterns and the presence of dikelike ridges. Areas between rocks, where there are no drifts or wind tails, appear to be littered with small fragments or clods a centimeter in size and smaller.

At Lander 2 (fig. 12), the horizon is monotonous and flat although a block-strewn ridge rises behind the lander, and a distant smooth-appearing ridge may represent one of the ejecta lobes of the crater Mie (Mutch and others, 1976c). Drifts are both scarce and small. They are present as wind tails among rocks and in small rilles. Though the rocks appear to be generally larger than those at Lander 1, they show the same general characteristics. Most rocks are coarsely pitted and fluted, but some appear to be layered. Smooth ventifacts are present (Mutch and others, 1977); some are perched on the surface and others are set in a matrix of surface materials. Rock sizes near the lander range from a few centimeters to a meter in diameter.

Typically, areas between rocks appear to be littered with small fragments or clods that are a centimeter in size or smaller. Locally, smooth surfaces of crusts transected by fractures and mosaics of surface-material units are present among the rocks. Interconnected troughs about $1 \mathrm{~m}$ wide are unique to the Lander 2 site and may represent the surface expression of ice-wedge polygons. One of these troughs, just beyond the sample field, is partly filled by small drifts.

\section{SAMPLE FIELDS}

The sample field of Lander 1 appears to be representative of the site in general with the possible exception of outcrops of rock (fig. 11). Materials in the sample field can be grouped into three categories: (1) drift material, (2) blocky material, and (3) rocks (Moore and others, 1977, 1979). Drift material is present as a thick continuous unit in the left part of the sample field (fig. 11A). This area is informally called "Sandy Flats". Both the local relief and thickness of the drift material decrease in a clockwise direction from the left edge of the sample field where rocks are absent and the thickness of drift material is 16 to $20 \mathrm{~cm}$ (see pl. 1A). In the areas where drift material thins in a clockwise direction, rocks become evident, but the spaces between them are partly filled with drift material. Rather large wind tails of drift material are present on the leeward sides and deflation hollows on the windward sides of rocks, such as Sponge (rock 2), where the local relief of the wind tail is about $6 \mathrm{~cm}$. The deflation hollow on the windward side of Sponge, $3 \mathrm{~cm}$ deep, may represent the thickness of drift material there. Orientations of the wind tails of drift material associated with the rocks here and elsewhere in the sample field are consistent with a wind from $11.5^{\circ}$ clockwise of north (Sagan and others, 1977), but local ridges on the drift materials with a variety of northerly orientations in areas of abundant rocks indicate that swirls and eddies of the wind have modified the surface. Near the central part of the sample field, rocks dominate the scene, but small wind tails and thin patches of drift material are found between the rocks. Locally, patches with millimeter- to centimeter-size objects are present between the rocks where drift material has been stripped away. In the right-hand part of the sample field, local relief again increases about $20 \mathrm{~cm}$, and large rocks, set in a matrix of drift and blocky materials, are particularly abundant.

Blocky material, a name derived from its behavior during sampling, underlies drift material throughout the sample field. It is found at or very near the surface in an area informally called "Rocky Flats", in local patches elsewhere, and in the vicinity of footpad 3 (fig. $11 B$ ). In these areas, the surface between the rocks is covered by small lumps and objects a few millimeters to a centimeter or so in diameter. Apparently, these lumps and objects are residues left by the wind that has removed the overlying drift material. The blocky nature of the subjacent materials is not readily apparent because of the lumps and objects. Apparently, the contact between substantial thicknesses of drift material and the thinly veneered blocky material passes beneath the lander near engine 2 . As will be discussed later, exhaust gases from engine 2 exposed blocky material.

Rocks, with intermediate diameters $3.5 \mathrm{~cm}$ and larger, make up a significant part of the sample field and, as noted previously, are particularly abundant in the righthand part of the sample field (pl. $1 A$ ). They occupy about 8 percent of the area that includes the entire sample field and the region immediately in front of the lander (fig. 13). Most of this area is represented by rocks between 5 and $20 \mathrm{~cm}$ for which the area of rocks in each diameter (D) increment of $\sqrt{2} \mathrm{D}$ is about 1.7 percent. Cumulative frequencies increase rapidly from $0.15 / \mathrm{m}^{2}$ for rocks $>20 \mathrm{~cm}$ to $14 / \mathrm{m}^{2}$ for rocks $>3.5 \mathrm{~cm}$ (fig. 14). Incremental frequen- 


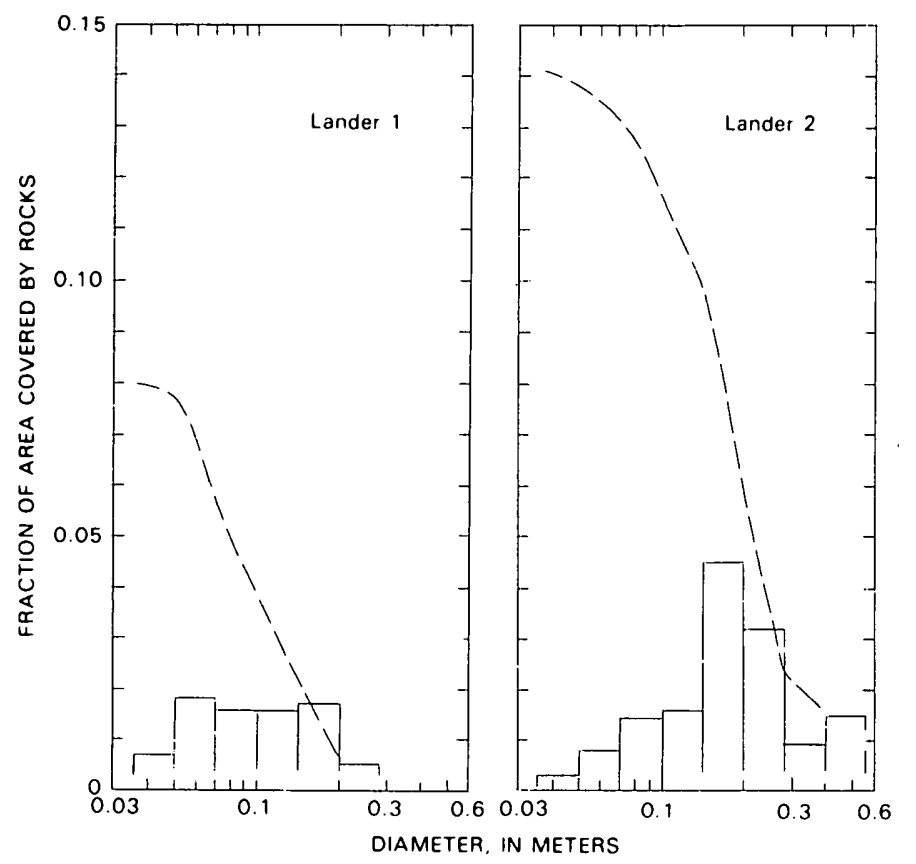

FiguRE 13.-Fraction of area covered by rocks with intermediate diameters larger than $3.5 \mathrm{~cm}$ for the sample fields of Lander 1 and Lander 2. Data for plots obtained from 1:10 scale maps of sample fields. Cumulative frequencies shown by dashed line.

cies in $\sqrt{2} \mathrm{D}$ bins also increase rapidly to the 5 - to $7-\mathrm{cm}$ size interval but those $3.5-5 \mathrm{~cm}$ in diameter are less frequent than those in the next larger interval. The two largest rocks (Metate 1 and 2) measure $22 \times 31 \times 18 \mathrm{~cm}$ and $15 \times 41 \times 15 \mathrm{~cm}$, respectively. Several others are comparable in size.

The sample field of Lander 2 also appears to be reasonably representative of the site in general (pl. $2 A$ ). Although there are no thick deposits of drift material in the sample field, drift material is present as fillets at the bases of some rocks, as thin veneers between rocks, and as small wind tails (fig. 12). The most abundant materials here are crusty to cloddy material and rocks. In contrast with the blocky material at Lander 1, evidence for crusty to cloddy material abounds at the surface. Most of the areas among the rocks have been stripped of fine-grained material. This produces two kinds of surfaces: knobby, lumpy surfaces that are perhaps more extensive in the left part of the sample field and light-colored surfaces of crusts and clods that are more extensive in the right part of the sample field. Particularly good occurrences of knobby and lumpy surfaces are present near the bases of rocks such as rock 15 (Doc) and rock 10 (Centaur). The light-colored surfaces of crust and clods are extensive in an area informally called "Physica Planitia". The crust exposed in "Bonneville Salt Flats" (near rock 6) is particularly striking. Here, the exposed crust, which is approximately $40 \mathrm{~cm}$ wide, is transected by open fractures. Elsewhere, such as the base of rock 1 (ICL), the crusty to cloddy material has been etched by the wind revealing fractures that outline units several centimeters wide. Crusty to cloddy material underlies the local patches of drift material and the knobby and lumpy surfaces and abuts against the rocks.

Rocks with intermediate diameters $3.5 \mathrm{~cm}$ and larger form a larger part of the sample field than at Lander 1 and are distributed more or less evenly throughout the sample field except in Physica Planitia in the right-hand part where they are rare ( $\mathrm{pl} .2 A$ ). About 14 percent of the sample field is occupied by rocks, and roughly 7.8 percent of the area is littered with rocks having diameters between 14 and $28 \mathrm{~cm}$ (fig. 13). Cumulative frequencies increase rapidly to $2.6 / \mathrm{m}^{2}$ at diameters of $14 \mathrm{~cm}$, but below this size the increase is less rapid. At a diameter near $5.5 \mathrm{~cm}$, cumulative frequencies of rocks are about the same as at the Lander 1 site, but above this size rocks are more frequent (fig. 14). The incremental distribution is bimodal with a peak of about $2.4 / \mathrm{m}^{2}$ in the diameter interval of $4.8-7.0 \mathrm{~cm}$ and about $1.7 / \mathrm{m}^{2}$ in the interval from 14 to $28 \mathrm{~cm}$. The largest rock in the sample field (Centaur, rock 10 ), roughly $40 \times 60 \times 23 \mathrm{~cm}$, is clearly larger than those in the sample field of Lander 1.

\section{LANDING}

The first information on the physical properties of martian surface materials for each site was the result of the landing and pictures taken soon after landing. In the pictures, the interactions of the footpads with the surface and the response of the surface materials to the engineexhaust gases could be seen, measured, and interpreted. In this section, data on footpad interactions and engineexhaust erosion are: (1) described for Mars and tests on Earth, (2) interpreted in terms of the physical properties of surface materials on Mars, and (3) discussed. For continuity and completeness of information, the section begins with a description of the last part of the descent to the surface.

\section{DESCENT}

Descent trajectories of both landers were nearly vertical and both passed over the sample fields (fig. 15, pls. 1 and 2). Ten seconds before touchdown, Lander 1 was $27 \mathrm{~m}$ above the surface traveling in the direction of leg 1 (in the landed configuration) and the body center was approximately $1.5 \mathrm{~m}$ uprange from its final location. Thus, the sample field was exposed to engine-exhaust gases to a greater degree than it would have been if the lander had approached in any other direction. One second before touchdown, Lander 1 was descending at $2.44 \mathrm{~m} / \mathrm{s}$ and moving about $0.15 \mathrm{~m} / \mathrm{s}$ in a horizontal direction toward leg 1. A linear least squares fit to velocity increments measured by the inertial reference unit during the last $2 \mathrm{~s}$ indicates a touchdown velocity of $2.30 \mathrm{~m} / \mathrm{s}$. Roll about 


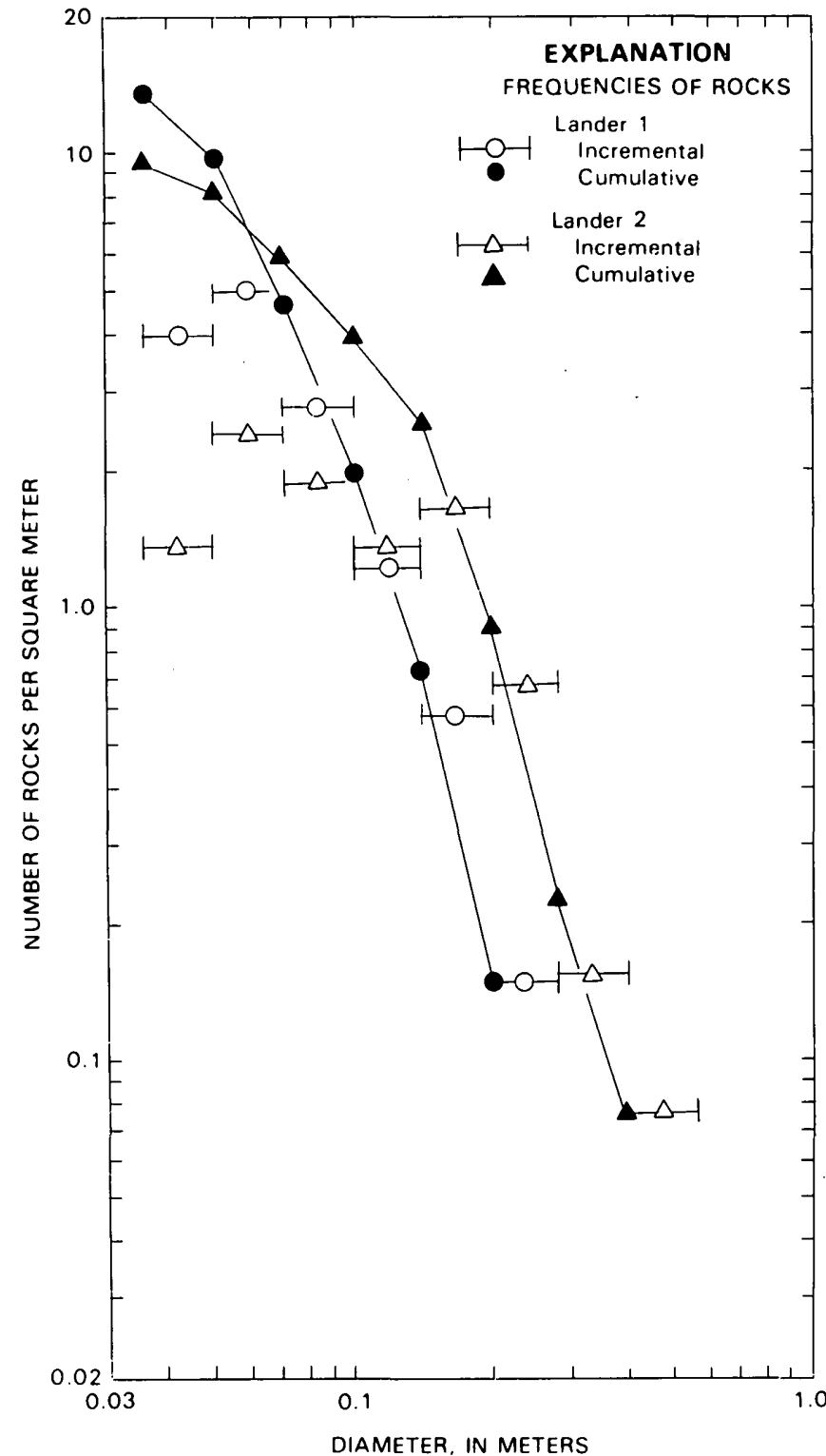

FIGURE 14.-Cumulative and incremental frequencies of rocks with intermediate diameters larger than $3.5 \mathrm{~m}$ for sample fields of Lander 1 and Lander 2. Data for plots obtained from 1:10 scale maps of sample fields.

the vertical axis was only $0.25 \%$ s. Inflight tilt at touchdown required that footpad 2 was about $1 \mathrm{~cm}$ lower and footpad 1 was about $1.5 \mathrm{~cm}$ higher than footpad 3 . When this information was combined with local surface tilt and topography, footpad 2 touched the surface first, followed by footpad 3 , and then footpad 1 .

Ten seconds before touchdown, Lander 2 was about $29 \mathrm{~m}$ above the surface and about $1.7 \mathrm{~m}$ uprange of its final location. It too passed over the sample field traveling in the direction of leg 1 . Lander 2 was descending at $2.44 \mathrm{~m} / \mathrm{s} 1 \mathrm{~s}$ before touchdown. A linear least-squares fit to velocity increments from the inertial reference unit dur-

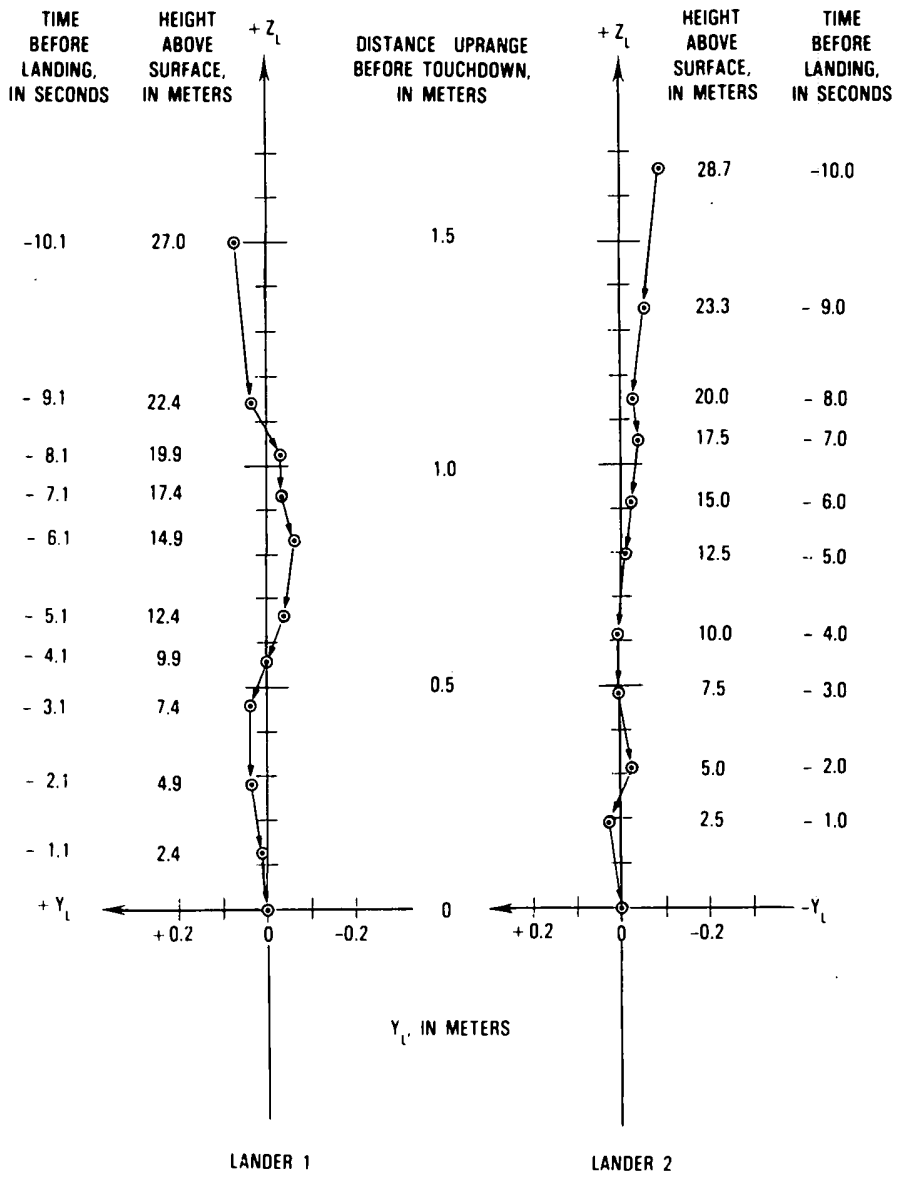

FIGURE 15.-Approximate traces of flight paths and trajectories of Landers 1 and 2 during final $10 \mathrm{~s}$ of descent to martian surface. Plane of projection is the spacecraft (LSCS) $Z_{L}-Y_{L}$ plane. Dots represent locations of spacecraft centers for 1-s intervals (see pls. 1 and 2 for locations). Numbers indicate time before landing and heights of centers above $Z_{L}-Y_{L}$ surface.

ing the last $2 \mathrm{~s}$ indicates a touchdown velocity of $2.34 \mathrm{~m} / \mathrm{s}$. Engine thrust levels increased during the last $0.4 \mathrm{~s}$ and probably reduced the velocity from $2.34 \mathrm{~m} / \mathrm{s}$ to a value between 1.85 and $1.95 \mathrm{~m} / \mathrm{s}$. This is discussed below in the section under Lander 2. At touchdown, the inflight tilt indicated that footpad 1 was $3.5 \mathrm{~cm}$ lower and footpad 2 was $7 \mathrm{~cm}$ higher than footpad 3 ; but local surface tilt and topography indicate that footpad 3 should have touched the surface first, followed by footpad 1 and then footpad 2 . This sequence could have been altered by the increased thrust levels during the last $0.4 \mathrm{~s}$.

Reconstructions of the flight paths show that nearly vertical descents were achieved (fig. 15). Average inclinations of the trajectories were about $3^{\circ}$ from the vertical but for short intervals of time these inclinations reached about $4.5^{\circ}$. The flight paths were reconstructed using data from the inertial reference units and terminal-descent landing radars, which were processed by the guidance control and sequencing computers; these computers also provided the orientation of the spacecraft with respect to the gravita- 
tional vector, spin rates about the axes, velocities along the spacecraft $X, Y$, and $Z$ axes, and time (Villyard and Ivers, 1978). The reconstructions are reasonably correct but not exact. They were prepared by computing displacements from components of in-flight velocities in the $Z_{L}$, $Y_{L}$, and $X_{L}$ directions for 1-s intervals starting from the time of landing and then to $10 \mathrm{~s}$ before landing. In-flight tilts about the $Z_{L}, Y_{L}$, and $X_{L}$ axes were usually within $2^{\circ}$ or less, and the direction of leg 1 was essentially constant. Indeed, the actual azimuths of leg 1 on the surface were within about $1.6^{\circ}$ (Lander 1 ) and $1.0^{\circ}$ (Lander 2) of the targeted azimuths (Martin Marietta Corp., 1976a). Projection of the flight-path traces from the $Z_{L}-Y_{L}$ plane to the surface would be displaced in the direction of tilt 1 to $2 \mathrm{~cm}$ for Lander 1 and 13 to $14 \mathrm{~cm}$ for Lander 2 .

\section{TOUCHDOWN}

The amount of footpad penetration into the surface materials during landing depends, in part, on the physical properties of the materials. When a knowledge of the penetration of the footpads is combined with data on the lander and landing conditions, mechanical properties of the surface materials may be estimated provided that there are no complicating factors. Analyses of footpad penetrations and landing data for Lander 1 show that there are two markedly different soillike materials at the site (Moore and others, 1977), and estimated vertical forces $^{2}$ on the footpads were consistent with the mechanical properties of the two materials obtained from surface-sampler data. For Lander 2, interactions of the footpads with rocks introduce complications that preclude sensible analyses of the mechanical properties of the surface materials. Interactions of the footpads of Landers 1 and 2 with the surface materials are described and interpreted below. Interpretations require that estimates of the maximum vertical forces on the footpads be made; the procedures used for estimating these forces are described later in the supplemental section on "Analysis of Landing Data."

\section{LANDER}

The first picture of the martian surface taken by Lander 1 included footpad 3 (fig. 16A). In the picture, the upper part of the footpad is visible and small amounts of material are present on it. The area of blocky material surrounding the footpad is littered with debris, clods, and rocks with diameters less than the limit of resolution

${ }^{2}$ The term vertical forces or vertical force is used loosely in this section to refer to forces on force parallel to the $X_{L}$ axis which was within a few degrees of vertical. $(\approx 0.07 \mathrm{~cm})$ to diameters about $7.0 \mathrm{~cm}$. Judging from shadows cast by the footpad in a number of pictures, footpad 3 penetrated blocky material to approximately $0.036 \mathrm{~m}$ (table 6). Evidence for deformation of the surface, such as open fractures and displaced wedges of cohesive material, is nonexistent, but such deformations could be present and hidden by the skirt of the footpad. According to both the stroke gauge and estimates of footpad travel, the amount of stroke of leg 3 is $0.083 \mathrm{~m}$ (table 6). The response of drift material to footpad 2 during landing was entirely different from that of blocky material to footpad 3. As shown in figure $16 B$, footpad 2 is completely buried and the base of the footpad is $0.165 \mathrm{~m}$ below the surface. A circular vertical scarp, produced by down-faulting of material on the footpad side, is present at distances of about $0.2 \mathrm{~m}$ from the perimeter of the buried footpad (pl. 1A). Within the circular scarp, lumpy masses of drift material fill the footpad to levels above the juncture of the primary and secondary struts. Beyond the scarp the deformed surface forms small stubby arcuate anticlines. A sinuous monocline, which may have resulted from footpad penetration, extends toward the right from the scarp (pl. $1 A$ ). The amount of stroking of leg 2 is smaller than that of leg 3 . According to the gauge, stroke is $0.032 \mathrm{~m}$; and, according to footpad travel, stroke is $0.028 \mathrm{~m}$. Footpad 1 is obscured by the lander body so that its penetration is unknown; but, the stroke gauge of leg 1 is visible. The amount of stroke of leg 1 is $0.07 \mathrm{~m}$.

Significantly different mechanical properties for drift material and blocky material are indicated because the deep penetration of footpad 2 is accompanied by a smaller stroke than that of footpad 3 , which penetrated less. The velocities of the two footpads should also have been nearly the same although footpad 2 touched down first.

In order to compare the forces and penetrations of the Lander 1 footpads with static tests of footpads on Earth, vertical forces on the footpads must be estimated. In the procedure for estimating the forces, the total work done in deformation of the primary shock absorbers, frictional sliding of the footpads, penetrations of the footpads into the surface materials, and the decaying engine thrusts from surface contact to the final rest position become equal to the kinetic energy of the spacecraft at surface contact plus the change of potential energy from surface contact to the final resting position when the effective coefficients of sliding friction for the footpads range from 0.20 to 0.5 . If these coefficients and other data are used, vertical forces on the footpads are $6.7-8.1 \mathrm{kN}(1), 4.4-5.6$ $\mathrm{kN}(2)$, and 7.5-8.9 kN (3). Because of uncertainties in the analyses, vertical forces for an effective coefficient of zero are also used. These forces are $6.2 \mathrm{kN}(1), 3.8 \mathrm{kN}(2)$, and $7.0 \mathrm{kN}(3)$. Comparison of these forces and the corresponding penetrations with results from static tests of a $3 / 8$-scale footpad in three surface-material simulants 


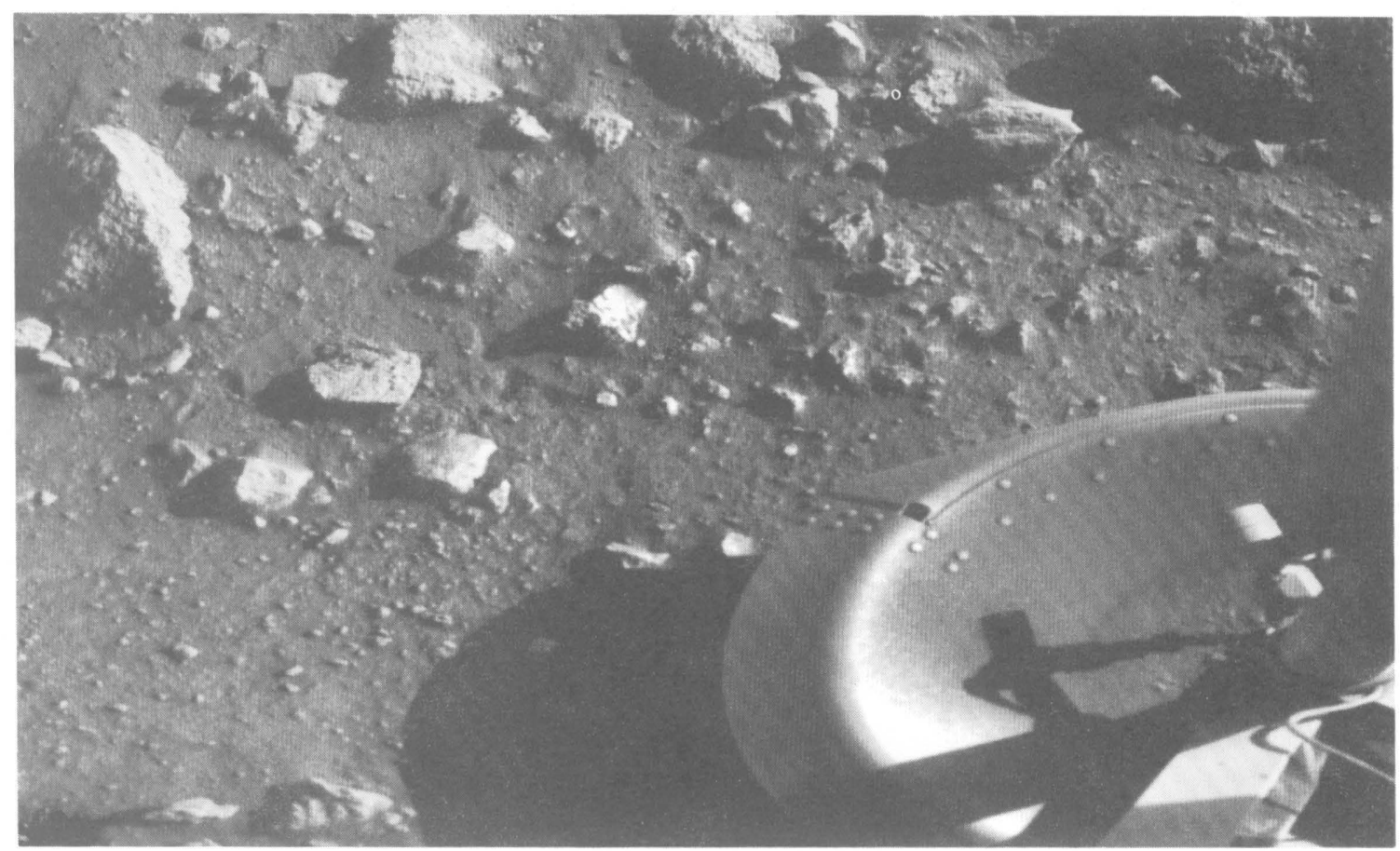

A

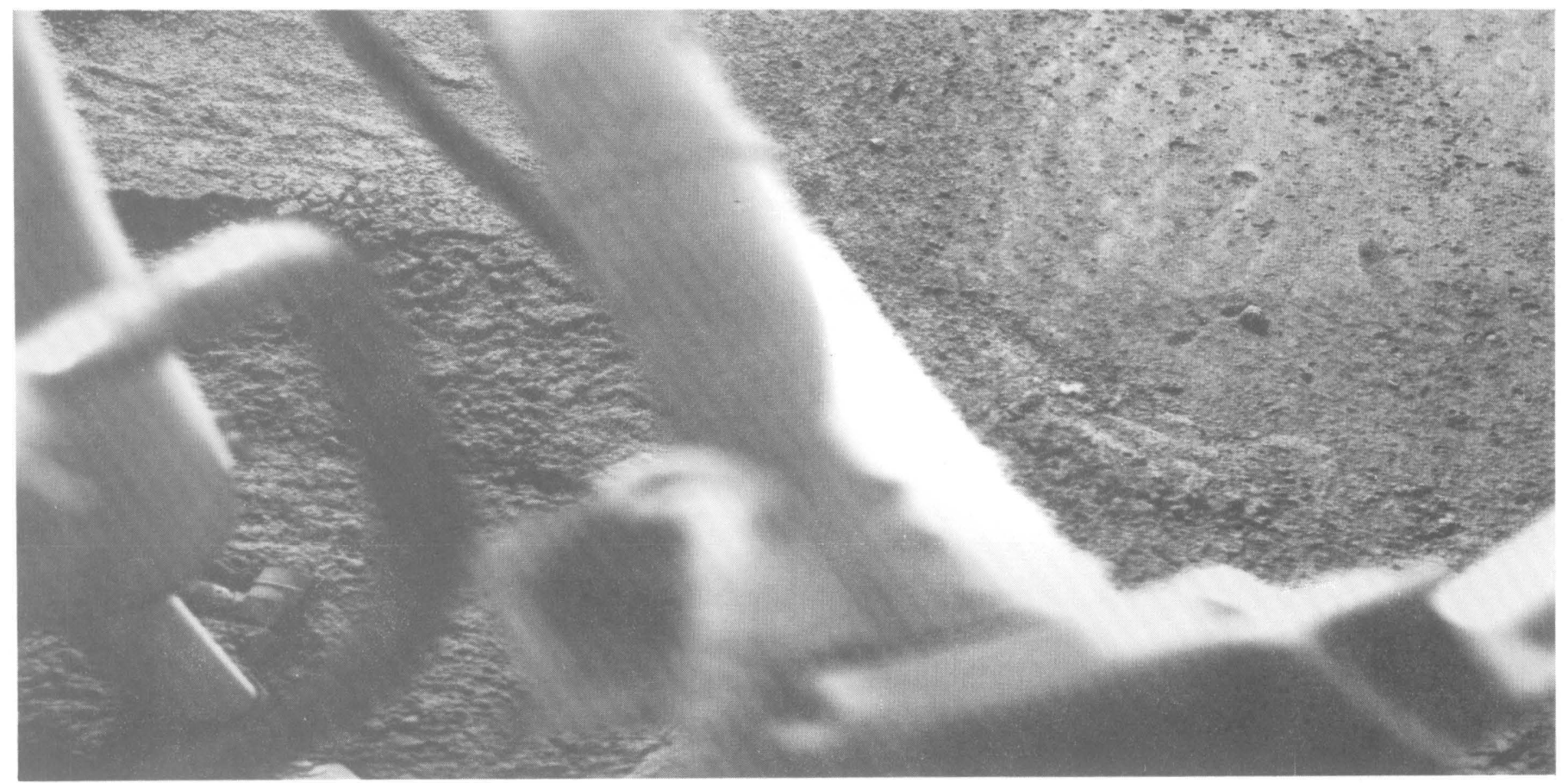

$\boldsymbol{B}$

Figure 16.-Penetration of footpads 3 and 2 of Lander 1. A, Footpad 3 penetrated about $0.036 \mathrm{~m}$ into blocky material and is unburied. Note surface is littered with small rocks and clods in contrast with drift material in figure $16 B$. Rock at upper left is $8 \mathrm{~cm}$ wide (frame $12 \mathrm{~A} 001 / 000) . B$, Primary strut at left slants down toward center of footpad 2, which is buried in drift material; base of footpad is about $0.165 \mathrm{~m}$ below surface. Light-gray meteorology boom (near center) and post of magnet cleaning brush (at right) converge to form a $\mathrm{V}$-shape in picture. Note arcuate scarp, $0.30 \mathrm{~m}$ long, produced by faults (between primary strut and meteorology boom) at upper left; small concentric depression below scarp is surface expression that outlines the buried rim of footpad. Note monoclinal folds of surface (between meteorology boom and post of brush and in lower right corner) and open fractures (in lower right corner) (frame 11A079/012). 
TABLE 6.-Touchdown conditions and landing data for Landers 1 and 2 [See also section on "Analysis of landing data"]

\begin{tabular}{lll} 
& Lander 1 & Lander 2 \\
\hline Vertical velocity (m/s) & & \\
Inertial reference unit & 2.30 & 2.34 \\
Engine thrusts & 2.44 & $1.85-1.95$ \\
Horizontal velocity (m/s) & 0.11 & 0.19 \\
Footpad 1 penetration (m) & - & - \\
Leg stroke (m) & .070 & $.025-0.032$ \\
Footpad 2 penetration (m) & .165 & .025 \\
Leg stroke (m) & $.032(0.028)$ & $.076(0.076)$ \\
Footpad 3 penetration (m) & .036 & $0-0.003$ \\
Leg stroke (m) & $.083(0.083)$ & $.013(0.013)$ \\
& & \\
\hline 1Numbers in parentheses are estimates from footpad travel; \\
others are from stroke gauge.
\end{tabular}

(table 7), appropriately scaled for the Viking lander on Mars (Moore and others, 1977), shows the contrasting difference in the penetration resistances of drift material, blocky material, and the simulants (fig. 17). Blocky material is stronger than the three simulants because the force and penetration for footpad 3 of Lander 1 lie to the upper left of the curves for the three simulants. Drift material is weaker and more penetrable than the three simulants because the force and penetration for footpad 2 of Lander 1 lie to the lower right of the curves for the three simulants. The data for the static tests stop at penetrations of about $0.07 \mathrm{~m}$; for penetrations between 0.07 and $0.099 \mathrm{~m}$, the cross-sectional area of the footpad changes in a complicated way (see fig. 2). When the penetration exceeds $0.099 \mathrm{~m}$, the full cross-sectional area presented by the penetrating footpad is constant $\left(0.135 \mathrm{~m}^{2}\right)$ so that the curves of the static data cannot necessarily be extrapolated in a simple way.

Vertical forces of footpads 2 and 3 were equated to static forces in the standard-bearing capacity equation for circular footings on horizontal surfaces (Scott, 1963;

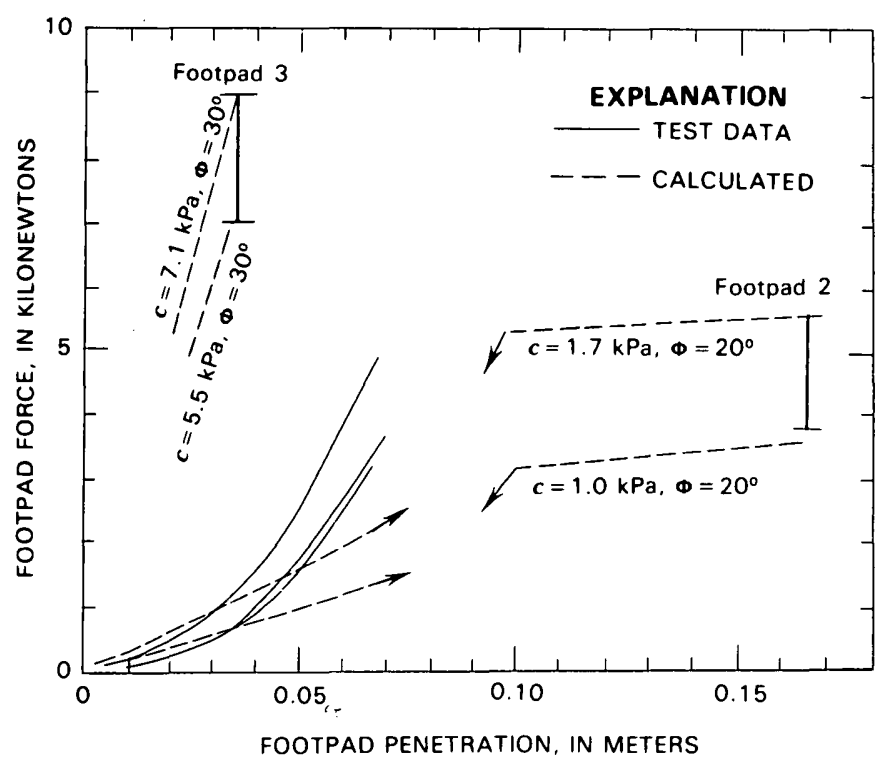

FIGURE 17.-Forces on footpads and footpad penetrations of Lander 1. Vertical I-bars indicate estimated forces and penetrations of footpads 3 and 2 during landing. Calculations of forces on footpads 3 and 2 based on assumed coefficients of sliding friction of $0-0.5$ (see supplemental section on "Analysis of landing data"); penetrations were obtained from Lander photographs. Solid lines indicate static forces on footpad and footpad penetration scaled to Mars conditions from $3 / 8$-scale tests. Dashed lines indicate static-bearing forces for materials with indicated cohesions $(c)$ and angles of internal friction $(\phi)$ computed using static-bearing capacity equation for circular footings, failure by general shear, and densities of $1,200-2,000$ $\mathrm{kg} / \mathrm{m}^{3}$. Space between arrows on dashed lines represent penetrations of footpad 2 where cross-sectional areas change in a complicated way because of footpad skirt (see fig. 2).

TABLE 7.-Mechanical properties of surface-material simulants used in footpad tests on Earth

[Lunar nominal and basalt dune sand were also used as surface-material simulants in the tests of erosion by the exhaust gases from the terminal-descent engines (Romine and others, 1973)]

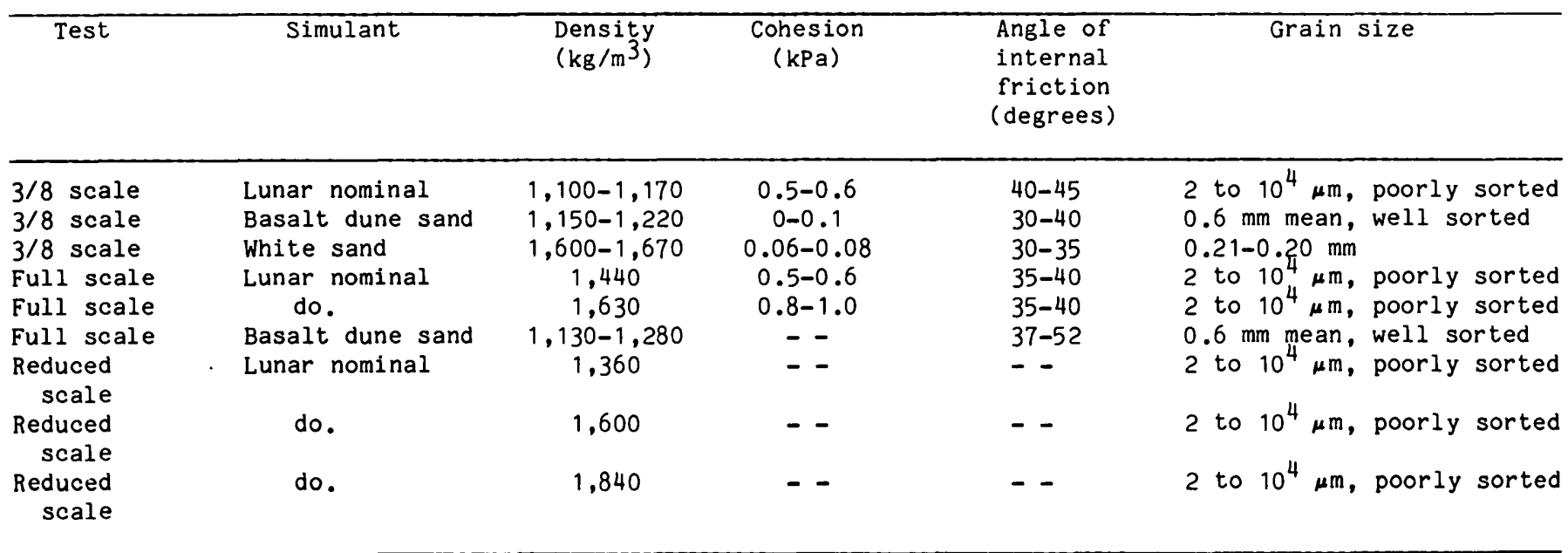


Terzaghi, 1948) in order to estimate the cohesions and angles of internal friction of drift and blocky materials (see supplemental section on "Analysis of landing data"). We found that the static forces for footpad 2 were consistent with a continuum of materials that fail by general shear and have a density of $1,200 \mathrm{~kg} / \mathrm{m}^{3}$ when the depth of footing was taken as $0.066 \mathrm{~m}$. At one extreme, the angle of internal friction was $0^{\circ}$ and the cohesion was between 4 and $6 \mathrm{kPa}$; at the other extreme, the cohesion was 0 and the angle of internal friction was about $32^{\circ}$ or $33^{\circ}$. On the basis of surface-sampler data to be discussed later, a material failing by general shear with a cohesion between 1.0 and $1.7 \mathrm{kPa}$, a density of $1,200 \mathrm{~kg} / \mathrm{m}^{3}$, and an angle of internal friction of $20^{\circ}$ is a reasonable model for drift material. Cohesions of material that fail by local shear become about 0.6 times smaller than materials that fail by general shear. Much stronger materials are required to account for the forces on footpad 3. Three models, based on failure by general shear for which staticbearing forces for circular footings (area $=0.028 \mathrm{~m}^{2}$ ) at 0 depth were equal to the forces during landing are:

$\begin{array}{cccc}\text { Model } & \text { Angle of } & & \\ 1 & \text { internal friction } & \text { Cohesion } & \text { Density } \\ 1 & 25 & 8.7-11.2 \mathrm{kPa} & 1,200-2,000 \mathrm{~kg} / \mathrm{m}^{3} \\ 2 & 30 & 5.5-7.1 \mathrm{kPa} & 1,200-2,000 \mathrm{~kg} / \mathrm{m}^{3} \\ 3 & 35 & 3.5-4.7 \mathrm{kPa} & 1,200-2,000 \mathrm{~kg} / \mathrm{m}^{3}\end{array}$

In these models, a density as large as $2,000 \mathrm{~kg} / \mathrm{m}^{3}$ is considered, on the basis of estimates of fragment densities in the XRFS chamber (Clark, B.C. and Weldon, R.J., written commun., 1980). Of the three models, model 2 is preferred because it agrees with the interpretation of surface-sampler data that is to be discussed later.

Comparison of footpad penetrations with dynamic tests on Earth are compatible with the analysis above. The dynamic tests of full-scale and reduced-scale footpads were performed because of the importance of two factors: the geometry of the penetrating object and positive poregas pressures resulting from compression of fine-grained materials during dynamic loading. A third factor that was not tested is the effect of acceleration of gravity on the behavior of the test materials. The effect of acceleration of gravity on footpad deceleration, however, was simulated. Full-scale tests of prototype footpads with skirts (table 8 ) were conducted in lunar nominal test materials with two bulk densities and basalt dune sand (table 7) at velocities of $2.44 \mathrm{~m} / \mathrm{s}$ in 1 atm of air (Martin Marietta Corp., 1971). In one test, penetration into lunar nominal material with a density of $1,440 \mathrm{~kg} / \mathrm{m}^{3}$ (run 14) was an astonishing $0.265 \mathrm{~m}$ and leg stroke was a mere $3 \mathrm{~mm}$ (fig. 18). This test result prompted an investigation of penetrations by reduced-scale footpads in both 5- and 1,000-mbar ambient pressures with lunar nominal test beds at densities estimated to be $1,360,1,600$, and 1,840 $\mathrm{kg} / \mathrm{m}^{3}$ (tables 7 and 8) (Clark, L.V., 1971). These tests at reduced pressures showed that penetrations were functions of the ambient pressure and density of the lunar nominal test material.

When data from the reduced-scale tests at reduced pressures were scaled to the dimensions of the Viking footpads, the velocity at touchdown, and a mass of $200 \mathrm{~kg}$ (about $1 / 3$ the mass of the spacecraft) using an equation developed for low-velocity impact (see fig. 18, Clark and McCarty, 1963), the results implied penetrations of about $0.15 \mathrm{~m}$ for a density of $1,360 \mathrm{~kg} / \mathrm{m}^{3}$ and $0.09 \mathrm{~m}$ for a density of $1,840 \mathrm{~kg} / \mathrm{m}^{3}$. Calculations for run 14 , adjusted for ambient pressure (Clark, L.V., 1971), indicated a penetration of almost $0.195 \mathrm{~m}$ (fig. 18). Penetrations of 0.15 and $0.195 \mathrm{~m}$ are in fair agreement with the actual penetration of footpad 2 on Mars. Thus, it does not seem that an extremely low-density material is required to explain the penetration of footpad 2 in drift material. A lunar nominal material with a density of about $1,300-1,500$ $\mathrm{kg} / \mathrm{m}^{3}$ would account for the large penetration. Acceleration of gravity (fig. 18) may also have some effect on the penetration. if the cohesion of the surface material is low enough, but this would increase the penetration by a factor of 1.14 (Pyrz, 1969) or less, depending on the cohesion. Footpad 3 penetration is consistent with lunar nominal material with a density of about $2,300 \mathrm{~kg} / \mathrm{m}^{3}$ because the extrapolated lines for the scaled Viking conditions on Mars indicate a penetration of $0.036 \mathrm{~m}$ at that density (fig. 18). In view of the large estimated fragment densities $\left(1,880-2,340 \mathrm{~kg} / \mathrm{m}^{3}\right)$ in the chamber of the X-ray fluorescence spectrometer, a large density for blocky material seems possible. However, the small penetration of footpad 3 could be the result of a large cohesion resulting from cementation or other induration processes and independent of density.

Although footpad penetrations and leg strokes of Lander 1 show that the two materials at the site have markedly differing mechanical properties, data on footpad penetration cannot be used to conclusively resolve the mechanical properties of drift material. It is entirely possible that a hard substrate or buried rock stopped penetration because drift material is superposed on blocky

TABLE 8.-Generalized conditions for footpad penetrations during tests on Earth and Mars

\begin{tabular}{|c|c|c|c|c|}
\hline \multirow{2}{*}{$\begin{array}{c}\text { Test } \\
\text { Parameter }\end{array}$} & \multicolumn{2}{|c|}{ Earth } & \multicolumn{2}{|c|}{ Mars } \\
\hline & $\begin{array}{c}\text { Full-scale } \\
\text { tests }\end{array}$ & $\begin{array}{l}\text { Reduced- } \\
\text { scale } \\
\text { tests }\end{array}$ & Lander 1 & Lander 2 \\
\hline $\begin{array}{l}\text { Maximum projected area } \\
\text { of footpad, m² } \\
\text { Mass, } \mathrm{kg} \\
\text { Velocity, m/s. } \\
\text { Pressure, mbar }\end{array}$ & $\begin{array}{c}0.151 \\
377 \\
2.44 \\
1.000\end{array}$ & $\begin{array}{c}0.092 \\
52.2204 \\
3.48 \\
57\end{array}$ & $\begin{array}{l}0.135 \\
204 \\
2.31 .9 \\
9\end{array}$ & 0.135 \\
\hline
\end{tabular}


material and thins to a centimeter or so in the vicinity of rock 2 (pl. $1 A$ ); however, no such difficulty occurred when excavating a trench $22 \mathrm{~cm}$ deep (Deep Hole 1) at $0.5 \mathrm{~m}$ from footpad 2 . When the coarse fraction from samples in the deep hole was purged, no blocky material was found; two $15-\mathrm{cm}$ rocks were excavated, however.

LANDER 2

The landing of Lander 2 was more complicated than

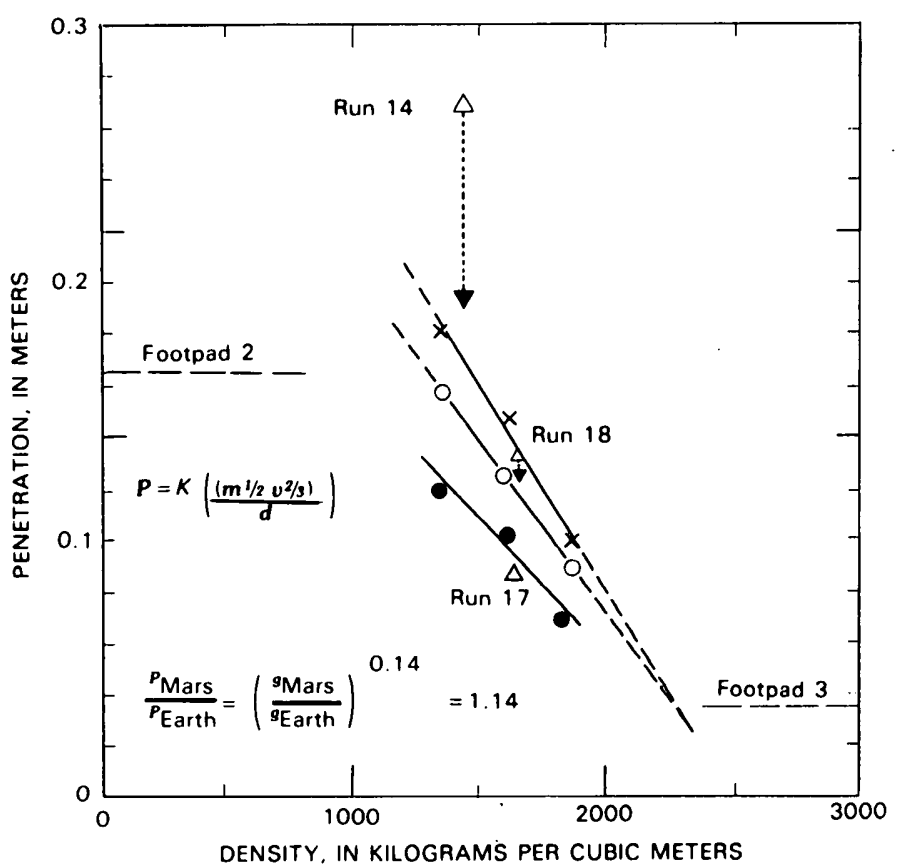

EXPLANATION

FULL-SCALE TEST OF PROTOTYPE FOOTPAD-Dotted line joins full-scale tests with corrected values of penetration

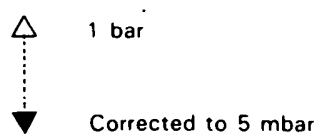

- ReduCED-SCALE TEST OF PROTOTYPE FOOTPAD $\cdots 5$ mbar SCALED TESTS Scaled to

- Viking Lander 1

$\times \quad$ Viking Lander 1 and Mars gravity (3/8g)

- PENETRATION DEPTH Footpad 2 and 3

FIgURE 18.-Density-penetration curves of full-scale and reduced-scale tests of Viking prototype footpads on Earth compared to penetrations of footpads 3 and 2 of Viking Lander 1 on Mars. Reduced-scaled tests were adjusted to full scale by using low-velocity impact penetration equation relating penetration $(P)$ to mass $(m)$, velocity $(v)$, and diameter (d) (Clark and McCarty, 1963). The parameter $K$ is related to the properties of the soillike materials used in the tests. Equation for effect of gravity $(g)$ on penetration is from Pyrz (1969). Adjustment of full-scale tests for reduced atmospheric pressure is from data of Clark (1971). Solid and long-dashed lines indicate trends of data. that of Lander 1, so the analyses of forces on the footpads of Lander 2 cannot be treated as they were for Lander 1 . The first picture taken by Lander 2, which includes footpad 3 , shows a rock adjacent to the footpad. The rock appears to have been overturned (fig. 19A). Thus, footpad 3 appears to have struck the rock during landing. A small penetration $(0-0.003 \mathrm{~m})$ was accompanied by a small leg stroke $(0.013 \mathrm{~m})($ table 6$)$. Footpad 2 also appears to have struck a rock (fig. 19B). Evidence for this is two-fold. First, an arcuate scarp with intervening disturbed material is present on the sample field side of the rock. Such a displacement of the rock would have been produced by a footpad and lander moving in the direction of leg 1 during landing. Second, open fractures near the footpad appear related to the rock rather than to the footpad (fig. 19B). Thus it appears that the rock transmitted the footpad forces to the surface material. Judging from shadows cast by the footpad, penetration of the footpad was $0.025 \mathrm{~m}$. Leg stroke was $0.076 \mathrm{~m}$ (see table 6). Again, footpad 1 cannot be seen but the leg stroke was between $0.025-0.032 \mathrm{~m}$.

A comparison of the leg strokes of the two landers indicates that the average leg stroke of Lander 1 was larger than that of Lander 2. Strokes of legs 2 and 3 determined from stroke gauges and leg travel for Lander 2 are the same, and the strokes imply that the load limiters at the secondary strut attachment on the lander body did not yield, so that energy dissipation in the landing gear was essentially due to irreversible crushing of the primary shock absorbers. For Lander 1, the work performed against the primary shock absorbers, 924-948 J, accounts for about 52-57 percent of the initial kinetic energy at surface contact, but the work performed against the primary shock absorbers of Lander 2 is almost half (487-525 J) that of Lander 1 (see supplemental section on "Analysis of Landing Data").

This problem can be resolved by considering data on valve settings of the descent engines just prior to touchdown. During the last $0.43 \mathrm{~s}$ before touchdown, thrust levels of engines 1,2 , and 3 increased by about 14, 81, and 91 percent, respectively (Martin Marietta Corp., 1976a). The impulse, which was about 1,360 N-s, produced an incremental velocity change of about 0.49 $\mathrm{m} / \mathrm{s}$. This makes the velocity of Lander 2 at surface contact about $1.85-1.95 \mathrm{~m} / \mathrm{s}$, and this velocity is smaller than the velocity measured by the inertial reference unit of $2.34 \mathrm{~m} / \mathrm{s}$ and nominal descent value of $2.44 \mathrm{~m} / \mathrm{s}$ that existed before the thrust suddenly increased (table 6). This smaller velocity is compatible with the smaller leg strokes on Lander 2, and calculations of the work performed against the primary strut shock absorbers are $42-45$ percent.

Vertical forces on the footpads cannot be interpreted in a manner similar to Lander 1 because the two visible 
footpads struck rocks. When such analyses are done, however, the reduced velocities of the spacecraft appear to be more probable because unusually large effective coefficients of sliding friction for the footpads (>1.1) would be required to account for the kinetic and potential energies at touchdown when the higher velocity is used.

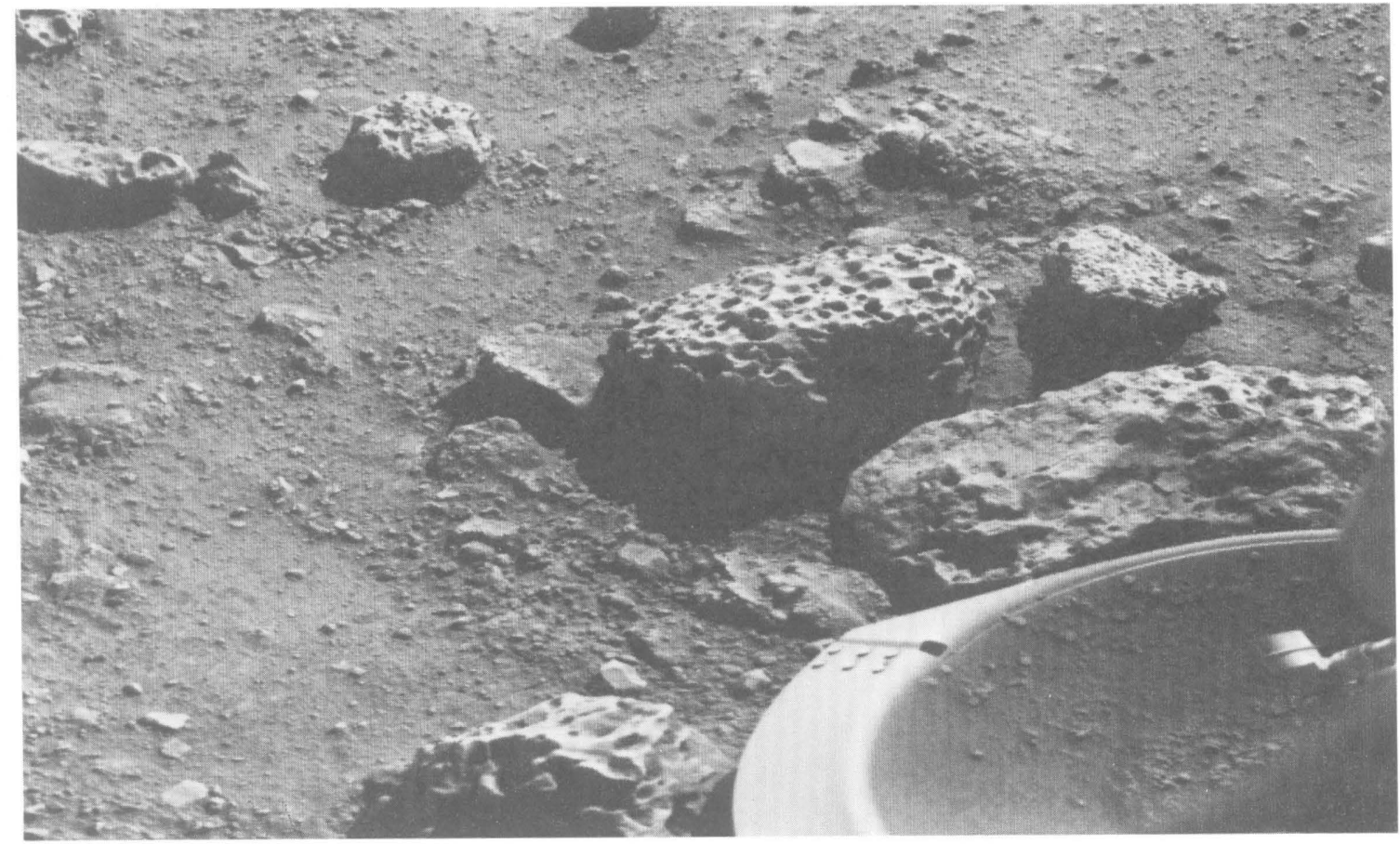

A

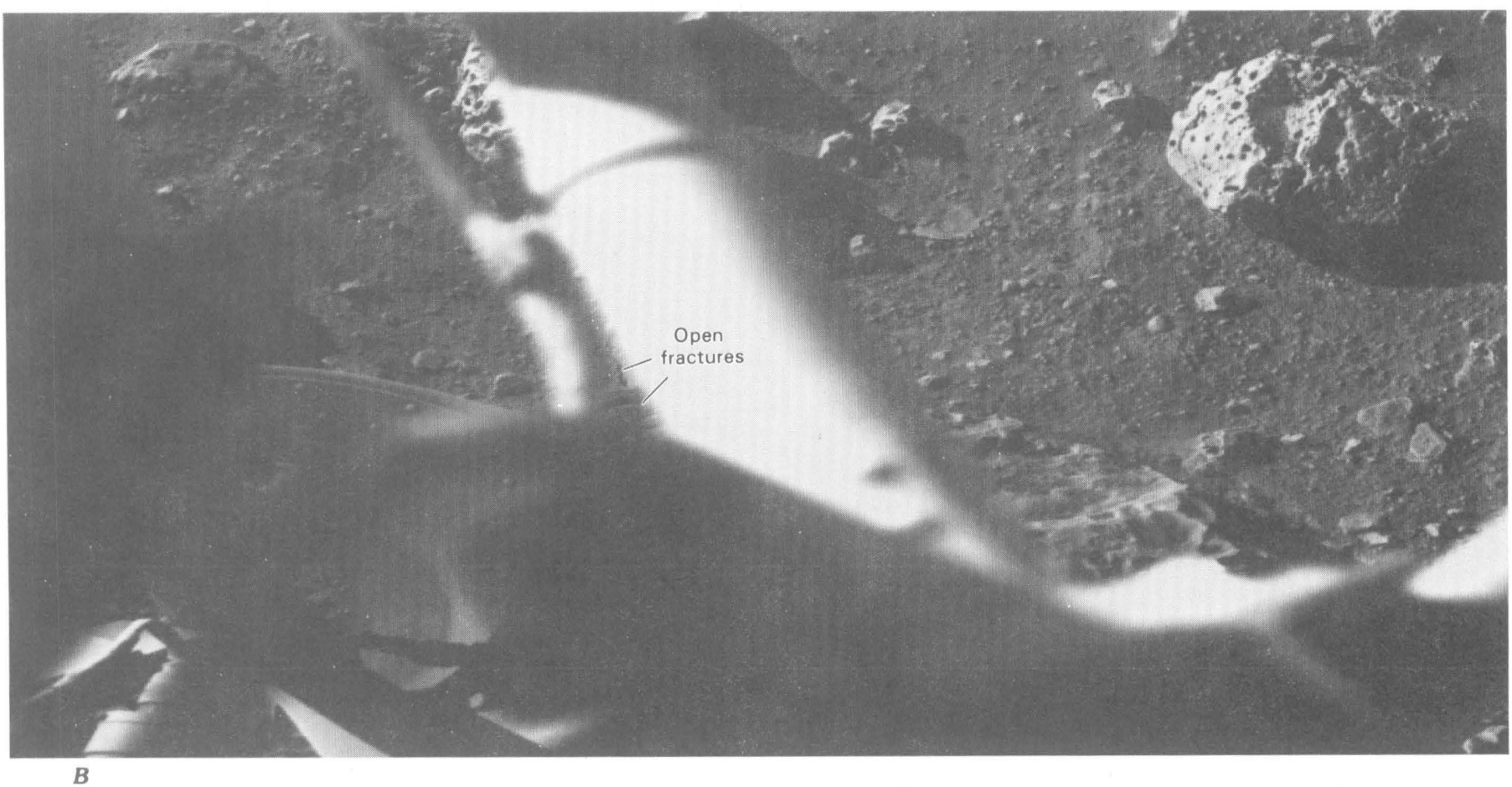

Figure 19.-Footpads 3 and 2 of Lander 2. A, Footpad 3 appears to have overturned rock, $32 \mathrm{~cm}$ wide, at its upper edge (frame $22 \mathrm{~A} 001$ ) 000). $B$, Footpad 2 has rock near right-hand edge that it probably struck during landing. Note concentric scarp and disturbed zone to right of rock and open fractures that appear to be related to rock rather than to footpad. Rock probably transmitted force of footpad to surface material which fractured. Rock at upper right is $26 \mathrm{~cm}$ wide (frame 21A031/004). 
For the reduced velocities, the effective coefficients of sliding friction range from 0.59 to 0.82 .

\section{DISCUSSION}

Measurements of static forces required to crush the shock absorbers in the primary struts were used for a static analysis of footpad penetrations. Dynamic forces required to crush the shock absorber appear to be about $1 \mathrm{kN}$ higher than static forces for the same amount of crushing or stroke (Pohlen and others, 1976); J.C. Pohlen (oral commun., 1980) suggested that this difference could be due to instrumental problems in measuring dynamic forces. If the difference is real, static forces on the footpad have been overestimated by roughly $1 \mathrm{kN}$. The difference has little effect on the estimated cohesions (see section on "Analysis of Landing Data").

Our experimental data on dynamic footpad penetrations are limited chiefly to tests using lunar nominal test materials. Other materials such as clay may have cohesions at lower densities that are the same as those of lunar nominal material at higher densities. For example, the cohesions of kaolinite with a density of $1,120 \mathrm{~kg} / \mathrm{m}^{3}$ and lunar nominal material with a density of $1,575 \mathrm{~kg} / \mathrm{m}^{3}$ are both about $1 \mathrm{kPa}$ (Ko, 1971; fig. 20). Thus, the densities

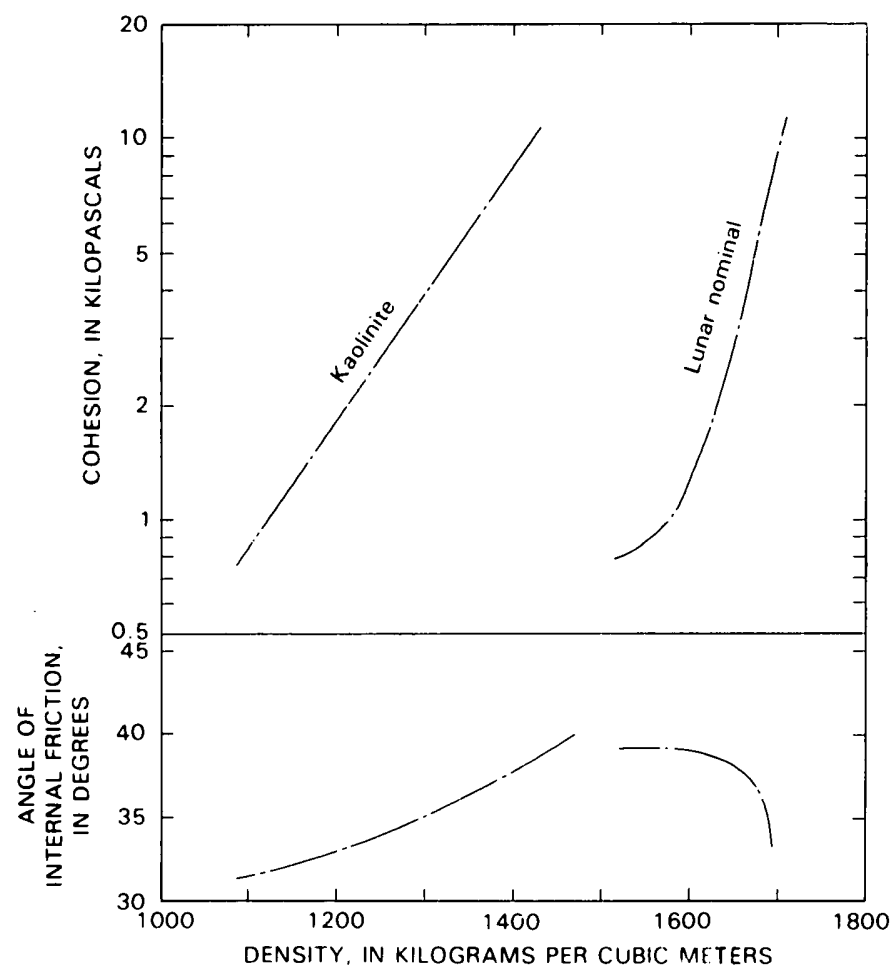

FIGURE 20.-Relation between cohesion, angle of internal friction, and bulk density for kaolinite and lunar nominal test materials (Ko, 1971). Note that densities of kaolinite are lower than those of lunar nominal materials for equal values of cohesion. Angles of internal friction of kaolinite increase with density whereas those of lunar nominal material decrease. of drift material, estimated by comparison with data from the dynamic tests of footpad penetration in the lunar nominal simulant, could be high by as much as 30 percent if the drift material has low densities similar to kaolinite and penetration resistances like lunar nominal material.

In any case, analyses of footpad penetrations of Lander 1 and comparisons with laboratory tests show that there is a significant difference in the mechanical properties of drift and blocky materials. Static analyses of the penetration of footpad 2 in drift material are consistent with a material that has a cohesion of $1.0-1.7 \mathrm{kPa}$, an angle of internal friction of about $20^{\circ}$, and a density of $1,200 \mathrm{~kg} / \mathrm{m}^{3}$. Static analyses of the penetration of footpad 3 in blocky material are consistent with a material that has a cohesion of about $5.5-7.1 \mathrm{kPa}$, an angle of internal friction of about $30^{\circ}$, and a density ranging from $1,200-2,000 \mathrm{~kg} / \mathrm{m}^{3}$. Interpretations of results from dynamic tests of the footpads do not require an extremely low density material for drift material $(1,300-1,500$ $\mathrm{kg} / \mathrm{m}^{3}$ for lunar nominal test material). Blocky material is clearly more resistant to penetration than drift material, and this resistance is probably the result of a larger angle of internal friction and cohesion. As noted previously, none of the analyses entirely resolve the problem of drift material because the footpad could have been stopped partly by a hard substrate of blocky material or a buried rock, but evidence that this happened is equivocal.

Conclusions or interpretations about the mechanical properties of the surface materials near Lander 2 cannot be made from analyses of footpad penetrations because the footpads struck rocks when Lander 2 landed. Analyses of the landing data do, however, indicate that the velocity of the lander was substantially reduced about $0.4 \mathrm{~s}$ before it came to its final position because of increases in thrusts of the engines.

\section{ENGINE-EXHAUST EROSION}

During the martian landings, the terminal-descent engine plumes of Landers 1 and 2 eroded the surface materials. This erosion had been anticipated and investigated both analytically and experimentally during the design phase of the Viking lander spacecraft. The goals of a successful engine design included minimal erosion of the surface and sample field by the engine exhausts (Hutton and others, 1980). Although the goals of engine design were appropriate for mission objectives, such as the detection of life and lander stability, they are in direct conflict with the goal of deducing physical properties of the martian surface materials from erosion data. For this latter goal, deep and extensive erosion is desirable. Nonetheless, interactions of the exhaust gases with the surface materials did provide significant information about their physical properties; and, more importantly, 
the engine design was one of the necessary factors for successful landings.

Pictures of the martian surface show clear evidence of the erosion produced during landing. The first evidence for erosion is found in the first pictures taken by both landers beginning $25 \mathrm{~s}$ after touchdown. For Lander 1 , the evidence is fourfold (fig. $21 A$ ). The initial $62 \mathrm{~s}$ of the first picture is brightly streaked, clear, and then dark. Bright streaks, which are caused by the motion of illuminated objects between the camera and the surface, are present during the first $18 \mathrm{~s}$ and then, the clearing lasts for about $17 \mathrm{~s}$. The dark part with 27 -s duration represents a cloud of dust and debris between the camera and the sun (Shorthill and others, 1976a). Elsewhere in the picture, there are small elongate pits or craters aligned along radials from engines 2 and 3 that were produced by the impacts of clods, grains, and fragments with the surface. These clods, grains, and fragments were propelled by entrainment in the engine-exhaust gases. Some rocks, about $6-\mathrm{cm}$ wide and $1.3 \mathrm{~m}$ from the engine, appear to have been displaced a few centimeters and more. Finally, fine-grained material and fragments as much as $7 \mathrm{~mm}$ in diameter have been deposited in and on footpad 3. For Lander 2, bright streaks are conspicuous during the initial $45 \mathrm{~s}$ of its first picture (fig. $21 B$ ). Like Lander 1, elongate pits are present, rocks were displaced, and fine-grained material and fragments as much as $7 \mathrm{~mm}$ in diameter were deposited in footpad 3.

Subsequent mapping revealed that erosion by the exhausts from engine 2 of each lander produced rather large craters with rims of mixed fine-grained material and platy to equidimensional clods, crusts, and fragments of surface material along with small rocks. For Lander 1 (pl. 1), erosion was extensive on the right side of engine 2 (fig. $22 A$ ) where blocky material is present beneath a thin layer of clods and fine-grained material; but on the left side, where drift material is present, erosion was small to nonexistent (fig. 22B). To the front and right, the sinuous rim of the erosion crater extends from 0.55 to $0.7 \mathrm{~m}$ from the engine centerline. Fragments about $2 \mathrm{~cm}$ long imbedded in the rim have intermediate diameters of about $1 \mathrm{~cm}$; the intermediate diameters of these fragements imply erosion to depths of $1 \mathrm{~cm}$ and perhaps $2 \mathrm{~cm}$. Within the crater, a veneer of overlying materials has been stripped away, exposing a planar surface underlain by fractured material. The flat crater floor extends $0.45-0.55 \mathrm{~m}$ away from the engine centerline. The material beneath the crater floor may represent exposed blocky material with the overlying fine-grained material, clods, and rocks removed; others have called the fractured material "duricrust"
(Binder and others, 1977). Beyond the rim, a lobe composed of thick ejecta of fine-grained material and centimeter-size objects extends to about $1 \mathrm{~m}$ from the engine centerline (fig. 23): Beyond the lobe, scattered fragments litter the surface and small elongate pits as much as 1 by $3 \mathrm{~cm}$ in size pock the surface. Elongations of the pits and aligned chains of them lie along radials from engines 2 and 3 . Some rocks, as much as $6 \mathrm{~cm}$ long and $1 \mathrm{~cm}$ thick, were moved several centimeters or more from their original positions at $1.2-1.4 \mathrm{~m}$ from the engine. One rock, estimated to be several centimeters wide and $1.3 \mathrm{~m}$ from the engine, was completely removed from its initial position in front of a wind tail. Direct views of the surface on the left side of engine 2 do not reveal the erosion crater seen on the right side except for a small part of the outermost limit of the rim (fig. $22 B$ ). Although the surface is partly hidden by spacecraft components, tilt of the spacecraft should have favored more extensive erosion on the left side, and the crater, by analogy with Lander 2 (see below), should be visible if it developed there. The engine pressures appear to have deformed the surface into a plateaulike surface bounded by monoclines and the partly hidden erosion crater. Locally, the plateaulike surface is deeply fissured and fractured. A lobe of thick ejecta extends across the plateau, monoclines, and surfaces beyond in a direction roughly opposite to the tilt direction to a distance of about $1.4 \mathrm{~m}$ from the engine centerline (fig. 23). Similar monoclinal structures appear to be related to the interaction of footpad 2 with the surface materials during penetration. Between the juncture of the two large monoclines, the surface is deeply fissured and fractured but not eroded. Pits produced by the impact of fragments with drift material appear to have larger depth to diameter ratios than those on the right side, and the impact pits are as large as 1.7 by $3.4 \mathrm{~cm}$ in plan view. Fragments in and around these pits indicate that the impacting fragments did not penetrate to depths significantly larger than their diameters. The pits and chains of pits appear to be related to engines 1 and 2 .

As shown by a mosaic of seven pictures taken with the boom mirrors (figs. 24 and 23), the surface beneath engine 2 is dominated by a single large crescent-shaped depression, about $10 \times 20 \mathrm{~cm}$, eroded to a depth of about $1 \mathrm{~cm}$ below its surroundings. The crater apparently lies within the large crater observed in the direct view of the surface in front of the lander because its center is about $0.3 \mathrm{~m}$ from the engine centerline. The corresponding total topographic relief is then estimated to be about $2-3 \mathrm{~cm}$ beneath the engine. Flat surfaces of cohesive materials have been exposed in the bottom of the crater, and the 


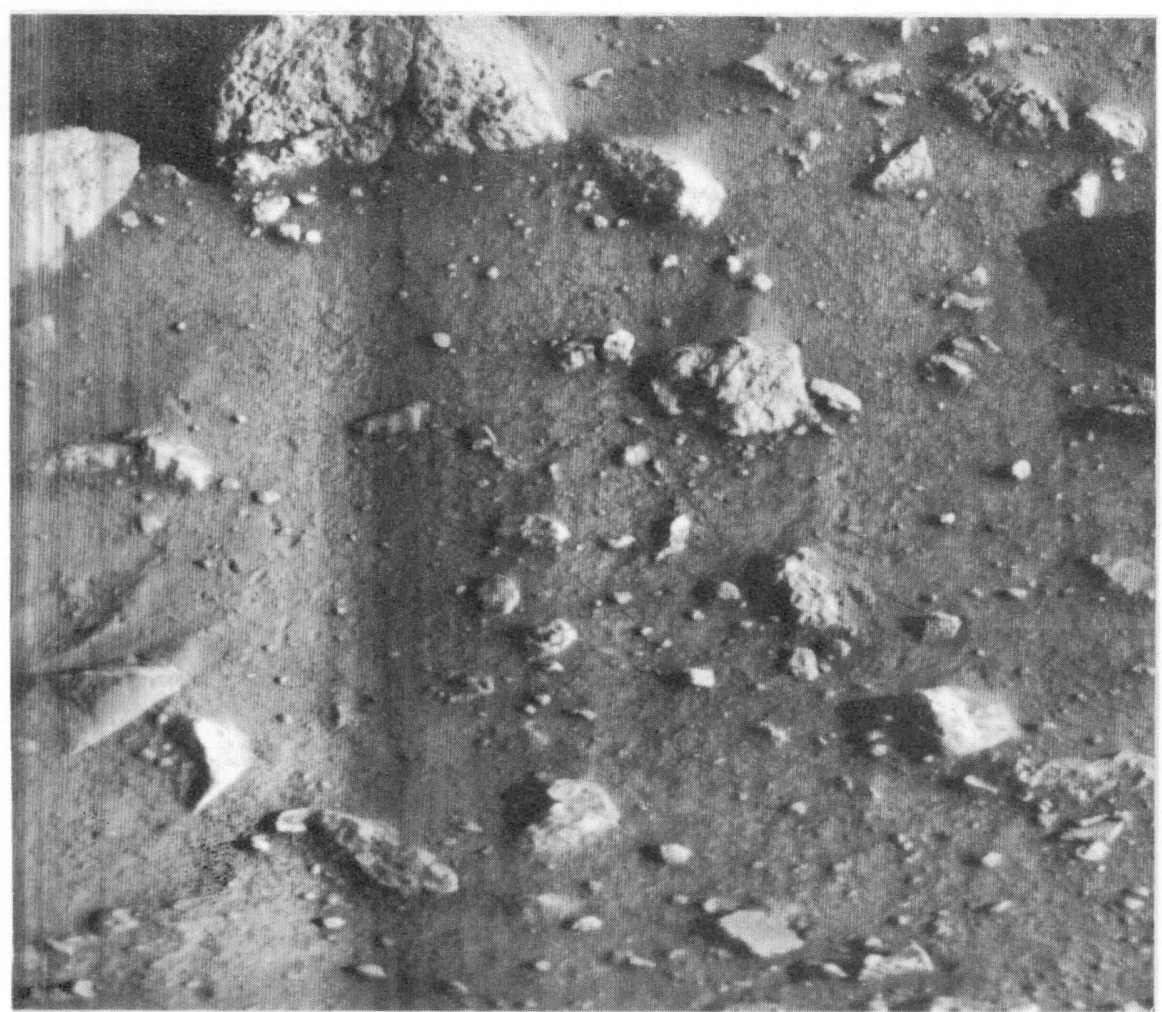

A

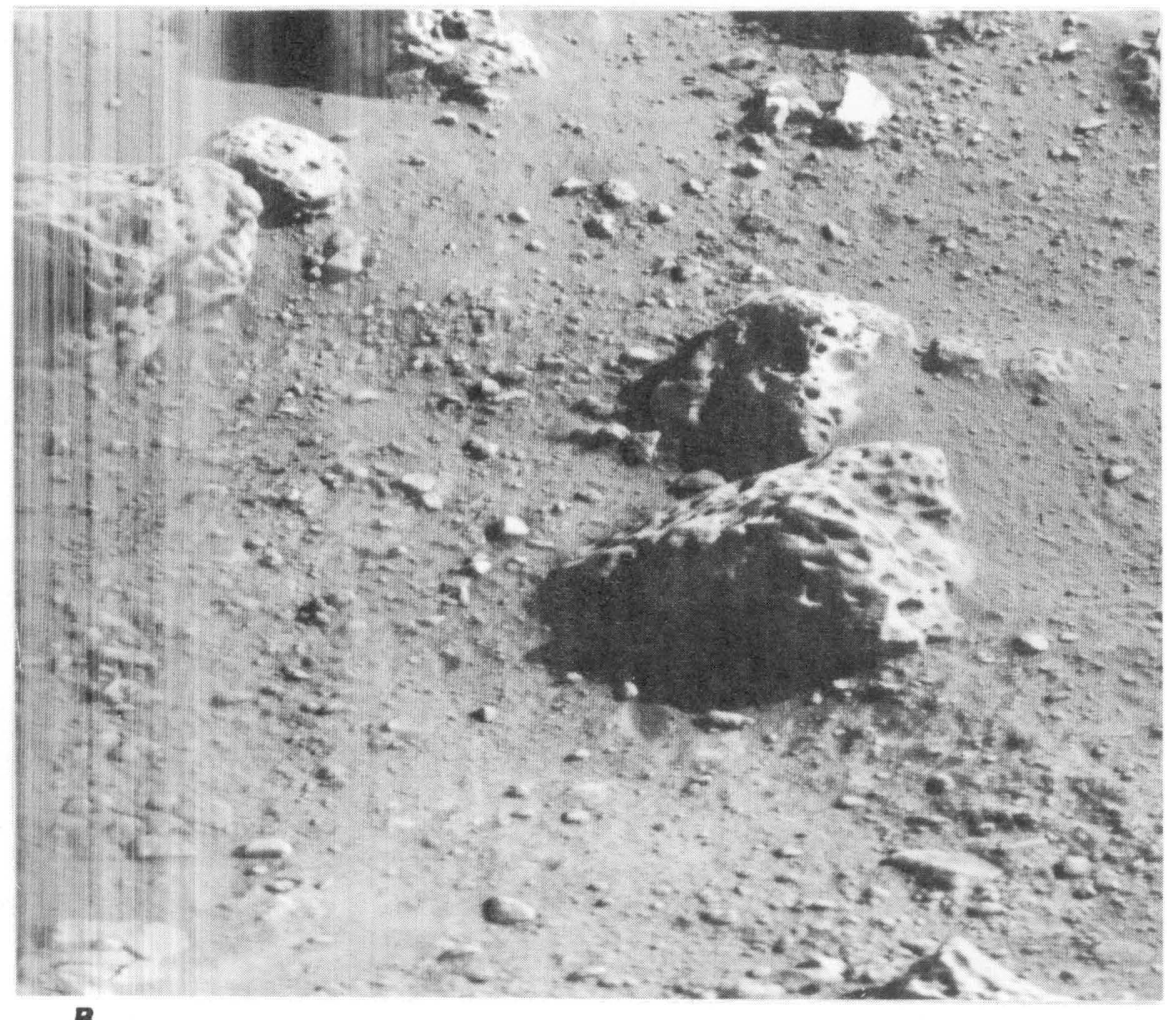

Figure 21.-First pictures taken by Viking landers. $A$, First part of Lander 1 picture of martian surface. Picture commenced $25 \mathrm{~s}$ after touchdown. Bright streaks during first $18 \mathrm{~s}$ of picture are due to motion of illuminated objects from surface initially set in motion by engine-exhaust gases; dark band appears $35 \mathrm{~s}$ after start of picture and lasts for about $27 \mathrm{~s}$. Dark band is shadow on surface caused by debris cloud from engine erosion that drifted between lander and Sun. Large rock at top is $22 \mathrm{~cm}$ wide. Figure $16 \mathrm{~A}$ shows last part of picture (frame 12A001/ $000)$. $B$, First Lander 2 picture of martian surface showing bright streaks during the initial $45 \mathrm{~s}$ of picture. Picture commenced $25 \mathrm{~s}$ after touchdown. Rock at right center is $16 \mathrm{~cm}$ wide. Figure $19 \mathrm{~A}$ shows last part of picture (frame 22A001/000). 
exhaust gases have preferentially eroded along joints and fractures in the materials. A residue of rocks and clods ranging in size from about $1 \mathrm{~mm}$ to $10 \mathrm{~cm}$ is shown in the mosaics.

In crusty to cloddy material at Lander 2, there appears to be more erosion on both sides of engine 2 than at Lander 1 . The rim of the crater, which is $0.5 \mathrm{~m}$ from the engine centerline on the left side and as much as $0.8 \mathrm{~m}$ on the right, is less clearly defined. The mapped elongation is consistent with lander tilt (pl. 2). At and near the rim, fragments of rocks and surface materials are roughly twice as large as those at Lander 1 (fig. 25). Thus, the exhaust gases have eroded to depths of $2 \mathrm{~cm}$ and perhaps 4. A larger erosion crater at Lander 2 was probably due, in part, to the sudden increase in thrust, 81 percent, that occurred about $0.4 \mathrm{~s}$ before touchdown (Shorthill and others, 1976c). A mosaic of seven individual pictures was also obtained using the boom mirror of Lander 2 (fig. 24); the mosaic covers essentially the same area as that of Lander 1 (fig. 23). The features shown in the mosaic of Lander 2 are strikingly different than those of Lander 1 because three small craters are present. Each crater, about $6 \mathrm{~cm}$ in diameter, was produced by exhaust gases from separate nozzles of the engine. These small depres-

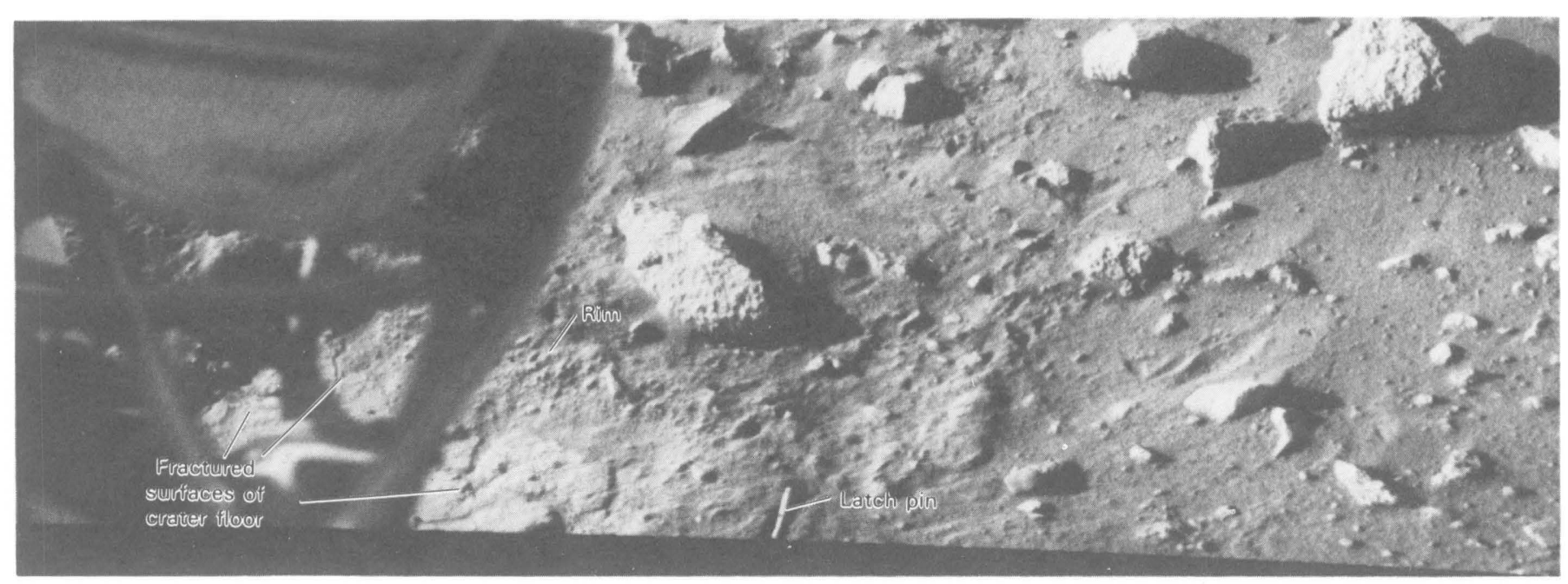

A

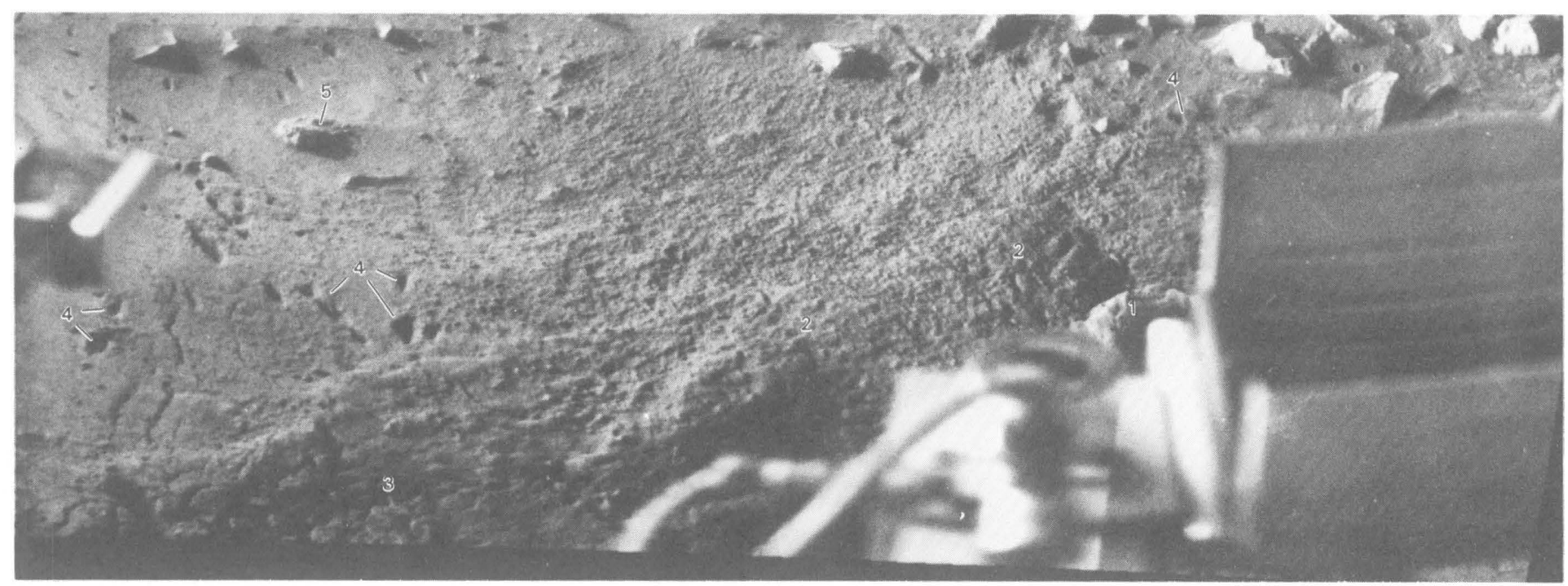

B

Figure 22.-Engine-exhaust erosion by Lander 1. A, Crater produced by engine-exhaust gases on right side of engine 2 . Base of surface sampler and its mounting bracket are at left part of picture. Note fractured surfaces of material of the flat crater floor (beneath surfacesampler base). Rod-shaped object in rim of erosion crater is the surfacesampler latch pin lying within the impact crater it produced. Note centimeter-size objects in rim of erosion crater. Lobe of ejecta extends from rim between rock and latch pin. Elongate rock near center is $23 \mathrm{~cm}$ long. $B$, View on left side of engine 2. Note tip of erosion crater (1), plateau bounded by monoclinal structure (2), fractured and fissured surface (3), and small pits (4) produced by impacts of debris propelled by engine exhausts. Rock at (5) is $8 \mathrm{~cm}$ wide. 


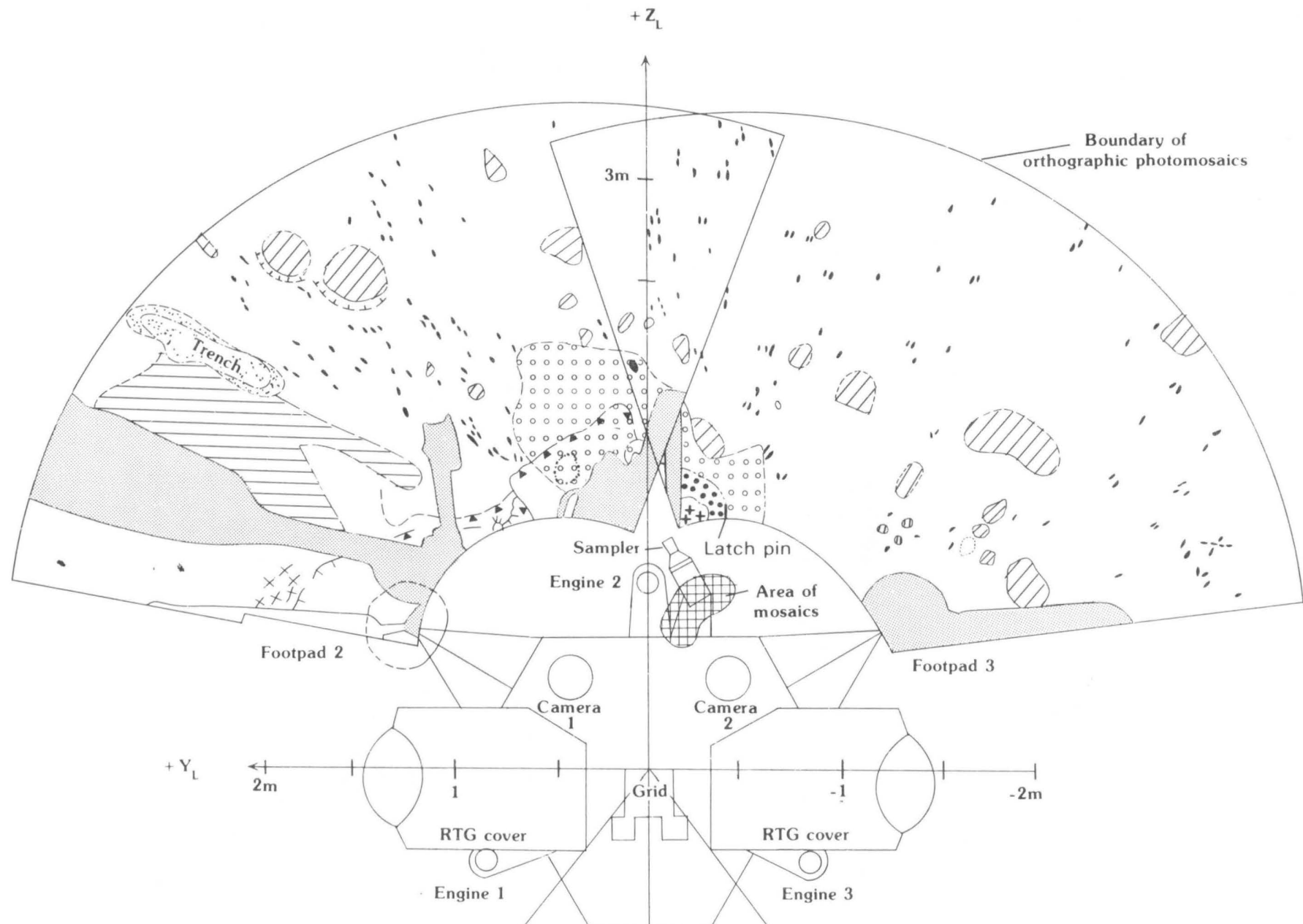

EXPLANATION +++
+++ FLOOR OF EROSION CRATER

$\because \because$ WALL OF EROSION CRATER

$\begin{array}{lll}\circ & 0 \\ 0 & \circ & \circ\end{array}$ EROSION CRATER

AREA COVERED BY MOSAICS-See figure 24

TRENCH AND TAILINGS

DROPPINGS AND WIND-BLOWN FINE-GRAINED MATERIAL FROM SURFACE SAMPLER

\section{AREA OBSCURED BY}

SPACECRAFT PARTS

- iMPACT PIT

ORIGINAL LOCATION OF ROCK

FIGURE 23.-Sketch map prepared from orthographic photomosaics showing the effects of erosion by engine exhausts of Lander 1 . Note that mapped distribution of ejecta is elongate in direction opposite to tilt direction, and erosion appears to be more extensive on right
EXPLANATION CONT.

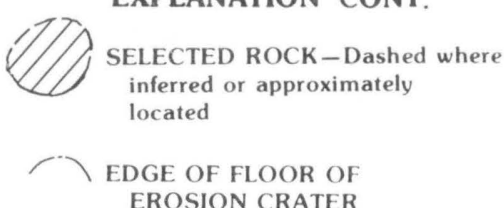

(.) RIM OF EROSION CRATER

CONTACT-Dashed where approximately located

$$
\begin{aligned}
& \text { X广 OPEN FRACTURE } \\
& \text { MONOCLINE-Barbs point } \\
& \text { in direction of slope } \\
& \measuredangle T \begin{array}{c}
\text { SCARP-Hachures point } \\
\text { downslope }
\end{array} \\
& \times \text { ANTICLINF }
\end{aligned}
$$

side of engine than on left side. Small impact pits appear to be aligned along radials from the three engines. Ejecta are superposed on monoclinal structure on left side of engine 2 . 
sions lie within the larger crater observed by direct viewing in front of the lander because they are about $0.3 \mathrm{~m}$ from the engine centerline. The crater near the top is about $1.9 \mathrm{~cm}$ deep, so the total depth of erosion is about 4-6 $\mathrm{cm}$ below the original surface when their depths are added to the estimated depth of the larger crater. Each small crater appears to have small elevated rims. The kidney-shaped depression in the Lander 2 mosaic was caused by exhaust gases flowing around the exposed corners of a rock (rock 12, Sneezy). Rocks and clods ranging in size from about $1 \mathrm{~mm}$ to $5 \mathrm{~cm}$ wide are visible in the mosaics. Farther from the engine, rocks 6.5 and $9.5 \mathrm{~cm}$ in diameter that were $2.2 \mathrm{~m}$ from the center of engine 2 were displaced several centimeters by the exhaust gases. A series of tests that were conducted during the enginedesign phase to assess the erosion characteristics of the various nozzle designs under consideration form the experimental basis for comparison of engine erosion on Mars (Romine and others, 1973; Hutton and others, 1980). In these tests, single-nozzle engines produced excessive erosion in particulate materials under Mars' atmospheric conditions because of the pressure distribution in the focused plume. In order to distribute the exhaust-gas pressures more uniformly, a cluster of small nozzles was designed and tested. The test sequences consisted of simulated landings while the engine exhaust gases im-

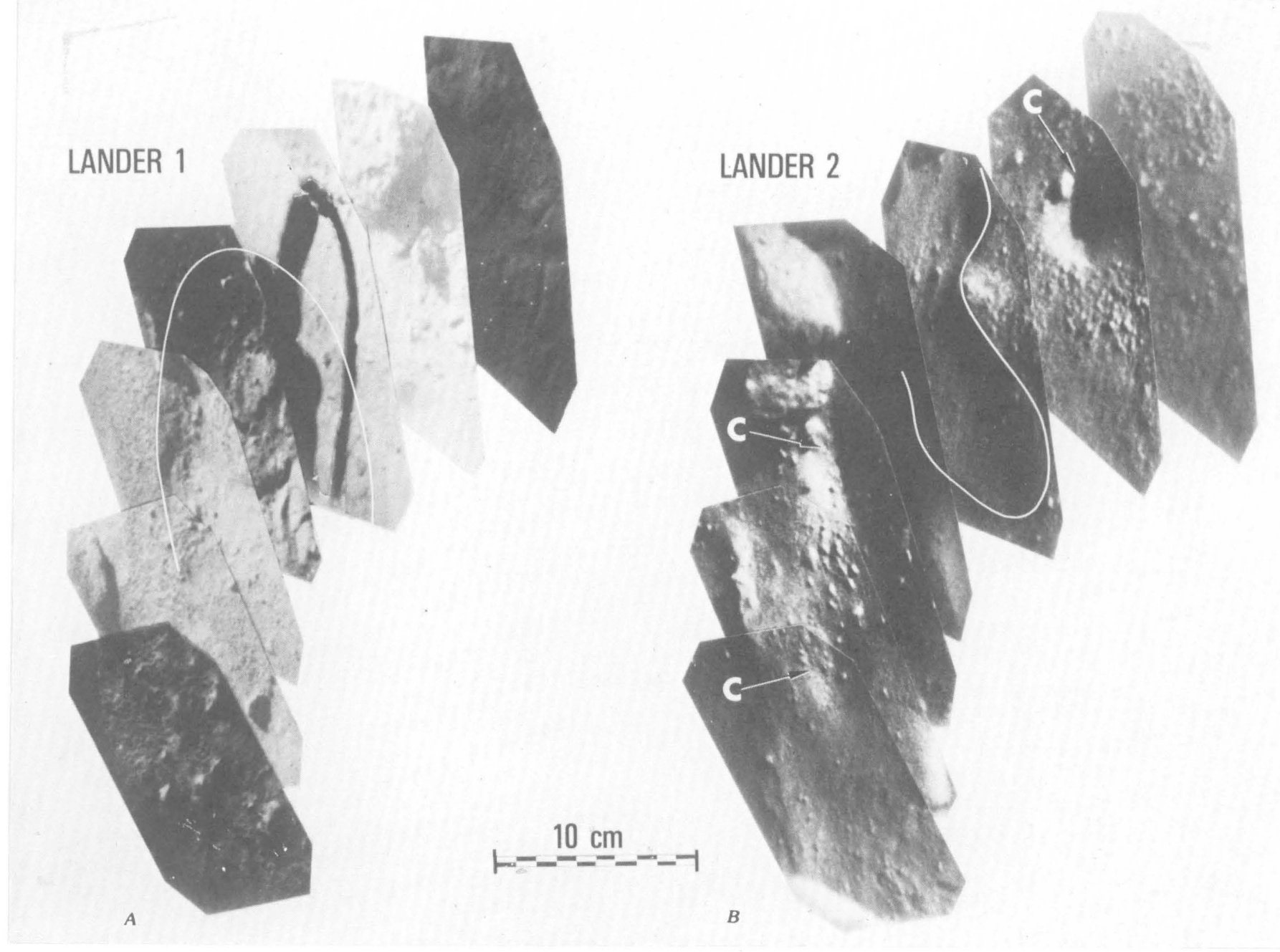

FIGURE 24.-Mosaics of pictures to right of engines 2 of Viking Landers 1 and 2 taken with mirror 2 on boom housing. Approximate location of pictures indicated in figure 23. Radial symmetry suggests that areas covered by mosaics are chiefly within rim of larger erosion crater. $A$, Surface near Lander 1 is dominated by crescent-shaped crater (outlined in white) about $1 \mathrm{~cm}$ deep and centered about $0.3 \mathrm{~m}$ from engine centerline; 8-cm rock occurs in upper part of second and third frames from top; dark area in third frame from top is shadow of a spacecraft part; note residue of lumps, clods, and fractured chunks over most of surface. Sun is to left (sun angles from top to bottom are $41.3^{\circ}, 42.5^{\circ}, 76.4^{\circ}, 53.0^{\circ}, 46.3^{\circ}, 39.5^{\circ}$, and $\left.25.8^{\circ}\right)$. $B$, Surface near Lander 2 shows three craters (labeled C) produced by gases from 3 of 18 nozzles; craters are about $6 \mathrm{~cm}$ wide and as deep as $2.5 \mathrm{~cm}$; large kidney-shaped depression (outlined in white), which was probably produced by gas flow across rock ( $12 \mathrm{in} \mathrm{pl.} 2 A$ ), can be seen in upper parts of fourth and fifth frames from bottom. Sun is to right (sun angles range from $26.7^{\circ}$ at top to $22.3^{\circ}$ at bottom). 
pinged onto surfaces covered with lunar nominal and basalt dune sand test "soils" (see table 7). Tests also included measurements of impingement pressures on a flat, instrumented metal plate.

The findings from these tests led to the selection of an 18-nozzle configuration for the Viking landers (fig. 1 and frontispiece). This configuration consists of nozzles 3.734 $\mathrm{cm}$ in exit diameter, arranged in two rings. The outer ring contains 12 nozzles canted $20^{\circ}$ from the engine centerline.
The inner ring contains 6 nozzles canted $12^{\circ}$ from the engine centerline.

Impingement pressures of a Viking engine were measured during its descent from $12.283 \mathrm{~m}$ down to 0.762 $\mathrm{m}$ at $1.56 \mathrm{~m} / \mathrm{s}$ (Romine and others, 1973). During descent, the engine thrust level was $685 \mathrm{~N}$ and the engine-chamber pressure was $451 \mathrm{kPa}$. The pressure in the vacuum sphere increased from an initial pressure of 10.32 mbar to a pressure of 19.30 mbar during the total 10.14-s engine

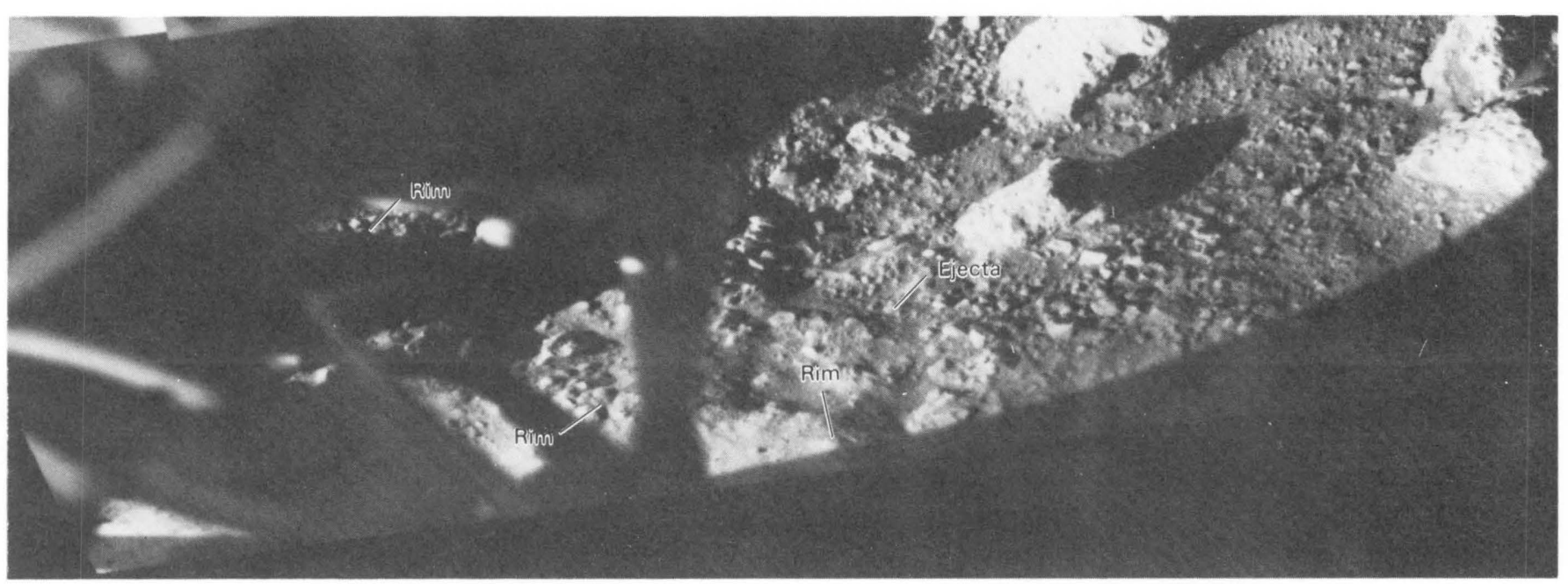

A

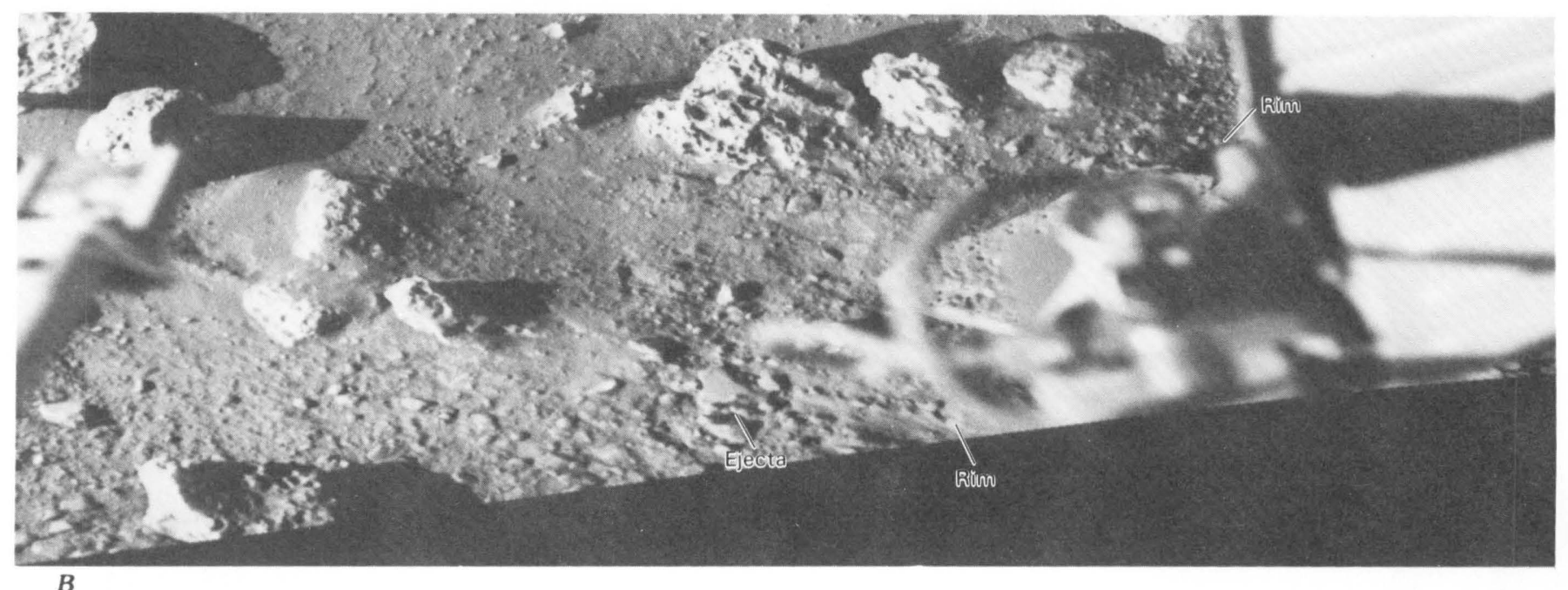

FIGURE 25.-Engine-exhaust erosion by Lander 2. A, Crater produced by engine-exhaust gases on right side of engine 2 . Base of surface sampler and its mounting bracket are at left part of picture. Note large fragments in rim of erosion crater. Rim is poorly defined. Ridges and valleys of ejecta are radial to erosion crater. Rock at far right is 15 $\mathrm{cm}$ wide. $B$, View on left side of engine 2 . Note rim and ejecta of erosion crater are readily identified. Rock at top center is $20 \mathrm{~cm}$ wide. 


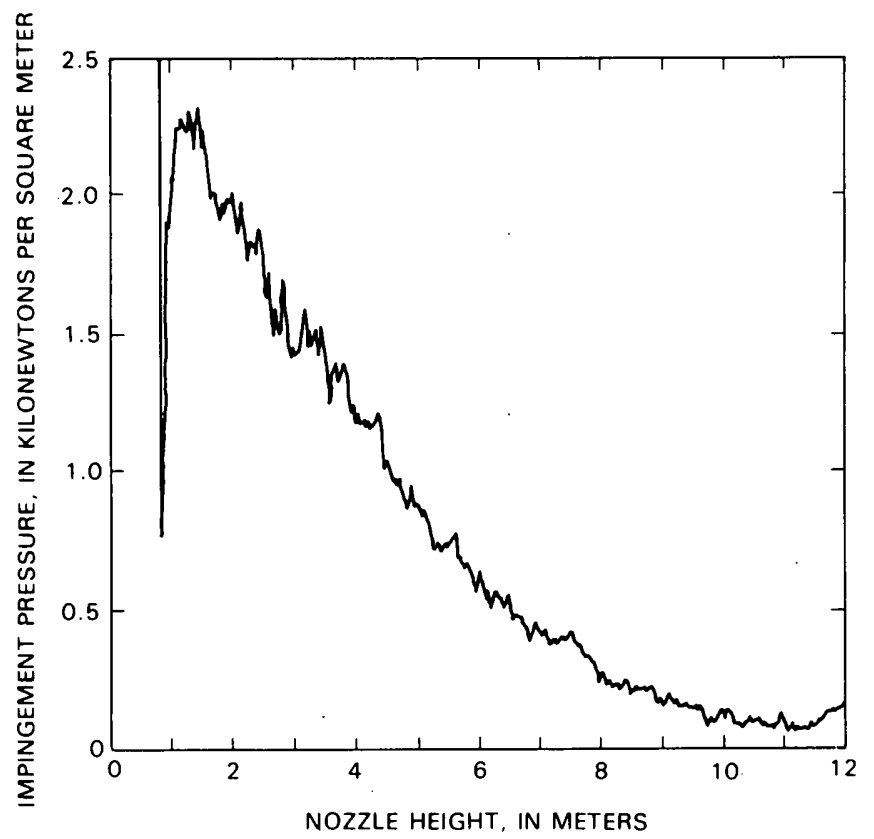

FIGURE 26.-Centerline-impingement pressures related to nozzle heights for Viking lander terminal-descent engine (Romine and others, 1973; White Sands Test Facility Phase II, Test 11K).

burn time. The pressure measured at the centerline impingement point increased as the engine descended (fig. 26). The peak impingement pressure of about $2.28 \mathrm{kPa}$ occurred when the nozzle was about $1.3 \mathrm{~m}$ above the surface. Pressures of similar magnitude were exerted by the Surveyor and Apollo spacecraft (Hutton and others, 1980).

The radial variation of impingement pressure, measured along a single radial line, is shown in figure 27 . The pressures shown for the higher nozzle heights should be representative of those at any azimuth because the flow from the 18 nozzles would have been well mixed and near-

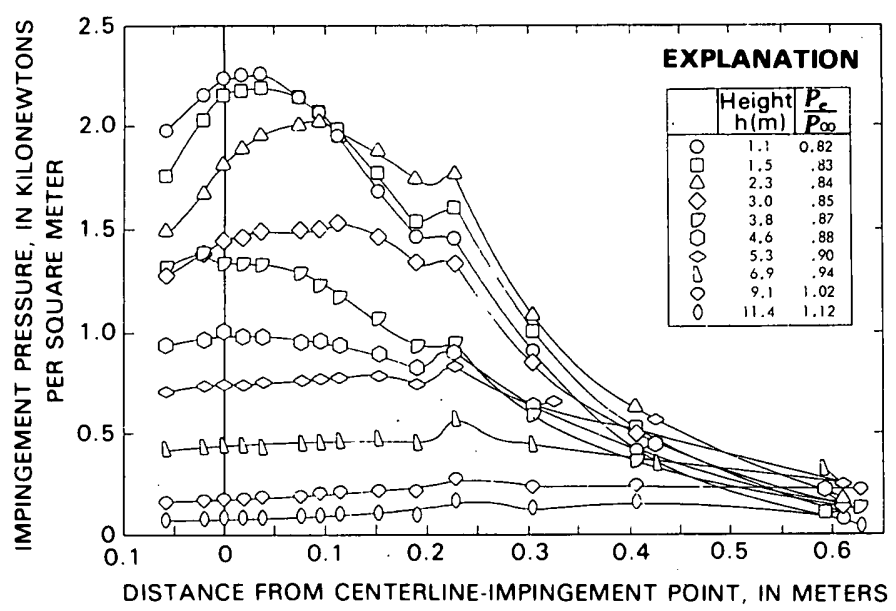

FIGURE 27.-Impingement pressure related to distance from centerlineimpingement point for Viking lander terminal-descent engine (Romine and others, 1973; White Sands Test Facility Phase II, Test 11K). ly axisymmetric. As the nozzles approach the surface, the flow pattern becomes less axisymmetric, and the pressures measured along the single radial line must be considered to represent estimates of the average impingement pressure over all azimuth angles.

Erosion depths measured after the engine descended to surfaces covered with lunar nominal test material and dune sand were a function of distance from the engine centerline (figs. $28 A$ and $B$ ). Most of the surface of the sample field was eroded less than $2 \mathrm{~mm}$, as required by the design criteria (fig. 28). For lunar nominal material, less than 5 percent of the sample field was eroded to depths greater than $2 \mathrm{~mm}$, and for dune sand about 20 percent was eroded to depths greater than $2 \mathrm{~mm}$. During the test with the lunar nominal "soil," the engine descended at $1.61 \mathrm{~m} / \mathrm{s}$ from $12.25 \mathrm{~m}$ to $0.65 \mathrm{~m}$ above the

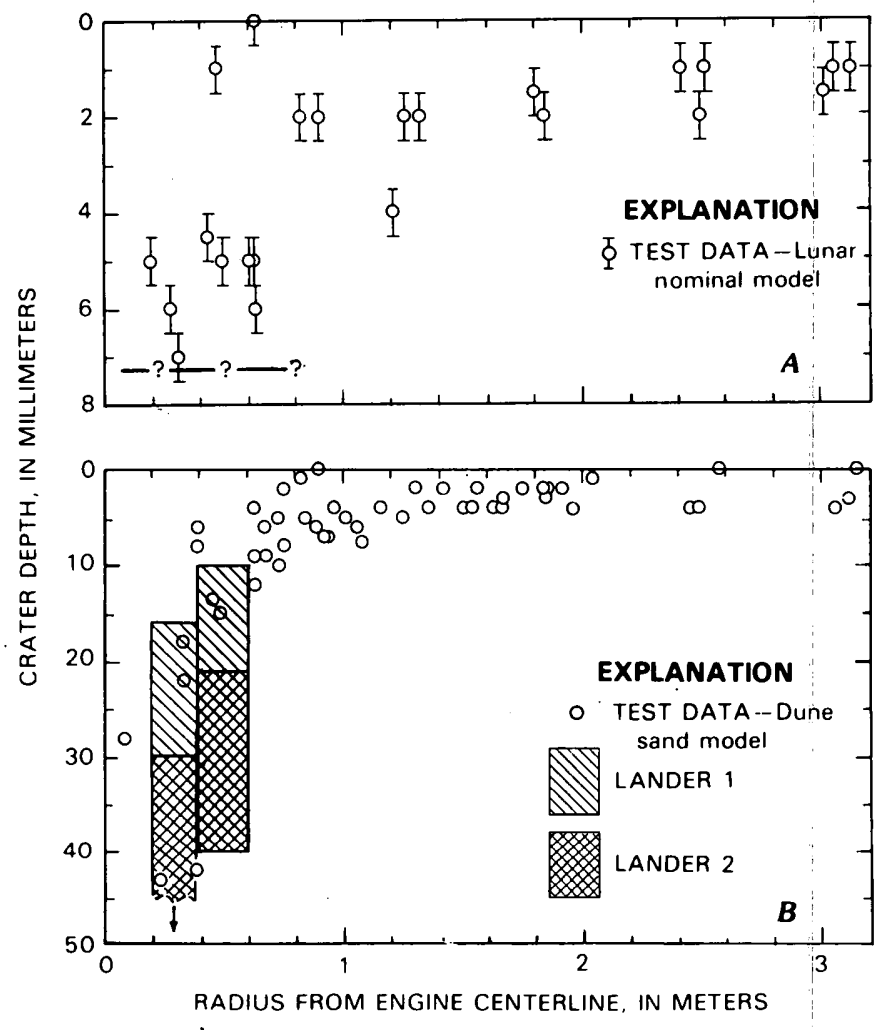

FIGURE 28.-Depths of craters produced by engine-exhaust erosion of Lander 1 and Lander 2 compared to depths of craters produced by gas-plume erosion tests of lunar nominal model and dune-sand model at White Sands Test Facility. $A$, Depths of crater produced by gas-plume erosion of lunar nominal model during test of Viking lander terminal-descent engine (Romine and others, 1973; White Sands Test Facility Phase II, Test 12E). Queried line indicates maximum estimated depth of erosion in drift material by Lander 1 engineexhaust gases. $B$, Depths of crater produced by gas-plume erosion of dune-sand model during test of Viking lander terminal-descent engine (Romine and others, 1973; White Sands Test Facility Phase II, Test 12F). Lined areas indicate estimates of depths of erosion in blocky material by Lander 1 and crusty to cloddy material by Lander 2 engine-exhaust gases. 
surface. During the descent at constant speed, the engine thrust level was $665 \mathrm{~N}$. The initial and final nozzle heights were the same in the dune-sand test, but the descent rate was $1.64 \mathrm{~m} / \mathrm{s}$ and the engine thrust level was $667.2 \mathrm{~N}$. Also shown in figures $28 \mathrm{~A}$ and $28 \mathrm{~B}$ are estimates of erosion depths produced on Mars during the landings.

On the lunar nominal surfaces, the area directly below the engine center was severely scrubbed and did not exhibit a discrete crater, nor were there smaller craters corresponding to the individual nozzles. In contrast, when the gases impinged onto the dune sand, a central area measuring $1.5 \mathrm{~m}$ in diameter was cratered by the gases. Within this area, 18 small craters corresponded to the 18 nozzles of the engine. The deepest small crater in the middle of the $1.5 \mathrm{~m}$ area was $3.8 \mathrm{~cm}$ below the original surface. Ridges formed between the small craters were $3.8 \mathrm{~cm}$ above the original surface; they produced a total topographic relief of $7.6 \mathrm{~cm}$.

Various rocks, wedge-shaped mounds of "soil," and craters were present in the test beds. Five of the 13 rocks on the lunar nominal test bed moved during the test. Both low- and nominal-density lunar nominal wedges showed similar changes; no change in surface relief was detected, but the surfaces of the wedges were slightly roughened by the exhaust gases. Facets of fluffy, low-density lunar nominal wedges facing the engine were extensively scrubbed, and pretest craters near the engine were partly destroyed by erosion and deposition. In the dune-sand test, the objects having greatest topographic relief suffered the most erosion. The features appeared to be sandblasted; near the engine the relief of surface features was cleanly cut away, and those at some distance away were neatly faceted. Some rocks actually tumbled radially inward because their bases were undercut by the faceting.

Comparisons of engine erosion on Mars with engine erosion during the tests on Earth permit some general conclusions concerning the martian surface materials. Generally, erosion by engine exhausts on Mars was not substantially different than that observed during the tests. Properties of the surface materials that affect the amount of erosion are cohesion, particle size, and structure. Large cohesions and particle sizes inhibit erosion. Structures within the surface materials, such as joints, fractures, and layering, may produce weak boundaries between more cohesive units, and the spacing of the structural elements results in an "effective" particle size. Because of variations in these properties, response of the martian surface materials to erosion by the exhaust gases varies in a manner unrelated to their relative strengths as determined from footpad penetrations and surface-sampler interactions with the materials.

Estimated depths of erosion near engine 2 for the region of blocky material at Lander 1 and for crusty to cloddy material at Lander 2 were about the same magnitude as that in the dune sand of the terrestrial test (fig. $28 B$ ), but the eroded materials on Mars were vastly different in their appearance from those on Earth. The dune sand used in the terrestrial tests was composed of cohesionless vesicular basalt sand with grains $0.06 \mathrm{~cm}$ in diameter. In contrast, materials in the rims of the erosion craters on Mars were composed of a continuum of fragment sizes. The particle size of most of this material was below the resolution of the cameras $(\approx 0.07 \mathrm{~cm})$, but fragments as much as 1 or $2 \mathrm{~cm}$ were abundant. Materials deposited in the footpad were also mostly fine-grained, but fragments as large as $0.7 \mathrm{~cm}$ were present. Most of the large fragments in the rims of the erosion craters appear to be cohesive clods and plates of crust that separated along fractures and planes of weakness during entrainment by the exhaust gases along with finer debris. Disaggregation and comminution of clods and crust must have occurred because other evidence indicates many of them were weak at Lander 1 and most of them were weak at Lander 2 . On Mars, materials with a uniform particle size of 0.01 or $0.18 \mathrm{~cm}$ would have eroded to depths of $2 \mathrm{~cm}$ and, for $0.07-\mathrm{cm}$ particles, erosion would have been about $3 \mathrm{~cm}$ (Romine and others, 1973). Thus, the erosion by engine exhausts of blocky and crusty to cloddy material is similar to that of coarse grained materials.

Another significant difference between the engine erosion of dune sand and that of martian surface materials was the shape and presence or absence of craters produced by individual nozzles. For the dune sand, these craters were deep (as much as $3.8 \mathrm{~cm}$ ) and surrounded by elevated rims $(3.8 \mathrm{~cm})$. Beneath the engine of Lander 1 small craters were absent because of the presence of a cohesive substrate that gave rise to the flat fractured floor of the erosion crater. The individual craters beneath the engine of Lander 2 were not so deep and their rims were low and rounded compared to the depths and rims of the dune sand of the terrestrial tests. The presence of craters at Lander 2 may be related to a more uniform character of the materials than at Lander 1 as well as to the "effective" grain size and cohesion.

The lack of erosion of the drift material seemed to be the result of the fine size of the grains, cohesion, and the absence of marked structural elements. The properties of the drift material were more like the lunar nominal test material than the dune sand of the terrestrial tests. An estimate of the specific surface of drift material using desorbed gases measured by the Gas Exchange Experiment is about $17 \mathrm{~m}^{2} / \mathrm{g}$ (Ballou and others, 1978); this figure corresponds to a particle size of $0.14 \mu \mathrm{m}$. This particle size is substantially smaller than that of the lunar nominal test material-80 percent of its particles (by number) are 3-9 $\mu \mathrm{m}$ in size. Cohesions of drift material, excluding bedding-plane weaknesses, are about $1-3 \mathrm{kPa}$; these cohesions are larger than the cohesions of the lunar 
nominal test material (Romine and others, 1973). Finally, evidence of structural elements in drift material comparable to those elements elsewhere is absent. Some fracturing is present but it is scarce.

More puzzling is the plateau bounded by monoclinal structures in drift material that appears to be related to the engine exhausts. We do not understand these structures. Perhaps the engine pressures of 2 or $3 \mathrm{kPa}$ compressed the drift material and after rapid cessation of the pressures the material expanded. Another explanation would be a small outward movement of a thin continuous layer of drift material (owing to viscous shear stresses and stresses within the erosion crater exerted by the exhaust gases) along weak bedding planes.

Finally, large rocks beneath the engines do not exhibit features suggesting that they were eroded by the exhaust gases, and, for Lander 2, the chief contribution of rock 12 (Sneezy, pl. 2) was to channel the flow of gases.

\section{SURFACE-SAMPLER DATA}

Activities of the surface samplers of the landers were impressive. Nearly all the activities were of interest to the Physical Properties Investigation and some activities were planned expressly for the investigation. Salient aspects of the activities were: (1) All sample trenches provided some information on the physical properties of the surface materials, but some were better suited for analyses than others. These analyses yielded estimates of

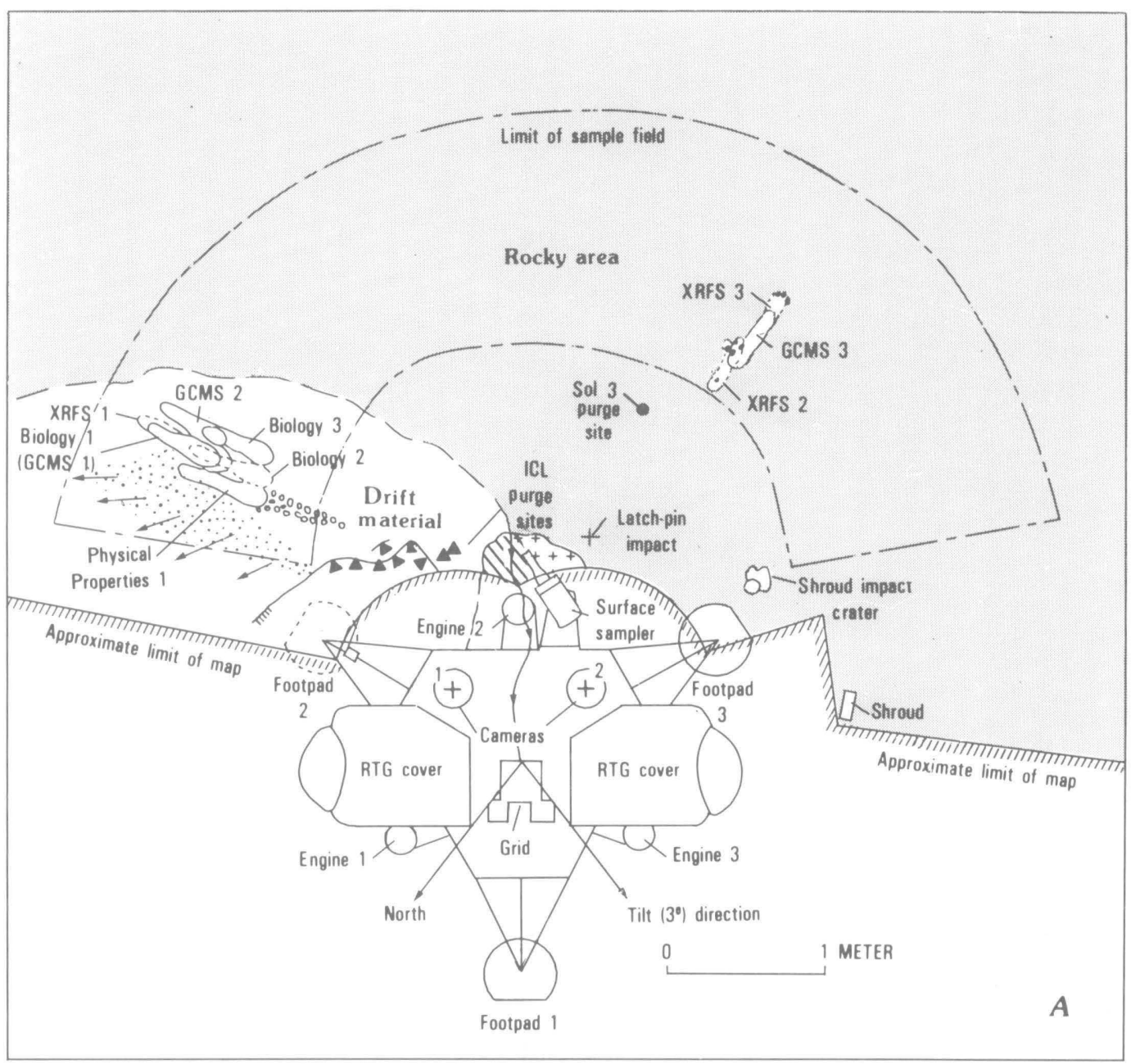

Figure 29.-Diagrammatic plan views of sample field of Lander 1 at end of Primary Mission and Extended Mission. Designations of trenches and other features correspond to those in plate 1 and in tables 38 and 39 of supplemental section on "Summary of surfacesampler activities". Sample fields are portrayed more accurately and in more detail in plate $1 . A$, Sample field at end of Primary Mission showing locations of sample trenches, purge sites, shroud and impact crater produced by shroud, surface eroded by engine 2 , and surface deformations near footpad 2. B, Sample field at end of Extended Mission showing locations of sample trenches, some rocks, purge sites, backhoe-touchdown sites, and conical piles. 
angles of internal friction and cohesions of the materials. (2) Three surface-bearing tests were conducted for the investigation, and analyses of them provided estimates of angles of internal friction and cohesions of the surface materials. (3) Backhoe-touchdown tests, conducted for the investigation, provided crude estimates of cohesions of the materials. (4) Surface impacts by sampler components and fragments intentionally dropped onto the surface pro-

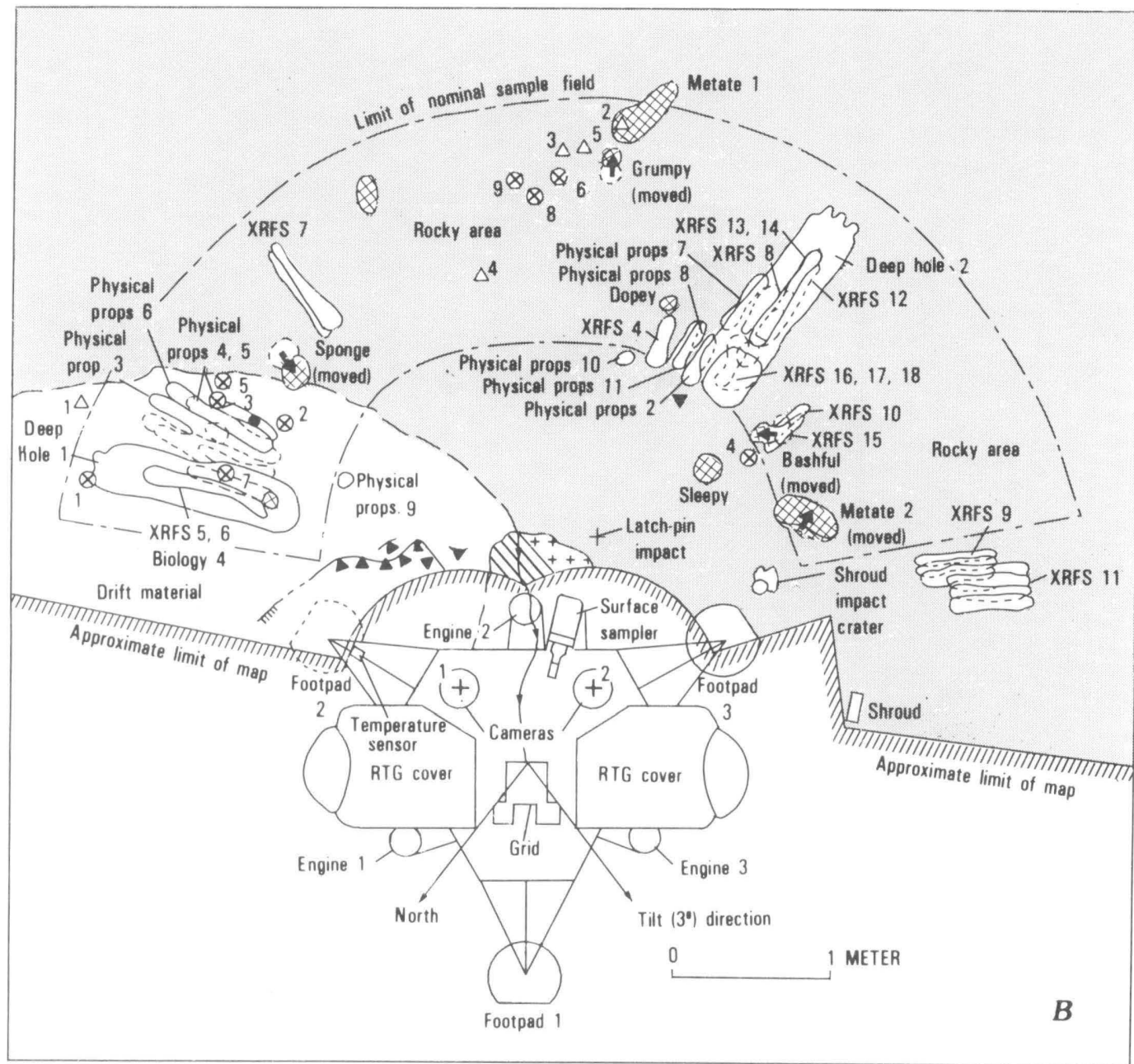

\section{EXPLANATION}

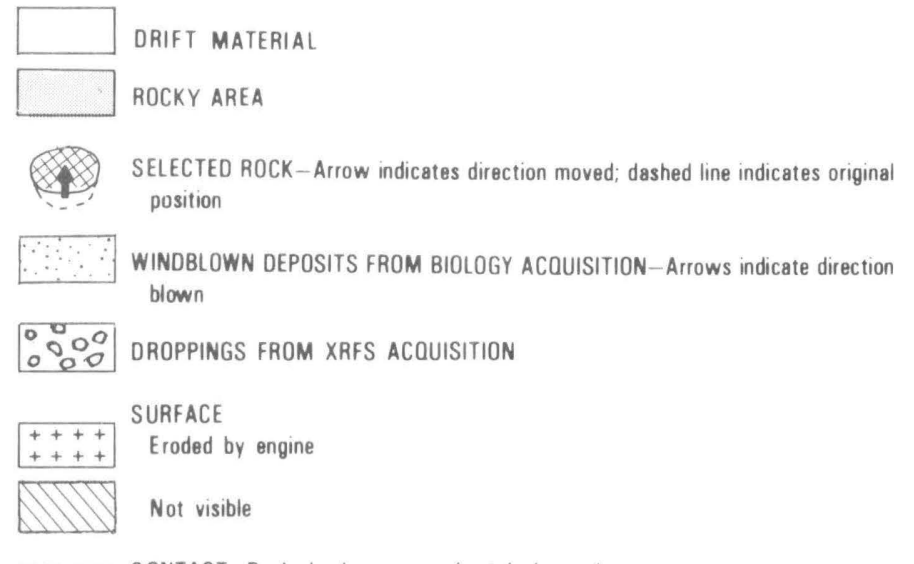

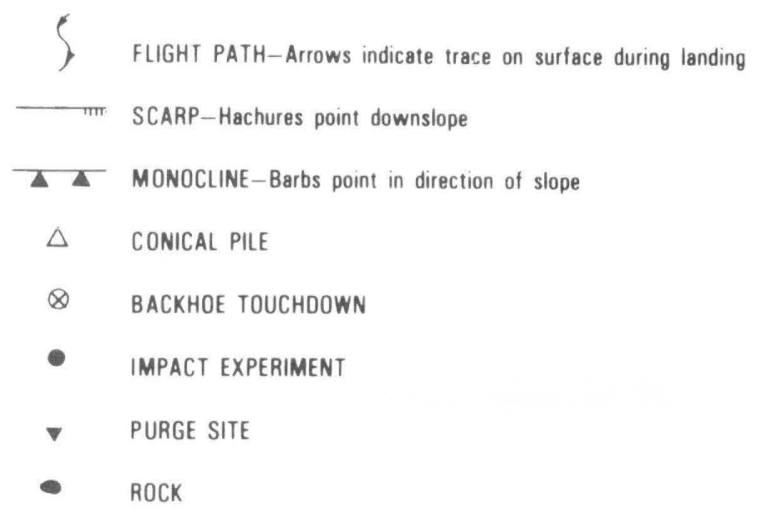

NOTE: Trenches shown by solid outline, except where destroyed or produced in Primary Mission, then shown by dashed outline 
vided some information on the physical properties. After a brief discussion of the surface-sampler activities, descriptions, interpretations, and analyses of results stemming from these activities are given and discussed below. Other activities that will be addressed in later sections are: (1) the conical piles of materials constructed by the samplers; (2) the stability of the walls of trenches and natural slopes; (3) attempts to acquire samples of rocks for analyses; (4) comminution of samples; (5) the pushing of rocks; and (6) diurnal temperatures measured by the sensors on the collector heads while immersed in the surface materials.

In our analyses of sample trenches and surface-bearing tests for angles of internal friction, we have used a theoretical model for plowing by narrow blades in soil (McKyes and Ali, 1977). Results from these analyses are outlined in a general way in this section. Equations and details of the procedure used appear in the supplemental section on "Analysis of Sample Trenches and SurfaceBearing Tests." Additionally, the equations for plowing in sand (pure friction) (Luth and Wismer, 1971) and wet clay (pure cohesion) (Wismer and Luth, 1972), which have been used empirically, also appear in the same supplemental section.

\section{ACTIVITIES}

The primary goals of the surface samplers were to acquire samples for the Biology, Molecular Analysis, and Inorganic Chemical Analysis experiments. These goals were achieved, along with others, during the Primary Mission. Additional samples were acquired for inorganic

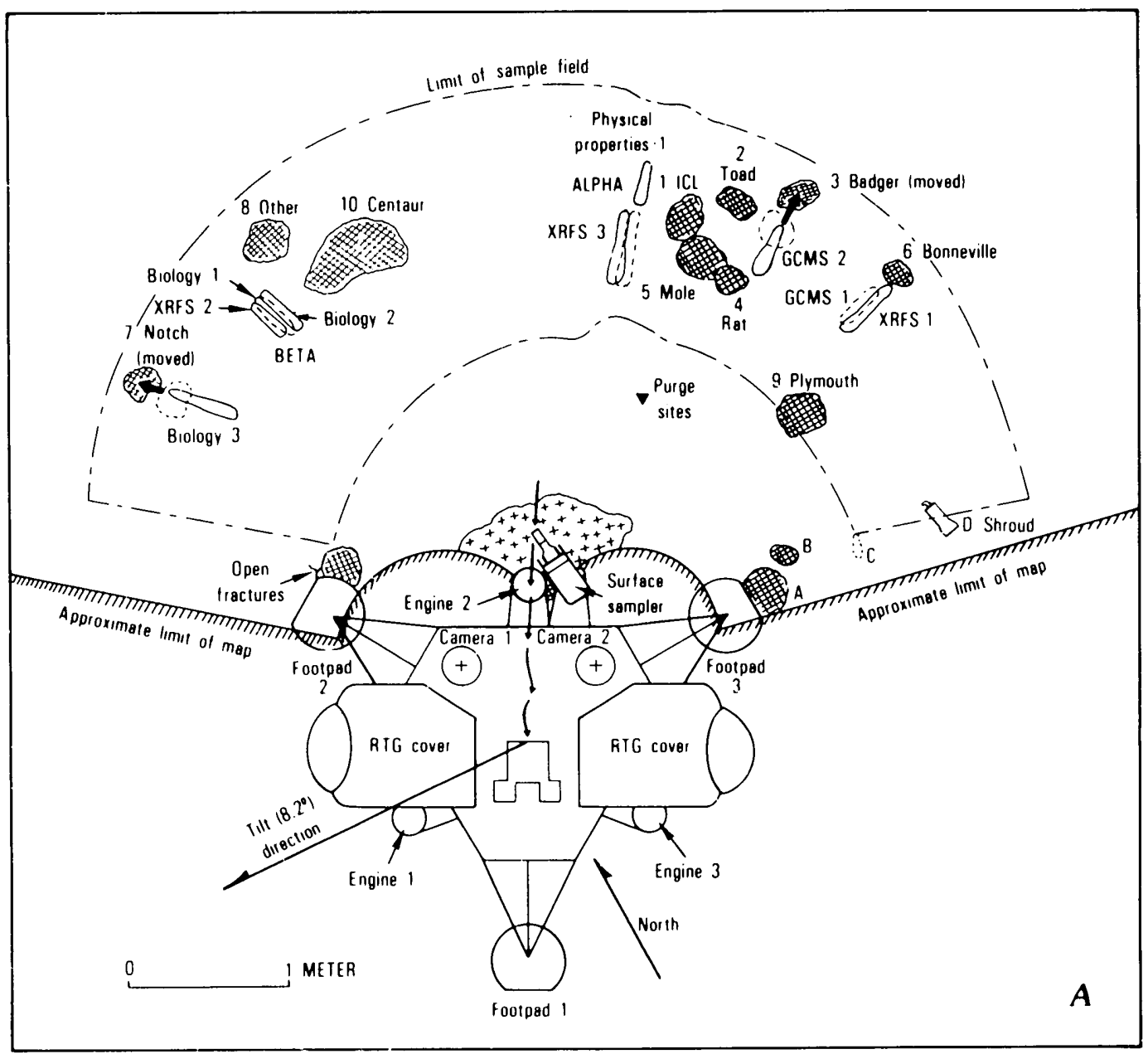

Ficiure 30.-Diagrammatic plan views of sample field of Lander 2 at end of Primary Mission and Extended Mission. Designations of trenches and other features correspond to those in plate 2 and in tables 40 and 41 of supplemental section on "Summary of surface. sampler activities". Sample fields are portrayed more accurately and in more detail in plate 2. A. Sample field at end of Primary Mission showing locations of sample trenches, purge sites, shroud, surface eroded by engine 2 , and some rocks. $B$. Sample field at end of Extended Mission showing locations of sample trenches. some rocks, purge sites, backhoe-touchdown sites, and conical piles. 


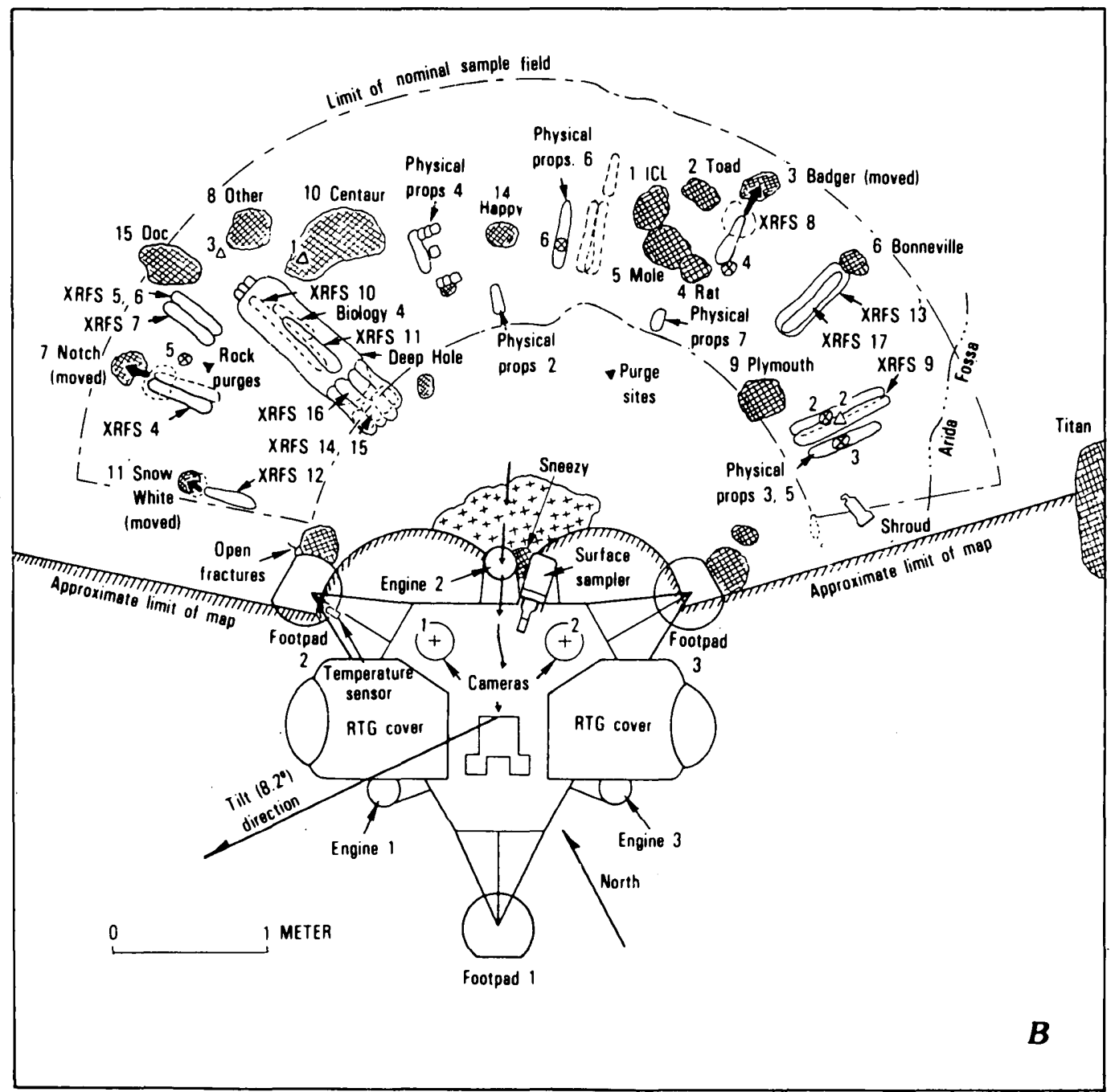

\section{EXPLANATION}

SELECTES ROCK-Arrow indicates direction moved; dashed line indicates original position. Numbers 1 through 8 were candidates for moving

Rock $A$ was struck by footpad 3 during landing. Shroud was ejected from surface semple, struck rock $B$, struck surface at $C$, and came to rest at D

$\left[\begin{array}{c}++7 \\ +++\end{array}\right.$ SURFACE-Eroded by engine 2

-..- SHALLOW DEPRESSION

FLIGHT PATH-Arrows indicaie trace on surface during landing

i CONICAL PILE

Q BACKHOE TOUCHDOWN

$\checkmark$ PURGE SITE

NOTE: Trenches shown by solid outline, except where destroyed or produced in Primary Mission, then shown by dashed outline 
chemical analyses and a variety of experiments were performed for the Physical and Magnetic Properties Investigation during the Extended Mission. Both surface samplers had been used so extensively that by the end of the Extended Mission it was difficult to find areas without rocks where additional trenches could be excavated or other experiments could be performed. The status of the sample fields at the end of the Primary and Extended Missions is shown diagrammatically in figure 29 (Lander 1) and figure 30 (Lander 2); the difference in the number of surface-sampler activities from the end of the Primary to the end of the Extended Mission is shown by the greater number of identified features portrayed in figures $29 B$ and $30 B$ than in figures $29 A$ and $30 A$. The sample fields are more accurately portrayed in plates 1 (Lander 1) and 2 (Lander 2).

In general, the surface-sampler activities conformed to the initial plans for sample acquisitions and other experiments, but a number of the activities were unplanned and unique. In unplanned but successful activities, the sampler of Lander 2 was used to push rocks and then to acquire samples from the materials that were originally beneath the rocks. Both samplers excavated large, deep trenches (deep holes) with the backhoes and acquired samples from the floors of the deep holes. Because of the difference in the character of the surface materials, the sampler of Lander 1 acquired and delivered samples of the coarse fraction for inorganic chemical analyses whereas Lander 2 did not. Finally, procedures were developed to acquire samples from very shallow depths by a skimming motion of the collector head.

A detailed chronological account of surface-sampler activities for each lander is presented in the supplemental section on "Summary of Surface-Sampler Activities". The tables contain an item number, a trench or activity designation, the Local Lander Times for the activity, generalized surface-sampler coordinates or positions that were achieved, and a brief description of the outcome for each lander in the Primary and Extended Missions. Additional information is presented in the supplemental section on "Outline Maps, Profiles, and Data on Trenches". Designations of trenches portrayed in figures 29 and 30, plates 1 and 2 , and as outline maps and profiles can be associated with those in the tables of the supplemental section on "Summary of Surface-Sampler Activities". Each designation indicates the experiment for which the trench was excavated and the sequential number of the excavations by each lander for that experiment. Biology 2 (or BIO 2) refers to the second trench excavated by Lander 1 (or 2) for the Biology Experiment. GCMS 1 refers to the first trench excavated by Lander 1 (or 2) for the Molecular Analysis Experiment. XRFS 12 refers to the twelfth trench excavated by Lander 2 (or 1) for the Inorganic Chemical Analysis Experiment. Physical Props (or PP) refers to the Physical Properties Investigation. Other designations appear in the explanations of the plates.

\section{SAMPLE TRENCHES}

As noted previously, there are three soillike materials at the landing sites: drift, blocky, and crusty to cloddy. Sample trenches in each of the materials are described and analyzed below. Procedures for excavation of trenches in these materials were essentially the same. In a typical sequence, the surface sampler was commanded to the desired azimuth, extended the proper amount, and then lowered to the surface. After surface contact, the collector head was extended about $15 \mathrm{~cm}$ into the surface material with the upper jaw open to acquire the sample. The collector head jaw was then closed and the collector head retracted and elevated, leaving a sample trench behind. This was followed by sample delivery. In some cases the sequence was repeated.

In plan view, most of the sample trenches were long and narrow; their lengths paralleled the directions of extensions away from and retractions toward the azimuth axis of the surface sampler. As used here, the tip is the narrow end of the trench farthest from the azimuth axis of the surface sampler (and from the lander) near the point where the collector head stopped extending to acquire a sample. Material was also excavated from the trench during retraction of the collector head and its backhoe. This material formed an elevated rim of waste or residue around the "tail" end of the trench nearest the azimuth axis of the surface sampler (and lander); thus these elevated rims of waste or residue have been termed "tailings" (Moore and others, 1977, 1979, 1982).

\section{DRIFT MATERIAL}

The rocky appearance of most of the sample field of Lander 1 put the fear of Ares in the Surface Sampler Team who were eager to acquire the first samples from the rock-free area of fine-grained drift material (fig. 29A) designated Sandy Flats (pl. 1A) (Clark, L.V., and others, 1977). In the sample field, drift material appears to be uniform; but farther away, differential erosion of drifts reveals cross bedding. Mechanical properties of drift material may vary with the dimensions and orientations of the applied loads with respect to those of the bedding. The five most consistent properties of trenches in idrift material are: (1) steep walls along much of their lengths as well as at their far tips, (2) lumpy appearing tailings and floors, (3) intact units of drift material that have slumped into the trench, particularly at the trench tips, (4) domed surfaces around the tips, and (5) highly reflective surfaces where the material has been tamped and rubbed by the sampler. The domed surfaces are particularly noteworthy and appear to have a plastic attribute not 


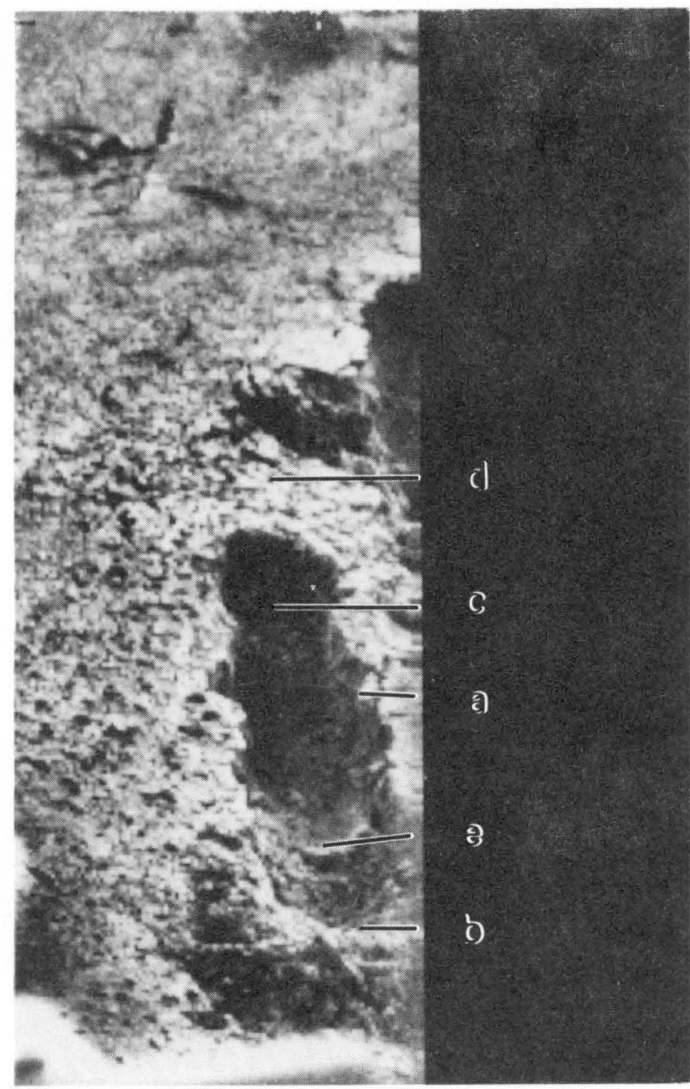

Figure 31.-Physical Properties 1 trench excavated by Lander 1 on Sol 41. Note steep walls (a), lumpy tailings (b), bench near tip (c), rim and domed surface (d), and highly reflective surface (e). Trench is 6 to $7 \mathrm{~cm}$ wide and $48 \mathrm{~cm}$ long from tip to rim crest of tailings (frame 12C043/183).

shared by most other trenches. All of these characteristics are present in the Sol 41 Physical Properties 1 trench (fig. 31). Near the far end of the trench, where it is $0.05-0.06$ $m$ deep, the upper walls have slopes of almost $85^{\circ}$ and down-dropped units of material rise from the floor to form a bench that abuts against the wall of the tip. A small highly reflective area about midway between the tip and rim crest of the tailings contrasts markedly with the less reflective lumpy floor and tailings. The domed and fractured surface at the tip of the trench is present, but that surface is obscured in monoscopic pictures by a complicated surface with pits and clods produced by windblown droppings from the sampler during earlier sample acquisitions. The domed surface with sparse fine fractures is particularly well illustrated by the first trench (fig. $32 B$; Biology 1); the surface is about $0.2 \mathrm{~m}$ wide and extends about $0.07 \mathrm{~m}$ outward from the tip of the trench. Relief of the dome at the trench edge is 0.01-0.02 m. Smooth reflective surfaces are particularly well illustrated by this trench. Subsequently, on the same Sol, acquisition of a sample deepened and extended the first trench (fig. 32C; XRFS 1). This trench exhibits all five of the typical characteristics, but unlike the majority of trenches in drift material, the left wall, about midway along the length, collapsed to produce a miniature lumpy landslide bounded by an arcuate scarp at the surface (fig. $32 C$ ). The characteristics of the trenches are a manifestation of the physical properties of drift material. Steep walls attest to the existence of cohesion, and local failures of the wall may reflect the presence of planes of weakness induced by the sampler during excavation of the trench, natural planes of weakness, or both. These properties will be discussed in a later section. Lumpy tailings and floors also indicate that drift material has cohesion, but its lumpy appearance does not suggest a large cohesion or the presence of indurated clods and rocks. The highly reflective surfaces tamped and rubbed by the surface sampler can only be produced if the material is fine grained and compressible, but only the upper limits of the grain size can be estimated from the pictures. During the Surveyor Program, it was found that the production of such smooth reflective surfaces requires compressible materials with substantial amounts ( $\approx 50$ percent) of silt- or clay-size particles (Christensen and others, 1967). Domed and fractured surfaces were the result of failure of the material in front of and to the sides of the collector head as it moved through the material, and the distance to which failure occurs depends on the angle of internal friction. These topics are discussed below.

Estimates of angles of internal friction require that the relative positions of the surface-sampler collector head, the sample trench, and the region of failure in front of the collector head be known. Thus, the relative accuracy of measurements that is inherent in the resolution of the cameras, the difficulty in identifying the region of failure, and the complex motions of the collector head result in a spread of the values of the estimates. Photographs of the positions of the collector head in trenches during sampling generally do not exist so that the positions must be inferred from the measured dimensions and locations of the sample trenches and achieved surface-sampler positions. The high rims of the trenches and one picture of the collector head inserted in drift material (fig. 33) indicate that the collector head becomes completely filled. For these reasons, the deformation of the surface beyond the collector head is assumed to be produced by a planar interface extending upward from the tip of the lower jaw toward the tip of the upper jaw. The interface has drift material trapped in the collector head on one side and drift material from the region of failure on the other side (fig. 34).

Angles of internal friction $(\phi)$ are estimated by comparison of theoretical or model values of the exterior wedge angle of the region of failure $(\beta)$ for conditions of 
least resistance of failure (McKyes and Ali, 1977) for three ratios of the upper prism depth $\left(d^{\prime}\right)^{3}$ and the collectorhead width and exterior wedge angles derived from data on actual measurements, inferred collector-head positions for each sample trench, and assumed angles of internal friction. These comparisons result in model curves and data curves that intersect; the estimate of the angle of internal friction (and exterior wedge angle) corresponds to the intersection of the model curves and data curve at the $d^{\prime} / w$ value appropriate for the trench. Results of these comparisons (fig. 35; table 9) show that the angles of

${ }^{3}$ In the theory of McKyes and Ali (1977), the soil in the region of failure is divided into an upper prism, a lower prism, and two pyramidal wings (see supplemental section on "Analysis of sample trenches and surface-bearing tests"). The upper prism depth $\left(d^{\prime}\right)$, which is used to estimate angles of internal friction, may be equal to, slightly larger than, or slightly smaller than the depth of the tip of the collector head. internal friction of drift material are between $14^{\circ}$ and $21^{\circ}$ and the average value is about $18.2^{\circ}$.

Cohesion of drift material can be calculated for those trenches that have motor-current records which may be used to estimate the forces exerted and that have sufficient data on the geometry of the trenches and the position of the collector head. The procedure for estimating the cohesion is to calculate the horizontal forces for a variety of cohesions until one of the values of the horizontal forces is reasonably close to the horizontal force exerted by the collector head. The horizontal force $\left(F_{T}\right)$ that can be exerted in deforming the surface material by the interface at the front of the collector head is substantially reduced because frictional forces on the base of the moving collector head resist its motion. The magnitude of the horizontal force is a function of the axial force of the sampler $\left(F_{A}\right)$, the elevation angle of the sampler $(\varepsilon)$,

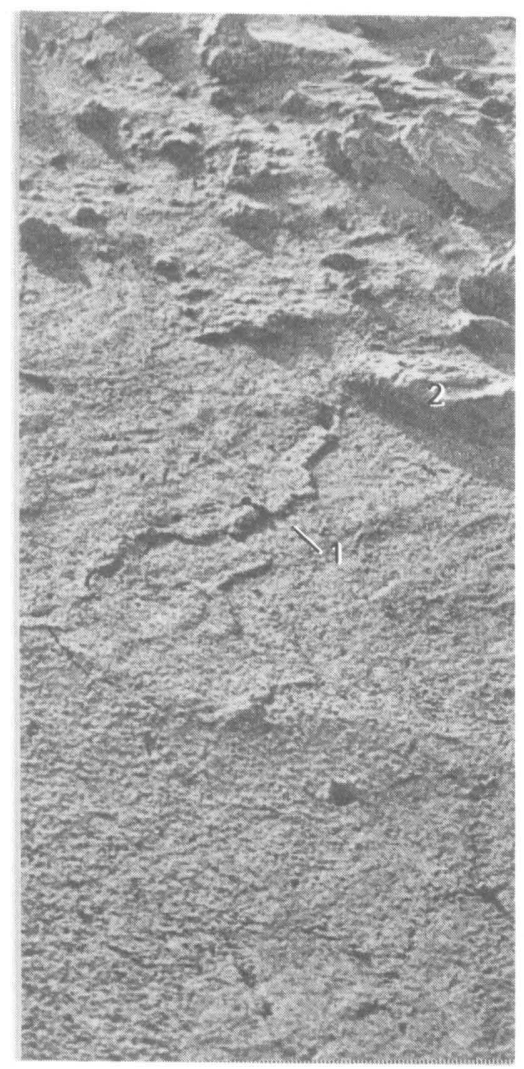

A

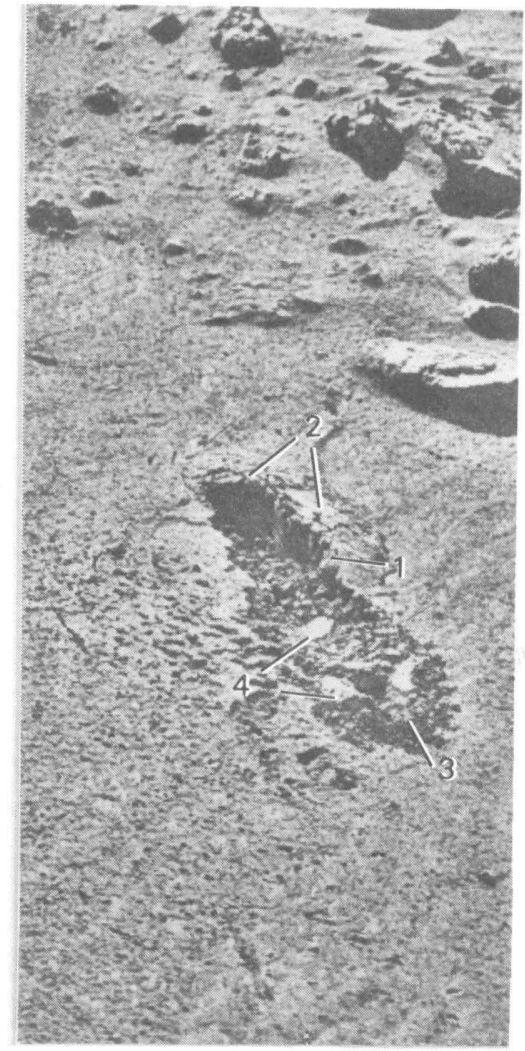

B

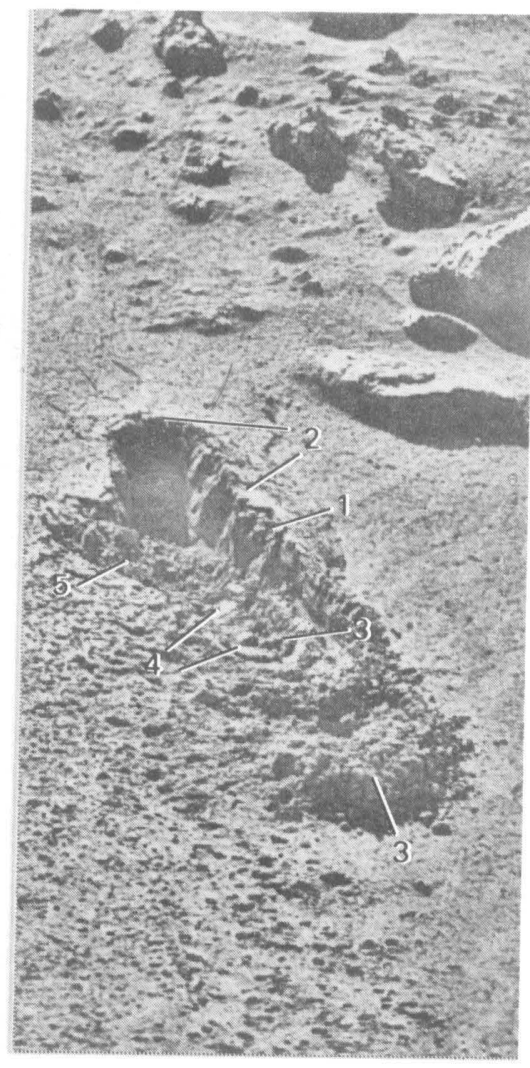

C
Figure 32.-Drift material of Sandy Flats, first sample trench (Biology 1), and first sample trench for Inorganic Chemical Analysis Experiment (XRFS 1). First sample for Molecular Analysis Experiment (GCMS 1) came from this area but no pictures of the trench were taken by Lander 1. $A$, Surface before samples were acquired is rock free; sinuous ridges (1) may be surface expressions of crossbedding; presample stereometric measurements showed that rock 1 (2) (Shadow; see pl. $1 A$ ) would not interfere with sample acquisition. Rock 1 is 18 cm wide (frame 11A055/008). B, Biology 1 trench showing steep walls
(1), domed and fractured surface above region of failure (2), lumpy tailings (3), and highly reflective surfaces that were tamped and rubbed by sampler (4). Trench is $8 \mathrm{~cm}$ wide and $45 \mathrm{~cm}$ long (frame 11A058/008). $C$, XRFS 1 trench after second acquisition stroke. Note steep walls (1), domed and fractured surface above region of failure (2), lumpy floor and tailings (3), highly reflective surfaces (4), and slump on left side of trench (5). Trench is $10 \mathrm{~cm}$ wide and $55 \mathrm{~cm}$ long; domed surface is about $20 \mathrm{~cm}$ wide and extends $7 \mathrm{~cm}$ outward from tip of trench (frame 11A078/012). 


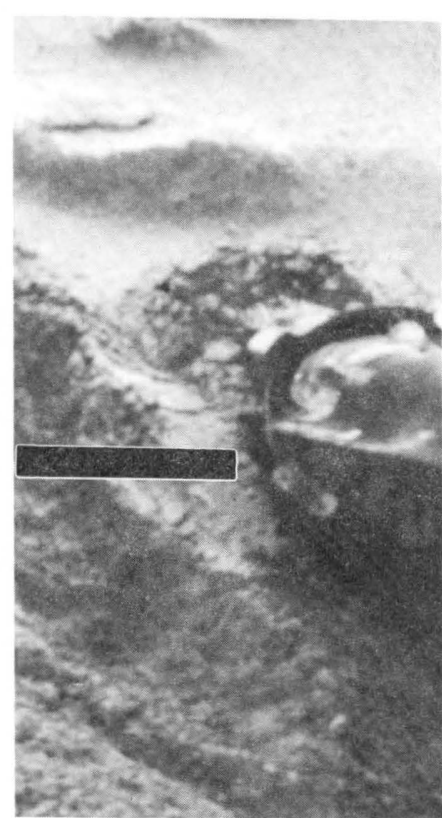

Figure 33.-Collector head was completely filled with drift material before end of the acquisition stroke. Resulting trench after retraction of collector head is Physical Properties 6. Temperatures measured by sensor on base of lower fixed jaw were recorded throughout a diurnal cycle. Bar to left of boom is $10 \mathrm{~cm}$ long (frame 11E041/343).

the rotation angle of the collector head $(\gamma)$, the angle between the base of the collector head and the interface of the leading edge $\left(\theta^{*}\right)$, and the coefficient of sliding fric$\operatorname{tion}(\tan \delta)$. Here the coefficient of sliding friction is assumed to equal the coefficient of internal friction of the material $(\tan \phi)$. The equations and model used to calculate the horizontal forces available to deform the surface material and to account for frictional sliding are illustrated and discussed in the supplemental section on "Analysis of Sample Trenches and Surface-Bearing Tests."

Cohesions calculated for drift material range from less than 0.4 to $5.2 \mathrm{kPa}$, depending on the angle of internal friction used (table 10). Our preferred values for the cohesions are, roughly, less than 0.8 to $2.3 \mathrm{kPa}$ because the average angle of internal friction is close to $20^{\circ}$. For the first trench (Physical Properties 1), only upper bound values for the cohesion of the drift material could be obtained because of the resolution of the motor-current record, and this value is $0.8 \mathrm{kPa}$ when the angle of internal friction is $20^{\circ}$. For the second trench (Physical Properties 3), motor-current records yielded both upper and lower bound forces and, hence, cohesions of $1.1-2.3 \mathrm{kPa}$ when the angle of internal friction is $20^{\circ}$. For the third

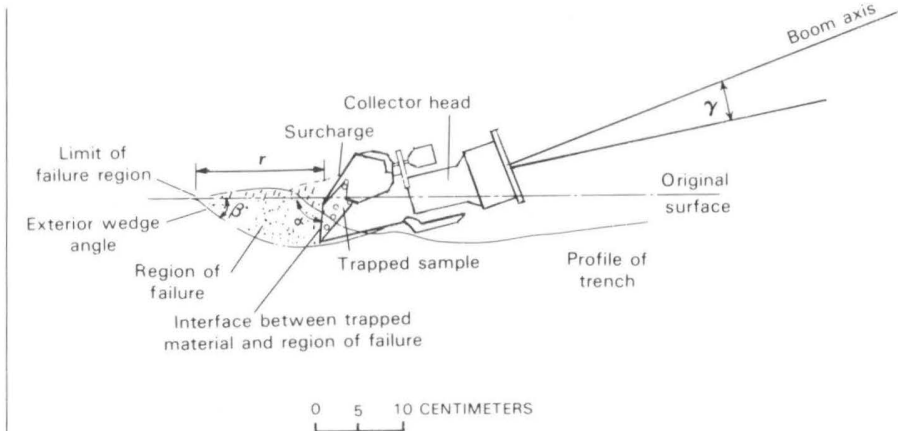

FIGURE 34.-Model used in analysis of sample trenches. Force exerted by collector head on surface material is assumed to be transmitted across a planar surface between trapped sample in collector head and material immediately in front of trapped sample. Locations of exterior wedge angle $(\beta)$, rake angle $(\alpha)$, and collector-head rotation angle $(\gamma)$ are indicated. Measure of limit of failure region is $r$. (See later supplemental section on "Analysis of sample trenches and surface-bearing tests.")

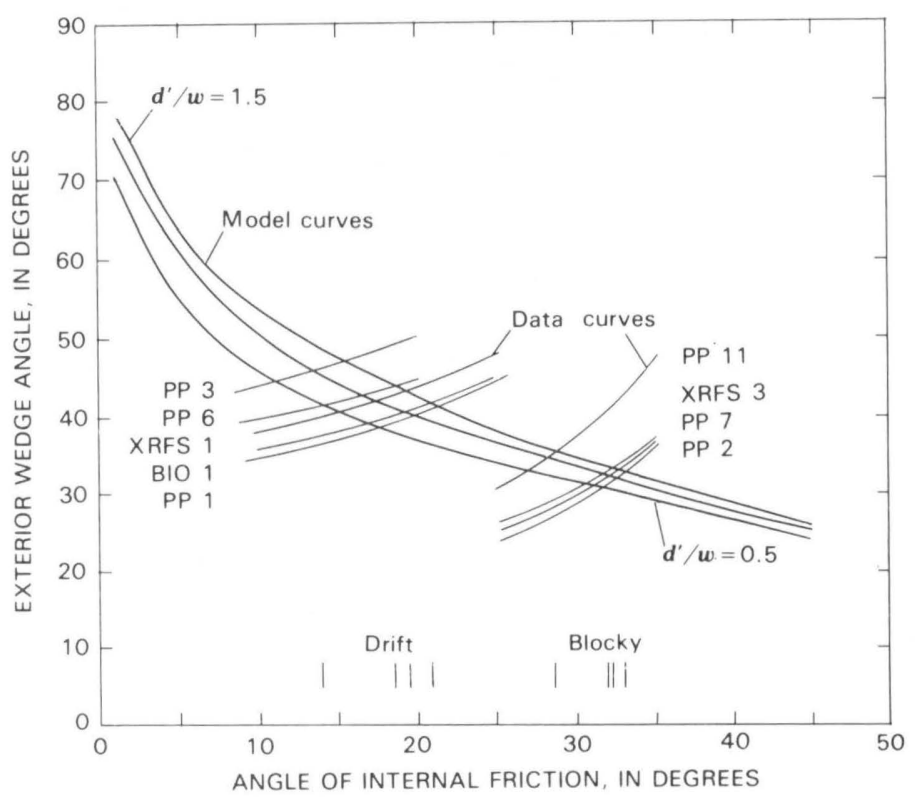

FIGURE 35.-Exterior wedge angles of region of failure predicted from theoretical model of plowing (McKyes and Ali, 1977) for three depthto-width ratios as a function of angle of internal friction (model curves) and exterior wedge angles as a function of assumed angles of internal friction for sample trenches in drift material and blocky material of Lander 1 (data curves). Estimate of angle of internal friction corresponds to intersection of model curves and data curve at $d^{\prime} / w$ value appropriate for trench. Note that average for drift material is about $18^{\circ}$ and for blocky material is about $31^{\circ}$. Equations and details of procedures appear in supplemental section on "Analysis of sample trenches and surface-bearing tests." Letters and numbers refer to trenches excavated for the Physical Properties Investigation (for example, PP 3), Biology Experiment (BIO 1), and Inorganic Chemical Analysis Experiment (for example, XRFS 3); trench locations are shown in plate 1; trench profiles appear in supplemental section on "Outline maps, profiles, and data on trenches"; see also tables 38 and 39 . 
TABLE 9.-Estimated angles of internal friction of drift material from data on sample trenches

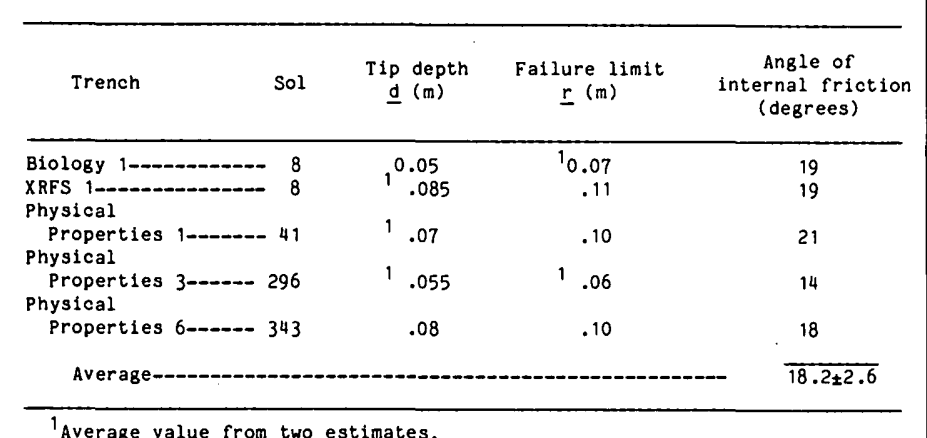

trench (Physical Properties 6), the results were similar to those for Physical Properties 3.

Motor-current records were obtained for two additional trenches in drift material (Physical Properties 4 and 5), but adequate measurements of the regions of failure in front of the collector-head tip could not be obtained. In order to compare these two trenches with the other three, apparent cohesions were estimated for all five trenches using the equations for clay or pure cohesion (Wismer and Luth, 1972), the same values for density and acceleration of gravity as used in the tests on Earth and an available horizontal force corresponding to $\phi=20^{\circ}$ (for example, table 10). The resulting apparent cohesions were somewhat larger than those obtained using the model of McKyes and Ali (1977) (see table 11), and the apparent cohesions of drift material were linearly related to those of McKyes and Ali when $\phi=20^{\circ}$ (fig. 36). This linear relation formed the basis of the probable cohesions given for the drift material found in the Physical Properties 4 and 5 trenches (table 11). Also included in table 11 are the expected horizontal forces for sand or pure friction; these calculations, based on the equation of Luth and Wismer (1971), suggest that the sand is not an analog for drift material because of the low values of the derived horizontal forces.

\section{BLOCKY MATERIAL}

Blocky material is present in the rocky area in front of Lander 1 (fig. 37). Most samples of it were taken from Rocky Flats (pl. 1A). Blocky material is rarely exposed at the surface because it is overlain by thin patches of drift material, small wind tails behind rocks, and patches of pebble-size clods. Rocks appear to rest upon blocky material and also to be set in a matrix of blocky material. Trenches in blocky material have several characteristics: (1) the materials at the far ends of the trenches are disrupted and consist of blocky clods, (2) surfaces around the tips of the trenches are displaced upward, (3) trench walls are locally steep near the tip of the trench but elsewhere they are gentle, (4) tailings and floors appear rubbly because they have distinct fragments and clodsin contrast to the lumpy appearance of drift material, and (5) highly reflective surfaces on the floors of the trenches are rare. The first trench excavated to acquire a sample

TABLE 10.-Cohesion of drift material as a function of angle of internal friction, using the model of McKyes and Ali (1977) [Calculations based on density of $1,200 \mathrm{~kg} / \mathrm{m}^{3}$ and acceleration of gravity of $3.72 \mathrm{~m} / \mathrm{s}^{2}$. Examples of detailed calculations are given in supplemental section on "Analysis of Sample Trenches and Surface-Bearing Tests"]

\begin{tabular}{|c|c|c|c|c|c|c|}
\hline Trench & Sol & $\begin{array}{c}\text { Rake } \\
\text { angle } \\
(\alpha, \text { degrees) }\end{array}$ & $\begin{array}{c}\text { Assumed } \\
\text { angle of } \\
\text { internal } \\
\text { friction } \\
(\phi, \text { degrees })\end{array}$ & $\begin{array}{c}\text { Cohesion } \\
\text { (kPa) }\end{array}$ & $\begin{array}{l}\text { Derived horizontal } \\
\text { force at collector } \\
\text { head interface } \\
\qquad \frac{F_{H}}{(N)}\end{array}$ & $\begin{array}{l}\text { Available surface- } \\
\text { sampler horizontal } \\
\text { force at interface } \\
\qquad \frac{F_{T}}{(N)}\end{array}$ \\
\hline $\begin{array}{l}\text { Physical } \\
\text { Properties } 1 \\
\phi=21^{\circ}\end{array}$ & 41 & 93.5 & $\begin{array}{r}9 \\
15 \\
120 \\
25\end{array}$ & $\begin{array}{l}<1.6 \\
<1.2 \\
1<.8 \\
<.4\end{array}$ & $\begin{array}{l}<24.0 \\
<22.4 \\
<20.3 \\
<20.3\end{array}$ & $\begin{array}{l}<24.2 \\
<22.9 \\
<21.4 \\
<19.6\end{array}$ \\
\hline $\begin{array}{l}\text { Physical } \\
\text { Properties } 3 \\
\phi=14^{\circ}\end{array}$ & 296 & 90.3 & $\begin{array}{r}9 \\
115 \\
20\end{array}$ & $\begin{array}{r}2.5-5.2 \\
11.8-3.7 \\
1.1-2.3\end{array}$ & $\begin{array}{l}23.8-48.1 \\
22.9-45.0 \\
21.7-41.9\end{array}$ & $\begin{array}{l}24.1-48.2 \\
22.6-45.2 \\
21.0-42.0\end{array}$ \\
\hline $\begin{array}{l}\text { Physical } \\
\text { Properties } 6^{2} \\
\phi=18\end{array}$ & 343 & 92.2 & $\begin{array}{r}9 \\
15 \\
120\end{array}$ & $\begin{array}{r}2.8-4 \cdot 3 \\
2.1-3 \cdot 3 \\
11.4-2.2\end{array}$ & $\begin{array}{l}47 \cdot 9-71 \cdot 9 \\
45.1-68.4 \\
42.6-63.0\end{array}$ & $\begin{array}{l}48 \cdot 4-72.6 \\
45 \cdot 5-68.3 \\
42.5-63.7\end{array}$ \\
\hline
\end{tabular}

\footnotetext{
${ }^{1}$ Cohesion and angle of internal friction are approximately compatible with model.

2 Collector head hit hard substrate or buried rock because motor decoupled, inferred forces represent a plateau in motor-current record just before a very rapid increase before decoupling.
} 
TABLE 11.-Apparent and probable cohesions of drift material and expected horizontal forces for sand

[Apparent cohesions calculated using equation for wet clay (pure cohesion) on Earth (Wismer and Luth, 1972). Probable cohesions extrapolated from linear relation between apparent cohesions and cohesions from model of McKyes and Ali (1977). Expected horizontal forces for sand calculated using equation for sand (pure friction) on Mars (Luth and Wismer, 1971). Definitions, equations, and discussion are given in supplemental section on "Analysis of Sample Trenches and Surface-Bearing Tests". Numbers in parentheses calculated using model of McKyes and Ali (1977); linear relation requires that angles of sliding $(\delta)$ and internal friction $(\phi)$ equal $20^{\circ}$ ]

\begin{tabular}{|c|c|c|c|c|c|c|c|c|c|}
\hline Trench & Sol & $\begin{array}{c}\text { Rake } \\
\text { angle } \\
\alpha \\
\text { (degrees) }\end{array}$ & $\begin{array}{l}\text { Depth } \\
\text { of } \\
\text { blade } \\
\frac{z}{(m)}\end{array}$ & $\begin{array}{l}\text { Length } \\
\text { of } \\
\text { blade } \\
\frac{1}{(m)}\end{array}$ & $\begin{array}{c}\text { Elevation } \\
\text { angle of } \\
\text { sampler } \\
\text { (degrees) }\end{array}$ & $\begin{array}{c}\text { Available } \\
\text { horizontal } \\
\text { force when } \\
\phi=20^{\circ} \\
(\mathrm{N})\end{array}$ & $\begin{array}{l}\text { Apparent } \\
\text { cohesion } \\
\quad(\mathrm{kPa})\end{array}$ & $\begin{array}{l}\text { Probable } \\
\text { cohesion } \\
(\mathrm{kPa})\end{array}$ & $\begin{array}{c}\text { Expected } \\
\text { horizontal } \\
\text { force for sand } \\
\text { (N) }\end{array}$ \\
\hline $\begin{array}{l}\text { Physical } \\
\text { Properties } 1\end{array}$ & 41 & 93.5 & 0.07 & 0.095 & 27.5 & 21.4 & 1.4 & $(<0.8)$ & 17 \\
\hline $\begin{array}{l}\text { Physical } \\
\text { Properties } 3\end{array}$ & 296 & 90.3 & .055 & .085 & 24.3 & $21.0-42.0$ & $1.7-3.1$ & $(1.1-2.3)$ & 10 \\
\hline $\begin{array}{l}\text { Physical } \\
\text { Properties } 4\end{array}$ & 324 & 92.9 & .06 & .09 & 26.9 & $42.6-64.0$ & $2.8-3.9$ & $2.0-2.9$ & 13 \\
\hline $\begin{array}{l}\text { Physical } \\
\text { Properties } 5\end{array}$ & 324 & 92.9 & .055 & .08 & 26.9 & 21.3 & 1.8 & $<1.2$ & 10 \\
\hline $\begin{array}{l}\text { Physical } \\
\text { Properties } 6\end{array}$ & 343 & 92.2 & .08 & .10 & 26.1 & $1_{42.5-63.7}$ & $2.2-3.1$ & $(1.4-2.2)$ & 21 \\
\hline
\end{tabular}

\footnotetext{
1 Motor decoupled suggesting that collector head hit hard substrate or buried rock; forces used correspond to a plateau in current record just before rapid increase that preceded decoupling.
}

for inorganic chemical analysis from Rocky Flats (XRFS 2) is a splendid illustration of the first characteristic of blocky material (fig. $37 B$ ). Here the entire region of the far end of the trench, about $0.1 \times 0.2 \mathrm{~m}$, has been disrupted to produce fine-grained material, fragments, and seven or so blocky clods as much as $0.04 \mathrm{~m}$ across. The tailings and the floor are rubbly appearing with fragments as much as $0.01 \mathrm{~m}$ wide. Unlike trenches in drift material, the tailings and trench rims are small and poorly developed. Upward deformation of the surface beyond the tip of the XRFS 2 trench and the previously formed trench (GCMS 3, fig. 37B) is evidenced by the greater reflection produced by surface tilt as compared with the lower reflection of the flatter undisturbed surfaces of the surroundings. Although generally uncommon, highly reflective surfaces appear in the tailings of XRFS 2 and midway along the trench floor of GCMS 3. The GCMS 3 trench does not have a particularly blocky tip, but a second sample trench excavated along the same azimuth (fig. 37C, XRFS 3) does reveal the blocky character of the material. One or two surface rocks are present among the blocks. The walls of the tip are steep because of the blocky nature of the material, but they do not look like the walls in drift material. By the end of Sol 350 (fig. 38), the blocky nature of the materials in the area of Rocky Flats was well established; every visible trench had abundant blocks at their tips and rubbly floors and tailings. Compared to drift material, the amounts of tailings were small. Blocky material was exposed elsewhere, such as to the left of rock 4 (rock 4 push, Sol 302), near rock 5 where both rocks and drift material were also present (XRFS 15), and at the base of the tailings of Deep Hole 2 (XRFS 18). At the end of the Extended Mission, the XRFS funnel was "filled to the brim" with blocky material chiefly from the base of the tailings of Deep Hole 2.

At the tips of four of the trenches in blocky material, it was possible to locate the limits of failure along the ground surface in the azimuthal planes (table 12) by comparing pictures of the surface before and after trenching. By using the same procedures for estimates (fig. 35) in drift material, angles of internal friction between $27^{\circ}$ and $33^{\circ}$ were obtained. The average of $31.0^{\circ}$ is considerably larger than the $18^{\circ}$ obtained for drift material. The larger angles of internal friction along with the blocky nature of the material differentiate it from drift material.

The overall strength of blocky material appears to be substantially greater than that of drift material. The strength is manifested not only in the difference in the penetration of the footpads but also in trenching operations. During normal sampling of drift material, the surface-sampler extend drive-motor decoupled only once during 16 acquisition strokes; and, for this one occurrence, the sampler could have hit a buried rock or a hard sub- 


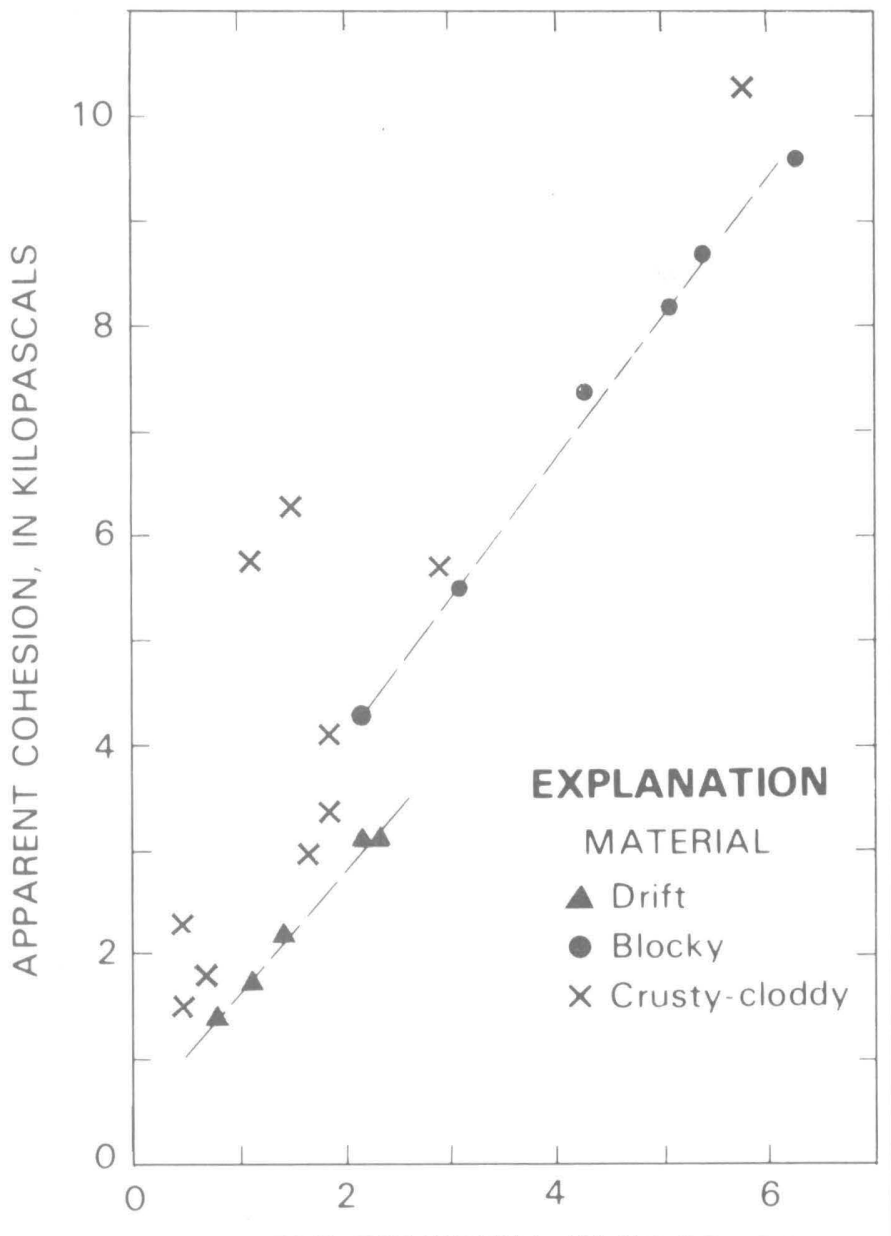

PROBABLE COHESION, IN KILOPASCALS

FiguRE 36.-Comparison between apparent cohesions and probable cohesions for sample trenches in drift, blocky, and crusty to cloddy materials. Apparent cohesions calculated with plowing model of Wismer and Luth (1972) for pure cohesion (wet clay). Probable cohesions calculated with plowing model of McKyes and Ali (1977). Note linear relations (dashed lines) for drift material and blocky material. Linear relation for crusty to cloddy material is poor. Graph used to estimate probable cohesions from apparent cohesions for trenches with insufficient data for analysis by model of McKyes and Ali (1977).

strate of blocky material (Physical Properties 6). In contrast, the surface-sampler extend drive-motor decoupled eight times during 15 acquisition strokes during normal sampling of blocky material. Excavation of the two deep holes leads to the conclusion that blocky material is stronger than drift material because the Deep Hole 1 in drift material was about $0.22 \mathrm{~m}$ deep and Deep Hole 2 in blocky material was about $0.10 \mathrm{~m}$ deep although essentially the same procedures and the same number of backhoe retractions were used for both.

Figure 37.-Rocky Flats, first and second trenches excavated in blocky material (GCMS 3, XRFS 2), and third trench excavated in blocky material (XRFS 3). A, Surface before samples were acquired appears pebbly, and small rocks are present on and around pebbly surfaces (see pl. 1A) (frame 12A140/024). B, Sample acquisition for inorganic chemical analysis (XRFS 2) disrupted surface at tip of trench (1). Disrupted area, about $0.1 \times 0.2 \mathrm{~m}$, contains seven or so blocky clods with diameters as much as $4 \mathrm{~cm}$. Note rubbly floor and tailings. Second trench (GCMS 3) has rubbly floor which is locally polished (2). Bright areas just beyond disrupted area and tip of second trench are result of doming of the surface. Second trench is about $7 \mathrm{~cm}$ wide (frame 12B030/034). C, Third sample trench for inorganic chemical analysis (XRFS 3) is extension of GCMS 3 trench. Here tip and wall show blocky nature of the material. Farthest trench is $9 \mathrm{~cm}$ wide (frame 12B163/073).

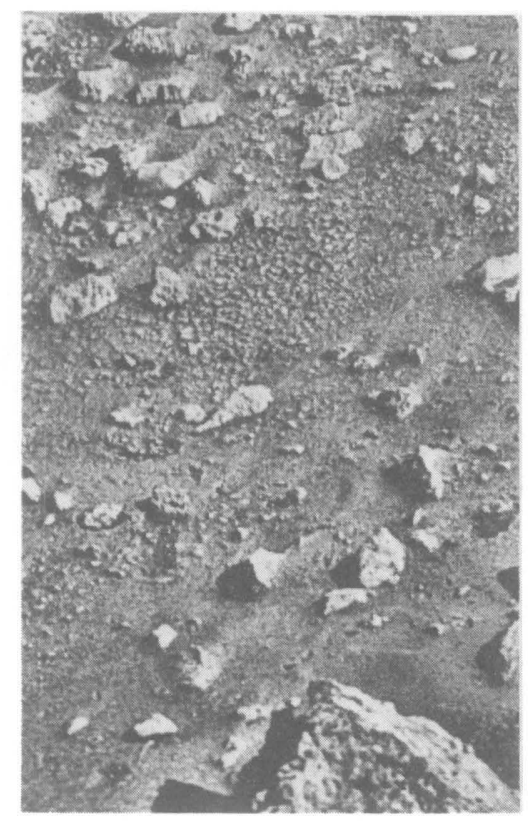

$\boldsymbol{A}$

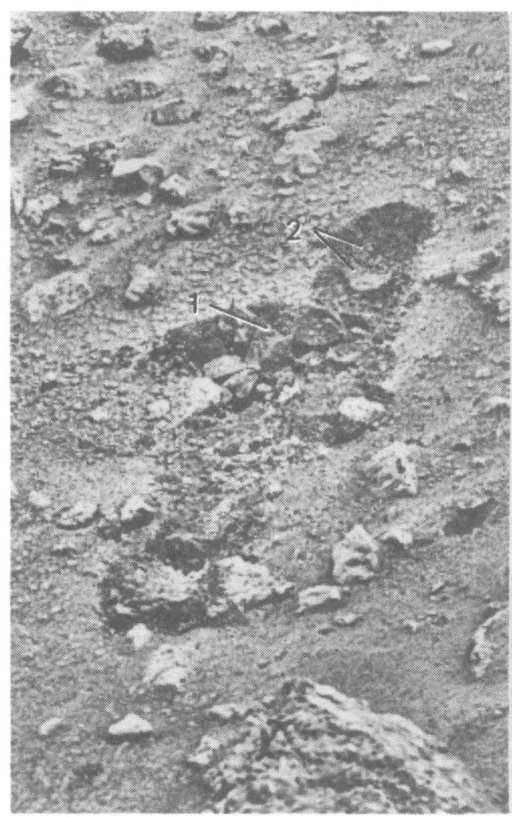

B

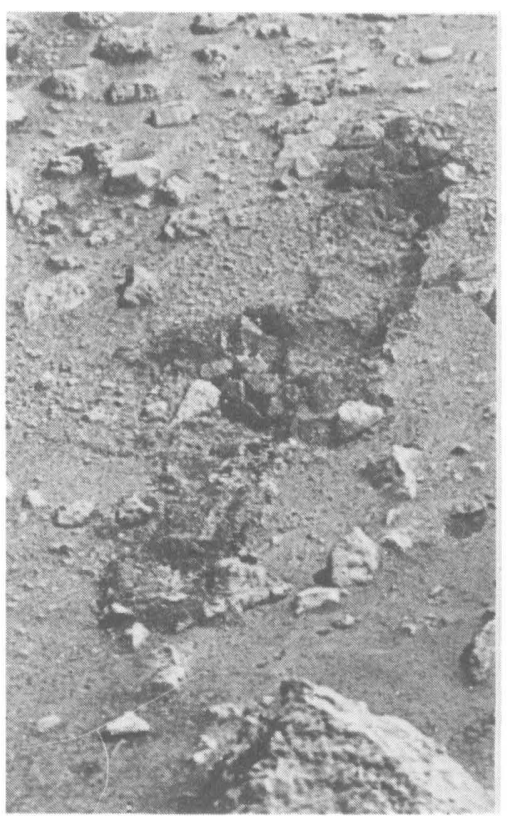

C 


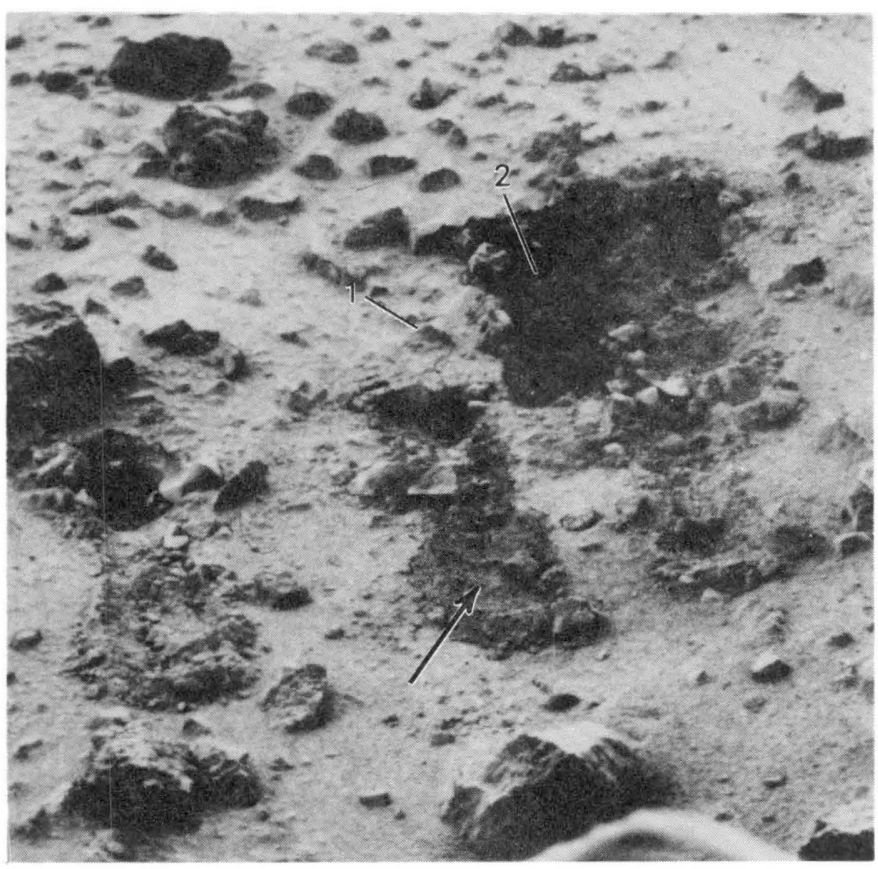

Figure 38.-Rocky Flats at the end of Sol 350, Lander 1. Trench near center is Physical Properties 2 trench (arrow). Surface has been deformed as far as rock (1). Displaced rocks are present about midway along trench. Note rubbly tailings. Trench to left is XRFS 4. All trenches to upper right show blocks at tip. Darkest trench (2) is youngest trench (Physical Properties 7, Sol 350) from which no sample was acquired. All of these trenches except XRFS 4 were destroyed when Deep Hole 2 was excavated. Physical Properties 2 trench is 7 to $12 \mathrm{~cm}$ wide and $46 \mathrm{~cm}$ long (frame 11E082/351).
TABLE 12.-Estimated angles of internal friction of blocky material from data on sample trenches

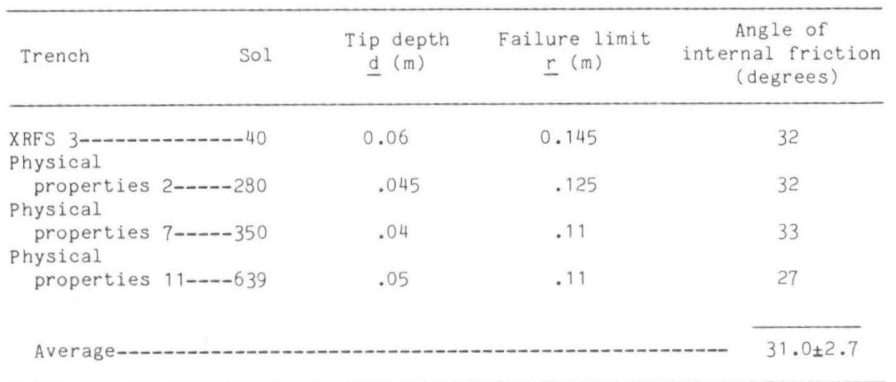

Motor-current records for trenches in blocky material, for which extend drive-motor decoupling did not occur, indicate that horizontal forces were uniformly higher by about 50-75 N than they were for drift material. Sufficient information on the regions of failure is available for three trenches in blocky material. For two of these trenches, the motor decoupled and one of these two has no motor-current record. Estimates of cohesions for these trenches vary considerably among themselves when the assumed angles of internal friction are the same (table 13). Such variable estimates are entirely consistent with the general appearance of the area. For an angle of internal friction of $30^{\circ}$, which is near the average value of $31^{\circ}$, cohesions range from 2.2 to $6.3 \mathrm{kPa}$.

Although data on horizontal forces from four additional trenches in blocky material were obtained, the region of failure could not be adequately measured. For these trenches, cohesions using the equation for pure cohesion

TABLE 13.-Cohesion of blocky material as a function of angle of internal friction, using the model of McKyes and Ali (1977) [Calculations assumed that the density was 1,200 and $2,000 \mathrm{~kg} / \mathrm{m}^{3}$ and the acceleration of gravity was $3.72 \mathrm{~m} / \mathrm{s}^{2}$. Examples of detailed calculations are given in supplemental section on "Analysis of Sample Trenches and Surface-Bearing Tests"]

\begin{tabular}{|c|c|c|c|c|c|c|}
\hline Trench & Sol & $\begin{array}{c}\text { Rake } \\
\text { angle } \\
\\
\alpha \\
\text { (degrees) }\end{array}$ & $\begin{array}{c}\text { Assumed } \\
\text { angle of } \\
\text { internal } \\
\text { friction } \\
\phi \\
\text { (degrees) }\end{array}$ & $\begin{array}{l}\text { Cohesion } \\
\qquad \text { (kPa) }\end{array}$ & $\begin{array}{l}\text { Derived horizontal } \\
\text { force at collector- } \\
\text { head interface } \\
\qquad \frac{\mathrm{F}}{(\mathrm{N})}\end{array}$ & $\begin{array}{c}\text { Available surface- } \\
\text { sampler horizontal } \\
\text { force at interface } \\
\frac{\mathrm{F}}{\mathrm{T}} \\
(\mathrm{N})\end{array}$ \\
\hline $\begin{array}{l}\text { XRFS } 3 \\
\quad \phi=32^{\circ}\end{array}$ & 40 & 94.8 & $\begin{array}{r}25 \\
130 \\
35\end{array}$ & $\begin{array}{r}6 \cdot 5-7 \cdot 5 \\
14 \cdot 3-5 \cdot 1 \\
1 \cdot 7-2 \cdot 3\end{array}$ & $\begin{array}{l}158.2-177.6 \\
143.7-161.0 \\
128.1-143.0\end{array}$ & $\begin{array}{l}158 \cdot 5-178 \cdot 3 \\
142 \cdot 9-160.7 \\
126.0-141.7\end{array}$ \\
\hline $\begin{array}{l}\text { Physical } \\
\text { Properties } 2 \\
\phi=32^{\circ}\end{array}$ & $2^{280}$ & 100.4 & $\begin{array}{r}25 \\
130 \\
35\end{array}$ & $\begin{array}{r}3 \cdot 3-4.4 \\
2.2-3 \cdot 1 \\
0.5-1.2\end{array}$ & $\begin{array}{l}62.8-79.8 \\
58.0-73.5 \\
49.9-66.0\end{array}$ & $\begin{array}{l}61 \cdot 2-81 \cdot 7 \\
58.8-74.4 \\
49.7-66.3\end{array}$ \\
\hline $\begin{array}{l}\text { Physical } \\
\text { Properties } 1 \\
\phi=27^{\circ}\end{array}$ & $11^{639}$ & 100.4 & $\begin{array}{l}125 \\
30 \\
35\end{array}$ & $\begin{array}{l}19 \cdot 3-10.6 \\
5.4-6.3 \\
0.7-1.1\end{array}$ & $\begin{array}{l}163 \cdot 6-183.0 \\
149.2-166.3 \\
133.6-147.9\end{array}$ & $\begin{array}{l}163 \cdot 4-183 \cdot 8 \\
148 \cdot 8-167 \cdot 3 \\
132 \cdot 5-149.1\end{array}$ \\
\hline
\end{tabular}

\footnotetext{
${ }^{1}$ Cohesion and angle of internal friction are approximately compatible with model.
} 
TABLE 14.-Apparent and probable cohesions of blocky material and expected horizontal forces for sand

|Apparent cohesions calculated using equation for wet clay (pure cohesion) on Earth (Wismer and Luth. 1972). Probable cohesions extrapolated from linear relation between apparent cohesions and cohesions from model of McKyes and Ali (1977). Expected horizontal forces for sand calculated using equation for sand (pure friction) on Mars (Luth and Wismer. 1971). Definitions,
equations, and discussion are given in supplemental section on "Analysis of Sample Trenches and Surface-Bearing Tests". Numbers in parentheses calculated with morel of McKyes and Ali (1977); linear relation requires that angles of sliding $(\delta)$ and internal friction $(\phi)$ equal $30^{\circ}$ )

\begin{tabular}{|c|c|c|c|c|c|c|c|c|c|}
\hline Trench & Sol & $\begin{array}{c}\text { Rake } \\
\text { Angle } \\
\boldsymbol{\alpha} \\
\text { (degrees) }\end{array}$ & $\begin{array}{l}\text { Depth } \\
\text { of } \\
\text { blade } \\
\frac{z}{(m)}\end{array}$ & $\begin{array}{l}\text { Length } \\
\text { of } \\
\text { blade } \\
\frac{1}{(m)}\end{array}$ & $\begin{array}{c}\text { Elevation } \\
\text { angle of } \\
\text { sampler } \\
\epsilon \\
\text { (degrees) }\end{array}$ & $\begin{array}{l}\text { Available } \\
\text { Horizontal } \\
\text { force when } \\
\phi=30^{\circ} \\
(N)\end{array}$ & $\begin{array}{c}\text { Apparent } \\
\text { cohesion } \\
\text { (kPa) }\end{array}$ & $\begin{array}{c}\text { Probable } \\
\text { cohesion } \\
\qquad(\mathrm{kPa})\end{array}$ & $\begin{array}{c}\text { Expected } \\
\text { horizontal } \\
\text { force for sand } \\
\text { (N) }\end{array}$ \\
\hline XRFS 3 & 40 & 94.8 & 0.06 & 0.09 & 28.8 & $1143-161$ & $7.4-8.2$ & $(4 \cdot 3-5 \cdot 1)$ & $13-22$ \\
\hline XRFS 4 & 180 & 101.1 & .065 & .10 & 35.1 & $1149-168$ & $6.6-7.2$ & $4.0-4.5$ & $18-30$ \\
\hline $\begin{array}{l}\text { Physical } \\
\text { Properties } 2\end{array}$ & 280 & 100.4 & .045 & .06 & 34.4 & $56-74$ & $4 \cdot 3-5 \cdot 5$ & $(2.2-3 \cdot 1)$ & $7-12$ \\
\hline XRFS 8 & 312 & 95.4 & .045 & .07 & 29.4 & $59-72$ & $4.4-5.6$ & $2.3-3.2$ & $7-12$ \\
\hline $\begin{array}{l}\text { Physical } \\
\text { Pruperties } 7\end{array}$ & 350 & 96.0 & .04 & .07 & 30.0 & $72-90$ & $5 \cdot 9-7.1$ & $3.4-4.4$ & $6-10$ \\
\hline $\begin{array}{l}\text { Physical } \\
\text { Properties } 8\end{array}$ & 608 & 101.1 & .04 & .06 & 35.1 & $149-168$ & $10.5-11.5$ & $6.9-7.7$ & $6-10$ \\
\hline $\begin{array}{l}\text { Physical } \\
\text { Properties } 11\end{array}$ & 639 & 100.4 & .05 & .07 & 34.4 & $149-167$ & $8.7-9.6$ & $(5.4-5.3)$ & $9-15$ \\
\hline
\end{tabular}

${ }^{1}$ No motor-current record; sampler failed to reach commanded extension so that motor must have decoupled.

and horizontal sampler forces for $\phi=30^{\circ}$ were calculated to test for compatibility with the three trenches analyzed previously (table 14). Three of the apparent cohesions fall within the range of the apparent cohesions for the three trenches analyzed in more detail; one apparent cohesion was high (Physical Properties 8). Apparent cohesions, which are larger than those obtained using the model of McKyes and Ali (1977), appear to be linearly related when $\phi=30$ (see fig. 36). This relation was used to estimate probable cohesions for the four trenches (table 14). The large probable cohesion of Physical Properties 8 suggests that local cohesions of blocky material may be as large as $6.9-7.7 \mathrm{kPa}$.

\section{CRUSTY TO CIOODDY MATIERIAI,}

Bonneville Salt Flats at the Lander 2 site (pl. $2 A$ ) was the place where the first samples were acquired for molecular and inorganic chemical analyses (fig. $30 \mathrm{~A}$, GCMS 1 and XRFS 1) because overlying materials there had been stripped away to expose a smooth, relatively bright patch with open fractures transecting the patch (fig. 39A). Many subsequent sample trenches in the Bonneville Salt Flats resulted in a vertical profile of the materials. The area around the tip of the first trench was deformed to produce uplifted plates of crust (fig. 39B) and the subsequent two trenches further substantiated the crusty nature of the material (fig. $39 C$ and $D$ ). For these two later-formed trenches, thin plates of crust $0.04 \mathrm{~m}$ wide and as much as $0.01 \mathrm{~m}$ thick were present near the tip of the trenches along with very fine-grained material. By the end of Sol 595 (fig. 39D), the far wall of the trench showed that the bright crust was a layer between two finegrained units. Elsewhere, the surface materials commonly break into thicker clods. This phenomena is well illustrated by the trench near rock 1 (ICL) (fig. 40, XRFS 3) where the surface around the end of the trench broke into clods as large as $0.07 \mathrm{~m}$ wide and $0.03 \mathrm{~m}$ thick. Surfaces adjacent to the trench have been deflated to expose the top of the clods and the joints that separate them. This trench and most of the Lander 2 sample trenches have rather small, low, poorly developed tailings similar to those in blocky material. Tailings are composed of fragments, crusts, and fine-grained material. Not all the Lander 2 sample trenches exhibit the large plates of crust

Figure 39.-Bonneville Salt Flats and trenches excavated by Lander 2. $A$, Surface prior to sample acquisitions showing smooth, relatively bright crust transected by fractures. Note that surrounding materials appear to overlie crust except where it is exposed. Rock 9 (Plymouth) at lower right is about $10 \mathrm{~cm}$ high and rock 6 (Bonneville) at center just beyond and to right of exposure of crust is about $25 \mathrm{~cm}$ long. Location of site is shown in figure 30 and plate 2 (frame 22A007/001). $B$, First sample trench (GCMS 1) exposes thin plates of crust at end of trench where surface has been domed upward (frame 22A162/021). $C$, Second sample trench (XRFS 1) has disrupted surface leaving a residue of plates of brighter crust mixed with darker fine-grained material. Largest plates are about $0.04 \mathrm{~m}$ in diameter and $0.01 \mathrm{~m}$ thick (frame 22A247/030). D, Final trench (XRFS 17) after multiple acquisitions. Note bright layer of crust in upper trench wall (arrow) is overlain and underlain by dark fine-grained material. Large slab of crust has fallen into trench at left. Base of rock 6 has been undermined (frame $22 \mathrm{H} 046 / 604)$. 


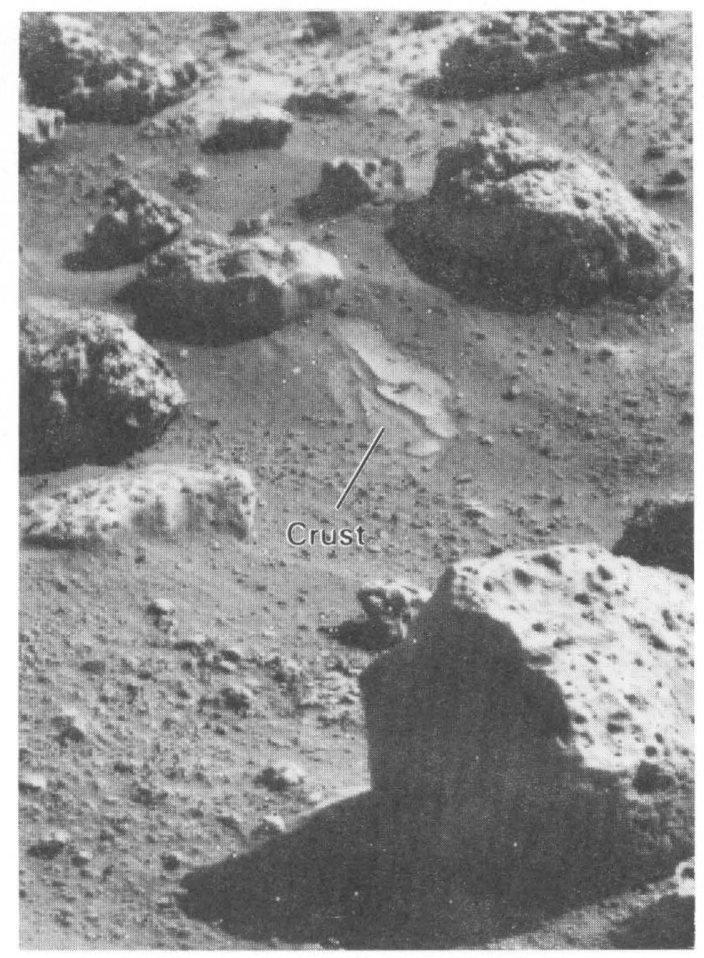

$\boldsymbol{A}$

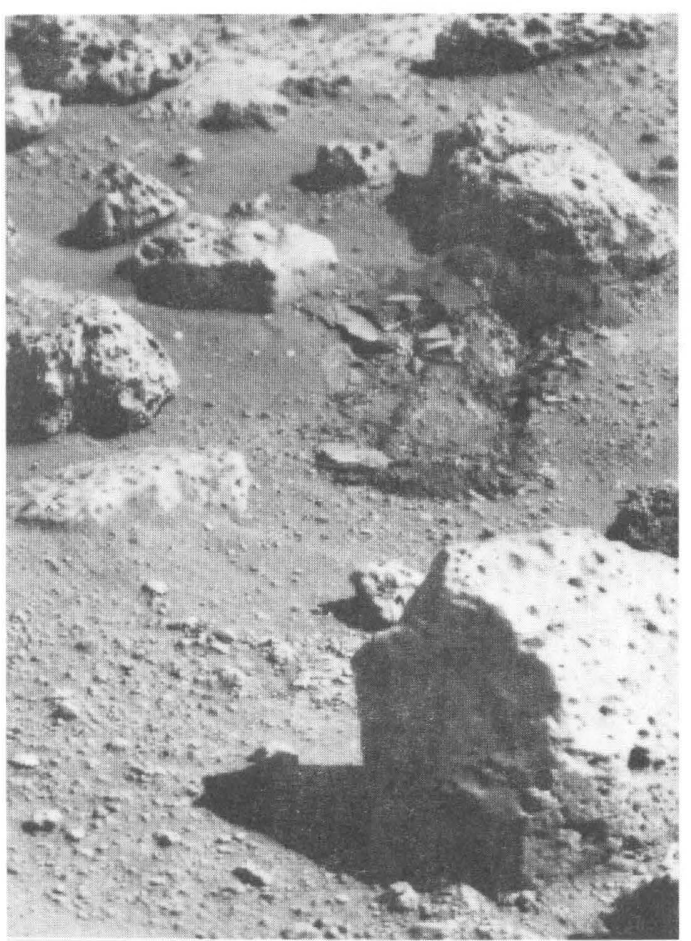

C

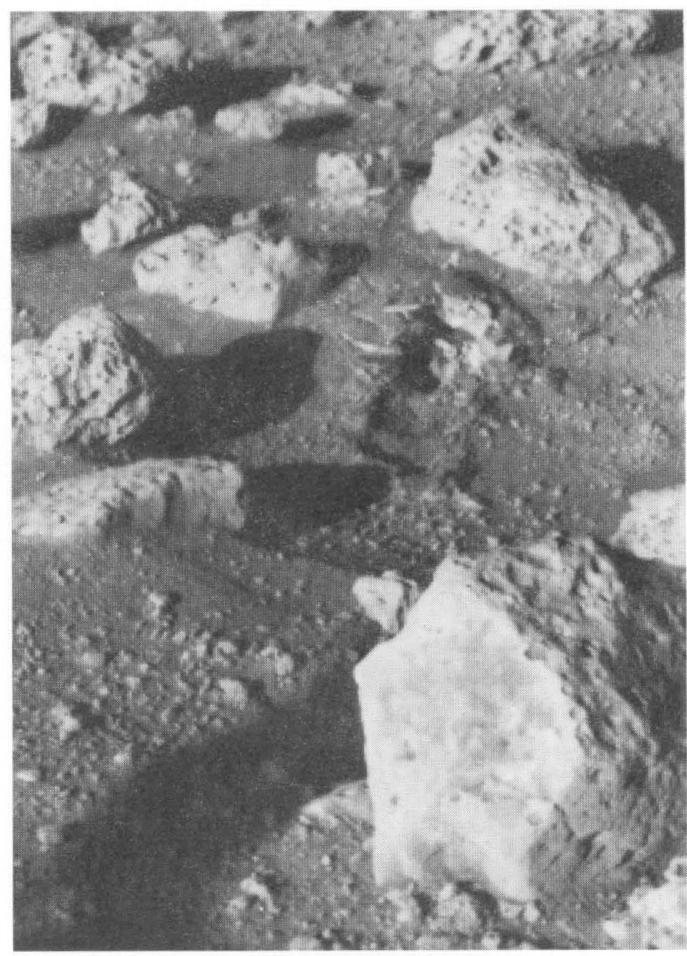

B

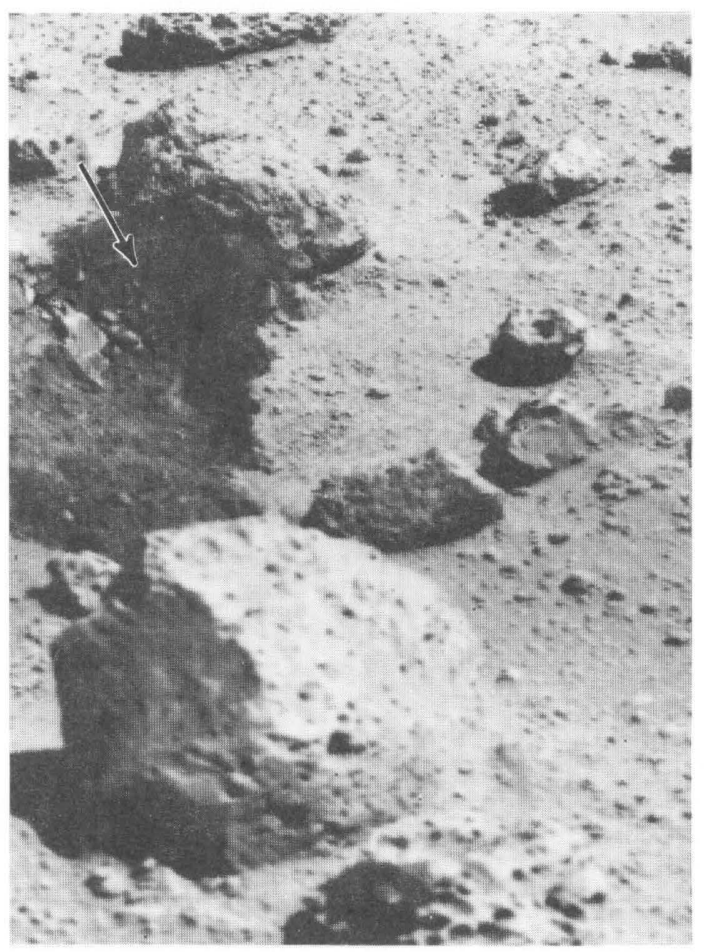

D 


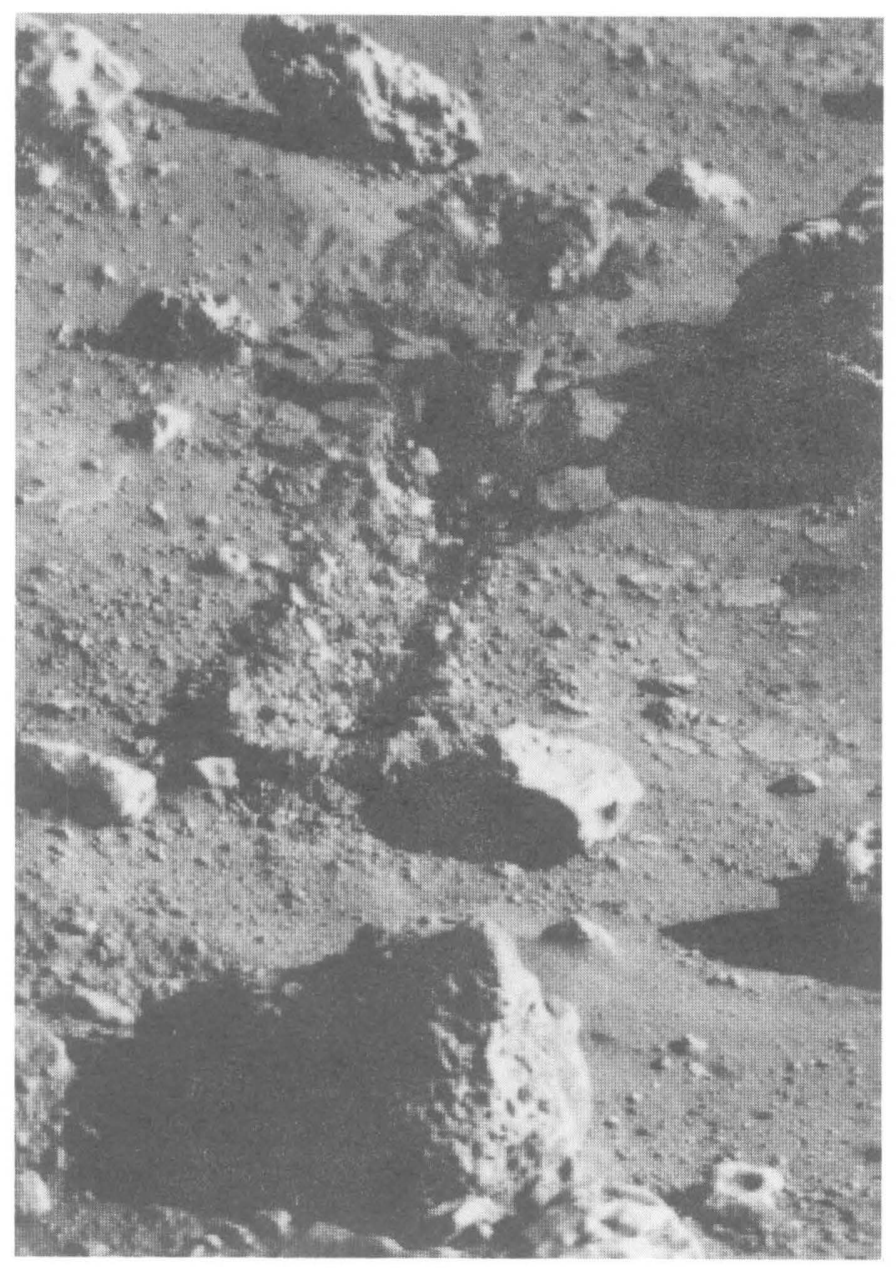

Figure 40.- Trench in cloddy material (XRFS 3). Note surface around tip of trench has been broken into angular clods as much as $0.07 \mathrm{~m}$ wide and $0.03 \mathrm{~m}$ thick. Tailings of this trench and others at Lander 2 site are poorly developed. Width of trench is about $7 \mathrm{~cm}$ (frame 22C045/57).

and clods. This is particularly true for the first trench (fig. 41A, Biology 1) where both the tailings and the tip of the trench are made of small equidimensional fragments as much as $0.02 \mathrm{~m}$ in diameter, but tailings of subsequent trenches in the same area have rather large slabs of crust in them (fig. $41 B$, Biology 2).

Although the average angles of internal friction of cloddy material seem to be about $5^{\circ}$ larger than those of blocky material, the larger values for the cloddy material must be viewed with skepticism (table 15, fig. 42). For example, an angle of internal friction of $35^{\circ}$ and a cohesion of 0 predicts that the force exerted by cloddy material at the collector-head interface of Physical Properties 5 is larger than the available horizontal force; and this is impossible. An angle of internal friction of $40^{\circ}$, however, yields a cohesion between 0.8 and 1.9 for the Physical
Properties 6 trench. The reason for this is not entirely clear. Perhaps, platy units of crust moved as large units or pushed one another during the Physical Properties 5 test to produce an apparent limit of failure that is larger than the actual one. In any case, angles of internal friction ranging from $28^{\circ}$ to $39^{\circ}$ seem probable.

Unfortunately, good estimates of the limit of failure for other trenches in materials that are mixed fine-grained material and crust or simply lumpy are not available so that the angles of internal friction cannot be determined. The limits of failure were roughly estimated for the material of two trenches (XRFS 1 and 2; table 16).

Cohesions of materials vary widely at the Lander 2 site, just as they do at the Lander 1 site, but they are typically lower for a given assumed angle of internal friction (table 16). For an angle of internal friction of 30, cohesions range from 0.5 to $1.7 \mathrm{kPa}$ when using the model of McKyes and Ali (1977), except for one shallow trench which contained only crust (Physical Properties 6). For this trench, the cohesion turned out to be rather largeabout $2.9-5.8 \mathrm{kPa}$ for an angle of internal friction of $30^{\circ}$. Additional trenches seem to have apparent cohesions that are the same magnitudes as the others when the available force corresponding to $\phi=30$ is used (table 17). The linear relation between apparent and probable cohesions is not well founded (see fig. 36).

\section{DISCUSSION}

Four conclusions can be drawn from the analyses of the sample trenches. First, the angles of internal friction of drift material are smaller than those of blocky material and crusty to cloddy material. For drift material, the angles of internal friction seem to range from $14^{\circ}$ to $21^{\circ}$; for blocky material, they range from $27^{\circ}$ to $33^{\circ}$; and for crusty to cloddy material they range from $28^{\circ}$ to $39^{\circ}$.

Second, cohesions of drift material typically seem to range from 1.4 to $3.0 \mathrm{kPa}$ for a nominal angle of internal friction of $18^{\circ}$; locally they are lower (fig. 43). Cohesions of blocky material range from 2.2 to $6.2 \mathrm{kPa}$ for a nominal angle of internal friction of $30^{\circ}$. Cohesions of crusty to cloddy material range from 0.5 to $5.8 \mathrm{kPa}$ for a nominal angle of internal friction of $30^{\circ}$.

Third, cohesions and angles of internal friction derived from the sample trenches can be used in the staticbearing-capacity equation (assuming general shear) to calculate forces on the footpads that are consistent with those estimated from landing data of Lander 1. For drift material, footpad forces can be predicted using

$$
\phi=15^{\circ} \text { and } c=1.6 \text { to } 2.5 \mathrm{kPa} \text {, and } \rho=1,200 \mathrm{~kg} / \mathrm{m}^{3}
$$

or

$$
\phi=20^{\circ} \text { and } c=1.0 \text { to } 1.7 \mathrm{kPa} \text {, and } \rho=1,200 \mathrm{~kg} / \mathrm{m}^{3} .
$$




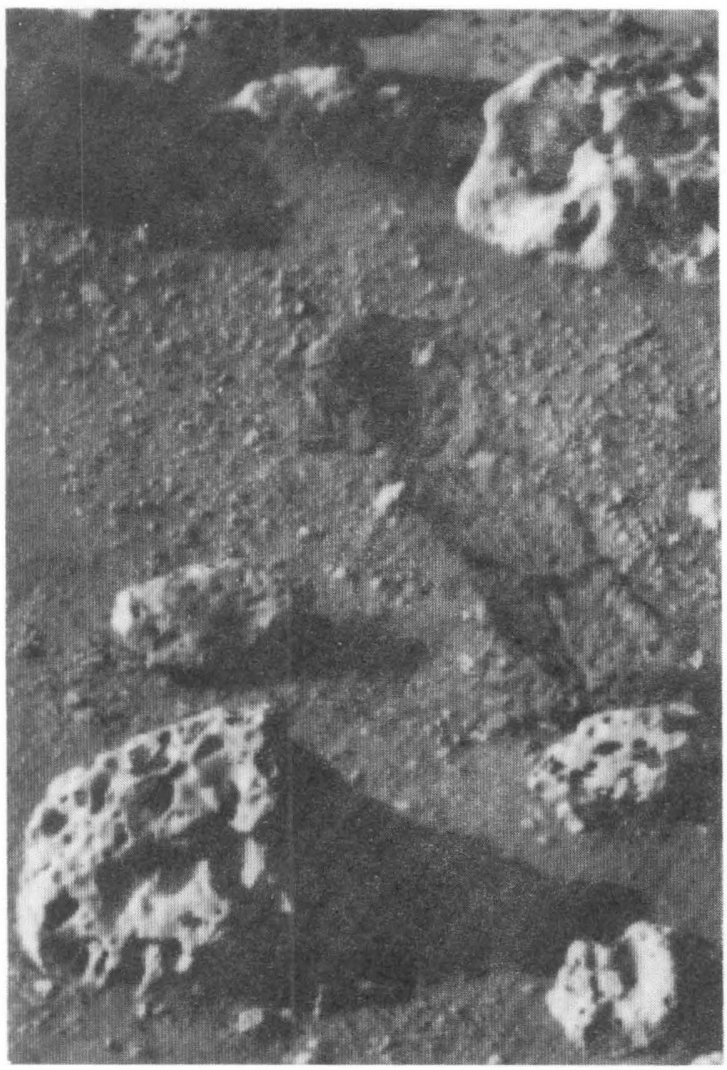

A

Figure 41.-Sample trenches in Beta site. A, First trench excavated by Lander 2 on Sol 8 (Biology 1) showing poorly developed rims and tailings. Tailings and rims contain equidimensional fragments of surface material as much as $2 \mathrm{~cm}$ wide. Form and size of rims and tailings suggest cohesionless fragments of surface material (frame

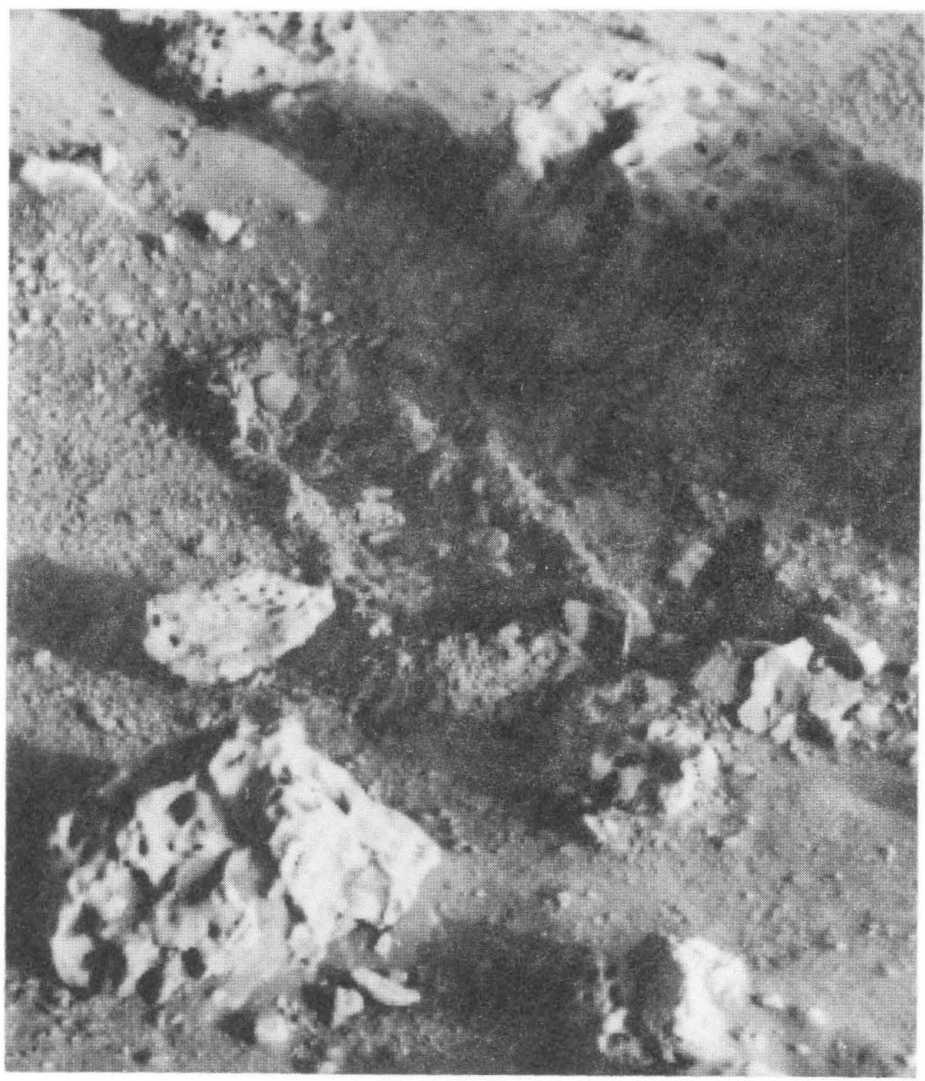

$\boldsymbol{B}$

$21 \mathrm{~A} 110 / 015)$. Trench is about $8 \mathrm{~cm}$ wide. $B$, Two later formed trenches have contrasting morphologies. Numerous slabs of crust are present in tailings of right-hand trench (Biology 2) but left-hand trench (XRFS 2) has more equidimensional fragments or clods in tailings that are as large as $3 \mathrm{~cm}$. Left-hand trench is $10 \mathrm{~cm}$ wide (frame 21C124/141)
For blocky material, footpad forces can be predicted using $\phi=30^{\circ}$ and $c=5.5-7.1 \mathrm{kPa}$, and $\rho=1,200-2,000 \mathrm{~kg} / \mathrm{m}^{3}$.

Fourth, angles of internal friction and cohesions may vary somewhat for each type of material (fig. 43) but each could be reasonably well characterized by:

Drift material,

$\phi=15^{\circ} \quad c=<1.2-3.7 \mathrm{kPa} \rho=1,200 \mathrm{~kg} / \mathrm{m}^{3}$, or $\phi=20^{\circ} \quad c=<0.8-2.9 \mathrm{kPa} \rho=1,200 \mathrm{~kg} / \mathrm{m}^{3}$.

Blocky material,

$$
\phi=30^{\circ} \quad c=2.2-7.8 \mathrm{kPa} \quad \rho=1,200 \text { to } 2,000 \mathrm{~kg} / \mathrm{m}^{3} \text {. }
$$

Mostly cloddy material,

$\phi=30^{\circ} c=0.5-5.8 \mathrm{kPa} \quad \rho=1,200$ to $2,000 \mathrm{~kg} / \mathrm{m}^{3}$, or $\phi=35^{\circ} \quad c=0-4.1 \mathrm{kPa} \quad \rho=1,200$ to $2,000 \mathrm{~kg} / \mathrm{m}^{3}$.
Mostly crusty material, $\phi=30^{\circ} \quad c=0.5-1.7 \mathrm{kPa} \quad \rho=1,200$ to $2,000 \mathrm{~kg} / \mathrm{m}^{3}$, or $\phi=35^{\circ} c=0.1-1.0 \mathrm{kPa} \quad \rho=1,200$ to $2,000 \mathrm{~kg} / \mathrm{m}^{3}$.

TABLE 15.-Estimated angles of internal friction of cloddy material from data on sample trenches, Lander 2

Limit of failure for Physical Properties 3 occurs on slope. Monoscopic estimate of limit of failur for Physical Properties 5 occurs on slope

\begin{tabular}{|c|c|c|c|c|}
\hline Trench & Sol & $\begin{array}{l}\text { Tip depth } \\
\underline{d}(\mathrm{~m})\end{array}$ & $\begin{array}{c}\text { Failure limit } \\
\underline{r}(\mathrm{~m})\end{array}$ & $\begin{array}{l}\text { Angle of } \\
\text { internal friction } \\
\text { (degrees) }\end{array}$ \\
\hline $\begin{array}{l}\text { XRFS } 3 \\
\text { Physical }\end{array}$ & 57 & 0.05 & 0.17 & 39 \\
\hline $\begin{array}{l}\text { Properties } 3 \\
\text { Physical }\end{array}$ & 479 & .065 & .16 & 34 \\
\hline $\begin{array}{l}\text { Properties } 5 \\
\text { Physical }\end{array}$ & 595 & .08 & .17 & 28 \\
\hline Properties 6 & 595 & .02 & .08 & 39 \\
\hline
\end{tabular}




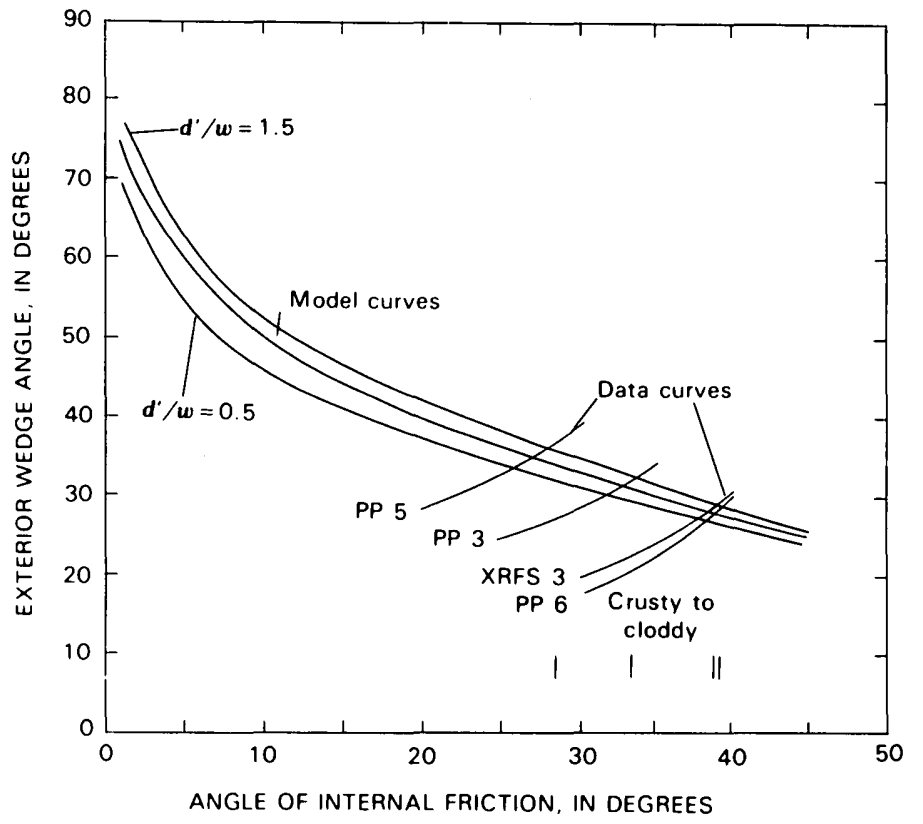

FIGURE 42.-Exterior wedge angles of region of failure predicted from theoretical model of plowing (McKyes and Ali, 1977) for three depthto-width ratios as a function of angle of internal friction and exterior wedge angles as a function of assumed angles of internal friction for sample trenches in crusty to cloddy material of Lander 2. Estimate of angle of internal friction corresponds to intersection of model curve and data curve at $d^{\prime} / w$ values appropriate for trench. Loci of calculated values are obtained by calculating wedge angles from assumed angles of internal friction. Note that average is near $35^{\circ}$. Equations and details of procedures appear in supplemental section on "Analysis of sample trenches and surface-bearing tests." Letters and numbers refer to trenches excavated for the Physical Properties Investigation (for example, PP 5) and Inorganic Chemical Analysis Experiment (XRFS 3); trench locations are shown in plate 2; trench profiles appear in supplemental section on "Outline maps, profiles, and data on trenches"; see also tables 40 and 41.

TABLE 16.-Cohesion of crusty to cloddy material as a function of angle of internal friction, using the model of McKyes and Ali (1977), Lander 2 |Calkulations assumed that the density was 1,200 and $2,000 \mathrm{~kg} / \mathrm{m}^{3}$ and the acceleration of gravity was $3.73 \mathrm{~m} / \mathrm{s}^{2}$. Examples of detailed calculations are given in supplemental section on "Analysis of Sample Trenches and Surface-Bearing Tests"]

\begin{tabular}{|c|c|c|c|c|c|c|}
\hline Trench & Sol & $\begin{array}{c}\text { Rake } \\
\text { angle } \\
\boldsymbol{\alpha} \\
\text { (degrees) }\end{array}$ & $\begin{array}{l}\text { Assumed } \\
\text { angle of } \\
\text { internal } \\
\text { friction } \\
\phi \\
\text { (degrees) }\end{array}$ & $\begin{array}{l}\text { Cohesion } \\
\text { (kPa) }\end{array}$ & $\begin{array}{c}\text { Derived horizontal } \\
\text { force at collector- } \\
\text { head interface } \\
\underline{\mathrm{F}}_{\underline{\mathrm{H}}} \\
(\mathrm{N})\end{array}$ & $\begin{array}{l}\text { Available surface- } \\
\text { sampler horizontal } \\
\text { force at interface } \\
\qquad \underline{F}^{-} \\
(\mathrm{N})\end{array}$ \\
\hline \multicolumn{7}{|c|}{ Chiefly cloddy material } \\
\hline $\begin{array}{l}\text { Physical } \\
\text { Properties } 3 \\
\phi=34^{\circ}\end{array}$ & 479 & 91.2 & $\begin{array}{r}25 \\
30 \\
135\end{array}$ & $\begin{array}{r}1.0-2.5 \\
0.5-1.7 \\
1 \begin{array}{r}0-0.7\end{array}\end{array}$ & $\begin{array}{l}39 \cdot 5-78 \cdot 1 \\
35 \cdot 6-70.4 \\
38.9-60.5\end{array}$ & $\begin{array}{l}38.6-77.2 \\
34.6-69.2 \\
30.3-60.6\end{array}$ \\
\hline $\begin{array}{l}\text { Physical } \\
\text { Properties } 5 \\
\phi=28^{\circ}\end{array}$ & 595 & 101.2 & $\begin{array}{r}25 \\
130 \\
35\end{array}$ & $\begin{array}{c}2.6-3.2 \\
1.1-1.6 \\
0\end{array}$ & $\begin{array}{c}182 \cdot 3-205.0 \\
169.9-188.2 \\
\text { exceeds horizontal force }\end{array}$ & $\begin{array}{l}181.4-204.1 \\
169.0-190.1 \\
154.5-173.8\end{array}$ \\
\hline $\begin{array}{l}\text { Physical } \\
\text { Properties } 6 \\
\phi=39^{\circ}\end{array}$ & 595 & 96.5 & $\begin{array}{r}25 \\
30 \\
35 \\
140\end{array}$ & $\begin{array}{l}3.7-7.4 \\
2.9-5.8 \\
1.9-4.1 \\
10.6-1.8\end{array}$ & $\begin{array}{l}40.4-79.8 \\
36.9-71.3 \\
31.9-63.0 \\
27.6-53.8\end{array}$ & $\begin{array}{l}40 \cdot 0-80.1 \\
36 \cdot 2-72.4 \\
32.0-64.0 \\
27 \cdot 6-55.2\end{array}$ \\
\hline \multicolumn{7}{|c|}{ Mixed crusty and fine-grained materials } \\
\hline XRFS $2^{2}$ & 47 & 93.4 & $\begin{array}{r}25 \\
130 \\
35\end{array}$ & $\begin{array}{r}0.9-2.0 \\
.5-1.4 \\
.1-0.7\end{array}$ & $\begin{array}{l}20.2-39.6 \\
17.2-35.7 \\
14.8-32.2\end{array}$ & $\begin{array}{l}19.6-39.2 \\
17.6-35.3 \\
15.8-32.2\end{array}$ \\
\hline XRFS $1^{2}$ & 30 & 88.7 & $\begin{array}{r}25 \\
130 \\
35\end{array}$ & $\begin{array}{r}1.1-2.3 \\
.7-1.7 \\
.3-1.0\end{array}$ & $\begin{array}{l}18 \cdot 2-38 \cdot 1 \\
16.7-33 \cdot 8 \\
15.2-30.0\end{array}$ & $\begin{array}{l}18.9-37.8 \\
16.9-33.7 \\
14.7-29.4\end{array}$ \\
\hline
\end{tabular}

${ }^{1}$ Cohesions and angle of internal friction are approximately compatible with model.

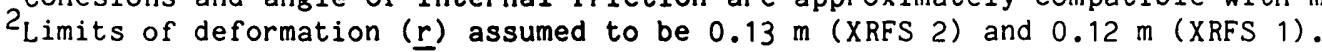


TABLE 17.-Apparent and probable cohesions of crusty to cloddy material and expected horizontal forces for sand, Lander 2

[Apparent cohesions calculated using equation for wet clay (pure cohesion) on Earth (Wismer and Luth, 1972). Probable cohesions estimated from relation between apparent cohesions and cohesions from model of McKyes and Ali (1977). Expected horizontal forces for sand calculated using equation for sand (pure friction) on Mars (Luth and Wismer, 1971). Definitions, equacohesions from model of McKyes and Ali (1977). Expected horizontal forces for sand calculated using equation for sand (pure friction) on Mars (Luth and Wismer, 1971). Definitions, equations, and discussion are given in supplemental section on "Analysis of Sample Trenches and Surface-Bearing Tests". Elevation angles of surface sampler corrected for lander tilt. Numbers
in parentheses calculated with model of McKyes and Ali (1977); relation used angles of sliding $(d)$ and internal friction $(\phi)$ of $30^{\circ}{ }^{\circ}$. Trench morphology of Physical Properties 2 and XRFS in parentheses calculated with model of McKyes and Ali (1977); relation used angles of sliding ( $d$ ) and internal friction $(\phi)$ of $30^{\circ}$. Trench morphology of Physical Properties 2 and XRFS
1 and 13 was made with monoscopic estimates. Trenches of Physical Properties 3 and 5 are in same location; collector head for second trench was elevated for $2 \mathrm{~s}$ just before extension for sampling. Sample trench of Physical Properties 6 is shallow and in surface crust]

\begin{tabular}{|c|c|c|c|c|c|c|c|c|c|}
\hline Trench & Sol & $\begin{array}{c}\text { Rake } \\
\text { angle } \\
\alpha \\
\text { (degrees) } \\
\end{array}$ & $\begin{array}{c}\text { Depth } \\
\text { of } \\
\text { blade } \\
\underline{z} \\
\end{array}$ & $\begin{array}{l}\text { Length } \\
\text { of } \\
\text { blade } \\
\frac{1}{(m)}\end{array}$ & $\begin{array}{c}\text { Elevation } \\
\text { angle of } \\
\text { sampler } \\
\epsilon \\
\text { (degrees) } \\
\end{array}$ & $\begin{array}{c}\text { Available } \\
\text { horizontal } \\
\text { force when } \\
\phi=30^{\circ} \\
(\mathrm{N})\end{array}$ & $\begin{array}{l}\text { Apparent } \\
\text { cohesion } \\
(\mathrm{kPa})\end{array}$ & $\begin{array}{c}\text { Probable } \\
\text { cohesion } \\
(\mathrm{kPa})\end{array}$ & $\begin{array}{c}\text { Expected } \\
\text { horizontal } \\
\text { force for sand } \\
\text { (N) }\end{array}$ \\
\hline \multicolumn{10}{|c|}{ Mainly crust and fine-grained material } \\
\hline $\begin{array}{l}\text { Biology } 2 \\
\text { XRFS } 2 \\
\text { XRFS } 1 \\
\text { XRFS } 13\end{array}$ & $\begin{array}{r}28 \\
47 \\
30 \\
506\end{array}$ & $\begin{array}{l}92.8 \\
.93 .4 \\
88.7 \\
88.7\end{array}$ & $\begin{array}{l}0.03 \\
.05 \\
.045 \\
.07\end{array}$ & $\begin{array}{l}0.045 \\
.07 \\
.06 \\
.09\end{array}$ & $\begin{array}{l}26.8 \\
27.4 \\
22.7 \\
22.7\end{array}$ & $\begin{array}{c}<18 \\
18-33 \\
19-36 \\
36-54\end{array}$ & $\begin{array}{l}<2.6 \\
1.6-2.9 \\
1.8-3.3 \\
2.2-3.0\end{array}$ & $\begin{array}{c}<0.9 \\
(0.5-1.4) \\
(0.7-1.7) \\
0.5-1.5\end{array}$ & $\begin{array}{r}3 \\
8 \\
6 \\
15\end{array}$ \\
\hline \multicolumn{10}{|c|}{ Mainly cloddy } \\
\hline $\begin{array}{l}\text { Physical } \\
\quad \text { Properties } 2\end{array}$ & 405 & 101.6 & .08 & 0.09 & 35.6 & $.150-169$ & $5.7-6 . .4$ & $1.1-3.2$ & 24 \\
\hline $\begin{array}{l}\text { Physical } \\
\text { Properties } 3\end{array}$ & 479 & 91.2 & .065 & .08 & 25.2 & $35-69$ & $2 \cdot 3-4 \cdot 1$ & $(0.5-1.7)$ & 13 \\
\hline $\begin{array}{l}\text { Physical } \\
\text { Properties } 5\end{array}$ & 595 & 101.2 & .08 & .14 & 25.2 & $169-190$ & $5 \cdot 7-6.3$ & $(1.1-1.6)$ & 27 \\
\hline $\begin{array}{l}\text { Physical } \\
\text { Properties } 6\end{array}$ & 595 & 96.5 & .02 & .045 & 30.5 & $36-72$ & $5.7-10.2$ & $(2.9-5.8)$ & 1.7 \\
\hline
\end{tabular}

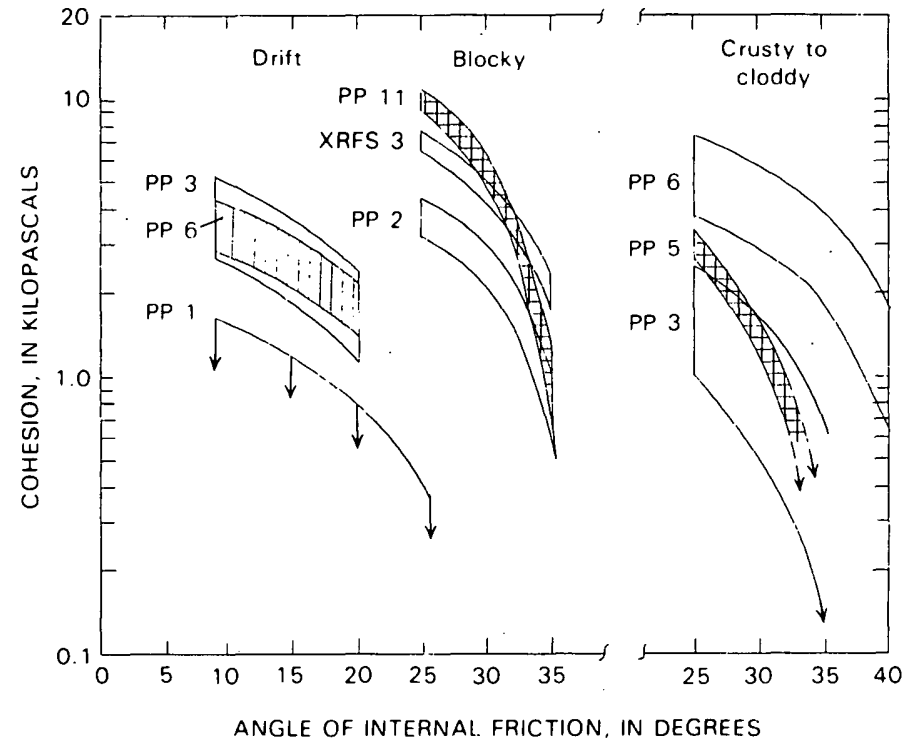

FIGURE 43.-Cohesion as a function of angle of internal friction for drift material, blocky material, and crusty to cloddy material as calculated with the model of McKyes and Ali (1977). Cohesions for nominal angle of internal friction for drift material $\left(\phi=18^{\circ}\right)$ are $\leqslant 3 \mathrm{kPa}$; those for each trench are: $2.0-4.0 \mathrm{kPa}\left(\mathrm{PP} 3, \phi=14^{\circ}\right), 1.7-2.6 \mathrm{kPa}\left(\mathrm{PP} 6, \phi=18^{\circ}\right.$, shown by pattern), and $0-0.7 \mathrm{kPa}\left(\mathrm{PP} 1, \phi=21^{\circ}\right)$. Cohesions for nominal angle of internal friction of blocky material $\left(\phi=30^{\circ}\right)$ are $2.2-6.2 \mathrm{kPa}$; those for each trench are: $7.8-9.0 \mathrm{kPa}\left(\mathrm{PP} 11, \phi=27^{\circ}\right.$, shown by pattern), $2.8-4.0 \mathrm{kPa}\left(\mathrm{XRFS} 3, \phi=32^{\circ}\right)$, and $1.5-2.3 \mathrm{kPa}\left(\mathrm{PP} 2, \phi=32^{\circ}\right)$. Cohesions for nominal angle of internal friction for crusty to cloddy material $\left(\phi=30^{\circ}\right)$ are $0.5-5.8 \mathrm{kPa}$; those for each trench are: $0.8-2.3$ $\mathrm{kPa}\left(\mathrm{PP} 6, \phi=39^{\circ}\right), 1.6-2.3 \mathrm{kPa}$ (PP $5, \phi=28^{\circ}$, shown by pattern), and $0.2-0.9 \mathrm{kPa}\left(\mathrm{PP} 3, \phi=34^{\circ}\right.$ ). Arrows on curve for PP 1 (drift material) indicate cohesions are maxima; arrows on curves for PP 3 and PP 5 indicate cohesions go to zero when angles of internal friction increase a few degrees. 


\begin{tabular}{|c|c|c|c|c|c|c|c|}
\hline & \multicolumn{4}{|c|}{ Local Lander Time } & \multicolumn{3}{|c|}{$\begin{array}{c}\text { Achieved } \\
\text { surface-sampler positions } \\
\end{array}$} \\
\hline & (Sol) & $(h r)$ & $(\min )$ & (s) & (degrees) & (in.) & (degrees) \\
\hline $\begin{array}{l}\text { Drift material (Sandy Flats) } \\
\quad \text { Viking Lander } 1\end{array}$ & 612 & 03 & 05 & 41 & 117.2 & $\begin{array}{r}56.0 \\
60.7 \\
4.1\end{array}$ & 40.1 .39 .5 \\
\hline Pretest picture & 611 & - & - & - & & & \\
\hline Test picture & 612 & 08 & 12 & 32 & & & \\
\hline Test picture & 612 & 08 & 14 & 59 & & & \\
\hline Posttest picture & 613 & 07 & 13 & 21 & & & \\
\hline Posttest picture & 613 & 07 & 15 & 21 & & & \\
\hline $\begin{array}{l}\text { Blocky inaterial (Rocky Flats) } \\
\text { Viking Lander } 1\end{array}$ & 612 & 15 & 19 & 22 & 184.1 & $\begin{array}{r}54.9 \\
58.3 \\
4.1\end{array}$ & $40.1,39.5$ \\
\hline Pretest picture & 611 & 16 & 54 & 43 & & & \\
\hline Test picture & 612 & 16 & 52 & 32 & & & \\
\hline Test picture & 612 & 16 & 54 & 32 & & & \\
\hline Posttest picture & 616 & 07 & 14 & 11 & & & \\
\hline Posttest picture & 618 & 07 & 15 & 00 & & & \\
\hline
\end{tabular}

$11 \mathrm{H} 164$

124186

$11 \mathrm{H} 187$

$12 \mathrm{H} 191$

$11 \mathrm{H} 192$

$12 \mathrm{H} 179$

$11 \mathrm{H} 188$

124189

$11 \mathrm{H} 200$

$12 \mathrm{H} 204$
Remarks

Commanded elevations were $40.1^{\circ}$ and $39.5^{\circ}$. Commanded extension was 68.8 in.; motor decoupled about $5.7 \mathrm{~s}$ after motor start; motor currents exceeded no-load currents $1.7 \mathrm{~s}$ af ter motor start and increased rapidly $4 \mathrm{~s}$ after motor start.

Local Lander time tag missing, should be about the same as for $11 \mathrm{H} 187$.

Collector head is closed; surface of deformation indicates sampler moved buried rock.

Backhoe dragged on surface during extension and thus folded.

Surface-sampler boom housing obscured surface.

Surface sampler smoothed part of tailings and produced reflective surface. Photographic measurements indicate that surface-sampler tip touched surface at $40.1^{\circ}$ elevation, then lifted to $39.5^{\circ}$ and should have achieved elevations of $40.1^{\circ}$ and $39.5^{\circ}$; and that the surface was contacted at an extension of 56.3 in. $\left(39.5^{\circ}\right.$ elevation); duration of motor currents indicates final extension was about $61.5 \mathrm{in.}$

Comnanded elevations were $40.1^{\circ}$ and $39.5^{\circ}$. Commanded extension was 67.0 in.; motorcurrent record difficult to interpret; motor did not clutch or decouple.

Shows undisturbed surface.

Backhoe is folded; no grooves were produced during extension.

Comparison with $12 \mathrm{H} 179$ reveals subtle surface deformation.

Part of posttest stereopair.

Part of posttest stereopair. Photographic measurements indicate that surfacesampler tip touched surface at $40.1^{\circ}$ elevation then lifted to $39.5^{\circ}$ and should have achieved elevations of $40.1^{\circ}$ and $39.5^{\circ}$; motor currents indicate surface sampler touched surface at motor start and extended to 53.4 in. 
음

峁苍芯。

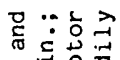

-

웡ㅎㅀ

वर岕的

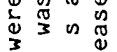

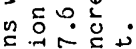

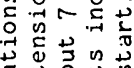

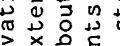

c o 돈

○过

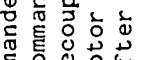

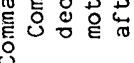

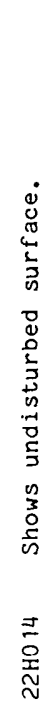

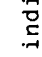

궁

$$
\text { 政 }
$$

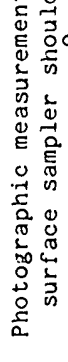

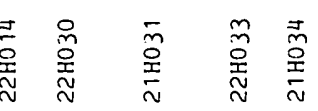

กิ

$\stackrel{n}{\infty}$

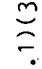

$\stackrel{\circ}{g}$

ลิว

งิํㅇㅁㅇ

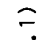

$\dot{\circ}$

$\stackrel{n}{n}$

$$
\begin{aligned}
& \text { 으 요 in } \\
& \text { 8 กิ ษี่ }
\end{aligned}
$$

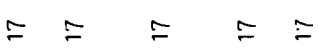

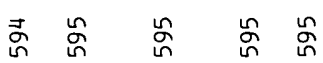

$\stackrel{\infty}{i}$

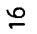

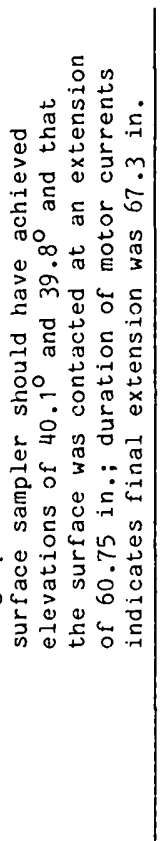

\section{SURFACE-BEARING TESTS}

Three surface-bearing tests were conducted just before the surface samplers were parked near the end of the Extended Mission. Two of the tests were conducted by Lander 1: one on drift material in Sandy Flats (Physical Properties 9) and the other on blocky material near Rocky Flats (Physical Properties 10). The third test was conducted by Lander 2 (Physical Properties 7). For a surface-bearing test, the collector head was pushed into the surface material at a steep angle with the maximum possible force (the maximum force occurs when the surface-sampler extend-drive motor decouples). The extent of surface deformation and penetration of the collector head was measured and established using pictures and surface-sampler data. The dimensions of the region of failure and the geometry of the inserted collector head were used to compute exterior wedge angles and to estimate the angle of internal friction. Cohesion was then calculated from the known force required to decouple the motor using the theory for narrow plow blades (McKyes and Ali, 1977). The calculation was corrected for frictional sliding, and the geometrical relations were established by mapping. For more detail, refer to to the supplemental section on, "Analysis of Sample Trenches and SurfaceBearing Tests."

Sequences of all the surface-bearing tests were essentially the same (table 18). The collector head was lowered to the surface along the commanded azimuth with the desired extension. After the downward motion was stopped by surface contact, alignment of the collectorhead axis with the boom axis was ensured by an elevation command in the upward direction for a period of $1 \mathrm{~s}$. The surface sampler was then extended into the surface material while the collector head was in the closed position. Initially, the collector head probably extended in the atmosphere before entering the surface material. Motor currents were monitored at the lander bus (Format 5 ) as the extending collector head penetrated into the surface material. These current records, here interpreted in terms of force, were used to show that the motor did, in fact, decouple; and the fact that it did indicates that maximum force $(200-225 \mathrm{~N})$ was exerted by the collector head. The procedure used to account for forces related to frictional sliding was the same as that used in the previous section except that the collector-head rotation angle $(\gamma)$ was 0 and the angle between the leading edge and base $\left(\theta^{*}\right)$ was $53^{\circ}$.

\section{DRIFT MATERIAL}

Pictures of the surface-bearing test in drift material show that the axis of the collector-head unit is aligned with the boom axis and that the collector head is in the closed position (fig. $44 B$ ). The backhoe is partly folded and lies 
flat against the surface. The style of deformation varies with the distance from the collector head. Near the deeply buried collector head, drift material was displaced to form a lumpy embankment that extends 0.03-0.04 $\mathrm{m}$ from the collector-head lid. Beyond the lumpy embankment, the surface has mostly been deformed upward to produce monoclinal flexures, but locally the surface is depressed. One monoclinal flexure extends from the edge of a buried rock that was also slightly displaced; this relation indicates that the collector-head tip may have contacted the rock during the test. It is also possible that the collector head disturbed a hard substrate which, in turn, disturbed the rock. These relations were well shown by difference pictures as well as by the normal pictures (fig. 44). The limit of failure extended $0.085 \mathrm{~m}$ from the collector head along the original ground surface in the azimuth plane; the failure region was $0.132 \mathrm{~m}$ wide (fig. 45).

Mapping and establishing the geometrical relations of the drift material was entirely successful, but the mapping was somewhat complicated because the post-test view of camera 2 was partly obscured by the boom hous- ing. Although the left-hand edge of the surface and deformed surface could be mapped with pictures of the test from cameras 1 and 2, mapping of the right-hand edge required the careful use of pretest and test pictures taken by both cameras and the camera 1 post-test picture. In the resulting map (fig. 45), the relation between the collector head, the surface deformations, and the local surface were well established and surface-sampler positions inferred from the map were in excellent agreement with the achieved positions derived from surface-sampler data (table 19). The depth of the collector-head tip below the original surface was $0.07 \mathrm{~m}$.

An angle of internal friction was estimated with the procedures used previously for the sample trenches. Comparison of the model curves and the data curve for drift material yielded an angle of internal friction of about $17^{\circ}$ (fig. 46).

The motor-current record (interpreted in terms of force) for the surface-bearing test was rather typical (fig. 47A), and it showed that the surface-sampler extend drivemotor decoupled so that a force of about 200-225 N was

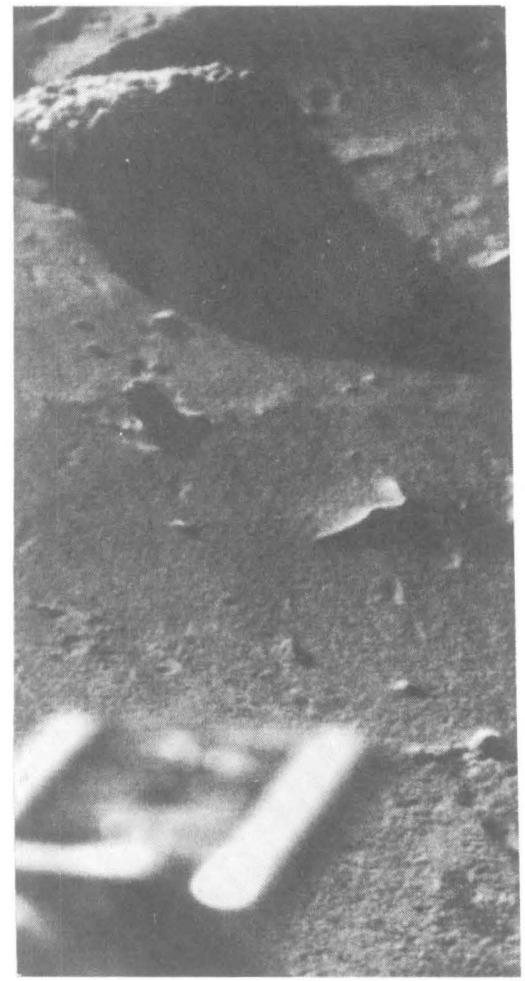

A

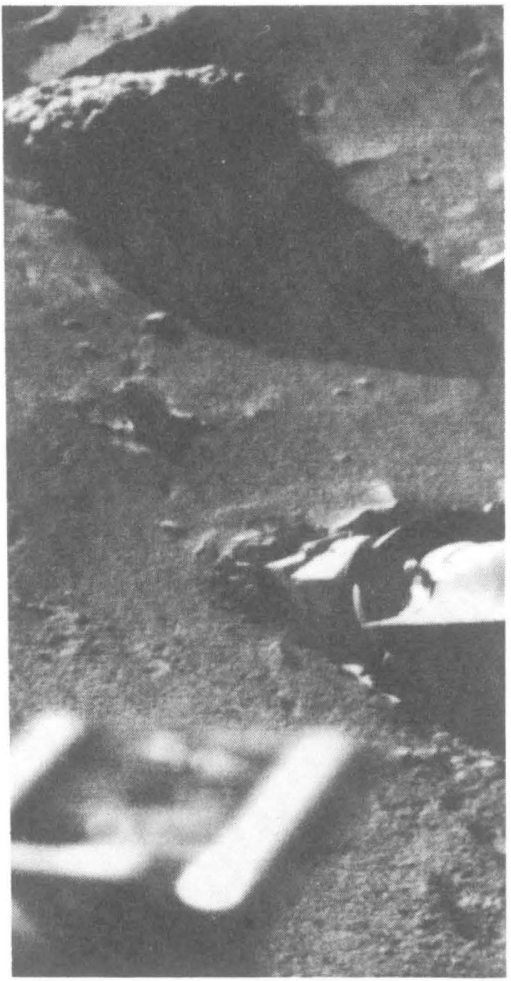

$\boldsymbol{B}$

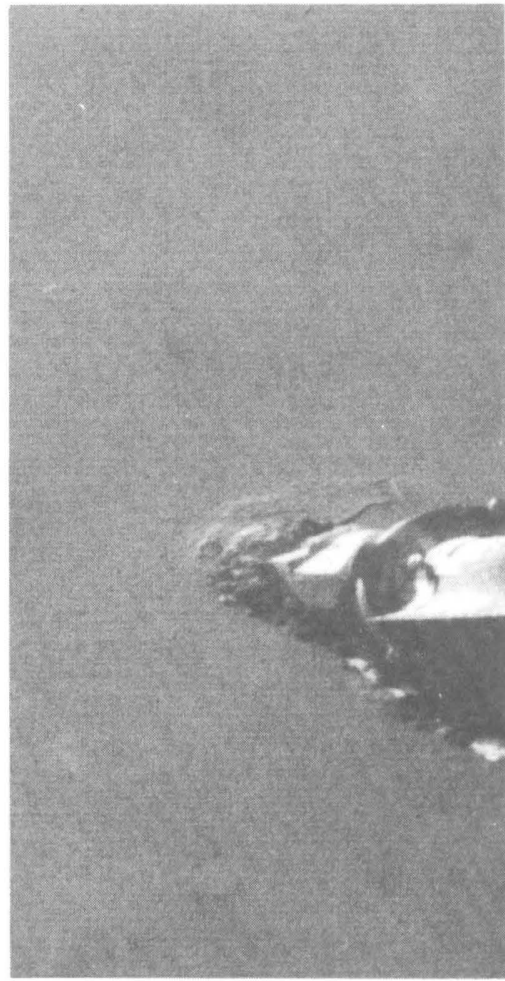

C
Figure 44.-Surface-bearing test in drift material (Lander 1). A, Surface before test; object in lower left is magnet cleaning brush. Note light V-shaped tip of buried rock (about $10 \mathrm{~cm}$ long) and bright pebble to left (frame 11H164/611). B, Collector head inserted in drift material at end of test. Note that collector head is aligned with boom axis (to right) and that lid is closed. Lumpy embankment of disturbed surface is adjacent to collector head and monoclinal flexure is bright band extending from $\mathrm{V}$-shaped rock toward pebble in front of collector head (frame $11 \mathrm{H} 187 / 612$ ). C, Difference picture made by computer processing of frames $A$ and $B$. Changes in surface are either lighter or darker than background. Note that V-shaped pattern of rock above collector head is visible. Monoclinal flexure of surface appears as sinuous light band from rock. Picture processed by E.A. Guinness, Lander Imaging Team. 
exerted by the collector head. For this amount of force, the horizontal component (allowing for friction) would be $236-265 \mathrm{~N}\left(\phi=15^{\circ}\right)$ or $224-252 \mathrm{~N}\left(\phi=20^{\circ}\right)$ (see table 20 ). From the mapping data and the plowing model, the cohesion required to produce horizontal forces ranging from $236-252 \mathrm{~N}$ is in the range from 12 to $20 \mathrm{kPa}$ (see table 20 ); this amount of cohesion is incompatible with cohesion estimates based on the sample trenches operations and backhoe touchdowns which are described later. For cohesions ranging from 1 to $2 \mathrm{kPa}$, horizontal forces should be $22-39 \mathrm{~N}\left(\phi=20^{\circ}\right.$; table 20$)$. Thus, the increase in force from the motor-current record above the no-load current should not have exceeded about $50 \mathrm{~N}$ (total force $100 \mathrm{~N}$, in fig. 47A). Therefore, it seems that the high currents were caused by an unanalyzed factor. This factor is probably a hard substrate or buried rock. In any case, the estimate of the angle of internal friction appears to be reasonable.

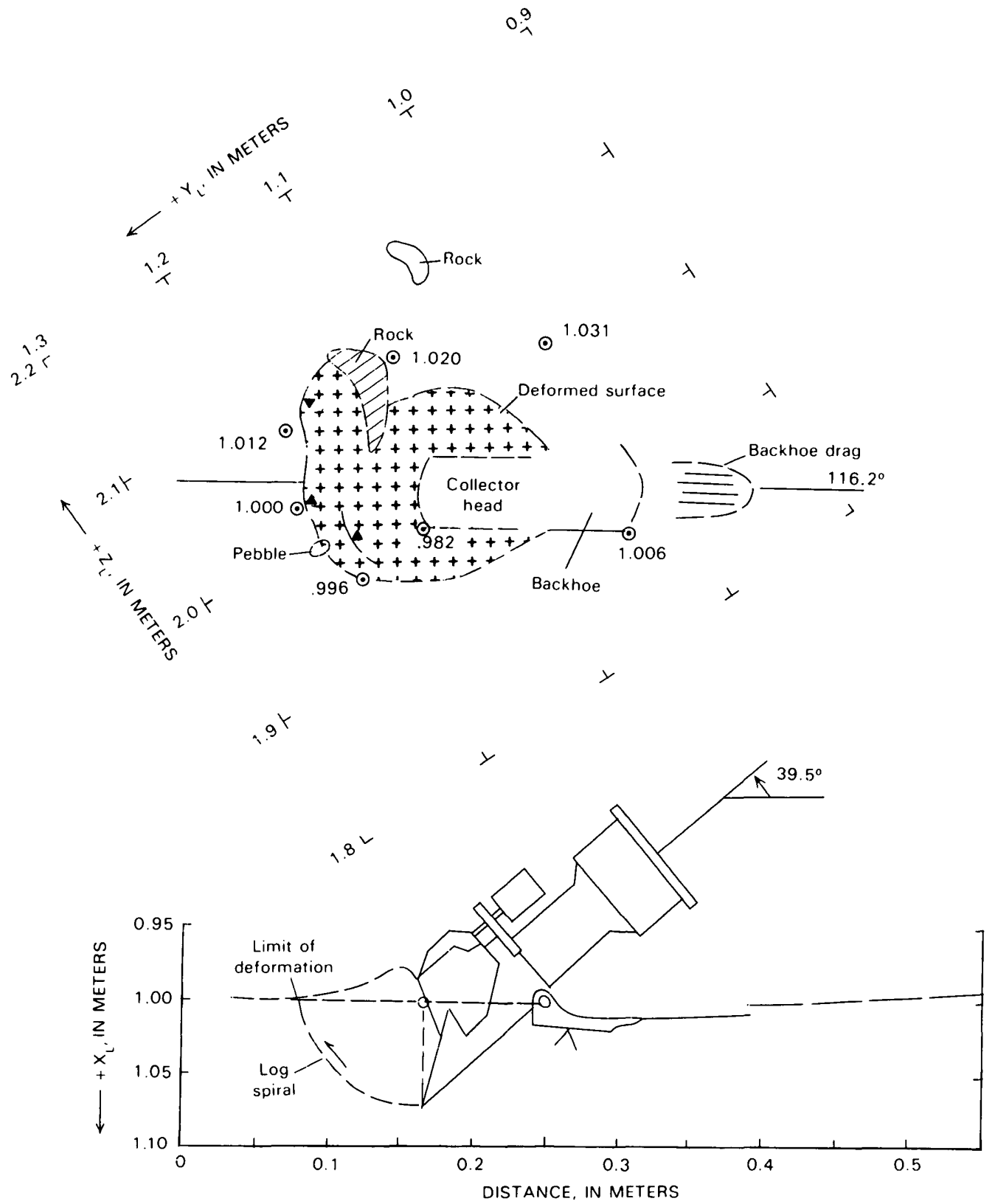

FIGURE 45.-Map and profile of surface-bearing test on drift material (see fig. 44). Note pebble and V-shaped tip of rock. Dashed lines outline disturbed surface. Solid triangles indicate monoclines. Exterior wedge angle and depth-to-width ratio indicate that angle of internal friction is approximately $17^{\circ}$. Numbers by open circles with dots indicate elevations in meters in direction of $+X_{L}$ axis. Surface-sampler elevation angle is $39.5^{\circ}$; surface-sampler azimuth is $116.2^{\circ}$. 


\section{BLOCKY MATERIAL}

Pictures of the surface-bearing test on blocky material show that the axis of the collector-head unit was aligned with the boom axis and that the collector-head lid was in the closed position because if either the unit was rotated $10^{\circ}$ or if the lid of the collector head was open, the lid would be visible (fig. 48). Only part of the deformed surface was visible in the test, and difference pictures (fig. 48) show this deformation as a lateral lobe to the right of the collector head. The remainder of the region of failure of surface material and the trench were seen by comparing pretest pictures and enhanced post-test pictures. Near the trench, the materials were displaced upward to form an

TABLE 19.-Commanded positions, surface-sampler data, and inferred positions from stereometric measurements and mapping for the surfacebearing test in drift material (Lander 1)

\begin{tabular}{|c|c|c|c|}
\hline $\begin{array}{l}\text { Surface-sampler } \\
\text { positions }\end{array}$ & $\begin{array}{l}\text { Azimuth } \\
\text { (degrees) }\end{array}$ & $\begin{array}{l}\text { Elevation } \\
\text { (degrees) }\end{array}$ & $\begin{array}{l}\text { Extension } \\
\text { (in.) }\end{array}$ \\
\hline $\begin{array}{l}\text { Commanded } \\
\text { Surface-sampler data } \\
\text { Inferred }\end{array}$ & $\begin{array}{l}117.2 \\
117.2 \\
116.2\end{array}$ & $\begin{array}{l}38.9 \\
39.5 \\
39.4\end{array}$ & $\begin{array}{l}68.8 \\
60.7 \\
60.8\end{array}$ \\
\hline $\begin{array}{l}\text { Spacecraft coordinates } \\
\text { of tip of collector head }(\mathrm{m})\end{array}$ & $\underline{z}_{\underline{L}}=1.980$ & $\underline{Y}_{\underline{L}}=1.132$ & $\underline{x}_{L}=1.070$ \\
\hline
\end{tabular}

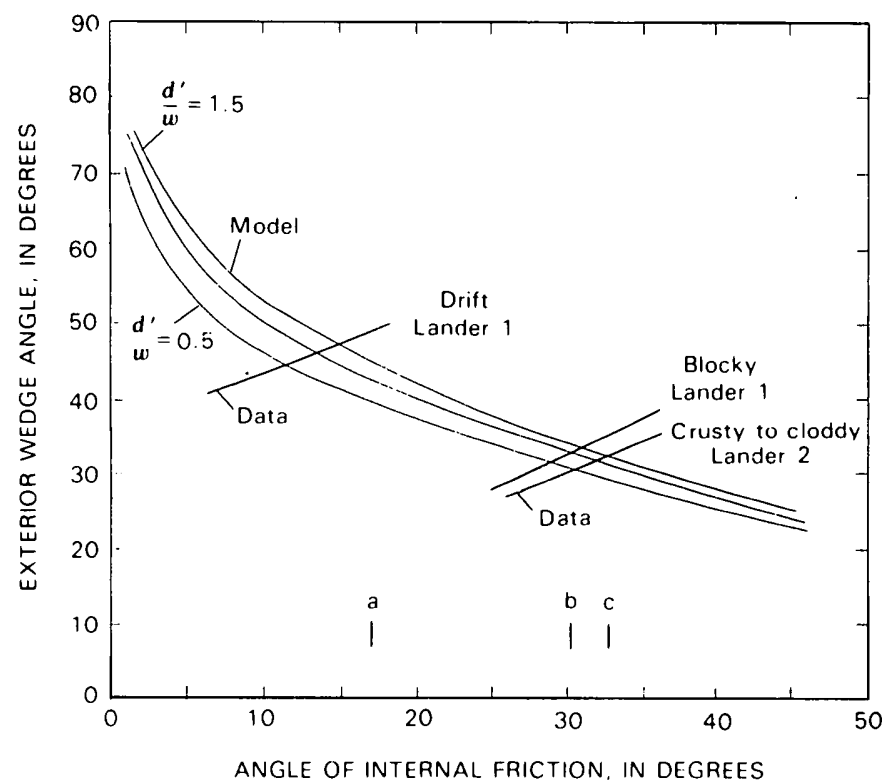

FIgURE 46.-Exterior wedge angles of the region of failure predicted from theoretical model of plowing (McKyes and Ali, 1977) for three depth-to-width ratios as a function of angle of internal friction and exterior wedge angles as a function of assumed angles of internal friction for surface-bearing tests. Estimate of angle of internal friction corresponds to intersection of model curves and data curve at depth to width ratio $\left(d^{\prime} / w\right)$ appropriate for test. Estimated angles of internal friction are: (1) Drift material $17^{\circ}$ (a, Lander 1); (2) Blocky material $30^{\circ}$ (b, Lander 1); and (3) Crusty to cloddy material $32^{\circ}$ (c, Lander 2$)$. embankment of fine-grained and blocky units. Beyond the embankment a second larger lobe was produced by the deformed surface. Thus, in contrast with drift material, the deformed surface had two lobes and more blocky clods. Within the trench and on the elevated rim of the
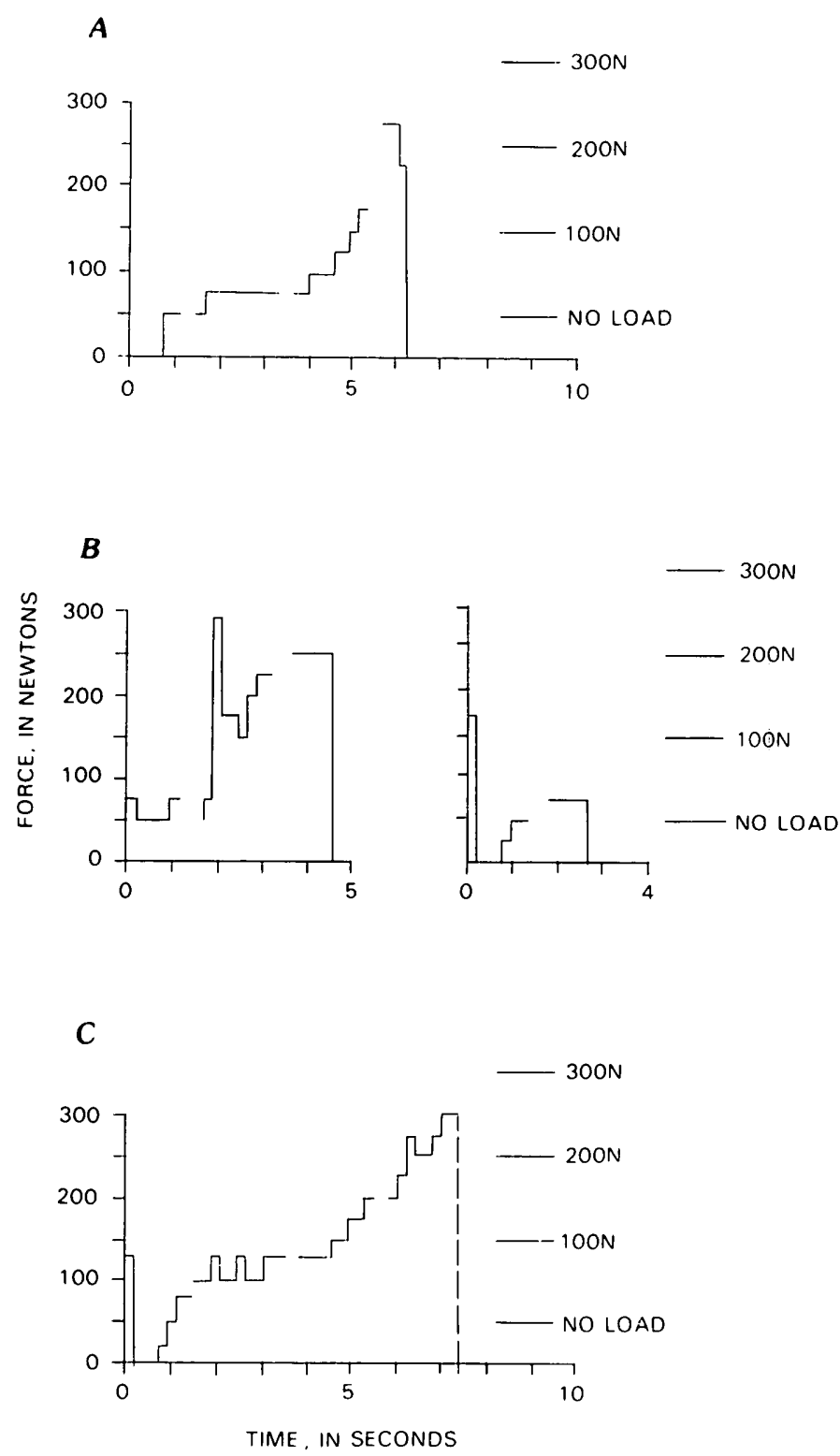

FiguRE 47.-Forces inferred from motor-current records of surfacebearing tests. $A$, Inferred forces for surface-bearing test (Sol 612) on drift material of Sandy Flats (Lander 1) above no-load condition $(200-225 \mathrm{~N})$ are consistent with surface-sampler test data for motor decoupling. $B$, Two interpretations of forces from motor-current record of surface-bearing test (Sol 612) on blocky material of Rocky Flats (Lander 1). One interpretation implies forces about $200 \mathrm{~N}$ above noload condition and the other interpretation implies forces of $25 \mathrm{~N}$ or less. See text for discussion. $C$, Inferred forces for surface-bearing test (Sol 595) on crusty to cloddy material (Lander 2). Inferred forces are $225-250 \mathrm{~N}$; this value indicates that surface-sampler motor decoupled. Because of coarse resolution of motor currents, force is taken as $200-225 \mathrm{~N}$. 
embankment, fragments a few centimeters in diameter were present. The elevated right-hand lobe, $0.06 \mathrm{~m}$ in diameter, was bounded by a fracture near the trench, but at its tip it was bounded by a monoclinal flexure. The left margin of the larger lobe was also bounded by a fracture near the trench and a monoclinal flexure at its farthest tip. Width of the region of failure was about $0.145 \mathrm{~m}$.

Mapping, chiefly with post-test enhanced pictures combined with surface-sampler data, indicated that the inferred positions are good (see table 21). From the inferred positions, the collector head penetrated 0.035 or $0.04 \mathrm{~m}$ below the surface, and the limit of deformation measured in the azimuthal plane along the original surface was 0.075 or $0.085 \mathrm{~m}$ (fig. 49). The intersection of the data curve and model curves indicated an angle of internal friction of about $30^{\circ}$ (fig. 46).

Motor currents and the forces inferred from them for the surface-bearing test on blocky material may be interpreted two ways, and there are justifications for both interpretations (fig. 47B). Neither interpretation is consistent with the surface-sampler control assembly (SSCA) currents unless $1.5-2.0 \mathrm{~s}$ of the Format 5 record is missing - this would validate the first interpretation in figure $47 B$. In the first interpretation (fig. $47 B$ ), the initial part of the record that includes motor start is missing but the no-load current corresponding to $50 \mathrm{~N}$ is present. Inferred forces increase to $200 \mathrm{~N}$ rapidly but a large transient current related to a short in the boom electrical cable could have occurred during extension. This large transient current phenomenon occurred during Backhoe Touchdown 8 of Lander 1 as well as earlier (Pike and others, 1978). Rates of travel from the first surface-bearing test were about $0.75 \mathrm{in} . / \mathrm{s}$ and the duration of the current record for this test ( $4.65 \mathrm{~s})$ indicates that the increase in extension should have been $3.5 \mathrm{in}$. for a total of $58.4 \mathrm{in}$. (see table 21); these figures are in good agreement with the inferred and achieved extensions. Motor-current measurements for other trenches in blocky material indicated that forces greater than $100 \mathrm{~N}$ were exerted and that the sampler frequently failed to reach the commanded extension during other trenching operations in blocky material. These two facts suggest that the motor probably decoupled during the test. In the second interpretation (fig. $47 B$ ), the large transient current is interpreted to be motor start followed by no-load forces $(50 \mathrm{~N})$ and then by current increases corresponding to $25 \mathrm{~N}$. Rates of travel from the first surface-bearing test and the duration of the current record of $2.0 \mathrm{~s}$ indicate an extension of $2.2 \mathrm{in}$. for a total of $56.9 \mathrm{in}$. and this is incompatible with the inferred and achieved extensions.

TABLE 20.-Cohesions from surface-bearing tests as functions of angles of internal friction, using the model of McKyes and Ali (1977) [Calculations assumed densities as indicated, and the accelerations of gravity were 3.72 (Lander 1) and 3.73 (Lander 2). For examples of detailed calculations and procedures, see supplemental section on "Analysis of Sample Trenches and Surface-Bearing Tests"|

\begin{tabular}{|c|c|c|c|c|c|c|}
\hline $\begin{array}{l}\text { Surface- } \\
\text { bearing test } \\
\text { location }\end{array}$ & $\begin{array}{l}\text { Assumed } \\
\text { density } \\
\qquad \\
\left(\mathrm{kg} / \mathrm{m}^{3}\right)\end{array}$ & $\begin{array}{c}\text { Rake } \\
\text { angle } \\
\\
\alpha \\
\text { (degrees) }\end{array}$ & $\begin{array}{l}\text { Assumed } \\
\text { angle of } \\
\text { internal } \\
\text { friction } \\
\quad \phi \\
\text { (degrees) }\end{array}$ & $\begin{array}{l}\text { Cohesion } \\
\qquad(\mathrm{kPa})\end{array}$ & $\begin{array}{l}\text { Derived horizontal } \\
\text { force at collector- } \\
\text { head interface } \\
\qquad \underline{\mathrm{F}}_{\mathrm{H}} \\
(\mathrm{N})\end{array}$ & $\begin{array}{l}\text { Available surface- } \\
\text { sampler horizontal } \\
\text { force at interface } \\
\qquad \frac{\mathrm{F}}{\mathrm{T}} \\
(\mathrm{N})\end{array}$ \\
\hline \multicolumn{7}{|c|}{ Drift material } \\
\hline $\begin{array}{l}\text { Lander 1, } \\
\text { Sandy Flats }\end{array}$ & $\begin{array}{l}1,200 \\
1,200 \\
1,200\end{array}$ & $\begin{array}{l}90.0 \\
\text { do. } \\
\text { do. } \\
\text { do. }\end{array}$ & $\begin{array}{l}10 \\
15 \\
20 \\
20\end{array}$ & $\begin{array}{c}22.0-24.7 \\
18.0-20.2 \\
12.6-14.2 \\
1.0-2.0\end{array}$ & $\begin{array}{c}244-274 \\
236-265 \\
224-252 \\
22-39\end{array}$ & $\begin{array}{l}244-275 \\
236-266 \\
224-252\end{array}$ \\
\hline \multicolumn{7}{|c|}{ Blocky material } \\
\hline $\begin{array}{l}\text { Lander 1, } \\
\text { Rocky Flats }\end{array}$ & $\begin{array}{l}1,200 \\
1,200 \\
1,200 \\
2,000\end{array}$ & $\begin{array}{l}90.0 \\
\text { do. } \\
\text { do. } \\
\text { do. }\end{array}$ & $\begin{array}{l}25 \\
30 \\
35 \\
30\end{array}$ & $\begin{array}{c}19.7-22.1 \\
13.9-15.6 \\
6.7-7.6 \\
13.8-13.5\end{array}$ & $\begin{array}{l}210-235 \\
192-216 \\
172-194 \\
193-216\end{array}$ & $\begin{array}{l}210-236 \\
192-216 \\
173-194 \\
192-216\end{array}$ \\
\hline \multicolumn{7}{|c|}{ Crusty to cloddy material } \\
\hline $\begin{array}{c}\text { Lander } 2 \text {, } \\
\text { Delta }\end{array}$ & $\begin{array}{l}1,200 \\
1,200 \\
1,200 \\
2,000\end{array}$ & $\begin{array}{l}84.4 \\
\text { do. } \\
\text { do. } \\
\text { do. }\end{array}$ & $\begin{array}{l}25 \\
30 \\
35 \\
35\end{array}$ & $\begin{array}{l}6.4-7.3 \\
4.7-5.2 \\
2.6-2.9 \\
2.3-2.7\end{array}$ & $\begin{array}{l}209-237 \\
195-214 \\
175-193 \\
173-197\end{array}$ & $\begin{array}{l}210-236 \\
192-216 \\
173-194 \\
173-194\end{array}$ \\
\hline
\end{tabular}


Although it is not possible to exclude either interpretation unequivocally, the interpretation that the extenddrive motor decoupled gives results compatible with other data and could accommodate $1.5 \mathrm{~s}$ of missing Format 5 data. The first and preferred interpretation indicates that the total horizontal forces exerted by the collector head should have been about $200 \mathrm{~N}$. The second interpretation indicates horizontal forces of about $23 \mathrm{~N}$.

According to calculations using the model of McKyes and Ali (1977), cohesions required to produce horizontal forces in the range from 192 to $216 \mathrm{~N}$ are 13.8 to 15.6 $\mathrm{kPa}$ when the angle of internal friction is $30^{\circ}$ (table 20 ); these values are compatible with the larger estimates of cohesion from sample trenches in blocky material and a backhoe touchdown experiment to be described later. Cohesions required by the second interpretation of the motor-current record are about $1.5 \mathrm{kPa}$ when the angle of internal friction is $30^{\circ}$.

\section{CRUSTY TO CLODDY MATERIAL}

The surface-bearing test of Lander 2 was the most successful test of the three. Pictures indicated that the collector-head lid was closed and aligned with the boom axis because no part of it was visible in the camera 1 picture. Failure of the surface material around the collector head was partly visible in both camera pictures taken during the test (fig. $50 \mathrm{~B}$ ), but the region of failure must be mapped on post-test pictures because the boom was in the way. Failure of the material around the collector head was divided into two zones (fig. 51). The first and innermost zone nearest the collector head was composed of a jumbled array of platy units of surface material as much as $0.04 \mathrm{~m}$ wide that were thrust upward and outward. This region extends about $0.06 \mathrm{~m}$ from the trench edge. Upper surfaces of some platy units slope outward toward the surrounding surfaces. A second zone surrounds the inner zone. In this second zone, which is about $0.242 \mathrm{~m}$ wide, the surface materials have been fractured, faulted, domed, and thrust outward over adjacent surface materials that include a rock. Displacements on faults between the undisturbed surface and deformed region were several millimeters. The limit of deformation along the original ground surface in the azimuthal plane was about $0.15 \mathrm{~m}$. For this test, the major mishap was that the surfacesampler data on achieved positions were not recovered,

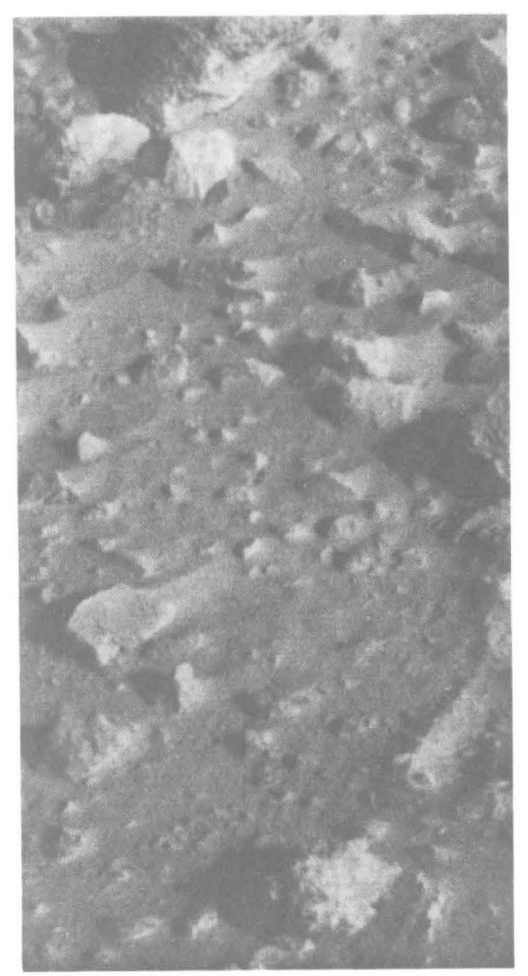

$\boldsymbol{A}$

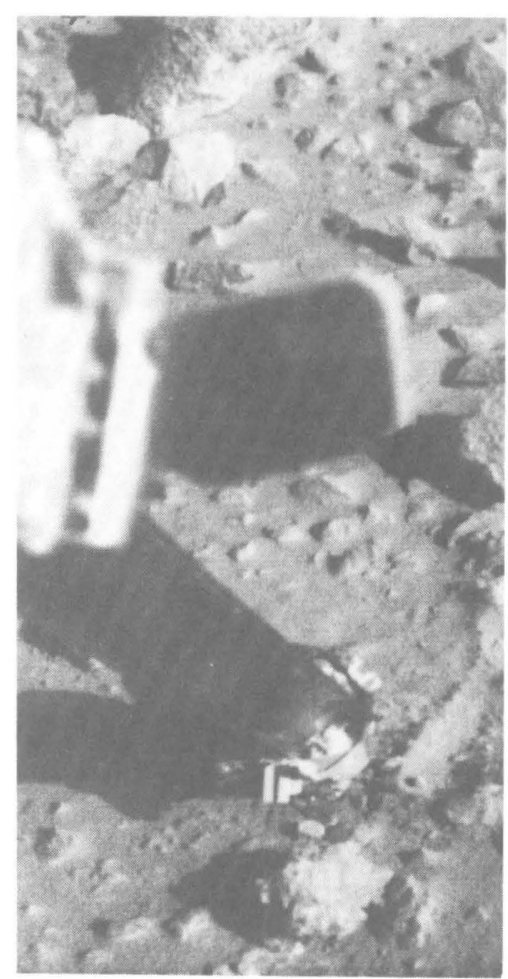

B

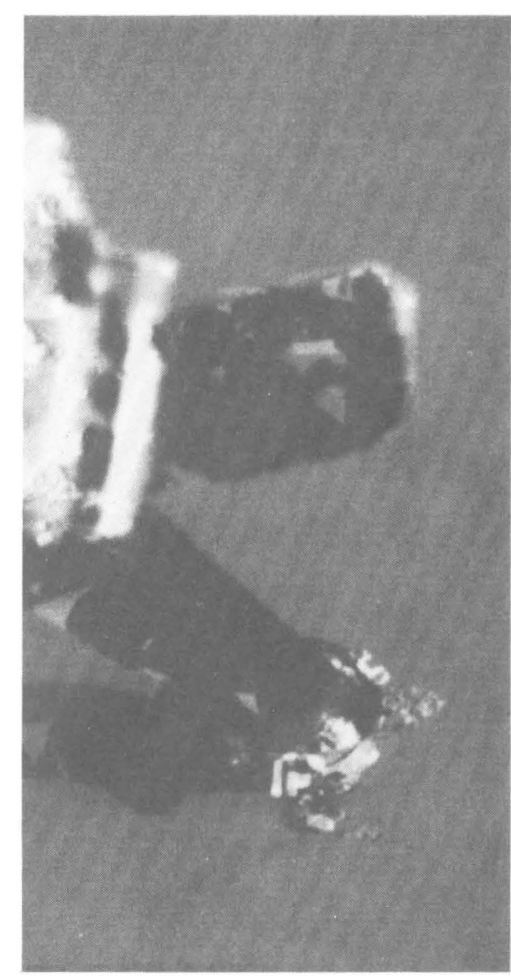

C
Figure 48.-Surface-bearing test on blocky material (Lander 1). A, Surface before test. Note heterogeneous appearance of surface with rocks and blocky units. Trench and tailings from XRFS 4 sample (Sol 177-180) acquisition at lower right. Rock at bottom right center is $8 \mathrm{~cm}$ wide (frame $12 \mathrm{H} 179 / 611$ ). $B$, Collector head inserted in blocky material. Note that lid of collector cannot be seen, which indicates that it is closed and aligned with boom axis. Comparing $B$ with $A$, lobe of deformed surface near tailings of preexisting trench (frame $12 \mathrm{H} 189 / 612$ ) is visible. $C$, Difference picture made by computer processing of frames in $A$ and $B$. Note lobe of deformed surface to upper right of boom collar. Picture processed by E.A. Guinness, Lander Imaging Team. 
TABLE 21.-Commanded positions, surface-sampler data, and inferred positions from stereometric measurements and mapping for the surfacebearing test in blocky material (Lander 1)

[a. first and preferred interpretation of inferred position; b. second interpretation of inferred position; see text]

\begin{tabular}{|c|c|c|c|c|}
\hline $\begin{array}{l}\text { Surface-sampler } \\
\text { positions }\end{array}$ & & $\begin{array}{c}\text { Azimuth } \\
\text { (degrees) }\end{array}$ & $\begin{array}{l}\text { Elevation } \\
\text { (degrees) }\end{array}$ & $\begin{array}{l}\text { Extension } \\
\text { (in.) }\end{array}$ \\
\hline $\begin{array}{l}\text { Commanded } \\
\text { Surface-sampler data } \\
\text { Inferred }\end{array}$ & $\begin{array}{l}\text { (a) } \\
\text { (b) }\end{array}$ & $\begin{array}{l}184.1 \\
184.1 \\
183.3 \\
182.2\end{array}$ & $\begin{array}{l}38.9 \\
39.5 \\
39.2 \\
39.5\end{array}$ & $\begin{array}{l}67.0 \\
58.3 \\
58.4 \\
58.2\end{array}$ \\
\hline Spacecraft coordinates & (a) & $\underline{z_{L}}=2.597$ & $\underline{Y}_{L}=(-) 0.640$ & $\underline{x}_{\underline{L}}=1.025$ \\
\hline of tip of collector head $(\mathrm{m})$ & (b) & $\underline{z}_{\underline{L}}=2.587$ & $\underline{Y}_{L}=(-) 0.636$ & $\underline{x}_{\underline{L}}=1.030$ \\
\hline
\end{tabular}

but the inferred positions are believed to be reliable (table 22).

Mapping on specially enhanced post-test pictures revealed that the deepest part of the trench was $0.065 \mathrm{~m}$ below the original surface, and the location of the far trench wall constrains the depth of penetration of the collector-head tip to $0.08 \mathrm{~m}$ below the original surface (fig. 51). If the collector-head tip was $0.065 \mathrm{~m}$ below the first original surface, the limit of failure in the azimuthal plane along the original surface was $0.16 \mathrm{~m}$ from the collector head, and, if the tip was $0.08 \mathrm{~m}$ below the original surface, the limit of failure was $0.14 \mathrm{~m}$. Because trench

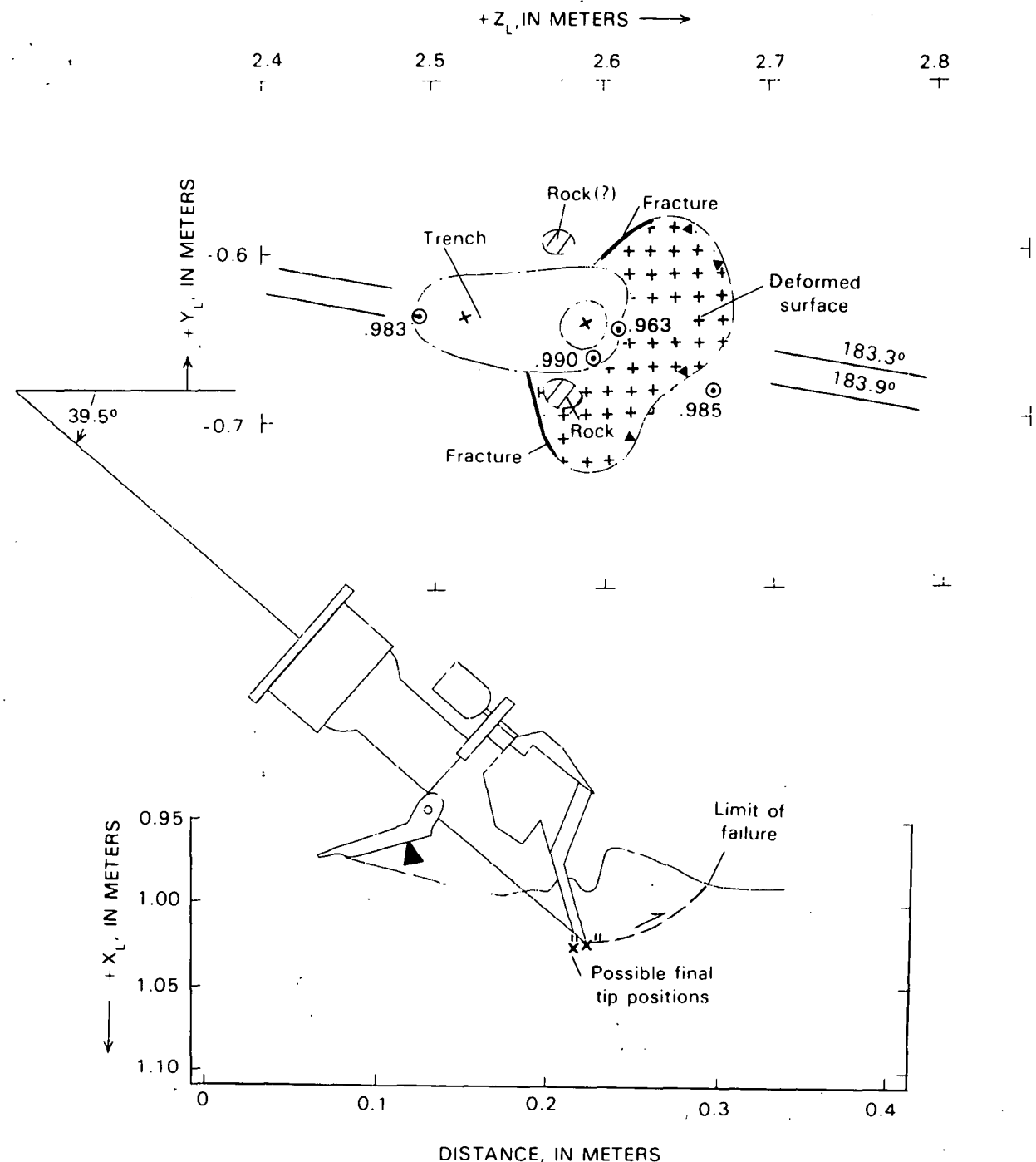

FIGURE 49.-Map and profile of surface-bearing test on blocky material (see fig. 48). Note that there are two lobes of deformed surface materials. Dashed lines outline disturbed surface, solid triangles indicate monoclines. Heavy lines indicate fractures with small displacements. The exterior wedge angle and depth-to-width ratio indicate that angle of internal friction is approximately $30^{\circ}$. Numbers by open circles with dots indicate elevations in meters in direction of $+X_{L}$ axis. Surface-sampler elevation angle is $39.5^{\circ}$; surface-sampler azimuths of $183.3^{\circ}$ and $183.9^{\circ}$ are indicated by lines. Small crosses in plan view indicate locations of tip of collector head at start and end of test; small crosses in profile indicate possible locations of tip of collector head at finish of test. 


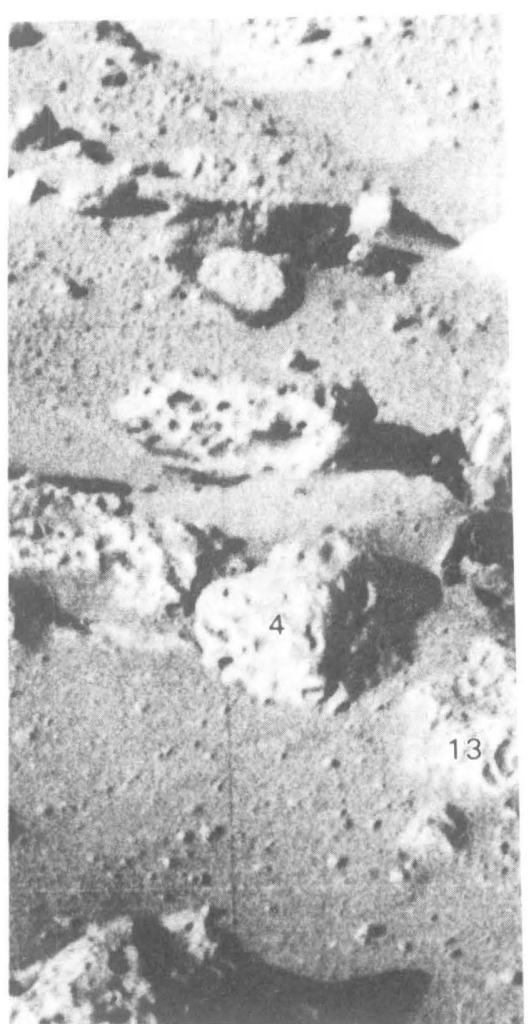

$A$

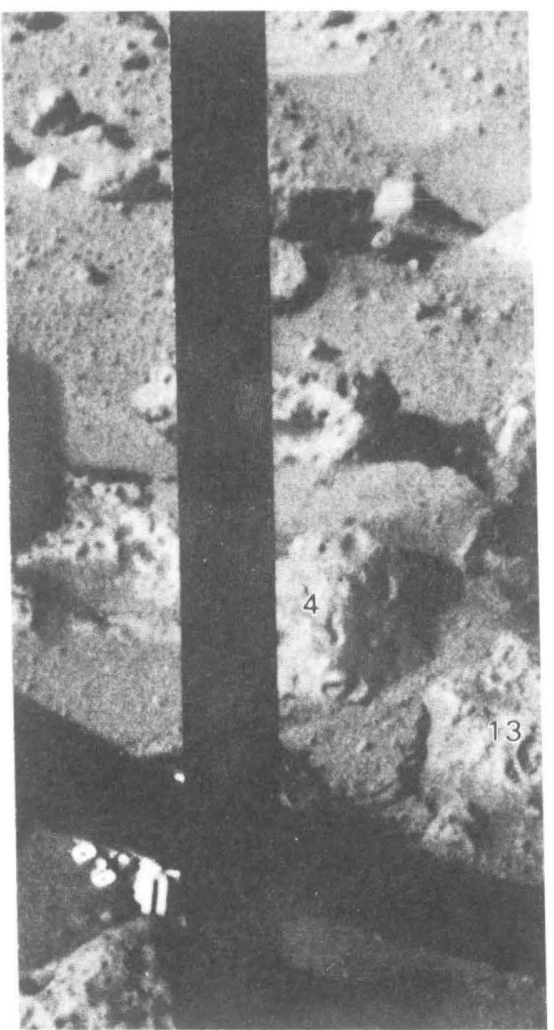

$B$

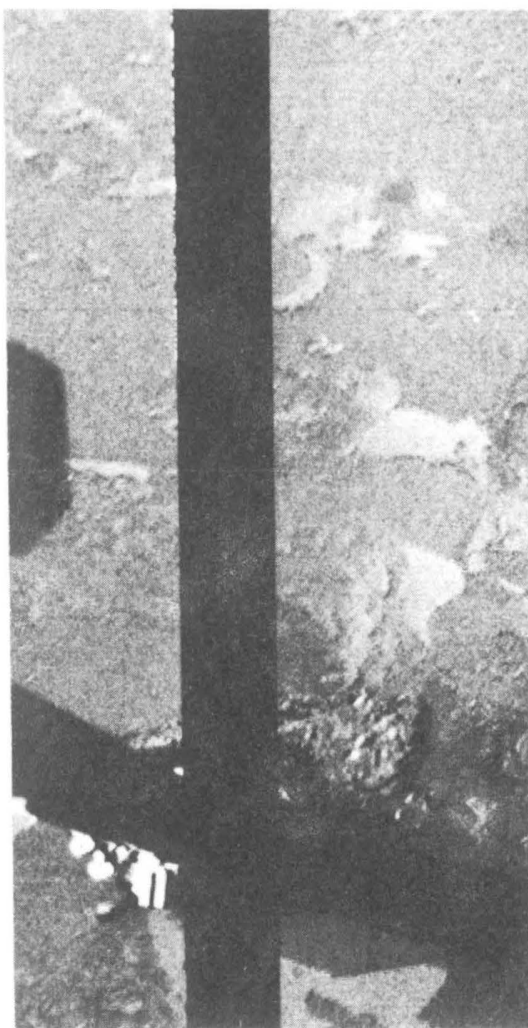

C
Figure 50.-Surface-bearing test on crusty to cloddy material (Lander 2). A, Surface before test. Note rocks near center (rock 4) and to right (rock 13). Rock 4 is $18 \mathrm{~cm}$ wide (frame $22 \mathrm{H} 014 / 594$ ). B, Collector head inserted in crusty to cloddy material. Note surface material has been thrust over edge of rock 13 and adjacent surfaces. Dark band represents missing data of picture (frame 22H030/595).
$C$, Difference picture made by computer processing of frames in $A$ and $B$. Patterns in background are due to $0.1^{\circ}$ difference in sun azimuth between pictures in $A$ and $B$ and not to surface movements. Deformed surface shown by strong variations in shades of gray from very light to very dark. Picture processed by E.A. Guinness, Lander Imaging Team. walls tend to collapse somewhat, filling the trench floor in the process, the best estimate was somewhere between-say, $0.070 \mathrm{~m}$ deep and $0.15 \mathrm{~m}$ for the limit of failure. The intersection of the data curve for this test with the model curves indicates that the angle of internal friction was about $32.5^{\circ}$ (fig. 46).

For this test, forces inferred from the motor currents were as large as $250 \mathrm{~N}$ above the no-load current and this amount of force indicates that the motor decoupled (see fig. $47 C$ ). We thought this value might be too high because of the coarse resolution of the motor currents; so we assumed the force to range from 200 to $225 \mathrm{~N}$, and the horizontal forces, after allowing for friction, range from 192 to $216 \mathrm{~N}\left(\phi=30^{\circ}\right)$. Cohesions, using the procedures of McKyes and Ali (1977), that were required to account for these forces were $4.5-5.0 \mathrm{kPa}$ when the angle of internal friction was $30^{\circ}$ (table 20 ). These values of cohesions are comparable with those from the analyses of one sample trench in crusty to cloddy material (see table 16).

\section{BACKHOE TOUCHDOWNS}

The amount of penetration of the backhoe on the collector head of surface samplers during normal touchdowns or de-elevations to the surface was, in part, related to the cohesion of the material. Because of this, a number of backhoe-touchdown tests were conducted on Mars and these tests were compared with similar tests made on Earth. For the test material used, cohesions from separate triaxial tests were found to be half the shearvane strengths measured for the backhoe tests when the bulk densities are the same. Thus, when the martian backhoe penetration is the same as that in a terrestrial test, the cohesion of the martian material is assumed to be half the shear-vane strength of the terrestrial test. In some tests, such as on rocks, only lower limits of cohesions can be obtained, and in others, such as on tailings, only upper limits can be obtained.

In the backhoe touchdown tests, the surface-sampler 


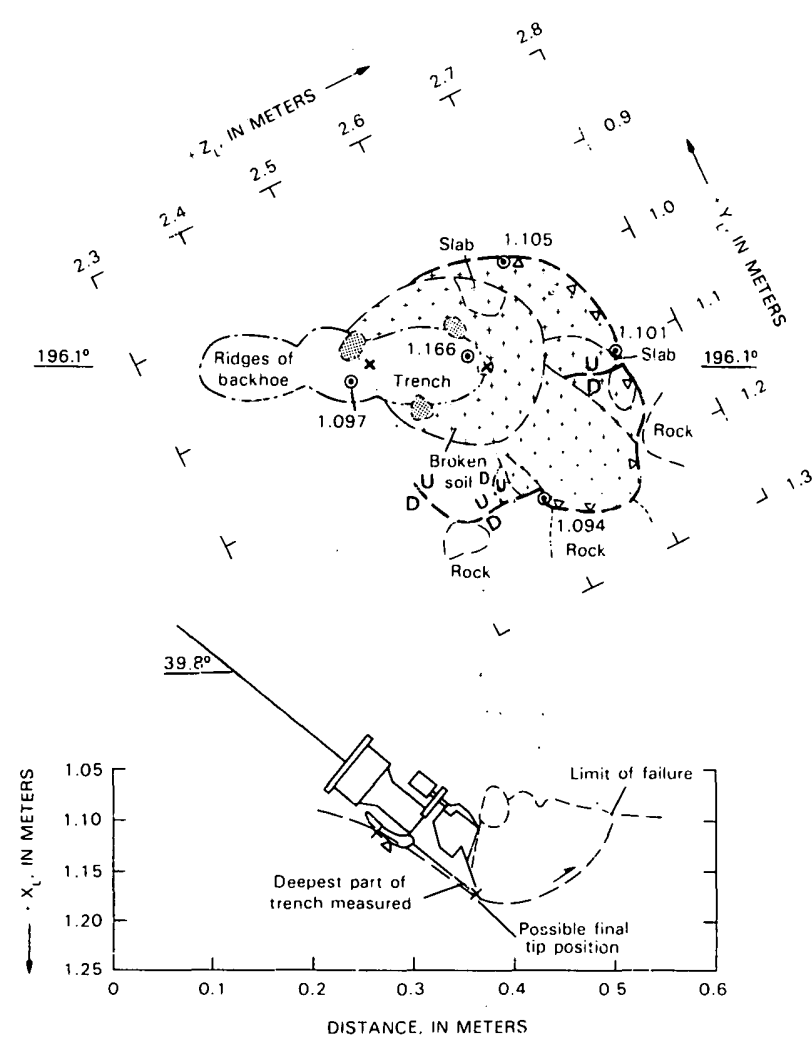

FIGURE 51.-Map and profile of surface-bearing test on crusty to cloddy material (see fig. 50). Note two zones of deformation. Dashed lines outline disturbed surface. Open triangles indicate edge of overthrust. U, upthrown side of fault; D, downthrown side of fault. Exterior wedge angle and depth-to-width ratio indicate that angle of internal friction is approximately $32.5^{\circ}$. Numbers by open circles with dots indicate elevations in meters in direction of $+X_{L}$ axis. Surface-sampler elevation angle is $39.8^{\circ}$; surface-sampler azimuth is $196.1^{\circ}$. Small crosses in plan view and profile indicate locations of tip of collector head at start and end of test.
TABLE 22.-Commanded positions, surface-sampler data, and inferred positions from stereometric measurements and mapping for the surfacebearing test in crusty to cloddy material (Lander 2)

(a and b are two possible interpretations; see text]

\begin{tabular}{|c|c|c|c|}
\hline $\begin{array}{l}\text { Surface-sampler } \\
\text { positions }\end{array}$ & $\begin{array}{l}\text { Azimuth } \\
\text { (degrees) }\end{array}$ & $\begin{array}{l}\text { Elevation } \\
\text { (degrees) }\end{array}$ & $\begin{array}{l}\text { Extension } \\
\text { (in.) }\end{array}$ \\
\hline $\begin{array}{l}\text { Conmanded } \\
\text { Surface-sampler data } \\
\text { Inferred }\end{array}$ & $\begin{array}{c}196.1 \\
\text { no data } \\
196.0 \\
196.0\end{array}$ & $\begin{array}{c}38.9 \\
\text { no data } \\
39.8 \\
39.8\end{array}$ & $\begin{array}{c}72.0 \\
\text { no data } \\
65.9 \\
66.9\end{array}$ \\
\hline Spacecraft coordinates & $\underline{z}_{L}=2.593$ & $\underline{y}_{L}=(-) 1.047$ & $\underline{x}_{\underline{L}}=1.165$ \\
\hline of tip of collector head $(\mathrm{m})$ & $\underline{z}_{\underline{L}}=2.610$ & $\underline{Y}_{L}=(-) 1.057$ & $\underline{x}_{\underline{L}}=1.180$ \\
\hline
\end{tabular}

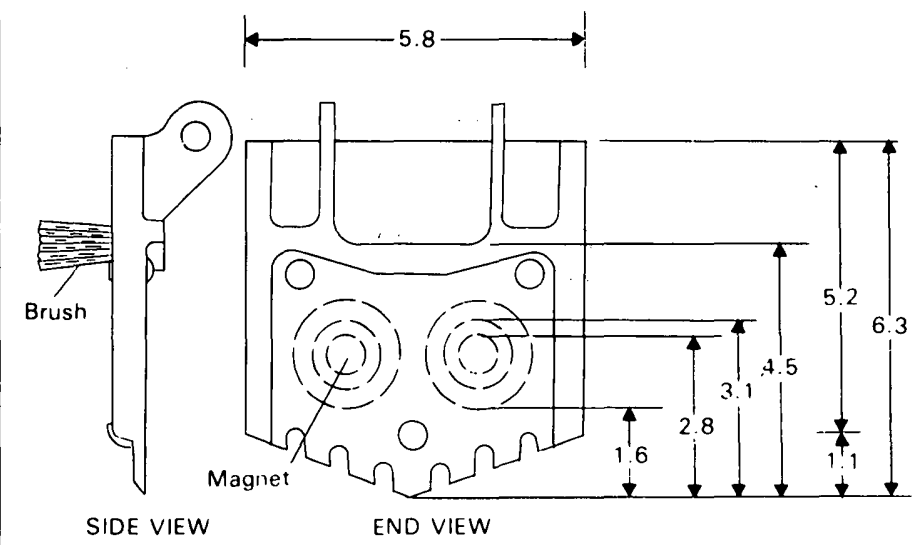

Figure 52.-Dimensions (in centimeters) of surface-sampler backhoes, Viking Landers 1 and 2.

collector head was commanded to the desired azimuth and extension and then lowered to the surface. Normally, the backhoe on the collector head penetrated the surface material, and forces exerted on it caused an upward rotation of the collector head through an angle of $10^{\circ}$. This rotation actuated a contact switch that stopped the elevation motor. About $10.6 \mathrm{~N}$ of force was required to produce the rotation, and this force was exerted against a spring so that gravitational forces were unimportant. Stresses exerted on the surface material by the backhoe were complex because the backhoe geometry is complex (fig. 52) and, in some touchdowns, the tip of the collector head became involved. Cross-sectional areas of the backhoe increased as the penetrations increased from a small value to $1.1 \mathrm{~cm}$ because, as the backhoe tip is both tapered and notched, the thickness of the tip increases. A very large increase in cross-sectional area occurs when penetrations are about $4.5 \mathrm{~cm}$ because of the presence of the ten wire tufts that make up the brush on the forward side of the backhoe (fig. 52). In some tests, only the backhoe touched and penetrated the surface (see type A in fig. 53). In other tests, the backhoe penetrated so deeply that the tip of the collector head touched the surface and the combined forces exerted on the tip and backhoe caused the rotation that activates the cut-off switch (see type B in fig. 53). In this latter case, cross-sectional areas and stresses on the surface were very complex. During a backhoe touchdown a final mishap could occur; the backhoe could fold back toward the lander (but not away from it) because it was held in position with a spring.

Backhoe-touchdown tests were conducted almost entirely during the Extended Mission because of the low priority given to the Physical Properties Investigation; however, one successful test was conducted by Lander 2 during the Primary Mission. All told, nine tests were performed by Lander 1 and six were performed by Lander 2 (table 23). 
Analyses of the backhoe-touchdown tests on Mars indicate that five kinds of materials were found. They are, in order of increasing strength, as follows: (1) disturbed material of tailings of trenches, (2) drift material of Lander 1, (3) crusty to cloddy material of Lander 2, (4) blocky material of Lander 1 , and (5) rocks at both sites. Locations for the backhoe touchdowns are shown in plates $1 B$ and $2 B$. Backhoe penetrations into tailings at both sites were clearly the largest. The backhoe penetrated the tailings of Deep Hole 1 of Lander 1 about $4.5 \mathrm{~cm}$ (table 23); mapping combined with surface-sampler data indicated that the collector-head tip penetrated the tailings $2.5 \mathrm{~cm}$ and that the wire brush penetrated the tailings (fig. $54 \mathrm{C}$ ). Although not mapped in detail, comparison of pretest, test, and post-test images showed that the tailings of Lander 2 responded in a similar way to those of Lander 1: backhoe penetration was about $4.5 \mathrm{~cm}$, the collector-head tip penetrated deeply into the tailings, and the wire brush penetrated the tailings (fig. $55 \mathrm{C}$ ); however, backhoe penetrations in drift material were not so great as those in the tailings nor did the tip of the collector head penetrate so deeply although one of them almost did (table 23). For backhoe touchdown 2 (fig. $54 A$ ), penetration was about $2.9 \mathrm{~cm}$, and mapping indicated that the tip penetrated about $1.5 \mathrm{~cm}$ and the brush may have touched the surface. For touchdown 3, penetration was about $4 \mathrm{~cm}$, and mapping indicated that the tip penetrated about $2 \mathrm{~cm}$ and the brush touched the surface. For touchdown 5 (fig. $54 B$ ), penetration was about $3.2 \mathrm{~cm}$, and mapping indicates that the tip penetrated about $1 \mathrm{~cm}$ and the brush probably touched the surface. In none of these tests did it appear that the wire brush was as deeply buried in the drift material as it was in the disturbed material of the tailings. Of the four tests in blocky material, only two were entirely successful. In test 4 , the backhoe only penetrated $0.6 \mathrm{~cm}$ and the tip touched the surface; and, in test 6 , the backhoe did not appear to touch the surface, suggesting that the tip hit a rock or clod or, more probably, the backhoe folded a little. Although the tip touched the surface initially for backhoe touchdowns 8 and 9 (fig. $54 D$ ), mapping combined with surfacesampler data indicated that the tip was not touching the

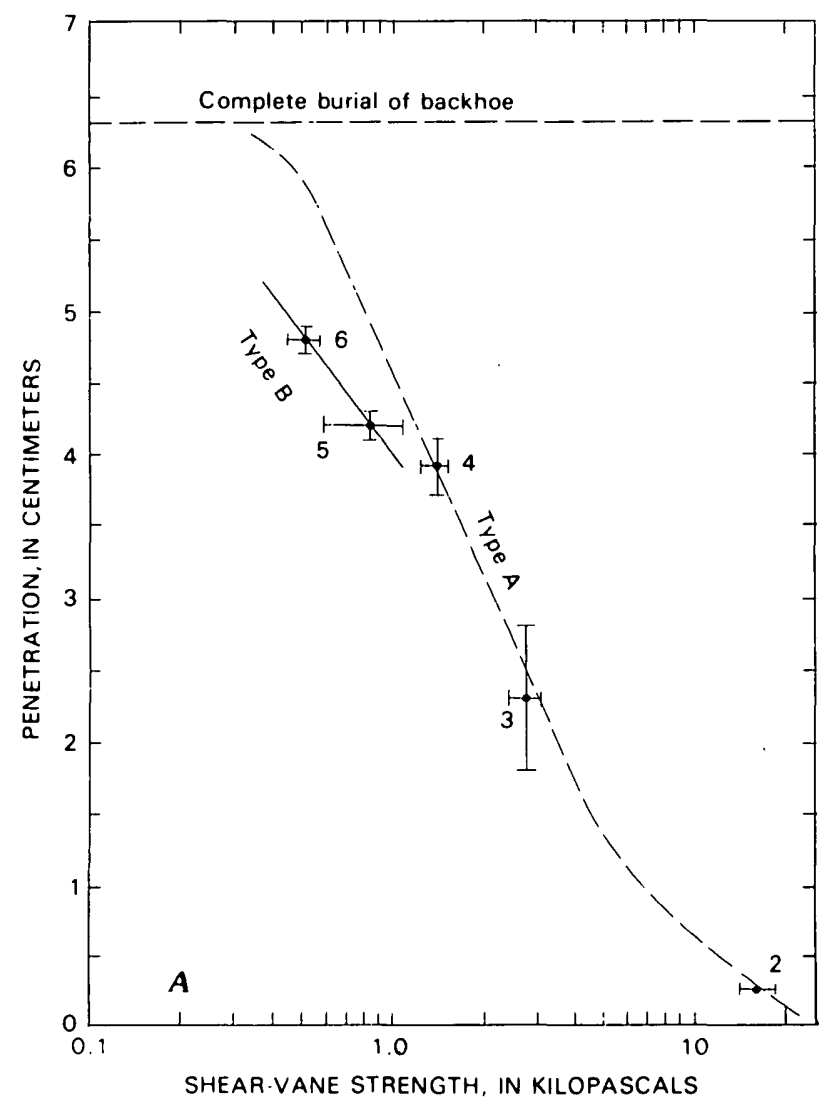

Figure 53.-Two types of backhoe touchdowns, $A$ and $B$; their amounts of penetration in soil, relation of penetration to shear-vane strength, and results for Mars. $A$, Comparison between backhoe penetration and shear-vane strength for tests using the Science Test Lander and lunar nominal material. Numbers correspond to tests

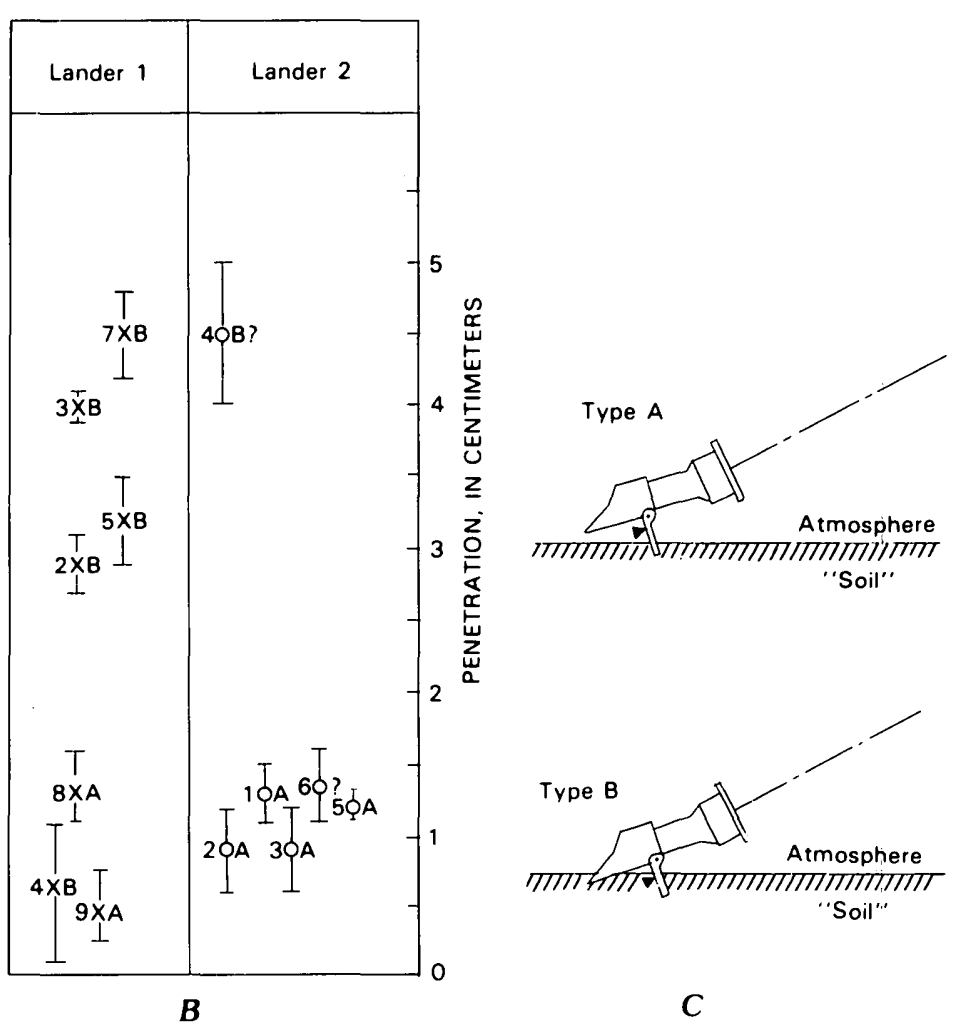

on Earth (table 24) except for test 1 which cannot be plotted. $B$, Penetration of backhoes of Landers 1 and 2 . Numbers correspond to backhoe-touchdown tests in table 23; letters refer to type of backhoe touchdown. Lander 1 indicated by X, Lander 2 indicated by $\mathrm{O}$. $C$, Two types of backhoe touchdowns, type A and type $\mathrm{B}$. 
TABLE 23.-Backhoe touchdowns and data on locations, times, surface-sampler positions, photographs, and remarks

\begin{tabular}{|c|c|c|c|c|c|c|c|c|c|c|}
\hline \multirow[t]{2}{*}{ It em } & \multirow{2}{*}{$\begin{array}{l}\text { Material or } \\
\text { location }\end{array}$} & \multicolumn{4}{|c|}{ Local Lander Time } & \multicolumn{3}{|c|}{ Surface-sampler positions } & \multirow{2}{*}{$\frac{\text { Photographs }}{\text { Frame/Sol }}$} & \multirow[t]{2}{*}{ Remarks } \\
\hline & & (Sol) & $(\mathrm{hr})$ & $(\min )$ & $(s)$ & $\begin{array}{l}\text { Azimuth } \\
\text { (degrees) }\end{array}$ & $\begin{array}{l}\text { Extension } \\
\text { (in.) }\end{array}$ & $\begin{array}{l}\text { Elevation } \\
\text { (degrees) }\end{array}$ & & \\
\hline \multicolumn{11}{|c|}{ Viking Lander 1} \\
\hline 1 & Drift material & 202 & 14 & 07 & 06 & 95.7 & 111.2 & 18.7 & $11 \mathrm{c075/202}$ & $\begin{array}{l}\text { Backhoe not visible; collar does not touch } \\
\text { surface. }\end{array}$ \\
\hline 2 & Drift material & 270 & 13 & 22 & 40 & 113.4 & 71.0 & 31.9 & $\begin{array}{l}11 \mathrm{C} 245 / 270 \\
\text { F } 1093 \mathrm{Fr} 6\end{array}$ & $\begin{array}{l}\text { Magnet center not visible; upper part of circle } \\
\text { visible; penetration is } 2.9 \pm 0.2 \mathrm{~cm} \text {; col- } \\
\text { lector-head tip touches surface; backhoe } \\
\text { partly folded. }\end{array}$ \\
\hline 3 & Drift material & 280 & 12 & 08 & 01 & 110.8 & 92.0 & 25.0 & $\begin{array}{l}110031 / 280 \\
\text { F } 1095 \mathrm{Fr} 19\end{array}$ & $\begin{array}{l}\text { Magnet circle not visible; penetration is } \\
4.0 \pm 0.1 \mathrm{~cm} \text {; collector-head tip touches } \\
\text { surface. }\end{array}$ \\
\hline 4 & Blocky material & 296 & 09 & 07 & 13 & 219.4 & 51.2 & 35.7 & $\begin{array}{l}120090 / 296 \\
\text { F } 1100 \mathrm{Fr} 1\end{array}$ & $\begin{array}{l}\text { Backhoe teeth partly visible; penetration is } \\
0.6 \pm 0.5 \mathrm{~cm} \text {; collector-head tip touches } \\
\text { surface. }\end{array}$ \\
\hline 5 & Drift material & 520 & 09 & 03 & 33 & 114.6 & 92.0 & 25.0 & $\begin{array}{l}11 \mathrm{G} 172 / 520 \\
\mathrm{~F} 1132 \mathrm{Fr} 11\end{array}$ & $\begin{array}{l}\text { Magnet circle not visible; bolts visible; pene- } \\
\text { tration is } 3.2 \pm 0.3 \mathrm{~cm} \text {; collector-head tip } \\
\text { touches surface. }\end{array}$ \\
\hline 6 & Blocky material & 527 & 15 & 07 & 43 & 165.8 & 97.8 & 24.3 & $11 \mathrm{G} 201 / 527$ & $\begin{array}{l}\text { Backhoe teeth visible and do not touch surface; } \\
\text { collector-head tip is on clod or rock. }\end{array}$ \\
\hline 7 & $\begin{array}{l}\text { Tailings of } \\
\text { Deep Hole } 1\end{array}$ & 550 & 10 & 37 & 20 & 103.3 & 87.8 & 24.3 & $\begin{array}{l}11 \mathrm{G} 252 / 550 \\
\mathrm{~F} 1141 \mathrm{Fr} 41\end{array}$ & $\begin{array}{l}\text { Magnet circle not visible; bolts not visible; } \\
\text { penetration of } 4.5 \pm 0.2 \mathrm{~cm} \text {; collector-head tip } \\
\text { touches surface. }\end{array}$ \\
\hline 8 & Blocky material & 639 & 13 & 07 & 05 & 163.9 & 88.6 & 26.9 & $\begin{array}{l}11 \mathrm{H} 237 / 639 \\
\text { F } 1154 \mathrm{Fr} 3\end{array}$ & $\begin{array}{l}\text { Magnet circle visible; backhoe teeth not visi- } \\
\text { ble; penetration is } 1.35 \pm 0.25 \mathrm{~cm} \text {; col- } \\
\text { lector-head tip does not touch surface. }\end{array}$ \\
\hline 9 & Blocky material & 639 & 13 & 35 & 40 & 162.0 & 97.8 & 23.7 & $\begin{array}{l}11 \mathrm{H} 238 / 639 \\
\text { F } 1154 \text { Fr } 5\end{array}$ & $\begin{array}{l}\text { Teeth of backhoe visible; penetration is } 0.5 \pm \\
0.25 \mathrm{~cm} \text {; collector-head tip does not touch } \\
\text { surface. }\end{array}$ \\
\hline & & & & & & ing Lander & $2--$ Crusty & to cloddy ma & rial & \\
\hline 1 & $\begin{array}{l}\text { Bonneville } \\
\text { Salt Flats }\end{array}$ & 021 & 10 & 08 & 58 & 216.3 & 93.6 & 30.0 & $\begin{array}{l}22 \mathrm{~A} 154 / 021 \\
\text { F } 2031 \mathrm{Fr} 57\end{array}$ & $\begin{array}{l}\text { Magnet circle visible; backhoe teeth not visi- } \\
\text { ble; penetration is } 1.3 \pm 0.20 \mathrm{~cm} \text {; collector- } \\
\text { head tip does not touch surface. }\end{array}$ \\
\hline 2 & Physica Planitia & 388 & 14 & 37 & 37 & 232.7 & 80.2 & 31.9 & $\begin{array}{l}\text { 22E207/388 } \\
\text { F } 2106 \text { Fr } 75\end{array}$ & $\begin{array}{l}\text { Comparative view with Sol } 479 \text { picture indicates } \\
\text { penetration is } 0.90 \pm 0.30 \mathrm{~cm} \text {. }\end{array}$ \\
\hline 3 & Physica Planitia & 479 & 10 & 09 & 16 & 238.4 & 74.9 & 33.2 & $\begin{array}{l}22 F 206 / 479 \\
\text { F } 2112 \text { Fr } 9\end{array}$ & $\begin{array}{l}\text { Magnet circle visible; backhoe teeth not visi- } \\
\text { ble; penetration is } 0.90 \pm 0.30 \mathrm{~cm} \text {; col- } \\
\text { lector-head tip does not touch surface. }\end{array}$ \\
\hline 4 & $\begin{array}{l}\text { Tailings near } \\
\text { Badger }\end{array}$ & 502 & 12 & 33 & 36 & 202.4 & 83.3 & 30.6 & $\begin{array}{l}22 \mathrm{G} 037 / 502 \\
\mathrm{~F} 2120 \mathrm{Fr} 11\end{array}$ & $\begin{array}{l}\text { Relief of tailings is } 4.0 \mathrm{~cm} \text {; backhoe appears } \\
\text { to have penetrated tailings to substrate; } \\
\text { penetration estimate is } 4.5 \pm 0.5 \mathrm{~cm} \text {. }\end{array}$ \\
\hline 5 & Near Notch & 522 & 10 & 10 & 23 & 110.2 & 88.0 & 21.8 & $\begin{array}{l}21 \mathrm{G} 087 / 522 \\
\mathrm{~F} 2131 \mathrm{Fr} 11\end{array}$ & $\begin{array}{l}\text { Magnet circle visible; backhoe teeth not visi- } \\
\text { ble; penetration is } 1.20 \pm 0.10 \mathrm{~cm} \text {; col- } \\
\text { lector-head tip does not touch surface. }\end{array}$ \\
\hline 6 & Near ICL & 595 & 10 & 37 & 05 & 173.3 & 75.2 & (33) & $\begin{array}{l}22 \mathrm{HO} 18 / 595 \\
\text { F } 2142 \mathrm{Fr} 15\end{array}$ & $\begin{array}{l}\text { Magnet circle visible; backhoe teeth not visi- } \\
\text { ble; penetration is } 1.35 \pm 0.25 \mathrm{~cm} \text {; col- } \\
\text { lector-head tip not visible. }\end{array}$ \\
\hline
\end{tabular}




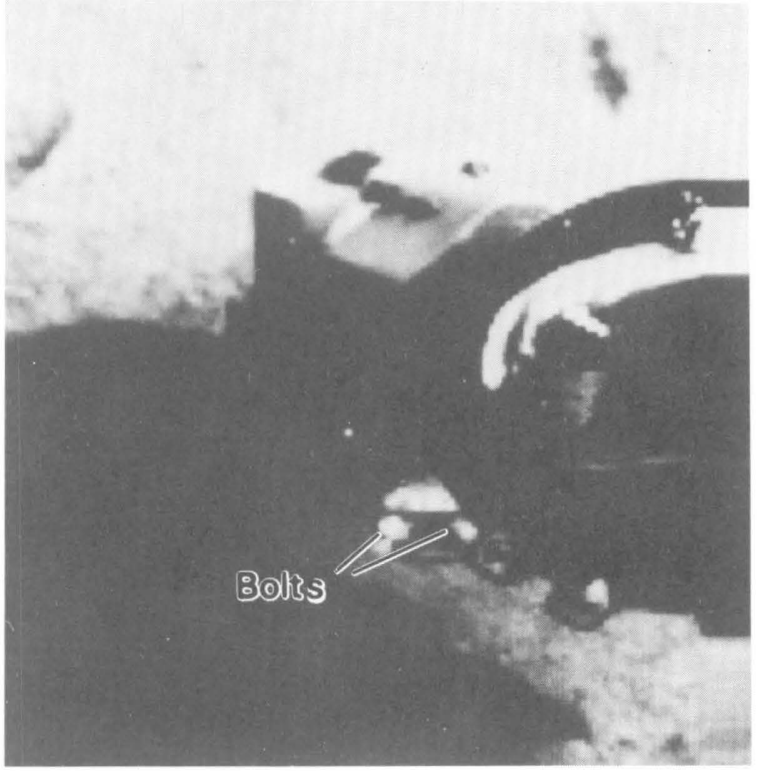

$\boldsymbol{A}$

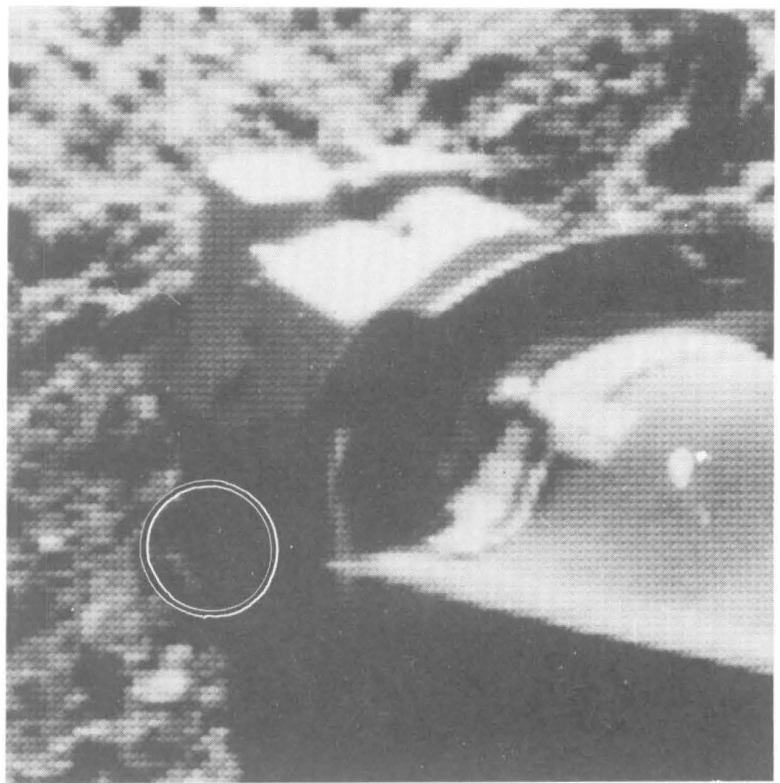

C

Figure 54.-Selected backhoe-touchdown tests of Lander 1. A, Backhoe Touchdown 2 on drift material at Sandy Flats. Collector head is near center of picture and collar (about $9 \mathrm{~cm}$ in diameter) is to right. Note magnetic material on magnets are not visible, but bolts that are higher on backhoe are (frame $11 \mathrm{C} 245 / 270$ ). $B$, Backhoe Touchdown 5 on drift material of Sandy Flats. Note that only upper $3 \mathrm{~cm}$ of left edge of backhoe is visible (circled) and tip of collector head touches surface

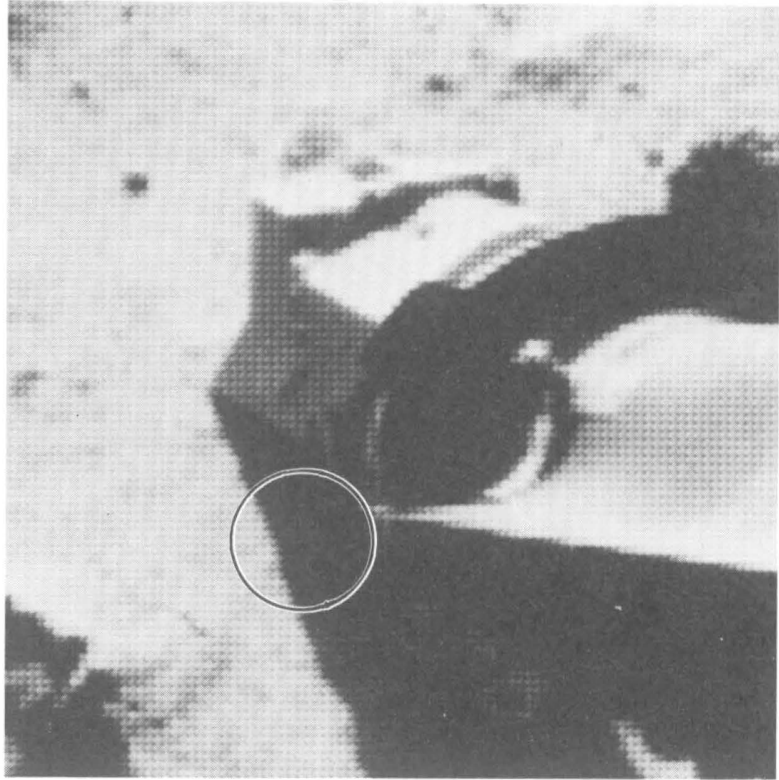

B

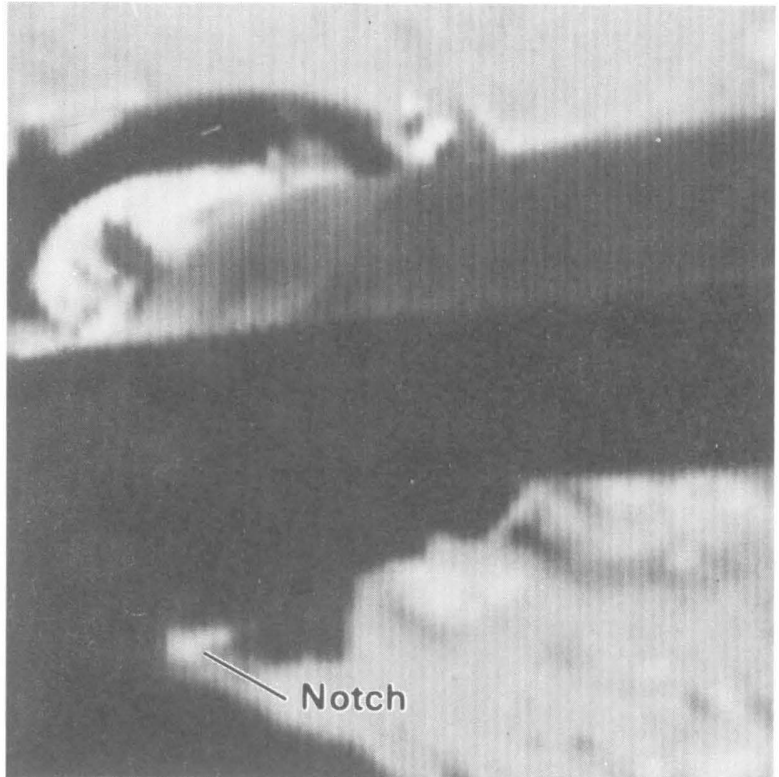

D

(frame 11G172/520). C, Backhoe Touchdown 7 on tailings of Deep Hole 1. Note that only upper $1.8 \mathrm{~cm}$ of left edge of backhoe is visible (circled). Mapping indicates that both tip of collector head and wire brush penetrated tailings (frame 11G252/550). D, Backhoe Touchdown 9 on blocky material. Dark furlable boom stretches across field of view; collector head is at left. Note notch on illuminated part of backhoe is visible beneath boom (frame 11H238/639). 


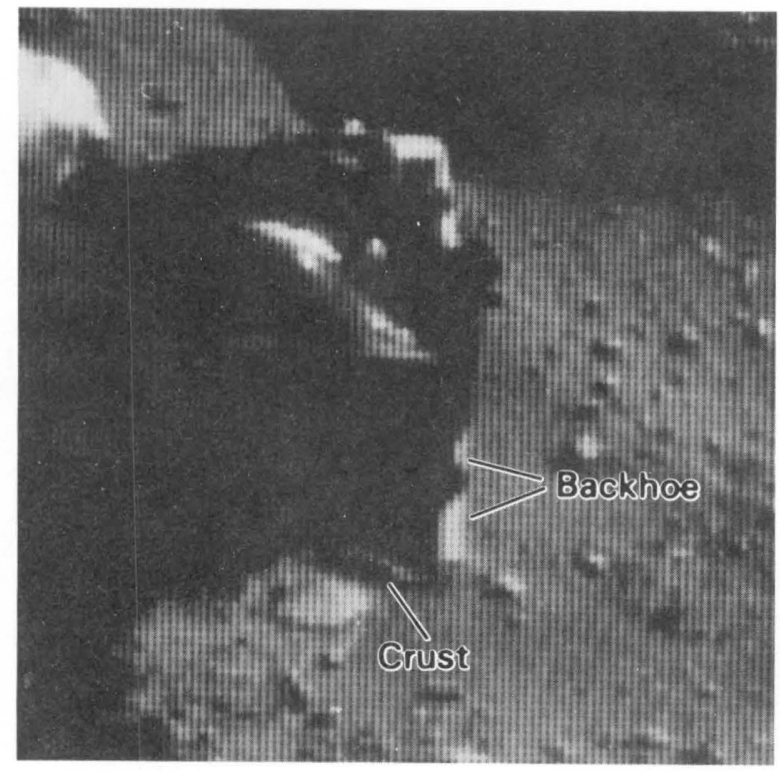

A

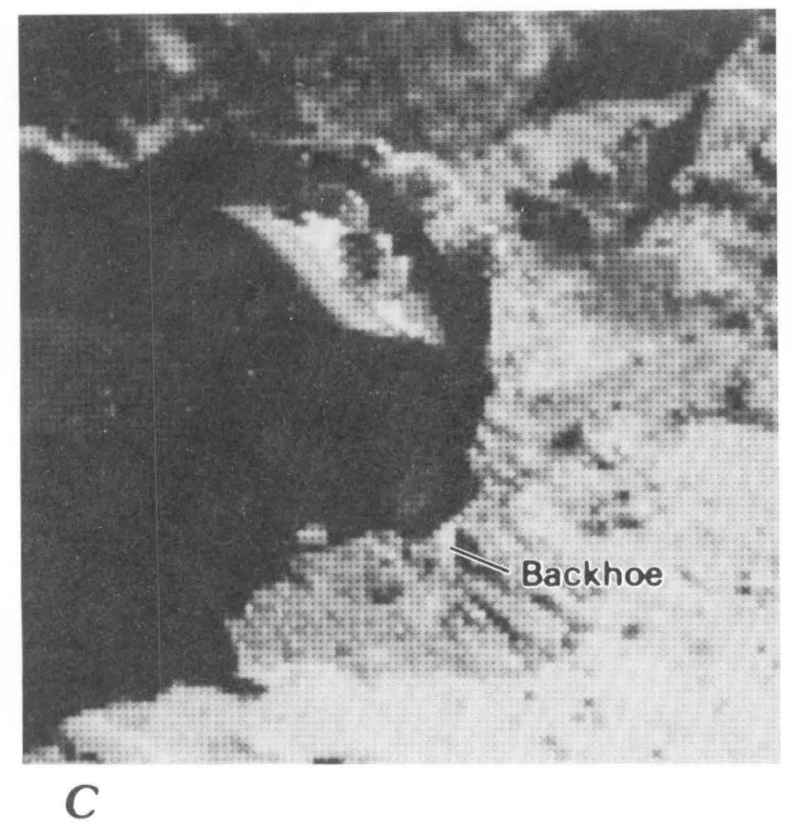

Figure 55.-Selected backhoe-touchdown tests of Lander 2. A, Backhoe Touchdown 1 on crusty material of Bonneville Salt Flats. Note tilted piece of crust near backhoe (frame 22A154/021). B, Backhoe Touchdown 2 on crusty to cloddy material of Physica Planitia. Note that tip of collector head does not touch surface (frame 22E207/388). C, Backhoe Touchdown 4 on tailings of trenches in front of rock 3

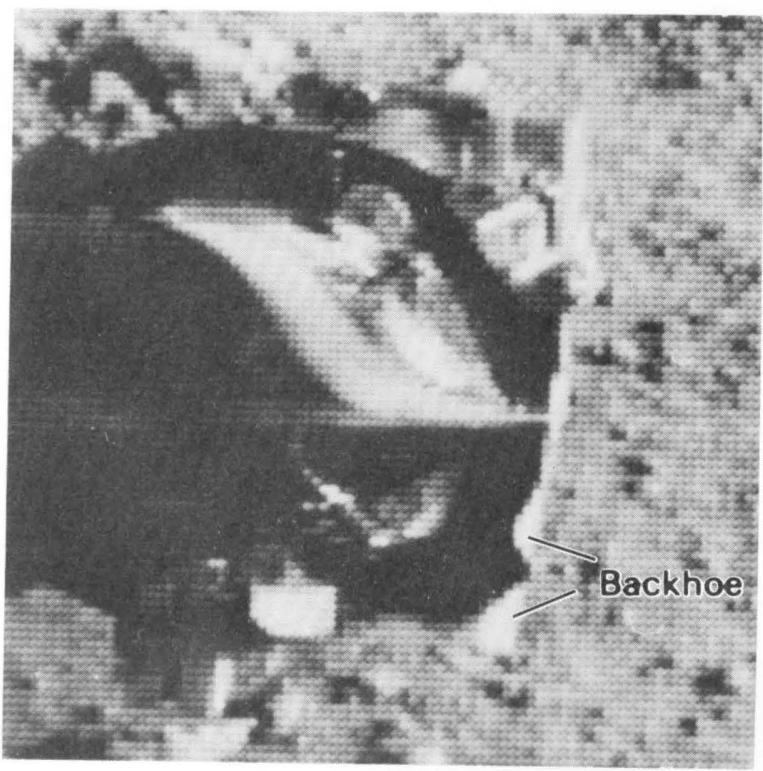

B

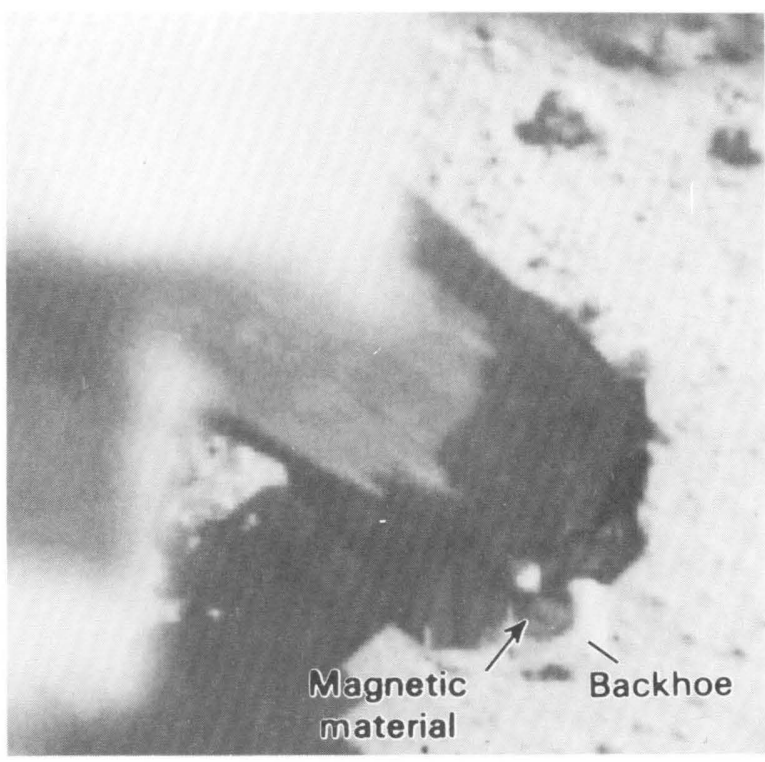

D

(Badger). Comparison of pretest and post-test pictures shows that tip of collector head and wire brush penetrated tailings (frame 22G037/502). D, Backhoe Touchdown 6 on cloddy material in front of Lander 2. Dark concentric circles (arrow) are magnetic materials on backhoe magnet. Outer dark circle is about $2 \mathrm{~cm}$ in diameter (frame 22H018/595). 
surface after the $10^{\circ}$ rotation of the collector head. Penetrations for these two tests were 1.35 and $0.5 \mathrm{~cm}$ (table 23). Because of the thin veneer of fine-grained materials on the surface, the smaller penetration probably indicates that the material was of pure blocky material. Backhoe penetrations in crusty to cloddy material were between 0.9 to $1.35 \mathrm{~cm}$ (table 23). In these tests, neither the collector-head tip nor the wire brush touched the surface when the collector head was rotated $10^{\circ}$ (fig. $55 \mathrm{~A}$, $B$, and $D$ ). Finally, penetrations of the backhoe into rocks were zero. For the backhoe touchdowns on rocks, the normal deelevation to the surface was followed by a second deelevation command of 2 -s duration to increase the

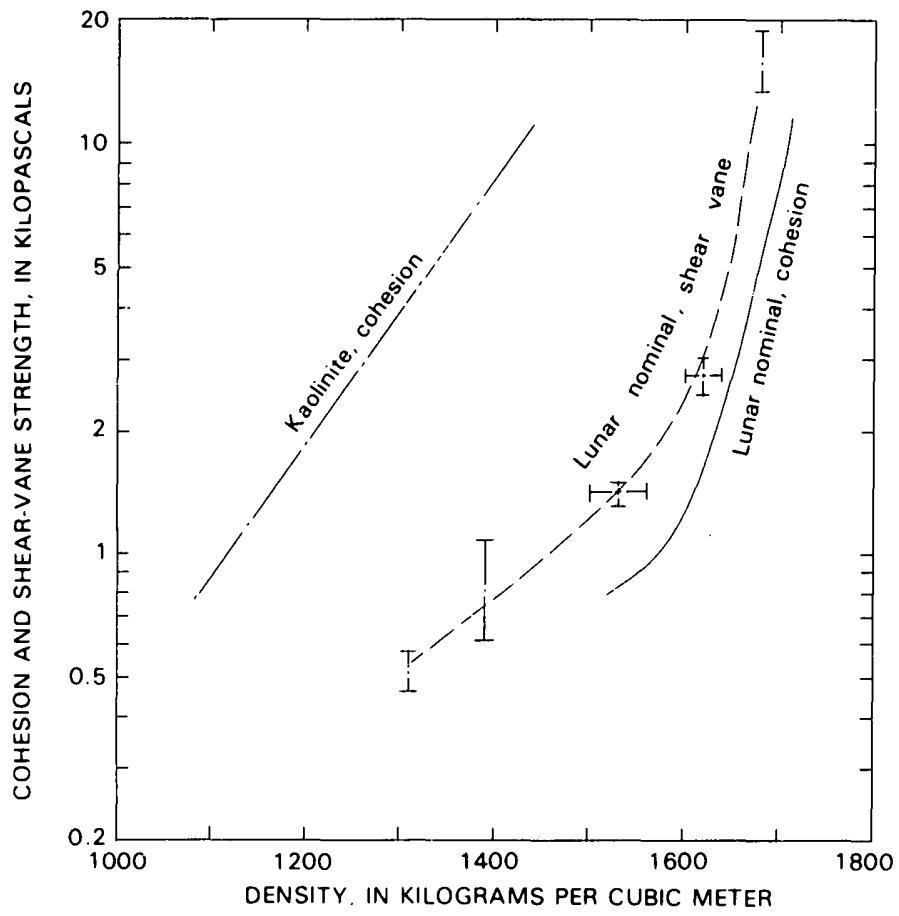

FIGURE 56.-Comparison between cohesions of lunar nominal material from triaxial tests $(\mathrm{Ko}, 1971)$ and shear-vane strengths of lunar nominal material as a function of density. Note that shear-vane strengths are about twice as large as cohesion for a given density. Cohesions of kaolinite from triaxial tests are included for comparison. stresses on the rocks. No evidence for chipping or spallation of the rocks was observed.

Seven backhoe touchdown tests were conducted using the Science Test Lander and lunar nominal simulants with a variety of densities and cohesions. For each test, densities were obtained by weighing the sample of material in a can of known volume that was inserted in the material. Torques on a shear vane at the time of failure were converted to a "shear-vane strength." For the same density of material, shear-vane strengths are about twice the cohesion from the triaxial tests for the same density (Ko, 1971) (fig. 56). As with the martian backhoe touchdowns, the tip of the collector head did not touch the surface for the stronger more cohesive materials (type A backhoe touchdown); but, for weaker materials, both the backhoe and collector-head tip touched the surface (type B, backhoe touchdown). Penetrations measured in these tests were an inverse function of the shear-vane strengths (table 24; fig. 53), but the measurements were somewhat invalidated by the type of touchdown (type A or B).

Rough estimates of cohesion, which are about half the shear-vane strength (fig. 56), can be made by comparing the penetrations from the Viking backhoe tests with those of the terrestrial tests. Backhoe touchdowns in the tailings on Mars were both type $B$ with a penetration of about $4.5 \mathrm{~cm}$; comparison with the terrestrial data (fig. 53) shows that this penetration corresponds to shear-vane strengths from 0.4 to $1.0 \mathrm{kPa}$ (Backhoe Touchdowns 7, Lander 1, and 4, Lander 2). For drift material at Lander 1 , the penetrations correspond to shear-vane strengths between about 1.0 and $2.5 \mathrm{kPa}$. For the backhoe touchdowns that best represent the blocky material of Lander 1, penetrations correspond to shear-vane strengths of $4-20 \mathrm{kPa}$ (backhoe touchdowns 4 and 9). For crusty to cloddy material at Lander 2, the penetrations correspond to shear-vane strengths between about 4-10 $\mathrm{kPa}$. Finally, rocks must have shear-vane strengths larger than $20 \mathrm{kPa}$.

TABLE 24.-Backhoe-touchdown tests conducted with the Science Test Lander

[Test material was lunar nominal (Hazen) soil. Data are plotted in figure 53]

\begin{tabular}{|c|c|c|c|c|c|c|c|c|c|}
\hline \multirow[t]{2}{*}{$\begin{array}{l}\text { Test } \\
\text { No. }\end{array}$} & \multirow[t]{2}{*}{$\begin{array}{l}\text { Type of } \\
\text { backhoe }\end{array}$} & \multirow{2}{*}{\multicolumn{2}{|c|}{$\begin{array}{l}\text { Test } \\
\text { data }\end{array}$}} & \multirow{2}{*}{$\begin{array}{c}\text { Density } \\
\rho \\
\left(\mathrm{kg} / \mathrm{m}^{3}\right)\end{array}$} & \multirow{2}{*}{$\begin{array}{c}\text { Backhoe } \\
\text { penetration } \\
\left(\frac{\mathrm{P}}{\mathrm{cm}}\right)\end{array}$} & \multirow{2}{*}{$\begin{array}{c}\text { Shear-vane } \\
\text { torque } \\
\text { (in.-lb) }\end{array}$} & \multirow{2}{*}{$\begin{array}{c}\text { Shear-vane } \\
\text { Strength } \\
\frac{\mathrm{S}}{\mathrm{kPa})}\end{array}$} & \multicolumn{2}{|c|}{$\begin{array}{l}\text { Surface-sampler } \\
\text { commands }\end{array}$} \\
\hline & & & & & & & & $\begin{array}{l}\text { EXT } \\
\text { (in.) }\end{array}$ & $\begin{array}{c}\text { ELEV } \\
\text { (degrees) }\end{array}$ \\
\hline 1 & A & 18 & May 1978 & 1,800 & 0 & large & large & 92 & 23.1 \\
\hline 2 & A & 18 & May 1978 & 1,680 & 0.25 & $67.5 \pm 9.57$ & $16.47 \pm 2.34$ & 92 & 23.1 \\
\hline 3 & A & 4 & Oct 1977 & $1,620 \pm 20$ & $2.3 \pm 0.5$ & $11.22 \pm 1.32$ & $2.74 \pm 0.32$ & 92 & 20.1 \\
\hline 4 & A & 4 & Oct 1977 & $1,530 \pm 30$ & $3.9 \pm 0.2$ & $5.83 \pm 0.38$ & $1.42 \pm 0.09$ & 92 & 20.1 \\
\hline 5 & B & 3 & Oct 1977 & 1,390 & $4.2 \pm 0.1$ & $3.50 \pm 1.0$ & $.85 \pm 0.24$ & 92 & 20.1 \\
\hline 6 & B & 4 & Oct 1977 & 1,310 & $4.8 \pm 0.1$ & $2.15 \pm 0.23$ & $.52 \pm 0.06$ & 92 & 20.1 \\
\hline 7 & A & 3 & Oct 1977 & 1,300 & $5.5 \pm 0.1$ & 2.5 & .61 & 92 & 20.1 \\
\hline
\end{tabular}




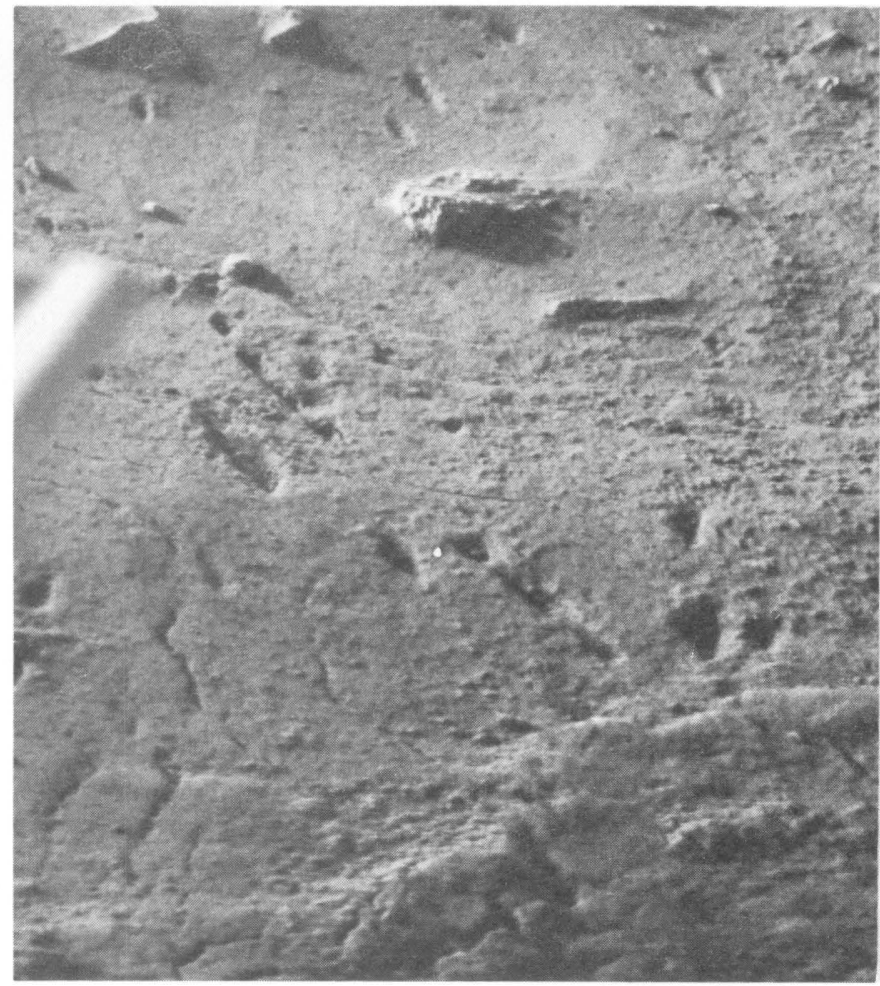

Figure 57.-Impact craters and pits in drift material produced by fragments propelled by engine-exhaust gases during landing. Note low to nonexistent rims of craters and pits and small fragments in craters and pits as well as on surface. Alignments and elongations of craters and pits are radial to engines 1 and 2 (frame 11A022/004). Rock in top center of photograph is approximately $8 \mathrm{~cm}$ wide.

\section{SURFACE IMPACTS}

Five kinds of impacts with the surface occurred during the Viking Missions: (1) fragments and debris propelled by engine exhaust gases produced small impact craters; (2) the surface-sampler shroud units struck the surface after they were ejected from the boom housing; (3) free fall of the surface-sampler restraint or latch pin produced an elongate crater in the rim of the engine-erosion crater of Lander 1 ; (4) materials that were accidentally dropped from the surface-sampler collector head produced craters, scattered fragments, and piles of debris; and (5) coarse fragments were intentionally dropped on drift material in impact tests. Inferences about the physical properties of the surface materials from these impacts are somewhat qualitative but informative.

Interpretation of surface impacts produced by fragments and debris propelled by engine-exhaust gases was hampered by the lack of knowledge of the velocities, angles of impact, and difficulties in associating small impact craters with the objects that produced them. Morphologies of the pits and craters that were produced revealed differences in the various materials in a qualita-

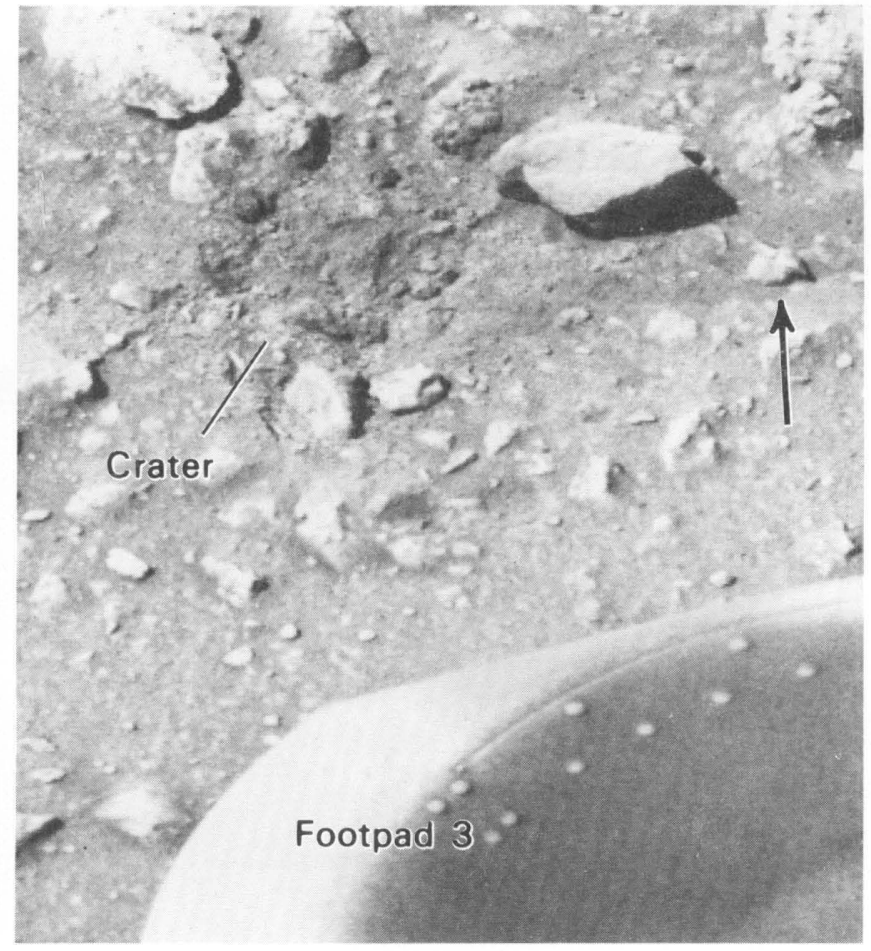

FiguRE 58.-Impact crater in blocky material produced by ejection of shroud unit on Lander 1 , which hit the surface at $3.6 \mathrm{~m} / \mathrm{s}$. Crater is $0.1 \mathrm{~m}$ in diameter. Note irregular hummocky floor and wide range of sizes of ejecta. Small ejected fragment, $2.5 \mathrm{~cm}$ wide, at right (arrow) is about $0.16 \mathrm{~m}$ from center of crater (frame 12A013/002).

tive way. Impact craters and pits in drift material (fig. 57) typically had low to nonexistent rims and large depth-todiameter ratios; these features imply failure during impact mostly by local shear or compression-a characteristic of low-density materials. The presence of scattered fragments on the surface and in the pits and craters suggests that the drift material had considerable strength because such fragments would have penetrated well below the surface into very weak materials. Pits and craters in the rocky area were mostly confined to thin veneers of drift material and wind tails. Pits and craters at the Lander 2 site were similar to those at Lander 1 . They were particularly abundant in the small drifts, but they did not appear to have such large depth-to-diameter ratios as those at the Lander 1 site. Rocks at both sites appeared to be devoid of impact craters.

The impacts of the shroud units of the two landers produced strikingly different results because the shroud of Lander 1 hit a surface underlain by blocky material and bounced away and that of Lander 2 hit a rock and bounced away. Although velocities of the shrouds at impact were well established by the combined use of high-speed photography of shroud ejection on Earth, the acceleration of gravity at the surface on Mars, and known 


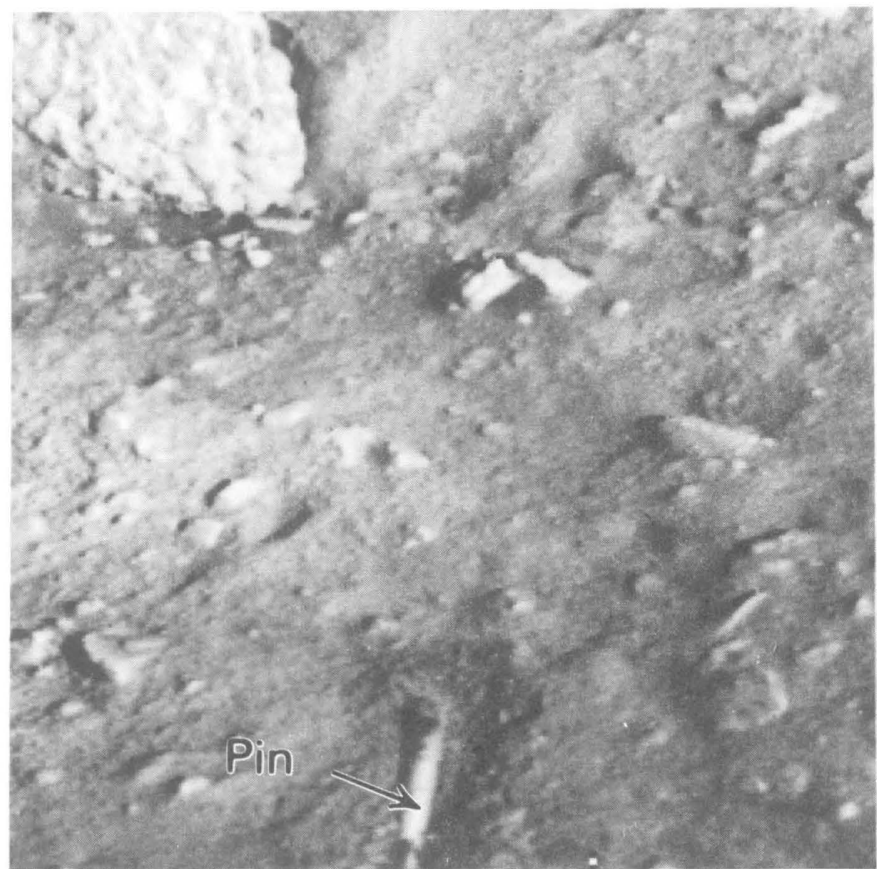

FIGURE 59.-Crater produced by impact of restraint or latch pin (arrow) on rim of crater that was produced by engine-exhaust gases, Lander 1 . Rim and elongate crater about $10 \mathrm{~cm}$ long, $2.4 \mathrm{~cm}$ wide, and $0.6 \mathrm{~cm}$ deep (frame 12A081/012).

distances to the surfaces of the sample fields, the complicated geometry of the tip of the shroud units introduced large uncertainties. Upon impact at $3.6 \mathrm{~m} / \mathrm{s}$, the shroud of Lander 1 produced a shallow crater $0.1 \mathrm{~m}$ wide by the ejection of rocks and clods with diameters from $0.03 \mathrm{~m}$ down to the limit of resolution as well as much finergrained material (fig. 58). Some objects were simply displaced. The floor of the crater was irregular and hummocky, and this irregularity may have been due to the heterogeneous character of the impacted material as well as to the complicated nature of the shroud. At Lander 2, the shroud struck a rock near footpad 3, scratched the surface beyond the rock, and came to rest about $1.1 \mathrm{~m}$ beyond the rock. Because there was no evidence that the shroud scratched or marred the surface of the rock, the rock does not have a weak punky rind produced by weathering or other processes.

Although unplanned, the restraint or latch pin of Lander 1 was released about 0.9-1.0 $\mathrm{m}$ above the surface and fell to the surface, impacting the rim of the crater that was produced by the engine-exhaust gases at 2.6-2.7 $\mathrm{m} / \mathrm{s}$ (fig. 59). An end of the elongate pin (8.2 cm long and $0.6 \mathrm{~cm}$ in diameter) may have struck first but the final crater was clearly elongate, and measuring $2.4 \mathrm{~cm}$ wide and about $10 \mathrm{~cm}$ long. Penetration of the $11.3 \mathrm{-g}$ pin was approximately equal to its diameter. The rimmed crater appeared to have formed mostly by the ejection of fine- grained debris. Because the pin did not penetrate several pin diameters, a fluffy, very low density model may be excluded for the material in the rim of the engine-erosion crater.

Impacts of debris and fragments accidentally dropped from the surface-sampler collector heads during sample acquisitions occurred throughout the missions and in many places in the sample fields. In general, the materials that fell from the collector heads simply littered the surface unless they fell on drift material where impact craters were formed. After sampling, the collector head was retracted, elevated, and then retracted toward the lander so that continua of impact velocities were possible. For the littered and pitted surface of drift material produced by droppings near the trenches excavated on Sol 8, the maximum free-fall velocity was about $3.9 \mathrm{~m} / \mathrm{s}$ because the maximum height of the collector head above the surface was $2.0 \mathrm{~m}$. Near the lander, the free-fall velocity was about $3.1 \mathrm{~m} / \mathrm{s}$ because the height of the collector head was about $1.3 \mathrm{~m}$ so that reasonable impact velocities could be estimated for impacts that were not close to the trenches. As would be expected, a variety of impact phenomena were produced by the continua of velocities and the variations in the mechanical properties of the droppings (fig. 60). Near the trench a dense array of small lumps and surface piles dominated; but nearer the lander, scattered clods were found along with low to rimless impact craters typically $0.01 \mathrm{~m}$ in diameter. Many of the craters contained objects that were probably the projectiles that produced them. The objects, which were $1 / 2-1 / 3$ the diameter of the crater they produced, appeared to have penetrated about one object diameter or less. Other craters with flat floors, central peaks, or central mounds were probably produced by weak clumps of drift material.

Fragments and debris were intentionally dropped on the surface during purges to clean the collector head prior to a subsequent sample acquisition and as part of impact experiments. Generally, purges were accomplished from small heights above the surface so that free-fall velocities were small and penetrations were undetectable. Clumps from the first purges at the Lander 1 site did penetrate drift materials. Here, the collector head was emptied in front of the lander after sample deliveries. Only results from the very first purge were visible because apparently no material was purged the second and third times, and the last purges were partly hidden by part of the spacecraft. The first purge produced a crater in drift material about $0.04 \mathrm{~m}$ wide by the impact of a clump of drift material at about $2 \mathrm{~m} / \mathrm{s}$ (fig. 61). Because of the large size and weakness of the clump of material and the small impact velocity, the crater was almost completely filled by the clump of material that produced it. Thus, the hummocky crater floor with clumps as large as $0.5 \mathrm{~cm}$ was underlain by the purged material. 


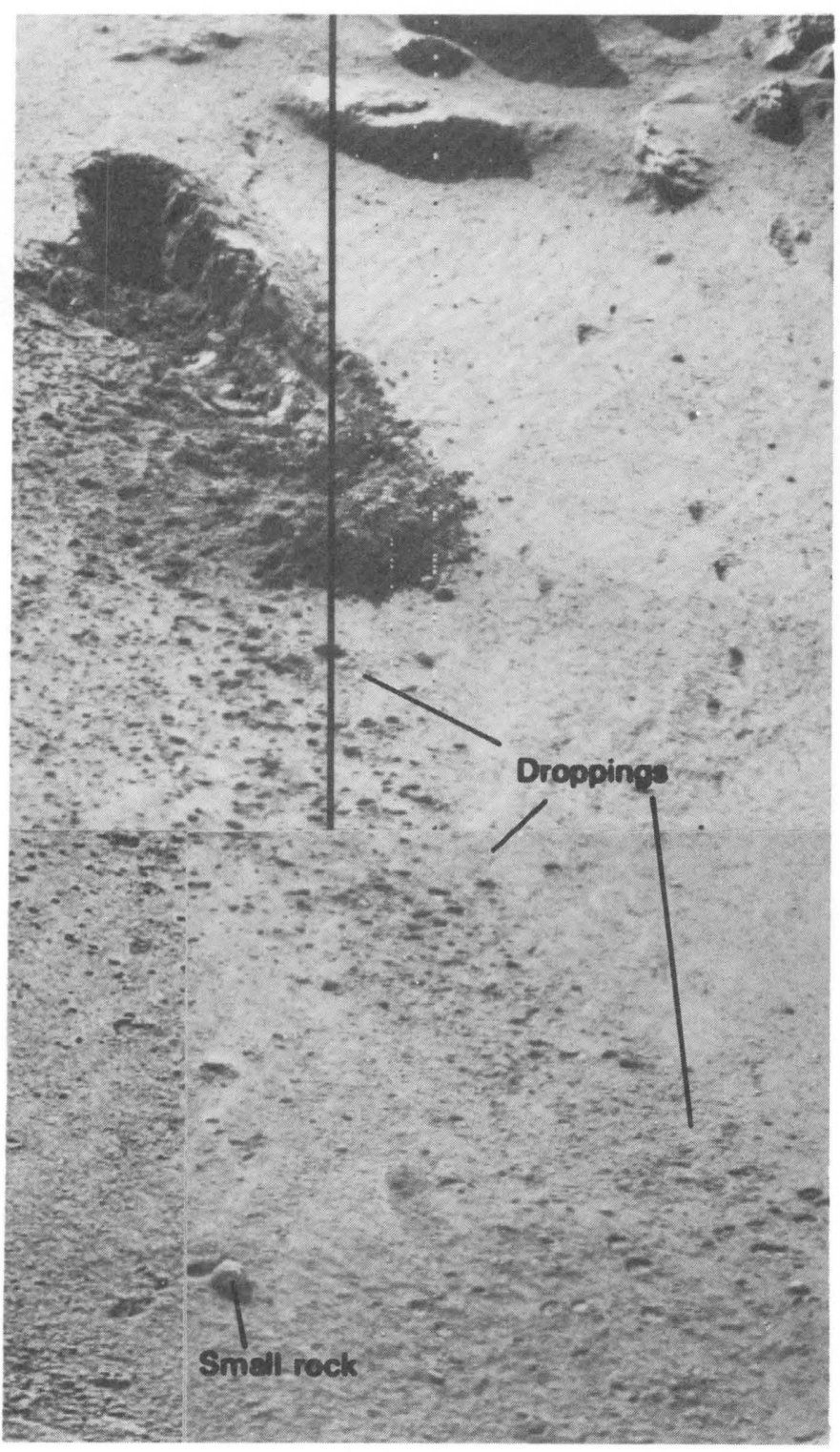

Figure 60.-Droppings on drift material from surface sampler during Sol 8 sample acquisitions, Lander 1 (see table 38). A dense array of lumps and clods cover surface near sample trench, which is $10 \mathrm{~cm}$ wide and $55 \mathrm{~cm}$ long. Nearer the lander, scattered debris and impact craters are present. Small rock or clod is $1.5 \mathrm{~cm}$ in diameter. Most craters have low to nonexistent rims. Objects in some craters are probably projectiles that produced them. Many craters have flat floors, central peaks, or central mounds suggesting that they were produced by weak clumps of material (frames 11A078/012 and 11A079/012). Dark vertical line represents missing data.

For the intentional impact experiments that were conducted only by Lander 1 , fragments larger than $0.2 \mathrm{~cm}$ were dropped onto drift material from a height of about $0.84 \mathrm{~m}$. Fragments were collected by sampling blocky material in Rocky Flats, purging the fine-grained material through the sieve in the collector head, and then drop-

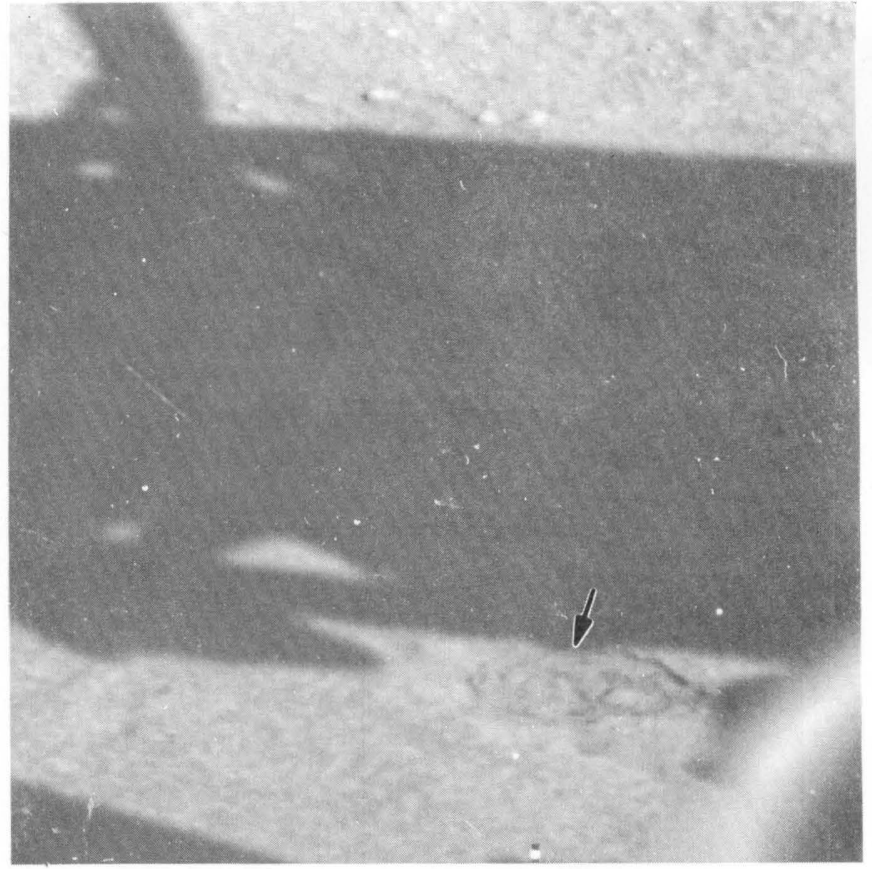

FIGURE 61.-Impact crater (arrow) produced by purging collector head on Sol 8, Lander 1. Drift material fell on drift material at about $2 \mathrm{~m} / \mathrm{s}$. Second pit is concealed by spacecraft cable and shadow of surfacesampler boom. Flat, lumpy floor of crater is underlain by deformed projectile of purged material that produced the crater. Crater is about $4 \mathrm{~cm}$ wide (frame 11B059/036).

ping the coarse residue onto drift material. In the first test during Sol 280, the coarse fragments ranged from about $1 \mathrm{~cm}$ to $0.2 \mathrm{~cm}$ in diameter and possibly smaller. The two largest fragments (about $1 \mathrm{~cm}$ wide) impacted undisturbed drift material and disturbed material in the area of the magnetic mineral abundance test at a velocity of about $2.52 \mathrm{~m} / \mathrm{s}$ (table 25; fig. 62). One fragment penetrated the undisturbed material about $1 / 4-1 / 2$ of its diameter, and the other penetrated the disturbed material about $1 / 2-1$ of its diameter. In the second subsequent impact test during Sol 350 (fig. 63), much smaller objects than those of Sol 280 were collected so that interpreting the results was difficult. For the largest objects, about $0.3 \mathrm{~cm}$ wide, maximum penetrations into undisturbed drift material appeared to be almost $0.15 \mathrm{~cm}$.

The major conclusion drawn from the surface impacts is that the drift material did not appear to be a weak under-dense material equivalent to a fluff because the fragments did not disappear beneath the surface on impact-a result that would have occurred for a very weak under-dense material. Penetration parameters can be calculated for the martian impacts and compared with terrestrial data of comparable impact velocities and projectile sizes using an equation developed for low velocity impacts (Clark and McCarty, 1963): 
TABLE 25.-Data from impact experiments on Mars and Earth and penetration parameters

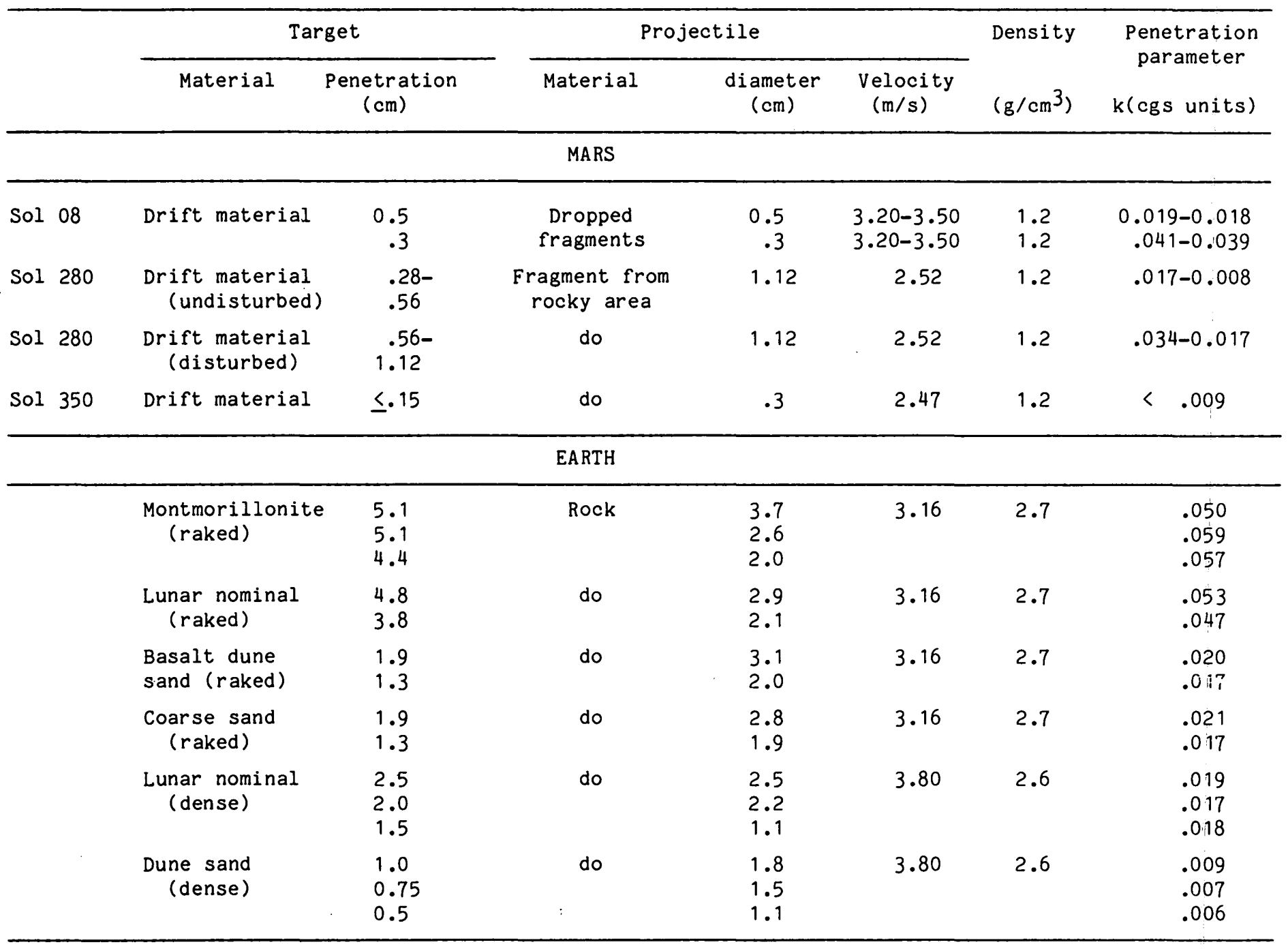

$$
k=\frac{P D}{m^{1 / 2} v^{2 / 3}}
$$

where

$k$ is the penetration parameter (cgs units),

$P$ is the projectile penetration $(\mathrm{cm})$,

$D$ is the projectile diameter $(\mathrm{cm})$,

$m$ is the projectile mass (g), and

$v$ is the projectile velocity $(\mathrm{cm} / \mathrm{s})$.

Values of the penetration parameter $(k)$ for the martian impacts at velocities between 2.47 and $3.50 \mathrm{~m} / \mathrm{s}$ range from 0.008 to 0.041 when the projectiles are assumed to be spheres with a density of $1.2 \mathrm{~g} / \mathrm{cm}^{3}$ (table 25). The assumption of a density of $2.0 \mathrm{~g} / \mathrm{cm}^{3}$ would reduce the value of $k$ by about 0.77 times; a density of $3.0 \mathrm{~g} / \mathrm{cm}^{3}$ would reduce $k$ by about 0.63 times. For impacts of rocks at $3.16 \mathrm{~m} / \mathrm{s}$ into low-density montmorillonite and lunar nominal on Earth after they were weakened and fluffed by raking, penetration parameters ranged from 0.047 to 0.059 (table 25) and the rocks, which penetrated more than their diameters, literally "sank out of sight." Penetrations of rocks at $3.80 \mathrm{~m} / \mathrm{s}$ into compacted lunar nominal on Earth were comparable with the rock diameters so that the parameters were about 0.018 and comparable to some of the parameters in the martian tests. Thus, impact tests on Mars suggest that undisturbed drift material has properties similar to compacted lunar nominal material in its penetration resistance, but disturbed material is somewhat weaker.

Comparison of the Viking impacts with those of Clark and McCarty (1963) conducted at atmospheric pressures yields a variety of possibilities. For impacts of $2.54-\mathrm{cm}$ metal spheres into $16 \mu \mathrm{m}$ particles at $4.48 \mathrm{~m} / \mathrm{s}$, penetration parameters ranged from 0.032 to 0.019 when materials were compacted with mass loads of 10 and 76 $\mathrm{g} / \mathrm{cm}^{2}$. Clark and McCarty (1963) also found a dependence 


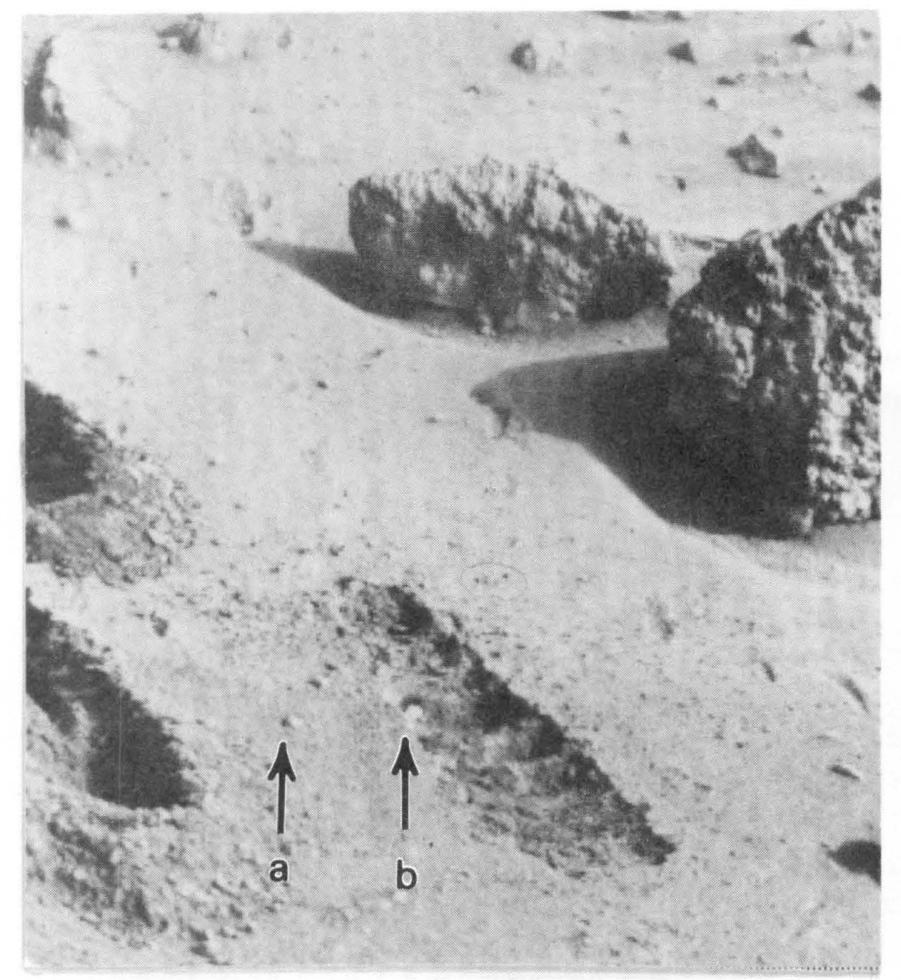

Figure 62.-Results of Sol 280 impact experiment on Mars, Lander 1. Largest two fragments impacted undisturbed drift material (a) and disturbed material of mineral-abundance test (b). Fragments, about $1 \mathrm{~cm}$ wide, impacted at $2.52 \mathrm{~m} / \mathrm{s}$. Fragment (a) penetrated $1 / 4^{-1 / 2}$ of its diameter and fragment (b) penetrated $1 / 2-1$ of its diameter. Drift material is not a fluffy, low-density, granular material because fragments only penetrated small amounts. Mineral-abundance test disturbed area is about $6 \mathrm{~cm}$ wide (frame 11D034/281).

of the penetration parameter and average particle size in tests at reduced pressures. For target particles coarser than about $32 \mu \mathrm{m}$, the parameter ranged from 0.019 to 0.022 and increased rapidly to about 0.042 for target particles of $16 \mu \mathrm{m}$. At $3 \mu \mathrm{m}$ the parameter was about 0.055 . In general, the Viking penetration parameters are like those for 16 to $32 \mu \mathrm{m}$ target particles.

From the data, it is not possible to separate variables such as grain size, cohesion, and density, but it is clear that a very low density material is not required to explain drift material.

\section{SLOPE STABILITY}

The behavior of slopes on Mars poses interesting problems concerning the mechanical properties of the materials and the processes that produce slope failures. Slope stability analyses may be applied to (1) trench walls, (2) natural slopes, and (3) slopes of tailings and conical piles. In the absence of factors other than stresses related to gravity, cohesion estimates derived from slope failures are significantly smaller than those derived from analyses

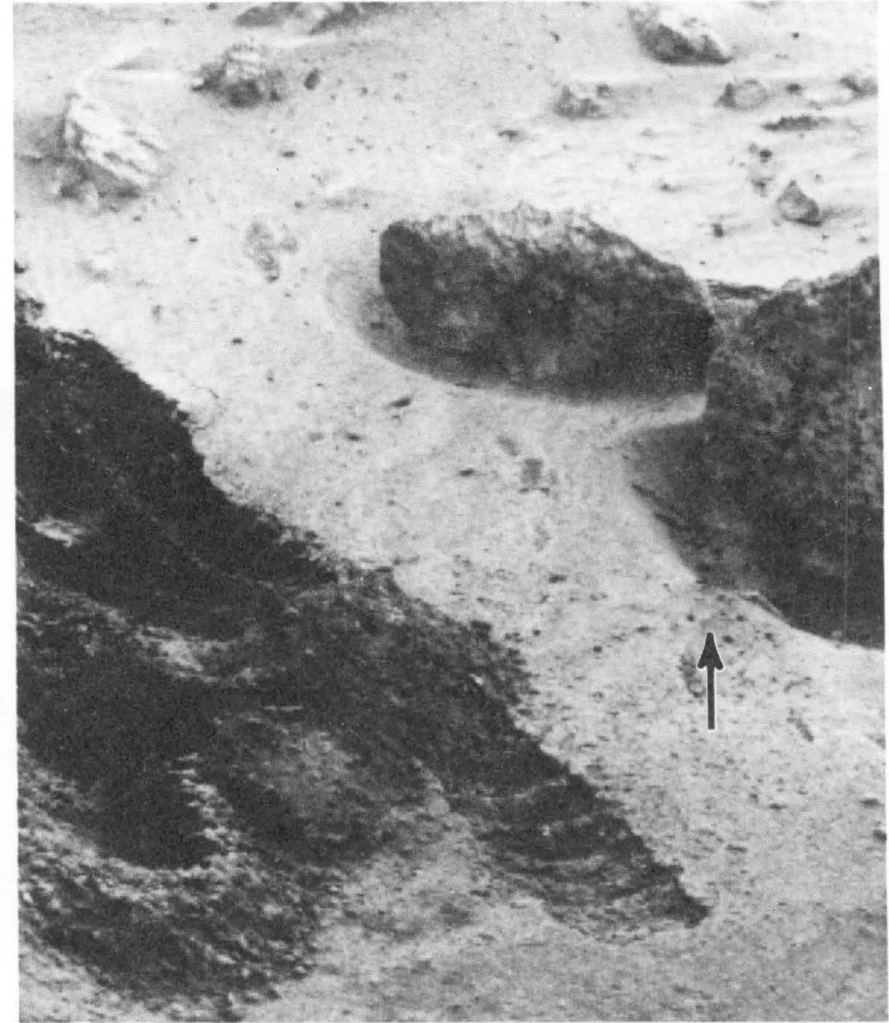

Figure 63.-Results of Sol 350 impact experiment on Mars, Lander 1. Largest fragment (arrow) is about $0.45 \mathrm{~cm}$ wide but others are about $0.3 \mathrm{~cm}$ to below resolution of pictures $(0.15 \mathrm{~cm})$. Fragments appear to have penetrated $1 / 2$ or less of their diameters. Pretest surface shown in figure 62 . Free-fall velocities were about $2.5 \mathrm{~m} / \mathrm{s}$ (frame 11E082/351).

of footpad penetrations, sample trenches, surface-bearing tests, and backhoe touchdowns. For slopes of trench walls, tailings, and conical piles, the materials have been weakened and disrupted as a result of surface-sampler activities but the slope failures of drift material that occurred at large distances from the sample field of Lander 1 at different times were not affected by surfacesampler activities. Natural phenomena initiated these slope failures. Drift material is both crossbedded and crusted so that variable strength properties, depending on the orientation of the applied loads, will affect the failure of the materials, and additional factors may be involved in producing the slope failures. The rather large slope angles of tailings and conical piles are much greater than the angles of internal friction derived from the surface-bearing tests and trenches. Cohesions inferred from slope-stability analyses of trench walls, slopes, and tailings and conical piles are discussed below.

\section{TRENCH WALLS}

Walls of some trenches in drift material with reliefs of about $0.09 \mathrm{~m}$ have failed whereas other trench walls with 

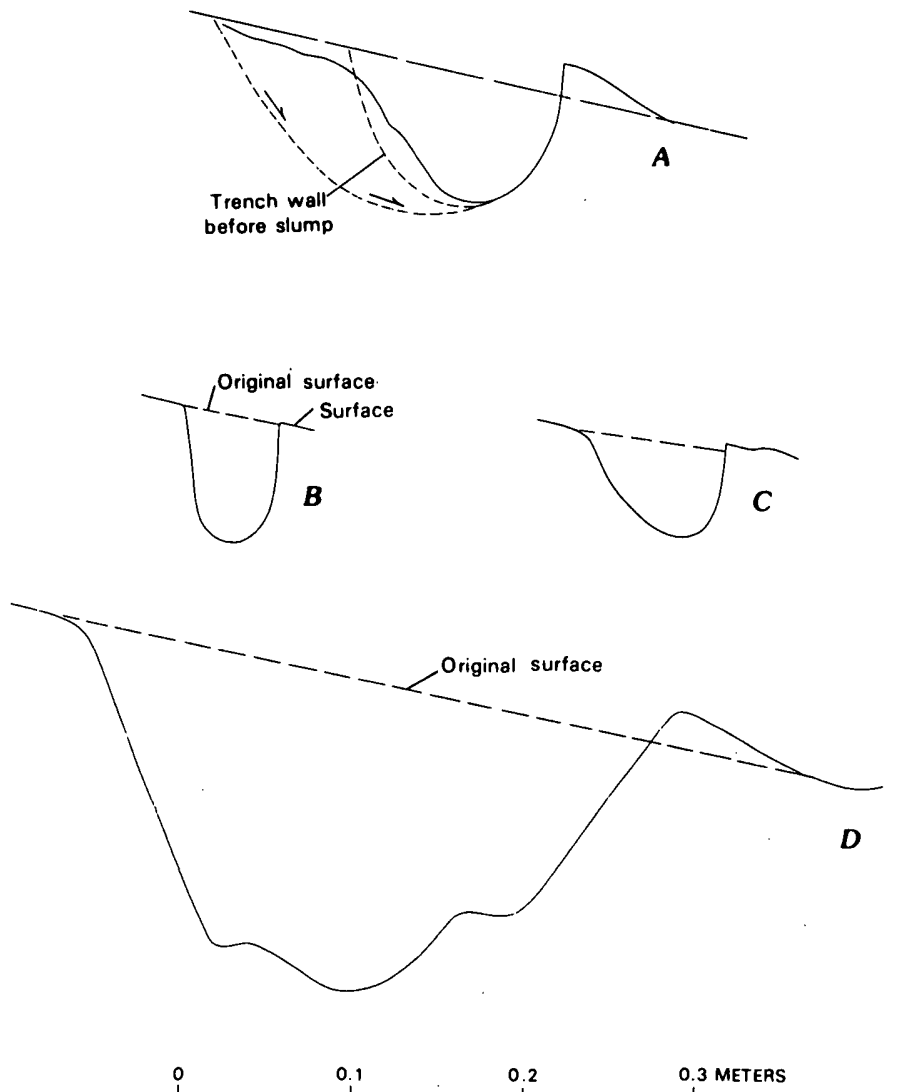

FIGURE 64.-Profiles of three trenches in drift material at right angles to the surface-sampler azimuth planes. $A$, Left wall of trench excavated by Lander 1 on Sol 8 appears to have slumped (see fig. 32C). Reconstructed trench wall shown by short dashed line. Original local surface shown by long dashed line. $B$, Side walls of tip of trench excavated by Lander 1 on Sol 41 did not slump. $C$, Failure of trench in $B$ may have occurred midway along left side of trench; scarp midway along right side may be surface expression of incipient failure (see fig. 31). $D$, Slope of left wall of Deep Hole 1 has not failed despite larger relief (see fig. 65A). Scale for all three profiles is the same; there is no vertical exaggeration.

comparable relief have not failed; one trench wall with $0.185 \mathrm{~m}$ of relief did not fail. Where trench walls failed, disruption of material by the collector head probably produced the planes of weakness along which the failures occurred because surfaces around the trenches are commonly domed and deformed and trenches normally have down-dropped units at their tips.

\section{XRFS 1 TRENCH}

The left wall of the first trench excavated by Lander 1, which was dug to acquire a sample of drift material for inorganic chemical analysis (XRFS 1; fig. $32 C$ ), failed im- mediately during or after the excavation. About midway along the left wall, a mass of drift material appears to have slumped downward toward the center of the trench. The slumped mass is bounded by the trench and an arcuate scarp that extends about $0.08 \mathrm{~m}$ upslope from a chord, $0.17 \mathrm{~m}$ long, along the projected outline of the trench. Profiles through the trench suggest that the slope angle, before failure, was about $59^{\circ}$ and that the relief was about $0.09 \mathrm{~m}$. Vertical displacements are small, $0.01 \mathrm{~m}$ or less (fig. $64 A$ ). Surfaces external to the opposite wall have been domed but the walls themselves have not collapsed.

The domed surfaces near the tip and on the right edge of the trench were obviously produced by the collector head pressing against the drift material, so the failed region along the left wall also could have been initially disrupted by the collector head. The site of the scarp is consistent with both compressional failure caused by the collector head and slope failure if the angle of internal friction is about $20^{\circ}$. If the failure was produced by compressional failure, angles of internal friction greater than $20^{\circ}$ would result in a scarp that extends to a greater distance from the trench wall than that of the observed scarp; for slope failure, angles of internal friction greater than $20^{\circ}$ would result in a scarp that is closer to the trench wall than that of the observed scarp. Thus, it appears that the angle of internal friction of drift material is about $20^{\circ}$.

\section{PHYSICAL PROPERTIES 1 TRENCH}

The walls of the Physical Properties 1 trench of Lander 1 with $0.08-0.09 \mathrm{~m}$ of relief were generally stable, although incipient and actual failures may have occurred midway along its walls (figs. 31 and $64 B$ and $C$ ). The position of the possible incipient failure is consistent with a material that has an angle of internal friction of $20^{\circ}$.

Figure 65.-Deep holes excavated on Mars. A, Deep Hole 1 of Lander 1 was excavated in drift material by backhoe using 48 retraction strokes. Trench is about $0.22 \mathrm{~m}$ deep, $0.30 \mathrm{~m}$ wide, and $1.6 \mathrm{~m}$ long. Maximum relief of longest slope of left wall is about $0.18 \mathrm{~m}$; slope is about $68^{\circ}$. Note rock, $0.15 \mathrm{~m}$ wide, in tailings (frame 11C134/221). B, Deep Hole 2 of Lander 1 was excavated in blocky material by backhoe using 47 retraction strokes. Trench is about $0.10 \mathrm{~m}$ deep, $0.30 \mathrm{~m}$ wide, and $1.10 \mathrm{~m}$ long. Note blocky appearance of tailings which include some rocks (frame 12G079/488). C, Deep Hole 1 of Lander 2 was excavated in crusty to cloddy material by backhoe using 48 retraction strokes. Trench is about $0.12 \mathrm{~m}$ deep, $0.27 \mathrm{~m}$ wide, and $0.80 \mathrm{~m}$ long. Note slabs of crusts near tip and clods and rocks in tailings (frame 21G061/512). 


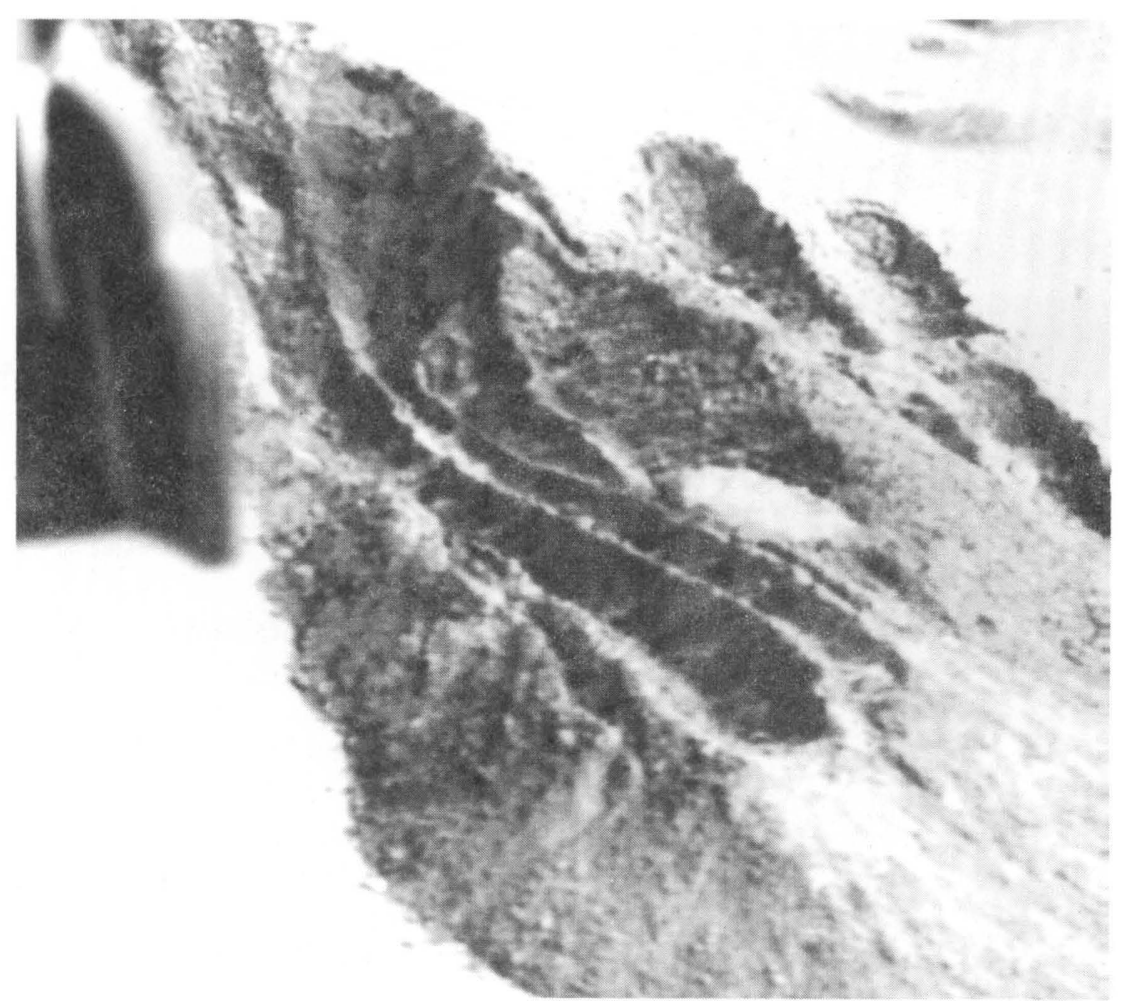

A

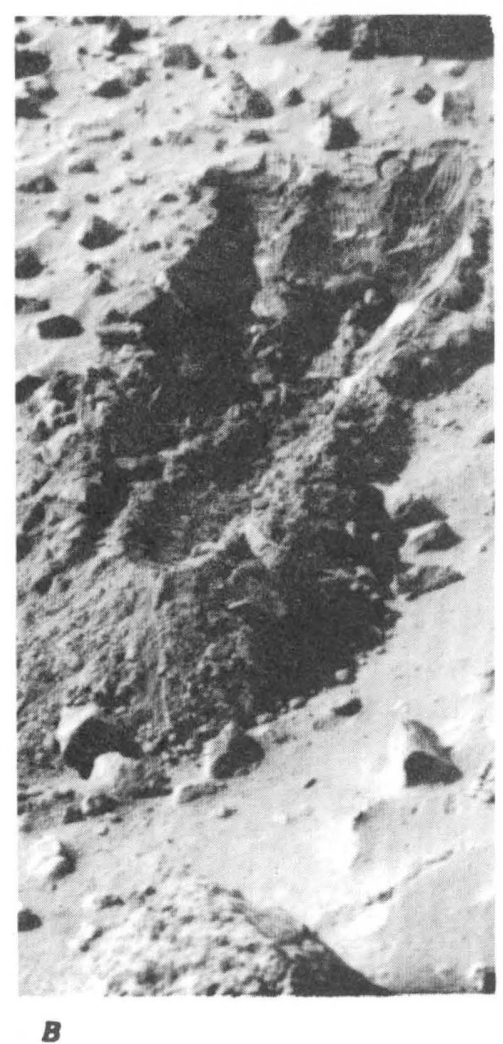

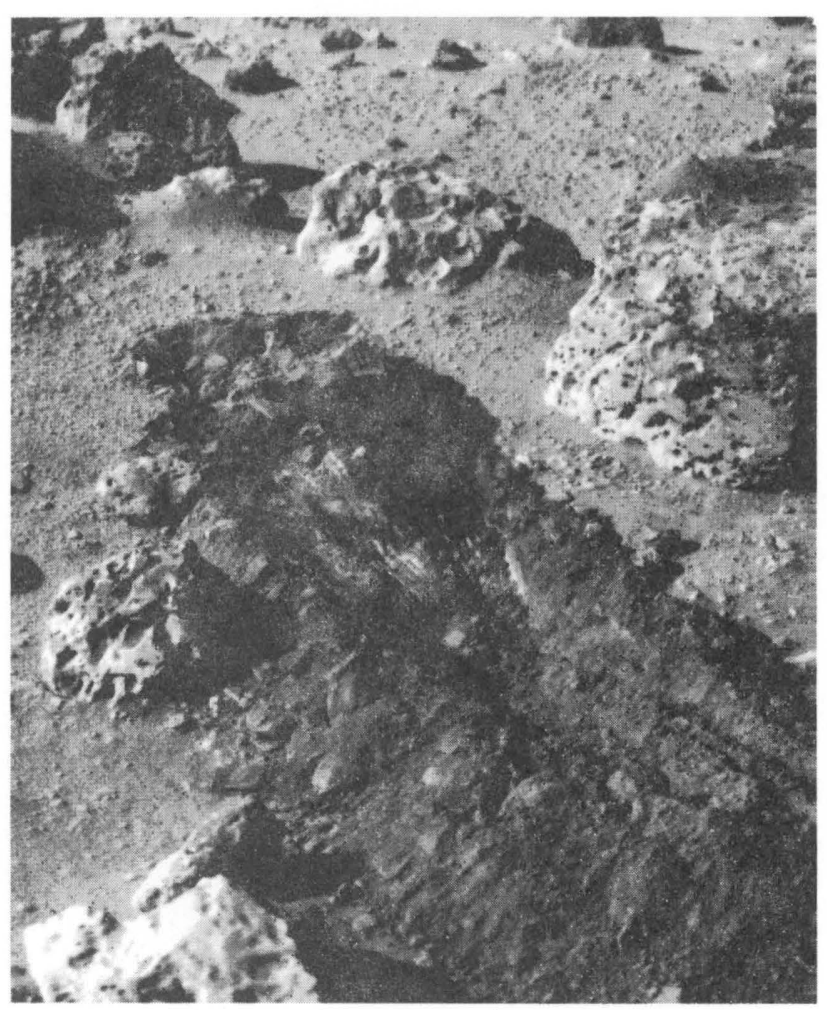

C 


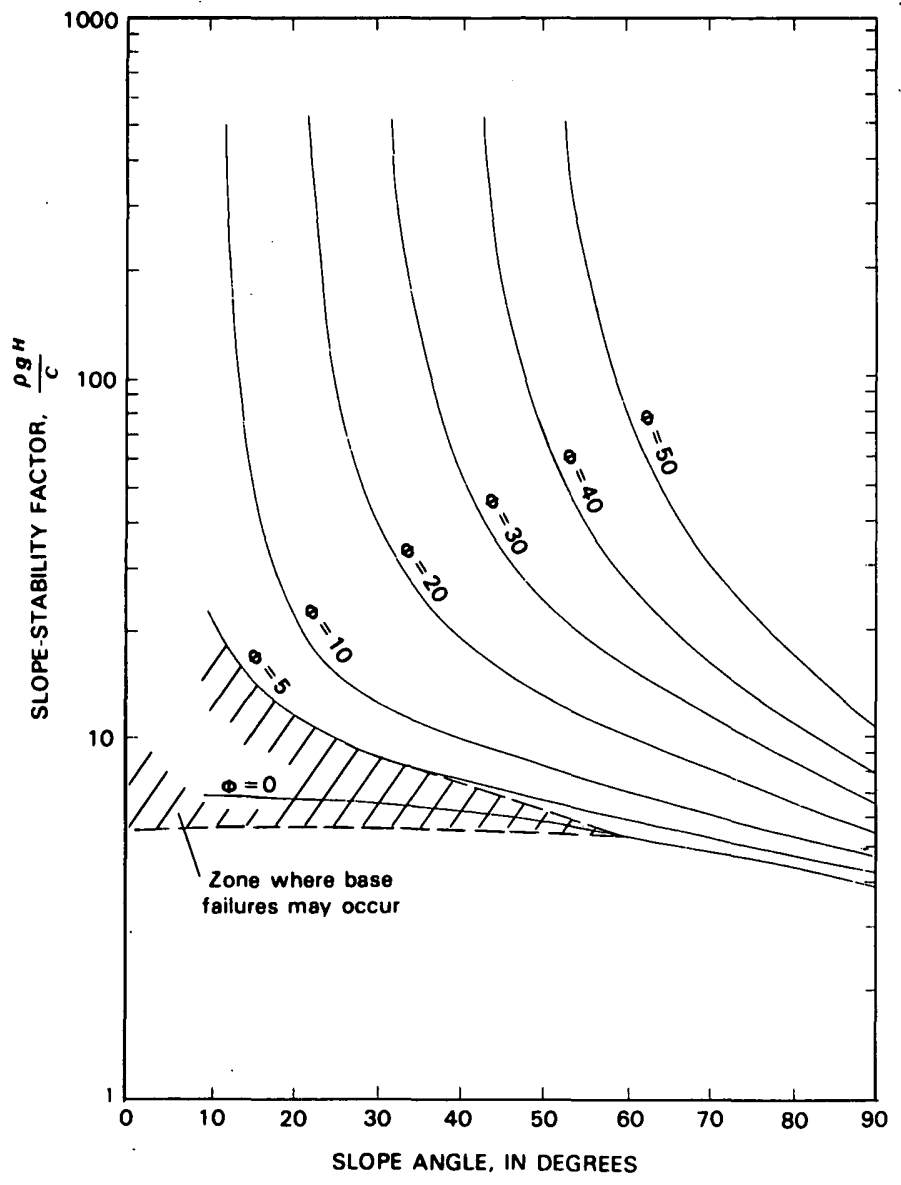

FIGURE 66.-Slope-stability factors for angles of internal friction from 0 to $50^{\circ}$ as a function of slope angle. Curves computed using theory of Baker and Garber (1978). For slope-stability factors see also Scott (1963) and equation 3.

\section{DEEP HOLE 1}

The excavation of Deep Hole 1 on Sols 202, 204, 218, and 219 by repeated retractions of the backhoe through drift material produced a stable slope of $68^{\circ}$ with a relief of $0.185 \mathrm{~m}$ (figs. $64 D, 65 A$ ). This slope appeared to be unchanged on Sol 1358 so that greater stability for drift material is implied by this wall than by the walls of other trenches.

\section{INFERRED COHESIONS}

Rough estimates of cohesions or of limits for cohesions can be obtained by using dimensionless slope-stability factors (Scott, 1963; fig. 66) for the various trench walls (table 26). Rigorous analyses (Baker and Garber, 1978) of trench slopes yield essentially the same values for the cohesions and limits as the rough estimates. Slope-stability factors are a function of the angle of internal friction and slope angle. The factors represent an equilibrium condition wherein the slope is marginally stable according to:

$$
\text { Slope-stability factor }=\frac{\rho g H}{c}
$$

where

$\rho=$ density of the material,

$g=$ the acceleration of gravity,

$H=$ the relief of the slope, and

$c=$ the cohesion of the material.

For an angle of internal friction of $20^{\circ}$, the slope-stability factors for the shallow trenches in drift material range from 8.2 to 11 (table 26). For drift material with a density of $1,200 \mathrm{~kg} / \mathrm{m}^{3}$, the failed wall of the XRFS 1 trench implies a cohesion less than $36 \mathrm{~Pa}$, whereas the stable walls of the Physical Properties 1 trench imply a cohesion greater than $44 \mathrm{~Pa}$. For the stable wall of Deep Hole 1, the cohesion must exceed $95 \mathrm{~Pa}$. On the basis of a cohesion of about $1,000 \mathrm{~Pa}$ derived from the sample trenches and backhoe touchdowns, slopes of $68^{\circ}$ with a relief of $2.5 \mathrm{~m}$ should be marginally stable; but $68^{\circ}$ slopes of drift material with $2.5 \mathrm{~m}$ of relief in the far field are rare if they exist at all.

The absence of slopes of drift material as steep as $68^{\circ}$ with $2.5 \mathrm{~m}$ of relief may be, partly, the result of local planes of weakness. Wind erosion has etched drift material revealing cross laminations, and the cross laminations alone indicate variable mechanical properties. In other places the presence of a thin crust also implies variable cohesions. Finally, exposures of drift material show that drift material is, at least locally, fractured in directions that form large angles with the cross laminations, and these fractures, which are open, imply zero cohesion on the scale length of the fractured areas (0.5-1.0 m) (fig. 67).

In general, the walls of trenches in other materials appear to be stable although a minor amount of slumping has occurred in the upper walls of Deep Hole 2 of Lander 1 and Deep Hole 1 of Lander 2 (fig. $65 B$ and $C$ ). Except for the minor failures, the walls of the deep holes and smaller trenches appear to be stable; therefore, the cohesions in table 26 are lower limits.

\section{NATURAL SLOPES}

Natural slopes of drift material failed at two places. The first failure occurred between Sols 74 and 183 near the leeward base of the rock called Big Joe (figs. $68 A$ and $B$ ), which is $8-10 \mathrm{~m}$ to the left of Lander 1 . The second failure occurred about 1 martian year later between Sols 767 and 771 near the leeward base of a rock called Whale (figs. $68 C$ and $D$ ) which is about $25 \mathrm{~m}$ from the lander. It is probably significant that both slides occurred in late summer $\left(\mathrm{L}_{\mathrm{s}}=148\right)$ during periods of reduced atmospheric pressure (Hess and others, 1980), high average surface and air temperatures (Kieffer, 1976; Ryan and Henry, 1979), and 
TABLE 26.-Slopes, reliefs, slope-stability factors, and limits for cohesions, assuming three angles of internal friction for selected trenches

\begin{tabular}{|c|c|c|c|c|c|c|c|c|c|c|}
\hline \multirow[t]{2}{*}{ Item } & \multirow[t]{2}{*}{$\begin{array}{l}\text { Trench or } \\
\text { feature }\end{array}$} & \multirow[t]{2}{*}{ Material } & \multirow{2}{*}{$\begin{array}{c}\text { Slope } \\
\text { angle } \\
\theta \\
\text { (degrees) }\end{array}$} & \multirow{2}{*}{$\begin{array}{c}\text { Vertical } \\
\text { relief } \\
\qquad \frac{\mathrm{H}}{(\mathrm{cm})}\end{array}$} & \multicolumn{3}{|c|}{$\begin{array}{c}\text { Slope stability } \\
\text { factors }\end{array}$} & \multicolumn{3}{|c|}{$\begin{array}{c}\text { Cohesions }(\rho=1200) * \\
(\mathrm{~Pa})\end{array}$} \\
\hline & & & & & $\phi=20^{\circ}$ & $\phi=30^{\circ}$ & $\phi=40^{\circ}$ & $\phi=20^{\circ}$ & $\phi=30^{\circ}$ & $\phi=40^{\circ}$ \\
\hline 1 & $\begin{array}{l}\text { XRFS 1, wall } \\
\text { failure }\end{array}$ & Drift & $59 \pm 1$ & 0.09 & 11 & 16 & 29 & 36 & $<25$ & $<14$ \\
\hline 2 & $\begin{array}{l}\text { Physical Properties } 1, \\
\text { wall }\end{array}$ & do & $\begin{array}{l}61 \pm 1 \\
71 \pm 1\end{array}$ & $\begin{array}{l}.055 \\
.080\end{array}$ & $\begin{array}{r}10 \\
8.2\end{array}$ & $\begin{array}{l}15 \\
11\end{array}$ & $\begin{array}{l}27 \\
16\end{array}$ & $\begin{array}{l}25 \\
44\end{array}$ & $\begin{array}{r}16 \\
>32\end{array}$ & $\begin{array}{l}9.1 \\
>22\end{array}$ \\
\hline 3 & $\begin{array}{l}\text { Deep Hole 1, } \\
\text { wall }\end{array}$ & do & $68 \pm 1$ & .85 & 8.7 & 12 & 19 & 95 & $>69$ & $>44$ \\
\hline 4 & $\begin{array}{l}\text { Deep Hole } 1 \text {, } \\
\text { tailings }\end{array}$ & $\begin{array}{l}\text { Drift, } \\
\text { disturbed }\end{array}$ & $43 \pm 2$ & .205 & 17 & 39 & 500 & 54 & 24 & 1.8 \\
\hline 5 & Conical pile 1 & do & $45 \pm 1$ & .036 & 16 & 33 & 180 & 10 & 4.9 & .89 \\
\hline & Conical pile 2 & do & & .057 & -- & -- & - & 16 & 7.7 & 1.4 \\
\hline & Conical pile 3 & do & & .034 & -- & -- & -- & 9.5 & 4.6 & .84 \\
\hline & Conical pile 4 & do & & .044 & -- & -- & - & 12 & 5.9 & 1.1 \\
\hline 6 & $\begin{array}{l}\text { XRFS } 4 \text {, } \\
\text { tailings }\end{array}$ & $\begin{array}{l}\text { Blocky, } \\
\text { disturbed }\end{array}$ & $34 \pm 2$ & .016 & 27 & 200 & $\infty$ & 2.6 & .4 & 0 \\
\hline 7 & $\begin{array}{l}\text { Physical Properties } 2, \\
\text { tailings }\end{array}$ & do & $40 \pm 2$ & .02 & 20 & 56 & $\infty$ & 4.7 & 1.7 & 0 \\
\hline 8 & $\begin{array}{l}\text { Physical Properties } 7 \text {, } \\
\text { tailings }\end{array}$ & do & $34 \pm 2$ & .20 & 27 & 200 & $\infty$ & 33 & 4.5 & 0 \\
\hline 9 & $\begin{array}{l}\text { Deep Hole 2, } \\
\text { wall }\end{array}$ & Blocky & $\begin{array}{l}73 \pm 2 \\
59 \pm 2\end{array}$ & $\begin{array}{l}.09 \\
.12\end{array}$ & $\begin{array}{r}7.8 \\
11\end{array}$ & $\begin{array}{l}11 \\
16\end{array}$ & $\begin{array}{l}15 \\
29\end{array}$ & $\begin{array}{l}>52 \\
>49\end{array}$ & $\begin{array}{l}>36 \\
>33\end{array}$ & $\begin{array}{l}>27 \\
>18\end{array}$ \\
\hline 10 & $\begin{array}{l}\text { Deep Hole } 2, \\
\text { tailings }\end{array}$ & $\begin{array}{l}\text { Blocky, } \\
\text { disturbed }\end{array}$ & $38 \pm 2$ & .10 & 22 & 76 & $\infty$ & 20 & 5.9 & 0 \\
\hline 11 & $\begin{array}{l}\text { Deep Hole } 1 \text {, } \\
\text { wall }\end{array}$ & Crusty-cloddy & $64 \pm 4$ & .10 & 9.5 & 14 & 23 & $>47$ & $>32$ & $>19$ \\
\hline 12 & $\begin{array}{l}\text { Deep Hole } 1 \\
\text { tailings }\end{array}$ & do & $35 \pm 4$ & .098 & 25 & 150 & $\infty$ & 18 & 2.9 & 0 \\
\hline
\end{tabular}

* Use of a density of $2,000 \mathrm{~kg} / \mathrm{m}^{3}$ would increase the cohesion by $3 / 2$.

large differences in maximum and minimum daily temperatures (Kieffer, 1976; Ryan and Henry, 1979). Unfortunately, the slopes on which these failures occurred cannot be measured with the two cameras. The first slope was visible from camera 1 but not from camera 2 , and the second was too distant for adequate description much less measurement.

The slope failure near the leeward base of Big Joe, which is close enough to be described, appears to have occurred along a plane within the drift material (fig. 68B). Both the runout and visible parts of the exposed scarp appeared to be more or less uniform in thickness. The runout appeared to be about $0.012-0.017 \mathrm{~m}$ thick (2-3 pixels) and the scarp about $0.006-0.012 \mathrm{~m}$ (1-2 pixels). Although the apparent distance from the scarp to the toe of the runout was $0.37 \mathrm{~m}$ and the apparent slope was $36^{\circ}$, the viewing conditions require that this distance is too small and this slope angle is too large. Judging from shadows cast by the drift here and elsewhere, the slope was probably about $30^{\circ}$; the distance from the scarp to the toe of the runout would then be about $0.47 \mathrm{~m}$ and the runout would be about $0.23 \mathrm{~m}$ along slope. Natural slopes of drift material, where measurable in and near the sample field, are usually less than $30^{\circ}$. Slopes are commonly about $20^{\circ} \pm 3^{\circ}$ for slope lengths about $0.1-0.2 \mathrm{~m}$; however, slopes may be as much as $30^{\circ}$ for slope lengths of 0.03 $\mathrm{m}$. Inspection of the drifts beyond the sample field suggests that long slopes are commonly about $20^{\circ}$.

Cohesion estimates for the failure at the base of Big Joe should be based on a sheetlike failure because the slopestability factors (fig. 66) apply to logarithmic spiral-failure surfaces. For planar-failure surfaces:

$$
\tau_{s}=c+(S-p) \tan \phi
$$

where

$\tau_{s}$ is the shearing strength on the base of the sheet, $c$ is the cohesion,

$S$ is the normal stress,

$p$ is the pore-fluid pressure, and

$\phi$ is the angle of internal friction.

Shear stress $(\tau)$ and normal stress $(S)$ on the base of the sheet are given by:

$$
\begin{gathered}
\tau=\rho g t \sin \theta \\
S=\rho g t \cos \theta
\end{gathered}
$$




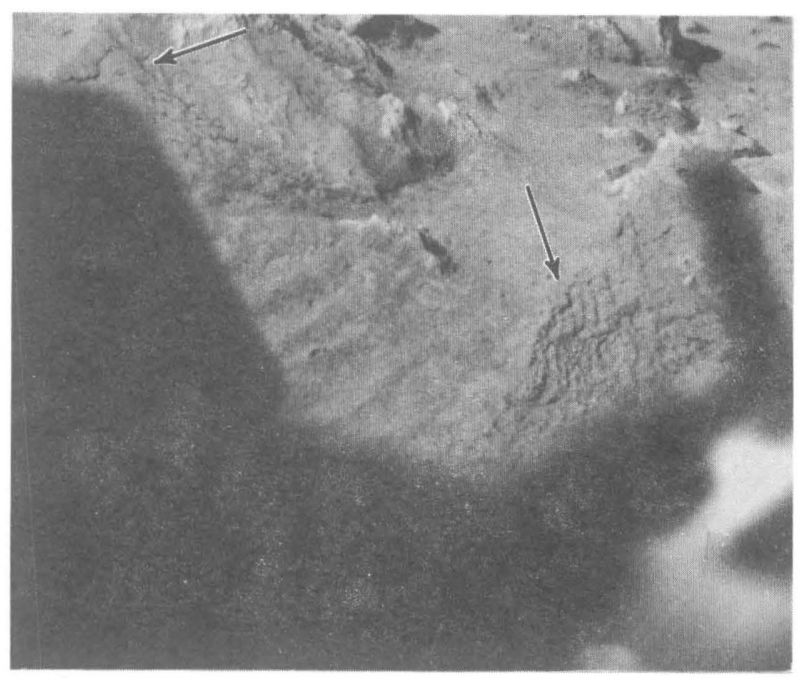

A

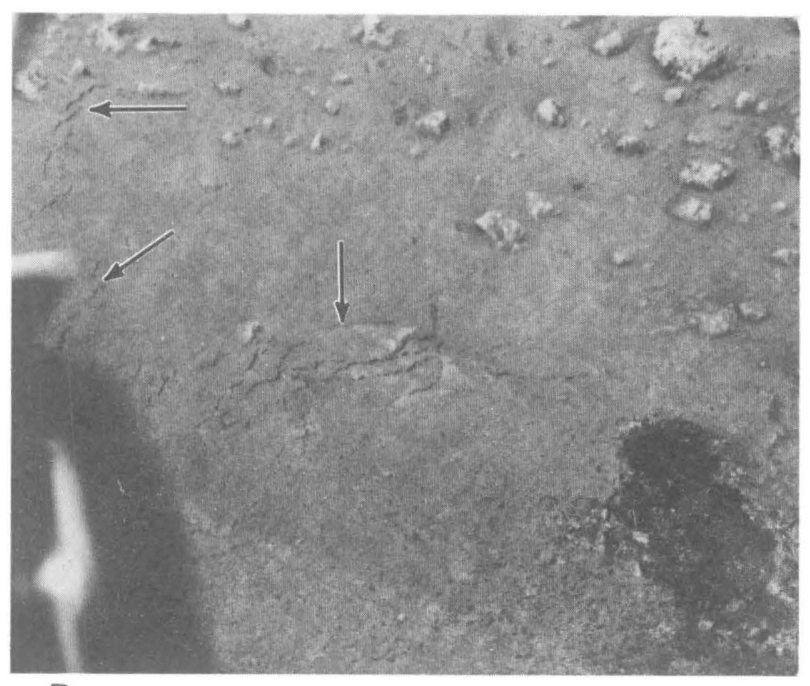

B

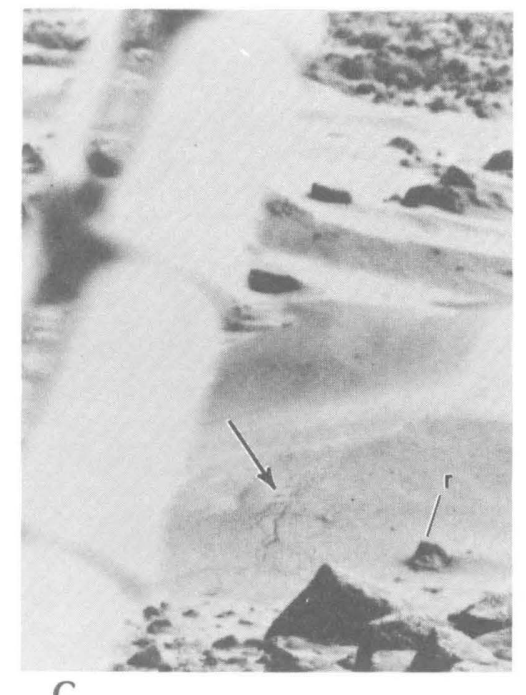

C where

$\rho$ is the density of the sheet,

$g$ is the acceleration of gravity,

$t$ is the thickness of the sheet, and

$\theta$ is the slope angle.

In the absence of pore pressures $(p)$ for marginally stable conditions (that is, $\tau=\tau_{s}$ ), cohesions are about 5 to $14 \mathrm{~Pa}$ when the slope angle is $30^{\circ}$, the angle of internal friction is $20^{\circ}$, and the density is $1,200-2,000 \mathrm{~kg} / \mathrm{m}^{3}$. Tau $(\tau)$ is between 13.4 and $22.3 \mathrm{~Pa}$ and $S$ is between 23.2 and 38.6 $\mathrm{Pa}$. If the cohesion of drift material is about $40 \mathrm{~Pa}$, as could be inferred from the slope-stability analyses of the shallow trenches, positive pore pressures of 87 to $96 \mathrm{~Pa}$ due to a gas acting against an impermeable base of the slab could initiate failure. Such pore pressures are within the magnitude of the seasonal atmospheric pressure changes on Mars. If the cohesion is $1,000-2,000 \mathrm{~Pa}$, as would be inferred from the analyses of trenches and backhoe touchdowns, the pore-gas pressures acting against an impermeable base of the slab would need to be more than 2,700-5,500 Pa.

Generation of positive pore gas pressures of $87-96 \mathrm{~Pa}$ (0.9-1.0 mbar) are certainly easier to achieve than 2,700 to $5,500 \mathrm{~Pa}$ ( 27 to 55 mbars) but the larger pressures should not be rejected outright. For martian springtime temperatures, isothermal desorption of $\mathrm{CO}_{2}$ per gram of basalt powder or nontronite could exceed $0.087 \mathrm{~cm}^{3} \mathrm{STP}$ (standard temperature and pressure) when total pressures change from $8.25-6.75$ mbar. Warming of $3^{\circ}$ or more under isobaric conditions could release more than 0.087 $\mathrm{cm}^{3}$ STP of $\mathrm{CO}_{2}$ per gram (Fanale and Cannon, 1979). This amount of $\mathrm{CO}_{2}$ is substantial and perfect-gas-law calculations suggest that pressures of trapped $\mathrm{CO}_{2}$ might reach 100-200 mbar. Thus, it appears possible that the combined winter to summer changes of higher to lower pressures, cooler to warmer average near-surface temperatures, and smaller to larger diurnal temperature differences could act in concert with wind eddies and local planes of weakness in drift material to produce small landslides on steep slopes. Final possibilities that might cause failures, but which cannot be adequately assessed, involve changes within drift material such as changes in moisture content and removal of a binding salt by solution.

Figure 67.-Open fractures in drift material, Lander 1. A, Open fractures (arrows) in drift material on surface to left of stroke gauge, and in upper left. Arrows are about $10 \mathrm{~cm}$ long (frame 11A144/025). $B$, Open fractures (arrows) in drift material to left of Sol 8 trench (frame 11A069/009). Trench is about $10 \mathrm{~cm}$ wide. $C$, Open fractures (arrow) can be seen on lee face of drift beyond sample field. Rock (r) is about $10 \mathrm{~cm}$ wide (frame $11 \mathrm{C} 162 / 239$ ). 


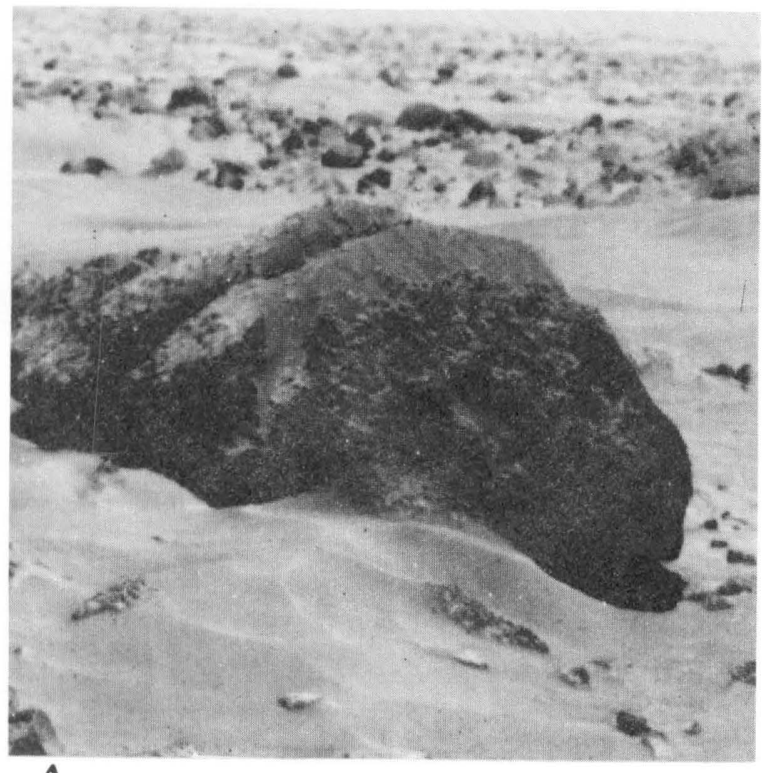

A

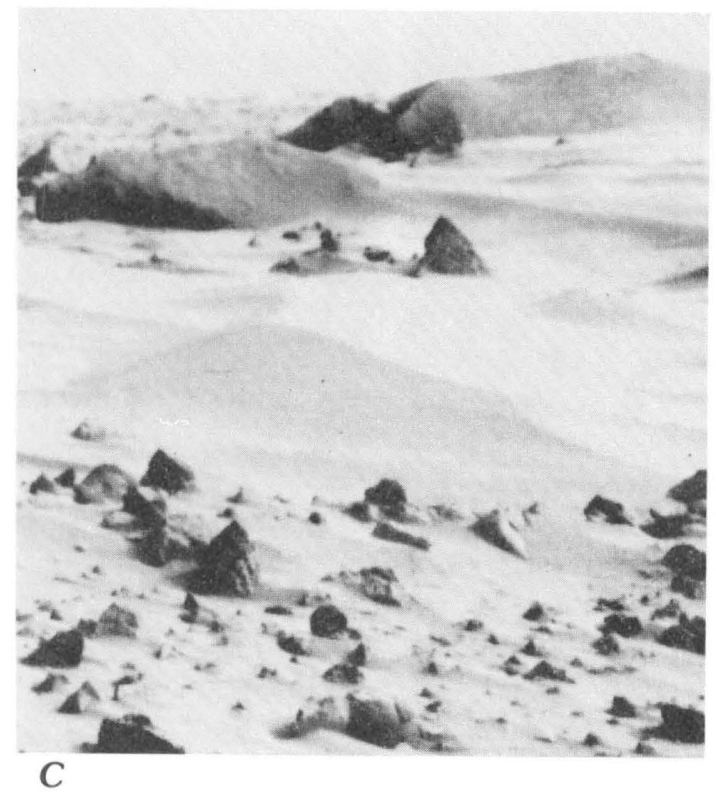

Figure 68.-Natural slope failures of drift material have occurred on leeward sides of large rocks. $A$, Surface near base of large rock called Big Joe prior to slope failure (frame 11B162/072). Big Joe is 8-10 m from Lander 1 and $1 \mathrm{~m}$ high. $B$, Slope failure at base of Big Joe (arrow).

Failures similar to those at the base of Big Joe and Whale rocks have probably occurred elsewhere; that they may be common is suggested by a drift about $10 \mathrm{~m}$ behind Lander 1 (fig. 69). The uppermost edge or crest of the
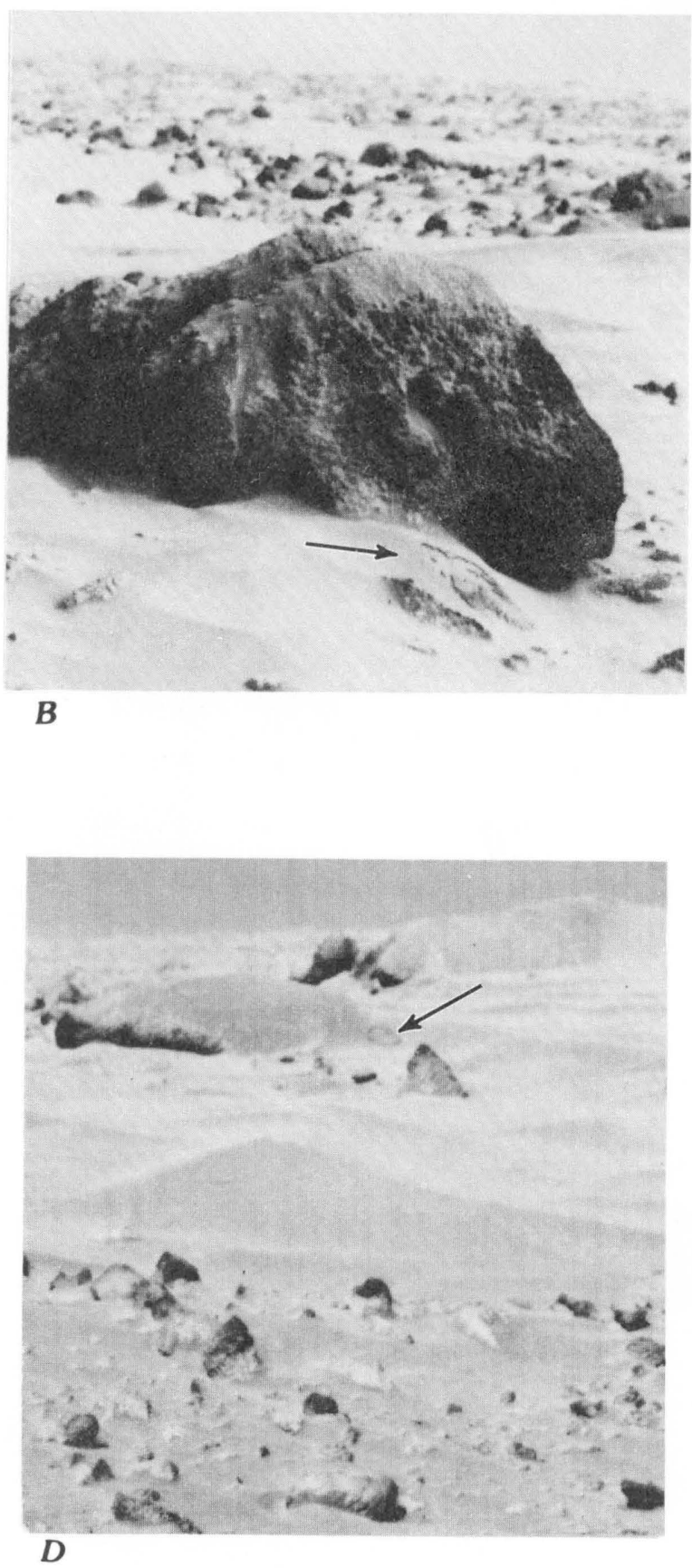

Note thin scarp and tabular form of runout (frame 11C162/239). C, Surface near Whale rock (about $25 \mathrm{~m}$ from Lander 1 and $2.5 \mathrm{~m}$ wide; frame 11I139/767). $D$, Slope failure at base of Whale appears as dark irregular ring (arrow) on drift (frame 11I141/771). steep right-hand side of the leeward face of the drift was outlined by a bright band produced by the illumination of a steep slope about $1 \mathrm{~cm}$ high. This steep slope may be the surface expression of a more cohesive zone or crust 


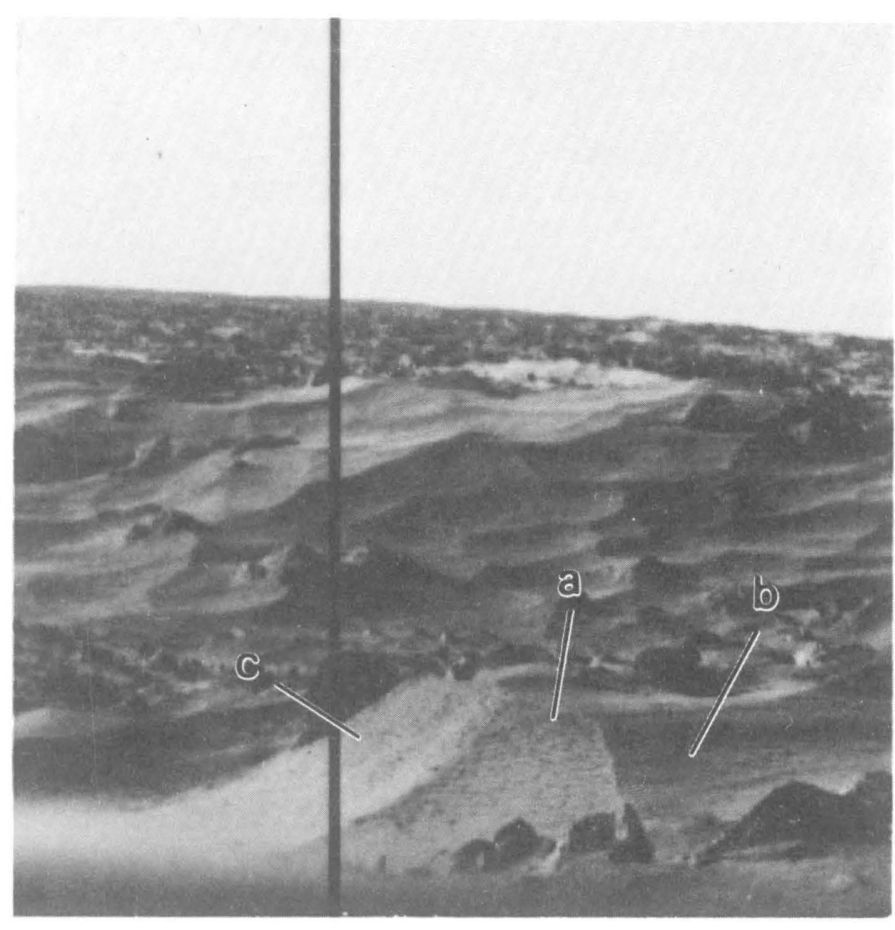

Figure 69.-Drift about $10 \mathrm{~m}$ behind Lander 1 . Drift is about $0.4 \mathrm{~m}$ high. a, Note bright outline of crest above nearest (right) steep face; the outline may represent steep scarp of surface crust about $(1 \mathrm{~cm}$ thick). b, Nearly horizontal bands on face and flanks are layers etched into relief by deflation. c, Dark streaks parallel to slope of left face may be residue of runouts from slope failures. Drift is too far away for accurate measurements of slopes of face (frame 11B098/040). Dark vertical line is missing data.

at the surface of the drift that was previously exposed by slope failures for which the runout has been removed. On the left-hand side of the face, darker streaks extending downslope on the face may be the residue of thin veneers of runout that have been partly removed by the wind.

\section{CONICAL PILES AND TAILINGS}

Slopes and reliefs of the conical piles and tailings should be marginally stable and reflect the properties of the disturbed materials. Typical slopes for disturbed drift material were about $43^{\circ}-45^{\circ}$, much larger than one would expect them to be for cohesionless materials with angles of internal friction about $20^{\circ}$. For conical piles and tailings of small trenches, slope angles were about $45^{\circ}$ and their reliefs are near $0.04 \mathrm{~m}$. In contrast, the tailings of Deep Hole 1 in drift material had a slope of almost $43^{\circ}$ and a relief of $0.20 \mathrm{~m}$.

Interpretations of these data depend on the initial assumptions. If the angle of internal friction is assumed to be $20^{\circ}$, slope-stability factors for all of the piles and tailings are about 16-17 and the derived cohesions for the low-relief tailings and conical piles are about 10-12 $\mathrm{Pa}$, but the derived cohesions are much less than the derived cohesion of $54 \mathrm{~Pa}$ for the high-relief tailings of the deep hole (table 26). An alternative initial assumption is that both the cohesion and angle of internal friction of disturbed drift material are the same for the low- and highrelief features. This assumption requires that the slopestability factors for the high- and low-relief tailings have the same ratio as their respective reliefs, or about 5 . This figure is only correct for slopes of $43^{\circ}$ and $45^{\circ}$ if the angle of internal friction is about $41^{\circ}$, in which case the cohesion must be very small-about $1 \mathrm{~Pa}$.

Similar results are obtained for the conical piles and tailings of trenches and deep holes in other materials although these slopes are slightly less. The slopes of tailings of Deep Hole 2 of Lander 1 are as much as $38^{\circ}$, but steeper slopes were measured after the tailings were sampled (XRFS 16, 17, and 18). Slopes of the Deep Hole 1 tailings of Lander 2 were about $35^{\circ}$.

\section{SUMMARY}

Interpretation of the stability of the walls of trenches is in doubt because the collector head could have disrupted the surface materials adjacent to the walls, and the materials could have planes of weakness. However, the locations of the failures and scarps in the trench walls are consistent with an angle of internal friction of $20^{\circ}$ for undisturbed drift material for both slope failure and compressional failure. For drift material, the $68^{\circ}$ slope with a relief of about $0.18 \mathrm{~m}$ for Deep Hole 1 suggests that undisturbed drift material has a cohesion greater than $95 \mathrm{~Pa}$ if the angle of internal friction is $20^{\circ}$.

The two natural slablike slope failures of drift material require the presence of very weak planes in drift material, or the generation of positive pore pressures, or both. Very large positive pore pressures would be required if the cohesion was $1-2 \mathrm{kPa}$, as inferred from the analysis of sample trenches.

The slopes of conical piles and tailings of drift material may be interpreted to be the result of small but variable cohesions and an angle of internal friction $20^{\circ}$ or, alternatively, a cohesion of $1 \mathrm{~Pa}$ and an angle of internal friction of about $41^{\circ}$.

\section{THE QUEST FOR ROCKS}

Considering the rocky appearance of both landing sites, it was surprising to discover that none of the samples had compositions like lunar or terrestrial rocks (Toulmin and others, 1977). Compositions of samples of the coarse fraction $(0.2-1.2 \mathrm{~cm})$ from blocky material at the Lander 1 site appeared to be similar to compositions of samples of fine-grained material except for larger amounts of sulfur 
TABLE 27.-Acquisitions for coarse samples by Lander 1 and Lander 2

\begin{tabular}{|c|c|c|c|c|c|c|c|c|c|c|c|}
\hline \multirow{2}{*}{ Item } & \multirow{2}{*}{ Designations } & \multirow[t]{2}{*}{ Location } & \multirow[t]{2}{*}{ Sol } & \multirow{2}{*}{$\begin{array}{l}\text { Sample } \\
\text { Strokes }\end{array}$} & \multirow{2}{*}{$\begin{array}{l}\text { Sample } \\
\text { Type }\end{array}$} & \multicolumn{4}{|c|}{ Sieving procedures } & \multirow[t]{2}{*}{ Samples } & \multirow[t]{2}{*}{ Comments } \\
\hline & & & & & & $\begin{array}{c}\text { Extension } \\
\text { (in.) }\end{array}$ & $\begin{array}{l}\text { Elevation } \\
\text { (degrees) }\end{array}$ & $\begin{array}{l}\text { Rate } \\
(\mathrm{Hz})\end{array}$ & $\begin{array}{c}\text { Duration } \\
\text { (s) }\end{array}$ & & \\
\hline \multicolumn{12}{|c|}{ Lander 1} \\
\hline 1 & XRFS $2(C-2)$ & Rocky Flats & 34 & 2 & Normal & 57.3 & 7.9 & 8.8 & 60 & Sample acquired & Sampled for rocks. \\
\hline 2 & XRFS $3(C-3)$ & Rocky Flats & 40 & 2 & Normal & 57.3 & 7.9 & 8.8 & 90 & $\begin{array}{l}\text { Partial sample; } \\
80 \text { percent full }\end{array}$ & Sampled for rocks. \\
\hline 3 & XRFS $4(C-5)$ & Atlantic City & $177-180$ & 4 & Normal & 56.5 & $(-) 15.4$ & 8.8 & 120 & Sample acquired & Sampled for rocks. \\
\hline 4 & XRFS 9 & Edge of sample field & $336-338$ & 3 & Skim & 53.9 & $(-) 5.3$ & 4.4 & $(120) \times 5$ & No sample & $\begin{array}{l}\text { Sampled for duri- } \\
\text { crust. }\end{array}$ \\
\hline 5 & XRFS $11(C-10)$ & Edge of sample field & $430-432$ & 4 & Skim & 49.9 & $(-) 7.9$ & 8.8 & 30 & $\begin{array}{l}\text { Partial sample; } \\
60 \text { percent full }\end{array}$ & $\begin{array}{l}\text { Sampled for duri- } \\
\text { crust. }\end{array}$ \\
\hline 6 & XRFS $16(C-13)$ & Deep Hole 2 tailings & $558-561$ & 16 & Normal & 58.1 & $(-) 7.9$ & 8.8 & 90 & $\begin{array}{l}\text { No new material } \\
\text { reported in XRFS }\end{array}$ & Sampled tailings. \\
\hline 7 & XRFS $17(\mathrm{C}-13)$ & Deep Hole 2 tailings & 581 & 16 & Normal & 58.1 & $(-) 7.9$ & 8.8 & 90 & $\begin{array}{l}\text { Sample acquired; } \\
\text { XRFS cavity full on } \\
\text { Sol } 581\end{array}$ & $\begin{array}{l}\text { Sampled tailings } \\
\text { and surface be- } \\
\text { low tailings. }\end{array}$ \\
\hline 8 & XRFS $18 \quad(\mathrm{C}-13)$ & Deep Hole 2 tailings & 586 & 16 & Normal & 58.1 & $(-) 7.9$ & 8.8 & 90 & $\begin{array}{l}\text { Sol } 652 \text { picture shows } \\
\text { clods and rocks (?), } \\
3 \mathrm{~cm} \text { across and less } \\
\text { on funnel; cavity } \\
\text { full on Sol } 581\end{array}$ & $\begin{array}{l}\text { Sampled original } \\
\text { surface material } \\
\text { below tailings. }\end{array}$ \\
\hline \multicolumn{12}{|c|}{ Lander 2} \\
\hline 1 & Biology 1 & Beta site & 8 & 1 & Normal & 10.2 & $(-) 3.4$ & 8.8 & 45 & No sample & Sampled for rocks. \\
\hline 2 & XRFS 2 & Beta site & $46-47$ & 4 & Normal & 83.1 & $(-) 11.0$ & 8.8 & 90 & No sample & Sampled for rocks. \\
\hline 3 & XRFS 3 & Alpha site & $57-58$ & 4 & Normal & 63.3 & $(-) 18.0$ & 8.8 & 90 & No sample & Sampled for rocks. \\
\hline 4 & XRFS 13 & Bonneville & 506 & 12 & Normal & 16.2 & (-) 10.4 & 8.8 & 90 & No sample & $\begin{array}{l}\text { Sampled for duri- } \\
\text { crust. }\end{array}$ \\
\hline 5 & XRFS 14 & Deep Hole tailings & $522-23$ & 16 & Normal & 16.2 & $(-) 7.9$ & 8.8 & 90 & No sample & $\begin{array}{l}\text { Sampled at base of } \\
\text { tailings. }\end{array}$ \\
\hline 6 & XRFS 15 & Deep Hole tailings & 535 & 16 & Normal & 16.2 & $(-) 7.9$ & 8.8 & 90 & No sample & $\begin{array}{l}\text { Sampled at base of } \\
\text { tailings. }\end{array}$ \\
\hline 7 & XRFS 16 & Deep Hole tailings & 545 & 12 & Normal & 16.2 & $(-) 7.9$ & 8.8 & 90 & No sample & Sampled tailings. \\
\hline
\end{tabular}


and chlorine (B.C. Clark and others, 1977). Samples of the coarse fraction were, in fact, never acquired at the Lander 2 site. After the experience gained from inorganic chemical analyses of samples by Lander 1 , the desire to analyze a sample containing demonstrable rock fragments became so pressing that an attempt was made to deliver the residuum of coarse material from the very first sample collected by Lander 2 to the XRFS (Baird and others, 1977). However, no coarse material was acquired, and after futilely attempting to collect some we concluded that little or no coarse material was strong enough to survive the sieving procedures. During the Extended Mission, major efforts were made to acquire rock fragments but to no avail.

A variety of techniques was employed in the quest for rocks. These techniques ranged from acquiring samples from surfaces littered with coarse objects to acquiring samples from the tailings of the deep holes. We even tried to construct piles of the coarse fraction and, we hoped, rock fragments. There were, of course, rocks at the landing sites. It is possible that the unusually high motor currents observed while comminuting samples of blocky material were due to the presence of millimeter-sized grains of dense rocks, silicates, or metal oxides. The larger rocks at both sites were pushed around by the sampler.

\section{SAMPLE ACQUISITIONS}

To obtain samples of coarse material (table 27), Lander 1 performed about 63 surface-sampler acquisition strokes. For each stroke, the acquired samples were sieved by vibrating the inverted collector head, typically at $8.8 \mathrm{~Hz}$, while it was elevated above the surface for durations of, commonly, 30-120 s. Nineteen of these samples apparently did not contain coarse material and six contained some material. Nearly all the samples of coarse material came from the upper $0.06 \mathrm{~m}$ or so because most of the trenches from which the coarse material was obtained were that deep. These samples included those designated as "Deep Hole 2 tailings" (XRFS 16, 17, and 18). The first 16 acquisition strokes in the tailings of Deep Hole 2 (Sols 558-561) did not acquire a sample. Subsequently, on Sol 581, coarse material was delivered to the XRFS chamber, but before the end of the sequence of 16 strokes, the surface sampler had removed all the tailings and begun excavating the original surface materials beneath the tailings. Samples of the coarse material acquired from the base of the Deep Hole 2 tailings on Sol 586 were probably entirely from materials originally near the surface, and fragments from this upper surface were in and on the XRFS funnel (fig. 70). The largest fragment may have come from the floor of Deep Hole 2 on Sol 502, because the fragment was present on the funnel in color pictures taken on Sol 506; but the fragment could also have

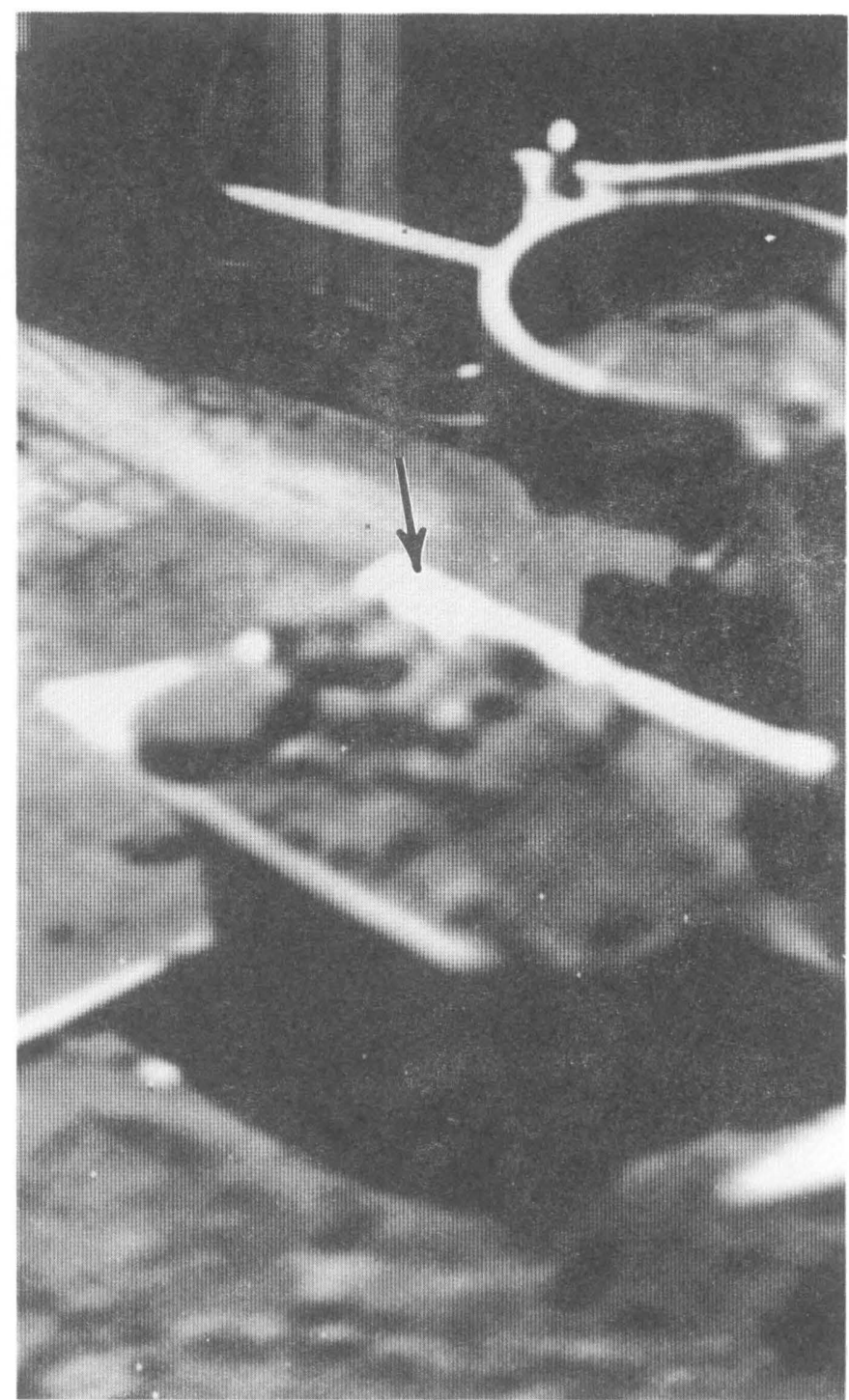

FIGURE 70.-Funnel (arrow) above chamber of the X-ray fluorescence spectrometer showing fragments collected by the end of Sol 586 . Chamber was filled by end of Sol 581. Most of material in chamber and funnel probably came from material at the surface instead of from the tailings, but largest fragment on left tip of funnel may have come from Deep Hole 2. Largest tabular fragment is about $3 \mathrm{~cm}$ wide. In color picture large tabular fragments are red; smaller fragments have both reddish and bluish hues (frame 12I026/652). Funnel dimensions are $10 \mathrm{~cm}$ by $15 \mathrm{~cm}$.

originated from near the surface.

Bulk densities of the individual coarse fragments analyzed by Lander 1 appear to vary considerably. Estimates of bulk densities of the coarse material in the XRFS chamber were between $570-940 \mathrm{~kg} / \mathrm{m}^{3}$ (B.C. Clark, A.J. Castro, and Ray J. Weldon, written commun., 1978) and for samples with typical porosities of 50-60 percent, densities of the individual fragments were $1,140-1,880 \mathrm{~kg} / \mathrm{m}^{3}$ and $1,400-2,300 \mathrm{~kg} / \mathrm{m}^{3}$. Such densities are incompatible 
with the densities of massive igneous rocks, but they are compatible with some dense soils, sedimentary rocks, and porous igneous rocks. Reported chemical analyses are incompatible with unweathered pure igneous rocks (Toulmin and others, 1977).

The experience of acquiring samples of coarse material by Lander 2 was different than that by Lander 1 . Lander 2 did not deliver samples of the coarse material although some 65 strokes were made attempting to do so (table 27). Samples from areas where the surface was littered with small objects $0.5 \mathrm{~cm}$ wide at the Alpha and Beta sites yielded no coarse materials even though eight sample strokes were executed. The obviously crusty material at Bonneville yielded no coarse material after 16 sample strokes. Neither the materials at the base of Deep Hole 1 nor in the tailings yielded a coarse fraction. Thus, there seemed to be little or no material at the Lander 2 site ranging from 0.2 to $1.2 \mathrm{~cm}$ that was capable of surviving the sieving procedures.

\section{PURGES AND ROCK PILES}

After a sample delivery, the procedure was to empty or purge the excess material onto the surface so that the collector head would be clean for the next sample acquisition. For all rock piles and most sample deliveries preceding purges, the collector head was inverted and almost always vibrated at $8.8 \mathrm{~Hz}$ for periods of $17-120 \mathrm{~s}$. During a subsequent purge, the collector head was deelevated in the upright position and vibrated at $4.4 \mathrm{~Hz}$ for $30-60 \mathrm{~s}$ so that the remaining material would bounce out of the collector head and fall to the surface. The purged material was mostly the coarse fraction because the material previously either had been sieved or a portion had been delivered to the biology and GCMS processors or the XRFS funnel through the inverted collector head. Initially, for Lander 1, materials were purged close to the lander in view of camera 1 , but not camera 2 . Subsequently purge sites were selected that were in the field of view of both cameras and as close to the landers as possible in order to achieve both stereoscopy and good resolution (table 28; fig. 71). Later, efforts were made to construct "rock piles" by purging at sites in the field of view of both cameras, but the sites were farther away in the sample field where they could be sampled. For both landers, the amounts of coarse material at the purge sites were unimpressive. At the initial purge site of Lander 1, globs of fine-grained drift material produced craters, and the largest clumps were about $0.5 \mathrm{~cm}$ in diameter (table 28; items 1-2). Very few objects were purged at the second purge site (table 28; items 4-9) because the samples either had been obtained from drift material or had been previously sieved in an attempt to collect the coarse fraction. The rock pile (table 28; items 10-13) was a dismal failure because most

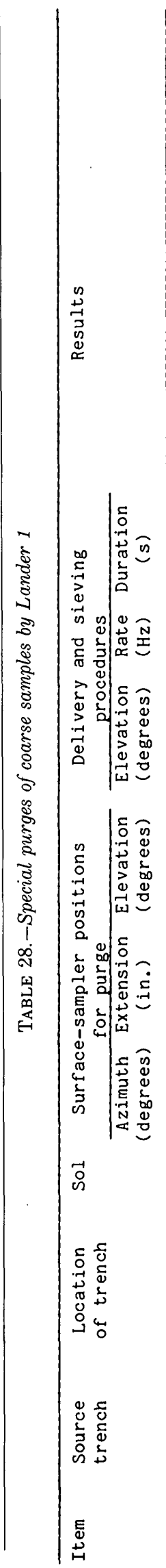

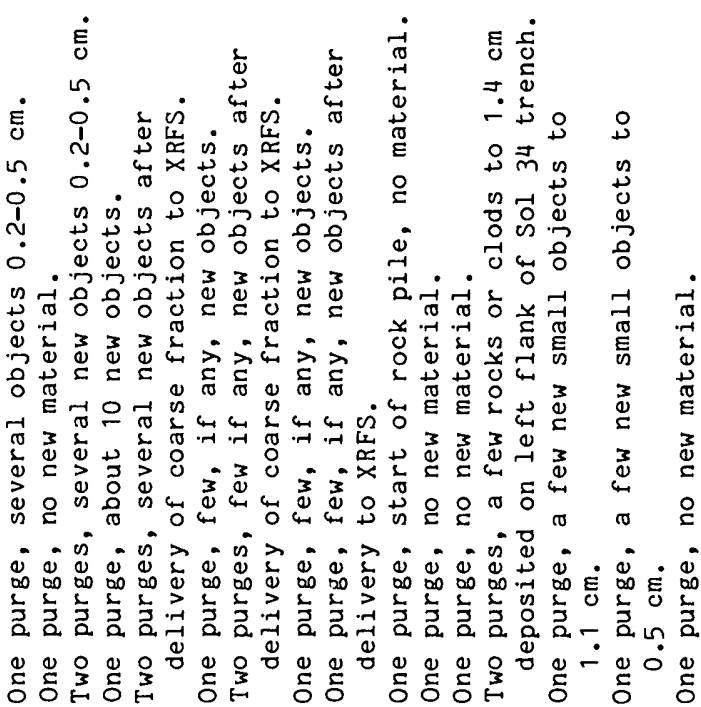

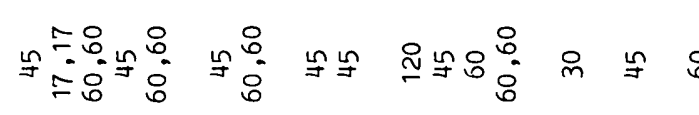

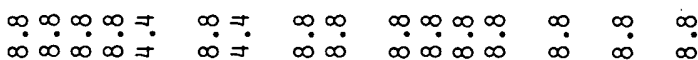

च.च̣

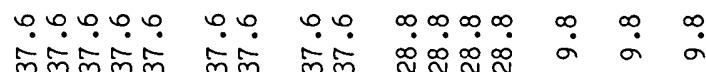

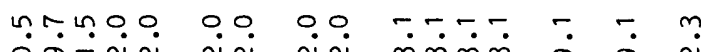

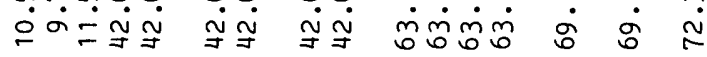

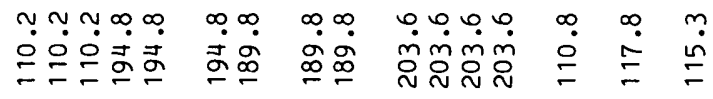

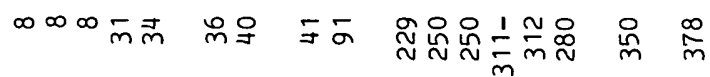

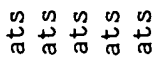

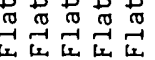

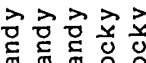

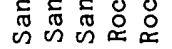

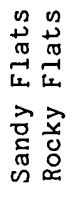

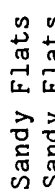

- -

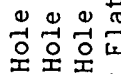

웡워웧ㅇ

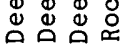

-

ad - - m N

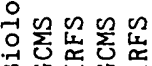

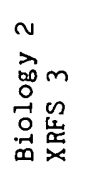

an

竞

$=$

ก

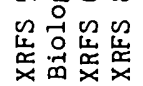

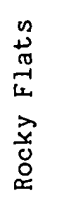

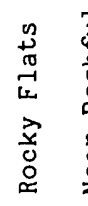

$\vec{Z}$
$\frac{1}{5}$
0
0
0
$\frac{1}{\pi}$
$\frac{0}{2}$

$\sim$.

然

空

- Nma un

or

으모 


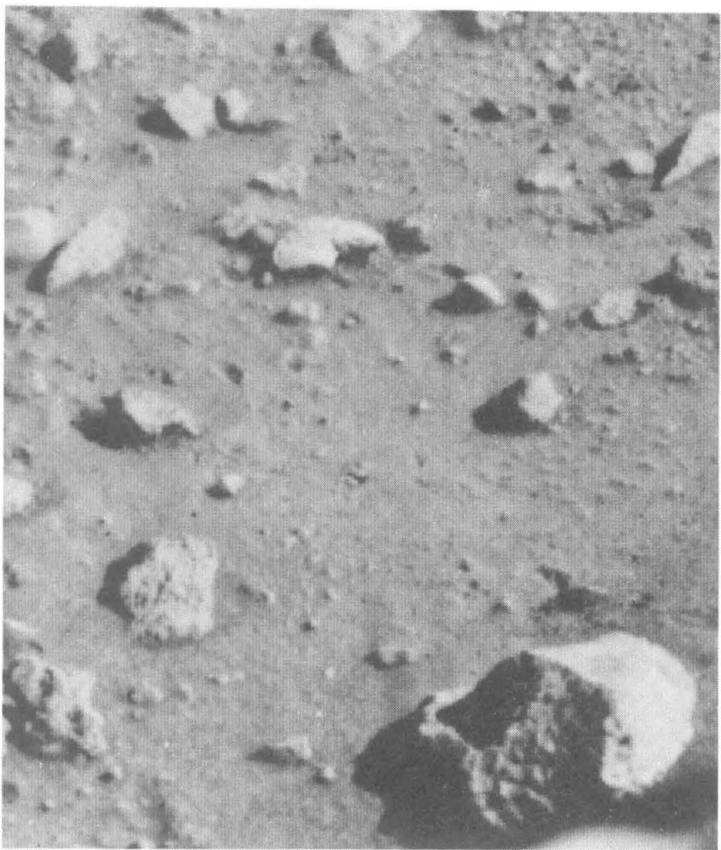

$\boldsymbol{A}$

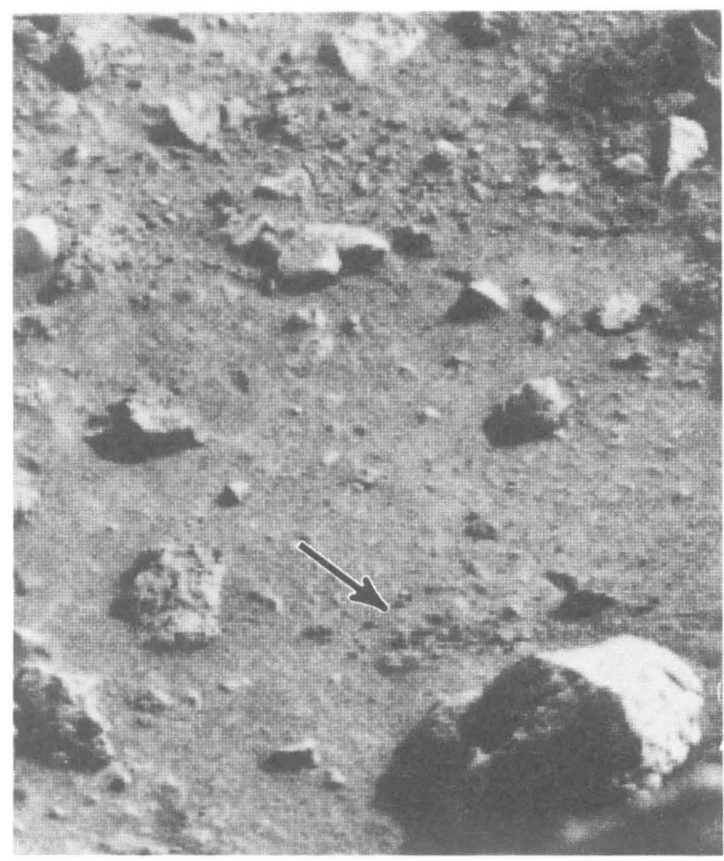

B samples were from drift material, but a few objects as much as $1.4 \mathrm{~cm}$ in diameter were purged from the one sample of blocky material from Rocky Flats. Of three purges onto drift material, two samples from blocky material had coarse material (table 28; items 15,16 ), but the sample near rock 5 (XRFS 10) appeared to have been from drift material between the rocks because no coarse material seemed to have been purged.

Purges of coarse material by Lander 2 were just as disappointing as those of Lander 1 . One object ( $2.7 \mathrm{~cm}$ wide) that could be called a rock because of its high reflectivity and the large pit in it, like rocks elsewhere, was purged at the nominal site early during the mission (fig. 72; table 29; item 1). This rock and others like it in the sample field would not have passed through the $1.25-\mathrm{cm}$ screen over the XRFS funnel. The subsequent seven purges at the nominal site resulted in a few additional fragments as much as $0.8 \mathrm{~cm}$ wide (table 29 ; items $2-6$ and 13). The experiment to construct a pile of rocks (table 29, items 7-12) by 14 purges of the coarse material resulted only depositing a few scattered fragments, one of which was $1.2 \mathrm{~cm}$ wide, on the surface.

At this point it is of interest to compare the results of the Viking purges with the composition of a lunar nominal sample. About 6-8 percent of lunar nominal material contained rock fragments with diameters ranging from 0.2 to $1.25 \mathrm{~cm}$. A full collector head will hold about $100 \mathrm{~cm}^{3}$ and about $25 \mathrm{~cm}^{3}$ of it will completely fill the XRFS chamber. Thus, roughly three or four $100-\mathrm{cm}^{3}$ samples of sifted lunar nominal would have filled the XRFS chamber with coarse material or would have produced a conical pile composed of coarse material $2-3 \mathrm{~cm}$ high and 4-5 cm wide. Even if the collector head were only onequarter full, one would have expected rather large amounts of coarse fraction from a lunar nominal material after acquiring samples or constructing a rock pile after 6-14 acquisition strokes.

\section{COMMINUTION}

The comminution experiments, like other independent data sources, show that the sampled materials are of three types. They are, in order of increasing strength: (1) drift material of Lander 1, (2) crusty to cloddy material of Lander 2, and (3) blocky material of Lander 1. No measurable increase in the motor currents of Lander 1 occurred

Figure 71.-Nominal purge site in stereoscopic field of view of Lander 1. $A$, Site prior to purges (frame 11A114/019). B, Site after eight purges. Note that additional material (arrow) is composed of a few small fragments (frame 11B210/151). Rock in lower right corner is $8 \mathrm{~cm}$ wide. 


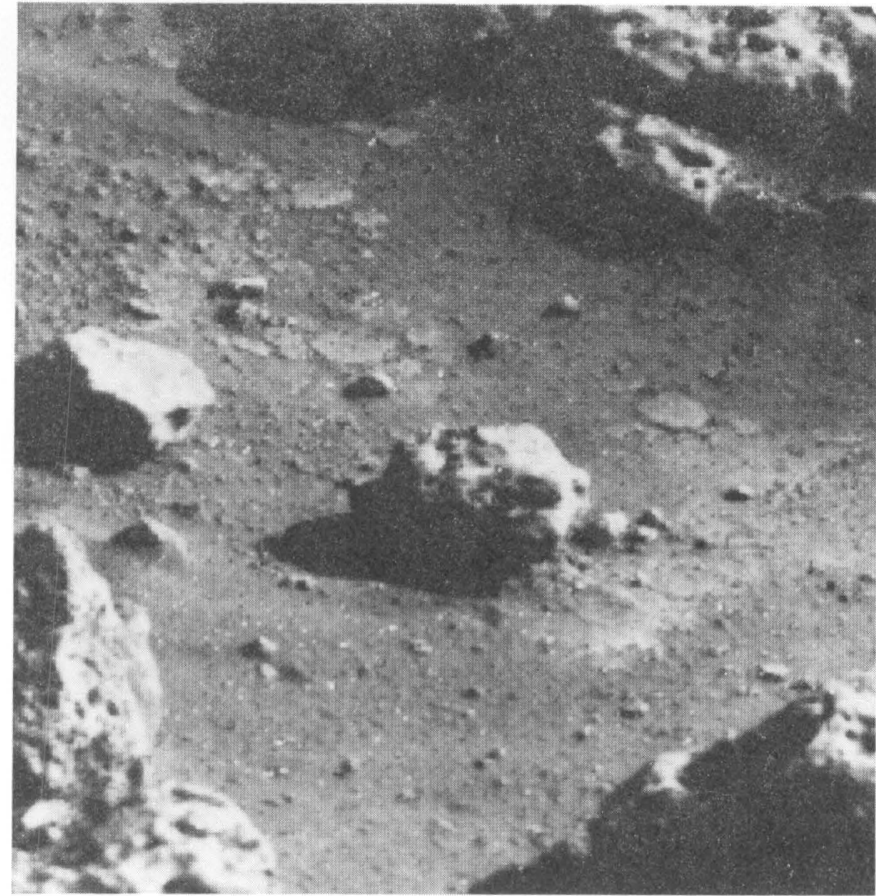

A

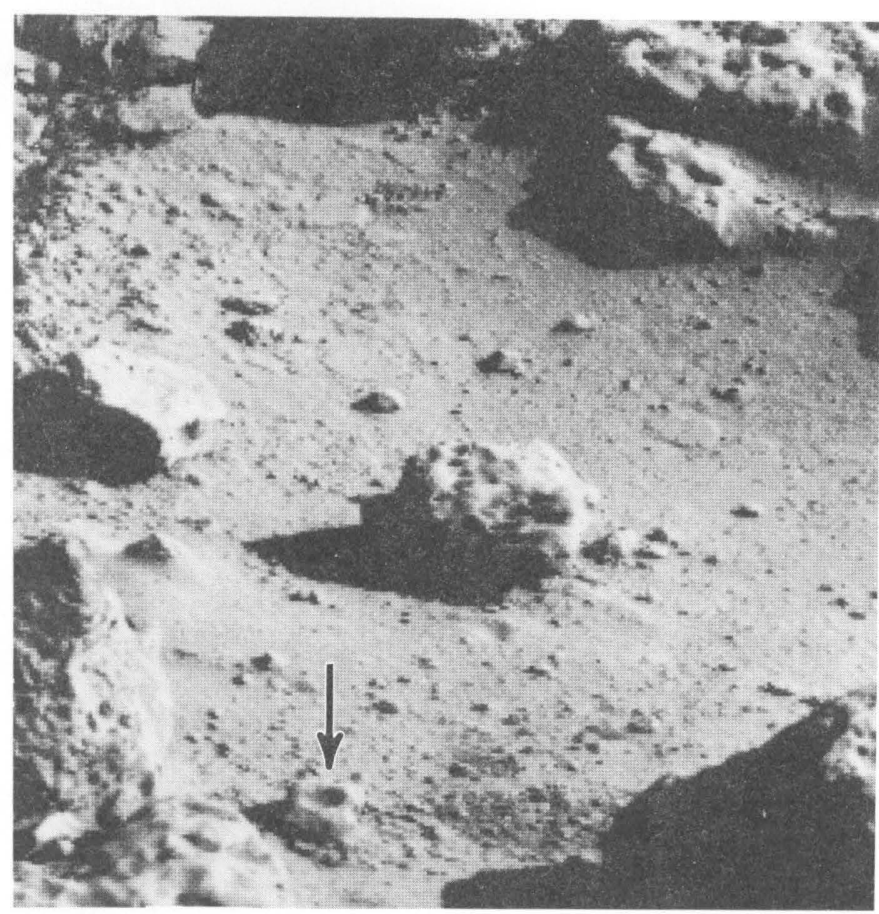

B while comminuting drift material; a modest increase in the motor currents of Lander 2 seems to have occurred while comminuting crusty to cloddy material. In contrast, surprisingly large increases in currents, 0.6-1.6 A, occurred while crushing blocky material. Terrestrial materials that will cause this range of motor currents are: (1) mixtures of powders, clods and rocks, (2) coarse (1-2 mm) grains of silicates, metal oxides, and dense rocks, or (3) rather strong clods or fragments of shale. The comminutor experiments are also consistent with the results of sampling the coarse fraction of surface materials and of purging of the coarse fraction.

The preliminary tests on Earth, in which $10-\mathrm{cm}^{3}$ samples of quartz and feldspar grains were poured into an operating comminutor, proved that increases in motor currents above the no-load current were a function of grain size as expected (fig. 73). Very coarse grains $(0.99-1.98 \mathrm{~mm})$ produced large increases in currents, ranging from about 0.9 to $1.5 \mathrm{~A}$, and fine-grains $(0.06-0.18 \mathrm{~mm})$ produced small increases ranging from about 0.01 to $0.05 \mathrm{~A}$. Because of the small size of the samples, the currents tended to decrease rapidly with comminution time. In later tests, larger samples weighing 30-40 g were ground in a flight-type comminutor in order to simulate more closely the experiments on Mars. These later tests verified the previous results and showed that coarse-grained material $(0.99-1.98 \mathrm{~mm})$ such as metal oxides, shale, serpentine, and some clods of indurated nontronite produced very large increases in motor currents that ranged from about 0.6 to $2.0 \mathrm{~A}$ (figs. 74 and $75 A$ ). Coarse fragments $(0.99-1.98 \mathrm{~mm})$ of porous materials such as diatomite did not increase motor currents whereas coarse $(1 \mathrm{~mm})$ silicon carbide, an unusually hard substance, sheared the comminutor drive-pin at a current increase of about 4.0 A. Excluding diatomite and silicon carbide, motor current increases for coarse materials, crushed to pass $0.99 \mathrm{~mm}$, generally ranged from 0.35 to $0.80 \mathrm{~A}$, and the current record for $40 \mathrm{~g}$ samples tended to maintain these values more or less uniformly for $50 \mathrm{~s}$ or more. Very weak clods and materials with uniform grain sizes less than about $0.2 \mathrm{~mm}$ produced small increases in motor currents, but these currents were so low that they probably would not be detected by the lander comminutors on Mars. Current increases from 0.1 to 0.16

FIGURE 72.-Nominal purge site in stereoscopic field of view of Lander 2 $(A)$ before first purge and $(B)$ after seven purges. Large object, about $2.7 \mathrm{~cm}$ wide (arrow), is best possibility for being a rock that was picked up and purged by surface sampler because of its high reflectivity and large pit on its surface (frames $(A) 22 \mathrm{~A} 007 / 001$ and $(B) 22 \mathrm{~F} 219 / 480$ ). 
TABLE 29.-Special purges of coarse samples by Lander 2

\begin{tabular}{|c|c|c|c|c|c|c|c|c|c|c|c|}
\hline \multirow[t]{2}{*}{ Item } & \multirow{2}{*}{\multicolumn{2}{|c|}{$\begin{array}{l}\text { Source } \\
\text { trench }\end{array}$}} & \multirow[t]{2}{*}{$\begin{array}{l}\text { Location } \\
\text { of trench }\end{array}$} & \multirow[t]{2}{*}{ Sol } & \multicolumn{3}{|c|}{$\begin{array}{l}\text { Surface-sampler positions } \\
\text { for purge }\end{array}$} & \multicolumn{3}{|c|}{$\begin{array}{c}\text { Delivery and sieving } \\
\text { procedures }\end{array}$} & \multirow[t]{2}{*}{ Results } \\
\hline & & & & & $\begin{array}{l}\text { Azimuth } \\
\text { (degrees) }\end{array}$ & $\begin{array}{l}\text { Extension } \\
\quad \text { (in.) }\end{array}$ & $\begin{array}{l}\text { Elevation } \\
\text { (degrees) }\end{array}$ & $\begin{array}{l}\text { Elevation } \\
\text { (degrees) }\end{array}$ & $\begin{array}{l}\text { Rate } \\
(\mathrm{Hz})\end{array}$ & $\begin{array}{l}\text { Duration } \\
(s)\end{array}$ & \\
\hline 1 & GCMS & 1 & Bonneville & 21 & 190.4 & 39.9 & 36.3 & -10.4 & 8.8 & 45 & $\begin{array}{l}\text { One purge, one rock fragment } 2.7 \mathrm{~cm} \\
\text { wide and a few smaller objects. }\end{array}$ \\
\hline $\begin{array}{l}2 \\
3\end{array}$ & $\begin{array}{l}\text { Biolc } \\
\text { XRFS }\end{array}$ & ogy 2 & $\begin{array}{l}\text { Beta } \\
\text { Bonneville }\end{array}$ & $\begin{array}{r}28 \\
29- \\
30\end{array}$ & $\begin{array}{l}190.4 \\
190.4\end{array}$ & $\begin{array}{l}39.9 \\
39.9\end{array}$ & $\begin{array}{l}36.3 \\
36.3\end{array}$ & $\begin{array}{r}-3.4 \\
8.5\end{array}$ & $\begin{array}{l}8.8 \\
8.8\end{array}$ & $\begin{array}{c}45 \\
90,90\end{array}$ & $\begin{array}{l}\text { One purge, no new material. } \\
\text { Two purges, small amount of new ma- } \\
\text { terial } 0.5 \mathrm{~cm} \text { and smaller. }\end{array}$ \\
\hline 4 & GCMS & 2 & Badger & 40 & 192.9 & 39.9 & 36.3 & -10.4 & 8.8 & 45,45 & One purge, no new material. \\
\hline 5 & Biolc & ogy 3 & Notch & 51 & 190.4 & 43.9 & 36.3 & -3.4 & 8.8 & 45 & $\begin{array}{l}\text { One purge, a few new objects } 0.2 \mathrm{~cm} \\
\text { and smaller. }\end{array}$ \\
\hline 6 & Phys & Prop 1 & Alpha & 57 & 192.3 & 39.9 & 36.3 & 1.0 & 8.8 & 60 & $\begin{array}{l}\text { One purge, a few new objects } 0.5 \mathrm{~cm} \\
\text { and smaller. }\end{array}$ \\
\hline 7 & XRFS & 4 & Notch, rock 7 & 131 & 111.5 & 79.9 & 19.9 & 8.5 & 8.8 & 90 & $\begin{array}{l}\text { One purge, start of rock pile, no } \\
\text { pictures. }\end{array}$ \\
\hline $\begin{array}{l}8 \\
9\end{array}$ & $\begin{array}{l}\text { Biolc } \\
\text { XRFS }\end{array}$ & $\log _{5} 4$ & $\begin{array}{l}\text { Beta site } \\
\text { Spalling Valley }\end{array}$ & $\begin{array}{l}145 \\
145\end{array}$ & $\begin{array}{l}111.5 \\
111.5\end{array}$ & $\begin{array}{l}79.9 \\
79.9\end{array}$ & $\begin{array}{l}19.9 \\
19.9\end{array}$ & $\begin{array}{r}-3.4 \\
-10.4\end{array}$ & $\begin{array}{l}8.8 \\
8.8\end{array}$ & $\begin{array}{c}45 \\
120,120 \\
120,120\end{array}$ & $\begin{array}{l}\text { One purge, no pictures } \\
\text { Four purges, a few fragments } 1.2 \mathrm{~cm} \\
\text { and smaller. }\end{array}$ \\
\hline 10 & XRFS & 6 & Spalling Valley & 161 & 111.5 & 79.9 & 19.9 & 8.5 & 8.8 & 90,90 & Two purges. \\
\hline 11 & XRFS & 7 & Spalling Valley & 172 & 111.5 & 79.9 & 19.9 & -10.4 & 8.8 & $\begin{array}{l}120,120 \\
120,120\end{array}$ & Four purges. \\
\hline 12 & XRFS & 8 & $\begin{array}{l}\text { Badger, } \\
\quad \text { rock } \# 3\end{array}$ & $\begin{array}{r}185- \\
186\end{array}$ & 111.5 & 79.9 & 19.9 & 8.5 & 8.8 & 90,90 & $\begin{array}{l}\text { Two purges, cumulative result is a } \\
\text { few scattered fragments } 1.2 \mathrm{~cm} \text { and } \\
\text { smaller after } 14 \text { purges. }\end{array}$ \\
\hline 13 & Phys & Prop 3 & $\begin{array}{l}\text { Physica } \\
\text { Planitia }\end{array}$ & 479 & 192.3 & 43.9 & 36.3 & -5.3 & 3.8 & 60 & $\begin{array}{l}\text { One purge, several new fragments } \\
\text { as large as } 0.8 \mathrm{~cm} \text {. }\end{array}$ \\
\hline
\end{tabular}


A were produced by medium sand $(0.12-0.50 \mathrm{~mm})$ and fine nontronite powder (figs. 74 and $75 B$ ). Current records for lunar nominal test material were very erratic because of the presence of fragments as much as $2 \mathrm{~mm}$ in diameter in a material mostly finer than $0.2 \mathrm{~mm}$ ( 75 percent finer), but sustained currents from 0.1 to $0.18 \mathrm{~A}$ were observed.

For several reasons, sample delivery and comminution procedures varied on Mars but in all cases comminution commenced after the sample was delivered through the $2 \mathrm{~mm}$ screen of the GCMS processor. Time intervals between delivery and comminution start ranged from $18 \mathrm{~s}$ (GCMS 1, Lander 1) to 15.74 sols (XRFS 13, Lander 2). These time intervals were partly dictated by surfacesampler operational procedures and the time available for

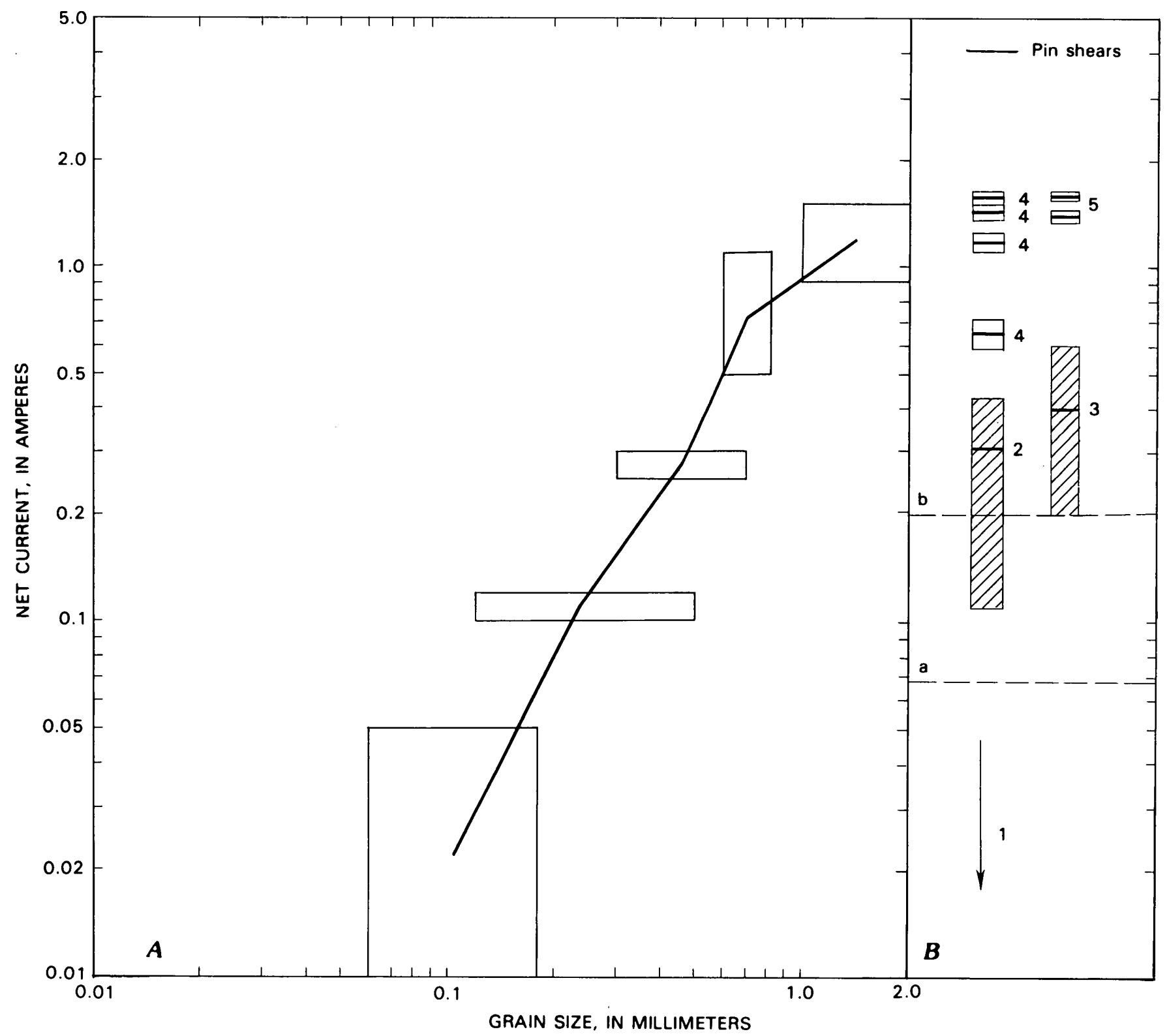

FIGURE 73.-Relation between grain size of comminuted materials and comminutor motor currents on Earth and on Mars. $A$, Terrestrial tests showing relation between grain size of well-sorted samples and net or increase in comminutor motor currents. Widths of boxes indicate range of grain size used in tests; heights of boxes indicate range of net currents; heavy solid line connects centers of boxes. $B$, Viking results: (a) standard error for SSCA no-load currents of Lander 1 , (b) standard error for SSCA no-load currents of Lander 2; (1) current increase from SSCA record for drift material is smaller than standard error as shown by downward pointing arrow, (2) SSCA current increase and standard error (bar) for crusty to cloddy material, (3) current increase from Format 5 record and record standard error from SSCA record (bar) for crusty to cloddy material, (4) four values of SSCA current increases above average no-load current and standard errors (bars) for blocky material, and (5) two values of current increases from Format 5 record and standard error from SSCA record (bar) for blocky material. The comminutor drive mechanism breaks when the net current exceeds 4.0 amperes. 
surface-sampler operations. The processor shuttle block was in the meter position, which allowed the comminuted material to pass into the GCMS ovens (Clark, L.V., and others, 1977), for all samples comminuted for organic chemical analyses. For the samples comminuted expressly for the Physical Properties Investigation (Physical Properties 8 and XRFS 13), the processor shuttle block was in the dump position to allow the comminuted material to pass into the dump cavity. Most of the samples passed through the screen of the inverted collector head by vibrating the upper lid at $8.8 \mathrm{~Hz}$. The rotation-position switch of the collector head of Lander 2 malfunctioned so the collector head was inverted by rotating it for 8-10 $\mathrm{s}$. This procedure caused additional vibrations of the collector head in the inverted position at the end of the rotation period because the rotation motor decoupled when the mechanism reached its mechanical limit. The same procedure for rotating the collector head was used for delivery of the last sample for comminution by Lander 1, and nine such rotation commands were accompanied by seven collector-head vibrations at $4.4 \mathrm{~Hz}$. The collector head was over the processor in the upright position and $5^{\circ}$ higher than the loading elevation for all but three of the no-load current readings: (1) the first of Lander 1 on Sol 8, (2) the second of Lander 1 on Sol 350, and (3) the no-load test for Lander 2 on Sol 502. We have no evidence that the procedural differences affected the results of the comminution experiments in any way.

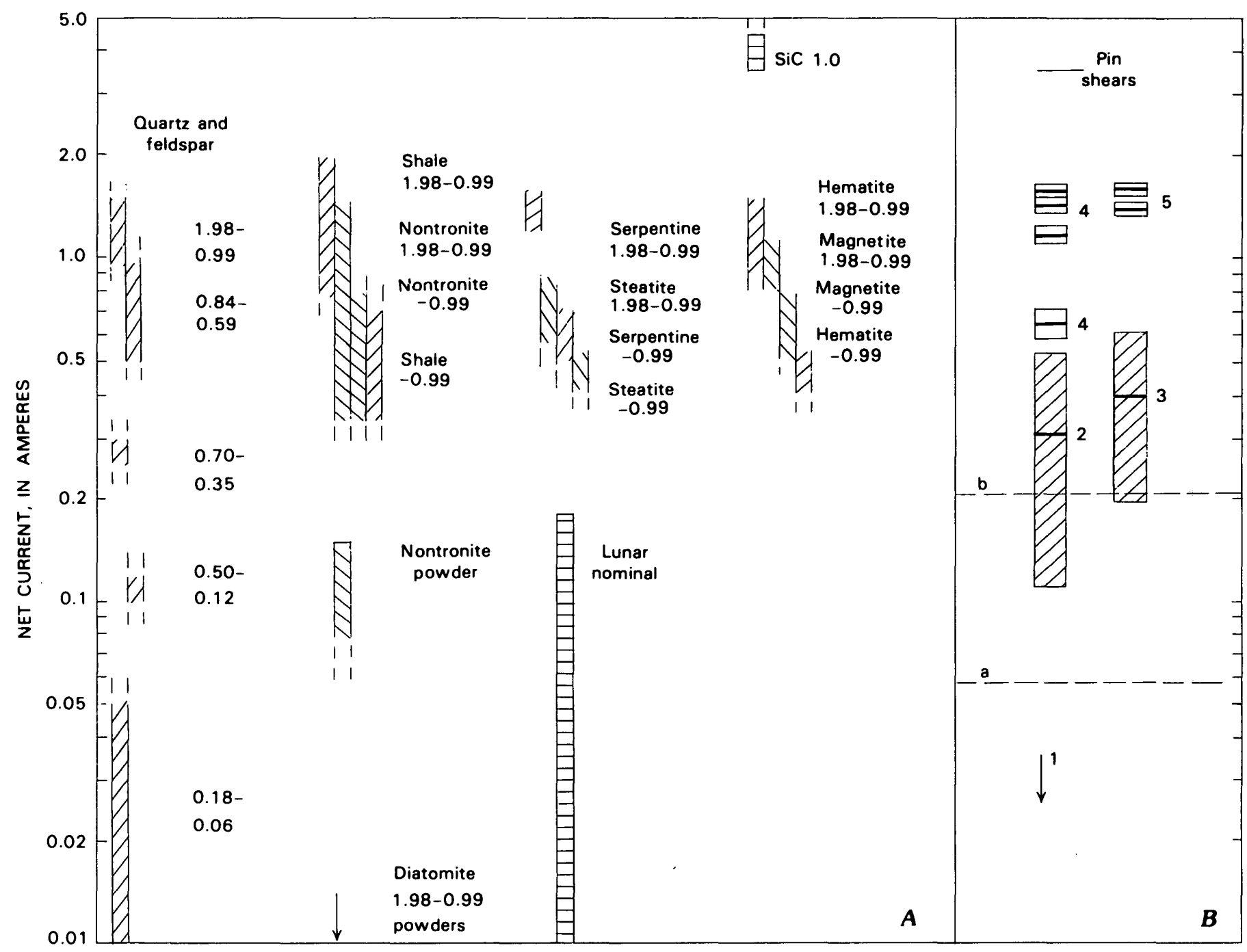

FIGURE 74.-Relation between comminuted fragmental materials of porous and dense materials and increases in comminutor motor currents on Earth and Mars. $A$, Terrestrial tests show that motor currents increase with a variety of well-sorted and poorly sorted materials, nontronite powder, and lunar nominal test material. Numbers indicate range of grain size of well-sorted materials in millimeters. Poorly sorted materials have a continuous range of grain sizes from 0.99 millimeters to smaller sizes [labeled (-) 0.99 in fig.]. $B$, Viking results indicated at right (see fig. 73 for explanation). The comminutor drive mechanism breaks when the net current exceeds 4.0 amperes. 
Although Viking motor-current data from the SSCA (surface-sampler control assembly) and from the lander electrical distribution system (Format 5) are consistent with each other, interpretation of them is restricted for several reasons (table 30 ). The most severe restriction is imposed by the comminutor of Lander 2 because the stirring wire loop below the auger somehow interfered with its operation. This interference produced large and variable no-load currents. For the SSCA currents measured at 2 and $15 \mathrm{~s}$ after motor start, average no-load currents of Lander 2 are $1.068 \pm 0.0211 \mathrm{~A}$. In contrast, average no-load currents for Lander 1 are $0.618 \pm 0.068 \mathrm{~A}$. The standard errors for the no-load currents for both landers exceed the resolution of current readings (0.028 A for SSCA currents and $0.039 \mathrm{~A}$ for Format 5 currents). The Format 5 no-load current record for Lander 1 was entirely consistent with those for flight-type comminutors on Earth (figs. $76 A$ and $B$ ). For Lander 1 and the Science Test Lander, the current increase $(0.194 \mathrm{~A}$ for $6.72 \mathrm{~s})$ due to the level detector can be seen in the motor-current records (figs. $76 A$ and $B$ ). This level detector current is not measured by the SSCA circuit for the comminutor motor. In contrast with Lander 1, Format 5 no-load current records for Lander 2 on Mars clearly show the variable record produced by the stirring wire-loop interference (fig. 76C). The same result was obtained during preflight checkouts on Earth. No-load currents for the Format 5 records are about $1.36 \mathrm{~A}$ for Lander 1 and $1.85 \mathrm{~A}$ for Lander 2.

There is no evidence of an increase in motor currents while drift material is being comminuted (table 30 ). The average current obtained with the SSCA circuit-about

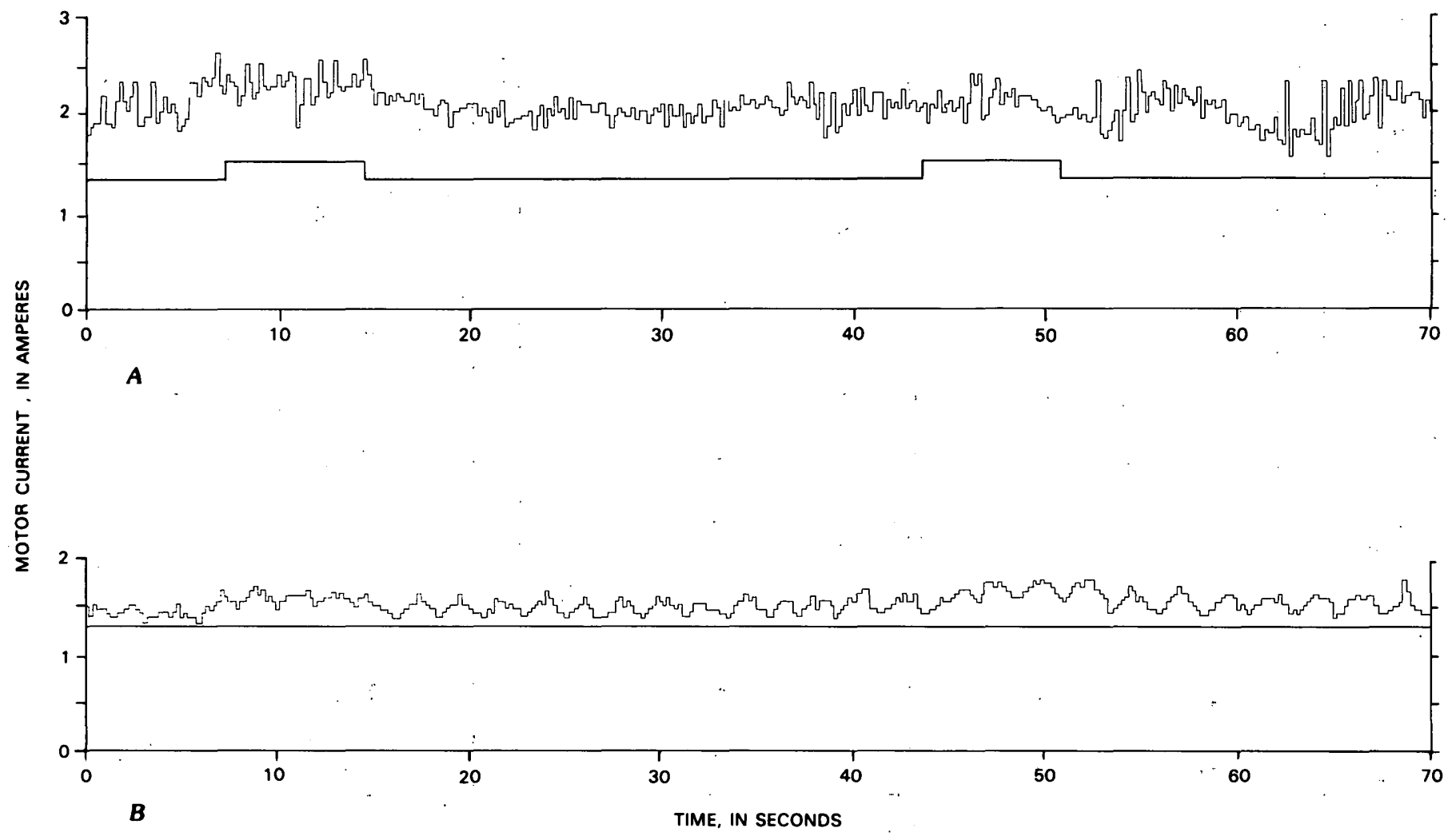

FIGURE 75.-Format 5 comminutor motor-current records for Science Test Lander show that current increased while crushing nontronite. $A$, Increase is about $0.62 \mathrm{~A}$ for $1 \mathrm{~mm}$ clods. No-load motor-current record shown by straight horizontal lines; increases near 10 and $45 \mathrm{~s}$ are due to level detector. $B$, Increase is about $0.12 \mathrm{~A}$ for powder. 
TABLE 30.-Comminution data for Viking Landers 1 and 2

[CH, Collector head; HF, high frequency; LF, low frequency]

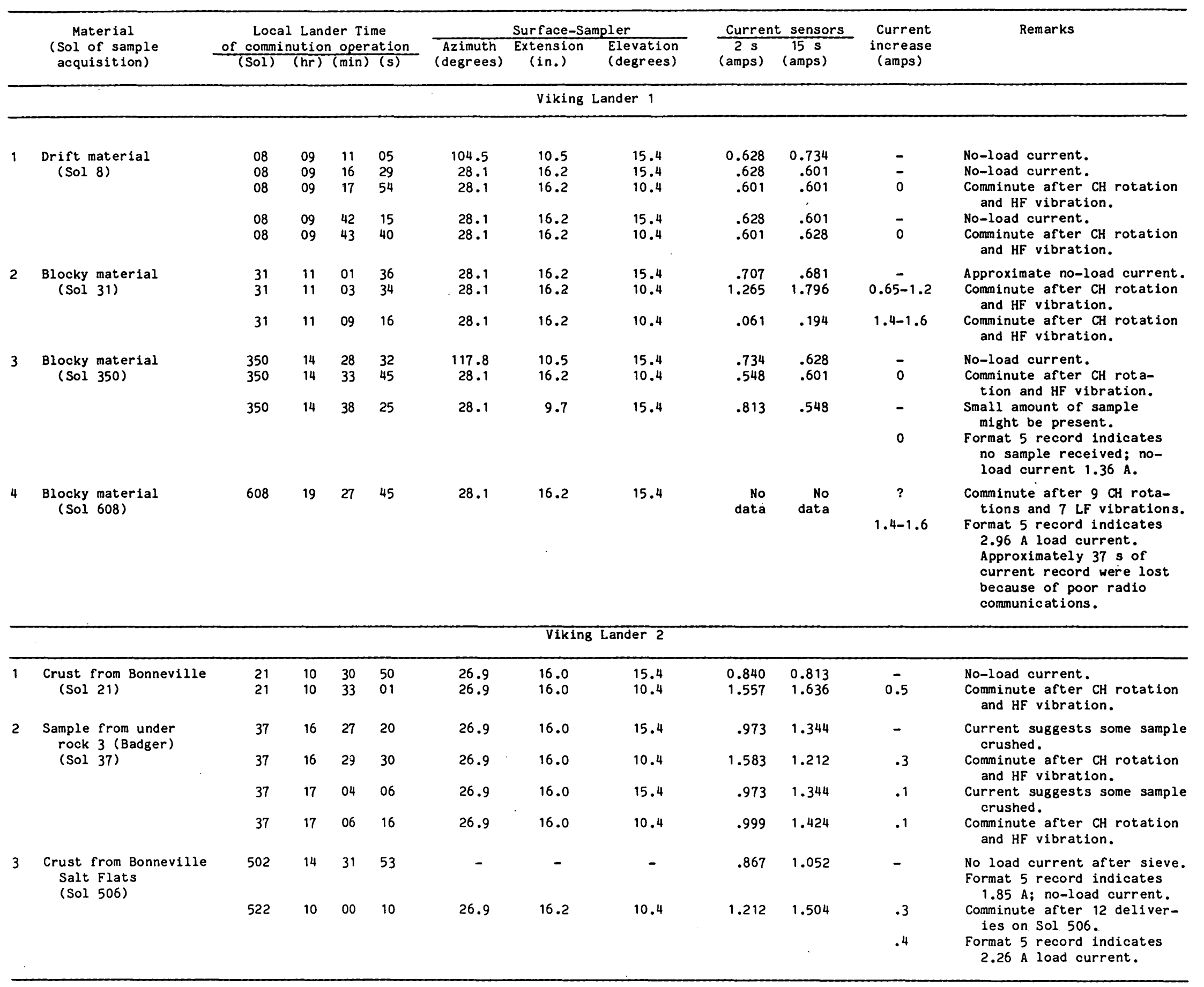

rift material

locky material

locky material

(Sol 350 )

locky material

28.

1.5

Viking Lander 1 

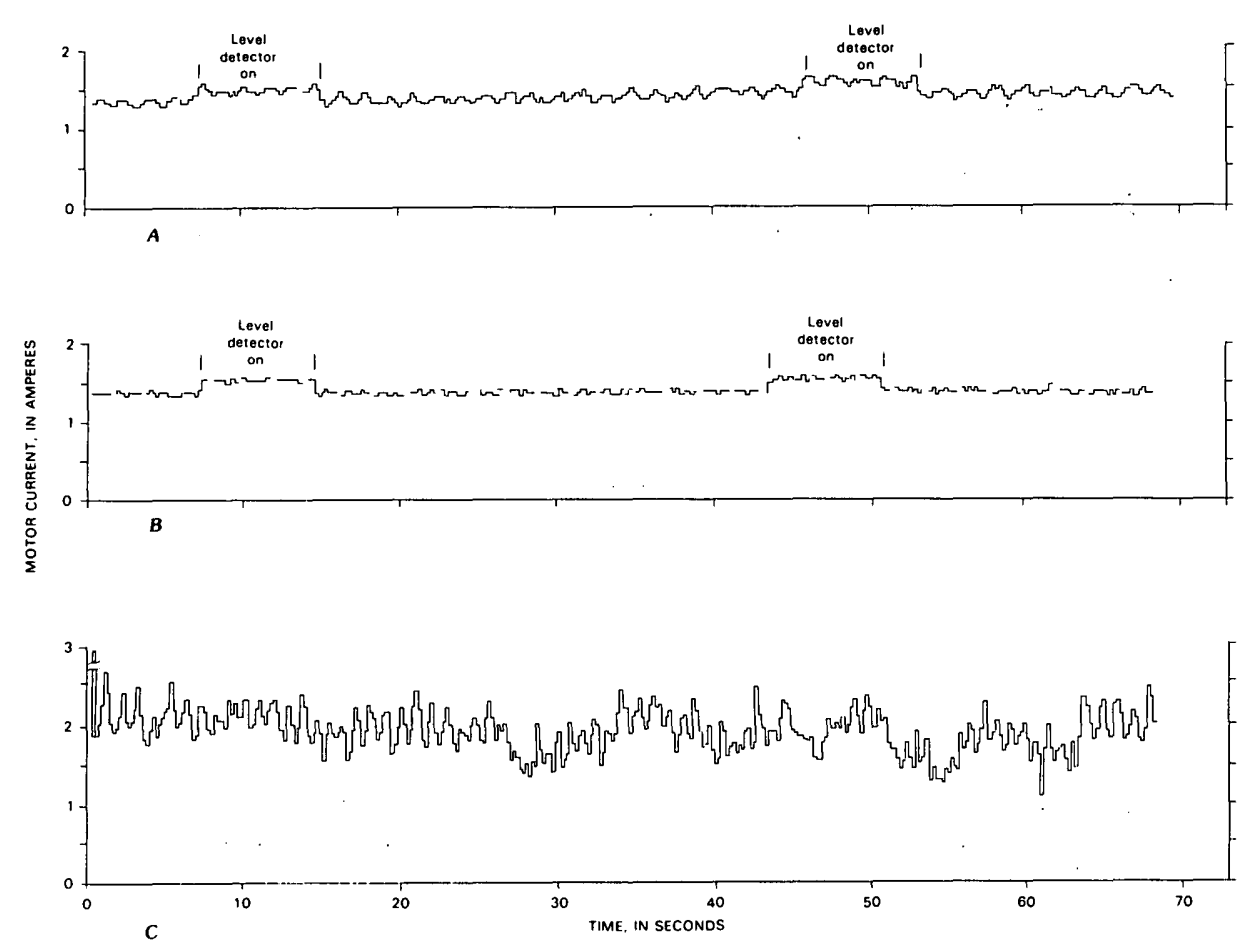

FIGURE 76.- Motor-current records for empty comminutors from Format 5. Note current increases corresponding to level detectors in $A$ and $B$. A, Science Test Lander (May 18, 1978). Transient amperage surge of 6.75 A occurred during motor start of Lander 1. B, Lander 1 (Sol 350 ). $C$, Lander 2 (Sol 502); erratic behavior of comminutor is due to interference by stirring mechanism.

$0.608 \mathrm{~A}-$ was entirely consistent with the average current for fine-grained material (fig. 73). Because of the rather large standard error for the SSCA currents and the terrestrial data, the comminutor currents indicate that the grain size of the drift material is probably less than about $0.2 \mathrm{~mm}$ (see solid line in fig. 73). In contrast with the drift material, strong coarse fragments must be present in the blocky material (table 30). On Sol 31, SSCA currents were initially $0.65 \mathrm{~A}$ above the no-load average; $15 \mathrm{~s}$ after motor start, currents rose to about $1.2 \mathrm{~A}$ above the no-load average, and continued comminution produced currents of 1.4 A and 1.6 A above the no-load. The Format 5 current record yields the same result (fig. 77A)the motor currents rose to about 1.4 to $1.6 \cdot \mathrm{A}$ above the no-load currents between 14 and $37 \mathrm{~s}$ after motor start. Unfortunately, a poor communications link resulted in the loss of $42 \mathrm{~s}$ of the Format 5 current record so that changes with time, if any, cannot be detected. The significance of the motor current increases for blocky material is that comparison with terrestrial data require that coarse indurated grains or clods be present.in blocky material. It is conceivable that the crushed fragments could have been clods of very cohesive nontronite or shale, but the con: sistent large currents favor the presence of coarse grains of silicates or metal oxides along with some finer grained material that passed through the comminutor ahead of the coarse material.
Interpretation of the Lander 2 currents is somewhat uncertain. The SSCA load currents average $1.391 \pm 0.226$ $\mathrm{A}$ and are about $0.3 \mathrm{~A}$ above the average no-load (table 30). The Format 5 current record of Lander. 2 becomes smoother while comminuting; and the average load current, which is about $2.26 \mathrm{~A}$ is larger than the average, although erratic, no-load current (1.85 A) (figs. $77 \mathrm{~B}$ and $76 \mathrm{C}$ ). Indeed, the no-load current rarely exceeds $2.26 \mathrm{~A}$. The difference in average currents $(0.4 \mathrm{~A})$ is more or less consistent with the SSCA current increase. We conclude, therefore, that fragments of clods or grains about $0.5 \mathrm{~mm}$ could have been comminuted at Lander 2 ; but rather large limits should be placed on this interpretation.

\section{ROCK PUSHING}

Initially, rocks were pushed by Lander 2 in order to collect samples from materials that were originally under the rocks. The biologists believed that biota and organic compounds; assumed to be sensitive to UV radiation, would be protected beneath the rocks from the photochemical reactions that would destroy them (Moore and others, 1978). However, evidence for biota was equivocal (Klein, 1977) and indigenous organic compounds were not detected (Biemann and others, 1977) in the sample materials; a sample from beneath rock 3 (Badger) ap- 


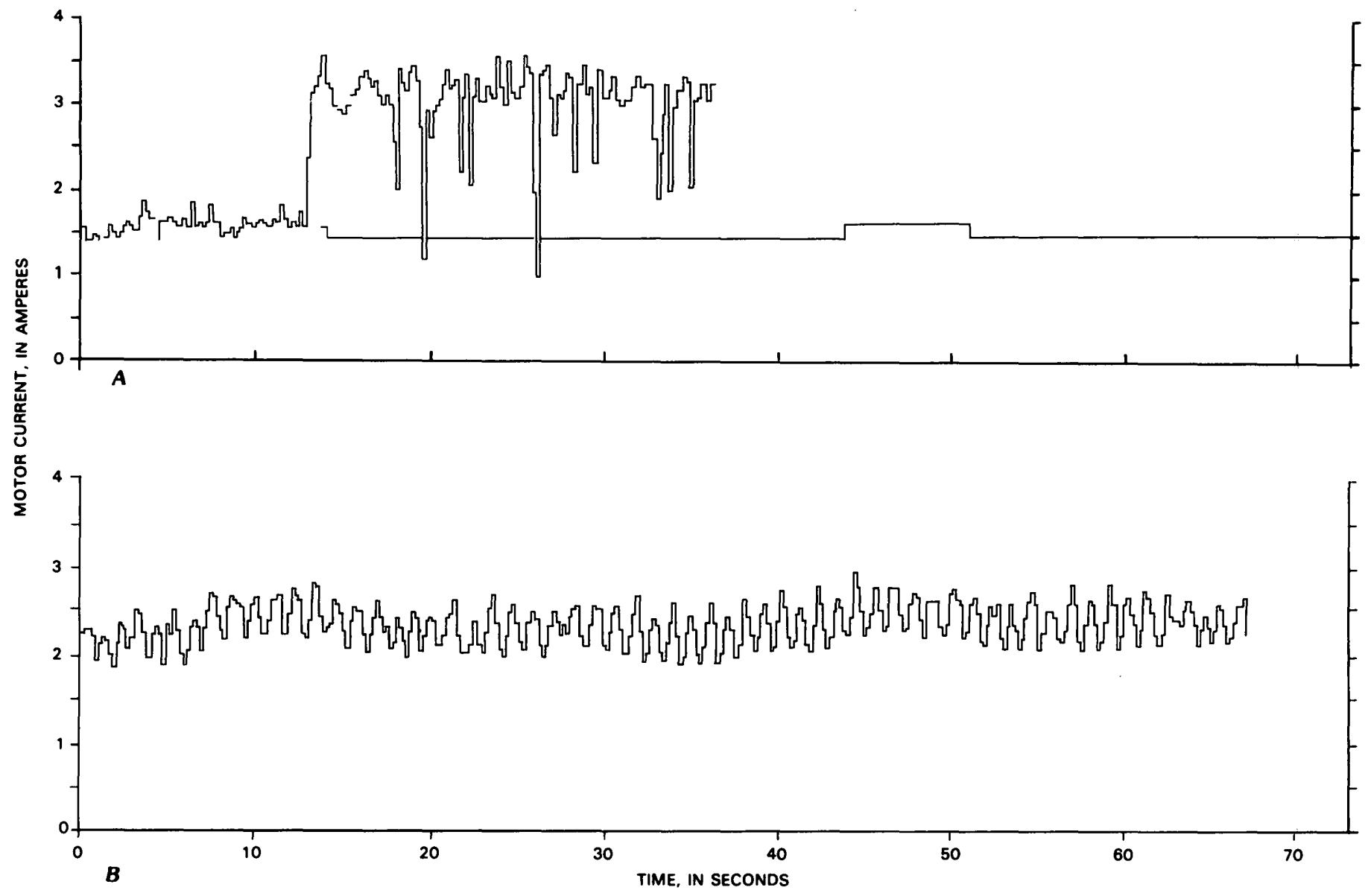

FIGURE 77.-Motor-current records for loaded comminutors from Format 5. $A$, Lander 1 (Sol 608) while crushing blocky material from Rocky Flats. Increase in current above no-load condition (see fig. 76) for Lander 1 is about 1.4-1.6 A. B, Lander 2 (Sol 522) while crushing crusty material from Bonneville Salt Flats. Amplitudes of current variations for Lander 2 appear to be smaller than those of no-load condition (see fig. 76); average current increase is about $0.4 \mathrm{~A}$. peared to give off more $\mathrm{H}_{2} \mathrm{O}$ in the GCMS than did a sample exposed to the atmosphere and sun (Biemann and others, 1977). Furthermore, the surfaces of the rocks were hard, because the surface sampler did not scratch, chip, or spall the rocks; a ledge of soil adhered to one side of the rock proving that a near-surface crust was present (Moore and others, 1978).

Later, during the Extended Mission, rocks were moved and scraped by the samplers of both landers in order to try to collect additional samples from materials originally beneath them and to see if the material would chip, spall, or scratch. For a variety of reasons, no additional samples from materials originally beneath the rocks were collected, but some information on the character of the rocks was obtained.

Four rocks were intentionally attacked by the surface sampler of Lander 1 and a number of others were inadvertently moved. During the excavation of Deep Hole 1 (Sols 202, 204, 218, 219; in drift material), two buried rocks, about $15 \mathrm{~cm}$ wide, were brought to the surface, and both of them appeared to be weakly pitted. The first, subsequently reburied, was subangular and the second appeared to be subrounded. Neither rock showed evidence of damage as a result of their excavation. Because the Lander 1 sampler glided over rock 4 (Grumpy, Sol 302), rock 4 rolled over in a direction away from the lander, slightly displacing two rocks nearby. Although none of the rocks, especially rock 4, appeared to have been damaged by the surface sampler, an area behind rock 4 was disrupted. Rock 5 (Bashful, Sols 369, 378, and 527) moved unpredictably during the episodes of pushing and sampling. Instead of pushing rock 5 , the collector head glided over the top of it and dug a trench behind it. While retracting the collector head, the backhoe pulled rock 5 toward the lander, causing it to rotate at the same time. Translations and rotations of rock 5 during sampling (XRFS 10) disturbed the nearby rocks to the left of it. Because of these complicated movements, combined with a variety 
of illumination conditions, pictures of rock 5 are difficult to interpret. The moving of the rock revealed that a fillet was present at its right edge, and material adhered to its upper surface. The marked change in the appearance of the tip of the rock nearest to the lander, shown in pictures taken on Sols 369,384 , and 528 , are the result of translation, clockwise rotation, and different illumination conditions. Thus, there is no evidence that rock 5 was damaged by the surface sampler during pulling and sampling activities. A "water-line" ledge of surface materials was revealed when a nearby rock was rotated a small amount. During the last push of rock 6 (Metate 2, Sol 376), the teeth of the lower jaw of the collector head contacted the rock, but the rock did not appear to have been chipped, spalled, or scratched. Because the rock rotated counterclockwise, the forces exerted on it only reached $100-125 \mathrm{~N}$. The backhoe was lowered on rock 2 (Sponge, Sol 441); the sampler was commanded downward an additional $2 \mathrm{~s}$ before retraction because it was known that pumice would chip when treated in the same way. Rock 2 simply toppled over toward the lander during retraction, and color pictures as well as black and white, show no evidence of chipping, spalling, or scratching in the area where the backhoe touched the rock. The surfaces of the exposed parts of the rocks seem to be strong and do not seem to have a weak weathered rind. Surface materials adhere to parts of some rocks that were originally buried.

Lander 2 was generally more successful at rock pushing than Lander 1-probably because the rocks pushed by Lander 2 were larger than those pushed by Lander 1 . Rock 1 (ICL, Sol 30), however, did not move and the extend-drive motor decoupled, so the surface sampler must have exerted about $200-225 \mathrm{~N}$ of force on it. The cross-sectional areas of the teeth on the lower jaw of the collector head, which contacted the rock, are about $1 \mathrm{~mm}^{2}$ so that loads of the order of $10^{8} \mathrm{~Pa}$ were achieved. Despite the large load, the rock surface did not chip or spall. During the pushes of rocks 3,6, and 7, which have been described previously (Moore and others, 1977, 1978), forces range from 50 to $100 \mathrm{~N}$. Again, these rocks did not appear to be chipped or spalled although some loose material may have been scraped from the bottom of rock 3 when the rock leaned on the sampler during the first attempted push (Moore and others, 1978). Forces of 200-225N were exerted on Rock 11 (Snow White, Sol 471) and it moved about $5 \mathrm{~cm}$. The upper jaw, rather than the teeth, probably touched rock 11 because surface materials were disturbed up to its face. Resulting stresses would have been small $\left(10^{5} \mathrm{~Pa}\right)$. The surface sampler decoupled while attempting to sample beneath rock 11 (XRFS 12 , Sol 483), so the force was about 200-225 $\mathrm{N}$ after $5 \mathrm{~cm}$ of extension. Yet, because no deformation of the surrounding surfaces occurred, the collector-head tip must have pushed on a buried rock without moving or break ing it. The backhoe of the surface sampler was lowered to touch rock 5 (Mole, Sol 559); it was lowered again for $2 \mathrm{~s}$ and then retracted. Although rock 5 did not move, spall, or chip, some fines from the collector head were deposited on it.

The pushing and rolling of the rocks proves that their surfaces are hard and strong and that there is no weak rind of weathered material on them although near-surface materials adhere to the buried sides of some of them. This conclusion should not be construed to mean that the rock surfaces are unweathered and pristine because the weathered surfaces of some terrestrial rocks in desert environments, such as basalt, can be as strong or even stronger than the rock itself.

\section{SUMMARY}

The surface sampler has provided important data on the physical properties of the surface materials at the landing sites. Drift material is virtually free of rock fragments and strong clods in the size range of the coarse fraction $(0.2-1.2 \mathrm{~cm})$ and significant amounts of strong mineral grains and rock fragments larger than about $0.2 \mathrm{~mm}$ are absent. Objects at the purge sites and in the sample field of Lander 2 that can be reasonably identified as rocks are typically $1.4-3.0 \mathrm{~cm}$ or larger; there is no evidence for rock fragments $0.2-1.2 \mathrm{~cm}$ wide or smaller. Objects in the coarse-fraction size range that were seen in the sample field were weak clods and crusts that broke up in the vibrating collector head. Small increases in the comminutor motor currents indicated that clods or silicate grains about $0.5 \mathrm{~mm}$ wide might be present. For blocky material at the Lander 1 site, the successful acquisition, delivery, and analysis of the coarse fraction combined with the large increases of motor currents during comminution showed that the coarse fraction was strong. Terrestrial comminution data suggest that significant amounts of strong, coarse (1-2 mm) fragments or grains were present in blocky material; dense silicates and metal oxides simulate the results most readily, but coarse, wellindurated aggregates such as chunks of shale cannot be excluded.

Rocks in the sample fields that were pushed, rolled, and scraped by the surface sampler did not break, chip, spall, or scratch. Though the rocks. do not have a weak, weathered rind, this does not mean that their surfaces are unweathered.." "Water-line" ledges of crust and soillike materials adhered to the sides and bottoms of some rocks.

\section{TEMPERATURES}

Temperatures were measured by the Viking Landers in the atmosphere about $1.6 \mathrm{~m}$ above the surface during the Meteorology Experiment (Hess and others, 1980), 
near the surface by the temperature sensors on footpad 2 (Shorthill and others, 1976b, 1976c), and beneath the surface by the temperature sensors on the collector heads (Moore and others, 1977, 1980). Additionally, temperatures were monitored by the science instruments and by other lander components for both scientific and engineering purposes. Surface temperatures measured from orbit (Kieffer and others, 1977) will be discussed later. Only the temperatures measured by the sensors on the collector heads throughout three diurnal cycles will be considered here because the scope and amount of other temperature data are much too large and much too complicated for this report.

\section{SURFACE-SAMPLER DIURNAL TEMPERATURES}

The surface-sampler collector heads were inserted into the martian surface materials, and temperatures were monitored throughout a diurnal cycle on Sols 343-344 (Lander 1), Sols 405-406 (Lander 2), and Sols 957-958 (Interim period of Lander 2). Temperatures were also monitored on Footpad 2 during these Sols as well as previous and subsequent Sols. As a standard practice, the collector head, in the upright position with the upper jaw open, penetrated the surface materials so as to surround the sensor with surface materials and to immerse it as deeply as possible. The solenoid was turned off during the temperature measurements because the lid was commanded to close after the final extension was achieved.

\section{LANDER 1}

Diurnal temperatures on Sols 343-344 were obtained when the collector head was buried in drift material (Physical Properties 6). These Sols corresponded to an areocentric longitude of the sun $\left(\mathrm{L}_{\mathrm{S}}\right)$ of 296 or about 40 sols after the winter solstice. The sample field of the southeast-facing lander should have been illuminated by the sun throughout the daylight hours. Pictures taken while the measurements were being made show that the collector head was well buried but the top of the lid was exposed to the atmosphere and sun (fig. 33). Stereometric measurements combined with the geometry of the collector head indicate that the sensor was $0.06-0.07 \mathrm{~m}$ below the surface. At the beginning of the measurements (at 10:30), 2.6 hours after sunrise, temperatures were about $197^{\circ} \mathrm{K}$; this temperature was about $5^{\circ} \mathrm{K}$ higher than the temperature at 10:30 on the following day (fig. 78). The difference was probably the result of the heating of the collector head when the head was exposed to the sun during maneuvers to reach the site. Test data indicate that heat from the solenoid that opens the jaw should not affect the temperature sensor significantly when the power is on for 8-10 s. A peak temperature of $215.4^{\circ} \mathrm{K}$ was attained on Sol 343 at $15: 13$, about 2.7 hours
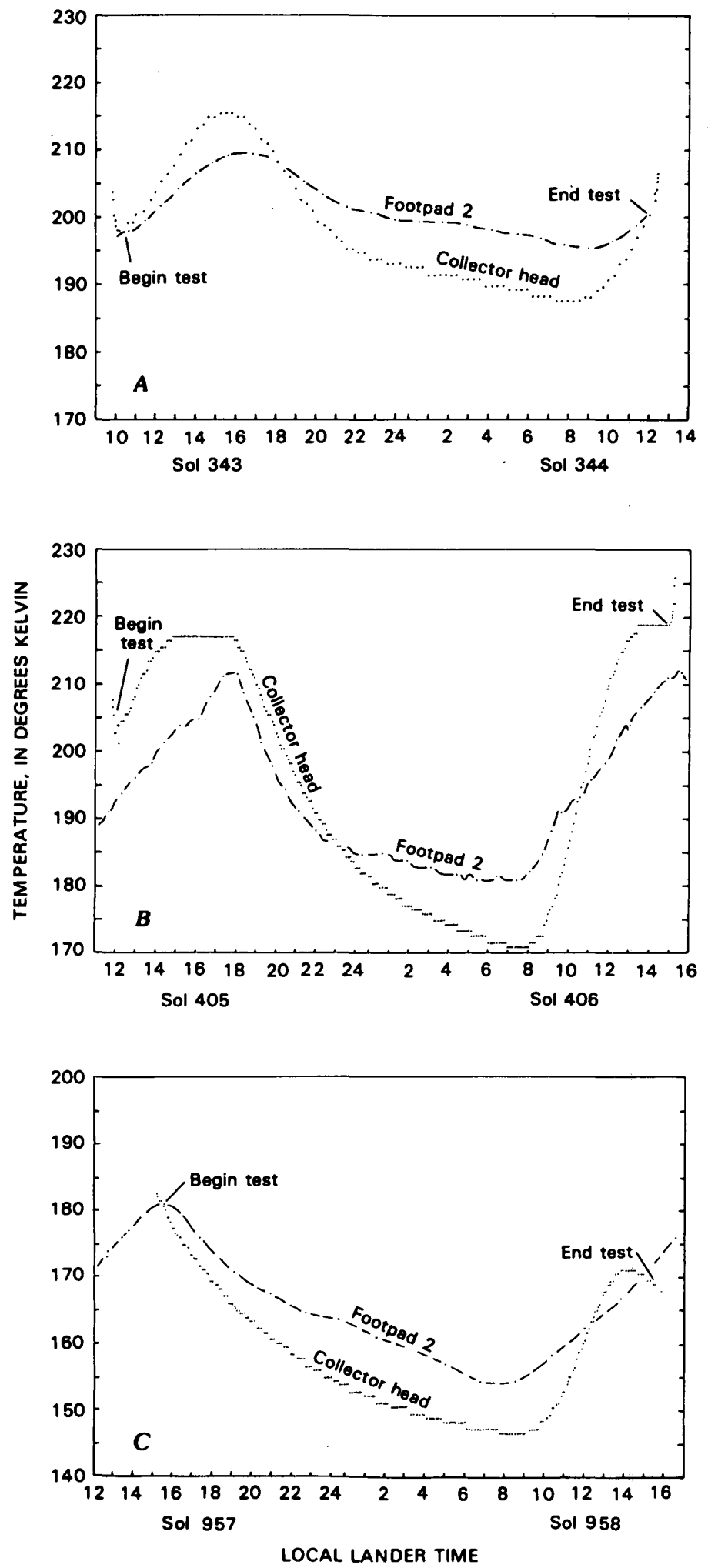

FIGURE 78.-Surface-sampler diurnal temperatures and footpad 2 temperatures. $A$, Lander 1 (Sols 343-344). Footpad 2 temperatures are chiefly from Sols 324-346. B, Lander 2 (Sols 405-406). Note that truncated peaks occur at different times of day. $C$, Interim period of Lander 2 (Sols 957-958). 


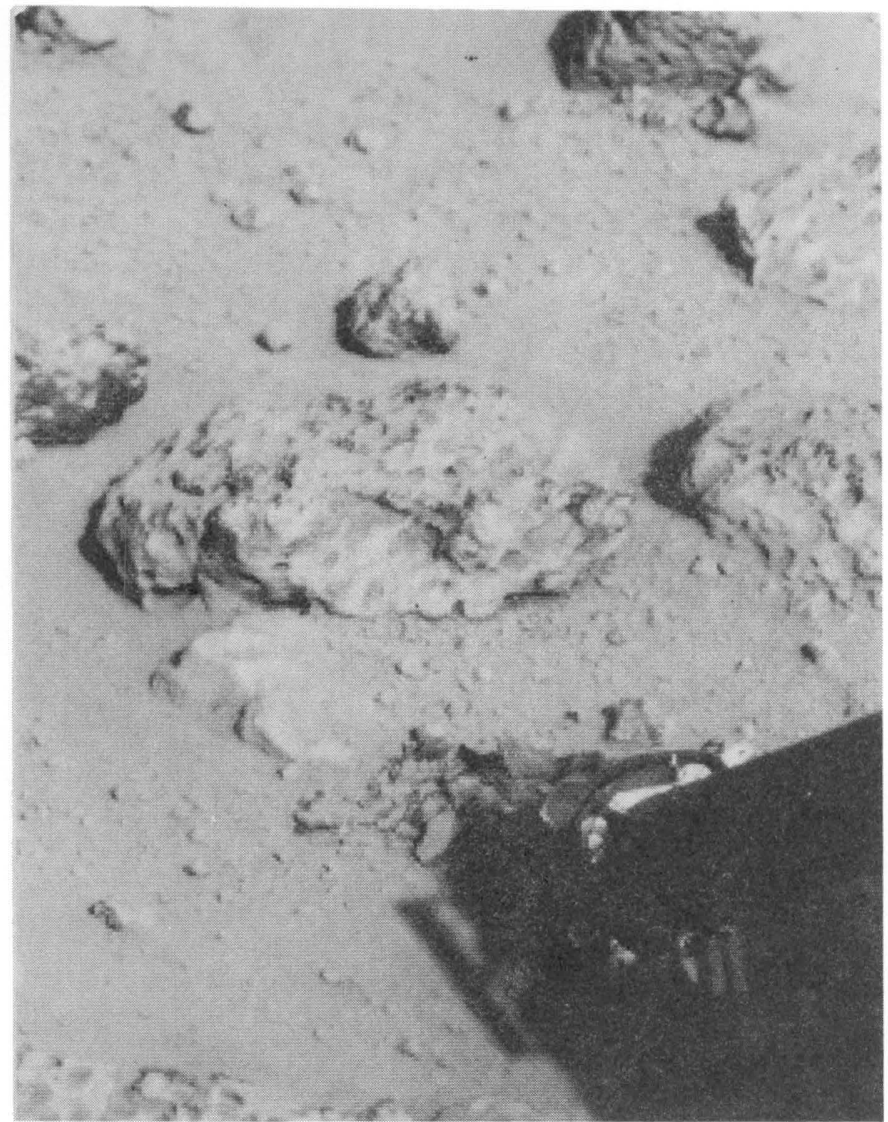

Figure 79.-Collector head of Lander 2 inserted in crusty to cloddy material during diurnal temperature measurements. Shadow of camera 2 is cast along azimuth of boom and collector head (frame 21E236/405). Large rock (rock 14) in center of picture, just beyond collector head, is about $22 \mathrm{~cm}$ wide.

before sunset. Minimum temperatures of about $187.6{ }^{\circ} \mathrm{K}$ were reached on Sol 344 between 07:23-07:58. The sensors on footpad 2, which were buried $1.2-2.4 \mathrm{~cm}$ in disturbed drift material did not measure temperatures as high or as low as those measured by the collector head. Because of their location, the sensors of footpad 2 are subjected to complicated insolation functions as well as heating by the spacecraft.

\section{LANDER 2}

Diurnal temperatures on Sols 405-406 were obtained when the collector head was buried in cloddy material (Physical Properties 2). These Sols corresponded to an $\mathrm{L}_{\mathrm{S}}$ of 355 or about 14 sols before the vernal equinox-during the late winter thaw. Photographic evidence shows that "snow" occurred as patches on the surface before and after the diurnal temperatures were recorded and that the aerial extent of the patches decreased with time. Depths of the final trench were estimated by monoscopic techniques to be about $0.04-0.06 \mathrm{~m}$ deep. All but $0.016 \mathrm{~m}$ of the inserted collector-head lid was immersed and the lid was held open by the materials in it. The base of the lower jaw was inclined $27^{\circ}$, the collector head tip was about 0.07 to $0.08 \mathrm{~m}$ below the surface (fig. 79). At this depth, the midpoint of the sensor was $0.05-0.06 \mathrm{~m}$ below the original surface. Insolation during the measurements was complicated because the lander faces in a northeasterly direction and sun elevation angles were generally low. The surface should have been illuminated from sunrise (07:30) until about 14:55 at which time the shadow of camera 2 passed across the buried collector head. The surface was again illuminated from about 15:04 until about 15:55 at which time it was in shadow. Subsequently, it was illuminated from about 16:12 until sunset (19:30).

An interesting result from these temperature measurements is the constant temperatures in the afternoon (fig. 78). Initial temperatures were $203^{\circ} \mathrm{K}$ near noon on Sol 405. By 14:49 they reached $217^{\circ} \mathrm{K}$ and remained there until 17:49. Temperatures decreased rapidly and then more slowly until 07:00-08:00 on Sol 406 when they were $170.9{ }^{\circ} \mathrm{K}$. Temperatures then increased rapidly to $218.7^{\circ} \mathrm{K}$ at $13: 28$ and remained there until 15:03 at which time the test ended. Again, the amplitude of the temperatures of footpad 2 (these sensors were not buried) was not as large as that of the collector head. The regular slope of the ascending curve of the footpad 2 temperature curve was due to the shadow of the spacecraft.

The constant temperatures may have been caused by a phase change in some component in the surface materials. Spacecraft shadowing is an unlikely reason to cause the constant temperatures because the constant temperatures occurred at different times on Sols 405 and 406. The sensors seemed to be working because temperatures changed rapidly near the beginning and end of the test (fig. 78). Changes in insolation related to cloud cover could produce constant temperatures (Ford and Anderson, 1967), but the temperature response was the reverse, in part, of what would be expected from relative optical densities obtained from changes in surface brightness before and during periods of constant temperature. On Sol 405, optical densities changed from 0.96 (11:00) to 0.45-0.58 (16:30 and 18:00) and, then, on Sol 406, they changed from $0.37(12: 20)$ to 0.56 (16.:30). Atmospheric temperatures on both Sol 405 and 406 were similar. Average peak ambient sensor temperatures were $213.6^{\circ} \mathrm{K}$ (near 16:17 on Sol 405) and $213.1^{\circ} \mathrm{K}$ (near 15:18 on Sol 406); reference sensor temperatures were $207.8^{\circ} \mathrm{K}$ and $211.5^{\circ} \mathrm{K}$, respectively (Conway Leovy, written commun., 1979). Reduced cloud cover would tend to increase the amplitude of the thermal wave, but this does not seem to have happened to any substantial degree. Wind directions and speeds varied during the tests, but not in a way that would account for the constant temperatures observed. During the times when the temperatures 
TABLE 31.-Maximum and mininum temperatures measured by the surface sampler in the surface materials, and predicted maximum and minimum surface temperatures

\begin{tabular}{|c|c|c|c|c|c|c|c|c|}
\hline & $\frac{\text { Local }}{\text { (Sol) }}$ & $\frac{\text { Lander }}{(\mathrm{hr})}$ & $\frac{\text { Time }}{(\mathrm{min})}$ & $\frac{\text { Measured }}{\substack{\text { Maximum } \\
\left({ }^{\circ} \mathrm{K}\right)}}$ & $\frac{\begin{array}{c}\text { temperature } \\
\text { Minimum } \\
\left({ }^{\mathrm{K}}\right)\end{array}}{}$ & $\frac{\text { Predicted }}{\substack{\text { Maximum } \\
\left({ }^{\left.O_{K}\right)}\right.}}$ & $\frac{\substack{\text { temperature } \\
\text { Minimum } \\
\left({ }^{\circ} \mathrm{K}\right)}}{{ }^{\circ}}$ & $\begin{array}{c}\text { Subsolar } \\
\text { longitude } \\
\mathrm{L}_{\mathrm{s}} \\
\text { (degrees) }\end{array}$ \\
\hline Lander & $\begin{array}{r}1--343 \\
344\end{array}$ & $\begin{array}{l}15 \\
07 \\
07\end{array}$ & $\begin{array}{l}13 \\
23- \\
58\end{array}$ & $\begin{array}{c}215.4 \\
-\end{array}$ & $18 \overline{7} .6$ & $\begin{array}{r}250 \\
-\end{array}$ & $18 \overline{0}$ & 296 \\
\hline Lander & $\begin{array}{r}406 \\
406\end{array}$ & $\begin{array}{l}14 \\
17 \\
13 \\
15 \\
06 \\
08\end{array}$ & $\begin{array}{l}49- \\
49 \\
28- \\
03 \\
59- \\
30\end{array}$ & $\begin{array}{l}217.0 \\
218.7 \\
-\end{array}$ & $\begin{array}{c}- \\
- \\
170.9\end{array}$ & $\begin{array}{r}240 \\
240 \\
-\end{array}$ & - & 355 \\
\hline Lander & $\begin{array}{r}2 \\
958\end{array}$ & $\begin{array}{l}13 \\
14 \\
07 \\
09\end{array}$ & $\begin{array}{l}45- \\
35 \\
40- \\
05\end{array}$ & $\begin{array}{c}170.5 \\
-\end{array}$ & $\begin{array}{c}- \\
146.5\end{array}$ & $\begin{array}{r}200 \\
-\end{array}$ & $\begin{array}{r}- \\
150\end{array}$ & 289 \\
\hline
\end{tabular}

were constant on Sol 405, winds were from the northwest at speeds of about 3-4 m/s and, on Sol 406, they were from the west at speeds of about $14 \mathrm{~m} / \mathrm{s}$ (Conway Leovy, written commun., 1979). Thus, the buried collector-head sensor may have been in the turbulent wake of Lander 2 on Sol 405 but not on Sol 406. Finally, the photographic evidence for the steady decrease in the sizes of the "snow" patches at the surface lends strong support for the notion that phase changes were occurring at the surface.

\section{INTERIM PERIOD}

In general, the surface-sampler activities of Lander 2 during the interim period are excluded from this report, but diurnal temperatures measured with the collector head and footpad sensors are reported here because they were obtained during the martian winter $\left(\mathrm{L}_{\mathrm{s}}=289\right)$ on Sols 957 and 958 and about 28 sols after the winter solstice. Casual inspection of the one complete picture of the final trench, which was along the same azimuth as, but nearer to the lander than the Physical Properties 6 trench, suggests that the sensor was immersed on Sols 597-958 to about the same depth as it was on Sols 405-406. Lower elevation angles of the sun attendant with the season must have created a complicated insolation function that included longer periods of shadows on Sols 957-958 than those on Sols 405-406. Ground cover of "snow" was more extensive on Sols 957-958 than on Sols 405-406; some "snow" was present on the surface adjacent to the immersed collector head and larger patches were illuminated by the sun beyond the shadows of the lander.
Temperatures at the time of insertion of the collector head at $15: 20$ on Sol 957 were about $181.5^{\circ} \mathrm{K}$. This time coincided with the time expected for the peak of the thermal wave. In the early morning on Sol 958 from 07:40-09:05, temperatures were $146.5^{\circ} \mathrm{K}$ and the peak of the wave $\left(170.5^{\circ} \mathrm{K}\right)$ occurred at $13.45-14: 35$ on Sol 958 . Here, footpad 2 temperatures exceeded those of the collector head at about 15:00 on Sol 958. Air temperatures peaked at $181^{\circ} \mathrm{K}$ on Sol 957 and at $168-174^{\circ} \mathrm{K}$ on Sol 958.

\section{DISCUSSION}

A detailed analysis of the temperatures of the collector head is beyond the scope of this report, but some comments are worth making. In general, the diurnaltemperature curves of the collector head resembled terrestrial and other martian diurnal-temperature curves. Temperatures were highest 2-4 h after noon and lowest in the early morning about 08:00. Peak temperatures were lower than predicted surface temperatures (table 31 ) but lows were reasonably close to predicted surface temperatures (Kieffer, 1976). A smaller range in diurnal temperatures would be expected for a buried probe, but clouded skies could also reduce the range.

The lowest collector-head temperatures on Sol 344 of Lander 1 and Sol 406 of Lander 2 were larger than the $\mathrm{CO}_{2}$ and $\mathrm{CO}_{2}-\mathrm{H}_{2} \mathrm{O}$-clathrate sublimation temperatures (152 ${ }^{\circ} \mathrm{K}$ and $158{ }^{\circ} \mathrm{K}$, respectively) for atmospheric pressures of $800-1,000 \mathrm{~Pa}$ (8-10 mbars) (Anonymous, 1957; Miller, 1973). Temperatures of the Lander 2 collector head during the interim period exceeded $152^{\circ} \mathrm{K}$ 
TABLE 32.-Conical piles of Viking Landers 1 and 2

[For locations in sample fields: see pl. $1 B$ and fig. $29 B$ for Lander 1 ; see pl. $2 B$ and fig. $30 B$ for Lander 2]

\begin{tabular}{|c|c|c|c|c|c|c|c|c|c|c|}
\hline \multirow{2}{*}{$\begin{array}{l}\text { Conical } \\
\text { Pile } \\
\text { No. }\end{array}$} & \multirow{2}{*}{$\begin{array}{l}\text { Source } \\
\text { of } \\
\text { material }\end{array}$} & \multicolumn{4}{|c|}{ Local Lander Time } & \multicolumn{3}{|c|}{ Surface-sampler positions } & \multirow{2}{*}{$\begin{array}{l}\text { Photographs } \\
\text { (Frame/Sol) }\end{array}$} & \multirow[t]{2}{*}{ Renarks } \\
\hline & & (Sol) & $(\mathrm{hr})$ & $(\min )$ & $(\mathrm{s})$ & $\begin{array}{l}\text { Azimuth } \\
\text { (degrees) }\end{array}$ & $\begin{array}{l}\text { Extension } \\
\text { (in.) }\end{array}$ & $\begin{array}{l}\text { Elevation } \\
\text { (degrees) }\end{array}$ & & \\
\hline \multicolumn{11}{|c|}{ Viking Lander 1} \\
\hline 1 & $\begin{array}{l}\text { Drift material } \\
\text { Physical Properties } 3\end{array}$ & 296 & 09 & 40 & 17 & 104.5 & 110.7 & 16.1 & $\begin{array}{l}11 \mathrm{D} 092 / 296 \\
12 \mathrm{~J} 008 / 921\end{array}$ & $\begin{array}{l}\text { Height of pile is } 3.6 \mathrm{~cm} \text {; half-width is } 3.5 \mathrm{~cm} \text {; } \\
\text { in plane of surface-sampler azimuth axis; } \\
\text { slope angle is } 45^{\circ} \text {. If material has been re- } \\
\text { moved by the wind, the amount is small. }\end{array}$ \\
\hline 2 & $\begin{array}{l}\text { Drift material } \\
\text { Physical Properties } 4\end{array}$ & $\begin{array}{l}324 \\
324\end{array}$ & $\begin{array}{l}11 \\
12\end{array}$ & $\begin{array}{l}02 \\
24\end{array}$ & $\begin{array}{l}11 \\
11\end{array}$ & 177.1 & 102.0 & 16.8 & $\begin{array}{l}110214 / 324 \\
11 \mathrm{~J} 013 / 921\end{array}$ & $\begin{array}{l}\text { Height of pile ranges from } 1.9 \text { to } 2.6 \mathrm{~cm} \text {; slope } \\
\text { angle is about } 45^{\circ} \text {. If any material has been } \\
\text { removed by the wind, the amount is small. }\end{array}$ \\
\hline 3 & $\begin{array}{l}\text { Drift material } \\
\text { Physical Properties } 5\end{array}$ & $\begin{array}{l}324 \\
324\end{array}$ & $\begin{array}{l}11 \\
12\end{array}$ & $\begin{array}{l}32 \\
54\end{array}$ & $\begin{array}{l}19 \\
19\end{array}$ & 168.9 & 96.7 & 21.8 & $\begin{array}{l}11 \mathrm{D} 214 / 324 \\
11 \mathrm{~J} 013 / 921\end{array}$ & $\begin{array}{l}\text { Height of pile is } 3.1-3.7 \mathrm{~cm} \text {; half }- \text { width is } \\
3.5-3.9 \mathrm{~cm} \text {; slope angle is about } 42^{\circ} \text {. If } \\
\text { any material has been removed by the wind, } \\
\text { the amount is small. }\end{array}$ \\
\hline 4 & $\begin{array}{l}\text { Drift material } \\
\text { Physical Properties } 6\end{array}$ & 344 & 12 & 26 & 30 & 144.2 & 77.0 & 25.6 & $\begin{array}{l}11 E 054 / 345 \\
11 \mathrm{~J} 007 / 921 \\
11 \mathrm{~J} 130 / 1765\end{array}$ & $\begin{array}{l}\text { No stereoscopic coverage; height of pile is } \\
\text { about } 4-5 \mathrm{~cm} \text {, width } 9-10 \mathrm{~cm} \text {; material has } \\
\text { been renoved by the wind between Sols } 921 \\
\text { and } 1765 \text {. }\end{array}$ \\
\hline 5 & $\begin{array}{l}\text { Blocky material } \\
\text { Physical Properties } 11\end{array}$ & 639 & 14 & 08 & 59 & 172.1 & 96.7 & 21.8 & $\begin{array}{l}111070 / 690 \\
11 \mathrm{~J} 013 / 921\end{array}$ & $\begin{array}{l}\text { First pictures obtained during Lander Continu- } \\
\text { ation Automatic Mission. Slope of pile is } \\
\text { shadowed when sun ang le is } 38.6^{\circ} \text {. If any } \\
\text { material has been removed by the wind, the } \\
\text { amount is small. }\end{array}$ \\
\hline \multicolumn{11}{|c|}{ Viking Lander 2} \\
\hline 1 & $\begin{array}{l}\text { Crusty to cloddy } \\
\text { material } \\
\text { Physical Properties } 2\end{array}$ & 406 & 15 & 08 & 15 & 133.6 & 82.8 & 17.4 & $\begin{array}{l}21 E 253 / 406 \\
211136 / 1193\end{array}$ & $\begin{array}{l}\text { Height of pile ranges from } 3.5 \text { to } 5.0 \mathrm{~cm} \text {; half- } \\
\text { width is } 2.7-5.2 \mathrm{~cm} \text {; slope angle is } 48.0 \pm \\
4.5^{\circ} \text {. If any material has been renoved by } \\
\text { the wind, the amount is small. }\end{array}$ \\
\hline 2 & $\begin{array}{l}\text { Crusty to cloddy } \\
\text { material } \\
\text { Physical Properties } 5\end{array}$ & $\begin{array}{l}595 \\
595\end{array}$ & $\begin{array}{l}08 \\
09\end{array}$ & $\begin{array}{l}28 \\
28\end{array}$ & $\begin{array}{l}49 \\
57\end{array}$ & 234.6 & 74.9 & 28.1 & $\begin{array}{l}22 \mathrm{H} 028 / 595 \\
22 \mathrm{I} 129 / 1177\end{array}$ & $\begin{array}{l}\text { Monoscopic coverage and illumination conditions } \\
\text { do not allow interpretation of pile morpholo- } \\
\text { gy. If any material has been removed by the } \\
\text { wind, the amount is small. }\end{array}$ \\
\hline 3 & $\begin{array}{l}\text { Crusty to cloddy } \\
\text { material } \\
\text { Physical Properties } 6\end{array}$ & 595 & 10 & 57 & 14 & 126.0 & 94.9 & 16.8 & $\begin{array}{l}21 \mathrm{H} 032 / 595 \\
211136 / 1193\end{array}$ & $\begin{array}{l}\text { Height of pile is } 1.4 \pm 0.2 \mathrm{~cm} \text {; slope angle is } \\
43-45^{\circ} \text {. If any material has been removed by } \\
\text { the wind, the amount is small. }\end{array}$ \\
\hline
\end{tabular}

for about $15 \mathrm{~h}$ and $158^{\circ} \mathrm{K}$ for about $11 \mathrm{~h}$ in the late morning and afternoon, but in the early morning temperatures were as low as $146^{\circ} \mathrm{K}$. Air temperatures never dropped below $152^{\circ} \mathrm{K}$. The "snow" cannot be entirely composed of solid $\mathrm{CO}_{2}$ and $\mathrm{CO}_{2}-\mathrm{H}_{2} \mathrm{O}$-clathrate because the temperatures of Lander 2 are too high in the late wintertime and generally too high in the early winter.

If the constant temperatures measured by the Lander 2 sensors were due to a phase change of a component in the surface materials, the minimum amount of heat required would not be large. The Sol 405 peak had been lowered by about $2^{\circ}$ or $3^{\circ} \mathrm{K}$. If the specific heat of the material is $0.14 \mathrm{cal} \mathrm{g}^{-1}{ }^{\circ} \mathrm{K}^{-1}$ (Kieffer, 1976), then the heat involved would be about $0.28-0.42 \mathrm{cal}^{-1}$. This amount of heat is comparable to latent heats of interfacial water on clay (Anderson and Tice, 1971), but the latent heats of interfacial water on clay were observed at temperatures much greater than $218.5^{\circ} \mathrm{K}$.

It seems possible that small amounts of eutectic solutions could produce the constant temperatures. Two eutectic solutions have been considered because the temperatures of the eutectics were reasonably close to the constant temperatures of Lander 2 on Sols 405-406 and there was some evidence that the substances could have been present in the surface materials: (1) $\mathrm{CaCl}_{2}-$
$\mathrm{MgCl}_{2}-\mathrm{H}_{2} \mathrm{O}$ brine has an eutectic at $218^{\circ} \mathrm{K}$ (Linke, 1958), and (2) $\mathrm{H}_{2} \mathrm{O}-\mathrm{H}_{2} \mathrm{O}_{2}$ has an eutectic at $217^{\circ} \mathrm{K}$ (Schumb and others, 1955). If $\mathrm{H}_{2} \mathrm{O}-\mathrm{H}_{2} \mathrm{O}_{2}$ is responsible for the constant temperatures observed on Mars, the postulate that $\mathrm{H}_{2} \mathrm{O}$. $\mathrm{H}_{2} \mathrm{O}_{2}$ in the regolith will naturally evolve to the eutectic (Nadeau, 1977) may be correct.

An additional explanation for the constant temperatures is evaporative cooling. Martian late winter is a season of strong winds and the sizes of the snow patches decrease. with time. For water ice, the equilibrium vapor pressure is about $2.0 \mathrm{~Pa}$ (500 precipitable microns) at $218{ }^{\circ} \mathrm{K}$.

\section{VISUAL OBSERVATIONS OF CHANGES}

Beginning with the arrival of the Viking Landers, the surfaces in the vicinities of the spacecraft were altered by the spacecraft so that they were no longer in equilibrium with the natural environment. The mere presence of the landers themselves altered the equilibrium configuration. Because of the disturbance of equilibrium conditions, changes might occur that could be recognized by observing the spacecraft, the altered surfaces of the sample fields, and natural surfaces over a period of time. The presence of the spacecraft and the effects of surfacesampler activities sometimes make separation of natural 


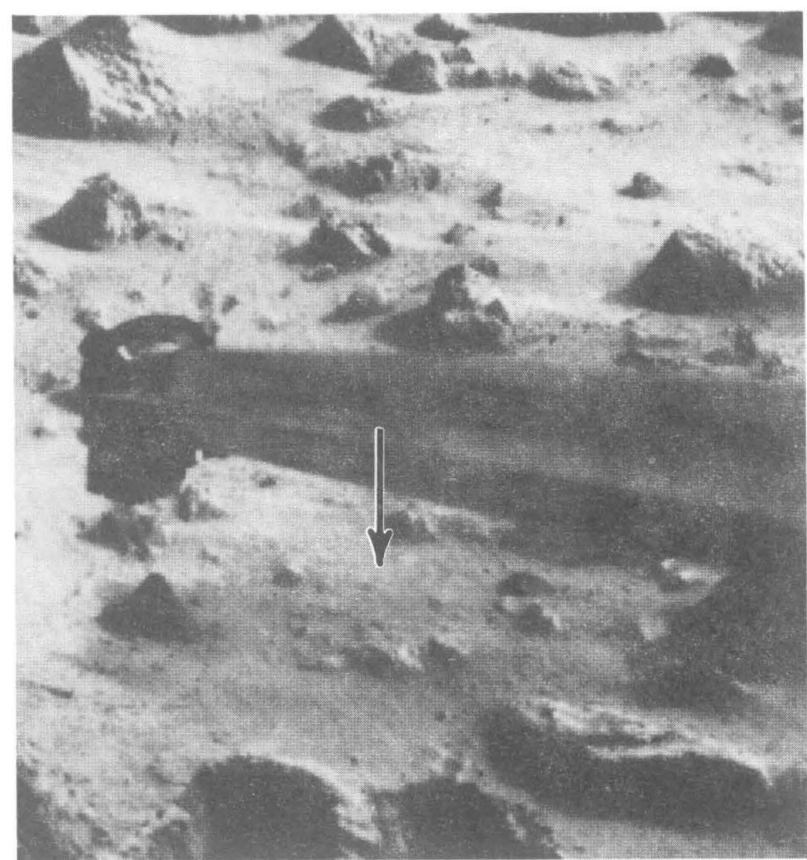

A

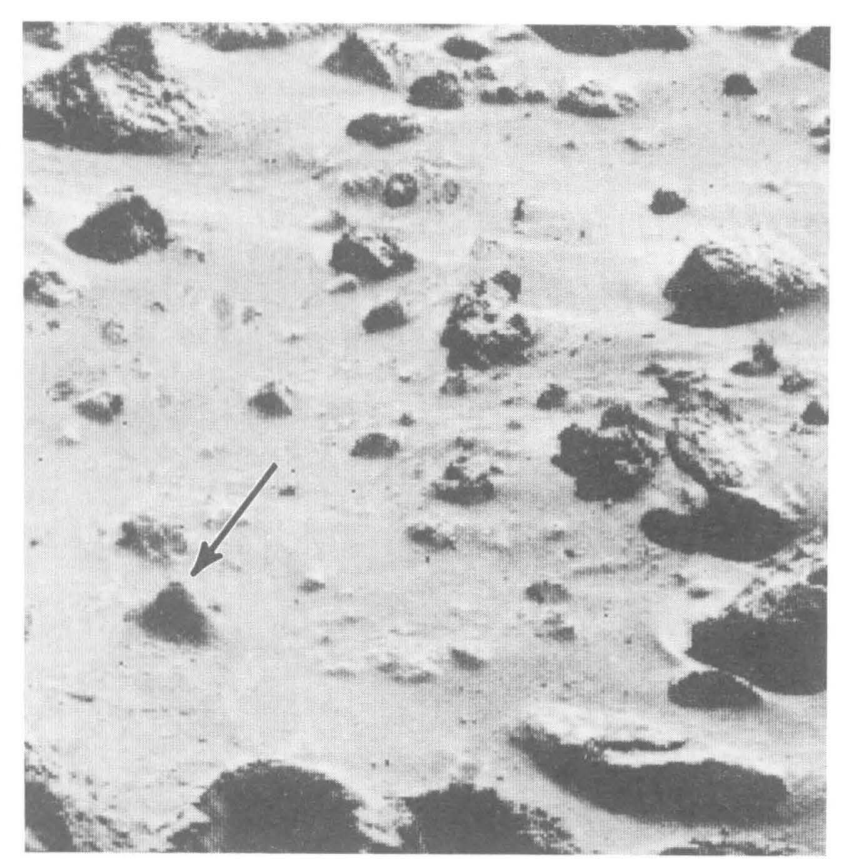

B and artificial changes difficult. The changes discussed below are, chiefly, those that can be identified readily in black and white pictures because the processing required for analyses of colors were not available for this project. For practical reasons, this project excludes data beyond about Sol 921 of Lander 1, except to note that exciting changes occurred by Sol 1757. The exciting changes were due to a local dust storm that was in progress on Sol 1742. These changes and others were noted by Moore (1982) and Wall (1982), and a more complete discussion of events beyond Sol 921 was given by Arvidson and others (1983).

Situations that required visual observations of changes were created intentionally, inadvertently, naturally, and by the presence of the spacecraft. (1) Conical piles of surface materials were intentionally constructed in the sample fields in order to detect changes induced by martian winds or other processes. (2) Debris and dust were deposited on the footpads and sample fields by engineexhaust gases during landing, inadvertently during surface-sampler activities, and naturally from the atmosphere. These materials could move for a variety of reasons such as wind eddies around the spacecraft, spacecraft vibrations, or other reasons. Disruption of material during penetration by the buried footpad 2 of Lander 1 could have produced an unstable situation. (3) Trenches with steep walls and tailings were excavated and fresh materials were exposed. (4) Natural materials containing planes of weakness on steep slopes could have changed because of natural processes. (5) Chips with paint that darken with exposure to ultraviolet (UV) radiation were placed on the landers to measure the intensity of UV radiation at the surface. (6) Finally, the landed spacecraft may have been unstable.

\section{CONICAL PILES}

Conical piles of fine-grained materials were constructed by the surface samplers of both landers during the Extended Mission to detect and measure erosion of the piles by the martian winds. Five piles were constructed at strategic positions in the sample field of Lander 1 (fig. $29 B$; pl. $1 B$, table 32 ), and one was placed on the lander body during the Primary Mission. Four of the piles

Figure 80.-Conical pile 1 of Lander 1. A, Conical pile 1 (approximately $3.6 \mathrm{~cm}$ high) on day it was emplaced. Note dark halo (arrow) and area to right produced by wind-wafted fine-grained material (frame 12D093/296). B, Conical pile 1 (arrow) after 505 sols of exposure to martian winds and other processes. Note that shape and form of pile are unchanged but darkened areas around base and to right of pile are no longer visible (frame 12I165/801). Pictures taken 625 sols after pile was emplaced also show that it was unchanged. 


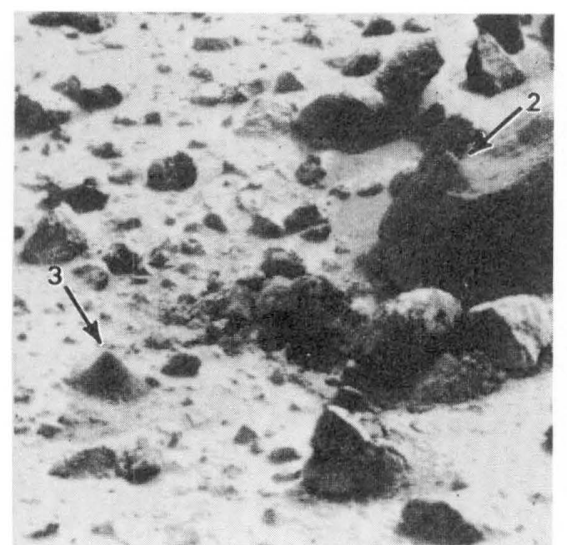

A

FIgure 81.-Conical piles 2, 3, and 5 of Lander 1. A, Conical piles 2 and 3 (approximately $3.5 \mathrm{~cm}$ high) after 55 sols on surface (frame 11E192/379). B, Conical piles 2 and 3 still retain their size and form after 366 sols of exposure to martian winds and other processes.

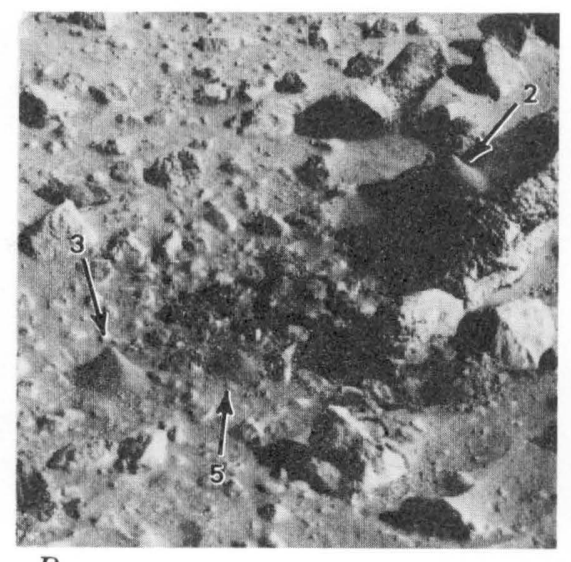

Conical pile 5 has been on surface for 41 sols (frame 111070/690). $C$, Conical piles 2, 3 , and 5 still retain their size and form after 597 , 597 , and 282 sols, respectively, of exposure to martian winds and other processes (frame 11J013/921) placed in the sample field were constructed with drift material because changes were observed for a pile of the drift material deposited earlier on the grid of the lander body. The fifth pile in the sample field was constructed with blocky material from the rocky area because blocky material was eroded by the engine exhausts. Three conical piles were constructed at strategic positions in the sample field of Lander 2 (fig. $30 B$; pl. $2 B$, table 32 ). There were no obvious choices of different materials to use at the Lander 2 site.

Locations of the conical piles were dictated by a combination of the capabilities of the surface sampler, the topography of the sample field, and the inferred directions of strong winds based on observations of natural features. The first conical pile of Lander 1 (fig. 80) was placed in a relatively open, rock-free area near the east edge of the sample field where wind turbulence caused by the spacecraft would be at a minimum if the dominant wind was, in fact, from $11.5^{\circ}$ east of north (Sagan and others, 1977). Locations of piles 2, 3, and 5 at Lander 1 were on and among rocks, so that their locations were partly dictated by the presence of a suitable rock (fig. 81; rock 3 or Metate 1 in pl. $1 B$ or fig. $29 B$ ). Conical pile 4 was placed next to a ventifact (fig. 82). All three of the conical piles at the Lander 2 site were on the upwind side of the spacecraft if the dominant wind came from $35^{\circ}$ east of north (Sagan and others, 1977), however, the orientations of the small drifts in the rill in front of Lander 2 suggested that the dominant wind direction was from $305^{\circ}$ east of north. If this is the case, conical pile 2 would have been in the turbulent wake of the lander, but piles 1 and 2 would have been upwind of the lander. Conical pile 1 was placed on the tallest rock available, and conical pile 3 was placed near the bases of three rocks (fig. 83; see rock 10 , Centaur, in fig. $30 B$ and pl. $2 B$ ). Conical pile 2 was within a very shallow trench (XRFS 9) in an open area near the ejected shroud (fig. 84; Physica Planitia; fig. 30B; pl. 2B).

All the conical piles in the sample fields at Lander 1 to Sol 1601 and at Lander 2 to Sol 1193 showed little or no evidence for deflation or removal of material subsequent to their emplacement. Rather, a very thin veneer of material appeared to have been deposited on them and their surroundings. During emplacement of the piles, a thin layer of material from the collector head, partly wafted by mild winds, was deposited around the flanks and on the leeward sides of the piles. These halos of finegrained material sometimes produced surfaces that appeared darker than the general area around them. With time, the darkened surfaces became lighter until they were no longer distinguishable from their surroundings. The gradual disappearance of the darkened areas was probably the result of deposition of very fine grained material stirred up by sampler operations and from the atmosphere because evidence for deflation elsewhere was lacking. Finally, pictures taken of conical pile 4 (Lander 1 ) on Sol 1765 (fig. 82D) revealed significant changes (Moore, 1982).

Conical pile 1 of Lander 1 , which was emplaced during Sol 296, does not appear to have diminished in size or changed in shape in pictures taken 505 sols later on Sol 801 (fig. 80). The initially darkened surface around the pile, which extended toward rock 1 (Shadow, fig. $80 \mathrm{~A}$, 


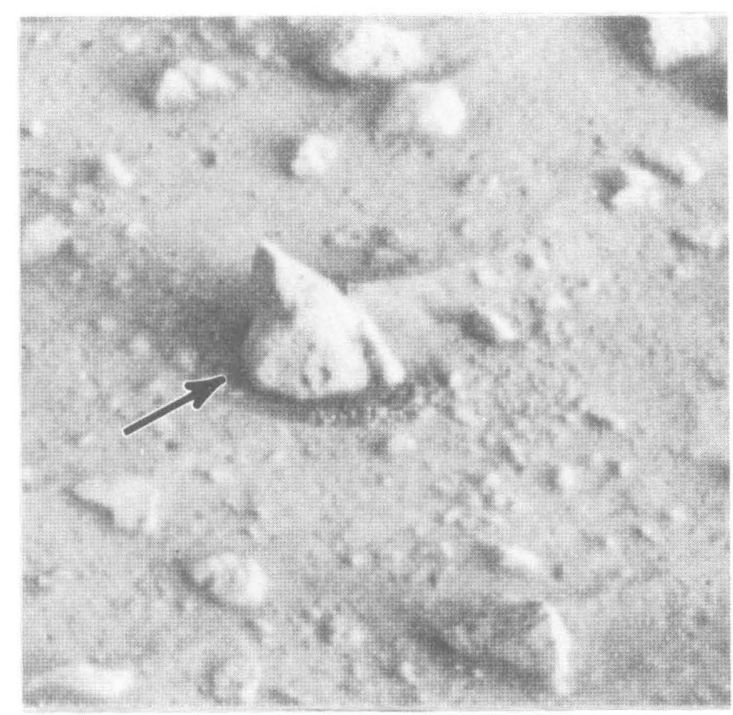

$A$

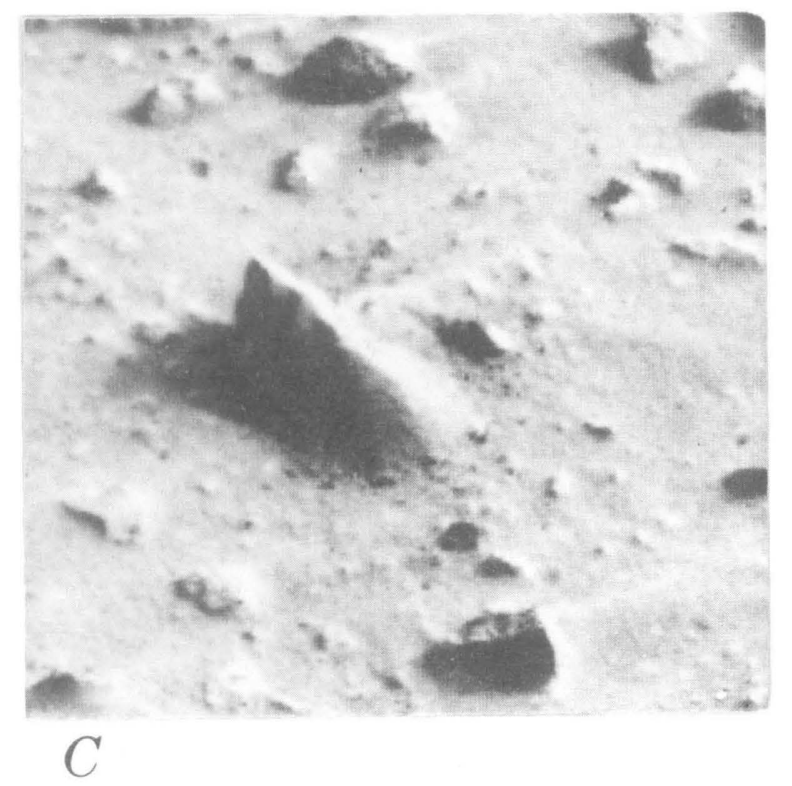

Figure 82.-Conical pile 4 of Lander 1 emplaced on Sol 344. A, Surface and ventifact (see arrow) before emplacement of conical pile 4. Note that two sharp edges of ventifact slant upward to left. Ventifact is $6-7 \mathrm{~cm}$ tall (frame 11D183/317). B, Conical pile 4 (approximately $5 \mathrm{~cm}$ high) after 7 sols on surface (see arrow). Note dark halo and area to right produced by wind-wafted fine-grained material during emplacement. Note that sharp edges of ventifact are buried (frame
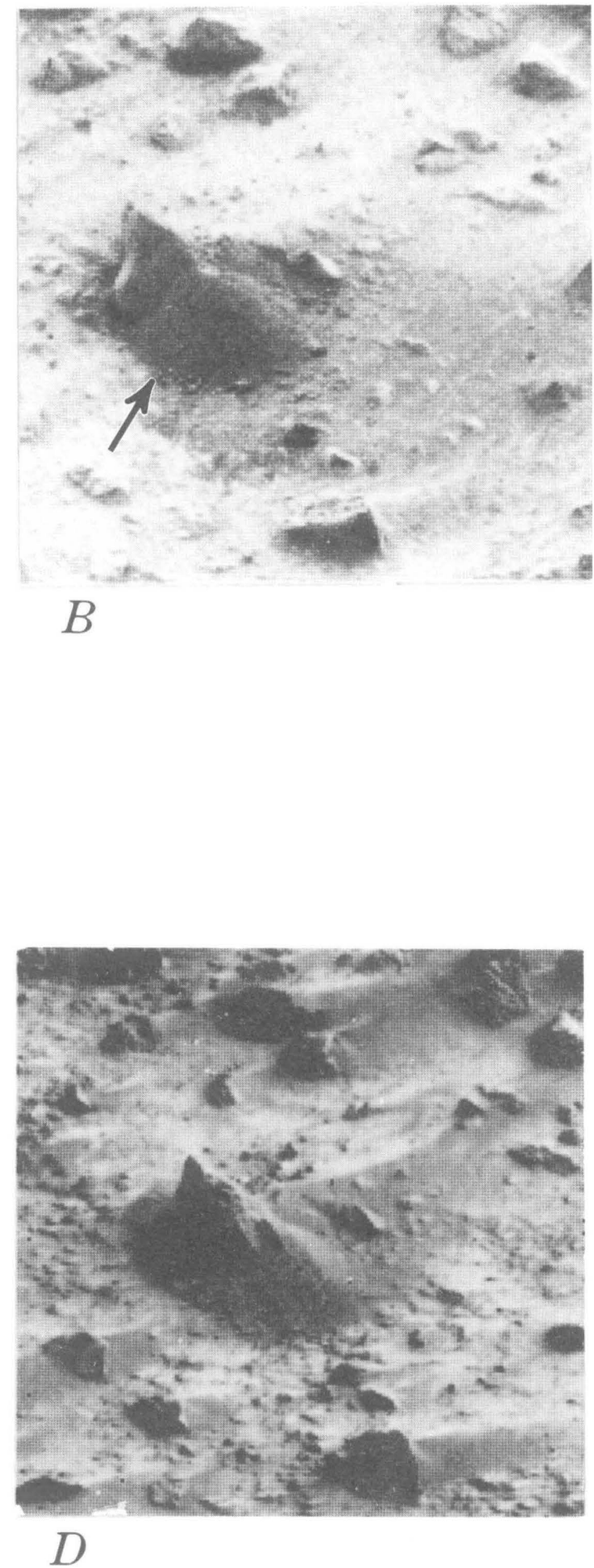

11E082/351). C, Conical pile 4 remains unchanged in size and form after 577 sols on surface but dark halo and area to right are no longer visible. Note that sharp edges of ventifact are buried (frame 11J007/921). $D$, Conical pile 4 after 1421 sols on surface. Note changes in pile; two sharp edges of ventifact are exposed; fine-grained material has been removed from surrounding surfaces (frame J130/1765). 


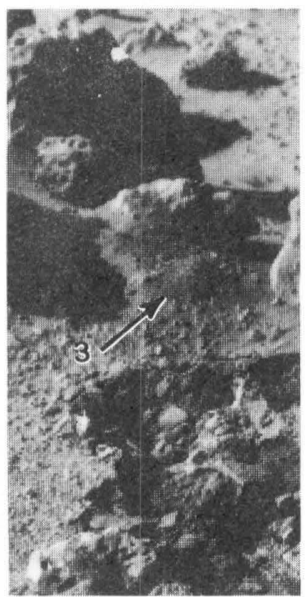

A

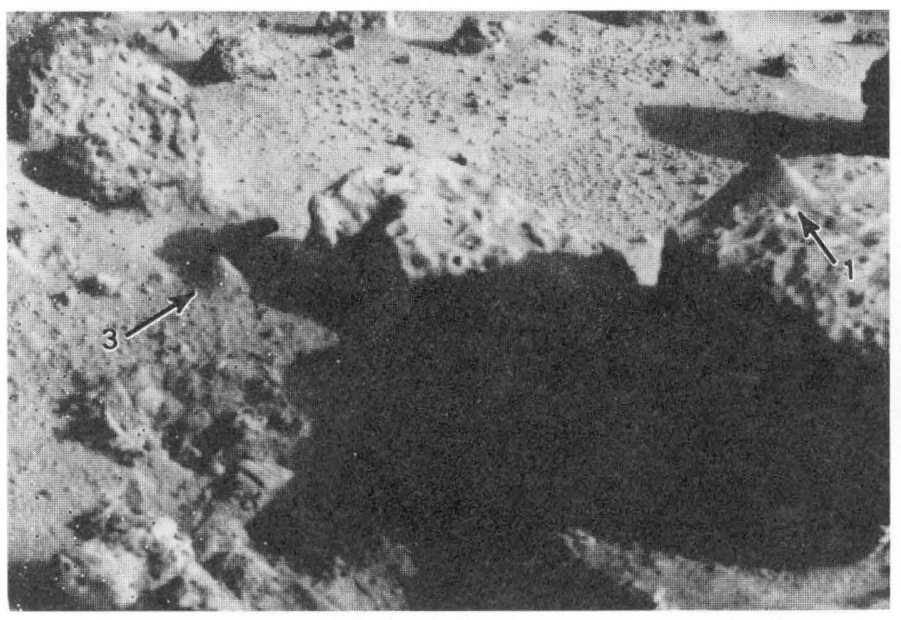

$B$

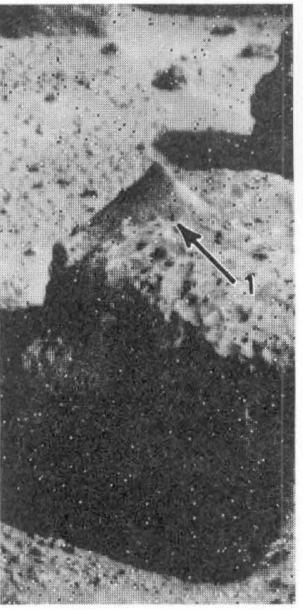

C
Figure 83.-Conical piles 1 and 3 of Lander 2. $A$, Conical pile 3 (approximately $1.4 \mathrm{~cm}$ high) shortly after emplacement. Note halo of dark material around pile (frame 21H032/595). B. Conical piles 1 and 3 after 734 and 545 sols of exposure to martian wind and other processes.
Halo of dark material around pile 3 is no longer visible. Pile 1 is approximately $4.5 \mathrm{~cm}$ high (frame 21I117/1140). $C$, Conical pile 1 after 787 sols of exposure to martian environment shows little or no change in size or form (frame 21I136/1193).

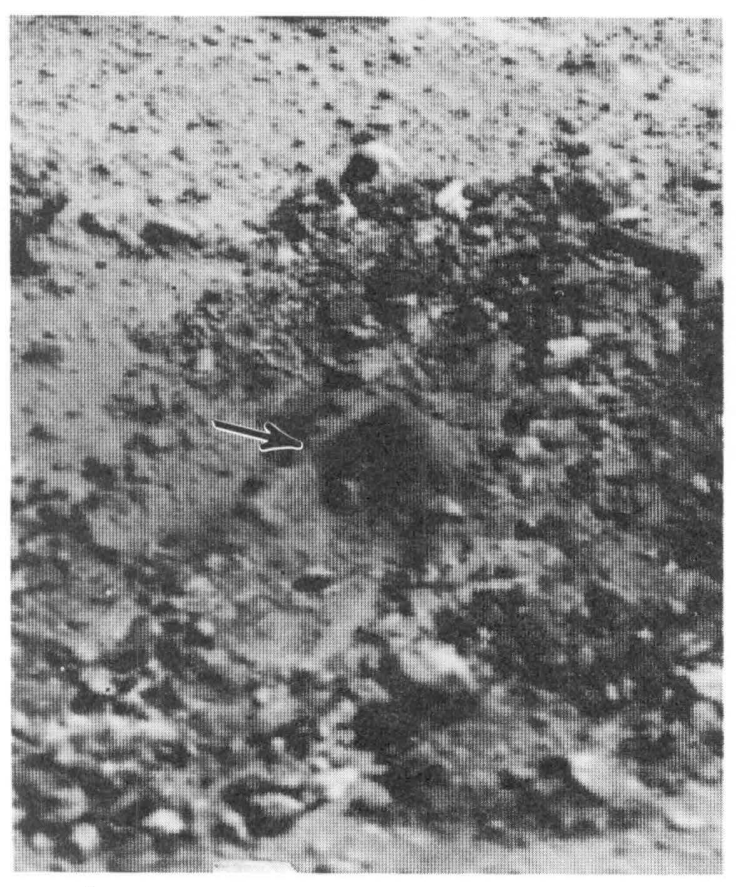

A

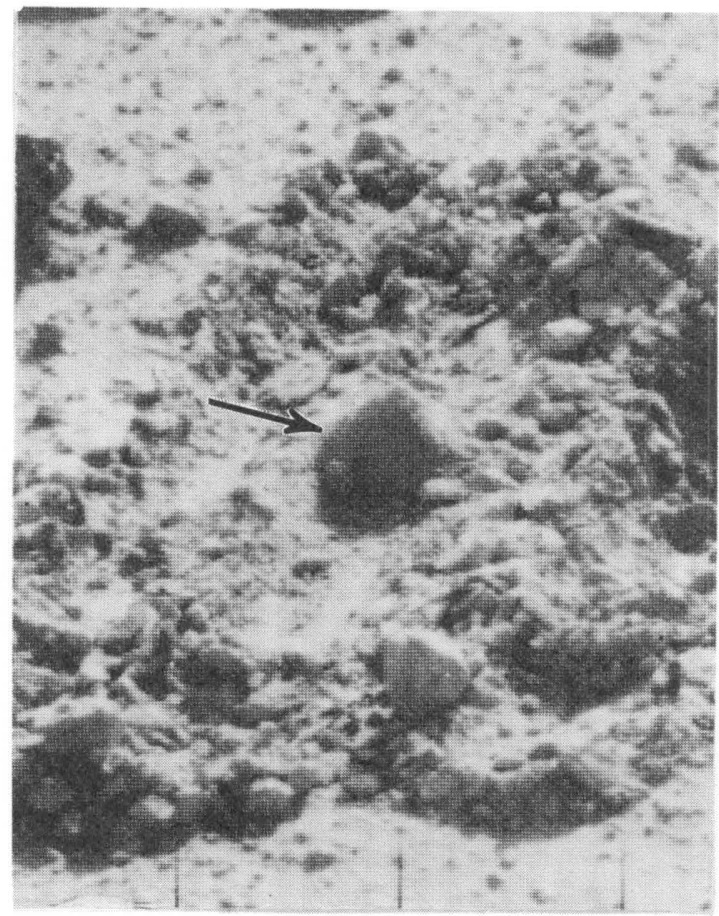

B

Figure 84.-Conical pile 2 (arrow) of Lander 2. A, Conical pile 2 (approximately $3-4 \mathrm{~cm}$ high) after 9 sols of exposure to martian winds and other processes (frame $22 \mathrm{H} 046 / 604$ ). $B$, Conical pile 2 appears unscathed despite 582 sols of exposure to martian winds (frame 22I129/1177). 


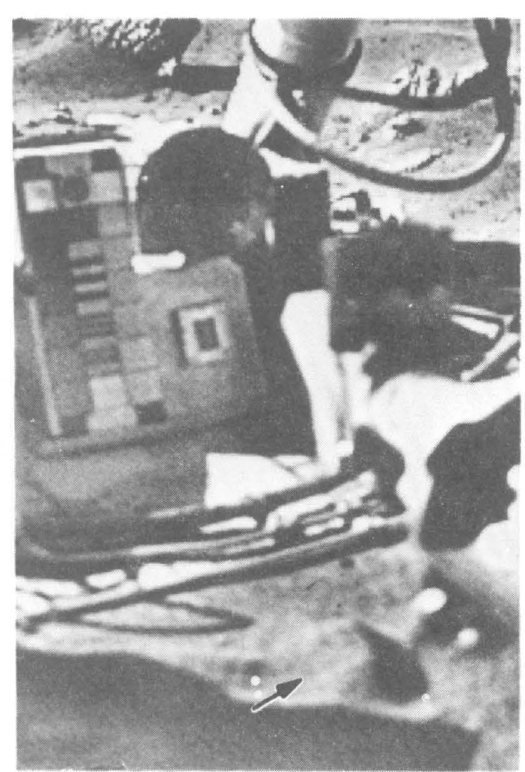

A

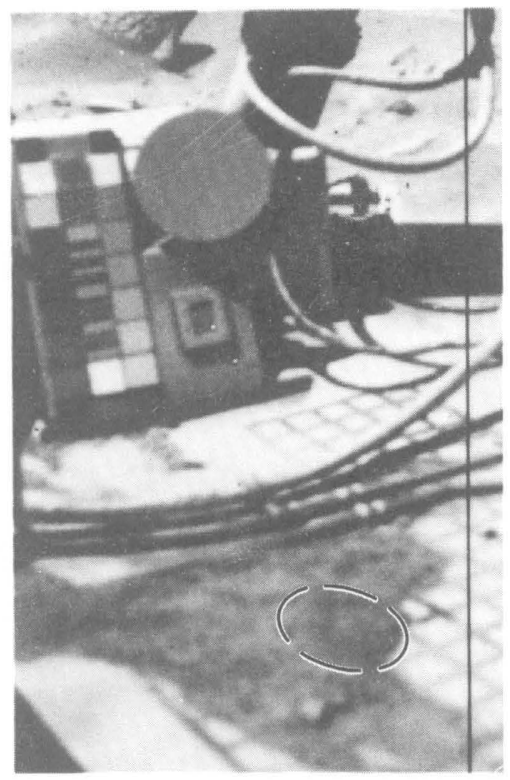

B

Figure 85.-Conical pile emplaced on grid of Lander 1 body. $A$, Conical pile (approximately $4 \mathrm{~cm}$ high, see arrow) after emplacement on Sol 41 (frame 12B107/041). B, Material of conical pile and other added materials on Sol 801. Note great change in appearance which is in marked contrast to conical piles in sample field. Dashed oval indicates original position of pile. Distribution of materials on Sol 921 was essentially the same as on Sol 801. Dark vertical line represents missing data (frame 12I167/801). pl. $1 B$ ), was still visible on Sol 330 but it gradually disappeared. By Sol 396, the darkened area was less noticeable and by Sols 457 and 458, it was barely discernible. The darkened area was no longer visible in Sol 550 pictures. On Sol 801, the pile appeared unchanged after 505 sols (fig. 80B). Evidence for significant changes in sizes or shapes of the remaining piles of Lander 1 was also lacking through Sol 1601 (see figs. 81 and 82). Conical pile 4 was unchanged on Sol 921 (fig. $82 C$ ); conical piles 3 and 5 were unchanged on Sol 1543; conical piles 2 and 5 were unchanged on Sol 1601. Darkened surfaces were difficult to recognize around some of the piles but, the dark surface around pile 3 had disappeared by Sol 379 and the dark surface around pile 4 was barely discernible by Sol 441 . Evidence that the dark halo surfaces were the result of deposition of materials from the collector head is indicated by the thin layer of material that covers the surface of the rock adjacent to pile 2 (fig 81 ). A picture taken of conical pile 4 on Sol 1765 revealed that it had finally changed (fig. $82 D$ ) about two martian years after it was emplaced; this time corresponds to the latter part of the third martian winter experienced by Lander 1 (Moore, 1982).

The conical piles of Lander 2, which were constructed after all but one of the Lander 1 piles, had not changed significantly as of Sol 1193. Conical pile 1, which was on a rock, appeared unscathed as late as Sol 1193 after more than one martian year (fig. $83 C$ ). A thin apron of finegrained material was deposited around it during its construction. In Sol 959 pictures, the condensate of the winter "snow-fall" covered the apron, but the south-facing surface of the cone appeared to be dark and uncovered. Neither piles 2 nor 3 appeared to have changed (figs. 83, 84). The dark halo around pile 3 was still visible on Sol 750 but it was gone by Sol 872 . In Sol 959 pictures, the relatively dark pile 3 rises majestically above its "snowy" surroundings.

It is difficult to ascertain the source of the materials that covered the dark halos because surface-sampler activities occurred during the interval when the dark halos became lighter. Sedimentation of fine-grained material during surface-sampler activities could have been considerable because only about $20 \mathrm{~cm}^{3}$ of fine-grained material are required to produce a $1 \mu \mathrm{m}$ layer of dust in the sample field, and this amount would be enough to obscure the dark halos (Guinness and others, 1979). Windblown materials derived from the upper gridded surfaces of the lander bodies are another possible source for the fine-grained material. The surface-sampler activities of Lander 1 continued to Sol 639-well beyond the dust storm period-and beyond the time when the darkened surfaces were obscured by lighter materials. Thus, the light-colored fine-grained materials could have been from the surface-sampler activities as well as from sedimentation from the atmosphere. Conical pile 5 of Lander 1 was 
among the last products of the surface sampler on Sol 639, and its halo lasted at least to Sol 750 before it disappeared by Sol 872 (fig. 81). In this case, it appears likely that the halos were mainly covered by fine-grained material deposited from the atmosphere, but it is possible that some material came from the upper surface of the lander body. In the case of Lander 2, some, if not most, of the material could have fallen from the atmosphere as is demonstrated by the wintertime "snow fall" (Jones and others, 1979).

The pile placed on the grid of the body of Lander 1 on Sol 41 changed greatly (Moore and others, 1979). Initially the pile was a cone about $3.6 \mathrm{~cm}$ high with steep slopes of about $45^{\circ}$. The large square of the grid around the pile was completely covered by drift material. By Sol 76, (1) the pile was a rounded dome but its horizontal position was essentially the same, (2) the surface of the dome appeared to be composed of cohesive units separated by fractures, (3) some lumps were better exposed than they were in previous pictures, and, (4) the right hand part of the large section of the grid was exposed. By Sol 268, the bulk of the material had shifted $30^{\circ}$ to the left in camera 2 pictures, the pile had flattened, and more of the grid was exposed. Subsequent minor changes occurred from Sol 268 to Sol 921. Cumulative changes to Sol 921 are shown in Figure 85.

Relative movement of the mass of materials on the grid of the body of Lander 1 was, generally, toward the upper left, as viewed in the pictures of camera 2. This direction corresponds to the direction of slope of the tilted lander. The movement appears to have been stopped, in part, by tubing near the surface of the grid. Movement of material on the lander body probably was partly due to the slope of the gridded surface because the direction of motion coincided with the direction of tilt. In view of the general stability of the conical piles in the sample fields, the conclusion that the wind removed the material from the grid by saltation "a la Bagnold" (Sagan and others, 1977) seems to be incorrect. The materials probably moved downslope primarily in response to vibrations of the flexible upper surface of the lander body. A wide variety of spacecraft vibration sources have been demonstrated by Anderson and others (1977). Small displacements of the flexible upper surface of the lander body $(1 \mathrm{~mm})$ could produce harmonic motion accelerations in excess of $3.73 \mathrm{~m} / \mathrm{s}^{2}$ if the frequency exceeds about $10 \mathrm{~Hz}$. Vibration frequencies larger than $10 \mathrm{~Hz}$ were reported during windy periods, and other vibrations larger than $10 \mathrm{~Hz}$ might have been due to temperature changes. The complicated wind eddies over the lander have contributed to the shape and form assumed by the materials on the grid because simple downslope motion would cause the materials to be neatly banked against the tubing.

\section{MATERIALS IN THE FOOTPADS}

Surface materials deposited in the visible footpads have changed in both large and small ways, and materials above the buried footpad of Lander 1 have slumped downward above the buried rim of the footpad. Considerable movement of materials occurred in footpad 3 of Lander 1 , material was added in footpad 2 of Lander 2 , and very little change occurred in footpad 3 of Lander 2 . It is not entirely clear why significant movements of materials occurred in the Lander 1 footpad but not in those of Lander 2, because the materials in both did not appear to differ significantly. Materials were initially deposited in the footpads after entrainment in engine-exhaust gases and ejection from the surface during touchdown. Modest amounts of fine-grained material were probably added to footpad 3 of both landers by atmospheric sedimentation (Pollack and others, 1977; Arvidson and others, 1979; Jones and others, 1979) and winds that wafted finegrained material during surface-sampler activities. Significant amounts of material were probably added to footpad 2 of Lander 2 and footpad 3 of Lander 1 as a result of surface-sampler activities. Initial amounts and areal distributions of materials in footpad 3 of Lander 1 and footpad 2 of Lander 2 appear to have been very similar (figs. $86 \mathrm{~A}$ and $87 \mathrm{C}$ ). For both, rather coarse objects, admixed with fine-grained material were present near the center of the floors of the dish-shaped footpads and were surrounded by a halo of much finer-grained material that also covered the floor. The walls were covered by a thin veneer of fine-grained material; locally the material was thick and lumpy. In contrast, the floor of footpad 3 of Lander 2 was covered by coarse objects without a halo of fine material and the walls generally had a thick, lumpy layer with admixed coarse objects instead of a thin veneer of fine material. Differences between the distribution of materials within and on the walls of the three footpads were probably the result of different conditions during landing. Maximum forces on the primary struts for the legs corresponding to footpad 3 of Lander 1 and footpad 2 of Lander 2 were nearly the same (9816 N and $9248 \mathrm{~N}$ ) whereas the force for footpad 3 of Lander 2 was about half as much $(4138 \mathrm{~N})$. The forces and accelerations on the first two footpads were probably large enough to cause materials to slide down the walls during touchdown and form the halos of fine-grained materials around the coarse materials on the floors, but the force and acceleration for footpad 3 at Lander 2 were not large enough to cause the material to slide down the walls during touchdown.

Rather striking changes occurred on footpad 3 of Lander 1 (fig. 86). Initial pictures show the floor of the dish-shaped footpad was filled with fine-grained material that surrounded mixed fines and clods with diameters as 
much as $0.7 \mathrm{~cm}$ (fig. $86 \mathrm{~A}$ ). The walls, which are inclined between $38^{\circ}$ and $48^{\circ}$, were covered with a veneer of finegrained material and a thicker patch with lumps or clods with diameters as much as $0.3 \mathrm{~cm}$. On Sol 171, changes on the inner walls were obvious-patches of uncovered metal had appeared. By Sol 347, much of the wall material was removed but deltoid-shaped patches were present on opposite sides of the footpad (fig. 86B). Parts of the floor were cleared of fine-grained material. The coarsest objects on the floor did not appear to have moved. Subsequent changes were little. Three clods or lumps, $0.1-0.3$ $\mathrm{cm}$ wide, appeared on the left wall where none were previously present. Lumps that slid down the inner walls appeared by Sol 580 (fig. 86C). These lumps probably

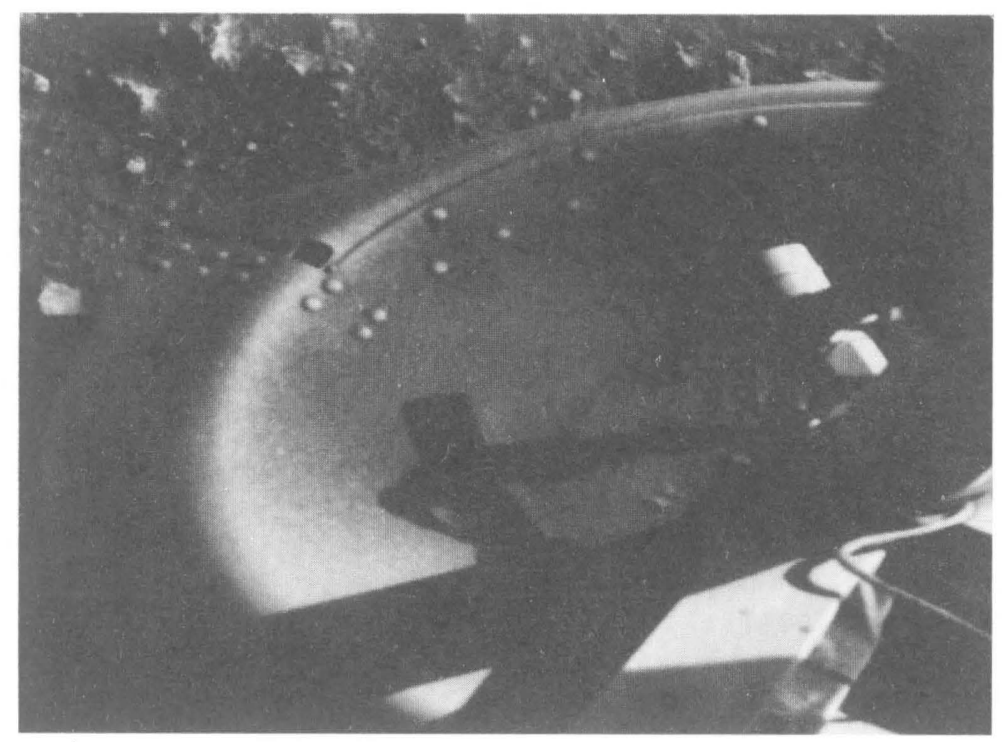

$A$
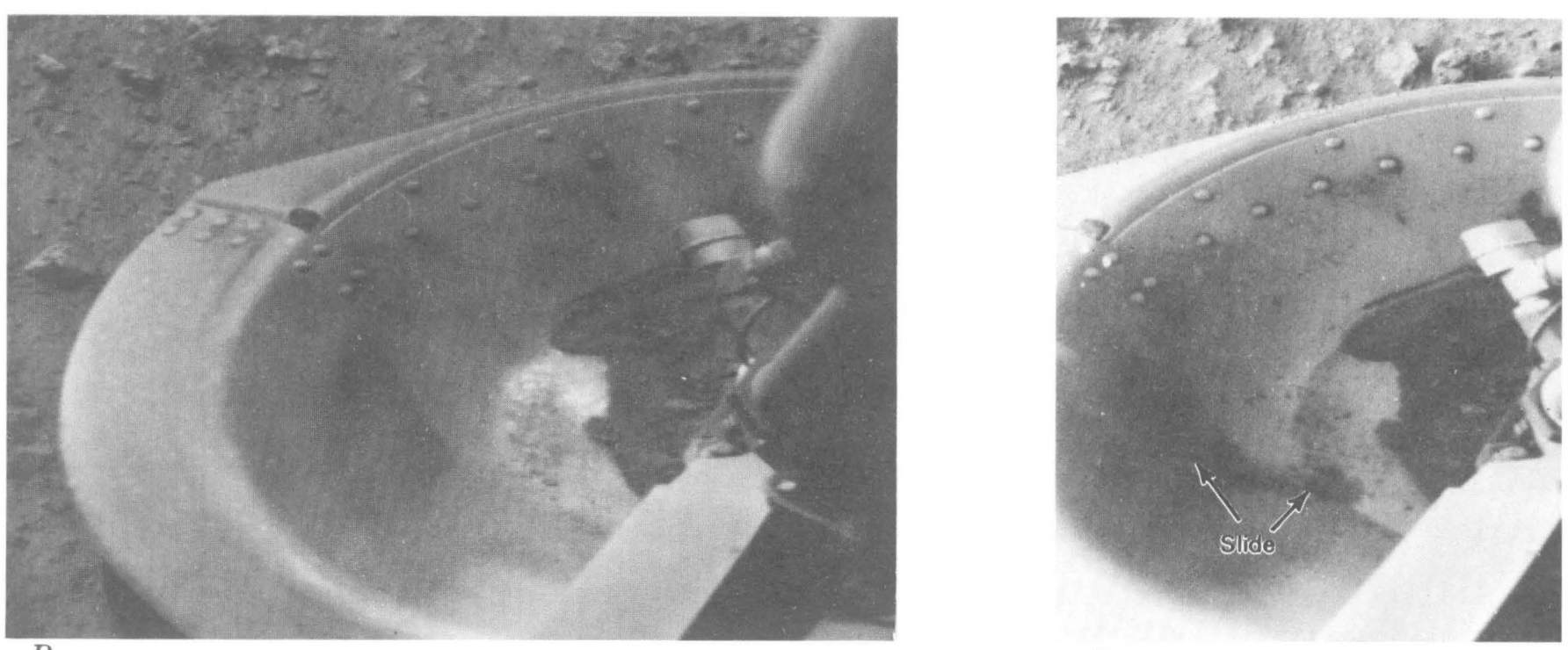

C

Figure 86.-Changes in footpad 3 (radius $0.206 \mathrm{~m}$ ) of Lander 1. A, Footpad 3 immediately after landing. Note thin veneer of fine-grained material on walls, halo of fine-grained material on floor, and core of mixed coarse and fine-grained material (frame 12A001/000). B, Footpad 3 after 476 sols on Mars. Note deltoid-shaped patch on left; materials have moved from left part of floor of footpad. Coarse ob- jects in deltoid patch have been added. Most of the coarsest objects near center are still in their same positions (frame 12G046/476). $C$, Footpad 3 after 921 sols on Mars. Note material in deltoid patch has slid downward toward center. Little or no change has occurred elsewhere on floor (frame 12J003/921). 
originated as the result of surface-sampler activities because the boom and collector head passed over and near footpad 3 on Sols 430, 431, and 550 during sample acquisitions. Added droppings are found on the surface near footpad 3. The material of the deltoid-shaped patch began sliding between Sols 476 and 580 and continued beyond the period of surface-sampler activities. Comparison of pictures taken on Sols 836 and 921, well after the sampler was parked, show that additional material slid down the wall. The thicknesses of the sliding units were about $0.3 \mathrm{~cm}$.

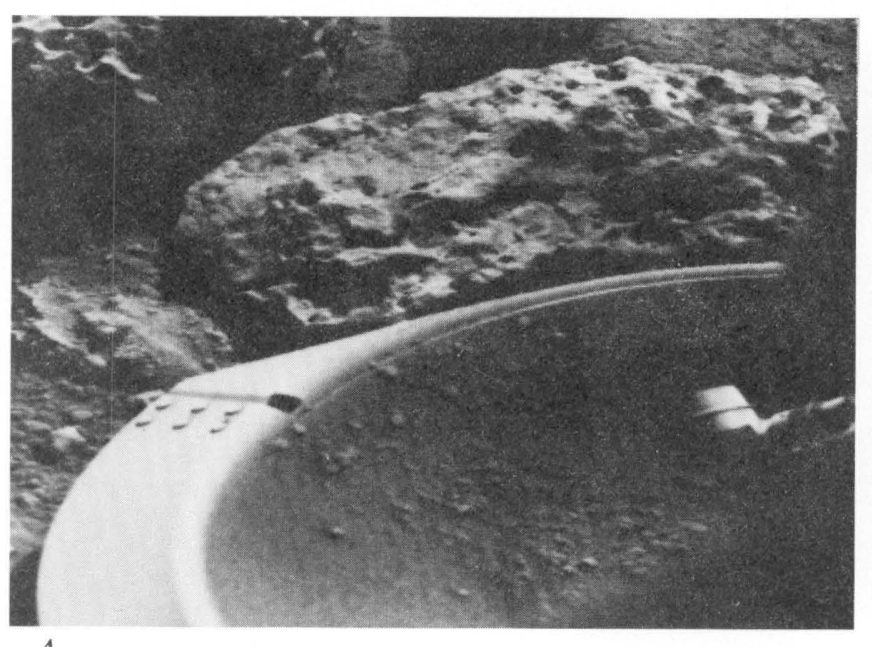

$A$

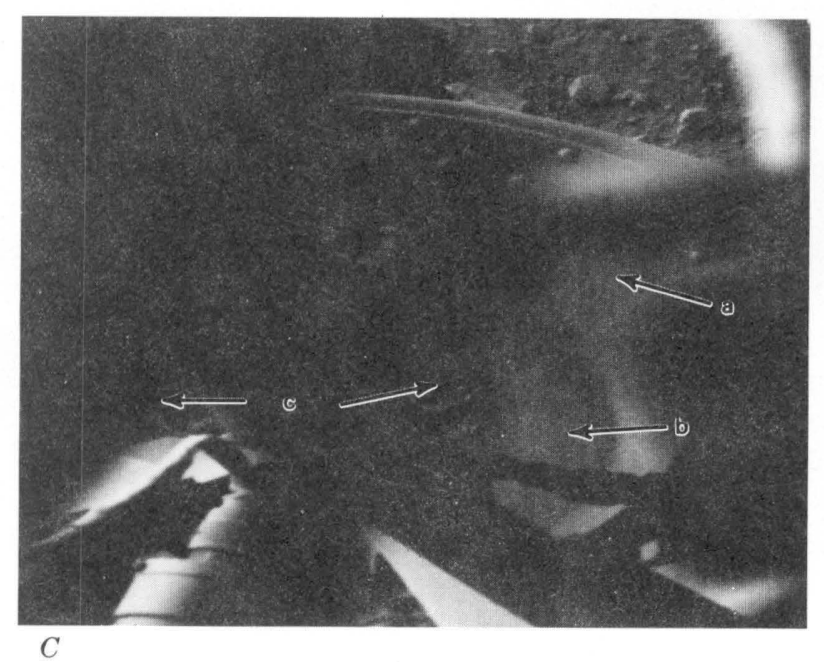

Figure 87.-Changes in footpads 3 and 2 of Lander 2. A, Footpad 3 of Lander 2 immediately after landing on Sol 0 (frame 22A001/000) Note homogeneous materials on walls and floor of footpad. $B$, Footpad 3 of Lander 2 on Sol 1140. Materials and objects within footpad dish do not appear to have moved nor have noticeable amounts of material been added. Small skiff of fine-grained material has been added to left skirt-probably during surface-sampler activities. Note that separation between upper edges of rock and footpad has in creased about $0.01 \mathrm{~m}$ (frame 22I118/1140): C, Footpad 2 of Lander
The distribution of fine-grained material and clods in footpad 3 of Lander 2 changed little or not at all (figs. $87 A$ and $B$ ). Although illumination conditions varied from picture to picture, all resolvable lumps and objects as much as $0.7 \mathrm{~cm}$ wide were visible in pictures of footpad 3 from Sol 0 to Sol 1140. No sliding or movement of material appears to have occurred despite the fact that slopes of the footpad walls range from about $38^{\circ}$ to $50^{\circ}$. Some finegrained material may have moved from the upper outboard rim. A thin skiff of fine-grained material was deposited on the skirt well after landing, but once

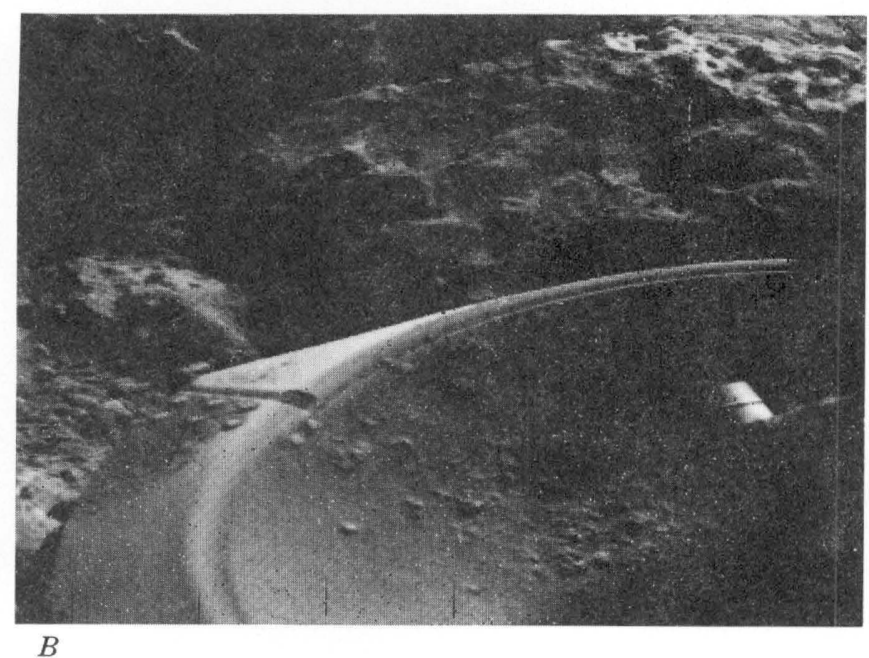

$B$

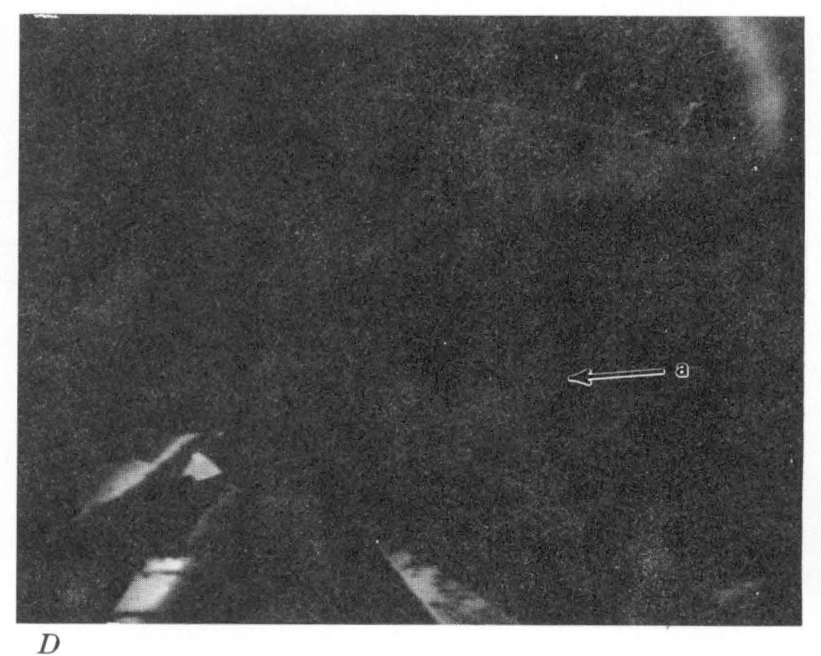

2 on Sol 4. Note thin veneer of fine-grained material on walls (a), halo of fine-grained material on floor (b), and core of mixed coarse and fine-grained material (c) (frame 21A031/004). D, Footpad 2 of Lander 2 on Sol 553. A large amount of fine-grained material has been deposited on footpads (a) between Sols 518 and 553; this interval corresponds to time when surface-sampler collector head was sifting material from deep-hole tailings in futile endeavor to collect coarse material (frame 21G153/553). 


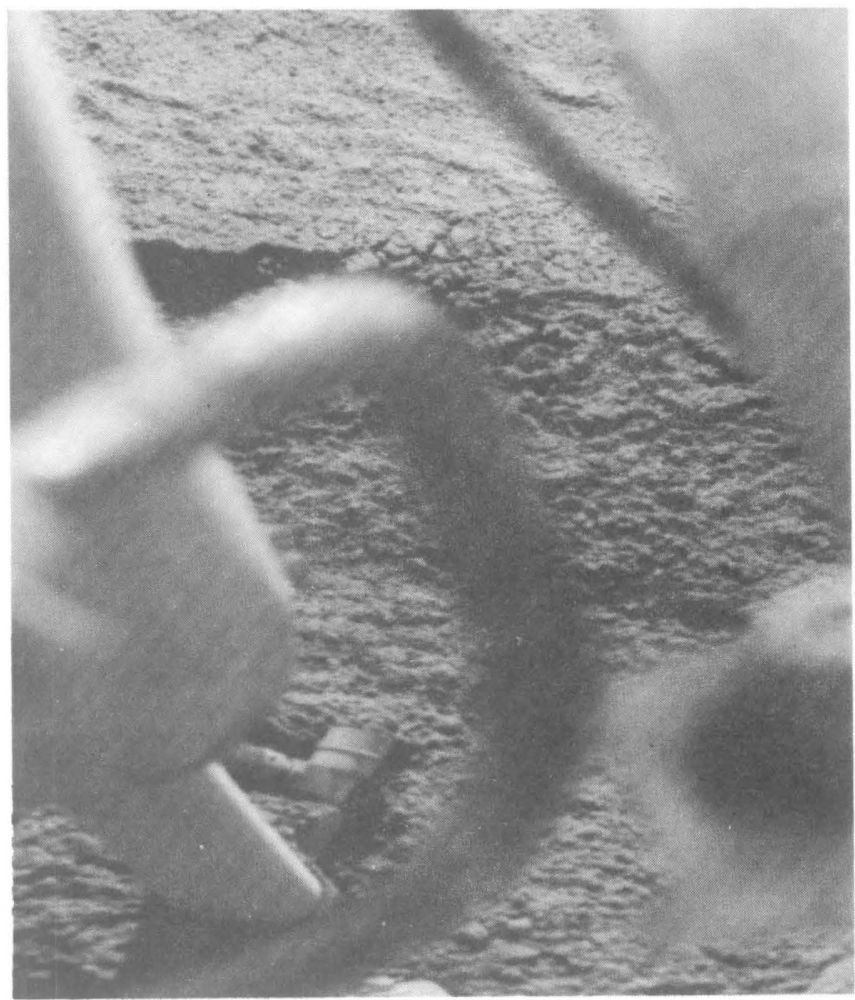

$A$

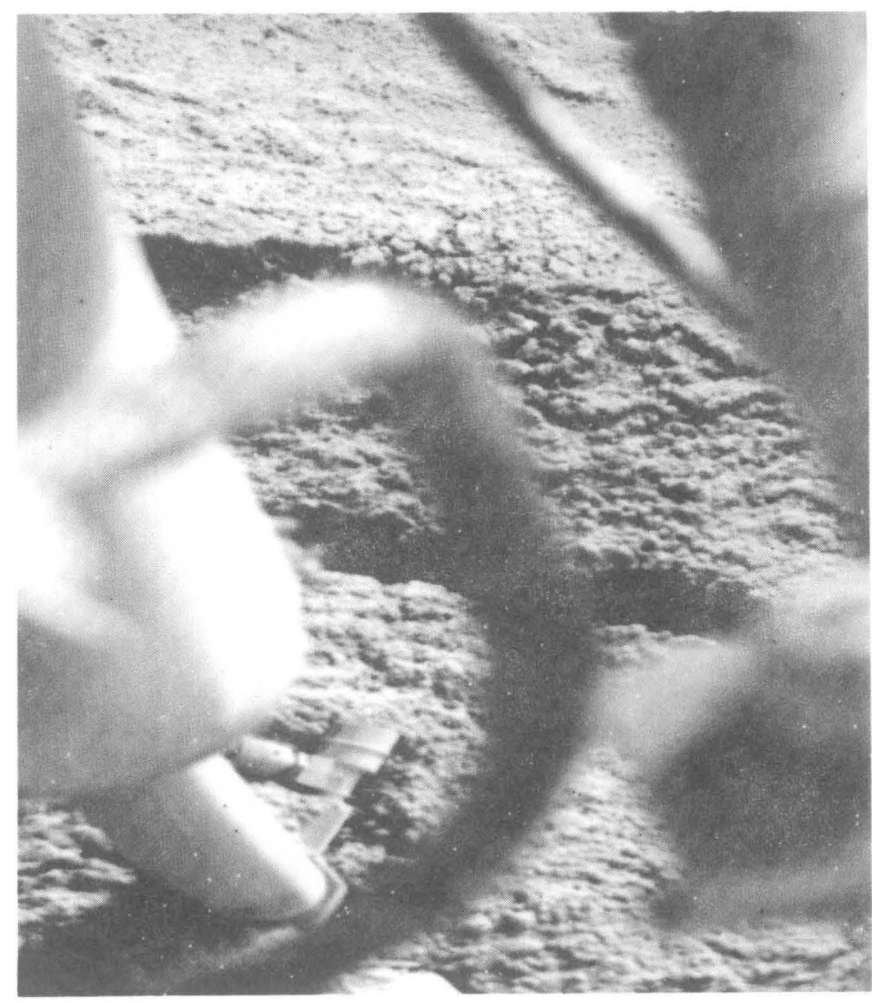

$B$

Figure 88.-Changes above footpad 2 of Lander 1. A, Footpad buried in drift material on Sol 12 (frame 11A079/012). B, Material has slumped vertically downward in region directly above footpad rim (frame 11I207/841). Slumping occurred as early as Sol 35.

deposited it remained unchanged. Thus, transportation of materials to and from footpad 3 of Lander 2 by the wind has been, at best, slight.

A different result is obtained when pictures of footpad 2 of Lander 2 taken from Sol 4 through Sol 553 are compared with one another (fig. $87 \mathrm{C}$ and $\mathrm{D}$ ). A large amount of fine-grained material was deposited on the footpad and on its walls, partly obscuring the original halo of finegrained material. The major increase in deposited materials coincided with the interval of time during which samples from the tailings of Deep Hole 2 (Sols 522, 535, and 545) were being sieved to separate the fine fraction from the coarse (but nonexistent) fraction. Despite the inclinations of the walls, which were $38^{\circ}$ to $47^{\circ}$, there was no evidence for slumping and sliding of materials down the walls, and preexisting lumps and objects identified in Sol 4 pictures were still present and in place on Sol 553. Thus, lack of slumping and sliding of materials on the footpads of Lander 2 was somewhat surprising in view of the slumping that occurred on footpad 3 of Lander 1.

The behavior of the materials on the footpads is related to a number of variables: (1) adhesion and friction between the aluminum of the footpads and surface materials tend to hold the materials in place, (2) the acceleration of gravity tends to cause sliding down steep walls,
(3) spacecraft vibrations induced by wind, surface-sampler activities, other spacecraft activities, and temperature changes tend to cause the material to move, and (4) highvelocity wind streams and eddies induced by the interaction of the wind with the spacecraft could cause the material to move. The symmetrical deltoid patterns on footpad 3 of Lander 1 and removal and deposition of skiffs of material on the other footpads suggest that highvelocity wind streams and eddies do, in fact, cause material to move; but, droppings from the surface sampler also contributed to the movement of material on footpad 3 of Lander 1.

Materials on the sloping walls remained there because gravitational stresses did not exceed those of adhesion and friction between the metal and surface materials. It does not appear to be possible to separate the effects of adhesion from those of friction. The stresses involved are:

where

$$
\begin{aligned}
& \tau=\rho g t \sin \theta \\
& s=\rho g t \cos \theta
\end{aligned}
$$

$\tau=$ gravitational shear stress parallel to the slope,

$s=$ gravitational normal stress across the slope,

$\rho=$ density of sliding material, and

$t=$ thickness of sliding material. 
When sliding occurs in the absence of adhesion:

$$
\tau=s \tan \delta
$$

where $\tan \delta=$ coefficient of friction between the metal wall and surface material. Therefore, in the absence of adhesion, the coefficient of friction for the materials in footpads 2 and 3 of Lander 2 exceed the tangent of $38^{\circ}-50^{\circ}$ and the marginally stable material on footpad 3 of Lander 1 has a coefficient of friction near the tangent of $41^{\circ}$. When adhesion is involved and failure occurs, the stress resisting sliding $\left(\tau_{s}\right)$ must be less than the gravitational stress $\left(\tau_{\alpha}\right)$ :

where

$$
\tau_{s}=\tau_{a}+s \tan \delta
$$

$\tau_{s}=$ the stress required for sliding, and

$\tau_{a}=$ the adhesion.

If $\delta=0$, only adhesion resists sliding so that an upper limit for the adhesion of the material on footpad 3 of Lander 1 is near $\tau$. For a thickness of $0.3 \mathrm{~cm}, \delta=0$, a density of $1,200 \mathrm{~kg} / \mathrm{m}^{3}$, a slope of $41^{\circ}$, and $g=3.72 \mathrm{~m} / \mathrm{s}$, the upper limit for adhesion is about $9 \mathrm{~Pa}$. For Lander 2 with lumps as much as $0.7 \mathrm{~cm}$ and slopes as much as $50^{\circ}$, adhesion must be greater than $24 \mathrm{~Pa}$. For smooth metals, soil-metal friction coefficients are commonly $0.36\left(\tan 20^{\circ}\right)$. When this coefficient is used, the adhesion of the material on footpad 3 of Lander 1 is about $5 \mathrm{~Pa}$ and that for Lander 2 is greater than $16 \mathrm{~Pa}$. For Lander 1, adhesions calculated here are well within the range of a previous estimate of 0.9-79 Pa (Moore and others, 1977), however, a unique determination of adhesion for Lander 2 was never achieved.

The surface above footpad 2 of Lander 1 , which was immersed in drift material, slumped downward along an arc that overlies the rim and skirt of the buried footpad (fig. 88). Elsewhere, on either side of the arc of slumping, there does not appear to have been either upward or downward displacement of material. Apparently, compression of the material produced a void beneath the surface into which the materials collapsed.

\section{TRENCHES}

Generally, the walls and tailings of trenches were stable. Little or no modification to the walls occurred except shortly after excavation when small disrupted units collapsed toward the floor and formed small talus cones. This is the case for the walls of the deep holes as well as small trenches. As a result of trenching in Bonneville Salt Flats, rock 6 was initially pushed upward on Sols 29-30 (Moore and others, 1977); during the Extended Mission, rock 6 was partly undermined, causing it to rotate back toward

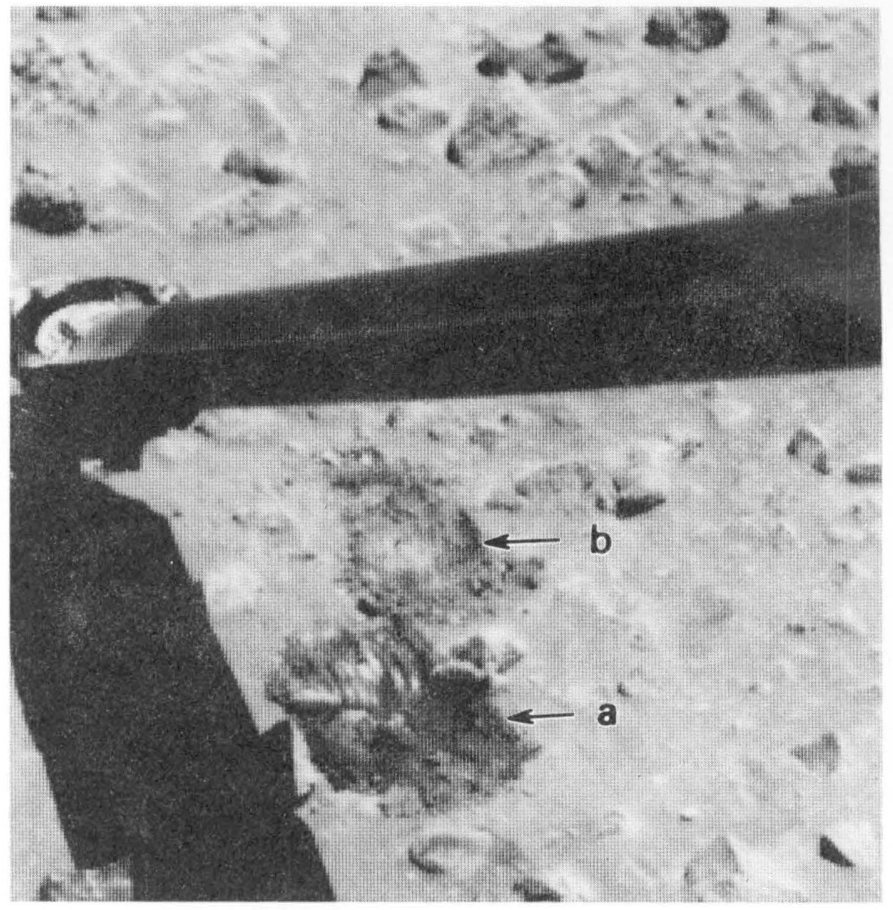

Figure 89.-Two backhoe-touchdown trenches (about $8 \mathrm{~cm}$ wide) in Rocky Flats showing effects of deposition of bright fine-grained material with time. Floor and tailings are darker for trench produced a few minutes before picture taken on Sol 639 (a) than they are for nearby trench produced on Sol 527 (b) or 112 sols earlier. Dark object extending across picture is boom extended for Backhoe Touchdown 8 (frame 11H238/639).

the spacecraft. Similar rotations of blocks in the disrupted region produced during the push of rock 4 on Sol 302 by Lander 1 occurred well after the initial disruption. Therefore, changes in and around trenches and other excavations were very slight.

Among the slight changes were sedimentation of finegrained material, from the atmosphere both naturally and as a result of surface-sampler activities. Because of this sedimentation of fine-grained material, trenches that appeared dark initially appeared lighter with time (Arvidson and others, 1979; Jones and others, 1979). This phenomenon is illustrated in figures 89 and 90 . A backhoetouchdown trench produced by Lander 1 on the same day as the picture was taken (fig. 89) appeared darker than its surroundings and a similar, nearby backhoe-touchdown trench produced about 112 sols earlier. Similarly, a trench produced by Lander 2 on Sol 595 appeared darker than its surroundings and other trenches produced 538 sols earlier at the end of the Primary Mission (fig. 90A).

In addition to the sedimentation of dust, a wintertime "snowfall" of dirty ice was observed at the Lander 2 site (Jones and others, 1979). Vestiges of the ice are visible, on surfaces near the lander, and on the floors of trenches around the bases in pictures taken on Sol 365 (fig. 90B). 


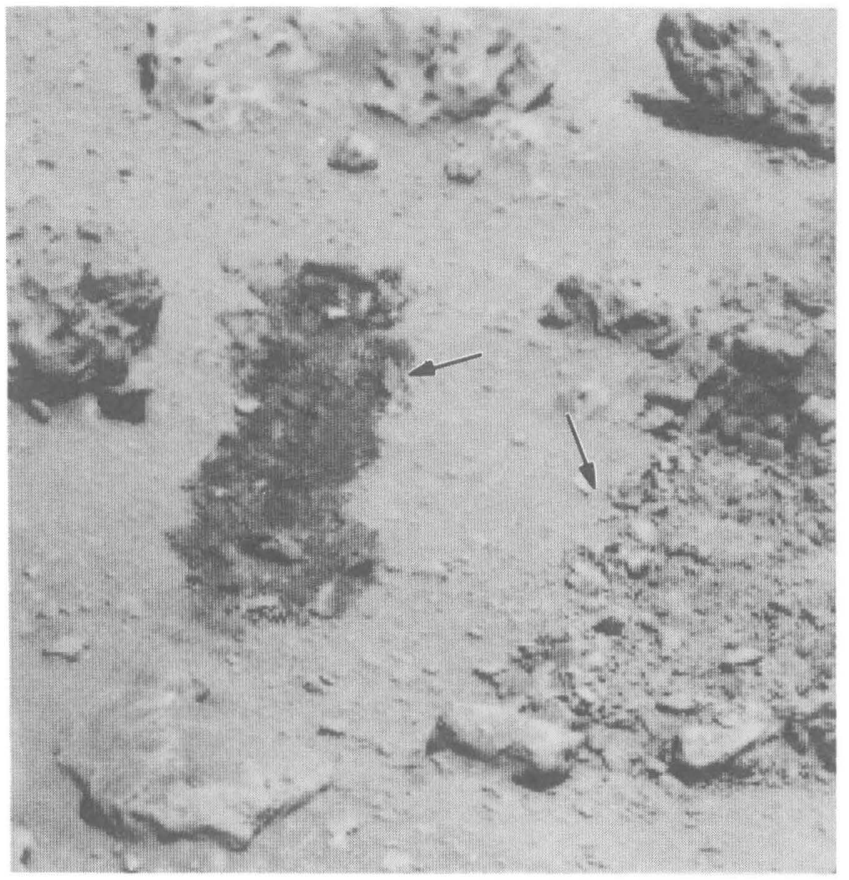

A

\section{NATURAL SURFACES}

Changes in the natural scene have been described previously and elsewhere by Arvidson and others (1979), Guinness and others $(1979,1982)$, and Jones and others (1979). Noteworthy is their observation that patches on a dune in front of Lander 1 changed from light to dark with time.

\section{CHIPS WITH ULTRAVIOLET SENSITIVE PAINTS}

Each Viking Lander has three reference test charts (RTC), and each test chart has two rectangular chips that darkened when exposed to UV (ultraviolet) radiation (Zent and others, 1980; Shorthill and others, 1972). On each test chart one chip is coated with antimony oxide and the other chip is coated with boron nitride. The first two test charts, mounted on the two RTG (radioisotope thermoelectric generator) covers were viewed by the corresponding camera with normal incidence, and the third test chart, mounted on the lander body, was viewed by both cameras.

Only 4 pictures of the 500 taken by Lander 1 proved useful for measuring the darkening of the UV chips. Most of the pictures were saturated and poorly illuminated; most Lander 2 pictures of the UV chips were not analyzed because they were taken in shadow (Zent and others, 1980). Furthermore, only the boron nitride chips were usable because pictures of the antimony oxide chips were saturated.

Changes in blue reflectances of the boron nitride chip (assumed to follow the Hapke-Irvine photometric function), which were viewed with normal incidence by camera 1 , changed from about 0.79 to about 0.60 after 1,500 hours of illumination. This change, when compared with laboratory data, indicates that the martian atmosphere is essentially transparent to UV radiation $(0.2-$ $0.4 \mu \mathrm{m})$ (Zent and others, 1980).

FIGURE 90.-Trenches of Lander 2 showing effects of deposition of bright fine-grained material and "snow fall." $A$, Arrows point to trenches. Dark trench (about $7.5 \mathrm{~cm}$ wide) was excavated on Sol 595 about 35 sols before picture was taken. Light-appearing trench to right was excavated 572 sols earlier on Sols 57 and 58 during Primary Mission (frame 22H090/630). B, Very bright patches of "snow" are present on floor of trench (about $50 \mathrm{~cm}$ long) excavated on Sol 172 (XRFS 7) (1), in foreground (2), and around bases of rocks in background (3). Earlier, "snow" was more extensive. View is toward north-northwest. Shadows of meteorology boom and sensors, camera 1, and other spacecraft parts are present in foreground (frame 21E153/365). 
TABLE 33.-Gases desorbed by humidification of samples in the Gas Exchange Experiment [After Oyama and Berdahl, 1977]

\begin{tabular}{|c|c|c|c|c|c|c|c|}
\hline \multirow{3}{*}{$\begin{array}{c}\text { Desorbed } \\
\text { gases }\end{array}$} & \multicolumn{2}{|c|}{ Lander 1} & & & \multicolumn{2}{|c|}{ Lander 2} & \\
\hline & \multicolumn{2}{|c|}{ Sandy Flats } & \multicolumn{2}{|c|}{ Beta Site } & & \multicolumn{2}{|c|}{ under Notch Rock } \\
\hline & (nanomols) & $(\mathrm{ppm})$ & (nanomols) & $(\mathrm{ppm})$ & & (nanomols) & (ppm) \\
\hline $\mathrm{Ar}$ & 13 & 0.40 & 4 & 0.12 & & 6 & 0.18 \\
\hline $\mathrm{N}_{2}$ & $8 \cdot 3$ & 1.8 & 30 & 0.6 & & 13 & 0.30 \\
\hline $\mathrm{O}_{2}^{c}$ & 790 & 9.7 & 190 & 2.3 & & $70-270$ & $0.9-3.3$ \\
\hline $\mathrm{CO}_{2}$ & 9,800 & 332 & 7,750 & 262 & & 6,110 & 207 \\
\hline
\end{tabular}

\section{SPACECRAFT MOVEMENT}

Lander 2 moved a small amount. Analyses of pictures of the footpads indicate that the lander rotated counterclockwise about $0.33^{\circ}$ and footpad 3 settled about $0.005 \mathrm{~m}$ by Sol 518 . The rotation increased to $0.46^{\circ}$ and footpad 3 was about $0.008 \mathrm{~m}$ lower by Sol 1212 than on Sol 0 (see fig. $87 A$ and $B$ ). This rotation affects stereometric and photogrammetric results when one picture was taken before and the other after a rotation. If no movement occurred between the times the two pictures were taken, the effects on stereometric and photogrammetric measurements were small for features in the sample field.

\section{OTHER VIKING EXPERIMENTS AND INVESTIGATIONS}

As noted previously, results from other scientific investigations provide information on the physical properties of the surface materials. Much of this information, which is briefly discussed below, could not be obtained with the tools available to the Physical Properties Investigation. Results from some investigations such as Viking Orbiter Imaging, Seismology, and Entry Science are excluded because the results were introduced previously.

\section{BIOLOGY}

The three biology experiments have yielded information on the physical and chemical properties of the martian surface materials; but, some doubts have arisen about the reliability of the information because the sample temperatures and (or) pressures in the experiment chambers were larger than those of the in situ surface material. For Lander 1, results were restricted to drift material because the samples that were analyzed came from Sandy Flats; one sample (Biology 4) was acquired from the Deep Hole 1 in Sandy Flats, the other samples were collected from normal sample trenches about $5 \mathrm{~cm}$ deep. For
Lander 2, three samples were acquired from the interlayered fine-grained material and crust of the Beta site and one sample from the material originally beneath rock 7 (Notch, pl. $2 A$ ).

\section{GAS EXCHANGE}

Gases desorbed (see table 33) by humidification of samples from drift material in the gas exchange instrument have been interpreted to come from physically and chemically adsorbed gases that were displaced by water adsorption (Ballou and others, 1978). The results of the analyses of $\mathrm{CO}_{2}, \mathrm{~N}_{2}$, and $\mathrm{CO}$ or Ar were found to lie along a straight line on an adsorption potential plot; this result is consistent with physical adsorption on grain surfaces of drift material. The amounts of evolved $\mathrm{O}_{2}$, though small (1-10 ppm), were larger than expected for physical adsorption, and this fact suggests that a chemical reaction occurred that liberated the $\mathrm{O}_{2}$. The specific surface area required for the physical adsorption of the evolved gases is about $1 \mathrm{~m}^{2} / \mathrm{g}$. For mineral grains of one size and a grain density of $2,600 \mathrm{~kg} / \mathrm{m}^{3}$, the corresponding size of the grains would be about $2 \mu \mathrm{m}$. This size is at the boundary between fine silt and clay. Extrapolating the data to the boiling point of liquid nitrogen and plotting the data using standard procedures suggest that the specificsurface area could be as large as $17 \mathrm{~m}^{2} / \mathrm{g}$ (Ballou and others, 1978). The corresponding grain size for this specific surface area is about $0.14 \mu \mathrm{m}$, which is in the claysize range.

For the samples of Lander 2, the amounts of desorbed gases were less than those of Lander 1, and the amounts of desorbed gases were larger for the samples from the exposed Beta site than they were for the sample from under the rock (Oyama and Berdahl, 1977). Reduced amounts of desorbed gases could be attributed to a larger (but still small) grain size, different mineral phases, or more adsorbed water. Oyama and Berdahl (1977) postu- 
lated that the mineralogy and specific surface areas were the same for both the Lander 1 and 2 samples, and then ascribed the differences in desorbed gases to the amount of adsorbed water. The interpretation based on their data seems reasonable because the amount of adsorbed water is a direct function of the aridity of the environment or atmosphere from which the samples were acquired. For the global scale environment, maximum amounts of atmospheric water vapor were less in the lower latitudes of Lander 1 than in the higher latitudes of Lander 2 (Farmer and others, 1977); and, for the local environment of Lander 2, smaller amounts of water would be expected to occur in materials exposed to the atmosphere than in materials covered by rocks (Jury and Bellantuoni, 1976a,b).

Anomalous amounts of evolved $\mathrm{O}_{2}$ were interpreted to be the result of chemical reactions of water vapor and reactive substances such as ozone, alkaline earth and alkali metal peroxides and superoxides such as those of $\mathrm{K}, \mathrm{Ca}$, and $\mathrm{Na}$ (Oyama and Berdahl, 1977). Subsequent tests in which a sample was heated to, apparently, $160^{\circ} \mathrm{C}$ and then humidified showed that the evolved $\mathrm{O}_{2}$ could not have been a biologic reaction because such a temperature would kill biota. However, superoxides such as $\mathrm{KO}_{2}$ and $\mathrm{Ca}\left(\mathrm{O}_{2}\right)_{2}$ could have been present because they are stable to temperatures of $425{ }^{\circ} \mathrm{C}$ and $290{ }^{\circ} \mathrm{C}$, respectively.

Thus, the results of the Gas Exchange Experiment showed that the surface materials analyzed were very fine grained and contained physically adsorbed $\mathrm{CO}_{2}, \mathrm{~N}_{2}$, and $\mathrm{CO}$ or Ar. Oxygen apparently was present as oxides, peroxides, and (or) superoxides of some sort.

The specific-surface areas do not prove or disprove the existence of clay minerals. The specific surface area $\left(17 \mathrm{~m}^{2} / \mathrm{g}\right)$ derived for drift material is compatible with surface areas of quartz crushed to pass a $10 \mu \mathrm{m}$ sieve (Brown and others, 1960) and of some natural clays (Mooney and others, 1952). The specific surfaces of kaolinite, measured by the adsorption of $\mathrm{N}_{2}$, range from 17 to $40 \mathrm{~m}^{2} / \mathrm{g}$, and the specific surfaces of montmorillonite range from 27 to $100 \mathrm{~m}^{2} / \mathrm{g}$. With water adsorption, the specific surface areas of some clays are much larger; some may be as large as $800-1,000 \mathrm{~m}^{2} / \mathrm{g}$. The larger specific-surface areas were interpreted to be the result of penetration of water between layers in unit cells of clay particles; the smaller areas were interpreted to be the result of nitrogen adsorption on the external areas of the clay particles (Mooney and others, 1952).

\section{LABELED RELEASE}

The results of the Labeled Release Experiment were consistent with the presence of life on Mars (Levin and Straat, 1977) as well as with the abiogenic reactions between the nutrients used in the experiment and a variety of substances. In simulation experiments with montmorillonite and nontronite clays containing adsorbed ions of iron (especially ferric) and hydrogen, it was possible to simulate the rapid release of ${ }^{14} \mathrm{C}$-labeled gas, followed by reduced reaction rates, prolonged slow release of gas, and the total amount of gas released (Banin and Rishpon, 1979). Montmorillonite and nontronite with adsorbed ions of calcium, sodium, and aluminum did not reproduce the reactions observed on Mars. Complete inhibition of the reactions by preheating the sample to $160^{\circ} \mathrm{C}$ for $3 \mathrm{~h}$ was never achieved as it was on Mars, but the addition of salts $\left(\mathrm{MgSO}_{4}\right.$ and $\left.\mathrm{CaCO}_{3}\right)$ postulated to exist on Mars did inhibit the reactions somewhat. Apparently the addition of maghemite $\left(\gamma-\mathrm{Fe}_{2} \mathrm{O}_{3}\right)$ inhibited the reaction. It is also questionable that montmorillonite-type clays are the only materials that will simulate the results. The results of Banin and Rishpon (1979) have been disputed by Levin and Straat (1981). They found that silica sand, $\mathrm{H}_{2} \mathrm{O}_{2}$, and $\gamma-\mathrm{Fe}_{2} \mathrm{O}_{3}$ will simulate the labeled release reactions observed on Mars.

\section{CARBON ASSIMILATION OR PYROLYTIC RELEASE}

Apparently, no entirely satisfactory analog has been found which duplicates the results of the Pyrolytic Release Experiment, but montmorillonite with adsorbed iron ions and maghemite produced reactions similar to those of the Viking tests on Mars (Hubbard, 1979). Hubbard believed that a model for martian soil can be selected with little confidence because of inadequate data about the minerals composing the materials.

\section{MOLECULAR ANALYSIS}

Although no organic compounds were identified in the gas chromatograph-mass spectrometer (GCMS) that could be related to the martian surface materials, rather crude estimates of the amounts of water and carbon dioxide in the samples were determined; nitrogen and sulfurcontaining substances were not detected at levels of mineralogical significance (Biemann and others, 1977). Samples for analyses came from a variety of environments. The two samples of Lander 1 came from Sandy Flats (GCMS 1) and Rocky Flats (GCMS 3). The two samples of Lander 2 came from Bonneville Salt Flats (GCMS 1) and from the surface originally under rock 3 (Badger).

Because analyses of the samples of Lander 1 were conducted using the ${ }^{13} \mathrm{CO}_{2}$ mode, it was only possible to obtain very crude estimates of water contents and no estimates of $\mathrm{CO}_{2}$. The sample from Sandy Flats, heated to $200^{\circ} \mathrm{C}$, produced less than 0.1 percent water, but the sample, heated to $500^{\circ} \mathrm{C}$, produced somewhere between 0.1-1.0 percent (table 34 ). The Rocky Flats sample pro- 
duced 0.1-1.0 percent water upon heating to $350{ }^{\circ} \mathrm{C}$, $500{ }^{\circ} \mathrm{C}$, and, then again, to $500^{\circ} \mathrm{C}$. For Lander 2, better but still crude estimates of water content were obtained because the GCMS was operated in the $\mathrm{H}_{2}$ mode as well as the ${ }^{13} \mathrm{CO}_{2}$ mode (table 34). For the Bonneville Salt Flats sample heated to $200^{\circ}, 350^{\circ}$, and $500{ }^{\circ} \mathrm{C}$, the amounts of $\mathrm{CO}_{2}$ were $<50,50-500$, and $50-500 \mathrm{ppm}$, and the amounts of water were $0.05,0.3$, and 1.0 percent by weight. Heating again to $500{ }^{\circ} \mathrm{C}$ in the ${ }^{13} \mathrm{CO}_{2}$ mode produced 0.25 percent water. For the sample under Badger heated to $50^{\circ}, 200^{\circ}, 350^{\circ}$, and $500^{\circ} \mathrm{C}$, the amounts of $\mathrm{CO}_{2}$ were $<50,50-500,40-400$, and $70-700 \mathrm{ppm}$ and the amounts of water were $<0.01,0.2,0.3$, and 0.8 percent by weight. Heating again to $500{ }^{\circ} \mathrm{C}$ in the ${ }^{13} \mathrm{CO}_{2}$ mode produced 0.6 percent water. The most distinctive differences among the samples were the larger amounts of $\mathrm{H}_{2} \mathrm{O}$ and $\mathrm{CO}_{2}$ obtained from the Badger sample when it was heated to $200^{\circ} \mathrm{C}$; and, perhaps the smaller amounts of water obtained from both Lander 2 samples when they were reheated to $500{ }^{\circ} \mathrm{C}$ (Biemann and others, 1977).

A number of factors contributed to the difficulty in interpreting the analyses: (1) The amounts of water and $\mathrm{CO}_{2}$ were crude estimates; (2) equilibrium was probably not attained because the samples were heated for such short intervals (30 s); (3) the chemical and mineralogical properties of the surface materials were poorly understood; and (4) the potential reactions involving $\mathrm{H}_{2}$ could not be definitely identified. Despite the lack of demonstrable equilibrium, it seems that the rather large sulfur concentrations, observed with the X-ray fluorescence spectrometer, were stable compounds such as sulfates, and the low vapor pressure of calcium carbonate suggests other sources of $\mathrm{CO}_{2}$ (Biemann and others, 1977). Samples from the Beta site and from under Notch rock, which were analyzed in the gas exchange instrument, contained about 7,750 and 6,110 nano-mols of $\mathrm{CO}_{2}$ adsorbed on their surfaces (Oyama and Berdahl, 1977).

TABLE 34.-Estimates of water and carbon dioxide in samples analyzed in the gas chromatograph-mass spectrometer for the Molecular Analysis Experiment

[After Biemann and others, 1977]

\begin{tabular}{ccccc}
\hline $\begin{array}{c}\text { Sample } \\
\text { location }\end{array}$ & $\begin{array}{c}\text { Oven heating } \\
\text { temperature, }\end{array}$ & Mode & $\begin{array}{c}\text { Water } \\
\text { (weight percent) }\end{array}$ & $\begin{array}{c}\mathrm{CO}_{2} \\
\text { (ppm) }\end{array}$ \\
\hline Lander 1, & 200 & $13 \mathrm{CO}_{2}$ & $<0.1$ & -- \\
$\begin{array}{c}\text { Sandy Flats } \\
\text { Lander 1, }\end{array}$ & 500 & $13 \mathrm{CO}_{2}$ & $.1-1.0$ & -- \\
Rocky Flats & 350 & $13 \mathrm{CO}_{2}$ & $.1-1.0$ & -- \\
& 500 & $13 \mathrm{CO}_{2}$ & $.1-1.0$ & -- \\
Lander 2, & 500 & $13 \mathrm{CO}_{2}$ & $.1-1.0$ & -- \\
Bonneville & 200 & $\mathrm{H}_{2}$ & .05 & $<50$ \\
Salt Flats & 350 & $\mathrm{H}_{2}$ & .3 & $50-500$ \\
& 500 & $\mathrm{H}_{2}$ & 1.0 & $50-500$ \\
Lander 2, & 500 & $13 \mathrm{CO}_{2}$ & .25 & -- \\
under Badger & 50 & $\mathrm{H}_{2}$ & $<.01$ & $<50$ \\
& 200 & $\mathrm{H}_{2}$ & .2 & $50-500$ \\
& 350 & $\mathrm{H}_{2}$ & .3 & $40-400$ \\
& 500 & $\mathrm{H}_{2}$ & .3 & $70-700$ \\
\hline
\end{tabular}

These amounts of $\mathrm{CO}_{2}$ correspond to about 262 and 207 ppm $\mathrm{CO}_{2}$. It is difficult to ascribe the $\mathrm{CO}_{2}$ obtained by heating to $200{ }^{\circ} \mathrm{C}$ (table 34) to adsorption because the amounts obtained from the exposed samples and from under the rock samples were so different; also the amounts for the exposed samples were much lower than the amounts inferred by the biologists. Amounts of $\mathrm{CO}_{2}$ that were obtained at all the temperatures in the GCMS could be considered consistent with the amounts of $\mathrm{CO}_{2}$ desorbed from the biology samples. Several thousand ppm (and even larger amounts) of $\mathrm{CO}_{2}$ were adsorbed by nontronite and crushed basalts in experiments at temperatures of the surface materials (Fanale and Cannon, 1979), so that the presence of nontronite and crushed basalts on Mars would seem to be unlikely. However, desorption of $\mathrm{CO}_{2}$ could have occurred prior to analyses of the Viking samples; before the samples were analyzed, the samples were subjected to temperatures that ranged from $282^{\circ}$ to $298^{\circ} \mathrm{K}$ for, in some cases, days.

The nature of the evolved water was also difficult to assess. The water might have come from the peroxides or superoxides (which were postulated to exist in the surface materials [Oyama and Berdahl, 1977]) by reactions with hydrogen, particularly when the samples were analyzed in the $\mathrm{H}_{2}$ mode of the GCMS at the higher temperatures. However, the water could also have been adsorbed in a clayey material. When the relative humidity of water vapor is 0.1 or less, the heat of desorption of water on clay exceeds $12.6 \mathrm{kcals}$ per mole of $\mathrm{H}_{2} \mathrm{O}$ (Mooney and others, 1952); this amount of heat of desorption is larger than the heat of vaporization of ice-12.2 kcals per mole. This fact suggests that adsorbed water in clays would be the stable form of water under some conditions. Using a nominal value of $220^{\circ} \mathrm{K}$ for the surface materials at Lander 2 (Kieffer, 1976), the vapor pressure of ice is about $2.4 \times 10^{-5}$ bars. If the water-vapor pressure at the site is $2.4 \times 10^{-6}$ bars (or $\approx 70$ precipitable microns), one obtains a relative humidity of 0.1 ; therefore, the surface material could have about 5 percent adsorbed water by weight. A relative humidity of 0.04 ( 28 precipitable microns) would reduce the amount of adsorbed water to about 2 percent (Anderson and others, 1978) and increase the heat of desorption to about $13.5 \mathrm{kcals}$ per mole of $\mathrm{H}_{2} \mathrm{O}$; thus it seems that the percent of water measured by the GCMS could have been adsorbed water. The hypothesis of adsorbed water is supported by analogy between the large amount of water from the sample beneath Badger that was obtained at $200{ }^{\circ} \mathrm{C}(0.2 \mathrm{wt}$ percent $)$ and the behavior of water in arid terrestrial environments; in such places water tends to migrate to areas beneath rocks (Jury and Bellantuoni, 1976a,b). The surface required to hold 0.2 wt percent of $\mathrm{H}_{2} \mathrm{O}$ is only $7.23 \mathrm{~m}^{2} / \mathrm{g}$ and less than the specific-surface of $17.2 \mathrm{~m}^{2} / \mathrm{g}$ obtained by Ballou and others (1978). There are, of course, other 
explanations such as dehydroxylation of a variety of minerals (Biemann and others, 1977).

\section{INORGANIC CHEMICAL ANALYSIS}

The four important results obtained from the Inorganic Chemical Analysis Experiment were: (1) the sampled materials can reasonably be inferred to be silicates and oxides so that unusually peculiar physical properties are improbable; (2) the chemical compositions of all the samples that were analyzed are remarkably similar; (3) the minor differences in compositions seem to correlate with the type of material; and (4) the inferred bulk densities of the in situ surface materials vary widely.

Toulmin and others (1977), in their summary of the composition of the sampled materials (table 35), stated that the most plausible mineralogical model is a mixture of predominately iron-rich clays (or their degradation products) with ferric oxide, stable carbonate, and sulfate minerals that locally cement the fine-grained material into a duricrust. Apparently, several clay minerals such as nontronite and bentonite would be required (Baird and others, 1977). Some other minerals that could be present include kieserite $\left(\mathrm{MgSO}_{4} \cdot \mathrm{H}_{2} \mathrm{O}\right)$, maghemite $\left(\gamma-\mathrm{Fe}_{2} \mathrm{O}_{3}\right)$, magnetite $\left(\mathrm{Fe}_{3} \mathrm{O}_{4}\right)$, halite $(\mathrm{NaCl})$, and leucoxene $\left(\mathrm{TiO}_{2}\right)$. Iron oxides would probably occur as particles admixed with other mineral particles rather than as coatings on other minerals. It is important to realize that there are no data to prove the existence of montmorillonite-type clays or any other minerals. Oxides, carbonates, sulfates, some feldspars, and kaolinite may be the minerals present because they should be thermodynamically stable in the martian environment (Gooding, 1978). The compositions of the surface materials are consistent with alteration products of mafic igneous rocks-possibly palagonitelike products produced by encounters of mafic magmas with ice-rich deposits (Toulmin and others, 1977; Allen and others, 1981).

Compositions of the samples at the two widely separated sites are similar. Drift, crusty to cloddy, and blocky materials have essentially the same compositions except for small differences in the content of sulfur and chlorine (table 35). Samples obtained from various depths by skimming, from normal trenches, and from the deep holes were similar to each other as well as to those taken from under rocks. The similarities are attributed to a homogenizing process, such as aeolian transport.

The small differences in sulfur and chlorine seem to correlate with the type of materials and their physical properties. The drift material had relatively small amounts of sulfur and chlorine, crusty to cloddy material had intermediate amounts, and blocky material had relatively large amounts. Thus, cementation by sulfur and chlorine minerals may have caused the large cohesions of 
blocky material; the large cohesions, in turn, permitted the acquisition of samples of coarse material. The relatively large abundance of crusts and clods of the Lander 2 material, in contrast with the drift material, might also reflect small differences in cohesion related to cementation by sulfur and chlorine minerals.

Estimates of bulk densities of materials in the analysis chamber were useful despite the fact that the materials were in the disturbed state. Apparently, a reasonable estimate of the bulk density of the fine-grained material in the XRFS analysis chamber of Lander 1 is $1,100 \pm 150$ $\mathrm{kg} / \mathrm{m}^{3}$ (table 36 ); this estimate corresponds to a material with a porosity of 58 percent if the density of the individual mineral grains was $2,600 \mathrm{~kg} / \mathrm{m}^{3}$. The in situ density of drift material may be fairly close to the density of the fine-grained material in the analytical chamber. Samples of the coarse fraction-composed of fragments of blocky material-ranged from 570 to $940 \mathrm{~kg} / \mathrm{m}^{3}$ (table 36). In the standard interpretation, the pore space between the fragments is assumed to be 50 percent. If this is so, the bulk densities of the individual fragments ranged from 1,140 to $1,880 \mathrm{~kg} / \mathrm{m}^{3}$, but if the porosity was 60 percent the bulk densities of the fragments would range from 1,400 to $2,300 \mathrm{~kg} / \mathrm{m}^{3}$ (table 36 ). Thus, bulk densities of in situ blocky material could range from about 1,100 to $2,300 \mathrm{~kg} / \mathrm{m}^{3}$.

\section{MAGNETIC PROPERTIES}

Magnetic materials that have the same color as the surface materials in the sample fields were attracted to the magnets on the reference test charts and backhoes of the surface samplers of both landers (Hargraves and others, $1977,1979)$. Materials initially attracted to the magnets of the reference test charts were probably acquired from settling dust introduced into the atmosphere by engineexhaust gases during landing, and subsequent material mostly came from surface-sampler activities-particularly the delivery of samples to the analytical experiments.
Somewhat larger amounts of material settled on the magnets of Lander 1 during the Primary Mission, than on the magnets of Lander 2, and the amounts of material seemed to relate to wind speeds and directions during landing and surface-sampler activities. For Lander 1, wind directions tended to propel dust toward the magnets on the test chart whereas, for Lander 2, the directions tended to propel dust away from the magnets. Wind speeds tended to be smaller for Lander 1 than Lander 2 .

The first high-resolution picture of the magnets on the backhoe of the collector head of the surface sampler of Lander 1, taken before sampling, revealed that the magnets were clean (Hargraves and others, 1977, 1979). Subsequent high-resolution pictures, which were not obtained until after six sample acquisitions, showed that both the weak and strong magnets were either saturated with magnetic material or nearly so. The backhoe magnets of Lander 2 were almost completely saturated after three sample acquisitions. This saturation of the backhoe magnets of both landers persisted throughout the Primary Mission, and thicknesses of the magnetic materials were, roughly, 2-4 $\mathrm{mm}$. Two objects about $2-3 \mathrm{~mm}$ in diameter remained attached to the strong magnets on the backhoes of both landers.

During the Extended Mission, a successful controlled experiment was conducted to determine how the magnets became saturated (Hargraves and others, 1979). First the backhoe magnets were cleaned by passing them through the wire brushes mounted on the lander body, and then the cleaned backhoe magnets were inserted into the tailings in front of rock 3 (Badger; Backhoe Touchdown 4, Lander 2) by deelevation of the collector head three times using three extensions $(83.3,86.5$, and $89.4 \mathrm{in}$.). After each surface contact, a second deelevation command of $1 \mathrm{~s}$ duration was executed to insure deep penetration of the backhoes. Pictures were taken of the backhoe in the tailings immediately after the first insertion and of the inverted backhoe elevated in front of the lander after each

TABLE 36.-Bulk densities of disturbed materials in the full-analytical chamber of the $X$-ray-fluorescence spectrometer and inferred fragment (or mineral) densities for two assumed porosities, and derived porosity of sample or fragments for a mineral density of $2,600 \mathrm{~kg} / \mathrm{m}^{3}$

[Lander 1 fine samples from Clark, B.C., and others, 1977. XRFS 2, 4, 17 coarse samples from data furnished by Clark, B.C., Weldon, Ray, and Castro, A.J., (15 May 1978)]

\begin{tabular}{|c|c|c|c|c|}
\hline $\begin{array}{c}\text { Sample location } \\
\text { and type }\end{array}$ & $\begin{array}{c}\text { Bulk density } \\
\text { of sample }\end{array}$ & $\begin{array}{l}\text { Density of } \\
\text { fragments at } \\
50 \text { percent } \\
\text { porosity }\end{array}$ & $\begin{array}{l}\text { Porosity of } \\
\text { fragments or } \\
\text { sample if } \\
\text { mineral grain } \\
\text { density is } \\
2,600 \mathrm{~kg} / \mathrm{m}^{3} \\
\text { (percent) }\end{array}$ & $\begin{array}{l}\text { Density of } \\
\text { Fragments or } \\
\text { mineral grains } \\
\text { at } 60 \text { percent } \\
\text { porosity } \\
\left(\mathrm{kg} / \mathrm{m}^{3}\right)\end{array}$ \\
\hline $\begin{array}{l}\text { Lander } 1, \text { fine } \\
\text { XRFS } 2(C-2), \\
\text { XRFS } 4(C-5) \\
\text { XRFS } 17(C-13),\end{array}$ & $\begin{array}{c}1.100 \pm 150 \\
570 \\
940 \\
850\end{array}$ & $\begin{array}{c}- \\
1,140 \\
1,880 \\
1,700\end{array}$ & $\begin{array}{c}58 \pm 6 \\
56 \\
31 \\
35\end{array}$ & $\begin{array}{l}2,750 \\
1,425 \\
2,350 \\
2,125\end{array}$ \\
\hline
\end{tabular}


insertion. The picture taken of the inserted backhoe showed that penetrations of the backhoe were deep enough to completely bury the magnets. In subsequent pictures, the strong magnet was saturated after the first insertion; both magnets were saturated after the second insertion, and both remained saturated after the third insertion (Hargraves and others, 1979). The areal extent of material covering both magnets after two or more insertions was almost unity-a characteristic unique to the martian material that was not observed in terrestrial tests of the magnets (Hargraves and others, 1979).

Hargraves and others $(1977,1979)$ suggest that grains with coatings (less than $0.25 \mu \mathrm{m}$ thick) or dispersed crystallites of the magnetic mineral maghemite $\left(\gamma-\mathrm{Fe}_{2} \mathrm{O}_{3}\right)$ in concentrations of 1-7 percent seem to possess the magnetic properties of the material attracted to the lander magnets. Large hematite grains would not have been attracted to the magnets on the reference test charts and $1-\mu \mathrm{m}$ grains would be removed by the winds. Both the color reflectance data and the slow accumulation rate of the material favor maghemite. The materials on the backhoe magnets probably are composite particles (coated grains or grains containing crystallites) because the spectral reflectivities of the magnetic fraction and of the surface materials from the trenches were the same; both magnets became saturated after two insertions, and the areas covered by material on each of the strong and weak materials were almost equal. The attraction and retention of 2- to 3-mm particles on the strong magnets suggests that magnetic fraction is 1-7 percent by weight of the surface materials. Apparently, the quantitative significance of rather large thicknesses $(2-4 \mathrm{~mm})$ of material that adhered to the weak magnets has not yet been fully assessed. There are other reasons why maghemite is the preferred magnetic mineral (Hargraves and others, 1977, 1979): (1) a ferric compound, such as maghemite, should be stable in the oxidizing environment inferred from the Gas Exchange Experiment, and the probable fine-grain size of the material would favor the oxidization of iron; (2) maghemite could serve as a catalyst, and a catalyst would help to explain the results of the Pyrolytic Release Experiment; (3) the spectral reflectivity of the material was yellowish brown, and was comparable with the surface material; and (4) maghemite, a dehydroxylation product of nontronite clay, has been postulated to be or to have been present in the surface material. Another possibility for the magnetic fraction is feroxyhyte ( $\delta-\mathrm{FeOOH})$ (Burns, 1980).

If the magnetic fraction is maghemite and does coat the grains uniformly, an upper limit to the grain size can be estimated. The amount of maghemite is limited by chemical analysis (18 percent $\gamma-\mathrm{Fe}_{2} \mathrm{O}_{3}$ ) and the requirements to cover both the weak and strong magnets (3-4 percent $\gamma-\mathrm{Fe}_{2} \mathrm{O}_{3}$ ); the thickness of the coating must be less than $0.25 \mu \mathrm{m}$ to explain the inorganic chemical analyses (Hargraves and others, 1979). Hargraves and others $(1979$, table 1$)$ showed that the grain size must be less than 50 or $60 \mu \mathrm{m}$ for 3-4 percent $\mathrm{Fe}_{2} \mathrm{O}_{3}$ and $10 \mu \mathrm{m}$ for 18 percent $\mathrm{Fe}_{2} \mathrm{O}_{3}$. Smaller grains with thinner coatings would also be possible.

\section{METEOROLOGY}

A wealth of information on the weather of Mars was provided by the Meteorology Experiment (Hess and others, 1980; Ryan and others, 1978; Ryan and Henry, 1979). Mean daily pressures, which were about $0.7-1.2$ mb smaller at the higher Lander 1 site than at the lower Lander 2 site, fluctuated with the season (Hess and others, 1980). The lowest pressures occurred in late summer $(6.8$ and $7.5 \mathrm{mb})$ and the highest pressures occurred around the winter solstice $(8.8$ and $10.0 \mathrm{mb})$. Pressures dropped ( 8.0 and $8.7 \mathrm{mb})$ just before the spring equinox, and then increased in early spring $(8.3$ and $9.0 \mathrm{mb})$ before returning to the seasonal lows in late summer. These seasonal changes were ascribed to seasonal evaporation and condensation of $\mathrm{CO}_{2}$-particularly at the south pole (Hess and others, 1979, 1980).

Air temperatures and winds were mentioned and summarized previously. Two global dust storms (at Lander 1 on Sols 209 and 312) were remarkable. The storms were accompanied by high winds and by peak gust velocities $(20-26 \mathrm{~m} / \mathrm{s})$ and sudden drops in the maximum daily temperature $\left(12^{\circ} \mathrm{K}\right)$ followed by rapid increases in the minimum daily temperature $\left(12^{\circ} \mathrm{K}\right)$ a few sols later. Also, mean seasonal temperatures decreased from about $220^{\circ} \mathrm{K}$ in the late summer to about $190^{\circ} \mathrm{K}$ in the winter. Later, during a local dust storm at the Lander 1 site on Sol 432, wind gusts ranged from 25 to $30 \mathrm{~m} / \mathrm{s}$ (Ryan and others, 1981).

\section{LANDER IMAGING}

Three observations by the Lander Imaging Team are relevant to the Physical Properties Investigation. First, the subtle color differences in the fine-grained surface materials at the sites were accounted for by differences in grain size (Guinness, 1981). Second, the thin layers of bright-reddish dust, several $\mu \mathrm{m}$ thick, were deposited on the surface during the global dust storms (Arvidson and others, 1979; Jones and others, 1979; Guinness and others, 1979, 1982). And third, the condensates or "snow" were deposited on surfaces at the Lander 2 site during the winter dust storms (Guinness and others, 1979, 1982; Jones and others, 1979).

\section{RADIO SCIENCE}

Relative dielectric constants for the uppermost $2.5 \mathrm{~m}$ of the martian surface were determined from reflection coefficients of a broad area of the surface at a distances 
between 1.7-10 $\mathrm{m}$ from the lander using the lander (transmitter) and orbiter (receiver) communications links (Tang and others, 1977). The area sampled by radio waves was determined by the location of the antenna (fig. 1, UHF antenna) so that the area obscured by spacecraft parts was excluded. The frequency was $381 \mathrm{MHz}$. A dielectric constant of $3.3 \pm 0.7$ was obtained for Lander 1 , and values for Lander 2 ranged from 2.8 to 12.5. Although physical interpretations of the dielectric constants were not unique, some possibilities could be excluded. A variety of materials with a variety of physical properties was measured in the laboratory (Campbell and Ulrichs, 1969), but none of the materials was dry clay. Dry rock powders with bulk densities of $1,000 \mathrm{~kg} / \mathrm{m}^{3}$ can be excluded for the martian materials sampled by the reflected radio transmissions because dielectric constants of such materials are about $2.0 \pm 0.2$ when measured at $450 \mathrm{MHz}$. Less dense material yielded even lower dielectric constants. Rock powders with porosities of 40 percent had dielectric constants between 2.5 and 3.5; powders of mafic rocks had higher relative dielectric constants than powders of silicic rocks. A few porous rocks such as pumice, semiwelded tuffs, shales, and volcanic ash had dielectric constants that ranged from 2.6 to 4.0 , but dense mafic rocks had dielectric constants as much as 9 or 10 .

Dielectric constants between 2.6 and 4.0 seemed reasonable for blocky material. For a silicic rock with a grain density of $2,600 \mathrm{~kg} / \mathrm{m}^{3}$ and a porosity of 40 percent (dielectric constant $\approx 2.6$ ), the bulk density would be about $1,560 \mathrm{~kg} / \mathrm{m}^{3}$, and for a mafic rock with a grain density of $2,900 \mathrm{~kg} / \mathrm{m}^{3}$ and a porosity of 40 percent (dielectric constant $\approx 3.4$ ), the bulk density would be about 1,800 $\mathrm{kg} / \mathrm{m}^{3}$. The values above are comparable with the high estimates of densities of fragments of blocky material in the XRFS chamber. The high range of dielectric constant (4.0) could be caused by rocks in blocky material or higher densities (that is, lower porosities), or both.

Dielectric constants from Lander 2 also seemed reasonable and compatible with the appearance of the surface and other results. The type of failure and the large angles of internal friction of the surface materials imply failure by general shear. General shear requires low porosities of granular materials. Thus, a porosity of 30-40 percent seems reasonable, and this value would yield dielectric constants of about 2.8-3.0. The upper values of the dielectric constant could be met by the abundance of rocks. The rock strewn surface, when viewed at a small angle of incidence, would appear to be all rocks to the radio waves, however, a dielectric constant of 12.5 does seem to be somewhat high for normal rocks.

Data on dielectric constants of terrestrial soils are scarce. Sandy, loamy, and clayey soils of unspecified density have dielectric constants about 2.4-2.5 when measured at $300 \mathrm{MHz}$ (Von Hippel, 1954). The presence of water causes marked increases in dielectric constants ranging from 3.5 to 4.5 for 2 to 4 percent water and as much as 20 for 19 to 20 percent water. Water ice, if present, would be the most probable phase at the low temperatures and pressures of the martian surface materials; water ice has a dielectric constant of 3.0 and would not be detected by the radio waves.

\section{INFRARED THERMAL MAPPING}

Analyses of orbital measurements of surface temperatures have yielded thermal inertias (I) ${ }^{4}$ of the landing sites that may be interpreted in terms of grain size alone or as mixtures of materials with different thermal inertias, such as rocks, powders, and other materials (Kieffer and others, 1977). Interpretation of the thermal inertias is, at best, difficult because several kinds of materials were present at both sites and atmospheric pressure influences the thermal inertias of different types of material in different ways (Wechsler and Glaser, 1965). On Mars, thermal inertia is influenced by atmospheric pressure, and atmospheric pressure varies with elevation, material types and layering, shadowing during insolation, viewing conditions, and atmospheric opacity (Jakosky, 1979).

Nevertheless, an attempt should be made to interpret the thermal inertias of the landing sites in the light of surface data collected by the landers. The thermal inertias for the Lander 1 and 2 sites are $9 \pm 0.5$ and $8 \pm 1.5$, respectively. Following Kieffer and others (1977, fig. 10), direct interpretation of the inertias would be consistent with granular cohesionless silicates in a 600-Pa atmosphere that have grain sizes of $800 \mu \mathrm{m}$ (Lander 1) and $600 \mu \mathrm{m}$ (Lander 2) (fig. 91). Lander data, however, require at least a two-component system because roughly 10 percent (Lander 1) and 15 percent (Lander 2) of the sample fields are covered by rocks (I. taken as 40 ). Thus, a twocomponent system at Lander 1 would have about 10 percent of the material with $\mathrm{I}=40$ (rocks) and 90 percent of the material with $I=7.6$, and a two-component system at Lander 2 would have about 15 percent of the material with $I=40$ (rocks) and 85 percent of the material with $I=6.3$. Although the inertias for most of the materials become lower in the two components systems than those in the one-component systems, the implied grain sizes $(500 \mu \mathrm{m}$ and $300 \mu \mathrm{m}$ ) seem to be too large when compared with the other information. Lander 1 requires a three-component model of drift material, blocky material, and rocks. The drift material appears to be the closest material to a powder $(I=3)$, and it covers roughly 15 percent of the sample field as thick deposits. Thus, the remaining 85 percent of the material has an inertia of about 10.8. The

\footnotetext{
4The thermal inertial $(I)$ is the square root of the product of bulk density of the material $(\rho)$, its specific heat $(c)$, and its thermal conductivity $(k)$, for example, $(k \rho c)^{t_{h}}$. It is reported here as $10^{-3} \mathrm{cgs}$ units.
} 
remaining 85 percent of the material has 12 percent rocks $(I=40)$ set in a matrix of blocky material with $I=9$.

Although the correctness of the thermal inertias cannot be verified, they seem to be reasonable. An inertia

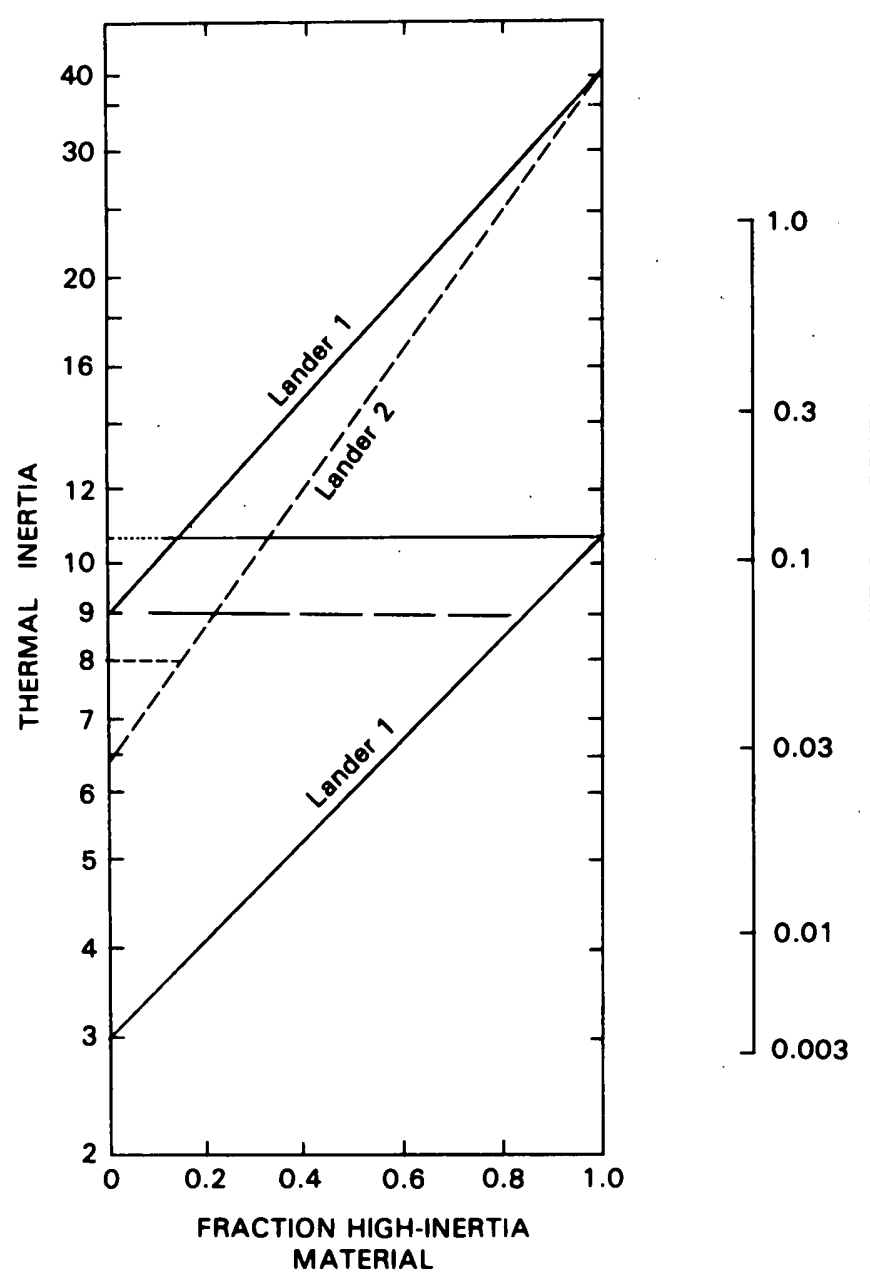

Figure 91.-Thermal inertias of material surfaces with one or more components on martian surfaces. Thermal inertias derived from orbital thermal measurements of Viking landing sites 1 and 2 are $9_{ \pm} 0.5$ and $8 \pm 1.5$ (in $10^{-3} \mathrm{cgs}$ units), respectively. One-component model of cohesionless material implies effective grain diameters of about 0.08 $\mathrm{cm}$ (Lander 1) and $0.06 \mathrm{~cm}$ (Lander 2). Two-component model for Lander 2 (short-dashed lines) has 15 percent rock $(I=40)$ and 85 percent matrix material $(I=6.3)$. Diagonal short-dashed line connects $I=40$ and $I=6.3$; length of horizontal short-dashed line from $I=8$ to diagonal short-dashed line corresponds to 15 percent ( 0.15 fraction) rock. Threecomponent model for Lander 1 has 15 percent drift material $(I=3)$ and 85 percent remaining material with $I=10.8$; remaining material has 12 percent rock $(I=40)$ set in matrix of blocky material $(I=9.2)$. Lower diagonal solid line connects $\mathrm{I}=10.8$ and $\mathrm{I}=3$; length of horizontal long-dashed line from $I=9$ to lower diagonal line corresponds to 85 percent $(0.85$ fraction) of remaining high inertial material. Upper diagonal solid line represents remaining high inertial material and connects $I=40$ and $I=9.2$; length of dotted horizontal line from $I=10.8$ corresponds to 12 percent ( 0.12 fraction) rocks; length of horizontal solid line corresponds to 88 percent ( 0.88 fraction) of blocky materials in remaining material (modified from Kieffer and others, 1977.) for crusty to cloddy material of 6.3 is reasonably close to that of compact lunar surface materials with bulk densities about $1,850 \mathrm{~kg} / \mathrm{m}^{3}$ in an atmosphere of $700 \mathrm{~Pa}$ of $\mathrm{CO}_{2}$ (Horai, 1979). For lunar materials, the porosity would be about 40 percent. The lower value of the dielectric constant (2.8) for Lander 2 is also consistent with a porosity of about 40 percent. The rather large angles of internal friction of crusty to cloddy material suggest a rather dense surface material that fails by general shear. The effect of the binding agent (that produces the crusts and clods) on the thermal inertia is, however, unclear.

A high inertia for blocky material $(I=9)$ might also be expected because of the large, although variable, cohesions derived from surface-bearing tests and sample trenches, rather large angles of internal friction which suggest failure by general shear, the origin of the only samples of the coarse fraction which come from blocky material, and the bulk densities of fragments of coarse fraction which could be as large as 1,800 to $2,300 \mathrm{~kg} / \mathrm{m}^{3}$. Again, the quantitative effects of cementing materials is unclear although they would tend to increase the inertia.

Finally, an inertia of about 3 appears to be reasonable for drift material. The lower angle of internal friction obtained from trenching and surface-bearing tests is related to some compression during deformation (that is, failure partly by local shear). This kind of failure is a property of porous granular materials. Because drift material has a bulk density of about $1,100 \mathrm{~kg} / \mathrm{m}^{3}$ in the XRFS analysis chamber and the relative dielectric constants do not appear to be unusually low, a bulk density of drift material, in situ, of about $1,200 \mathrm{~kg} / \mathrm{m}^{3}$ is possible; this density is compatible with a thermal inertia of about 3 .

\section{WATER-VAPOR MAPPING}

Water vapor appears to be distributed in the lowest several kilometers of the atmosphere, which generally contains as much vapor as it can hold (it reaches saturation at night) (Davies, 1979a). Average water-vapor abundances are chiefly a function of the season, latitude, and elevation (Farmer and Doms, 1979; Farmer and others, 1977). For the latitude of Lander 1, average column water-vapor abundances range from several pr $\mu \mathrm{m}$ (precipitable microns) in midwinter to roughly $15 \mathrm{pr} \mu \mathrm{m}$ in summer and late summer. For the latitude of Lander 2 , the average amount of water vapor cannot be computed for the winter because the atmosphere is so dusty that the water vapor cannot be measured, but it is low. In the spring and fall, abundances are a few $\mathrm{pr} \mu \mathrm{m}$ and, in the summer, peaks are about $22 \mathrm{pr} \mu \mathrm{m}$. The total global inventory of atmospheric water vapor reaches a maximum in northern midsummer.

Apparently, diurnal variations of water vapor abun- 
dances at specific locations have not been established because of variations in atmospheric opacity with time of day and air-mass thicknesses, both of which depend on observational opportunities (Farmer and others, 1977). These constraints may result in apparent variations that produce large apparent abundances near midday (Davies, 1979b).

Water vapor is transported across latitudes by meridional winds of general atmospheric circulation and from the surface by poorly understood processes. Transfer of water vapor by meridional winds is supported by the fact that water vapor velocities have the same magnitude and direction in the northern summer as those of the meridional winds (Farmer and Doms, 1979). The increase in total global inventory of atmospheric water vapor during the northern summer (Farmer and Doms, 1979) suggests that water vapor is exchanged between the atmosphere and the martian surface and regolith (Jakosky and Farmer, 1980). This exchange is unique to the northern hemisphere; in the southern hemisphere water-vapor abundances during the southern summer do not exceed those at the equator despite the fact that the atmosphere could hold more water vapor.

On the one hand, the changes in the total global inventory of water vapor can be accounted for with onedimensional models in which water vapor diffuses from the upper $1.0 \mathrm{~m}$ of a regolith with $0.22 \mathrm{~g}$ of water (Jakosky and Farmer, 1980); the model regoliths have a thermal inertia of 6 , albedo of 0.3 , an emissivity of $0.95,0-20$ percent rocks, and diffusivities ranging from 1 to $5 \mathrm{~cm}^{2} \mathrm{~s}^{-1}$. On the other hand, detailed studies of the diurnal variation of water vapor at the Lander 1 site did not require the daily exchange between the regolith and atmosphere (Davies, 1979b) as might be expected if water-vapor adsorption in the regolith were important-as proposed by Fanale and Cannon (1974). The winter "snow fall" at the Lander 2 site and its subsequent disappearance proves that water vapor is exchanged between the surface and the atmosphere. Apparently, the "snow fall" at the Lander 2 site can be explained as a fall of atmospheric dust coated with $\mathrm{CO}_{2}, \mathrm{CO}_{2}-\mathrm{H}_{2} \mathrm{O}$, and $\mathrm{H}_{2} \mathrm{O}$ ices (Guinness and others, 1979). Water (and possibly $\mathrm{H}_{2} \mathrm{O}-\mathrm{H}_{2} \mathrm{O}_{2}$ ) ice must be present because patches of the snow persisted beyond Sols 405-406 during the late winter. On Sols 405-406, air temperatures ranged from $210^{\circ}$ to $165^{\circ} \mathrm{K}-$ both of which exceed the frost point temperatures for $\mathrm{CO}_{2}$ and its water clathrate at 10 mbar pressure. Measured near-surface temperatures, as well as theoretical surface temperatures, also usually exceed the frost points of $\mathrm{CO}_{2}$ and $\mathrm{CO}_{2}$-water clathrate. Indeed, the peak temperatures of the collector-head temperatures of Lander 2 in the surface materials on Sol 405 corresponded to an equilibrium water-vapor pressure about $2 \mathrm{~Pa}(500 \mathrm{pr} \mu \mathrm{m})$ and winter temperatures on Sols 957-958 were above the frost points of $\mathrm{CO}_{2}$ and $\mathrm{CO}_{2}$-water clathrates a significant amount of time.

\section{DISCUSSION}

The chief goals of the Physical Properties Investigation were to deduce the bearing strength, cohesion, angle of internal friction, bulk density (porosity), thermal properties, grain size distribution, adhesion, and heterogeneity of surface materials. Because some aspects of these subjects are covered in the text, the discussion below is restricted to bulk density, grain-size distribution, angle of internal friction, cohesion, adhesion, composition, and seasonal changes. Thermal properties of the surface materials are not discussed because they were never assessed using Viking lander data. Some goals, such as heterogeneity of the surface materials, are included in the discussions of other goals. Bearing capacity also is excluded here because it can be calculated from variables related to the footing (such as size, shape, roughness, and depth) and the surface material (such as bulk density, angle of internal friction, cohesion, and failure mechanism of the surface material).

\section{DENSITY}

There were a variety of sources of information on the densities of the surface materials on Mars, but before the densities are discussed, the concept should be defined and the interpretation of density should be discussed. In a general sense, density is a readily understood concept that refers to the ratio of mass to volume of a material, however, when applied to granular materials, such as rocks and soils, the concept is more complex because the voids or pores between the grains contain fluids instead of solids, therefore the term bulk density is used. If a natural material, such as a rock, is made of grains of a mineral with a density of $2,600 \mathrm{~kg} / \mathrm{m}^{3}$ and there are no. voids or pores between the mineral grains, the bulk density of the rock will be the same as the density of the grains of the mineral, or $2,600 \mathrm{~kg} / \mathrm{m}^{3}$. If the same mineral grains are disaggregated and poured into a container in such a way that half the volume is occupied by grains and the other half is occupied by voids or pores between the grains, the bulk density of the material in the container is $1,300 \mathrm{~kg} / \mathrm{m}^{3}$. If small porous fragments with a bulk density of $1,300 \mathrm{~kg} / \mathrm{m}^{3}$ are poured into a container in such a way that half the volume is occupied by the porous fragments and the other half is occupied by voids in between the porous fragments, the bulk density of the material in the container will be $650 \mathrm{~kg} / \mathrm{m}^{3}$. When the fluid in the voids has the same density as the martian or terrestrial atmospheres, the contribution of the fluid to the bulk density of the material is negligible, but for other 
fluids, such as water, the contribution to the bulk density is significant and must be included.

Density was never uniquely determined by any instrument or experiment on Mars, but there were a number of lines of evidence that permitted reasonable estimates of the densities of various materials. Here, it is necessary to distinguish between the bulk densities of surface materials as they occurred naturally in the undisturbed state and the bulk densities of materials in the disturbed state after they were sampled and delivered to the XRFS analysis chamber. The disturbed bulk density of martian fine-grained material in the XRFS chamber was $1,100 \pm 150 \mathrm{~kg} / \mathrm{m}^{3}$, and the disturbed bulk densities of the coarse fractions of Lander 1 ranged from 480 to 940 $\mathrm{kg} / \mathrm{m}^{3}$. In the case of the fine-grained material, a mineral or grain density of $2,600 \mathrm{~kg} / \mathrm{m}^{3}$ indicates that the fraction of voids was $0.58 \pm 0.06$. In the case of the coarse fraction, when the fraction of voids between the individual fragments is assumed to be 0.58 , the bulk densities implied for the individual fragments range from 1,143 to $2,238 \mathrm{~kg} / \mathrm{m}^{3}$; these values correspond to the fraction of voids within the individual fragments that range from 0.56 to 0.14 . Terrestrial data for the XRFS chamber suggested that the fraction of voids may be 0.50 ; if so, this amount would lower the bulk densities of the individual fragments and they would range from 960 to $1,880 \mathrm{~kg} / \mathrm{m}^{3}$. Thus, the bulk densities of disturbed materials in the XRFS chamber suggested that the bulk densities of in situ undisturbed material between about 1,000 and $2,200 \mathrm{~kg} / \mathrm{m}^{3}$ were plausible.

Three lines of evidence indicated that the bulk densities of undisturbed material in the vicinity of the landers, on a scale of meters, were neither unusually low nor unusually high: (1) dielectric constants, (2) thermal inertias, and (3) the sampler and lander data. If we assume that the dielectric constants for all solid dense mineral and rock grains are the same, the Rayleigh mixing formula will predict the ratio of bulk densities to that of their solid phase. For the Lander 1 site with a dielectric constant of $3.3 \pm 0.7$, this ratio turned out to be $0.64 \pm 0.20$ for a solid phase with a dielectric constant that ranged from 6 to 12.4 and corresponded to a bulk density of 1,600 $\mathrm{kg} / \mathrm{m}^{3}$ (range from 1,080 to 2,020 ). For Lander 2, the lower dielectric constant (2.8) corresponded to a density ratio of $0.54 \pm 0.06$ and a bulk density of $1,400 \mathrm{~kg} / \mathrm{m}^{3}$ (range from 1,250 to 1,680). Needless to say, these values did not solve the problem at the level of detail that one would have preferred, but these values were compatible with the range of bulk densities inferred from the XRFS results.

Although the thermal inertias may be interpreted in a variety of ways, the rather large thermal inertias reported for the sites were compatible with rather moderate bulk densities for the surface materials at a grand scale.
Interpretation of the results from sampler and lander data was compatible with moderate bulk densities. The magnitudes of the angles of internal friction obtained for blocky material and crusty to cloddy material suggested failure by general shear. This kind of failure, in turn, implied that these materials should have bulk densities compatible with medium dense terrestrial soils that ranged from 1,400 to $1,600 \mathrm{~kg} / \mathrm{m}^{3}$. Drift material, in contrast, could have a low bulk density. The low angle of internal friction suggested that the material may have failed by local shear, and this kind of failure is typical for low density, porous soils. However, comparisons of the penetration of footpad 2 with dynamic tests on Earth did not require unusually low bulk densities. We know of no sensible way to determine the bulk density of undisturbed drift material, but it seems that $1,200 \mathrm{~kg} / \mathrm{m}^{3}$ is as good an estimate as any.

A model that seemed reasonable for the site at Lander 1 consisted of 0.08 fraction of rock $\left(\rho=2,600 \mathrm{~kg} / \mathrm{m}^{3}\right), 0.13$ fraction of drift material $\left(\rho=1,200 \mathrm{~kg} / \mathrm{m}^{3}\right)$, and $0.79 \mathrm{frac}-$ tion of blocky material $\left(\rho=1,600 \mathrm{~kg} / \mathrm{m}^{3}\right)$; this model gives a bulk density of $1,628 \mathrm{~kg} / \mathrm{m}^{3}$ for the entire sample field. For the site at Lander 2, 0.14 fraction of rock $(\rho=2,600$ $\left.\mathrm{kg} / \mathrm{m}^{3}\right)$ and 0.82 of cloddy material $\left(\rho=1,400 \mathrm{~kg} / \mathrm{m}^{3}\right)$ gives a bulk density of about $1,570 \mathrm{~kg} / \mathrm{m}^{3}$ for the entire sample field.

\section{GRAIN SIZE}

Grain size, like density, is a relatively straightforward concept until other variables, particularly cohesions, are introduced. Rocks are made of mineral grains that are strongly bound together by cohesive forces. The rocks may be broken into a variety of fragments ranging in size from meters down to the sizes of individual mineral grains or even smaller. The small rock or mineral grains may lack cohesion and form loose sand, or they may be held together by small to large cohesive forces and form clods with a variety of sizes and strengths. The objects larger than about $0.035 \mathrm{~m}$ in the sample fields of both landers appeared to be almost entirely rocks and rock fragments made of smaller grains bound together by very strong cohesive forces. Blocky material broke into clods with a variety of sizes that were $0.04 \mathrm{~m}$ wide or smaller. The mineral grains and rock fragments in each clod were bound together with moderate cohesive forces. Judging from the comminutor motor-current records, significant amounts of very cohesive coarse material $(\approx 1.0 \mathrm{~mm}$ in diameter) were present in the larger blocky clods as mineral grains, rock fragments, and (or) very strong "clodlets". Moderate cohesion of the blocky clods was implied by the successful acquisition, sieving, and delivery of the coarse fraction for inorganic chemical analyses. Crusty to cloddy material also broke into slablike and 
blocky clods, but these clods were much weaker than those of blocky material because no samples survived the acquisition, sieving, and delivery procedures used to collect the coarse fraction. Strong mineral grains, rock fragments, or "clodlets" ( $1 \mathrm{~mm}$ in diameter) also appeared to be absent because of the small increases in comminutor motor currents. The amounts of gases evolved in the Gas Exchange Experiment, interpreted as desorbed gases, implied that the individual mineral grains making up the crusts, clods, and less coherent fine-grained material ranged from about $0.1 \mu \mathrm{m}$ to $2 \mu \mathrm{m}$-the clay sizes. Drift material, which appeared to be made of mineral grains with sizes ranging from $0.1 \mu \mathrm{m}$ to $2 \mu \mathrm{m}$, formed lumps of weakly coherent mineral grains.

Variations from the generalizations above occurred in the sample fields of the landers. At Lander 2, more coherent crusts formed layers that produced slablike to nearly equidimensional clods, and materials above and below the layers formed much finer grained material. In some places, small millimeter-sized "clodlets" dominated the surface. The same kinds of variations occurred for blocky material. Drift material appeared to be more uniform than the crusty to cloddy and blocky materials, but deflated dune-forms of drifts were etched to reveal cross-laminations, and natural slope failures and slope profiles suggested that a weak crust may be present near the surface in drift material. Open fractures were present locally. Variations in grain size and character of the rocks were indicated by the variety of shapes and surfaces seen. Some rocks were pitted; others were not. Some broke into regular prisms; others were irregular in shape. Some appeared to be layered or banded; others were not. Thus, the rocks on Mars exhibited the same kinds of grain phenomena as those observed on Earth. The surface materials consisted of tiny mineral or mineralloid grains that were held together by coherent forces of varying magnitude to form lumps, crusts, clods, and rocks.

\section{ANGLE OF INTERNAL FRICTION}

Angles of internal friction of the martian surface materials appeared to vary with the type of material and to a lesser degree within each type. Little can be said about the angles of internal friction of the rocks in the sample fields. Although some of them had prismatic forms because they were bounded by fractures, lack of knowledge about the origin of the fractures precluded the use of angular relations to establish the angles of internal friction of the rocks. Dense terrestrial rocks commonly have angles of internal friction of $40^{\circ}-60^{\circ}$.

Most of the soillike materials on Mars had angles of internal friction that ranged from $27^{\circ}$ to $39^{\circ}$; these values were compatible with those of many terrestrial dense to medium dense, nonplastic sands and silts (Hough, 1957;
Gibbs and others, 1960). Though the blocky material appeared to have an average angle of internal friction of $31.0 \pm 2.7$, it: is possible that the angles for the material vary from $27^{\circ}$ to $33^{\circ}$. Such variability is supported by the heterogeneous appearance of the sample field where the blocky material was found as well as variations in the morphologies of the trenches excavated in blocky material.

Variations of angles of internal friction might also be expected for the soillike materials in the Lander 2 sample field. The layer of crusty to cloddy material exposed in some sample trenches appeared different than the fine materials below the layer and some above the layer. Although the average angle of internal friction was $35^{\circ} \pm 5.2^{\circ}$, actual angles may have depended on their locations in the sample field and whether or not the material was chiefly crusty to cloddy or was chiefly the less cohesive materials above and below it. Thus, the range of angles of internal friction may have reflected real differences in the mechanical properties of the materials. The larger angles of internal friction of $39^{\circ}$ were obtained entirely, or almost entirely, from trenches with crusty to cloddy material, whereas the lower angles of $28^{\circ}-34^{\circ}$ were obtained from trenches with mixed amounts of less cohesive fine materials and crusty material. No estimate of angle of internal friction was obtained from trenches that were excavated solely in material composed of millimeter-sized clods with admixed fine material, this material is believed to represent a local upper layer of material derived by weathering or alteration of crusts and clods that is neither thick nor widespread on Mars.

The low values for the angle of internal friction of drift material $\left(18.2^{\circ} \pm 2.6^{\circ}\right)$ were rather puzzling. Maybe the angle of internal friction was, in fact, small. Few materials other than smooth glass spheres have such low angles of internal friction although conventional interpretation of data on lunar fine-grained material yielded angles of internal friction as low as $13^{\circ}-19^{\circ}$ (Jaffe, 1973). However, it is possible that the derived angle of internal friction is an apparent one. Apparent angles of internal friction of wet clay on Earth may be low owing to positive pore pressures that developed during loading (Gibbs and others, 1960), but large amounts of water are not likely to be present in drift material. If positive pore pressures were involved, the only available pore fluid was $\mathrm{CO}_{2}$ and other gases in the atmosphere. Although compression of drift material and concomitant development of positive pore pressures by a footpad descending with a velocity of about $2.3 \mathrm{~m} / \mathrm{s}$ seems possible, it is difficult to imagine how compression of drift material could have produced significant positive pore pressures during the excavation of a sample trench about $0.06 \mathrm{~m}$ deep by a collector head at a speed of $2.54 \mathrm{~cm} / \mathrm{s}$. A more likely explanation is that the drift material failed by a combination of general shear and local shear. In general shear, strain in the soil that 
precedes failure by plastic flow is small whereas strain is large for local shear (Terzaghi, 1948).

The mode of failure depends on the porosity of the material. Dense to medium dense soils fail, mostly, by general shear; consequently the regions of failure ahead of the collector head were relatively large. Low-density soils fail partly by compaction and partly by shearing. Thus, one possible implication of the derived angles of internal friction is that the densities of drift material were lower than those of blocky and crusty to cloddy materials.

\section{COHESION}

Cohesions of martian surface materials possessed the same wide range in magnitude that was exhibited by terrestrial and lunar surface materials. Although little can be said about the cohesions of the martian rocks, it appears likely that they are similar to dense terrestrial and lunar rocks, which range from 1 to $50 \mathrm{MPa}$. The reddish hue of some of the rocks suggests that their surfaces might have been altered by weathering or possibly by the addition of materials. If the weathered surfaces are present, they are relatively strong and not weak punky rinds because the surface sampler did not chip, spall, or scratch them. Weathering or additive processes on Earth can produce surfaces which are just as strong as the rock itself.

Blocky material, judging from the analyses of the sample trenches, the surface-bearing test, footpad penetration, and backhoe touchdowns, had variable cohesions that ranged from about 2.2 to $15.6 \mathrm{kPa}$. This range is probably real and is supported by the range of bulk densities or fragments or clods (coarse fraction) estimated from radiation backscatter in the XRFS analysis chamber (that is, $960-2,240 \mathrm{~kg} / \mathrm{m}^{3}$ ). In general, cohesion tends to increase with bulk density.

Cohesions of crusts and clods, in contrast to materials above and below the crusts, are difficult to assess, but behavior of the crusts and clods during the excavation of sample trenches suggests that their cohesions were relatively large compared with the materials above and below them. Yet, the inability to collect samples of coarse fraction by Lander 2 implies that their cohesions were low-lower than much of the blocky material. For material that is chiefly crusty or cloddy, cohesions probably ranged from 0.8 to $5 \mathrm{kPa}$ and 0.2 to $2.3 \mathrm{kPa}$ for materials with smaller amounts of crust admixed with less cohesive fine material.

The cohesion of drift material is problematic, just as the low angles of internal friction are problematic. For nominal angles of internal friction of $18^{\circ}-20^{\circ}$, footpad penetration and sample trench analyses were compatible with cohesions of $0.7-3.0 \mathrm{kPa}$ and slope stability analyses of the deep hole in drift material indicated a cohesion in excess of about $0.1 \mathrm{kPa}$. The problem arises when the natural slope failures are considered because it is difficult to invoke failure mechanisms that do not include very low cohesions $(5-14 \mathrm{~Pa})$. Although it is conceivable that very large pore pressures could be generated by desorption of $\mathrm{CO}_{2}$ from mineral grains in drift material, the better alternative at this time seems to be that there were planes of weakness in drift material with small cohesive forces across them that enable natural slope failures to be induced by small changes in equilibrium. Unless future missions to Mars are accomplished, the magnitude of these cohesive forces and the causes of these small slope failures will be a matter of conjecture.

\section{ADHESION}

Earlier estimates of adhesion based on the vibration of the surface-sampler collector head ranged from 0.9 to 79 $\mathrm{Pa}$ (Moore and others, 1977). For the material that slid down the wall of footpad 3 of Lander 1, adhesion would be about $9 \mathrm{~Pa}$ in the absence of metal-surface material friction. For a common metal-soil friction coefficient (0.36), the adhesion is about $5 \mathrm{~Pa}$. Suitable experiments and situations were never achieved by Lander 2 for a unique determination of adhesion, but material remaining on the steep walls of the footpad indicated that the adhesion was probably greater than $16 \mathrm{~Pa}$. During the mission, the collector head never appeared to be dirty so that an upper bound consistent with Lander 1 results $(79 \mathrm{~Pa})$ seems to be reasonable.

\section{COMPOSITION}

Although the analytical results of the Viking Missions represent a large increase in our knowledge of the surface materials on Mars, significant gaps and uncertainties exist. The inorganic chemical compositions of the martian soillike materials, which were remarkably similar at two sites separated by about $6,500 \mathrm{~km}$, were comparable with a mixture dominated by iron-rich clays (or their degradation products) containing ferric oxides and stable carbonates and sulfates that locally cement the surface material (Toulmin and others, 1977). Because no data were obtained to prove the existence of any particular mineral, the mixture could be dominated by more stable phases such as oxides, carbonates, sulfates, some feldspars, and kaolinite (Gooding, 1978). Although an argument for the presence of iron-rich clays based on experiments designed to simulate the results of the Labeled Release Experimental on Mars has been offered (Banin and Rishpon, 1979), these experiments and their success have been disputed (Levin and Straat, 1981). The experiments failed to account for total inhibition of the release of radioactive gas by preheating the sample to 
$160{ }^{\circ} \mathrm{C}$, total inhibition after retention of the sample in the instrument for 2 or 3 months, and there is a possibility that biota may have been present in the samples of iron-rich clays used in the experiments (Levin and Straat, 1981). Even the mineralogy of the magnetic fraction has been challenged, because it seems that $\delta$-FeOOH (feroxyhyte) is just as viable a magnetic fraction as $\gamma$ - $\mathrm{Fe}_{2} \mathrm{O}_{3}$ (maghemite). Samples of demonstrable rock fragments were never analyzed by the landers unless the compositions of the rock fragments, represented by the coarse fraction obtained from blocky material at the Lander 1 site, were nearly the same as the fine fraction. The rather large comminutor-motor currents favored the presence of significant amounts of strong millimeter-sized fragments in blocky material. In any case, the mineralogy of the soillike surface materials and rocks will remain a matter of conjecture until samples are returned to Earth or subsequent landers obtain mineralogical data.

The origin of the water detected and crudely measured by the gas chromatograph-mass spectrometer was also unclear. Although adsorbed water was a reasonable postulate for at least some of the water, other possibilities, such as dehydroxylation or reaction of the $\mathrm{H}_{2}$ gas of the instrument with oxides, peroxides, and superoxides, simply cannot be excluded. Even the presence of $\mathrm{H}_{2} \mathrm{O}_{2}$ cannot be entirely dismissed because of the success of experiments using $\mathrm{H}_{2} \mathrm{O}_{2}$ with silica sand and $\gamma-\mathrm{Fe}_{2} \mathrm{O}_{3}$ that successfully simulated the labeled release reactions on Mars (Levin and Straat, 1981) and the constant temperatures measured by Lander 2 during the late winter thaw that matched eutectic temperatures of $\mathrm{H}_{2} \mathrm{O}-\mathrm{H}_{2} \mathrm{O}_{2}$. Furthermore, it seems likely that the winter "snowfall" at the Lander 2 site included water ice because the snow persisted on illuminated surfaces when temperatures were too high for dry ice $\left(\mathrm{CO}_{2}\right)$ and solid $\mathrm{CO}_{2}-\mathrm{H}_{2} \mathrm{O}$ clathrates. It is possible that the large seasonal changes of water vapor in the northern atmosphere were related to adsorption and desorption of water.from mineral surfaces in the regolith as well as evaporation of "snow".

Finally, the cohesions of the surface materials may be related, in part, to cementation by compounds of sulfur and chlorine because the amounts of sulfur and chlorine in the soillike materials appeared to increase with the cohesion.

\section{CHANGES}

The Viking landers observed important and exciting changes at the surface of Mars. The winter "snow falls" and the resulting snow covers at the Lander 2 site that survived into the spring were important scientific discoveries. Lander 2 data on temperatures measured by the collector head immersed in surface materials and those of the near-surface atmosphere lend strong support to the theory that the snows were, at least partly, water ice because the temperatures exceeded those required for stable solids of $\mathrm{CO}_{2}$ and $\mathrm{CO}_{2}-\mathrm{H}_{2} \mathrm{O}$ clathrate during the late winter thaw and during most of the time in the winter.

Lander 1 documented two slope failures in drift material that occurred 1 year apart at times when seasonal pressures were minima, high temperatures were maxima, and diurnal temperature differences were maxima. Although the cause of the slope failures is conjectural, some changes may have occurred in the drift material as a result of seasonal changes. One possibility is that reduced pressures, higher temperatures, and larger temperature differences caused desorption of $\mathrm{CO}_{2}$ from mineral surfaces, and this desorption generated pore pressures in drift material to cause the slope failures.

Although the trenches and dark halos around the conical piles became covered by a thin layer of bright dust from the atmosphere, significant changes were not produced at the Lander 1 site until about Sol 1742 when a dust storm eroded the surface. Evidence for these changes was illustrated by one conical pile that was emplaced shortly at the first martian winter solstice experienced by Lander 1 , and it survived about 2 martian years before it was eroded.

\section{CONCLUSIONS}

As in the previous section, the conclusions will be confined to the principal goals of the Physical Properties Investigation and our best estimates of the physical properties of the dominant materials at the sites will be given and summarized (table 37 ).

\section{ROCKS}

Very little is known about the rocks on the surface of Mars. Rocks as much as $0.4-0.6 \mathrm{~m}$ in diameter were present in the sample fields. The rocks did not chip, spall, or scratch when the surface sampler pushed them or when the backhoe scraped them. Judging from terrestrial experience, the angles of internal friction of the rocks should range from $40^{\circ}$ to $60^{\circ}$ and their cohesions should range from 1 to $50 \mathrm{MPa}$. Perhaps the strongest evidence for the rocks being dense and strong was the large upper bound of dielectric constants obtained from the analyses of the echoes of Lander 2 radio transmissions; however, the upper bound of the dielectric constant, 12.5 , was somewhat larger than most terrestrial rocks reported in the literature.

\section{DRIFT MATERIAL}

Crossbedding, open fractures, and crusts can produce variations in the response of drift material to different 
loads. In general, the responses of drift material to the surface sampler-collector head and footpad were consistent with a material that had angles of internal friction from $14^{\circ}$ to $21^{\circ}$ and cohesions from 0.7 to $3.0 \mathrm{kPa}$. Because of planes of weakness evidenced by crossbedding, crusts, and open fractures, cohesions were locally very small. For loads that have rather large areas, such as the Viking footpads, an angle of internal friction about $18^{\circ}$ to $20^{\circ}$ and cohesions in the range of $1-3 \mathrm{kPa}$ seem to be reasonable parameters for design purposes. Although there were no unique measurements of the bulk density of drift material, a value of $1,200 \mathrm{~kg} / \mathrm{m}^{3}$ seems to be reasonable because it is close to the bulk density of the material in the disturbed state $\left(1,100 \pm 150 \mathrm{~kg} / \mathrm{m}^{3}\right)$. By all criteria, the grain size of drift materials is very small. Response to engine-exhaust gases during landing suggested a grain size typically smaller than 3 to $9 \mu \mathrm{m}$, and gases evolved during the gas exchange experiments, interpreted to be desorbed, suggested an average grain size between $0.14 \mu \mathrm{m}$ and $2 \mu \mathrm{m}$. Grains of disturbed drift material cohere to form lumps. Although two large rocks were brought to the surface during the excavation of the deep hole, comminutor-motor currents and purges of the collector head indicated rock fragments greater than $0.2 \mathrm{~cm}$ are virtually absent in drift material.

\section{BLOCKY MATERIAL}

Blocky material is more heterogeneous than drift material. The response of blocky material to the surface sampler and footpad was consistent with a material that had angles of internal friction from $27^{\circ}$ to $32^{\circ}$ and cohesions from 1.5 to $16 \mathrm{kPa}$. In general, an angle of internal friction of $30^{\circ}$ and cohesions between 2.2 and $16 \mathrm{kPa}$ seem to be reasonable parameters for design purposes. Bulk densities of blocky material may be quite large judging from estimates of bulk densities of clods in the XRFS analysis chamber. On the basis of these bulk densities, the rather large average dielectric constant for Lander 1 site, and the dynamic interpretation of footpad penetration, $1,600 \pm 400 \mathrm{~kg} / \mathrm{m}^{3}$ seems to be a reasonable estimate for the bulk density of blocky material. There are no estimates of grain size of blocky material because no samples for the Biology Experiment were acquired from it. In view of the close similarity in chemical composition with drift material, a very small and comparable grain size seems likely. The mineral grains cohered to form strong clods from $2 \mathrm{~mm}$ to $4 \mathrm{~cm}$ in diameter that are capable of surviving the sieving procedures used to collect the coarse fraction. Significant quantities of millimeter-size rock fragments, mineral oxides, or very strong clods must have

TABLE 37.-Summary of physical properties of the surface materials at the Viking Landing sites [Dashes indicate no data]

\begin{tabular}{|c|c|c|c|c|c|c|}
\hline Material & Drift & Blocky & $\begin{array}{l}\text { Mixed crusts } \\
\text { and fines }\end{array}$ & $\begin{array}{l}\text { Crusty to } \\
\text { cloddy }\end{array}$ & Tailings & Rocks \\
\hline $\begin{array}{l}\text { Angle of internal } \\
\text { friction, } \phi \text {, degrees } \\
\text { Sample trenches } \\
\text { Surface bearing tests }\end{array}$ & $\begin{array}{c}14-21 \\
17\end{array}$ & $\begin{array}{c}27-32 \\
30\end{array}$ & $\begin{array}{c}28-34 \\
--\end{array}$ & $\begin{array}{c}39 \\
32.5\end{array}$ & -- & $\begin{array}{l}40-60 \\
-- \\
--\end{array}$ \\
\hline $\begin{array}{l}\text { Cohesion ( } \mathrm{kPa} \text { ) } \\
\text { Footpad penetration } \\
\text { Sample trenches } \\
\text { Surface-bearing test } \\
\text { Backhoe touchdowns } 1 \\
\text { Trench slopes } \\
\text { Natural slopes }\end{array}$ & $\begin{array}{c}1.1-1.7 \\
<0.7-3.0 \\
- \\
0.5-1.3 \\
>0.1 \\
0.01\end{array}$ & $\begin{array}{c}5.5-7.1 \\
1.5-9.0 \\
14-16 \\
2-10 \\
-- \\
--\end{array}$ & $\begin{array}{c}0.2-2.3 \\
-- \\
-- \\
- \\
--\end{array}$ & $\begin{array}{c}-- \\
0.5-2.3 \\
4.7-5.2 \\
2-5 \\
-- \\
--\end{array}$ & $\begin{array}{c}-- \\
-- \\
\overline{-} \\
<0.2-0.5 \\
0-0.05 \\
--\end{array}$ & $\begin{array}{c}\text { large } \\
-- \\
\text { large } \\
\text { large } \\
-- \\
--\end{array}$ \\
\hline $\begin{array}{l}\text { Bulk density }\left(\mathrm{kg} / \mathrm{m}^{3}\right) \\
\text { and range }\left(\mathrm{kg} / \mathrm{m}^{3}\right)\end{array}$ & $\begin{array}{r}1,200 \\
\pm 100\end{array}$ & $\begin{array}{r}1,600 \\
\pm 400\end{array}$ & $\begin{array}{r}1,400 \\
\pm 200\end{array}$ & $\begin{array}{r}1,400 \\
\pm 200\end{array}$ & $\begin{array}{r}1,100 \\
\pm 150\end{array}$ & $\begin{array}{c}2,600 \\
-\end{array}$ \\
\hline $\begin{array}{l}\text { Sizes } \\
\text { Grains }(\mu \mathrm{m}) \\
\text { Clods }(\mathrm{m}) \\
\text { Rock fragments (m) }\end{array}$ & $\begin{array}{c}0.1-2.0 \\
\text { lumpy } \\
\text { none }\end{array}$ & $\leq \frac{\leq 0.04}{\leq 0.001-0.035}$ & $\begin{array}{l}0.1-2.0 \\
\leq 0.04 \\
>0.035\end{array}$ & $\begin{array}{l}0.1-2.0 \\
\leq 0.04 \\
>0.035\end{array}$ & $\begin{array}{l}0.1-2.0 \\
\leq 0.04\end{array}$ & $\begin{array}{c}\overline{-} \\
0.035-1.0\end{array}$ \\
\hline
\end{tabular}

${ }^{1}$ Values are one-half the shear-vane strengths (see section on "Backhoe touchdowns" and figs. 53 and 56). 
been present in order to account for the large comminutor-motor currents. The presence of such strong large objects would help to explain the rather large "effective particle size" deduced from engine-exhaust erosion $(0.01-0.18 \mathrm{~cm})$.

\section{CRUSTY TO CLODDY MATERIAL}

Again, heterogeneity seemed to characterize the materials at the Lander 2 site where layers of crusts and cloddy units were present at the surface. Materials composed chiefly of crusts and clods appeared to have angles of internal friction that ranged from $32^{\circ}$ to $39^{\circ}$ and cohesions from 0.5 to $5.2 \mathrm{kPa}$, and those of mixed crusts and fine-grained material had angles of internal friction from $28^{\circ}$ to $34^{\circ}$ and cohesions from 0.2 to $2.3 \mathrm{kPa}$. Judging from the fact that no samples of the coarse fraction of surface materials were delivered for analysis in the XRFS, it seems probable that cohesions of the Lander 2 materials were typically lower than those of blocky material. The lower value of the dielectric constant (2.8) obtained using the radio echoes indicated that $1,400 \mathrm{~kg} / \mathrm{m}^{3}$ was a reasonable estimate for the bulk density of crusty to cloddy material. As for drift material, the evolved gases in the gas exchange experiments implied a grain size ranging from 0.1 to $2.0 \mu \mathrm{m}$. Weak cohesive forces between these grains formed thin layers of crusty and cloddy units with dimensions as much as $0.04 \mathrm{~m}$ in diameter. Above and below the crusty and cloddy units cohesions were less. Although pieces of rock about $0.03 \mathrm{~m}$ across were present, comminutor-motor currents and purges of the coarse fraction of material indicated that $\mathrm{mm}$-size to $\mathrm{cm}$-size rock fragments or strong clods were essentially absent.

\section{TAILINGS}

The tailings of the trenches and the conical piles placed upon and among the rocks were disturbed materials for which cohesive forces between the grains had been reduced. The combined effects of angle of internal friction and cohesion determined the slopes of the tailings; and the slopes were rather large $\left(38^{\circ}-45^{\circ}\right)$. We prefer the interpretation that the tailings have the same angle of internal friction as the parent material but with variable low cohesions. According to backhoe touchdowns, the cohesions were less than $0.6 \mathrm{kPa}$ and according to slope stability considerations they were from 10 to $54 \mathrm{~Pa}$. Because the material was disturbed, a bulk density $\left(1,100 \pm 150 \mathrm{~kg} / \mathrm{m}^{3}\right)$ comparable to that in the XRFS chamber was implied. Grain and clod sizes were the same as those of the parent materials although rather large rocks were sometimes found in the tailings.

\section{CONCLUDING REMARKS}

The angles of internal friction and cohesions that we obtained were based chiefly on a model for plowing by narrow blades in soil and a two-dimensional model assessing the effects of frictional sliding. There were a large number of variables involved in the application of the models that include angles, measured dimensions, and assumed densities. For example, reduction of the angle between the base and leading edge of the interface in front of the collector head $\left(\theta^{*}\right)$ of about $9^{\circ}$ would increase our estimates of the angles of internal friction of blocky and crusty to cloddy materials about $3^{\circ}$ with a concomitant 10 percent reduction of cohesions; such a change would not alter the angles of internal friction or cohesions of drift material. Any adjustments to our models seem futile because suitable experiments using the surface sampler and realistic surface-material simulants have not been undertaken at this time. Despite these drawbacks, our estimates seem reasonable; they represent our best estimates with the resources available to us.

\section{REFERENCES CITED}

Allen, C.C., Gooding, J.L., Jercinovic, Michael, and Keil, Klaus, 1981, Altered basaltic glass: A terrestrial analog to the soil of Mars: Icarus, v. 45 , no. 2 , p. $347-369$.

Anderson, D.M., and Tice, A.R., 1971, Low temperature phases of interfacial water in clay-water systems: Soil Science Society of America Proceedings, v. 35 , no. 1 , p. 47-54.

Anderson, D.M., Schwarz, M.J., and Tice, A.R., 1978, Water adsorption by sodium montmorillonite at $-5{ }^{\circ} \mathrm{C}$ : Icarus, v. 34 , no. $3, \mathrm{p}$. 638-644.

Anderson, D.L., Miller, W.F., Latham, G.V., Nakamura, Y., Toksoz, M.N., Dainty, A.M., Duennebier, F.K., Lazarewicz, A.R., Kovach, R.L., and Knight, T.C.D., 1977, Seismology on Mars: Journal of Geophysical Research, v. 82 , no. 28 , p. 4524-4546.

Anonymous, 1957, Handbook of chemistry and physics: Cleveland, Ohio, Chemical Rubber Publ. Co., 3213 p.

Arvidson, R.E., Guinness, E.A., and Lee, S., 1979, Differential aeolian re-distribution rates on Mars: Nature, v. 278, no. 5704, p. 533-535.

Arvidson, R.E., Guinness, E.A., Moore, H.J., Tillman, James, and Wall, S.D., 1983, Three Mars years: Mutch Memorial Station (Viking Lander 1) imaging observations: Science, v. 222, no. 4623, p. 463-468.

Baird, A.K., Castro, A.J., Clark, B.C., Toulmin, Priestley, III, Rose, H.J., Jr., Keil, Klaus, and Gooding, J.L., 1977, The Viking X-ray Fluorescence Experiment: sampling strategies and laboratory simulations: Journal of Geophysical Research, v. 82 , no. 28, p. 4595-4624.

Baker, R., and Garber, M., 1978, Theoretical analysis of the stability of slopes: Geotechnique, v. 28, no. 4, p. 395-411.

Ballou, E.V., Wood, P.C., Wydeven, T., Lehwalt, M.E., and Mack, R.E., 1978, Chemical interpretation of Viking Lander 1 life detection experiment: Nature, v. 271 , no. 5646 , p. $644-645$.

Banin, Amos, and Rishpon, J., 1979, Smectite clays in Mars soil: evidence for their presence and role in Viking Biology experimental results: Journal of Molecular Evolution, v. 14, no. 1-3, p. 133-152.

Biemann, Klaus, Oro, John, Toulmin, Priestley, III, Orgel, L.E., Nier, A.O., Anderson, D.M., Simmonds, P.G., Flory, Donald, Diaz, A.V., 
Rushneck, D.R., Biller, J.E., and Lafleur, A.L., 1977, The search for organic substances and inorganic volatile compounds in the surface of Mars: Journal of Geophysical Research, v. 82, no. 28, p. 4641-4658.

Binder, A.B., Arvidson, R.E., Guinness, E.A., Jones, K.L., Morris, E.C., Mutch, T.A., Pieri, D.C., and Sagan, Carl, 1977, The geology of the Viking Lander 1 site: Journal of Geophysical Research, v. 82, no. 28, p. 4439-4451.

Brown, J.H., Mitchell, S.R., and Weissman, M., 1960, Energy-size reduction relationship for the grinding of quartz: Transactions of the American Institute of Mining Engineers, v. 217, p. 203-206.

Burns, R.G., 1980, Does feroxyhyte occur on the surface of Mars?: Nature, v. 285 , no. 5767 , p. 647.

Campbell, M.J., and Ulrichs, Juris, 1969, Electrical properties of rocks and their significance for lunar radar observations: Journal of Geophysical Research, v. 74, no. 25, p. 5867-5881.

Carr, M.H., Masursky, Harold, Baum, W.A., Blasius, K.R., Briggs, G.A., Cutts, J.A., Duxbury, Thomas, Greeley, Ronald, Guest, J.E., Smith, B.A., Soderblom, L.A., Veverka, Joseph, and Wellman, J.B., 1976, Preliminary results from the Viking Orbiter Imaging Experiment: Science, v. 192 , no. 4255 , p. 766-776.

Christensen, E.M., Batterson, S.A., Benson, H.E., Choate, R., Jaffe, L.D., Jones, R.H., Ko, H.Y., Spencer, R.L., Sperling, F.B., and Sutton, G.H., 1967, Lunar surface mechanical properties: Surveyor III Preliminary Report: U.S. National Aeronautics and Space Administration Special Publication, SP-146, p. 94-120.

Clark, B.C., III, Baird, A.K., Rose, H.J. Jr., Toulmin, Priestley, III, Christian, R.P., Kelliher, W.C., Castro, A.J., Rowe, C.D., Keil, Klaus, and Huss, G.R., 1977, The Viking X-ray Fluorescence Experiment: analytical methods and early results: Journal of Geophysical Research, v. 82, no. 28, p. 4577-4594.

Clark, B.C., Baird, A.K., Weldon, R.J., Tsusaki, D.M., Schnabel, Lorraine, and Candelaria, M.P., 1982, Chemical composition of martian fines: Journal of Geophysical Research, v. 87, no. B12, p. 10,059-10,067.

Clark, L.V., 1971, Effect of ambient pressure on Viking Lander footpad penetration in nominal lunar soil: Letter 159 (PM-5751-LVC) Viking Project Office, U.S. National Aeronautics and Space Administration Langley Research Center, Hampton, Va:, Oct. 12, 1971, 8 p.

Clark, L.V., and McCarty, J.L., 1963, The effect of vacuum on the penetration characteristics of projectiles into fine particles: U.S. National Aeronautics and Space Administration Technical Note D-1519, $26 \mathrm{p}$.

Clark, L.V., Crouch, D.S., and Grossart, R.D., 1977, Summary of Primary Mission Surface Sampler Operations: Viking ' 75 Project Document VFT-019, 224 p., 3 appendices.

Cooley, C.G., and Lewis, J.G., 1977, Viking '75 Project-Viking Lander System Primary Mission Performance Report: U.S. National Aeronautics and Space Administration Contractors Report 145148, $103 \mathrm{p}$.

Crouch, D.S., 1976, PTC Surface Sampler Boom Loading Tests with Format 5 and SSCA TM Data: Viking Flight Team Memorandum SST-17870-DSC (25 June 1976), 4 p., 3 tables, 3 figures.

1977, Mars Viking surface sampler subsystem: Proceedings 25th Conference on Remote Systems Technology, 1977, The American Nuclear Society, Anniversary issue, p. 141-152.

Davies, D.W., 1979a, The relative humidity of Mars' atmosphere: Journal of Geophysical Research, v. 84, no. B14, p. 8335-8340.

$1979 \mathrm{~b}$, The vertical distribution of Mars water vapor: Journal of Geophysical Research, v. 84, no. B6, p. 2875-2879.

Davies, M.E., 1978, The control net of Mars, in Strom, Robert and Boyce, Joseph, compilers, Reports of Planetary Geology Program, 1977-1978, U.S. National Aeronautics and Space Administration Technical Memorandum 79729, p. 328-329.
Dial, A.L., Jr., 1978, The Viking 1 landing site crater diameter-frequency distribution, in Strom, Robert and Boyce, Joseph, compilers, Reports of Planetary Geology Program, 1977-1978, U.S. National Aeronautics and Space Administration Technical Memorandum 79729, p. 179-181.

Dycus, R.D., 1969, The meteorite flux at the surface of Mars: Publication of Astronomical Society of the Pacific, v. 81, no. 481, p. 399-414.

Fanale, F.P., and Cannon, W.A., 1974, Exchange of adsorbed $\mathrm{H}_{2} \mathrm{O}$ and $\mathrm{CO}_{2}$ between the regolith and atmosphere of Mars caused by changes in surface insolation: Journal of Geophysical Research, v. 79, no. 24 , p. 3397-3402.

1979, Mars: $\mathrm{CO}_{2}$ adsorption and capillary condensation on clays-significance for volatile storage and atmospheric history: Journal of Geophysical Research, v. 84, no. B14, p. 8404-8414.

Farmer, C.B., Davies, D.W., and LaPorte, D.D., 1976, Viking: Mars atmospheric water vapor mapping experiment-preliminary report of results: Science, v. 193 , no. 4255 , p. 776-780.

Farmer, C.B., Davies, D.W., Holland, A.L., LaPorte, D.D., and Doms, P.E., 1977, Mars: water vapor observations from the Viking Orbiters: Journal of Geophysical Research, v. 82, no. 28, p. $4225-4248$.

Farmer, C.B., and Doms, P.E., 1979, Global seasonal variations of water vapor on Mars and the implications for permafrost: Journal of Geophysical Research, v. 84, no. B6, p. 2881-2888.

Ford, A.B., and Anderson, B., 1967, Sorted talus aprons and the movement of debris in Antarctica by repeated thermal contraction and rupture of underlying ice: Journal of Geology, v. 75, no. 6, p. 722-732.

Gibbs, H.J., Hilf, J.W., Holtz, W.G., and Walker, F.C., 1961, Shear strength of cohesive soils: American Society Civil Engineers Research Conference on Shear Strength of Cohesive Soils, held at University of Colorado, Boulder Colo., June 1960, p. 33-162.

Gooding, J.L., 1978, Chemical weathering on Mars: Icarus, v. 33, no. 3, p. 483-513.

Guinness, E.A., 1981, Spectral properties ( 0.40 to 0.75 microns) of soils exposed at the Viking 1 landing site: Journal of Geophysical Research, v. 86, no. B9, p. 7983-7992.

Guinness, E.A., Arvidson, R.E., Gehret, D.C., and Bolef, L.K., 1979, Color changes at the Viking landing sites over the course of a'Mars year: Journal of Geophysical Research, v. 84, no. B14, p. 8355-8364.

Guinness, E.A., Leff, C.E., and Arvidson, R.E., 1982, Two Mars years of surface changes seen at the Viking lander sites: Journal of Geophysical Research, v. 87, no. B12, p. 10,051-10,058.

Hargraves, R.B., Collinson, D.W., Arvidson, R.E., and Cates, P.M., 1979, The Viking Magnetic Properties Experiment: Extended Mission results: Journal of Geophysical Research, v. 84, no. B14, p. 8379-8384.

Hargraves, R.B., Collinson, D.W., Arvidson, R.E., and Spitzer, C.R., 1977, The Viking Magnetic Properties Experiment: Primary Mission results: Journal of Geophysical Research, v. 82, no. 28, p. 4547-4558.

Hargraves, R.B., and Petersen, N., 1972, Magnetic Properties Investigation: The Viking Mars Lander: Icarus, v. 16, no. 1, p. 223-227.

Hess, S.L., Henry, R.M., Leovy, C.B., Ryan, J.A., and Tillman, J.E., 1977, Meteorological results from the surface of Mars: Viking 1 and 2: Journal of Geophysical Research, v. 82, no. 28, p. 4559-4574.

Hess, S.L., Henry, R.M., and Tillman, J.E., 1979, The seasonal variation of atmospheric pressure on Mars as affected by the south polar cap: Journal of Geophysical Research, v. 84, no. B6, p. 2923-2927.

Hess, S.L., Ryan, J.A., Tillman, J.E., Henry, R.M., and Leovy, C.B., 1980 , The annual cycle of pressure on Mars measured by Viking Landers 1 and 2: Geophysical Research Letters, v. 7, no. 3, p. 197-200.

Horai, Ki-iti, 1979, Loose and compacted soils: Two basic units composing the martian surface?: Lunar and Planetary Science X 
(abstracts of papers submitted to the Tenth Lunar and Planetary Science Conference, March 19-23, 1979), p. 564-566.

Horowitz, N.H., Hobby, G.L., and Hubbard, J.S., 1977, Viking on Mars: The Carbon-assimilation experiments: Journal of Geophysical Research, v. 82 , no. 28 , p. $4659-4662$.

Horowitz, N.H., Hubbard, J.S., and Hobby, G.L., 1972, The CarbonAssimilation Experiment: The Viking Mars Lander: Icarus, v. 16, no. 1 , p. $147-152$.

Hough, B.K., 1957, Basic Soils Engineering: New York, Ronald Press Co., $513 \mathrm{p}$.

Hubbard, Jerry S., 1979, Laboratory simulations of the pyrolytic release experiments: an interim report: Journal of Molecular Evolution, v. 14 , no. $1-3$, p. $211-221$.

Huck, F.O., Jobson, D.J., Park, S.K., Wall, S.D., Arvidson, R.E., Patterson, W.R., and Benton, W.D., 1977, Spectrophotometric and color estimates of the Viking Lander sites: Journal of Geophysical Research, v. 82 , no. 28 , p. 4401-4411.

Hutton, R.E., Moore, H.J., Scott, R.F., Shorthill, R.W., and Spitzer, C.R., 1980, Surface erosion caused on Mars from Viking descentengine plume: The Moon and Planets; v. 23, no. 3, p. 293-305.

Jaffe, L.D., 1973, Shear strength of lunar soil from Oceanus Procellarum: The Moon, v. 8, no. 1 , p. 58-72.

Jakosky, B.M., 1979, The effects of nonideal surfaces on the derived thermal properties of Mars: Journal of Geophysical Research, v. 84, no. B14, p. 8252-8262.

Jakosky, B.M., and Farmer, C.B., 1980, Seasonal buffering of atmospheric water by the martian regolith [abs.]: Proceedings of the Third Colloquium on Planetary Water, Niagara Falls, N.Y., Oct. 1980, convened by Department of Geology, State University of New York at Buffalo, p. 46-50.

Jones, K.L., Arvidson, R.E., Guinness, E.A., Bragg, S.L., Wall, S.D. Carlston, C.E., and Pidek, D.G., 1979, One Mars year: Viking Lander imaging observations: Science, v. 204, no. 4395, p. 799-806.

Jury, W.A., and Bellantuoni, B., 1976a, I. Thermal effects, Heat and water movement under surface rocks in a field of soil: Soil Science Society of America Journal, v. 40, no. 4, p. 505-509.

$1976 \mathrm{~b}$, II. Moisture effects, Heat and water movement under surface rocks in a field of soil: Soil Science Society of America Journal, v. 40 , no. 4 , p. $509-513$.

Kieffer, H.H., 1976, Soil and surface temperatures at the Viking landing sites: Science, v. 194, no. 4271, p. 1344-1346.

Kieffer, H.H., Chase, S.C., Jr., Miner, E.D., Palluconi, F.D., Munch, Guido, Neugebauer, Gerry, and Martin, T.Z., 1976, Infrared thermal mapping of the martian surface and atmosphere: first results: Science, v. 193 , no. 4255 , p. $780-786$.

Kieffer, H.H., Martin, T.Z., Peterfreund, A.R., Jakosky, B.M., Miner, Ellis D., and Palluconi, Frank Don, 1977, Thermal and albedo mapping of Mars during the Viking Primary Mission: Journal of Geophysical Research, v. 82 , no. 28, p. 4249-4291.

Klein, H.P., 1977, The Viking biological investigation: general aspects: Journal of Geophysical Research, v. 82, no. 28, p. 4677-4680.

Ko, Hon-Yim, 1971, Soil properties study: Martin Marietta Corp., Denver, Colo., Document VER-181, Oct. 15, 1971, 118 p.

Lee, B.G., 1976, Mission operations strategy for Viking: Science, v. 194, no. 4260 , p. $59-62$.

Levin, G.V., 1972, Detection of metabolically produced labeled gas: The Viking Mars Lander: Icarus, v. 16, no. 1, p. 153-166.

Levin, G.V., and Straat, P.A., 1977, Recent results from the Viking Labeled Release Experiment on Mars: Journal of Geophysical Research, v. 82 , no. 82 , p. $4663-4667$.

1981, A search for a non-biological explanation of the Viking Labeled Release Life Detection Experiment: Icarus, v. 45, no. 2, p. 494-516.

Levinthal, E.C., and Jones, K.L., 1980, The mosaics of Mars as seen by the Viking Lander cameras: U.S. National Aeronautics and Space
Administration Contractor Report 3326, 52 p., 20 figures.

Levinthal, E.C., Green, William, Jones, K.L., and Tucker, Robert, 1977 , Processing the Viking Lander Camera data: Journal of Geophysical Research, v. 82 , no. 28 , p. $4412-4420$.

Liebes, Sidney, Jr., 1982, Viking lander atlas of Mars: U.S. National Aeronautics and Space Administration Contractor Report 3568, $290 \mathrm{p}$.

Liebes, Sidney Jr., and Schwartz, A.A., 1977, Viking 1975 Mars Lander interactive computerized video stereophotogrammetry: Journal of Geophysical Research, v. 82, no. 28, p. 4421-4429.

Linke, W.F., 1958, Seidell's solubilities of inorganic and metal organic compounds: American Chemical Society, Washington D.C., 4th ed. D. Van Nostrand Co., v. 1 of 2 , p. 1486.

Luth, H.J., and Wismer, R.O., 1971, Performance of plane soil cutting blades in sand: Transactions of the American Society of Agriculture Engineers, v. 14 , no. 2, p. 255-262.

Martin Marietta Corp., 1971, Footpad soil penetration tests. Part I, Data Summary: Vehicle Engineering Report VER 188, 56 p., 97 figs., 9 drawings.

1976a, Entry data analysis for Viking Landers 1 and 2: Final Report, Martin Marietta Corp., Denver Division, Denver, Colo., TN-3770218, $147 \mathrm{p}$.

1976b, Viking Lander "as built" capabilities: Martin Marietta Corp., Denver Division, Denver, Colo., NAS 1-9000, June 1976, 121 p.

Masursky, H., and Crabill, N.L., 1976, Search for the Viking 2 landing site: Science, v. 194 , no. 4260 , p. $62-68$.

Mayo, A.P., Blackshear, W.T., Tolson, R.H., Michael, W.H., Jr., Kelly, G.M., Brenkle, J.P., and Komarek, T.A., 1977, Lander locations, Mars physical ephemeris, and solar system parameters: determination from Viking Lander tracking data: Journal of Geophysical Research, v. 82 , no. 28 , p. $4297-4303$.

McGetchin, T.R., Settle, M., and Head, J.W., 1973, Radial thickness variation in impact crater ejecta: Earth and Planetary Science Letters, v. 20 , no. 2 , p. 226-236.

McKyes, Edward, and Ali, O.S., 1977, The cutting of soil by narrow blades: Journal of Terramechanics, v. 14, no. 2, p. 43-58.

Michael, W.H., Jr., Tolson, R.H., Brenkle, J.P., Cain, D.L., Fjeldbo, Gunnar, Stelzried, C.T., Grossi, M.D., Shapiro, I.I., and Tyler, G.L., 1977, The Viking Radio Science Investigations: Journal of Geophysical Research, v. 82 , no. 28 , p. 4293-4295.

Miller, S.L., 1973, Nature and occurrence of clathrate hydrates, in Whalley, E., Jones, S.J., and Gold, L.W., eds., Physics and Chemistry of Ice, Ottawa, Royal Society of Canada, p. 42-50.

Mooney, R.W., Keenan, A.G., and Wood, L.A., 1952, Adsorption of water vapor by montmorillonite. I. Heat of desorption and application of BET Theory: Journal of American Chemical Society, v. 74, no. 6 , p. 1367-1371.

Moore, H.J., 1982, Erosion of surface materials at the Mutch Memorial Station (Lander 1), Mars: in Holt, H.E., compiler, Reports of the Planetary Geology Program-1982, U.S. National Aeronautics and Space Administration Technical Memorandum 85127, p. 180-181.

Moore, H.J., Clow, G.D., and Hutton, R.E., 1982, A summary of Viking sample-trench analyses for angles of internal friction and cohesions: Journal of Geophysical Research, v. 87, no. B12, p. 10,043-10,050.

Moore, H.J., Hutton, R.E., Scott, R.F., Shorthill, R.W., and Spitzer C.R., 1980, Viking surface sampler diurnal temperatures, in Wirth, P., Greeley, R., and D'Alli, R., compilers, Reports of the Planetary Geology Program, 1979-1980: U.S. National Aeronautics and Space Administration Technical Memorandum 81776, p. 166-168.

Moore, H.J., Hutton, R.E., Scott, R.F., Spitzer, C.R., and Shorthill, R.W., 1977, Surface materials of the Viking landing sites: Journal of Geophysical Research, v. 82, no. 28, p. 4497-4523.

Moore, H.J., Liebes, S. Jr., Crouch, D.S., and Clark, L.V., 1978, Rock pushing and sampling under rocks on Mars: U.S. Geological Survey Professional Paper 1081, 21 p. 
Moore, H.J., Spitzer, C.R., Bradford, K.Z., Cates, P.M., Hutton, R.E., and Shorthill, R.W., 1979, Sample fields of the Viking landers, physical properties, and aeolian processes: Journal of Geophysical Research, v. 84, no. B14, p. 8365-8377.

Morris, E.C., and Jones, K.L., 1980, Viking 1 Lander on the surface of Mars: revised location: Icarus, v. 44, no. 1, p. 217-222.

Mutch, T.A., Arvidson, R.E., Binder, A.B., Guiness, E.A., and Morris, E.C., 1977, The geology of the Viking Lander 2 site: Journal of Geophysical Research, v. 82 , no. 28 , p. 4452-4467.

Mutch, T.A., Arvidson, R.E., Binder, A.B., Huck, F.O., Levinthal, E.C., Liebes, Sidney, Jr., Morris, E.C., Nummedahl, Dag, Pollack, J.B., and Sagan, Carl, 1976a, Fine particles on Mars: observation with the Viking 1 Lander cameras: Science, v. 194, no. 4260, p. 87-91.

Mutch, T.A., Binder, A.B., Huck, F.O., Levinthal, E.C., Liebes, Sidney, Jr., Morris, E.C., Patterson, W.R., Pollack, J.B., Sagan, Carl, and Taylor, G.R., 1976b, The surface of Mars: The view from the Viking 1 Lander: Science, v. 193, no. 4255, p. 791-801.

Mutch, T.A., Grenander, S.V., Jones, K.L., Patterson, W.R., Arvidson, R.E., Guinness, E.A., Avrin, P., Carlston, C.E., Binder, A.B., Sagan, Carl, Dunham, E.W., Fox, P.L., Pieri, D.C., Huck, F.O., Rowland, C.W., Taylor, G.R., Wall, S.D., Kahn, Ralph, Levinthal, E.C., Liebes, S., Jr., Tucker, R.B., Morris, E.C., Pollack, J.B., Saunders, R.S., and Wolf, M.R., 1976c, The surface of Mars: The view from the Viking 2 lander: Science, v. 194, no. 4271, p. 1277-1283.

Mutch, T.A., Binder, A.B., Huck, F.O., Levinthal, E.C., Morris, E.C., Sagan, Carl, and Young, A.T., 1972, Imaging experiment: The Viking Lander: Icarus, v. 16 , no. 1, p. 92-110.

Nadeau, P.H., 1977, UV radiational effects on martian regolith water: Hanover, N .H., Dartmouth College, M. A. Thesis, 89 p.

Neukum, Gerhard, and Wise, D.U., 1976, Mars: a standard crater curve and possible new time scale: Science, v. 194, no. 4272, p. 1381-1387.

Neukum, Gerhard, Hiller, Konrad, Henkel, J., and Bodechtel, J., 1978, Mars chronology, in Strom, Robert, and Boyce, Joseph, compilers, Reports of the Planetary Geology Program, 1977-1978: U.S. National Aeronautics and Space Administration Technical Memorandum 79729, p. 172-174.

Nier, A.O., Hanson, W.B., McElroy, M.B., Sieff, Alvin, and Spencer, N.W., 1972, Entry science experiments for Viking 1975: Icarus, v. 16 , no. 1 , p. $74-91$

Nier, A.O., Hanson, W.B., Sieff, A., McElroy, M.B., Spencer, N.W., Duckett, R.J., Knight, T.C.D., and Cook, W.S., 1976, Composition and structure of the martian atmosphere: preliminary results from Viking 1: Science, v. 193, no. 4255, p. 786-788.

Oyama, V.I., 1972, The Gas Exchange Experiment for life detection: The Viking Mars Lander: Icarus, v. 16, no. 1, p. 167-184.

Oyama, V.I., and Berdahl, B.J., 1977, The Viking Gas Exchange Experiment results from Chryse and Utopia surface samples: Journal of Geophysical Research, v. 82 , no. 28, p. 4669-4676.

Patterson, W.R., III, Huck, F.O., Wall, S.D., and Wolfe, M.R., 1977, Calibration and performance of the Viking Lander cameras: Journal of Geophysical Research, v. 82, no. 28, p. 4391-4400.

Pike, D.D., Coulson, P.M., Crouch, D.S., and Yarbrough, J.P., 1978 Viking '75 Project Summary of Extended Mission Surface Sampler Operations: VFT-023, 3 v., 2057 p.

Pohlen, J.C., Maytum, B.D., Ramsey, I.W., and Blanchard, U.J., 1976, The evolution of the Viking landing gear: in JPL Tenth Aerospace Mechanisms Symposium, April 22-23, 1976, Jet Propulsion Laboratory, Pasadena, Calif., p. 218-228.

Pollack, J.B., Colburn, D., Kahn, Ralph, Hunter, J., Van Camp, W., Carlston, C.E., and Wolfe, M.R., 1977, Properties of aerosols in the martian atmosphere, as inferred from Viking lander imaging data: Journal of Geophysical Research, v. 82, no. 28, p. 4479-4496.

Pyrz, A.P., 1969, Gravity effects on low velocity penetration of a projectile into a cohesionless medium: Report GSF/MC/69-6, School of Engineering, Wright-Patterson Air Force Base, Ohio, 135 p.
Romine, G.L., Reisert, T.D., and Gliozzi, J., 1973, Site alteration effects from rocket exhaust impingement during a simulated Viking Mars landing, part 1 of 2, nozzle development and physical alteration: U.S. National Aeronautics Space Administration Contractor Report 2252; $155 \mathrm{p}$.

Ryan, J.A., Henry, R.A., Hess, S.L., Leovy, C.B., Tillman, J.E., and Walcek, C., 1978, Mars meteorology: Three seasons at the surface: Geophysical Research Letters, v. 5, no. 8, p. 715-718.

Ryan, J.A., and Henry, R.M., 1979, Mars atmospheric phenomena during major dust storms, as measured at surface: Journal of Geophysical Research, v. 84, no. B6, p. 2821-2829.

Ryan, J.A., Sharman, R.D., and Lucich, R.D., 1981, Local Mars dust storm generation mechanism: Geophysical Research Letters, v. 8, no. 8, p. 899-901.

Sagan, Carl, Pieri, David, Fox, Paul, Arvidson, R.E., and Guinness, E.A., 1977, Particle motion on Mars inferred from the Viking Lander cameras: Journal of Geophysical Research, v. 82, no. 28, p. 44304438.

Schumb, W.C., Satterfield, C.N., and Wentworth, R.L., 1955, Hydrogen peroxide: American Chemical Society Monograph: New York, Reinhold Publishing Co., 759 p.

Scott, R.F., 1963, Principles of Soil Mechanics: Reading, Mass., AddisonWesley, $550 \mathrm{p}$.

Sieff, Alvin, and Kirk, D.B., 1976, Structure of Mars' atmosphere up to 100 kilometers from entry measurements of Viking 2: Science, v. 194 , no. 4271 , p. $1300-1302$.

Shorthill, R.W., Hutton, R.E., Moore, H.J., and Sêott, R.F., 1972, Martian physical properties experiments: The Viking Mars lander: Icarus, v. 16 , no. 1 , p. 217-222.

Shorthill, R.W., Hutton, R.E., Moore, H.J., II, Scott, R.F., and Spitzer, C.R., 1976a, Physical properties of the martian surface from the Viking 1 lander: preliminary results: Science, v. 193 , no. 4255 , p. 805-809.

Shorthill, R.W., Hutton, R.E., Moore, H.J., II, Scott, R.F., Liebes, Sidney, Jr., and Spitzer, C.R., 1976b, The "soil" of Mars (Viking 1): Science, v. 194 , no. 4260 , p. 91-97.

Shorthill, R.W., Moore, H.J., II, Hutton, R.E., Scott, R.F., and Spitzer, C.R., 1976c, The environs of Viking 2 lander: Science, v. 194, no. 4271 , p. $1309-1318$.

Snyder, C.W., 1977, The missions of the Viking Orbiters: Journal of Geophysical Research, v. 82, no. 28, p. 3971-3983.

1979, The Extended Mission of Viking: Journal of Geophysical Research, v. 84, no. B14, p. 7917-7933.

Soderblom, L.A., 1977, Historical variations in the density and distribution of impacting debris in the inner solar system: evidence from planetary imaging: Impact and Explosion Cratering, New York, Pergamon Press, p. 629-633.

Soderblom, L.A., Condit, C.D., West, R.A., Herman, B.M., and Kreidler, T.J., 1974, Martian planet-wide crater distribution: implications for geologic history and surface processes: Icarus, v. 22, no. 3, p. 239263.

Soffen, G.A., 1977, The Viking Project: Journal of Geophysical Research, v. 82 , no. 28 , p. $3959-3970$.

Tang, C.H., Boak, T.I.S., III, and Grossi, M.D., 1977, Bistatic radar measurements of electrical properties of the martian surface: Journal of Geophysical Research, v. 82, no. 28, p. 4305-4315.

Terzaghi, Karl, 1948, Theoretical soil mechanics: New York, John Wiley and Sons, $509 \mathrm{p}$.

Toulmin, Priestley, III, Baird, A.K., Clark, B.C., Keil, Klaus, and Rose, H.J., Jr., 1973, Inorganic chemical investigation by X-ray fluorescence analysis: The Viking Mars Lander: Icarus, v. 20, no. 2, p. $153-178$.

Toulmin, Priestley, III, Baird, A.K., Clark, B.C., Keil, Klaus, Rose, H.J., Jr., Christian, R.P., Evans, P.H., and Kelliher, W.C., 1977, Geochemical and mineralogical interpretation of the Viking inorganic 
chemical results: Journal of Geophysical Research, v. 82 , no. 28 , p. 4625-4634.

Tucker, R.B., 1978, Viking Lander Imaging Investigation picture catalog of Primary Mission Experiment Data Record: U.S. National Aeronautics and Space Administration Reference Publication 1007, $558 \mathrm{p}$.

Tyler, G.L., Cambell, D.B., Downs, G.S., Green, R.R., and Moore, H.J., 1976, Radar characteristics of Viking 1 landing sites: Science, v. 193, no. 4255 , p. $812-815$.

U.S. National Aeronautics and Space Administration, 1970, Viking '75 Project, Viking Mission Definition, Langley Research Center, Viking Project Office M 75-123-1 (RS-3703001, Appendix D), 35 p.

Villyard, K.W., and Ivers, W.S., 1978, Design and performance characteristics of the Viking Lander inertial reference unit: Journal of Guidance and Control, v. 1, no. 4, p. 248-253.

Von Hippel, Arthur R., 1954, V tables of dielectric materials, in von Hippel, A.R., ed., Dielectric materials and applications, New York,
John Wiley and Sons, $430 \mathrm{p}$.

Wall, S.D., 1982, Viking lander monitor mission imaging investigation, in Holt, H.E., compiler, Reports of the Planetary Geology Program 1982: U.S. National Aeronautics and Space Administration Technical Memorandum 85127, p. 379-381.

Wechsler, A.E., and Glaser, P.E., 1965, Pressure effects on postulated lunar materials: Icarus, v. 4, no. 4, p. 335-352.

Wismer, R.O., and Luth, H.J., 1972, Performance of plane cutting blades in clay: Transactions of the American Society of Agricultural Engineers, v. 15, no. 2, p. 211-216.

Wu, S.S.C., 1979, Photogrammetric portrayal of Mars topography: Journal of Geophysical Research, v. 84, no. B14, p. 7955-7959.

Zent, A.P., Guinness, E.A., and Arvidson, R.E., 1980, Brightness degradation of Viking lander ultraviolet chips, in Holt, H.E., and Kosters, E.C., compilers, Reports of Planetary Geology Program-1980: U.S. National Aeronautics and Space Administration Technical Memorandum 82385, p. 426-428. 

SUPPLEMENTAL INFORMATION 


\section{MEASUREMENTS AND COORDINATES}

\section{INTRODUCTION}

The purposes of this section are to describe procedures, to provide equations that can be used by readers who wish to locate and determine dimensions of features in the sample fields of the landers using the surface samplers or cameras, and to note some problems. The procedures and equations yield or require coordinates in the LSCS (lander science coordinate system), which was described earlier and illustrated in figure 5. LSCS coordinates can be derived from surface-sampler coordinates or camera coordinates which were also described earlier.

\section{SURFACE SAMPLER}

Three surface-sampler coordinates are required to establish LSCS coordinates: (1) extension or the increase in length of the surface-sampler collector head from its original position in the boom housing, (2) elevation or the angle between the boom axis and a plane parallel to the upper surface of the lander body $\left(Y_{L}-Z_{L}\right.$ plane), and (3) the azimuth or angle between a plane passing through the azimuth axis and reference direction and a plane passing through the boom axis and azimuth axis (see fig. 5). Surface-sampler coordinates for trenching and other activities of Landers 1 and 2 are given in the section on "Summary of Surface-Sampler Activities".

where

$E$ is extension (in meters),

$\varepsilon$ is elevation (in degrees and positive downward), $\Gamma$ is azimuth (in degrees), and

$Z_{L}, Y_{L}$, and $X_{L}$ are LSCS coordinates (in meters).

In using these equations, it should be realized that the calculated LSCS coordinates may be in error by a small amount because of (1) boom azimuth override $\left(1.0^{\circ}\right.$ or less $)$ owing to spacecraft tilt, (2) boom sag at large extensions, (3) the complicated nature of the collector-head unit with its backhoe and $10^{\circ}$ collector-head rotation that occurred upon surface contact, and (4) the extensions are usually given in inches and have to be converted to meters. Additionally, it is necessary to understand the surface-sampler motions and sequence. According to our experience, calculated coordinates were good to within a few centimeters or less, depending on the amount of extension, the relations between the boom axis and tilt direction of the spacecraft, and the suitable selection of surface-sampler coordinates.

Equations for calculating surface-sampler coordinates from known LSCS coordinates are:

$$
\begin{gathered}
\Gamma=\tan ^{-1}\left[\frac{\left(Z_{L}-0.965\right)}{\left(Y_{L}+0.254\right)}\right]+80 \\
\varepsilon=\tan ^{-1}\left\{\frac{X_{L}+0.0444}{\left[\left(Z_{L}-0.965\right)^{2}+\left(Y_{L}+0.254\right)^{2}\right]^{1 / 2}-0.0254}\right\}
\end{gathered}
$$

Equations that may be used to obtain approximate LSCS coordinates of the collector-head tip from known surface sampler-coordinates are:

$$
\begin{aligned}
Z_{L}= & {[0.0254+(E+0.472+0.215 \tan \varepsilon) \cos \varepsilon] } \\
& \times \sin (\Gamma-80)+0.965 \\
Y_{L}= & {[0.0254+(E+0.472+0.215 \tan \varepsilon) \cos \varepsilon] } \\
& \times \cos (\Gamma-80)-0.254 \\
X_{L}= & {[(E+0.472+0.215 \tan \varepsilon) \sin \varepsilon] } \\
& -\frac{0.215}{\cos \varepsilon}-0.444
\end{aligned}
$$

0.215

$\left.\left.\left.4]^{1 / 2}-0.0254\right\}^{2}+\left(X_{L}+0.0444\right)^{2}\right]\right]^{1 / 2}$

$$
E=\left[\frac{\left(X_{L}+0.0444\right)+\frac{0.215}{\cos \varepsilon}}{\sin \varepsilon}\right]-0.472-0.215 \tan \varepsilon
$$

\section{CAMERAS}

Two camera coordinates of the same point, or a conjugate image point, for each camera are required in the stereometric method of obtaining coordinates in the LSCS. The coordinates may be obtained by identifying the conjugate images of points in camera 1 and 2 photographs. The conjugate images, which should be as small as possible, are best identified when the two photographs have similar illuminations. Camera scan-line numbers (recorded along the top of a photograph) and scan-sample numbers (recorded along the left side of a photograph) 
are determined for each conjugate image and multiplied by the angular size of a pixel $\left(0.04^{\circ}\right.$ for high resolution broadband pictures). The angles computed from the scanline numbers are then added to the starting azimuth angles of the picture to yield the camera azimuth. Starting azimuths and other data are recorded in a data block for each picture. These derived angles are then adjusted for bolt down corrections. The angles computed from scan-sample numbers are subtracted from the starting elevation angles (taken as positive downward) of the pictures (which are usually $60.24^{\circ}, 40.24^{\circ}$, or $20.24^{\circ}$ ).

Equations that may be used to locate objects approximately in the LSCS system from the camera coordinates are:

$$
\begin{aligned}
& Z_{L}=0.8219\left[\frac{1}{\left.\frac{1}{\tan (A+a)}-\frac{1}{\tan (B-b)}\right]+0.4717}\right. \\
& Y_{L}=0.411\left[\frac{\tan (B-b)+\tan (A+a)}{\tan (B-b)-\tan (A+a)}\right] \\
& X_{L}=0.8219\left[\frac{1}{\left.\frac{\cos (A+a)}{\tan \left(\varepsilon_{A}\right)}-\frac{\cos (B-b)}{\tan \left(\varepsilon_{B}\right)}\right]-0.4872}\right.
\end{aligned}
$$

where

$A=$ the camera azimuth of camera 2 in degrees,

$B=$ the camera azimuth of camera 1 in degrees,

$\varepsilon_{A}=$ the camera elevation of camera 2 in degrees,

$\varepsilon_{B}=$ the camera elevation of camera 1 in degrees,

$a=5.30^{\circ}$ for Lander 1 and $5.45^{\circ}$ for Lander 2,

$b=171.29^{\circ}$ for Lander 1 and $171.37^{\circ}$ for Lander 2 , and

$Z_{L}, Y_{L}$, and $X_{L}$ are LSCS coordinates in meters.

Corrections for diode displacements are made by adding to $A$ or $B$ (for even diodes) or by subtracting from $A$ or $B$ (for odd diodes) the angle computed from:

$$
\text { correction }=\tan ^{-1}\left(\frac{\tan 0.48^{\circ}}{\cos \varepsilon}\right)-0.48^{\circ}
$$

In using these equations, it should be realized that: (1) errors increase rapidly with distance away from spacecraft and when the lines of sight approach the plane that includes the azimuth axes of the cameras (Liebes and Schwartz, 1977), and (2) conjugate images must be used. In the case of Lander 2, there were small but significant movements of the spacecraft. Errors owing to this movement can be reduced by selecting pairs of photographs taken at times between which there has been no movement.
Equations that may be used to approximately determine camera coordinates of objects in the sample field with known LSCS coordinates are:

$$
\begin{gathered}
A=\tan ^{-1}\left(\frac{Z_{L}-0.4717}{Y_{L}+0.4110}\right)-a \\
B=\tan ^{-1}\left(\frac{Z_{L}-0.4717}{Y_{L}-0.4110}\right)+b \\
\varepsilon_{A}=\tan ^{-1}\left[\frac{\left(X_{L}+0.4872\right)}{\left\{\left(Z_{L}-0.4717\right)^{2}+\left(Y_{L}+0.4110\right)^{2}\right\}^{1 / 2}}\right] \\
\varepsilon_{B}=\tan ^{-1}\left[\frac{\left(X_{L}+0.4872\right)}{\left\{\left(Z_{L}-0.4717\right)^{2}+\left(Y_{L}-0.4110\right)^{2}\right\}^{1 / 2}}\right]
\end{gathered}
$$

\section{SUMMARY OF SURFACE-SAMPLER ACTIVITIES}

Two tables summarize the Primary (table 38 ) and Extended (table 39) Missions of Lander 1 and two tables summarize the Primary (table 40) and Extended (Table 41) Missions of Lander 2. The activities are listed in chronological order for each lander. Each item corresponds to a surface-sampler activity. A local lander-event time, achieved surface-sampler positions, and a brief description of the outcome of the activity are given for each item. Designations of the activities and rocks correspond to those portrayed on maps of the sample fields (fig. 29 and pl. 1 for Lander 1; fig. 30 and pl. 2 for Lander 2). Designations of sample trenches for experiments are shown on the plates as follows: Biology (BI0), Molecular Analysis (GCMS), Inorganic Chemical Analysis (XRFS), and Physical Properties (PP). Numbers associated with the map designations represent, sequentially, the number of the sample trench for each experiment (that is, BIO 3 is the third trench excavated to acquire a sample for the Biology Experiment). Designations of samples analyzed in the Inorganic Chemical Analyses Experiment that appear widely in the literature (that is, $\mathrm{C}-1, \mathrm{U}-4$, etc.) are given under the heading of "Activity". Other designations appear in the explanations of the plates or are selfexplanatory. Additional information appears in the headnotes of tables $38,39,40$, and 41 . Outline maps and profiles for most of the trenches excavated by the surface sampler are portrayed in the next section on "Outline maps, profiles, and data on trenches". The achieved surface-sampler positions are given in surface-sampler coordinates; these coordinates may be used with equations 9,10 , and 11 in the previous section.

Detailed information on surface-sampler activities and sequences is found in Viking Flight Team documents (Clark, L.V., and others, 1977; Pike and others, 1978). 
TABLE 38. -Surface-sampler activities related to the Physical Properties Investigation during the Primary Mission of Viking Lander 1

[Listed Local Lander Times correspond to: (a) time of extension command for shroud ejection, (b) collector-head open command just before extension, and (c) vibration commands for purges. Positions are from surface-sampler potentiometer readouts. Azimuths measured from a line $80^{\circ}$ counterclockwise from the $(+) Y_{L}$ direction (direction from camera 2 to camera 1). Azimuths should be reduced by about $1.0^{\circ}$ because of boom overide due to lander tilt. Extensions are

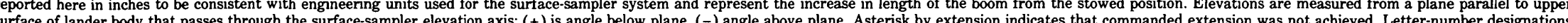
(Isted under "Activity" refer to sample analysis numbers used by Inorgnic ( Ch)

\begin{tabular}{lllll}
\hline Item Activity & Local Lander Time & & Surface-sampler positions & Description of activity \\
& Sol (hr) (min) (s) & $\begin{array}{c}\text { Azimuth } \\
\text { (degrees) }\end{array}$ & $\begin{array}{c}\text { Extension } \\
\text { (in.) }\end{array}$ & $\begin{array}{c}\text { Elevation } \\
\text { (degrees) }\end{array}$ \\
\hline
\end{tabular}

1 Shroud ejection and engine 2 picture via boom
mirror 2 .

2 Restraint (latch) pin ejection

05

$\begin{array}{llll}02 & 10 & 28 & 02\end{array}$

255.4

6.0

40.1

1. Shroud e jected at $3.2 \mathrm{~m} / \mathrm{s}$; struck surface near footpad 3 at $3.6 \mathrm{~m} / \mathrm{s}$ producing shallow crater $1 \mathrm{~cm}$ deep and $9 \mathrm{~cm}$ in diameter by displacement of rocks and e jection of fine de bris, then ricocheted and came to rest about $1 \mathrm{~m}$ from cra= ter. Picture under engine 2 via boom mirror 2 showed. small rock and small pebble-size objects.

2. Restraint (latch) pin $(8.2 \mathrm{~cm}$ long, $0.6 \mathrm{~cm}$ in diameter, and $11.3 \mathrm{~g}$ ) fell from $0.9-1.0 \mathrm{~m}$ to the surface reaching a velocity of $2.6-2.7 \mathrm{~m} / \mathrm{s}$. Pin impacted materials in rim of erosion crater produced by engine exhausts. Pin impact produced an elongate crater. Very fine-grained material

$\begin{array}{cllllllll}3 \text { Biology 1 sample } & 08 & 07 & 05 & 10 & 104.5 & 90.2 & 25.0 \\ \text { (Sandy Flats) } & & & & & 96.7 & 90.2\end{array}$

4 Purge Biology 1

(coarse fraction)

5 GCMS 1 sample (Sandy Flats)

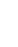

08

$08 \quad 08 \quad 39 \quad 09$

10.5

37.6

90.2

96.7

90.2

08

$09 \quad 31 \quad 56$

104.5

90.2
96.7

96.7

6 Purge GCMS 1

(coarse fraction) $\begin{array}{llllll}08 & 09 & 53 & 25 & 110.2 & 9.7\end{array}$
3. Trenched by extending in drift material after surface contact and then retracting. Trench is $4.0-4.5 \mathrm{~cm}$ deep, $8.0 \mathrm{~cm}$ wide, and about $45 \mathrm{~cm}$ long from tip to rim crestfo tailings; small lumps in and around trench are chiefly rubbed and tamped by surface-sampler parts are smooth and reflective. Deformed surface extends to $7.5 \mathrm{~cm}$ from tip reflective. Deformed surface extends to $7.5 \mathrm{~cm}$ from tip
of trench; collector-head tip reached depth of $5.0 \mathrm{~cm}$.

4. Purged material produced crater $0.04 \mathrm{~m}$ wide by impact a about $2 \mathrm{~m} / \mathrm{s}$. Purged material fills impact crater.

Largest clods $0.2-0.5 \mathrm{~cm}$ wide.

5. Trenched by extending in drift material after surface contact and then retracting with identical commands as in neering data indicate amount of sample delivered was unusually small after two attempts.

6. Available data suggest there was no purged material. 


\begin{tabular}{|c|c|c|c|c|c|c|c|c|}
\hline \multirow[t]{2}{*}{ Item } & \multirow[t]{2}{*}{ Activity } & \multicolumn{4}{|c|}{ Local Lander Time } & \multicolumn{3}{|c|}{ Surface-sampler positions } \\
\hline & & Sol & $(\mathrm{hr})$ & $(\min )$ & (s) & $\begin{array}{c}\text { Azimuth } \\
\text { (degrees) }\end{array}$ & $\begin{array}{l}\text { Extension } \\
\text { (in.) }\end{array}$ & $\begin{array}{l}\text { Elevatio } \\
\text { (degrees }\end{array}$ \\
\hline 7 & $\begin{array}{l}\text { XRFS } 1 \text { sample } \\
\text { (Sandy Flats) }\end{array}$ & 08 & 10 & 45 & 55 & 104.5 & $\begin{array}{r}94.1 \\
100.7 \\
94.1\end{array}$ & 24.3 \\
\hline & $c-1$ & 08 & 11 & 32 & 55 & 104.5 & $\begin{array}{r}94.1 \\
100.7 \\
94.1\end{array}$ & 25.0 \\
\hline 8 & $\begin{array}{l}\text { Purges XRFS } 1 \\
\quad \text { (coarse fraction) }\end{array}$ & $\begin{array}{l}08 \\
08\end{array}$ & $\begin{array}{l}11 \\
11\end{array}$ & $\begin{array}{l}10 \\
57\end{array}$ & $\begin{array}{l}18 \\
18\end{array}$ & $\begin{array}{l}110.2 \\
110.2\end{array}$ & $\begin{array}{l}11.5 \\
11.5\end{array}$ & $\begin{array}{l}37.6 \\
37.6\end{array}$ \\
\hline 9 & $\begin{array}{l}\text { GCMS } 2 \text { sample } \\
\quad \text { (Sandy Flats) }\end{array}$ & 14 & 06 & 35 & 42 & 107.7 & $\begin{array}{l}90.2 \\
96.7 \\
90.2\end{array}$ & 25.6 \\
\hline 10 & $\begin{array}{l}\text { Purge GCMS } 2 \\
\quad \text { (coarse fraction) }\end{array}$ & 22 & 12 & 27 & 49 & 87.5 & 9.9 & 37.6 \\
\hline 11 & $\begin{array}{l}\text { GCMS } 3 \text { sample } \\
\text { (Rocky Flats) }\end{array}$ & 31 & 10 & 51 & 02 & 204.9 & $\begin{array}{l}70.2 \\
76.5 \\
64.4\end{array}$ & 30.0 \\
\hline 12 & $\begin{array}{l}\text { Purge GCMS } 3 \\
\quad \text { (coarse fraction) }\end{array}$ & 31 & 11 & 18 & 02 & 194.8 & 42.0 & 37.6 \\
\hline 13 & $\begin{array}{l}\text { XRFS } 2 \text { sample } \\
\text { (Rocky Flats) }\end{array}$ & 34 & 10 & 19 & 21 & 203.6 & $\begin{array}{l}62.0 \\
67.5 \\
56.2\end{array}$ & 33.2 \\
\hline & $c-2$ & 34 & 11 & 19 & 21 & & $\begin{array}{l}62.0 \\
68.6 \\
56.2\end{array}$ & 33.8 \\
\hline 14 & $\begin{array}{l}\text { Purge XRFS } 2 \\
\quad \text { (fine fraction) }\end{array}$ & $\begin{array}{l}34 \\
34\end{array}$ & $\begin{array}{l}10 \\
11\end{array}$ & $\begin{array}{l}24 \\
24\end{array}$ & $\begin{array}{l}11 \\
11\end{array}$ & $\begin{array}{l}194.8 \\
194.8\end{array}$ & $\begin{array}{l}57.3 \\
57.3\end{array}$ & $\begin{array}{l}7.9 \\
7.9\end{array}$ \\
\hline 15 & $\begin{array}{l}\text { Purges XRFS } 2 \\
\text { (coarse fraction) }\end{array}$ & $\begin{array}{l}34 \\
34\end{array}$ & $\begin{array}{l}10 \\
11\end{array}$ & $\begin{array}{l}41 \\
41\end{array}$ & $\begin{array}{l}45 \\
45\end{array}$ & $\begin{array}{l}194.8 \\
194.8\end{array}$ & $\begin{array}{l}42.0 \\
42.0\end{array}$ & $\begin{array}{l}37.6 \\
37.6\end{array}$ \\
\hline 16 & $\begin{array}{l}\text { Biology } 2 \text { sample } \\
\text { (Sandy Flats) }\end{array}$ & 36 & 11 & 21 & 02 & 104.5 & $\begin{array}{l}78.3 \\
84.9 \\
72.8\end{array}$ & 27.5 \\
\hline
\end{tabular}

7. Trenched by extending in drift material after surface ontact then retracting. Trench is $6.5 \mathrm{~cm}$ deep, $10 \mathrm{~cm}$ wide and $55 \mathrm{~cm}$ long from tip to rim crest of tailings. Surface domed to about $10 \mathrm{~cm}$ from tip of trench. urements and images indicate wall at end of trench lapsed and incipient wall failure occurred on left side of trench. Sample of fine-grained material delivered to XRFS.

8. Purged material produced crater about $0.04 \mathrm{~m}$ wide by impact at about $2 \mathrm{~m} / \mathrm{s}$. Crater partly obscured by spacecraft.

9. Trenched by extending in drift material after surface contact then retracting. Trench is about $3.5 \mathrm{~cm}$ deep, $7.5 \mathrm{~cm}$ wide at tip, and $35 \mathrm{~cm}$ long from tip to rim crest of tailings. Surface domed to about $5 \mathrm{~cm}$ from $\mathrm{rim}$. Septum between XRFS 1 and this trench was displaced toward XRFS trench during sampling. Sample was not anal yzed by GCMS.

10. Purged material on surface obscured by lander body.

11. Trenched by extending in blocky material after surface contact then retracting. No ster eoscopic pictures were trench is $6-7 \mathrm{~cm}$ wide and about $40 \mathrm{~cm}$ long.

12. Purged material has grain size a few millimeters acros and may be small clods; no impact pits produced by purged materiais.

13. Trenched by extending in blocky material after surface contact and then retracting. Trench depth cannot be measured; trench is $5-8 \mathrm{~cm}$ wide; disrupted region at far end attain the command extension of $68.6 \mathrm{~cm}$. Sample of coarse fraction delivered to XRFS.

14. Purged fine-grained material dispersed.

15. Some fragments a few millimeters across were purged.

16. Trenched by extending in drift material after surface contact and then retracting. Trench is 4 to $5 \mathrm{~cm}$ deep, 7 to $8 \mathrm{~cm}$ wide, and $46 \mathrm{~cm}$ long from tip to rim crest of tailings; trench transects tailings of previous Sol 8 trenches. Trench partly obscured by spacecraft which precludes mapping of entire trench. 
TABLE 38. -Surface-sampler activities related to the Physical Properties Investigation during the Primary Mission of Viking Lander 1-Continued

\begin{tabular}{|c|c|c|c|c|c|c|c|c|c|}
\hline \multirow[t]{2}{*}{ Item } & \multirow[t]{2}{*}{ Activity } & \multicolumn{4}{|c|}{ Local Lander Time } & \multicolumn{3}{|c|}{ Surface-sampler positions } & \multirow[t]{2}{*}{ Description of activity } \\
\hline & & Sol & $(\mathrm{hr})$ & $(\min )$ & (s) & $\begin{array}{l}\text { Azimuth } \\
\text { (degrees) }\end{array}$ & $\begin{array}{c}\text { Extension } \\
\text { (in.) }\end{array}$ & $\begin{array}{l}\text { Elevation } \\
\text { (degrees) }\end{array}$ & \\
\hline 17 & $\begin{array}{l}\text { Purge Biology } 2 \\
\quad(\text { coarse fraction) }\end{array}$ & 36 & 13 & 22 & 40 & 194.8 & 42.0 & 37.6 & 17. Little, if any, material was purged. \\
\hline 18 & $\begin{array}{l}\text { XRFS } 3 \text { sample } \\
\text { (Rocky Flats) } \\
\text { C-3 }\end{array}$ & 40 & 10 & 38 & 39 & 204.9 & $\begin{array}{l}74.6 \\
80.9^{*} \\
69.1\end{array}$ & 28.8 & $\begin{array}{l}\text { 18. Trenched by extending in blocky material after surface } \\
\text { contact and then retracting. Trench is } 5 \mathrm{~cm} \text { deep, } 9 \mathrm{~cm} \\
\text { wide and } 45 \mathrm{~cm} \text { long from tip to rim crest of tailings; } \\
\text { tip of collector head probably reached a depth of } 6 \mathrm{~cm} \text {. } \\
\text { Trench extends } 8-9 \mathrm{~cm} \text { beyond GCMS } 3 \text { trench along same } \\
\text { azimuth. clod and rock framents are } 2-5 \mathrm{~cm} \text { wide. First } \\
\text { pass achieved commanded extension, second pass did not } \\
\text { achieve commanded extension. Sample of coarse fraction } \\
\text { delivered to XRFS. }\end{array}$ \\
\hline 19 & $\begin{array}{l}\text { Purge XRFS } 3 \\
\text { (fine fraction) }\end{array}$ & $\begin{array}{l}40 \\
40\end{array}$ & $\begin{array}{l}10 \\
12\end{array}$ & $\begin{array}{l}42 \\
22\end{array}$ & $\begin{array}{l}29 \\
29\end{array}$ & $\begin{array}{l}204.9 \\
204.9\end{array}$ & $\begin{array}{l}69.1 \\
69.1\end{array}$ & $\begin{array}{l}7.9 \\
7.9\end{array}$ & 19. Purged fine-grained material dispersed. \\
\hline 20 & $\begin{array}{l}\text { Purge XRFS } 3 \\
\quad \text { (coarse fraction) }\end{array}$ & $\begin{array}{l}40 \\
40\end{array}$ & $\begin{array}{l}11 \\
12\end{array}$ & $\begin{array}{l}14 \\
54\end{array}$ & $\begin{array}{l}18 \\
18\end{array}$ & $\begin{array}{l}189.8 \\
189.8\end{array}$ & $\begin{array}{l}42.0 \\
42.0\end{array}$ & $\begin{array}{l}37.6 \\
37.6\end{array}$ & 20. Few, if any, coarse fragments were purged. \\
\hline 21. & $\begin{array}{l}\text { Picture of footpad } \\
2 \text { temperature } \\
\text { sensor via boom } \\
\text { mirror } 1\end{array}$ & 40 & 11 & 06 & 02 & 125.4 & NA & 35.1 & $\begin{array}{l}\text { 21. Picture included part of temperature sensor housing; } \\
\text { subsequent picture taken during Extended Mission shows } \\
\text { temperature sensing elements are buried but entire } \\
\text { housing is not. }\end{array}$ \\
\hline 22 & $\begin{array}{l}\text { Physical Properties } 1 \\
\text { sample, temperatures } \\
\text { (Sandy Flats) }\end{array}$ & 41 & 15 & 37 & 45 & 101.4 & $\begin{array}{l}78.3 \\
84.9 \\
72.8\end{array}$ & 27.5 & $\begin{array}{l}\text { 22. Trenched by extending in drift material after surface } \\
\text { contact and then retracting. Trench is } 5-6 \mathrm{~cm} \text { deep, } \\
6-7 \mathrm{~cm} \text { wide, and } 48 \mathrm{~cm} \text { long from tip to } \mathrm{rim} \text { crest of } \\
\text { tailings; tip of trench collapsed to form a bench; trench } \\
\text { is unusually narrow. visual picture dif erencing shows } \\
\text { that septum between this trench and the Biology } 3 \text { trench } \\
\text { moved toward Physical Properties trench rather than toward } \\
\text { the previously formed Biology } 3 \text { trench. Surface-sampler } \\
\text { collectorhead temperatures were } 272^{\circ} \mathrm{K} \text { at extension, } \\
269^{\circ} \mathrm{K} \text { in surface material at } 10.7 \text { and } 11 \text { min after } \\
\text { extension. }\end{array}$ \\
\hline 23 & $\begin{array}{l}\text { Magnification } \\
\text { mirror image of } \\
\text { collector-head tip }\end{array}$ & 41 & 15 & 58 & 04 & 4.2 & 24.7 & 0.3 & $\begin{array}{l}\text { 23. Picture of material on tip of collector head; no } \\
\text { evidence for coarse fragments. }\end{array}$ \\
\hline 24 & $\begin{array}{l}\text { Purge of fine-grained } \\
\text { material on lander } \\
\text { grid } \\
\text { (Phys. Props. 1) }\end{array}$ & 41 & 16 & 03 & 42 & 4.2 & 24.7 & 3.5 & $\begin{array}{l}\text { 24. Purge of fine-grained material on lander grid produced } \\
\text { more or less conical mound. Picture taken with a sun } \\
\text { elevation angle of } 38^{\circ} \text { shows slopes of mound are shadowed } \\
\text { so that angle of repose is greater than } 38^{\circ} \text {. }\end{array}$ \\
\hline 25 & Purge Phys. Props. 1 & 41 & 16 & 17 & 41 & 189.8 & 42.0 & 37.6 & 25. Few, if any, coarse fragments were purged. \\
\hline
\end{tabular}


TABLE 38.-Surface-sampler activities related to the Physical Properties Investigation during the Primary Mission of Viking Lander 1-Continued 
TABLE 39.-Surface-sampler activities related to the Physical Properties Investigation during the Extended Mission of Viking Lander 1

[Listed Local Lander Times correspond to: (a) time of extension command for shroud ejection, (b) collector-head open command just before extension, (c) extension commands for nudges and pushes; (d) vibration commands for purges, and (e) elevation down and final retraction commands for deep holes. Positions are from surface-sampler potentiometer readouts. Azimuths measured from a line $80{ }^{\circ}$ counterclockwise from the $(+) Y_{L}$ direction (direction from camer

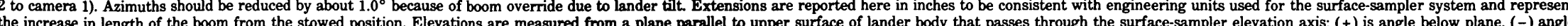
the increase in length of the boom from the stowed position. Elevations are measured from a plane paralle to upper surface of lander body that passes through the surface-sampler elevation axis; (+) is angle below plane, (-) angle
above plane. Asterisk by extension indicates that commanded extension was not achieved. Letter-number designations listed under "Activity" refer to sample analysis numbers used by Inorganic Chemical Analysis Team (XRFS) (for example, C-5, C-6, etc.)]

\begin{tabular}{|c|c|c|c|c|c|c|c|c|c|}
\hline \multirow{5}{*}{ Item } & \multirow{2}{*}{$\begin{array}{l}\text { Activity } \\
\text { XRFS } 4 \text { sample } \\
\text { (Atlantic City) }\end{array}$} & \multicolumn{4}{|c|}{$\frac{\text { Local Lander Time }}{\text { Sol (hr) (min) }(\mathrm{s})}$} & $\frac{\text { Surfac }}{\text { Azimuth }}$ & $\begin{array}{l}\text {-sampler po } \\
\text { Extension } \\
\text { (1n.) }\end{array}$ & $\begin{array}{l}\text { 1tions } \\
\text { Elevation } \\
\text { (degrees) }\end{array}$ & Description of activity \\
\hline & & 177 & 14 & 40 & 49 & 189.1 & \multirow{4}{*}{$\begin{array}{l}62.0 \\
67.0^{*} \\
56.5 \\
62.0 \\
67.5^{*} \\
56.5 \\
62.0 \\
67.3^{*} \\
56.5 \\
62.0 \\
67.8^{*} \\
56.5\end{array}$} & 35.1 & \multirow{4}{*}{$\begin{array}{l}\text { 1. Trenched by extending in blocky material after surface } \\
\text { contact and then retracting. Trench is } 6-7 \mathrm{~cm} \text { deep, } 9 \mathrm{~cm} \\
\text { wide, and } 45 \mathrm{~cm} \text { long from tip to rim crest of tailings. } \\
\text { Slopes of tailings nearest the lander are about } 36^{\circ} \text {. Sur- } \\
\text { face sampler failed to reach commanded extensions ( } 68.6 \\
\text { in.) on Sols 177, 178, } 179 \text {, and } 180 \text { Sample of coarse } \\
\text { fraction of blocky material was delivered to XRFS. }\end{array}$} \\
\hline & \multirow[t]{3}{*}{$c-5$} & 178 & 14 & 50 & 49 & 189.1 & & 35.7 & \\
\hline & & 179 & 10 & 40 & 49 & 189.1 & & 35.1 & \\
\hline & & 180 & 10 & 40 & 49 & 189.1 & & 35.1 & \\
\hline 2 & $\begin{array}{l}\text { Purge XRFS } 4 \\
\text { (fine fraction) }\end{array}$ & $\begin{array}{l}177 \\
178 \\
179 \\
180\end{array}$ & $\begin{array}{l}14 \\
14 \\
10 \\
10\end{array}$ & $\begin{array}{l}45 \\
55 \\
45 \\
45\end{array}$ & $\begin{array}{l}56 \\
56 \\
56 \\
56\end{array}$ & $\begin{array}{l}133.6 \\
133.6 \\
133.6 \\
133.6\end{array}$ & $\begin{array}{l}56.5 \\
56.5 \\
56.5 \\
56.5\end{array}$ & $\begin{array}{l}-15.4 \\
-15.4 \\
-15.4 \\
-15.4\end{array}$ & $\begin{array}{l}\text { 2. Purged fine-grained material scattered by wind and not } \\
\text { identified. Sample of coarse fraction of blocky material } \\
\text { delivered to XRFS. }\end{array}$ \\
\hline 3 & Backhoe touchdown 1 & 202 & 14 & 07 & 06 & 95.7 & 111.2 & 18.7 & $\begin{array}{l}\text { 3. Backhoe not visible in picture but collar of collector } \\
\text { head does not touch surface of drift material. }\end{array}$ \\
\hline \multirow[t]{12}{*}{4} & \multirow{6}{*}{$\begin{array}{l}\text { Deep Hole } 1 \\
\text { (Sandy Flats, } \\
\text { Part } 1,1 \text { st } \\
\text { pass) }\end{array}$} & \multirow[t]{6}{*}{202} & \multirow[t]{5}{*}{14} & \multirow[t]{5}{*}{07} & \multirow[t]{5}{*}{06} & 95.7 & 111.2 & $18.7,20.5$ & \multirow{12}{*}{$\begin{array}{l}\text { 4. Trenched by retracting backhoe in drift material after } \\
\text { surface contact and a } 2-s \text { deelevation. Excavation of } \\
\text { deep hole began with six retraction strokes on first } \\
\text { pass and six retraction strokes on second pass. }\end{array}$} \\
\hline & & & & & & 96.3 & $\begin{array}{r}105.1 \\
61.2\end{array}$ & $20.5,22.4$ & \\
\hline & & & & & & 99.5 & $\begin{array}{r}111.2 \\
68.8\end{array}$ & $19.3,21.8$ & \\
\hline & & & & & & 98.9 & $\begin{array}{r}105.1 \\
61.2\end{array}$ & $21.2,24.3$ & \\
\hline & & & & & & 97.6 & $\begin{array}{r}111.2 \\
68.8\end{array}$ & $18.7,21.2$ & \\
\hline & & & 14 & 39 & 05 & & $\begin{array}{r}105.1 \\
20.2\end{array}$ & $19.9,22.4$ & \\
\hline & \multirow{6}{*}{$\begin{array}{l}\text { Deep Hole } 1 \\
\text { (Sandy Flats, } \\
\text { Part 1a, 2nd } \\
\text { pass) }\end{array}$} & \multirow[t]{6}{*}{202} & \multirow[t]{6}{*}{15} & \multirow[t]{5}{*}{07} & \multirow[t]{5}{*}{06} & 95.7 & $\begin{array}{r}111.2 \\
68.8\end{array}$ & $18.0,20.5$ & \\
\hline & & & & & & 96.3 & $\begin{array}{r}105.1 \\
61.2\end{array}$ & $21.2,23.1$ & \\
\hline & & & & & & 99.5 & $\begin{array}{r}111.2 \\
68.8\end{array}$ & $19.3,21.8$ & \\
\hline & & & & & & 98.9 & $\begin{array}{r}105.1 \\
61.2\end{array}$ & $19.3,21.2$ & \\
\hline & & & & & & 97.6 & $\begin{array}{r}111.2 \\
68.8\end{array}$ & $19.3,21.2$ & \\
\hline & & & & 39 & 05 & & $\begin{array}{r}105.1 \\
20.2\end{array}$ & 19.9 .22 .4 & \\
\hline
\end{tabular}




\begin{tabular}{|c|c|c|c|c|c|c|c|c|c|}
\hline \multirow[t]{2}{*}{ Item } & \multirow[t]{2}{*}{ Activity } & \multicolumn{4}{|c|}{$\frac{\text { Local Lander Time }}{\text { Sol }(\mathrm{hr})(\min )(\mathrm{s})}$} & \multicolumn{3}{|c|}{ Surface-sampler positions } & \multirow[t]{2}{*}{ Description of activity } \\
\hline & & Sol & $(h r)$ & (min) & (s) & $\begin{array}{c}\text { Azimuth } \\
\text { (degrees) }\end{array}$ & $\begin{array}{l}\text { Extension } \\
\text { (in.) }\end{array}$ & $\begin{array}{l}\text { Elevation } \\
\text { (degrees) }\end{array}$ & \\
\hline $5 P$ & $\begin{array}{l}\text { Position for footpad } \\
2 \text { temperature } \\
\text { sensor picture } \\
\text { (picture taken } \\
\text { on Sol 203) }\end{array}$ & 202 & 15 & 42 & 45 & 122.8 & na & 40.1 & $\begin{array}{l}\text { 5. Picture of temperature sensor on footpad } 2 \text { shows } \\
\text { that the sensor tips are buried } 1.2-1.5 \mathrm{~cm} \text { by drift } \\
\text { material of Sandy Flats. }\end{array}$ \\
\hline $6 \mathrm{D}$ & $\begin{array}{l}\text { Deep Hole } 1 \\
\text { (Sandy Flats, } \\
\text { Part } 1 \mathrm{~b}, 3 \mathrm{rd} \\
\text { pass) }\end{array}$ & 204 & 12 & 07 & 06 & $\begin{array}{l}95.7 \\
96.3\end{array}$ & $\begin{array}{r}111.2 \\
68.8 \\
105.1 \\
61.2\end{array}$ & $\begin{array}{l}18.0,20.5 \\
21.2,23.1\end{array}$ & $\begin{array}{l}\text { 6. Excavation of deep hole in drift material continued as } \\
\text { in item } 4 \text { above for third and fourth passes. Trench about } \\
15 \mathrm{~cm} \text { deep, } 30 \mathrm{~cm} \text { wide, and } 104 \mathrm{~cm} \text { long from tip to rim of } \\
\text { tailings at end of fourth pass. Rock about } 14 \mathrm{~cm} \text { wide was } \\
\text { excavated. }\end{array}$ \\
\hline & & 204 & 12 & 39 & 05 & $\begin{array}{l}99.5 \\
98.9 \\
97.6\end{array}$ & $\begin{array}{r}111.2 \\
68.8 \\
105.1 \\
61.2 \\
111.2 \\
68.8 \\
105.1 \\
20.2\end{array}$ & $\begin{array}{l}19.3,21.2 \\
21.2,23.7 \\
18.7,21.2 \\
21.8,23.7\end{array}$ & - nom \\
\hline & $\begin{array}{l}\text { Deep Hole } 1 \\
\text { (Sandy Flats, } \\
\text { Part } 1 b, 4 \text { th } \\
\text { pass) }\end{array}$ & 204 & 13 & 07 & 06 & $\begin{array}{l}95.7 \\
96.3 \\
99.5 \\
98.9 \\
97.6\end{array}$ & $\begin{array}{r}111.2 \\
68.8 \\
105.1 \\
61.2 \\
111.2 \\
68.8 \\
105.1 \\
61.2 \\
111.2 \\
68.8 \\
105.1 \\
20.2\end{array}$ & $\begin{array}{l}18.7,20.5 \\
21.2,23.1 \\
19.3,21.8 \\
21.2,23.1 \\
19.3,21.2 \\
21.8,24.3\end{array}$ & \\
\hline $7 \quad \mathrm{D}$ & $\begin{array}{l}\text { Deep Hole } 1 \\
\text { (Sandy Flats, } \\
\text { Part 2a, 1st } \\
\text { pass }\end{array}$ & 218 & 12 & 07 & 06 & $\begin{array}{l}95.7 \\
96.3 \\
99.5 \\
98.9 \\
97.6\end{array}$ & $\begin{array}{r}106.2 \\
63.9 \\
100.1 \\
56.2 \\
106.2 \\
63.9 \\
100.1 \\
56.2 \\
106.2 \\
63.9 \\
100.1 \\
20.2\end{array}$ & $\begin{array}{l}20.5,23.1 \\
23.1,25.6 \\
22.4,25.0 \\
23.1,25.6 \\
21.2,23.1 \\
23.1,25.6\end{array}$ & $\begin{array}{l}\text { 7. Excavation of deep hole in drift material continued } \\
\text { using six retraction strokes for first pass and six } \\
\text { retraction strokes for second pass. Sequence was } \\
\text { similar to items } 4 \text { and } 6 \text { but with shorter extensions. }\end{array}$ \\
\hline & $\begin{array}{l}\text { Deep Hole } 1 \\
\text { (Sandy Flats, } \\
\text { Part 2, 2nd } \\
\text { pass) }\end{array}$ & 218 & 13 & 07 & 06 & $\begin{array}{l}95.7 \\
96.3 \\
99.5 \\
98.9 \\
97.6\end{array}$ & $\begin{array}{r}106.2 \\
63.9 \\
100.1 \\
56.2 \\
106.2 \\
63.9 \\
100.1 \\
56.2 \\
106.2 \\
63.9 \\
100.1 \\
20.2\end{array}$ & $\begin{array}{l}20.5,23.1 \\
23.1,25.6 \\
21.8,24.3 \\
23.7,25.6 \\
21.2,23.1 \\
23.7,26.2\end{array}$ & \\
\hline
\end{tabular}




\begin{tabular}{|c|c|c|c|c|c|c|c|c|c|}
\hline \multirow[t]{2}{*}{ Item } & \multirow[t]{2}{*}{ Activity } & \multicolumn{4}{|c|}{ Local Lander Time } & \multicolumn{3}{|c|}{ Surface-sampler positions } & \multirow[t]{2}{*}{ Description of activity } \\
\hline & & Sol & $(\mathrm{hr})$ & $(\min )$ & $(\mathrm{s})$ & $\begin{array}{c}\text { Azimuth } \\
\text { (degrees) }\end{array}$ & $\begin{array}{l}\text { Extension } \\
\text { (in.) }\end{array}$ & $\begin{array}{l}\text { Elevation } \\
\text { (degrees) }\end{array}$ & \\
\hline \multirow[t]{12}{*}{8} & \multirow{6}{*}{$\begin{array}{l}\text { Deep Hole } 1 \\
\text { (Sandy Flats, } \\
\text { Part 2b, 3rd } \\
\text { pass) }\end{array}$} & \multirow[t]{6}{*}{219} & \multirow[t]{6}{*}{12} & \multirow[t]{5}{*}{07} & \multirow[t]{6}{*}{06} & 95.7 & \multirow{6}{*}{$\begin{array}{r}106.2 \\
63.9 \\
100.1 \\
56.2 \\
106.2 \\
63.9 \\
100.1 \\
56.2 \\
106.2 \\
63.9 \\
100.1 \\
20.2\end{array}$} & $20.5,23.1$ & \multirow{12}{*}{$\begin{array}{l}\text { 8. Excavation of deep hole in drift material continued } \\
\text { as in item } 7 \text { above for third and fourth passes. Final } \\
\text { trench was } 22 \mathrm{~cm} \text { deep, } 30 \mathrm{~cm} \text { wide, and } 160 \mathrm{~cm} \text { iong from } \\
\text { tip to rim crest of tailings at end of Sol } 219 \text {. Maximum } \\
\text { relief of longest continuous slope on left wall is 18- } \\
20 \mathrm{~cm} \text {; slope angle is } 67.5^{\circ} \text {. Maximum relief of longest } \\
\text { continuous slope of right wall is } 15 \mathrm{~cm} \text {; slope angle is } \\
53^{\circ} \text {. }\end{array}$} \\
\hline & & & & & & 96.3 & & $23.1,25.6$ & \\
\hline & & & & & & 99.5 & & $21.8,25.0$ & \\
\hline & & & & & & 98.9 & & $23.1,25.6$ & \\
\hline & & & & & & 97.6 & & $21.2,23.7$ & \\
\hline & & & & 39 & & & & $23.7,26.2$ & \\
\hline & \multirow{6}{*}{$\begin{array}{l}\text { Deep Hole } 1 \\
\text { (Sandy Flats, } \\
\text { Part 2b, 4th } \\
\text { pass) }\end{array}$} & \multirow[t]{6}{*}{219} & \multirow[t]{6}{*}{13} & \multirow[t]{5}{*}{07} & \multirow[t]{5}{*}{06} & 95.7 & \multirow{6}{*}{$\begin{array}{r}106.2 \\
63.9 \\
100.1 \\
56.2 \\
106.2 \\
63.9 \\
100.1 \\
56.2 \\
106.2 \\
63.9 \\
100.1 \\
20.2\end{array}$} & $20.5,23.1$ & \\
\hline & & & & & & 96.3 & & $23.1,25.6$ & \\
\hline & & & & & & 99.5 & & $20.5,22.4$ & \\
\hline & & & & & & 98.9 & & $23.7,25.6$ & \\
\hline & & & & & & 97.6 & & $21.2,23.7$ & \\
\hline & & & & 39 & 05 & & & $23.7,26.2$ & \\
\hline \multirow[t]{2}{*}{9} & \multirow{2}{*}{$\begin{array}{l}\text { XRFS } 5 \text { sample } \\
\text { (Deep Hole 1) } \\
\text { C-6 }\end{array}$} & 229 & 08 & 09 & 28 & 97.6 & \multirow{2}{*}{$\begin{array}{r}100.1 \\
56.2 \\
90.9 \\
98.6 \\
56.2\end{array}$} & $24.3,26.9$ & \multirow{2}{*}{$\begin{array}{l}\text { 9. Trenched by retracting in drift material of deep } \\
\text { hole, followed by extending after surface contact, and a } \\
2-\mathrm{s} \text { deelevation, and then retracting. Sample trench not } \\
\text { mapped because only monoscopic view available; minor } \\
\text { slumping of trench walls precludes good depth estimate. } \\
\text { Inadequate sample of fine fraction of drift material de- } \\
\text { livered to XRFS because of surface-sampler mal function. }\end{array}$} \\
\hline & & 229 & 08 & 17 & 10 & 97.6 & & $26.2,28.1$ & \\
\hline 10 & $\begin{array}{l}\text { Purge XRFS } 5 \\
\text { (coarse fraction) }\end{array}$ & 229 & 08 & 40 & 33 & 203.6 & 63.1 & 28.8 & $\begin{array}{l}\text { 10. Purged in Sol } 34 \text { trench to build rock pile. No } \\
\text { evidence for purged coarse fraction of drift material. }\end{array}$ \\
\hline \multirow[t]{2}{*}{11} & \multirow[t]{2}{*}{$\begin{array}{c}\text { Biglogy } 4 \text { sample } \\
\text { (Deep Hole 1) }\end{array}$} & 250 & 08 & 09 & 22 & 97.6 & 100.1 & $24.3,26.9$ & \multirow{2}{*}{$\begin{array}{l}\text { 11. Trenched by retracting in drift material of deep hole, } \\
\text { followed by extending after surface contact, and a } 2-5 \\
\text { deelevation, and then retracting. No pictures were taken } \\
\text { after this sample. See item } 13 \text { below. Surface-sampler } \\
\text { data indicate sample of fine fraction of drift material } \\
\text { was delivered after one acquisition stroke. }\end{array}$} \\
\hline & & 250 & 08 & 17 & 12 & 97.6 & $\begin{array}{l}56.2 \\
90.9 \\
99.6 \\
56.2\end{array}$ & $26.2,28.8$ & \\
\hline 12 & Purge Biology 4 & 250 & 10 & 10 & 27 & 203.6 & 63.1 & 28.8 & $\begin{array}{l}\text { 12. Purged in Sol } 34 \text { trench to build rock pile. No } \\
\text { evidence (coarse fraction) for purged coarse fraction of } \\
\text { drift material. }\end{array}$ \\
\hline \multirow[t]{2}{*}{13} & \multirow{2}{*}{$\begin{array}{l}\text { XRFS-6 sample } \\
\text { (Deep Hole 1) } \\
\text { C-6 }\end{array}$} & 250 & 11 & 09 & 28 & 97.6 & 100.1 & $23.7,26.2$ & \multirow{2}{*}{$\begin{array}{l}\text { 13. Trenched by retracting in drift material of deep hole, } \\
\text { followed by extending after surface contact, and a } 2-\mathrm{s} \\
\text { deelevation, and then retracting. Deep Hole at sample } \\
\text { site is about } 2 \mathrm{~cm} \text { shallower than it was on Sol } 219 \\
\text { because of talus from walls; nearer the spacecraft trench } \\
\text { it is deeper than on Sol } 219 \text {. Sample from } 22-24 \mathrm{~cm} \text { below } \\
\text { original surface. Sample of fine fraction of drift } \\
\text { material delivered to XRFS. }\end{array}$} \\
\hline & & 250 & 11 & 17 & 10 & 97.6 & $\begin{array}{l}56.2 \\
90.9 \\
99.6 \\
56.2\end{array}$ & $26.2,28.8$ & \\
\hline
\end{tabular}




\begin{tabular}{|c|c|c|c|c|c|c|c|c|c|}
\hline Item & Activity & $\frac{\text { Loc }}{\text { Sol }}$ & $\frac{a l \quad L}{(h r)}$ & $\frac{\text { ander } T}{(\min )}$ & $\frac{i m e}{(s)}$ & $\frac{\text { Surfac }}{\text { Azimuth }}$ & $\begin{array}{l}\text {-sampler po } \\
\text { Extension } \\
\text { (in.) }\end{array}$ & $\begin{array}{l}\text { itions } \\
\text { Elevation } \\
\text { (degrees) }\end{array}$ & Description of activity \\
\hline 14 & $\begin{array}{l}\text { Purge XRFS } 6 \\
\quad \text { (coarse fraction) }\end{array}$ & 250 & 11 & 39 & 39 & 203.6 & 63.1 & 28.8 & $\begin{array}{l}\text { 14. Purged in Sol } 34 \text { trench to build rock pile. No } \\
\text { evidence for purged coarse fraction of drift material. }\end{array}$ \\
\hline 15 & $\begin{array}{l}\text { Backhoe touchdown } 2 \\
\text { (Magnetic Properties } \\
\text { (mineral abundance } \\
\text { experiment) } \\
\text { (Sandy Flats) }\end{array}$ & 270 & 13 & 22 & 40 & 113.4 & 71.0 & 31.9 & $\begin{array}{l}\text { 15. Backhoe penetrated drift material } 2.9 \pm 0.2 \mathrm{~cm} \text {. } \\
\text { Collector-head tip touched surface; backhoe partly } \\
\text { folded. }\end{array}$ \\
\hline 16 & $\begin{array}{l}\text { Pictures under } \\
\text { engine } 2 \text { via } \\
\text { mirror } 2\end{array}$ & 280 & $\begin{array}{l}11 \\
11 \\
11\end{array}$ & $\begin{array}{l}06 \\
12 \\
17\end{array}$ & $\begin{array}{l}48 \\
00 \\
17\end{array}$ & $\begin{array}{l}255.4 \\
251.6 \\
247.8\end{array}$ & $\begin{array}{l}\text { na } \\
\text { na } \\
\text { na }\end{array}$ & $\begin{array}{l}38.9 \\
38.9 \\
38.9\end{array}$ & $\begin{array}{l}\text { 16. Pictures beneath terminal-descent engine } 2 \text { via mirror } \\
\text { 2. One picture missed mirror; two pictures show surface } \\
\text { is scoured by engine-exhaust gases; more extensive erosion } \\
\text { occurs where corners of exposed rocks have channeled } \\
\text { flow of gases. }\end{array}$ \\
\hline 17 & $\begin{array}{l}\text { Physical Properties } 2 \\
\text { sample } \\
\text { (Rocky Flats) }\end{array}$ & 280 & 11 & 31 & 54 & 198.6 & $\begin{array}{l}62.0 \\
68.6 \\
44.4\end{array}$ & 34.4 & $\begin{array}{l}\text { 17. Trenched by extending in blocky material after surface } \\
\text { contact and then retracting. Trench is } 2-2.5 \mathrm{~cm} \text { deep, } 7 \\
\text { to } 12 \mathrm{~cm} \text { wide, and } 46 \mathrm{~cm} \text { long from tip to rim crest of } \\
\text { tailings. Tip of collector head probably penetrated to } \\
4.5 \mathrm{~cm} \text {. }\end{array}$ \\
\hline 18 & $\begin{array}{l}\text { Purge Phys. Props. } 2 \\
\text { (fine fraction) }\end{array}$ & 280 & 11 & 46 & 39 & 198.6 & 44.4 & 9.8 & $\begin{array}{l}\text { 18. Fine fraction purged. Coarse fraction used for drops } \\
\text { test in item } 19 \text { below. Dispersed fines not observed. }\end{array}$ \\
\hline 19 & $\begin{array}{l}\text { Purge Phys. Props. } 2 \\
\text { (coarse fraction) }\end{array}$ & 280 & 11 & 58 & 11 & 110.8 & 69.1 & 9.8 & $\begin{array}{l}\text { 19. Coarse fraction purged from } 0.85 \mathrm{~m} \text { above surface onto } \\
\text { drift material. Purged fragments were } 1.1 \mathrm{~cm} \text { wide and } \\
\text { smaller. Resolvable fragments penetrated less than one- } \\
\text { half their diameters. }\end{array}$ \\
\hline 20 & $\begin{array}{l}\text { Backhoe touchdown } 3 \\
\text { (Sandy Flats) }\end{array}$ & 280 & 12 & 08 & 01 & 110.8 & 92.0 & 25.0 & $\begin{array}{l}\text { 20. Backhoe penetrated drift material } 4.0 \pm 0.1 \mathrm{~cm} \text {; } \\
\text { collector-head tip touches surface. }\end{array}$ \\
\hline 21 & $\begin{array}{l}\text { XRFS } 7 \text { sample } \\
\text { (Wind tail) } \\
\text { C-7 }\end{array}$ & 286 & 11 & 13 & 08 & 129.8 & $\begin{array}{r}91.5 \\
94.4 \\
93.3 \\
99.4 \\
7.0 \\
91.5 \\
94.4 \\
93.3 \\
99.4 \\
7.0\end{array}$ & $\begin{array}{l}25.6 \\
26.2\end{array}$ & $\begin{array}{l}\text { 21. Trenched by small extensions after surface contact, } \\
\text { deelevations, small retractions, and then extensions to } \\
\text { produce skimming motion during sampling. Second trench in } \\
\text { drift material of wind tail superposed on first trench; } \\
\text { second trench is } 4 \mathrm{~cm} \text { deep, } 10 \mathrm{~cm} \text { wide, and } 67 \mathrm{~cm} \text { long } \\
\text { from tip to rim crest of tailings. Sample came from } \\
\text { depths between } 1-2 \mathrm{~cm} \text {. Sufficient sample was delivered } \\
\text { to XRFS. }\end{array}$ \\
\hline 22 & $\begin{array}{l}\text { Backhoe touchdown } 4 \\
\quad \text { (Bashful) }\end{array}$ & 296 & 09 & 07 & 13 & 219.4 & 51.2 & 35.7 & $\begin{array}{l}\text { 22. Backhoe penetrated blocky material } 0.6 \pm 0.5 \mathrm{~cm} \text {. } \\
\text { Collector-head tip touched surface and was partly } \\
\text { buried. }\end{array}$ \\
\hline 23 & $\begin{array}{l}\text { Physical Properties } 3 \\
\text { sample } \\
\text { (Sandy Flats) }\end{array}$ & 296 & 09 & 51 & 20 & 107.7 & $\begin{array}{r}95.2 \\
100.1 \\
56.2 \\
97.3 \\
102.3 \\
56.2\end{array}$ & 24.3 & $\begin{array}{l}\text { 23. Trenched by extending in drift material after } \\
\text { surface contact and then retracting. Trench is } 5 \mathrm{~cm} \\
\text { deep, } 9.5 \mathrm{~cm} \text { wide, and } 67 \mathrm{~cm} \text { long from tip to rim } \\
\text { crest of tailings. Collector-head tip probably reached } \\
\text { depth of } 5-6 \mathrm{~cm} \text {. Material of tip slumped into trench. } \\
\text { First sample of drift material used for conical pile } 1 \text {. }\end{array}$ \\
\hline 24 & Conical Pile 1 & 296 & 09 & 40 & 17 & 104.5 & $\begin{array}{r}100.7 \\
56.2\end{array}$ & 16.1 & $\begin{array}{l}\text { 24. Conical pile of drift material is } 3.6 \mathrm{~cm} \text { high; angle } \\
\text { of slope is } 45^{\circ} \text {. Only one of two samples was delivered } \\
\text { to form pile because of surface-sampler mal function. }\end{array}$ \\
\hline
\end{tabular}




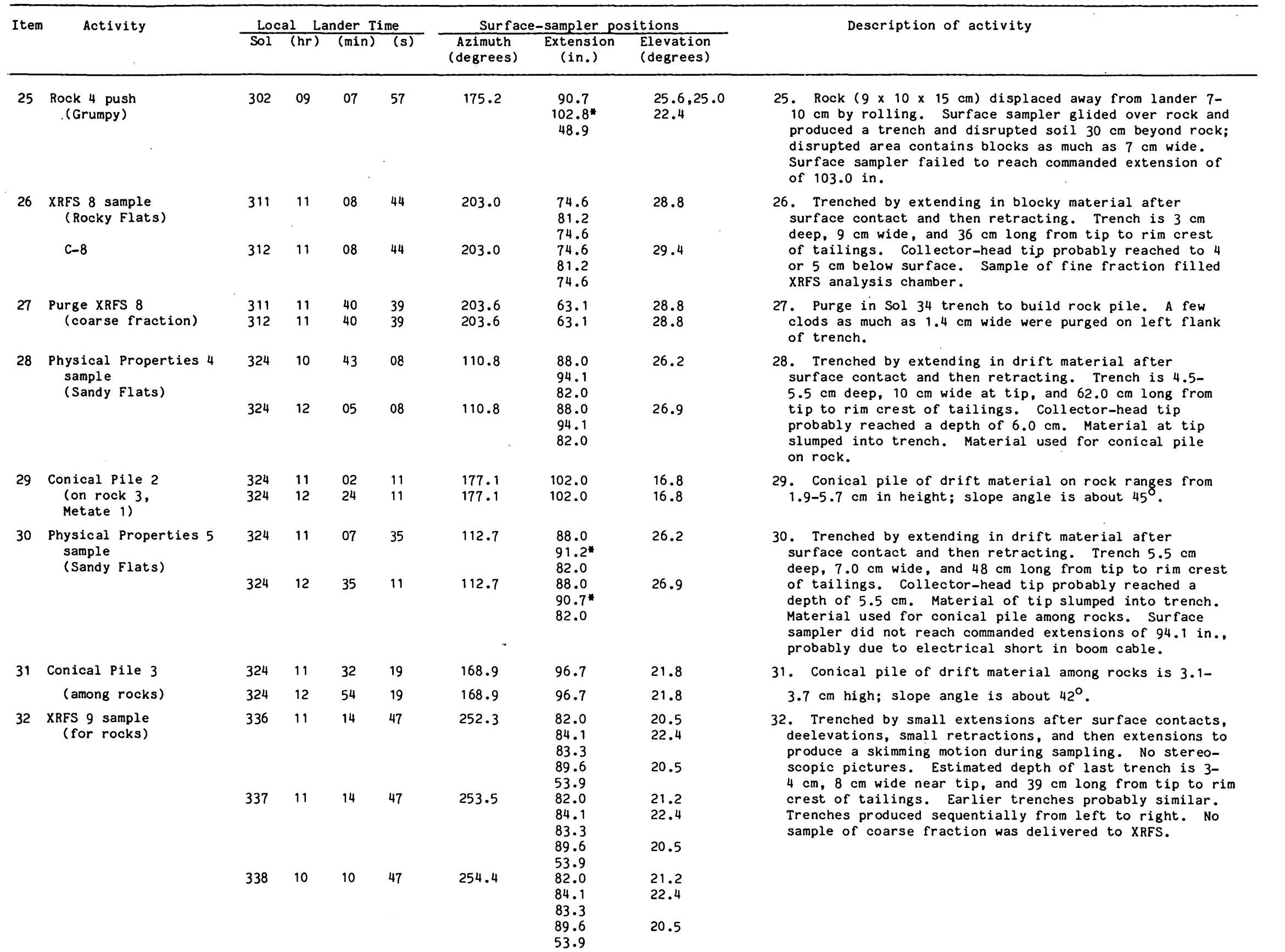


TABLE 39.-Surface-sampler activities related to the Physical Properties Investigation during the Extended Mission of Viking Lander 1-Continued

\begin{tabular}{|c|c|c|c|c|c|c|c|c|}
\hline \multirow[t]{2}{*}{ Item } & \multirow[t]{2}{*}{ Activity } & \multicolumn{4}{|c|}{ Local Lander Time } & \multicolumn{3}{|c|}{ Surface-sampler positions } \\
\hline & & Sol & $(\mathrm{hr})$ & $(\min )$ & (s) & $\begin{array}{l}\text { Azimuth } \\
\text { (degrees) }\end{array}$ & $\begin{array}{l}\text { Extension } \\
\text { (in.) }\end{array}$ & $\begin{array}{l}\text { Elevation } \\
\text { (degrees) }\end{array}$ \\
\hline 33 & $\begin{array}{l}\text { Purge XRFS } 9 \\
\text { (fine fraction) }\end{array}$ & $\begin{array}{l}336 \\
337 \\
338\end{array}$ & $\begin{array}{l}11 \\
11 \\
10\end{array}$ & $\begin{array}{l}56 \\
56 \\
52\end{array}$ & $\begin{array}{l}44 \\
44 \\
44\end{array}$ & $\begin{array}{l}252.3 \\
253.5 \\
254.8\end{array}$ & $\begin{array}{l}53.9 \\
53.9 \\
53.9\end{array}$ & $\begin{array}{l}-5.3 \\
-5.3 \\
-5.3\end{array}$ \\
\hline 34 & $\begin{array}{l}\text { Physical Properties } 6 \\
\text { Diurnal temperatures } \\
\text { (Sandy Flats) }\end{array}$ & $\begin{array}{l}343 \\
344\end{array}$ & $\begin{array}{l}10 \\
12\end{array}$ & 10 & $\begin{array}{l}41 \\
11\end{array}$ & 110.8 & $\begin{array}{l}92.0 \\
98.8^{*} \\
14.9\end{array}$ & $\begin{array}{l}19.9,26.2 \\
10.4\end{array}$ \\
\hline 35 & $\begin{array}{l}\text { Conical pile } 4 \\
\text { (next to ventifact) }\end{array}$ & 344 & 12 & 26 & 30 & 144.3 & 77.0 & 25.6 \\
\hline 36 & $\begin{array}{l}\text { Physical Properties } 7 \\
\text { (Special comminute) } \\
\text { (Rocky Flats) }\end{array}$ & 350 & 14 & 10 & $\begin{array}{r}51 \\
45\end{array}$ & 201.0 & $\begin{array}{l}72.0 \\
80.2 \\
72.0 \\
16.2\end{array}$ & -10.5 \\
\hline 37 & $\begin{array}{l}\text { Purge Phys. Props. } 7 \\
\text { (coarse fraction) }\end{array}$ & 350 & 14 & 46 & 20 & 117.8 & 69.1 & 9.8 \\
\hline 38 & $\begin{array}{l}\text { Rock } 5 \text { push } \\
\text { (Bashful) }\end{array}$ & 369 & 12 & 02 & 43 & 219.4 & $\begin{array}{l}53.3 \\
63.3 \\
59.1 \\
13.9\end{array}$ & $\begin{array}{l}31.3,31.9 \\
30.0\end{array}$ \\
\hline 39 & $\begin{array}{l}\text { Push-chip rock } 6 \text {, } \\
\text { (Metate 2) }\end{array}$ & 376 & $\begin{array}{l}11 \\
11\end{array}$ & $\begin{array}{l}31 \\
39\end{array}$ & $\begin{array}{l}35 \\
49\end{array}$ & 239.6 & $\begin{array}{l}49.6 \\
44.6 \\
50.4\end{array}$ & $\begin{array}{l}29.4 \\
30.0\end{array}$ \\
\hline & & 376 & 11 & 45 & 23 & 239.6 & $\begin{array}{l}44.0 \\
49.6 \\
44.6\end{array}$ & $\begin{array}{l}23.1 \\
28.8\end{array}$ \\
\hline & & & 11 & 46 & 30 & . & $\begin{array}{l}50.4 \\
44.6\end{array}$ & 23.1 \\
\hline & & 376 & 11 & 52 & 25 & 242.8 & $\begin{array}{l}51.0 \\
46.0\end{array}$ & 27.5 \\
\hline & & & 11 & 53 & 38 & & $\begin{array}{r}52.0 \\
4.1\end{array}$ & \\
\hline 40 & $\begin{array}{l}\text { XRFS } 10 \text { sample } \\
\text { (near Bashful) } \\
\text { C-9 }\end{array}$ & 378 & 14 & 16 & 15 & 219.4 & $\begin{array}{l}55.4 \\
60.4 \\
48.3\end{array}$ & 30.6 \\
\hline
\end{tabular}

33. Purged fine-grained material dispersed.

34. Collector-head temperatures obtained throughout diurnal cycle (high of $215.5^{\circ} \mathrm{K}$ near $15: 30$ and low of $187.5^{\circ} \mathrm{x}$ near $\left.7: 30\right)$. collector head appears to be sensor is 6-7 cm below surface. Trenched by extension in drift material after surface contact and then retracting. Trench is $6 \mathrm{~cm}$ deep, $9 \mathrm{~cm}$ wide near tip. and $65 \mathrm{~cm}$ long from tip to rim crest of tailings. Surface sampler failed to reach commanded extension of 100.1 in.; probably hit buried rock.

35. Conical pile of drift material adjacent to ventifact is about 4 to $5 \mathrm{~cm}$ high; no stereoscopic pictures of pile.

36. Trenched by extending in blocky material after surface contact and then retracting. Trench is probably $3 \mathrm{~cm}$ deep, $8 \mathrm{~cm}$ wide, and $37 \mathrm{~cm}$ long from tip to $\mathrm{rim}$ crest of tailings. Clods and rocks as much as $5 \mathrm{~cm}$ wide occupy far end of trench; slope of tailings nearest lander is $33^{\circ}$. Comminutor motor currents indicate that no sample was obtained.

37. A few small objects as much as $0.5 \mathrm{~cm}$ were purged rom a height of $0.82 \mathrm{~m}$ onto drift material and none of to have penetrated a significant amount.

38. Collector-head tip touched rock during deelevation; subsequent elevation caused collector head to glide over rock during extension. Trench produced beyond rock by extension and subsequent retraction. Backhoe of collector head pulled rock toward lander during retractions to 59.1 and 13.9 in. Rock toppled to left of commanded azimuth during final retraction to 13.9 in. Trench depth about $2.0 \mathrm{~cm}$, width $8 \mathrm{~cm}$ at tip. Removal of rock exposed fillet on right side of rock.

39. Rock was displaced by rotating counterclockwise during attempted chip. There is no evidence that the
rock chipped, scratched, or spalled during the three rock chipped

40. Trenched by extending in material of wind tail after surface contact and then retracting. Analyses of pictures indicate sample came from area behind rock rather than area originall y beneath rock. Trench is about $3 \mathrm{~cm}$ deep and $7.0 \mathrm{~cm}$ wide at tip. Bashful was analysis chamber. 
TABLE 39.-Surface-sampler activities related to the Physical Properties Investigation during the Extended Mission of Viking Lander 1-Continued

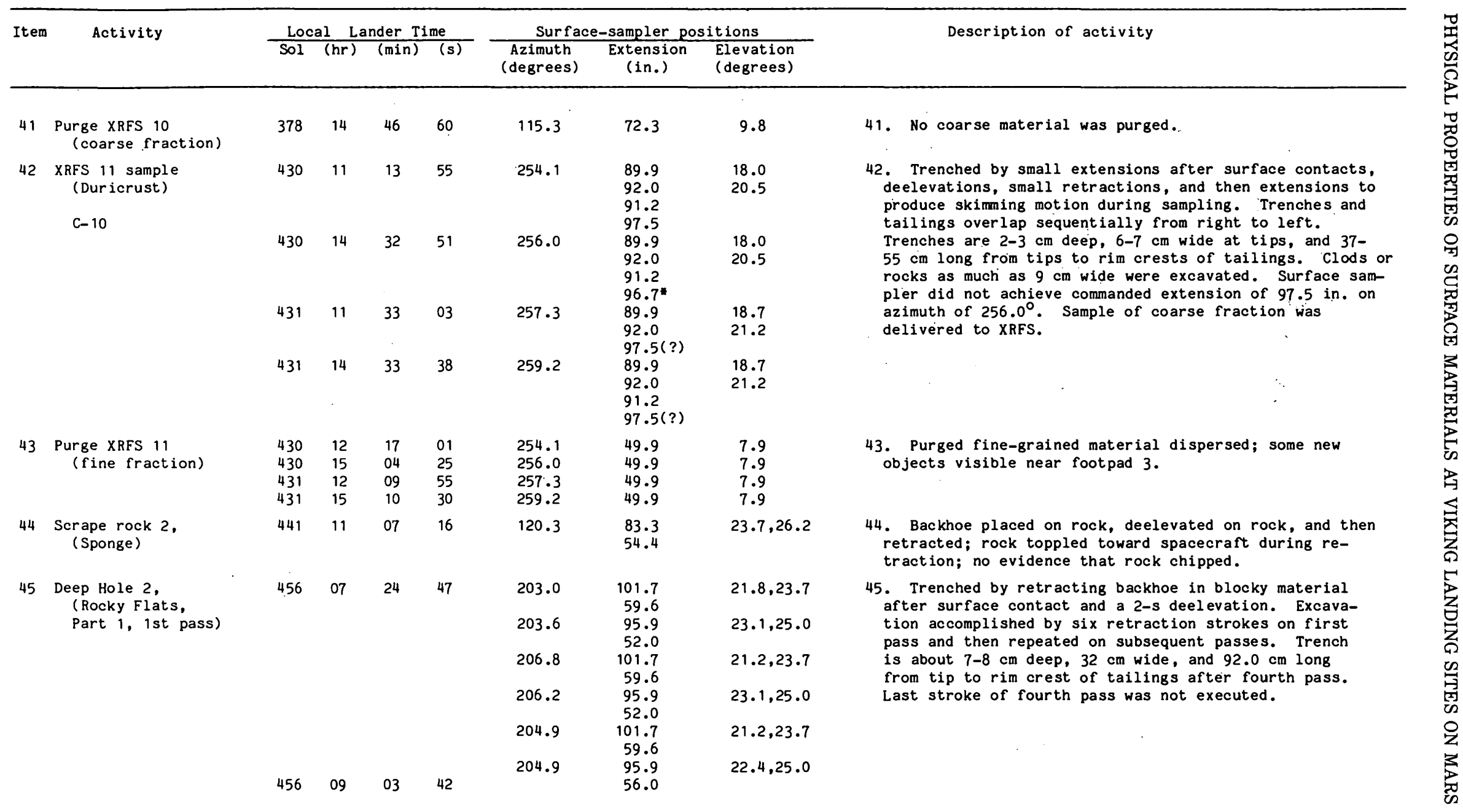


TABLE 39.-Surface-sampler activities related to the Physical Properties Investigation during the Extended Mission of Viking Lander 1-Continued

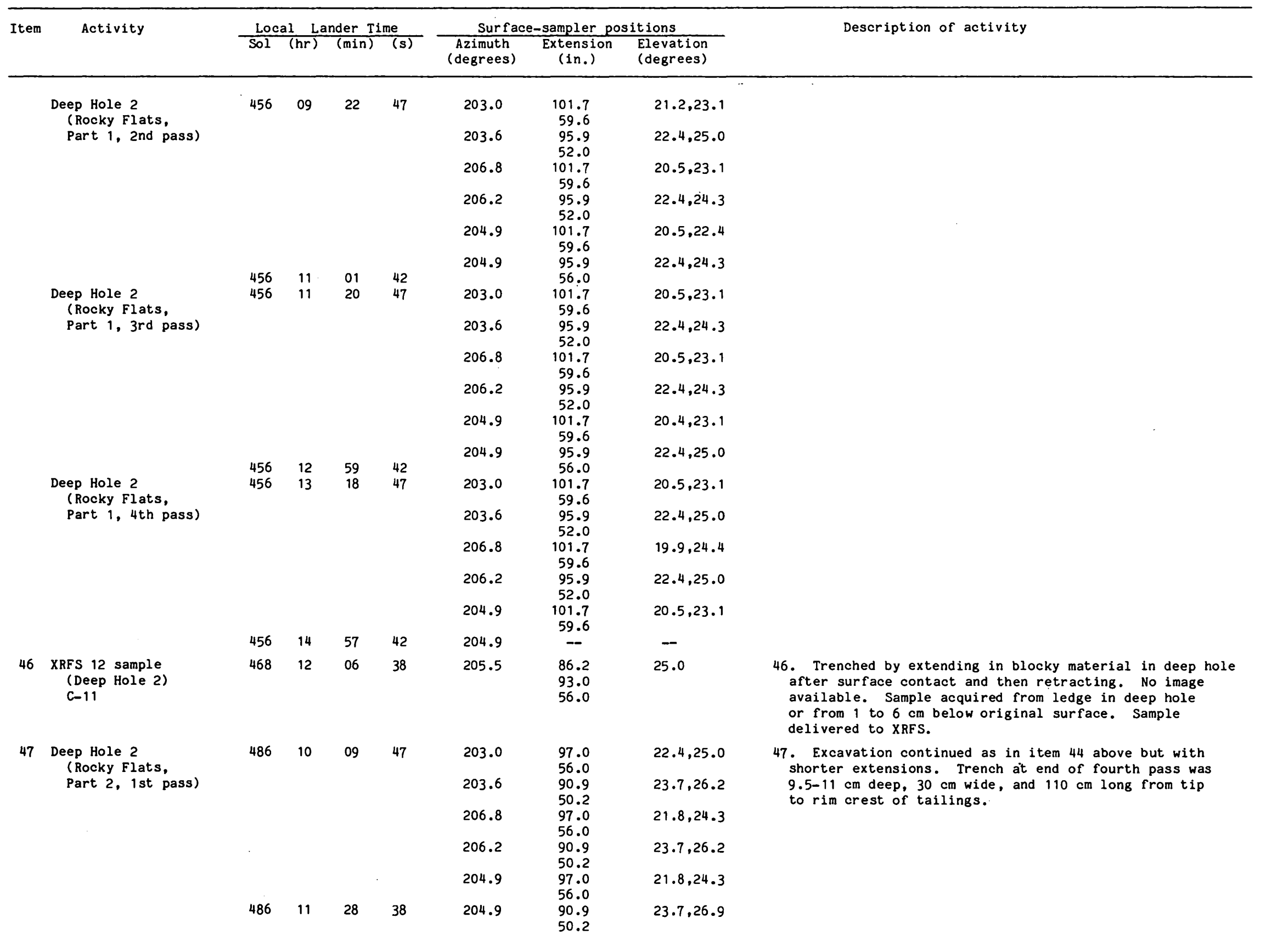


TABLE 39.-Surface-sampler activities related to the Physical Properties Investigation during the Extended Mission of Viking Lander 1-Continued

\begin{tabular}{|c|c|c|c|c|c|c|c|c|c|}
\hline \multirow[t]{2}{*}{ Item } & \multirow{2}{*}{ Activity } & \multicolumn{4}{|c|}{ Local Lander Time } & \multicolumn{3}{|c|}{ Surface-sampler positions } & \multirow{2}{*}{ Description of activity } \\
\hline & & Sol & $(\mathrm{hr})$ & $(\min )$ & (s) & $\begin{array}{c}\text { Azimuth } \\
\text { (degrees) }\end{array}$ & $\begin{array}{l}\text { Extension } \\
\text { (in.) }\end{array}$ & $\begin{array}{l}\text { Elevation } \\
\text { (degrees) }\end{array}$ & \\
\hline & \multirow{6}{*}{$\begin{array}{l}\text { Deep Hole } 2 \\
\text { (Rocky Flats, } \\
\text { Part 2, 2nd pass) }\end{array}$} & \multirow[t]{5}{*}{486} & \multirow[t]{5}{*}{11} & \multirow[t]{5}{*}{49} & \multirow[t]{5}{*}{47} & 203.0 & $\begin{array}{l}97.0 \\
56.0\end{array}$ & $21.8,24.3$ & \\
\hline & & & & & & 203.6 & $\begin{array}{l}90.9 \\
50.2\end{array}$ & $22.4,25.6$ & \\
\hline & & & & & & 206.8 & $\begin{array}{l}97.0 \\
56.0\end{array}$ & $21.8,24.3$ & \\
\hline & & & & & & 206.2 & $\begin{array}{l}90.9 \\
50.2\end{array}$ & $23.7,26.9$ & \\
\hline & & & & & & 204.9 & $\begin{array}{l}97.0 \\
56.0\end{array}$ & $21.8,24.3$ & \\
\hline & & 486 & 13 & 08 & 38 & 204.9 & $\begin{array}{l}90.9 \\
50.2\end{array}$ & $23.7,26.2$ & \\
\hline & \multirow{6}{*}{$\begin{array}{l}\text { Deep Hole } 2 \\
\text { (Rocky Flats, } \\
\text { Part 2, 3rd pass) }\end{array}$} & \multirow[t]{5}{*}{486} & \multirow[t]{5}{*}{13} & \multirow[t]{5}{*}{29} & \multirow[t]{5}{*}{47} & 203.0 & $\begin{array}{l}97.0 \\
56.0\end{array}$ & $22.4,24.3$ & \\
\hline & & & & & & 203.6 & $\begin{array}{l}90.9 \\
50.2\end{array}$ & $23.7,26.2$ & \\
\hline & & & & & & 206.8 & $\begin{array}{l}97.0 \\
56.0\end{array}$ & $21.8,24.3$ & \\
\hline & & & & & & 206.2 & $\begin{array}{l}90.9 \\
50.2\end{array}$ & $25.0,27.5$ & \\
\hline & & & & & & 204.9 & $\begin{array}{l}97.0 \\
56.0\end{array}$ & $21.8,24.3$ & \\
\hline & & 486 & 14 & 48 & 38 & 204.9 & $\begin{array}{l}90.9 \\
50.2\end{array}$ & $24.3,26.9$ & \\
\hline & \multirow{6}{*}{$\begin{array}{l}\text { Deep Hole } 2 \\
\text { (Rocky Flats, } \\
\text { Part 2, } 4 \text { th pass) }\end{array}$} & \multirow[t]{5}{*}{486} & \multirow[t]{5}{*}{15} & \multirow[t]{5}{*}{09} & \multirow[t]{5}{*}{47} & 203.0 & $\begin{array}{l}97.0 \\
56.0\end{array}$ & $21.8,24.3$ & \\
\hline & & & & & & 203.6 & $\begin{array}{l}90.9 \\
50.2\end{array}$ & $24.3,26.9$ & \\
\hline & & & & & & 206.8 & $\begin{array}{l}97.0 \\
56.0\end{array}$ & $21.8,24.3$ & \\
\hline & & & & & & 206.2 & $\begin{array}{l}90.9 \\
50.2\end{array}$ & $25.0,27.5$ & \\
\hline & & & & & & 204.9 & $\begin{array}{l}97.0 \\
56.0\end{array}$ & $21.8,24.3$ & \\
\hline & & 486 & 16 & 25 & 43 & 204.9 & $\begin{array}{l}90.9 \\
50.2\end{array}$ & $24.3,26.9$ & \\
\hline 48 & $\begin{array}{l}\text { XRFS } 13 \text { sample } \\
\text { (Deep Hole 2) }\end{array}$ & 486 & 16 & 47 & 30 & 204.9 & $\begin{array}{l}85.2 \\
92.8 * \\
56.0\end{array}$ & 26.2 & $\begin{array}{l}\text { 48. Trenched by extending in blocky material in deep } \\
\text { hole after surface contact and then retracting. } \\
\text { Shadows preclude measurements of trench. Comparisons } \\
\text { with other images suggest trench is similar to Sol } 502 \\
\text { trench (see below). Surface sampler did not achieve } \\
\text { commanded extension of } 94.9 \text { in. No sample delivered } \\
\text { to XRFS. }\end{array}$ \\
\hline \multirow[t]{2}{*}{49} & \multirow{2}{*}{$\begin{array}{l}\text { XRFS } 14 \text { sample } \\
\text { (Deep Hole 2) } \\
\text { C-12 }\end{array}$} & 502 & 10 & 08 & 39 & 204.9 & $\begin{array}{l}84.9 \\
91.7^{*}\end{array}$ & 26.9 & \multirow{2}{*}{$\begin{array}{l}\text { 49. Trenched by extending in blocky material in deep } \\
\text { hole after surface contact and then retracting. Sample } \\
\text { trench reached a maximum depth of } 9.5-11 \mathrm{~cm} \text { below origi- } \\
\text { nal surface. Local relief at tip is about } 5 \mathrm{~cm} \text {, width } \\
\text { is } 8.5 \mathrm{~cm} \text {, and length is } 15 \mathrm{~cm} \text {. Far end of trench has } \\
\text { overhang. Surface sampler did not achieve commanded } \\
\text { extensions of } 95.9 \text { in. Sample was delivered to XRFS. }\end{array}$} \\
\hline & & 502 & 12 & 07 & 24 & 204.9 & $\begin{array}{c}9.1 \\
84.9 \\
92.5^{*} \\
9.1\end{array}$ & 26.9 & \\
\hline
\end{tabular}


TABLE 39.-Surface-sampler activities related to the Physical Properties Investigation during the Extended Mission of Viking Lander 1-Continued

\begin{tabular}{|c|c|c|c|c|c|c|c|c|c|}
\hline \multirow[t]{2}{*}{ Item } & \multirow[t]{2}{*}{ Activity } & \multicolumn{4}{|c|}{ Local Lander Time } & \multicolumn{3}{|c|}{ Surface-sampler positions } & \multirow[t]{2}{*}{ Description of activity } \\
\hline & & Sol & $(\mathrm{hr})$ & $(\min )$ & $(s)$ & $\begin{array}{c}\text { Azimuth } \\
\text { (degrees) }\end{array}$ & $\begin{array}{l}\text { Extension } \\
(\text { in.) }\end{array}$ & $\begin{array}{l}\text { Elevation } \\
\text { (degrees) }\end{array}$ & \\
\hline 50 & Backhoe touchdown 5 & 520 & 09 & 03 & 33 & 114.6 & $\begin{array}{l}92.0 \\
57.0\end{array}$ & 25.0 & $\begin{array}{l}\text { 50. Backhoe penetrated drift material } 3.2 \pm 0.3 \mathrm{~cm} \text {. } \\
\text { Collector-head tip touches surface. }\end{array}$ \\
\hline 51 & $\begin{array}{l}\text { Picture of footpad } 2 \\
\text { via mirror } 1\end{array}$ & 520 & 10 & 03 & 36 & 124.1 & 4.1 & 40.1 & $\begin{array}{l}\text { 51. Picture of temperature sensor on footpad } 2 \text { shows en- } \\
\text { tire housing of temperature sensors. Temperature sensors } \\
\text { remained buried } 0.2 \text { and } 1.5 \mathrm{~cm} \text { in drift material. }\end{array}$ \\
\hline 52 & $\begin{array}{l}\text { XRFS } 15 \text { sample } \\
\text { (Bashful) }\end{array}$ & 527 & 09 & 00 & 10 & 220.7 & $\begin{array}{l}53.3 \\
59.9 \\
54.6\end{array}$ & 33.8 & $\begin{array}{l}\text { 52. Trenched by extending in area originally beneath } \\
\text { Bashful after surface contact and then retracting. } \\
\text { Sample trench is about } 6 \mathrm{~cm} \text { deep, } 6.5 \mathrm{~cm} \text { wide, and } 25 \mathrm{~cm} \text {. } \\
\text { long. } 11 \mathrm{~cm} \text { rock to left displaced } 5 \mathrm{~cm} \text { away from lander; } \\
\text { second rock to left rotated } 25^{\circ} \text { counterclockwise; rota- } \\
\text { tion revealed ledge of soil adhered to base of rock. } \\
\text { Further displacement of Bashful toward lander plowed soil. } \\
\text { No sample delivered to XRFS. }\end{array}$ \\
\hline 53 & Backhoe touchdown 6 & 527 & 15 & 07 & 43 & 165.8 & $\begin{array}{r}97.8 \\
4.1\end{array}$ & 24.3 & $\begin{array}{l}\text { 53. Backhoe teeth visible but do not touch surface. Col- } \\
\text { lector-head tip is on rock or clod or backhoe is folded. }\end{array}$ \\
\hline 54 & $\begin{array}{l}\text { Picture under } \\
\text { engine } 2 \text { via } \\
\text { mirror } 2 \text { (picture } \\
\text { (taken on Sol 528) }\end{array}$ & 527 & 16 & 05 & 02 & 249.1 & 4.1 & 40.1 & $\begin{array}{l}\text { 54. Picture beneath terminal-descent engine } 2 \text { via mirror } 2 \text {. } \\
\text { Picture shows surface scoured by engine-exhause gases left } \\
\text { a residue of fragments } 2 \mathrm{~cm} \text { wide and smaller. One of } \\
\text { seven pictures used in mosaic. }\end{array}$ \\
\hline 55 & $\begin{array}{l}\text { Pictures under } \\
\text { engine } 2 \text { via } \\
\text { mirror } 2\end{array}$ & $\begin{array}{l}550 \\
550 \\
550\end{array}$ & $\begin{array}{l}09 \\
09 \\
10\end{array}$ & $\begin{array}{l}00 \\
30 \\
00\end{array}$ & $\begin{array}{l}10 \\
21 \\
38\end{array}$ & $\begin{array}{l}254.1 \\
259.2 \\
264.2\end{array}$ & 4.1 & 40.1 & $\begin{array}{l}\text { 55. Three pictures beneath terminal-descent engine } 2 \text { via } \\
\text { mirror 2. Pictures show surface scoured by engine- } \\
\text { exhaust gases produced oval crater } 10 \times 20 \mathrm{~cm} \text {; exposed } \\
\text { blocky units of cohesive materials, and left. a residue } \\
\text { of fragments } 2-3 \mathrm{~cm} \text { wide and smaller. Three of seven } \\
\text { pictures used in mosaic. }\end{array}$ \\
\hline 56 & Backhoe touchdown 7 & 550 & 10 & 37 & 20 & 103.3 & 87.8 & 24.3 & $\begin{array}{l}\text { 56. Backhoe penetrated tailings of Deep Hole } 1 \text { (drift } \\
\text { material) } 4.5 \pm 0.2 \mathrm{~cm} \text {. Collector-head tip touches the } \\
\text { surface. }\end{array}$ \\
\hline 57 & $\begin{array}{l}\text { Pictures under } \\
\text { engine } 2 \text { via } \\
\text { mirror } 2\end{array}$ & 550 & 11 & 37 & 29 & 264.2 & 4.1 & 36.3 & $\begin{array}{l}\text { 57. Picture beneath terminal-descent engine } 2 \text { via mirror } 2 . \\
\text { Picture shows surface scoured by engine-exhaust gases and } \\
\text { part of } 6-\mathrm{cm} \text { fragment. Dark area is shadow of spacecraft } \\
\text { part. One of seven pictures used in mosaic. }\end{array}$ \\
\hline 58 & $\begin{array}{l}\text { XRFS } 16 \text { sample } \\
\text { (Deep Hole } 2 \\
\text { tailings) } \\
\text { C-13 }\end{array}$ & 558 & 08 & 06 & 49 & 207.4 & $\begin{array}{l}56.7 \\
63.3 \\
56.2 \\
56.7 \\
63.3 \\
56.2 \\
56.7 \\
62.0\end{array}$ & $\begin{array}{l}35.7 \\
-7.9 \\
35.7 \\
-7.9 \\
35.7\end{array}$ & $\begin{array}{l}\text { 58. Trenched by extending into tailings of deep hole after } \\
\text { surface contact and then retracting. No high resolution } \\
\text { stereoscopic coverage. Floor of longest trench about } 8- \\
10 \mathrm{~cm} \text { below original surface of tailings and largest } \\
\text { trench is about } 13 \mathrm{~cm} \text { wide. Surface sampler failed to } \\
\text { achieve-commanded extension of } 63.3 \text { in. } 12 \text { out of } 16 \\
\text { extension strokes. No sample of coarse fraction } \\
\text { delivered to XRFS. }\end{array}$ \\
\hline
\end{tabular}


TABLE 39.-Surface-sampler activities related to the Physical Properties Investigation during the Extended Mission of Viking Lander 1-Continued

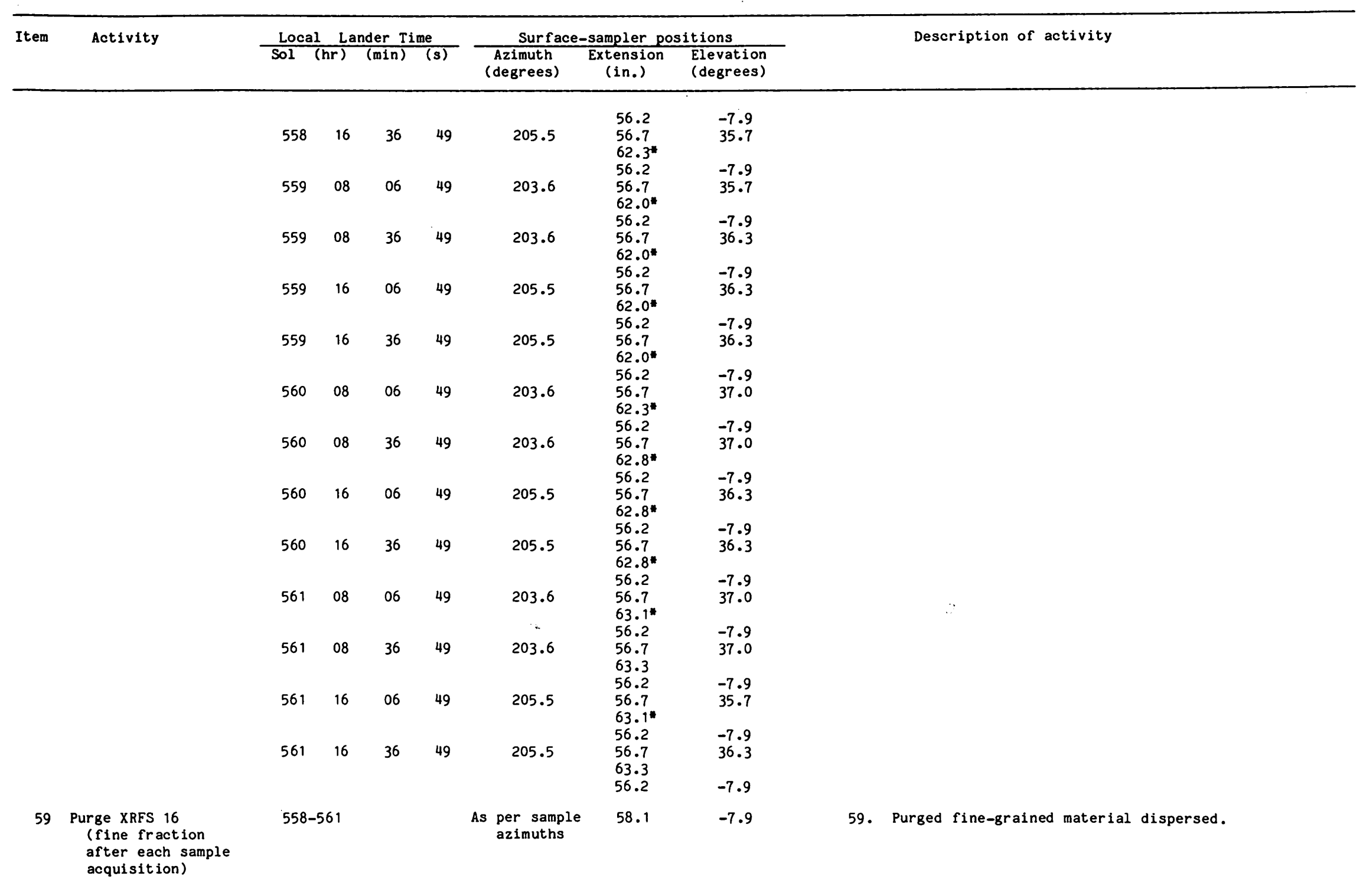


TABLE 39.-Surface-sampler activities related to the Physical Properties Investigation during the Extended Mission of Viking Lander 1-Continued

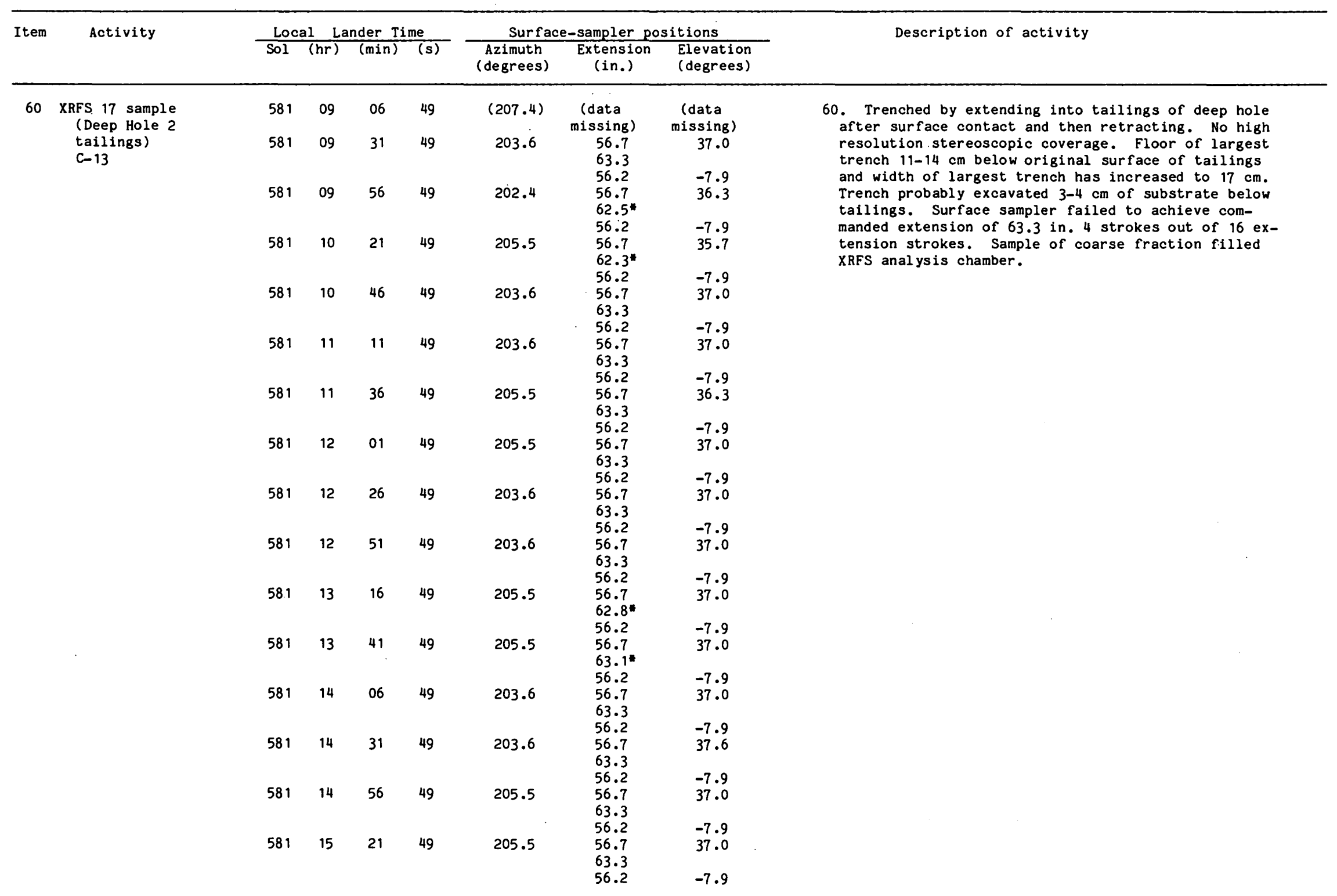




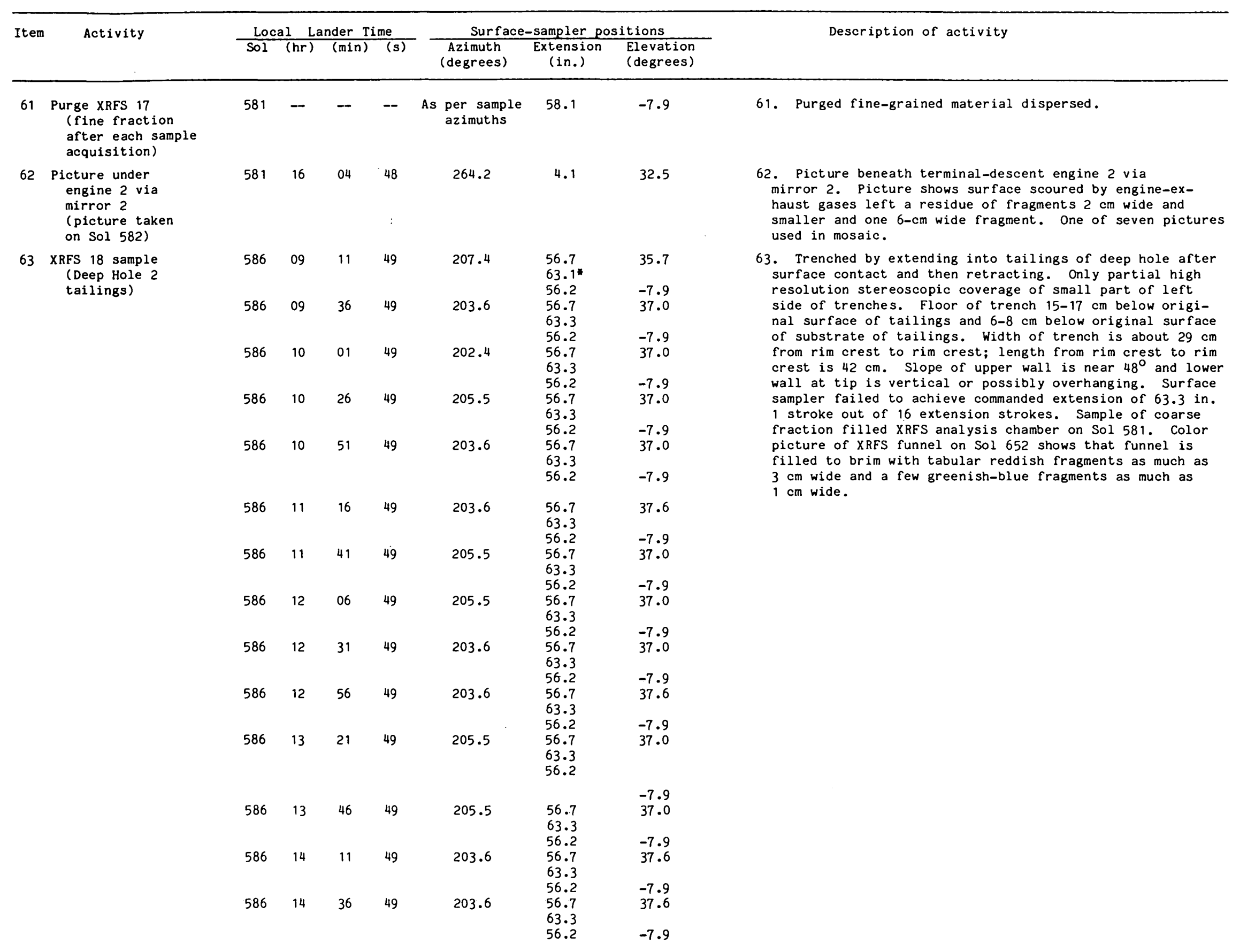


TABLE 39.-Surface-sampler activities related to the Physical Properties Investigation during the Extended Mission of Viking Lander 1-Continued

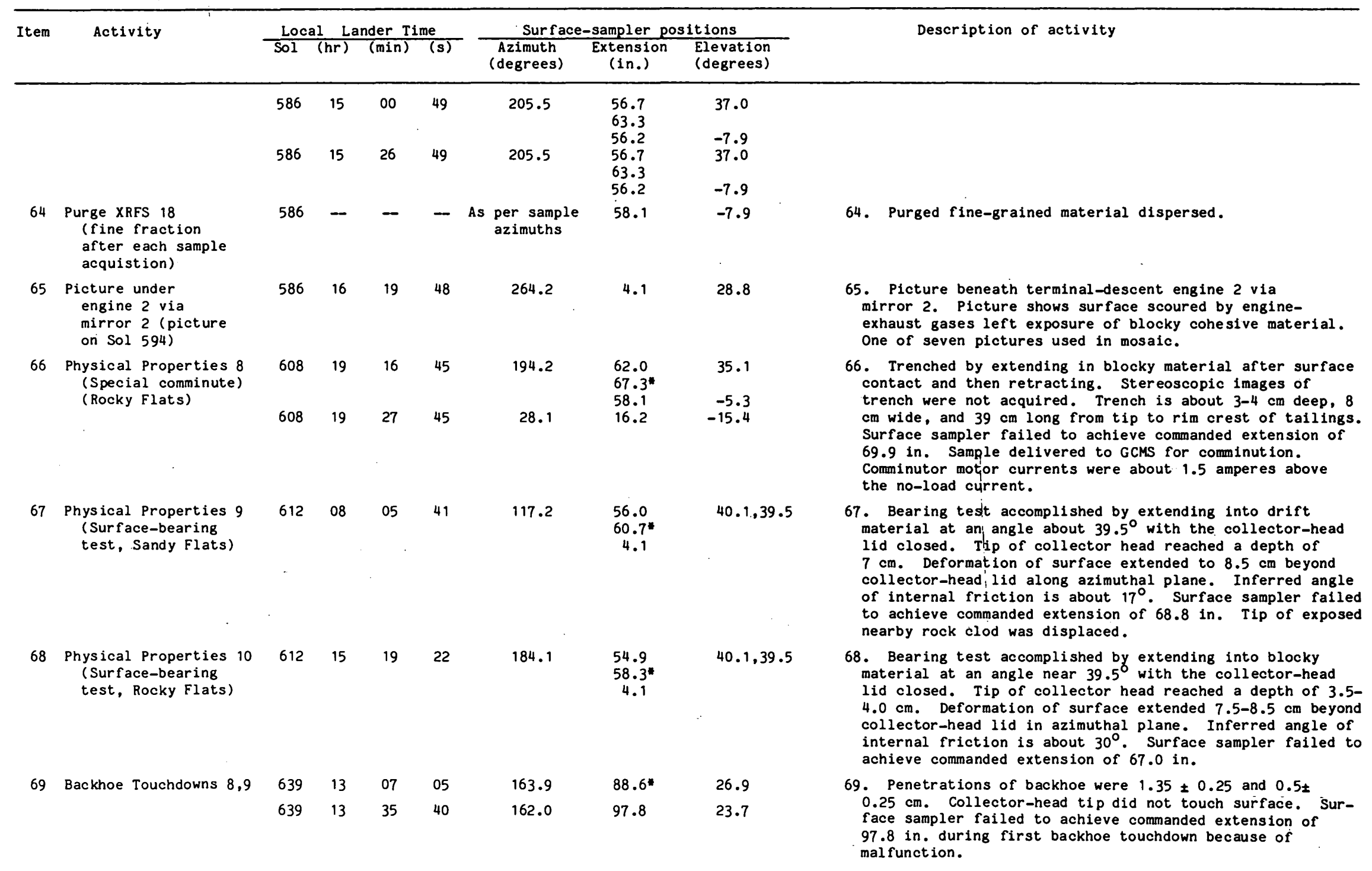


TABLE 39.-Surface-sampler activities related to the Physical Properties Investigation during the Extended Mission of Viking Lander 1-Continued

\begin{tabular}{|c|c|c|c|c|c|c|c|c|c|}
\hline \multirow[t]{2}{*}{ Item } & \multirow{2}{*}{ Activity } & \multicolumn{4}{|c|}{ Local Lander Time } & \multicolumn{3}{|c|}{ Surface-sampler positions } & \multirow[t]{2}{*}{ Description of activity } \\
\hline & & Sol & $(\mathrm{hr})$ & )$(\min )$ & $(s)$ & $\begin{array}{c}\text { Azimuth } \\
\text { (degrees) }\end{array}$ & $\begin{array}{l}\text { Extension } \\
\text { (in.) }\end{array}$ & $\begin{array}{l}\text { Elevation } \\
\text { (degrees) }\end{array}$ & \\
\hline 70 & $\begin{array}{l}\text { Physical Properties } 11 \\
\text { sample } \\
\text { (Rocky Flats) }\end{array}$ & 639 & 14 & 03 & 47 & 194.8 & $\begin{array}{l}62.0 \\
67.5^{\star} \\
58.1\end{array}$ & 34.4 & $\begin{array}{l}\text { 70. Trenched by extending in blocky material after suface } \\
\text { contact and then retracting. Trench is } 4 \mathrm{~cm} \text { deep, } 8 \mathrm{~cm} \\
\text { wide, and } 40 \mathrm{~cm} \text { long from tip to rim crest of tailings. } \\
\text { Collector head probably penetrated to } 5 \mathrm{~cm} \text { below surface. } \\
\text { Sample collected was used to form conical pile. Surface } \\
\text { sampler failed to achieve commanded extension of } 69.9 \text { in. }\end{array}$ \\
\hline 71 & $\begin{array}{l}\text { Conical Pile } 5 \\
\text { (between rocks) }\end{array}$ & 639 & 14 & 08 & 59 & 172.1 & 96.7 & 21.8 & $\begin{array}{l}\text { 71. Conical pile of blocky material; no stereoscopic image } \\
\text { available. Base is about } 7 \mathrm{~cm} \text { wide; relief is about } 3 \mathrm{~cm} \text {; } \\
\text { slope of pile is shadowed when sun angle is } 38.6^{\circ} \text {. }\end{array}$ \\
\hline 72 & Park & 639 & 14 & 16 & 75 & 3.5 & 4.1 & 14.9 & \\
\hline
\end{tabular}


TABLE 40.-Surface-sampler activities related to the Physical Properties Investigation during the Primary Mission of Viking Lander 2

[Listed Local Lander Times correspond to: (a) time of extension command for shroud ejection, (b) collector-head open command just before extension, (c) extension commands for nudges and pushes, and (d) vibration commands for purges.
Positions are from surface-sampler potentiometer readouts. Azimuths measured from a line $80^{\circ}$ counterclockwise from the $(+) Y_{L}$ direction (direction from camera 2 to camera 1). Azimuths should be reduced by about $0.3^{\circ}$ because Positions are from surface-sampler potentiometer readouts. Azimuths measured from a line $80^{\circ}$ counterclockwise from the $(+) Y_{L}$ direction (direction from camera 2 to camera 1). Azimuths should be reduced by about $0.3^{\circ}$ because
of boom override due to lander tilt. Extensions are reported here in inches to be consistent with engineering units used for the surface-sampler system and represent the increase in length of the boom from the stowed position. Elevations are measured from a plane parallel to upper surface of lander body that passes through the surface-sampler elevation axis; $(+)$ is angle below plane, $(-)$ angle above plane. Asterisk by extension indicates that commanded extension was not achieved. Letter-number designations listed under "Activity" refer to sample analysis numbers used by Inorganic Chemical Analysis Team (XRFS) (for example, U-1)]

\begin{tabular}{|c|c|c|c|c|c|c|c|c|c|}
\hline \multirow[t]{2}{*}{ Item } & \multirow[t]{2}{*}{ Activity } & \multicolumn{4}{|c|}{ Local Lander Time } & \multicolumn{3}{|c|}{ Surface-sampler positions } & \multirow[t]{2}{*}{ Description of activity } \\
\hline & & Sol & $(\mathrm{hr})$ & $(\min )$ & $(s)$ & $\begin{array}{l}\text { Azimuth } \\
\text { (degrees) }\end{array}$ & $\begin{array}{l}\text { Extension } \\
\text { (in.) }\end{array}$ & $\begin{array}{l}\text { Elevation } \\
\text { (degrees) }\end{array}$ & \\
\hline 1 & $\begin{array}{l}\text { Shroud } \\
\quad \text { ejection }\end{array}$ & 01 & 10 & 52 & 02 & 255.4 & 10.2 & 38.9 & $\begin{array}{l}\text { 1. Shroud ejected at } 3.2 \mathrm{~m} / \mathrm{s} \text {, struck rock near footpad } 3 \text { at } \\
3.7 \mathrm{~m} / \mathrm{s} \text {, ricocheted from rock impacting surface } 0.6 \mathrm{~m} \text { be- } \\
\text { yond rock, and came to rest } 1.1 \mathrm{~m} \text { beyond rock. Rock near } \\
\text { footpad } 3 \text { moved a small amount as a result of the impact. }\end{array}$ \\
\hline 2 & $\begin{array}{l}\text { Biology } 1 \text { sample } \\
\text { (Beta) }\end{array}$ & 08 & 16 & 10 & 20 & 124.7 & $\begin{array}{l}85.2 \\
91.7 \\
79.6\end{array}$ & $\begin{array}{l}23.1 \\
--\end{array}$ & $\begin{array}{l}\text { 2. Trenched by extending in crusty to cloddy material after } \\
\text { surface contact and then retracting. Trench is about } 3.5 \\
\mathrm{~cm} \text { deep, } 7.6 \mathrm{~cm} \text { wide, and } 41 \mathrm{~cm} \text { long from tip to rim crest } \\
\text { of tailings. Sample delivered to Biology; coarse fraction } \\
\text { purged to XRFs funnel but no sample was received. Small } \\
\text { lumps in and around trench are probably clods. Collector- } \\
\text { head tip reached about } 5 \mathrm{~cm} \text { below surface. }\end{array}$ \\
\hline 3 & Backhoe touchdown 1 & 21 & 10 & 08 & 58 & 216.3 & 93.6 & 30.0 & $\begin{array}{l}\text { 3. Backhoe penetrated crusty to cloddy material } 1.3 \pm 0.2 \mathrm{~cm} \text {. } \\
\text { Collector-head tip does not touch surface. }\end{array}$ \\
\hline 4 & $\begin{array}{l}\text { GCMS } 1 \text { sample } \\
\text { (Bonneville } \\
\text { Salt Flats) }\end{array}$ & 21 & 10 & 10 & 19 & 216.3 & $\begin{array}{l}93.6 \\
88.8 \\
97.3 \\
91.2\end{array}$ & $\overline{30.0}$ & $\begin{array}{l}\text { 4. Trenched by retracting after surface contact, extending } \\
\text { in crusty to cloddy material, and then retracting. Trench } \\
\text { is about } 2-3 \mathrm{~cm} \text { deep, } 6.0 \mathrm{~cm} \text { wide at far tip, and } 28 \mathrm{~cm} \\
\text { long. Collector head tunneled beneath crust forming dome } \\
\text { of crust near tip. Platy fragment of crust near } 5 \mathrm{~cm} \text {, wide } \\
\text { and } 1 \mathrm{~cm} \text { thick was moved by backhoe. }\end{array}$ \\
\hline 5 & $\begin{array}{l}\text { Purge GCMS } 1 \\
\text { (coarse fraction) }\end{array}$ & 21 & 10 & 53 & 46 & 190.4 & 39.9 & 36.3 & $\begin{array}{l}\text { 5. Purged material was greater than } 2.0 \mathrm{~mm} \text {; fragment } 2.7 \mathrm{~cm} \\
\text { wide and additional finer grains or clods } 2 \mathrm{~mm} \text { wide and } \\
\text { larger were purged. }\end{array}$ \\
\hline 6 & $\begin{array}{l}\text { Biology } 2 \text { sample } \\
\text { (Beta) }\end{array}$ & 28 & 16 & 10 & 31 & 126.0 & $\begin{array}{l}85.2 \\
91.7 \\
79.6\end{array}$ & 23.1 & $\begin{array}{l}\text { 6. Trenched by extending in crusty to cloddy material } \\
\text { after surface contact and then retracting. Trench is } \\
\text { about } 2.5-3.0 \mathrm{~cm} \text { deep, } 8-9 \mathrm{~cm} \text { wide, and } 45 \mathrm{~cm} \text { long from } \\
\text { tip to rim crest of tailings. Sample delivered to } \\
\text { Biology. }\end{array}$ \\
\hline 7 & $\begin{array}{l}\text { Purge Biology } \cdot 2 \\
\text { (coarse fraction) }\end{array}$ & 28 & 17 & 44 & 46 & 190.4 & 39.9 & 36.3 & $\begin{array}{l}\text { 7. Purged material should be greater than } 2.0 \mathrm{~mm} \text {; no } \\
\text { evidence for purged coarse particles. }\end{array}$ \\
\hline 8 & $\begin{array}{l}\text { XRFS } 1 \text { sample } \\
\text { (Bonneville } \\
\text { Salt Flats) } \\
U-1\end{array}$ & 29 & 13 & 39 & 56 & 217.5 & $\begin{array}{l}93.0 \\
99.4 \\
90.9 \\
93.0 \\
99.4 \\
90.9\end{array}$ & $\begin{array}{l}29.4 \\
-- \\
30.0 \\
=-\end{array}$ & $\begin{array}{l}\text { 8. Trenched by extending in crusty to cloddy material after } \\
\text { surface contact and then retracting. First acquisition } \\
\text { extension lifted rock } 6 \text { at far end of trench about } 0.4 \mathrm{~cm} \text {; } \\
\text { large platy fragments of crust are present in and around } \\
\text { trench with fine-grained material in rim at tip. Irench } \\
\text { is } 4-5 \mathrm{~cm} \text { deep, } 8 \mathrm{~cm} \text { wide near tip, and } 29 \mathrm{~cm} \text { long from } \\
\text { tip to rim crest of tailings. Material at far end has } \\
\text { spread laterally to trench azimuth because of rock; } \\
\text { rectangular fragment on rim is about } 3 \mathrm{~cm} \text { on an edge } \\
\text { and } 1 \mathrm{~cm} \text { thick. Sample of fine-grained material delivered } \\
\text { to XRFS. }\end{array}$ \\
\hline 9 & $\begin{array}{l}\text { Purge XRFS } 1 \\
\quad \text { (coarse fraction) }\end{array}$ & $\begin{array}{l}29 \\
30\end{array}$ & $\begin{array}{l}14 \\
11\end{array}$ & $\begin{array}{l}05 \\
05\end{array}$ & $\begin{array}{l}45 \\
45\end{array}$ & $\begin{array}{l}190.4 \\
190.4\end{array}$ & $\begin{array}{l}39.9 \\
39.9\end{array}$ & $\begin{array}{l}36.3 \\
36.3\end{array}$ & $\begin{array}{l}\text { 9. Purged material greater than } 2.0 \mathrm{~mm} \text {; large fragment has } \\
\text { been displaced and purged material } 5 \mathrm{~mm} \text { and smaller has } \\
\text { been added. }\end{array}$ \\
\hline 10 & $\begin{array}{l}\text { Rock } 1 \text { nudge } \\
\quad(I C L)\end{array}$ & 30 & 11 & 29 & 34 & 186.4 & $\begin{array}{l}75.4 \\
78.6\end{array}$ & $\begin{array}{l}33.2 \\
30.6\end{array}$ & $\begin{array}{l}\text { 10. Nudged by elevating after surface contact, extending } \\
\text { and then retracting. Surface sampler did not achieve } \\
\text { commanded extension which was } 83.1 \text { in. Rock did not move. }\end{array}$ \\
\hline
\end{tabular}


TABLE 40.-Surface-sampler activities related to the Physical Properties Investigation during the Primary Mission of Viking Lander 2-Continued

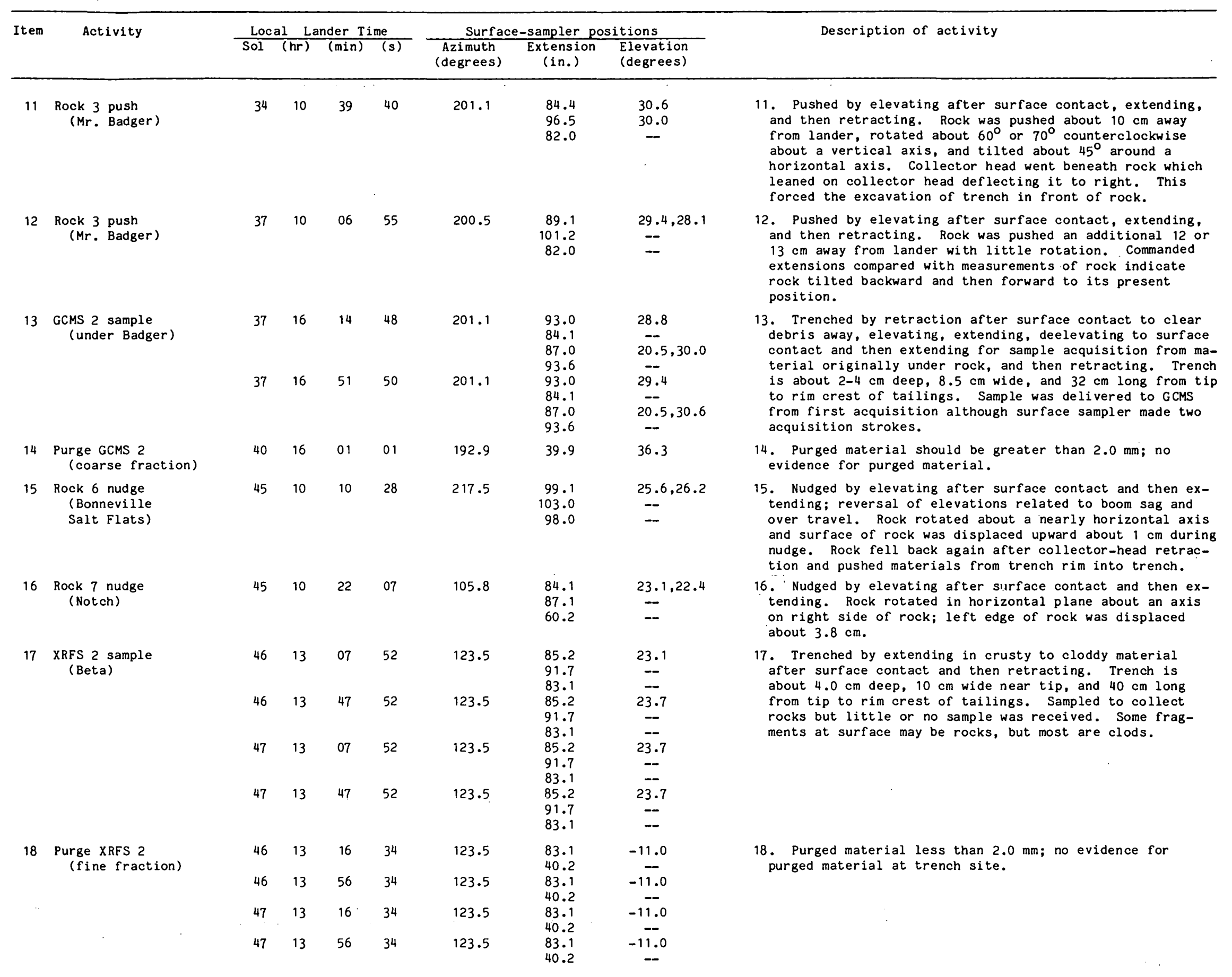


TABLE 40.-Surface-sampler activitine related to the Physical Properties Investigation during the Primary Mission of Viking Lander 2-Continued

\begin{tabular}{|c|c|c|c|c|c|c|c|c|c|}
\hline \multirow[t]{2}{*}{ Item } & \multirow[t]{2}{*}{ Activity } & \multicolumn{4}{|c|}{ Local Lander Time } & \multicolumn{3}{|c|}{ Surface-sampler positions } & \multirow[t]{2}{*}{ Description of activity } \\
\hline & & Sol & $(\mathrm{hr})$ & $(\min )$ & $(s)$ & $\begin{array}{l}\text { Azimuth } \\
\text { (degrees) }\end{array}$ & $\begin{array}{l}\text { Extension } \\
\text { (in.) }\end{array}$ & $\begin{array}{l}\text { Elevation } \\
\text { (degrees) }\end{array}$ & \\
\hline 19 & $\begin{array}{l}\text { Rock } 7 \text { push } \\
\quad(\text { Notch) }\end{array}$ & 51 & 06 & 22 & 49 & 106.4 & $\begin{array}{l}86.7 \\
98.0 \\
60.2\end{array}$ & $\begin{array}{l}21.8,21.8 \\
=\end{array}$ & $\begin{array}{l}\text { 19. Pushed by elevating after surface contact and then } \\
\text { extending. Rock rotated about } 50^{\circ} \text { counterclockwise in } \\
\text { horizontal plane; translated about } 24 \mathrm{~cm} \text { from original } \\
\text { position by plowing and sliding away from surface sampler. }\end{array}$ \\
\hline 20 & $\begin{array}{l}\text { Biology } 3 \text { sample } \\
\text { (under Notch } \\
\text { rock) }\end{array}$ & 51 & 06 & 55 & 35 & 107.1 & $\begin{array}{l}93.6 \\
78.1 \\
88.0 \\
94.6 \\
82.0\end{array}$ & $\begin{array}{l}20.5 \\
-- \\
15.5 .21 .8 \\
- \\
-\end{array}$ & $\begin{array}{l}\text { 20. Trenched by retracting to clear away possible contam- } \\
\text { inating debris, elevating, extending, deelevating to } \\
\text { surface contact and then extending in material originally } \\
\text { under rock. Backhoe trench about } 7.6 \mathrm{~cm} \text { wide. Sample } \\
\text { trench is about } 2.5 \mathrm{~cm} \text { deep, } 9 \mathrm{~cm} \text { wide, and } 42 \mathrm{~cm} \text { long } \\
\text { from tip to rim crest of tailings. Sample was delivered } \\
\text { to Biology. }\end{array}$ \\
\hline $\begin{array}{l}21 \\
\text { (coars }\end{array}$ & $\begin{array}{l}\text { Purge Biology } 3 \\
\text { rse fraction) }\end{array}$ & 51 & 09 & 03 & 49 & 190.4 & 43.9 & 36.3 & $\begin{array}{l}\text { 21. Purged material greater than } 2.0 \mathrm{~mm} \text {. A few small } \\
\text { grains }(\geq 2.0 \mathrm{~mm}) \text { were purged. }\end{array}$ \\
\hline 22 & $\begin{array}{l}\text { Physical Properties } 1 \\
\text { sample, temperatures } \\
\text { (Alpha) }\end{array}$ & 56 & $\begin{array}{l}14 \\
14\end{array}$ & $\begin{array}{l}15 \\
33\end{array}$ & $\begin{array}{l}58 \\
12\end{array}$ & 180.9 & $\begin{array}{l}88.0 \\
95.2 \\
88.0\end{array}$ & $\begin{array}{l}28.8 \\
-- \\
--\end{array}$ & $\begin{array}{l}\text { 22. Trenched by extending in crusty to cloddy material } \\
\text { after surface contact and then retracting. Trench is } \\
\text { about } 2.0 \mathrm{~cm} \text { deep, } 7.5 \mathrm{~cm} \text { wide near tip, and } 26 \mathrm{~cm} \text { long. } \\
\text { Trench is very shallow because of local slope and } \\
\text { interference by excavated rock which is about } 4 \mathrm{~cm} \text { by } \\
7 \mathrm{~cm} \text {. Surface-sampler temperatures were } 272^{\circ} \mathrm{K} \text { at } \\
\text { extension }(t=0), 272^{\circ} \mathrm{K}(\mathrm{t}=4.7 \mathrm{s.}), 273^{\circ} \mathrm{K}(\mathrm{t}= \\
8.7 \mathrm{s.}), 273^{\circ} \mathrm{K}(\mathrm{t}=12.7 \mathrm{~s}) \text {, and } 271^{\circ} \mathrm{K}(t=16.7 \mathrm{s.}) \text {. }\end{array}$ \\
\hline 23 & $\begin{array}{l}\text { Magnification } \\
\text { mirror image of } \\
\text { collector-head } \\
\text { tip and picture } \\
\text { of footpad } 2 \\
\text { via mirror } 2\end{array}$ & 56 & $\begin{array}{l}14 \\
14 \\
15 \\
15\end{array}$ & $\begin{array}{l}41 \\
58 \\
01 \\
03\end{array}$ & $\begin{array}{l}58 \\
44 \\
18 \\
48\end{array}$ & $\begin{array}{r}3.5 \\
120.9 \\
120.9 \\
118.4\end{array}$ & $\begin{array}{l}18.6 \\
\text { N/A } \\
\text { N/A } \\
\text { N/A }\end{array}$ & $\begin{array}{r}1.0 \\
28.1 \\
33.2 \\
33.2\end{array}$ & $\begin{array}{l}\text { 23. A picture of collector-head tip to study surface ma- } \\
\text { terial with magnification mirror. Three pictures intended } \\
\text { to determine conditions of footpad } 2 \text { temperature sensor } \\
\text { did not include sensor. Pictures of sensor taken during } \\
\text { Extended Mission show that temperature sensors are } \\
\text { unburied. }\end{array}$ \\
\hline 24 & $\begin{array}{l}\text { Physical Properties } \\
\text { engine } 2 \text { pictures } \\
\text { via boom mirror } 2\end{array}$ & 57 & $\begin{array}{l}06 \\
06 \\
06\end{array}$ & $\begin{array}{l}49 \\
52 \\
54\end{array}$ & $\begin{array}{l}37 \\
4 \\
31\end{array}$ & $\begin{array}{l}255.4 \\
251.6 \\
247.8\end{array}$ & $\begin{array}{l}\text { N/A } \\
\text { N/A } \\
\text { N/A }\end{array}$ & $\begin{array}{l}40.1 \\
40.1 \\
40.1\end{array}$ & $\begin{array}{l}\text { 24. A series of three pictures showing craters produced by } \\
\text { erosion by terminal-descent engine exhausts. }\end{array}$ \\
\hline 25 & $\begin{array}{l}\text { Purge (Physical } \\
\text { Properties } 1 . \\
\text { coarse) }\end{array}$ & 57 & 07 & 00 & 16 & 192.3 & 39.9 & 36.3 & $\begin{array}{l}\text { 25. A few objects, } 0.5 \mathrm{~cm} \text { and smaller, were purged from } \\
\text { collector head. }\end{array}$ \\
\hline \multirow[t]{4}{*}{26} & $\begin{array}{l}\text { XRFS } 3 \text { sample } \\
\text { (Alpha) }\end{array}$ & 57 & 08 & 08 & 29 & 180.3 & $\begin{array}{l}75.2 \\
81.7 \\
63.3\end{array}$ & $\frac{33.2}{--}$ & \multirow{4}{*}{$\begin{array}{l}\text { 26. Trenched by extending in crusty to cloddy material } \\
\text { after surface contact and then retracting. Sol } 57 \text { trench } \\
\text { is } 5.0 \mathrm{~cm} \text { deep, } 7.3 \mathrm{~cm} \text { wide, and } 53 \mathrm{~cm} \text { long from tip of } \\
\text { trench to rim crest of tailings; deformation at tip ex- } \\
\text { tends } 7.0 \mathrm{~cm} \text { in lateral directions; largest clods are } \\
4.0 \mathrm{~cm} \text { wide. Sol } 58 \text { trench is } 6.5 \mathrm{~cm} \text { deep, } 8.0 \mathrm{~cm} \text { wide, } \\
\text { and } 50 \mathrm{~cm} \text { long from tip of trench to rim crest of tail- } \\
\text { ings. Sol } 58 \text { trench overlaps Sol } 57 \text { trench on left side } \\
\text { of Sol } 57 \text { trench. Sample of coarse fraction to XRFS was } \\
\text { insufficient for analysis. }\end{array}$} \\
\hline & & 57 & 08 & 53 & 29 & 180.3 & $\begin{array}{l}75.2 \\
81.7 \\
63.3\end{array}$ & 33.2 & \\
\hline & & 58 & 08 & 08 & 29 & 179.0 & $\begin{array}{l}75.2 \\
81.7 \\
63.3\end{array}$ & $\begin{array}{l}32.5 \\
--\end{array}$ & \\
\hline & & 58 & 08 & 53 & 29 & 179.0 & $\begin{array}{l}75.2 \\
81.7 \\
63.3\end{array}$ & $\begin{array}{l}32.5 \\
-- \\
--\end{array}$ & \\
\hline 27 & $\begin{array}{l}\text { Purges (XRFS 3, } \\
\text { fine-grained } \\
\text { material) }\end{array}$ & $\begin{array}{l}57 \\
57 \\
58 \\
58\end{array}$ & $\begin{array}{l}08 \\
08 \\
08 \\
08\end{array}$ & $\begin{array}{l}12 \\
57 \\
12 \\
57\end{array}$ & $\begin{array}{l}40 \\
40 \\
40 \\
40\end{array}$ & $\begin{array}{l}180.3 \\
180.3 \\
179.0 \\
179.0\end{array}$ & $\begin{array}{l}63.3 \\
63.3 \\
63.3 \\
63.3\end{array}$ & $\begin{array}{l}-18.0 \\
-18.0 \\
-18.0 \\
-18.0\end{array}$ & 27. Purged fine-grained material dispersed. \\
\hline 28 & Park & 58 & 09 & 12 & 09 & 4.2 & 4.1 & 14.9 & \\
\hline
\end{tabular}


TABLE 41.-Surface-sampler activities related to the Physical Properties Investigation during the Extended Mission of Viking Lander 2

(Listed Local Lander Times correspond to: (a) time of extension command for shroud ejection, (b) collector-head open command just before extension, (c) extension commands for nudges and pushes, (d) vibration commands for purges, and (e) elevation down and final retraction commands for deep hole. Positions are from surface-sampler potentiometer readouts. Azimuths measured from a line $80^{\circ}$ counterclockwise from the $(+) Y_{L}$ direction (direction from camer 2 to camera 1). Azimuths shoul be reduced by abou 0.3 because of boom override due to lander tit. Extensions are reported here in inches to be consistent with engineering units used for the surface-sampler system and represent above plane. Asterisk by extension indicates that commanded extension was not achieved. Letter-number designations listed under "Activity" refer to sample analysis numbers used by Inorganic Chemical Analysis Team (XRFS) (for example, U-2, U-3, etc.)]

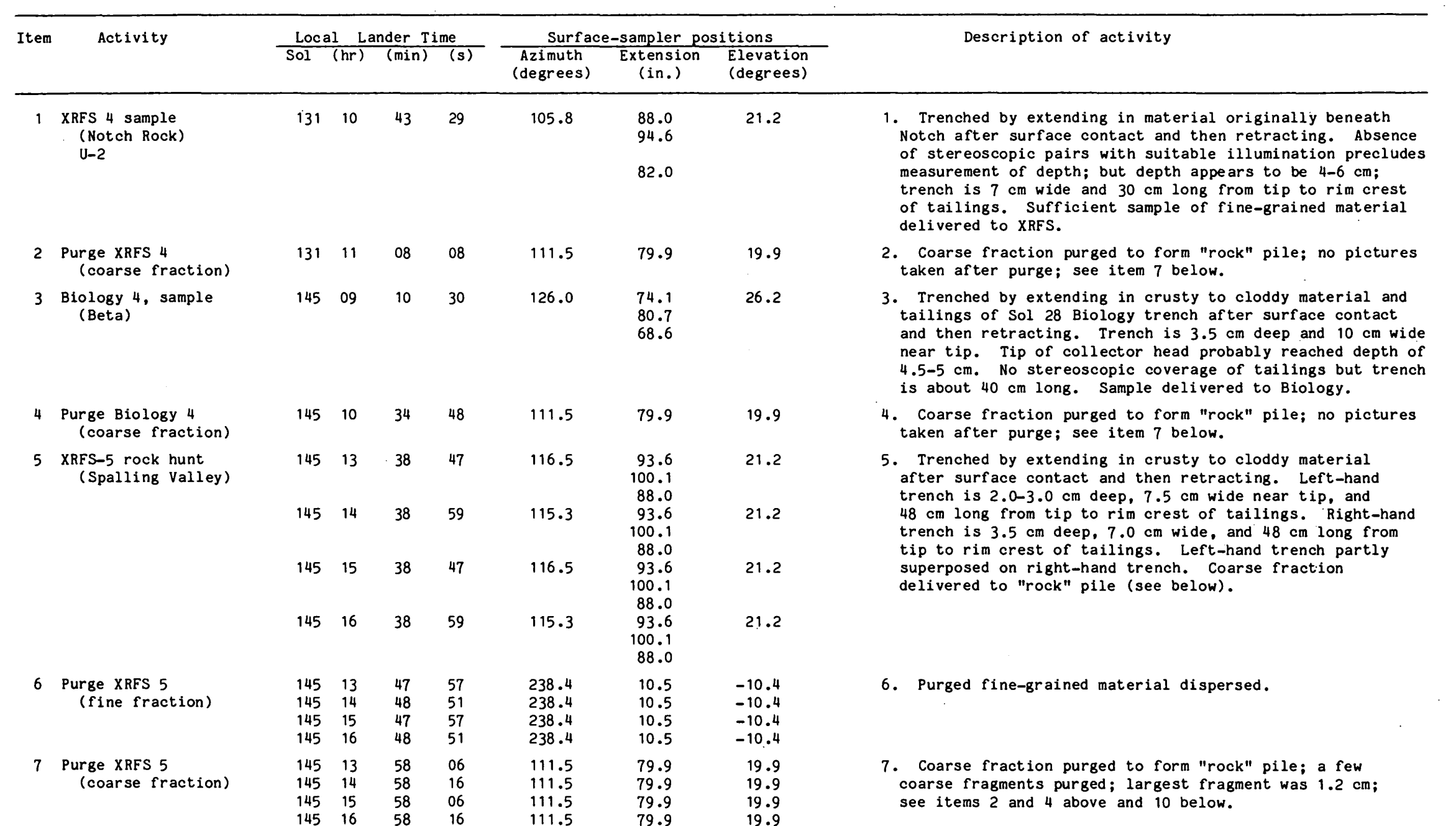

8 Picture of footpad 2 temperature

$\begin{array}{lllllll}154 & 16 & 08 & 32 & 114.6 & \text { na } & 38.9\end{array}$

8. Picture did not include temperature sensor. 


\begin{tabular}{|c|c|c|c|c|c|c|c|c|c|}
\hline \multirow[t]{2}{*}{ Item } & \multirow[t]{2}{*}{ Activity } & \multicolumn{4}{|c|}{ Local Lander Time } & \multicolumn{3}{|c|}{ Surface-sampler positions } & \multirow[t]{2}{*}{ Description of activity } \\
\hline & & Sol & $(\mathrm{hr})$ & $(\min )$ & $(s)$ & $\begin{array}{l}\text { Azimuth } \\
\text { (degrees) }\end{array}$ & $\begin{array}{l}\text { Extension } \\
(\text { in. })\end{array}$ & $\begin{array}{l}\text { Elevation } \\
\text { (degrees) }\end{array}$ & \\
\hline 9 & $\begin{array}{l}\text { XRFS } 6 \text { sample } \\
\text { (Spalling Valley) } \\
\text { U-3 }\end{array}$ & 161 & 11 & 18 & 28 & 116.5 & $\begin{array}{r}93.6 \\
100.1 \\
88.0 \\
93.6 \\
100.1 \\
88.0\end{array}$ & $\begin{array}{l}21.2 . \\
21.2\end{array}$ & $\begin{array}{l}\text { 9. Trenched by extending in crusty to cloddy material } \\
\text { after surface contact and then retracting. Right-hand } \\
\text { trench is } 4.0-4.5 \mathrm{~cm} \text { deep, } 8.5 \mathrm{~cm} \text { wide near tip, and } 50 \\
\text { cm long from tip to rim crest of tailings. Left-hand } \\
\text { trench partly filled in by debris from right-hand trench. } \\
\text { Sufficient sample of fine-grained material delivered to } \\
\text { XRFs. Coarse fraction delivered to "rock" pile } \\
\text { (see below). }\end{array}$ \\
\hline 10 & $\begin{array}{l}\text { Purge XRFS } 6 \\
\quad \text { (coarse fraction) }\end{array}$ & $\begin{array}{l}161 \\
161\end{array}$ & $\begin{array}{l}11 \\
12\end{array}$ & $\begin{array}{l}42 \\
27\end{array}$ & $\begin{array}{l}24 \\
24\end{array}$ & $\begin{array}{l}111.5 \\
111.5\end{array}$ & $\begin{array}{l}79.9 \\
79.9\end{array}$ & $\begin{array}{l}19.9 \\
19.9\end{array}$ & $\begin{array}{l}\text { 10. Coarse fractions purged to form "rock" pile; a few } \\
\text { additional small fragments purged; see item } 13 \text { below. }\end{array}$ \\
\hline 11 & $\begin{array}{l}\text { XRFS } 7 \text { rock hunt } \\
\text { (Spalling Valley) }\end{array}$ & 172 & 12 & 08 & 47 & $\begin{array}{l}114.0 \\
114.0\end{array}$ & $\begin{array}{r}93.6 \\
100.1 \\
88.0 \\
93.6 \\
100.1 \\
88.0 \\
93.6 \\
100.1 \\
88.0 \\
93.6 \\
100.1 \\
88.0\end{array}$ & $\begin{array}{l}20.5 \\
20.5 \\
21.2\end{array}$ & $\begin{array}{l}\text { 11. Trenched by four extensions into crusty to cloddy } \\
\text { material after surface contacts and four retractions. } \\
\text { Trench is } 2.5-3.0 \mathrm{~cm} \text { deep, } 8-10 \mathrm{~cm} \text { wide, and } 51 \mathrm{~cm} \\
\text { long from tip to rim crest of tailings. }\end{array}$ \\
\hline 12 & $\begin{array}{l}\text { Purge XRFS } 7 \\
\text { (fine fraction) }\end{array}$ & $\begin{array}{l}172 \\
172 \\
172 \\
172\end{array}$ & $\begin{array}{l}12 \\
12 \\
13 \\
14\end{array}$ & $\begin{array}{l}17 \\
57 \\
37 \\
17\end{array}$ & $\begin{array}{l}57 \\
57 \\
57 \\
57\end{array}$ & $\begin{array}{l}238.4 \\
238.4 \\
238.4 \\
238.4\end{array}$ & $\begin{array}{l}10.5 \\
10.5 \\
10.5 \\
10.5\end{array}$ & $\begin{array}{l}-10.4 \\
-10.4 \\
-10.4 \\
-10.4\end{array}$ & 12. Purged fine-grained material dispersed. \\
\hline 13 & $\begin{array}{l}\text { Purge XRFS } 7 \\
\text { (coarse fraction) }\end{array}$ & $\begin{array}{l}172 \\
172 \\
172 \\
172\end{array}$ & $\begin{array}{l}12 \\
13 \\
13 \\
14\end{array}$ & $\begin{array}{l}28 \\
08 \\
48 \\
28\end{array}$ & $\begin{array}{l}06 \\
06 \\
06 \\
06\end{array}$ & $\begin{array}{l}111.5 \\
111.5 \\
111.5 \\
111.5\end{array}$ & $\begin{array}{l}79.9 \\
79.9 \\
79.9 \\
79.9\end{array}$ & $\begin{array}{l}19.9 \\
19.9 \\
19.9 \\
19.9\end{array}$ & $\begin{array}{l}\text { 13. Coarse fraction purged to form "rock" pile; only a } \\
\text { few additional fragments purged; see item } 15 \text { below. }\end{array}$ \\
\hline 14 & $\begin{array}{l}\text { XRFS } 8 \text { sample } \\
\text { (Badger) } \\
\text { U-4. }\end{array}$ & 185 & 14 & 09 & 44 & $\begin{array}{l}201.1 \\
201.1\end{array}$ & $\begin{array}{l}87.0 \\
93.6 \\
84.1 \\
87.0 \\
93.6 \\
84.1\end{array}$ & 30.6 & $\begin{array}{l}\text { 14. Trenched by extending into material originally under } \\
\text { rock after surface contact and then retracting. Trench is } \\
\text { about } 3 \mathrm{~cm} \text { deep, } 7 \mathrm{~cm} \text { wide at tip, and } 40 \mathrm{~cm} \text { long from tip } \\
\text { to rim crest, of tailings. Sample clearly came from area } \\
\text { originally beneath rock. Surface of trench floor inclined } \\
22.5^{\circ} \text { away from lander. Sample of fine-grained material } \\
\text { filled the XRFS analysis chamber. }\end{array}$ \\
\hline 15 & $\begin{array}{l}\text { Purge XRFS } 8 \\
\text { (coarse fraction) }\end{array}$ & $\begin{array}{l}185 \\
186\end{array}$ & $\begin{array}{l}14 \\
13\end{array}$ & $\begin{array}{l}35 \\
35\end{array}$ & $\begin{array}{l}02 \\
02\end{array}$ & $\begin{array}{l}111.5 \\
111.5\end{array}$ & $\begin{array}{l}79.9 \\
79.9\end{array}$ & $\begin{array}{l}19.9 \\
19.9\end{array}$ & $\begin{array}{l}\text { 15. Coarse fraction purged to form "rock" pile; only ten } \\
\text { or so fragments accumulated in "rock" pile; largest } \\
\text { fragment is } 1.2 \mathrm{~cm} \text {. }\end{array}$ \\
\hline 16 & Backhoe touchdown 2 & 388 & 14 & 37 & 37 & 232.7 & 80.2 & 31.9 & $\begin{array}{l}\text { 16. Backhoe penetrated } 0.9 \pm 0.3 \mathrm{~cm} \text {. Collector-head tip } \\
\text { does not touch surface. Touchdown site altered by } \\
\text { subsequent trench. }\end{array}$ \\
\hline 17 & $\begin{array}{l}\text { XRFS } 9 \text { sample } \\
\text { (Physica Planitia) } \\
\text { U-5 }\end{array}$ & 388 & 15 & 41 & 55 & 234.6 & $\begin{array}{l}80.2 \\
83.3 \\
82.3 \\
90.4 \\
44.4 \\
80.2 \\
83.3 \\
82.3 \\
90.4 \\
44.4\end{array}$ & $\begin{array}{l}31.9 \\
33.2\end{array}$ & $\begin{array}{l}\text { 17. Trenched by small extensions after surface contact, } \\
\text { deelevations, retractions, and then extensions to produce } \\
\text { skimming motion during sampling. Second trench partly } \\
\text { superposed on first trench; second trench } 2.0-2.5 \mathrm{~cm} \text { deep, } \\
9 \mathrm{~cm} \text { wide, and } 79 \mathrm{~cm} \text { long; sample came from depths of } 2.3 \\
\mathrm{~cm} \text { and less. First trench not mapped but appears to have } \\
\text { same dimensions as second trench. Sufficient sample } \\
\text { delivered to XRFS. }\end{array}$ \\
\hline
\end{tabular}




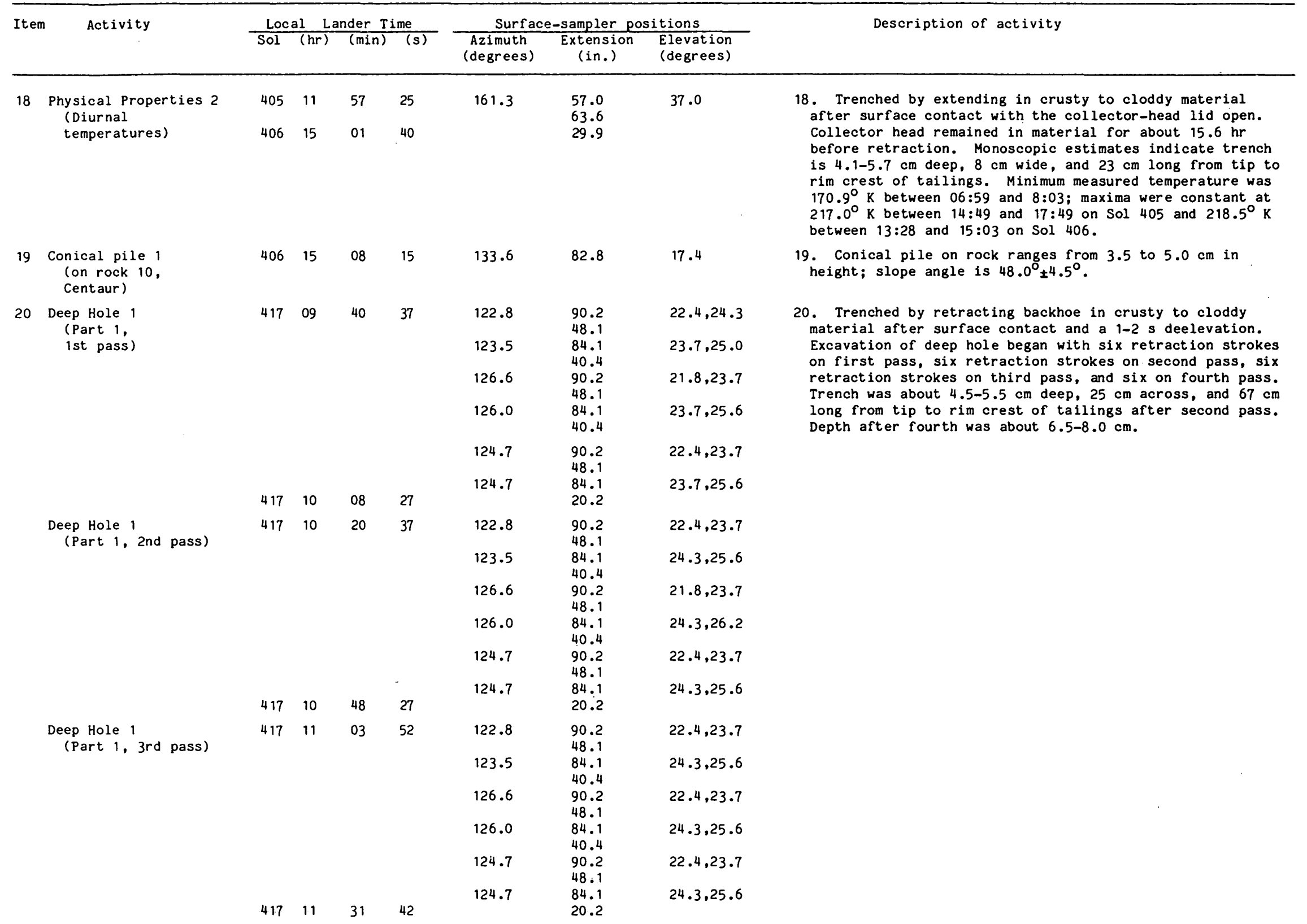


TABLE 41.-Surface-sampler activities related to the Physical Properties Investigation during the Extended Mission of Viking Lander 2-Continued

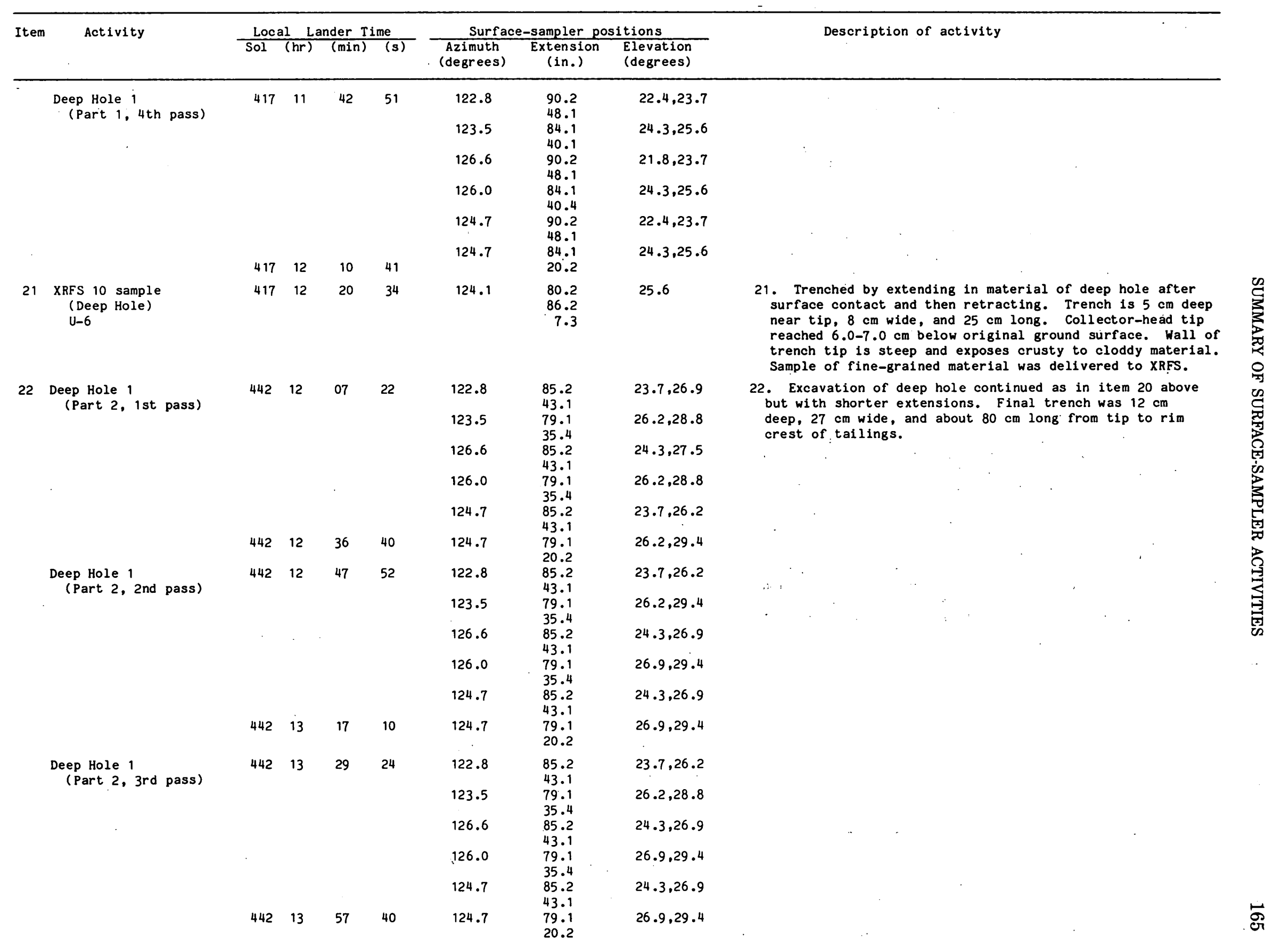


TABLE 41.-Surface-sampler activities related to the Physical Properties Investigation during the Extended Mission of Viking Lander 2-Continued

\begin{tabular}{|c|c|c|c|c|c|c|c|c|}
\hline \multirow[t]{2}{*}{ Item } & \multirow{2}{*}{ Activity } & \multicolumn{4}{|c|}{ Local Lander Time } & \multicolumn{3}{|c|}{ Surface-sampler positions } \\
\hline & & Sol & $(\mathrm{hr})$ & $(\min )$ & (s) & $\begin{array}{c}\text { Azimuth } \\
\text { (degrees) }\end{array}$ & $\begin{array}{l}\text { Extension } \\
(\text { in. })\end{array}$ & $\begin{array}{l}\text { Elevation } \\
\text { (degrees) }\end{array}$ \\
\hline & $\begin{array}{l}\text { Deep Hole } 1 \\
\text { (Part 2, 4th pass) }\end{array}$ & 442 & 14 & 12 & 52 & 122.8 & $\begin{array}{l}85.2 \\
43.1\end{array}$ & $24,3,26$ \\
\hline & & & & & & 123.5 & $\begin{array}{l}79.1 \\
35.4\end{array}$ & $26.9,29$ \\
\hline & & & & & & 126.6 & $\begin{array}{l}85.2 \\
43.1\end{array}$ & $24 \cdot 3,26$ \\
\hline & & & & & & 126.0 & $\begin{array}{l}79.1 \\
35.4\end{array}$ & $26.9,29$ \\
\hline & & & & & & 124.7 & $\begin{array}{l}85.2 \\
43.1\end{array}$ & $23.7,26$ \\
\hline & & 442 & 14 & 42 & 10 & 124.7 & $\begin{array}{l}79.1 \\
20.2\end{array}$ & $26.9,29$ \\
\hline 23 & XRFS 11 sample & 442 & 14 & 54 & 17 & 124.7 & 73.1 & 28.1 \\
\hline & $\begin{array}{l}\text { (Deep Hole) } \\
\text { U-7 }\end{array}$ & & & & & & $\begin{array}{l}77.3^{*} \\
7.3\end{array}$ & \\
\hline & & 442 & 15 & 14 & 02 & 124.7 & $\begin{array}{l}73.1 \\
77.8 \\
7.3\end{array}$ & 28.8 \\
\hline 24 & $\begin{array}{l}\text { Rock } 11 \text { push } \\
\text { (Snow White) }\end{array}$ & 471 & 12 & 10 & 57 & 92.5 & $\begin{array}{c}66.0 \\
72.0 \\
4.1\end{array}$ & $27.5,27$ \\
\hline 25 & Backhoe touchdown 3 & 479 & 10 & 09 & 16 & 238.4 & 74.9 & 33.2 \\
\hline 26 & $\begin{array}{l}\text { Physical Properties } 3 \\
\text { sample } \\
\text { (Physica Planitia) }\end{array}$ & 479 & 10 & 13 & 33 & 238.4 & $\begin{array}{r}74.9 \\
84.9 \\
4.1\end{array}$ & 33.2 \\
\hline 27 & $\begin{array}{l}\text { Purge Phys. Props. } 3 \\
\text { (fine fraction) }\end{array}$ & 479 & 10 & 46 & 34 & 180.9 & 43.9 & 5.3 \\
\hline 28 & $\begin{array}{l}\text { Purge Phys. Props. } 3 \\
\text { (coarse fraction) }\end{array}$ & 479 & 10 & 53 & 46 & 192.3 & 43.9 & 36.3 \\
\hline 29 & $\begin{array}{l}\text { Pictures of footpad } \\
2 \text { temperature } \\
\text { sensors via boom } \\
\text { mirror }\end{array}$ & $\begin{array}{l}479 \\
479\end{array}$ & $\begin{array}{l}11 \\
11\end{array}$ & $\begin{array}{l}04 \\
09\end{array}$ & $\begin{array}{l}38 \\
05\end{array}$ & $\begin{array}{l}\$ 25.4 \\
122.8\end{array}$ & $\begin{array}{l}4.1 \\
4.1\end{array}$ & $\begin{array}{l}40.1 \\
40.1\end{array}$ \\
\hline 30 & $\begin{array}{l}\text { XRFS } 12 \text { sample } \\
\text { (Snow White) }\end{array}$ & 483 & 12 & 06 & 37 & 92.5 & $\begin{array}{l}66.0 \\
68.1 \\
7.3\end{array}$ & 27.5 \\
\hline
\end{tabular}

Description of activity

$24.3,26.9$

$6.9,29.4$

$.3,26.9$

$9,29.4$

$3.7,26.2$

$6.9,29.4$

23. Trenched by extending in deep hole after surface contact and then retracting. Floor of trench is $12 \mathrm{~cm}$ below original surface. Tip of collector head probably reached to $14 \mathrm{~cm}$ below original surface. Trench is $3.5-$ $4.0 \mathrm{~cm}$ deep, $5 \mathrm{~cm}$ wide, and $9 \mathrm{~cm}$ long. Tip has overhang. Surface sampler failed to achieve commanded extensions of 81.2 in. Samples of fine-grained material delivered to XRFS.

24. Pushed by elevating after surface contact, then extending, and finally retracting. Rock was pushed about $5 \pm 1 \mathrm{~cm}$ away from lander with a small amount of rotation. Surface 76.6 in. There is no evidence that rock chipped or spalled.

25. Backhoe penetrated $0.9 \pm 0.3 \mathrm{~cm}$. Collector-head tip does not touch surface. Backhoe-touchdown site altered by subsequent trench.

26. Trenched by extending in crusty to cloddy material after surface contact and then retracting. Trench is about $3.0 \mathrm{~cm}$ deep, $8.0 \mathrm{~cm}$ wide, and $61 \mathrm{~cm}$ long from tip to rim crest of tailings. Collector-head tip probably attained depth of 5.3-7.3 cm. Surface at far end of trench is domed and fractured.

27. Purged fine-grained material dispersed.

28. Several additional fragments as much as $0.8 \mathrm{~cm}$ wide were purged.

29. Picture shows that temperature sensors are exposed to the atmosphere and are not buried.

30. Trenched by extending toward rock after surface conand then retracting. Trench is about $1 \mathrm{~cm}$ or less deep, $5 \mathrm{~cm}$ wide, and $21 \mathrm{~cm}$ long from tip to rim crest of tailings. Surface sampler failed to attain commanded extension of 72.5 in.; probably hit buried rock and never reached area under rock. No sample delivered to XRFS. 
TABLE 41.-Surface-sampler activities related to the Physical Properties Investigation during the Extended Mission of Viking Lander 2-Continued

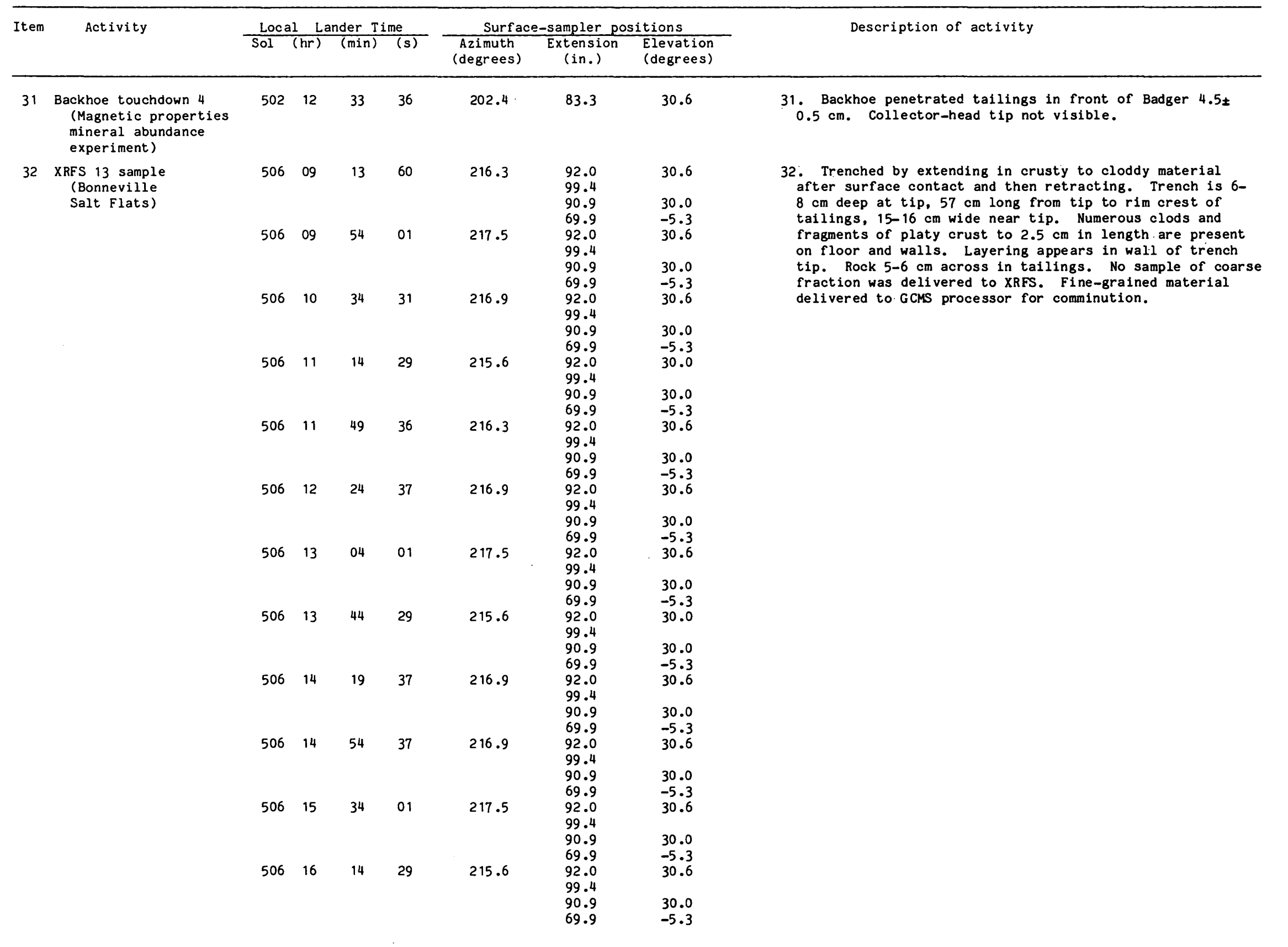


TABLE 41.-Surface-sampler activities related to the Physical Properties Investigation during the Extended Mission of Viking Lander 2-Continued

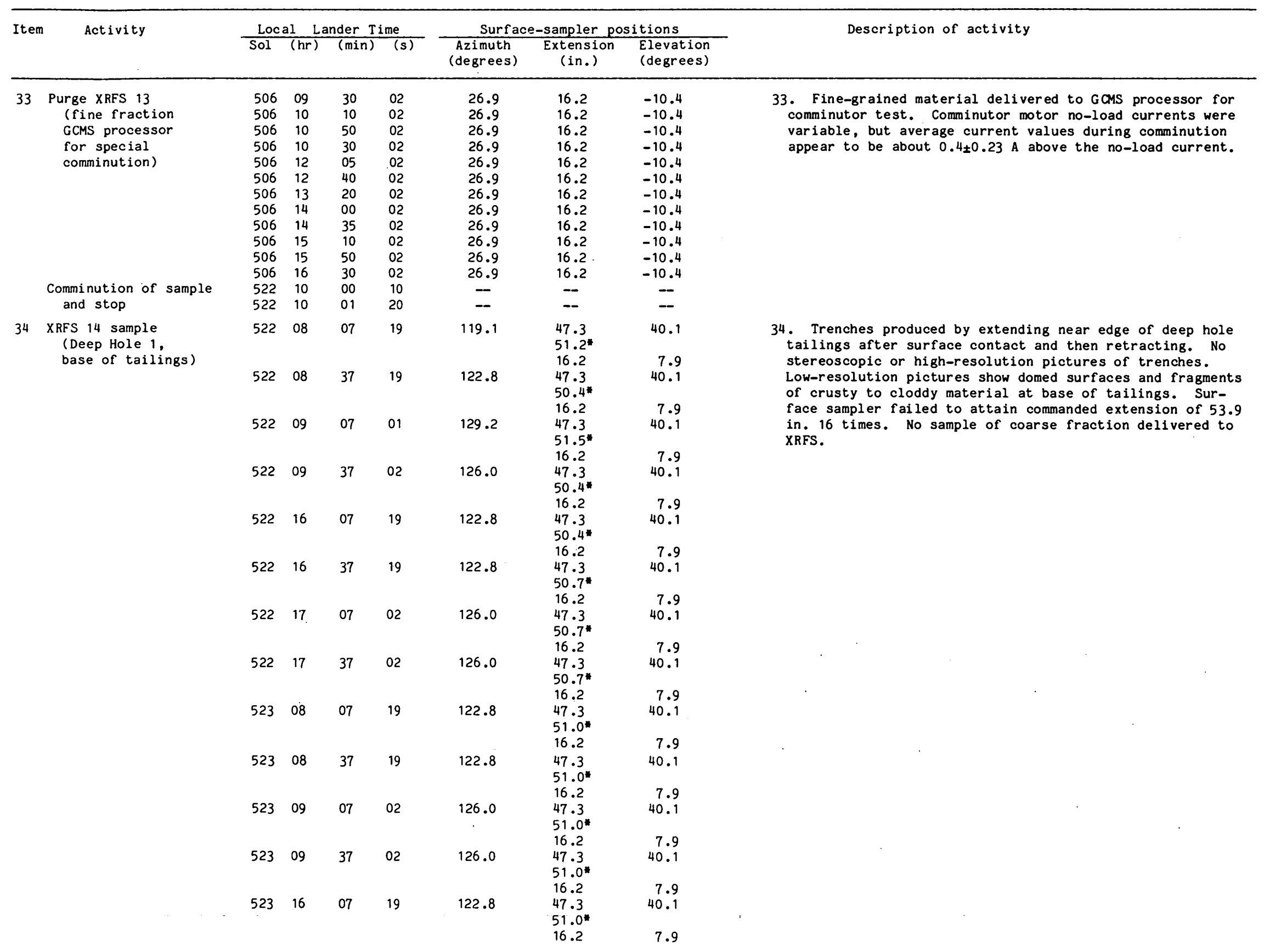


TABLE 41.-Surface-sampler activities related to the Physical Properties Investigation during the Extended Mission of Viking Lander 2-Continued

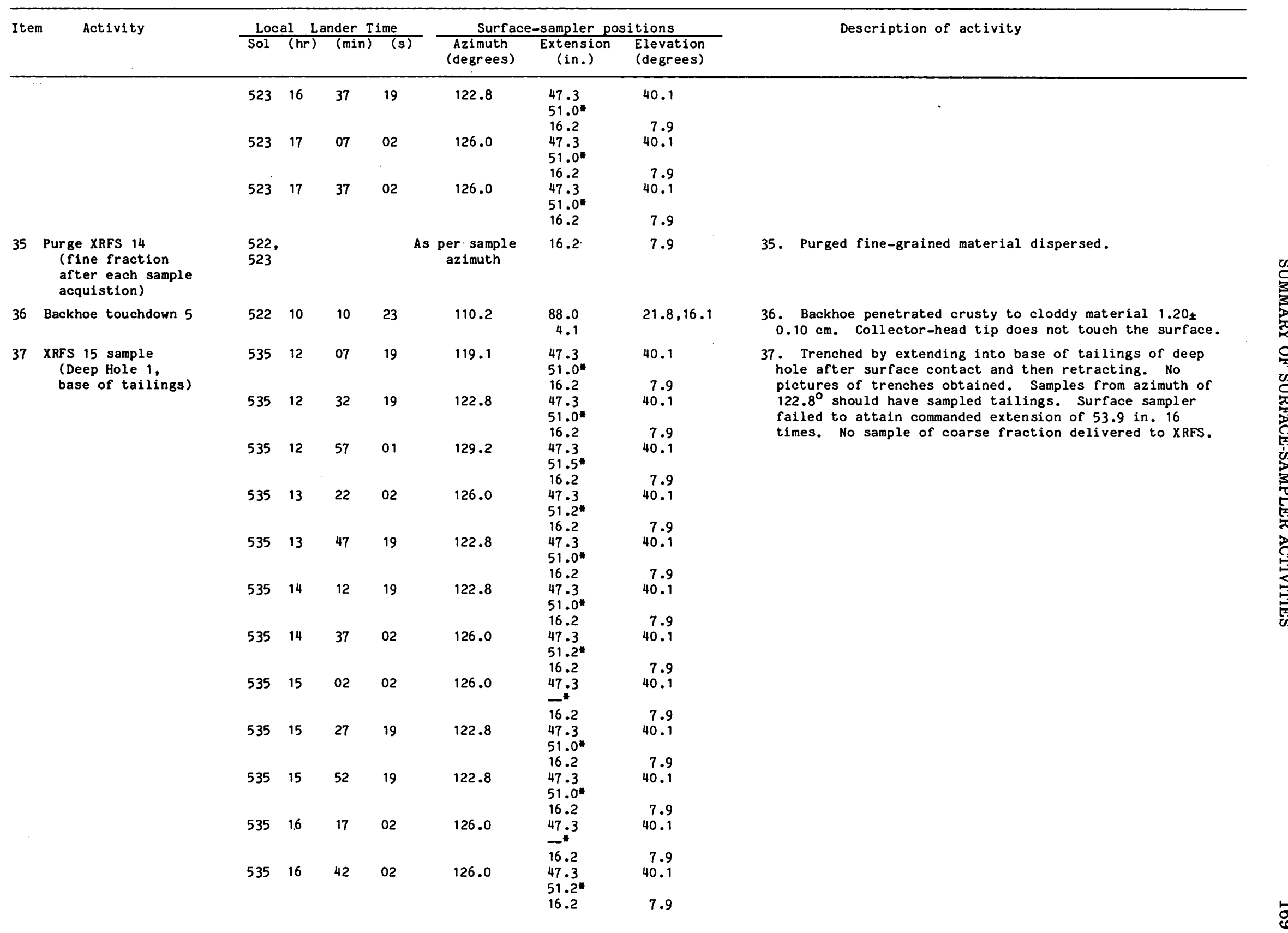


TABLE 41.-Surface-sampler activities related to the Physical Properties Investigation during the Extended Mission of Viking Lander 2-Continued

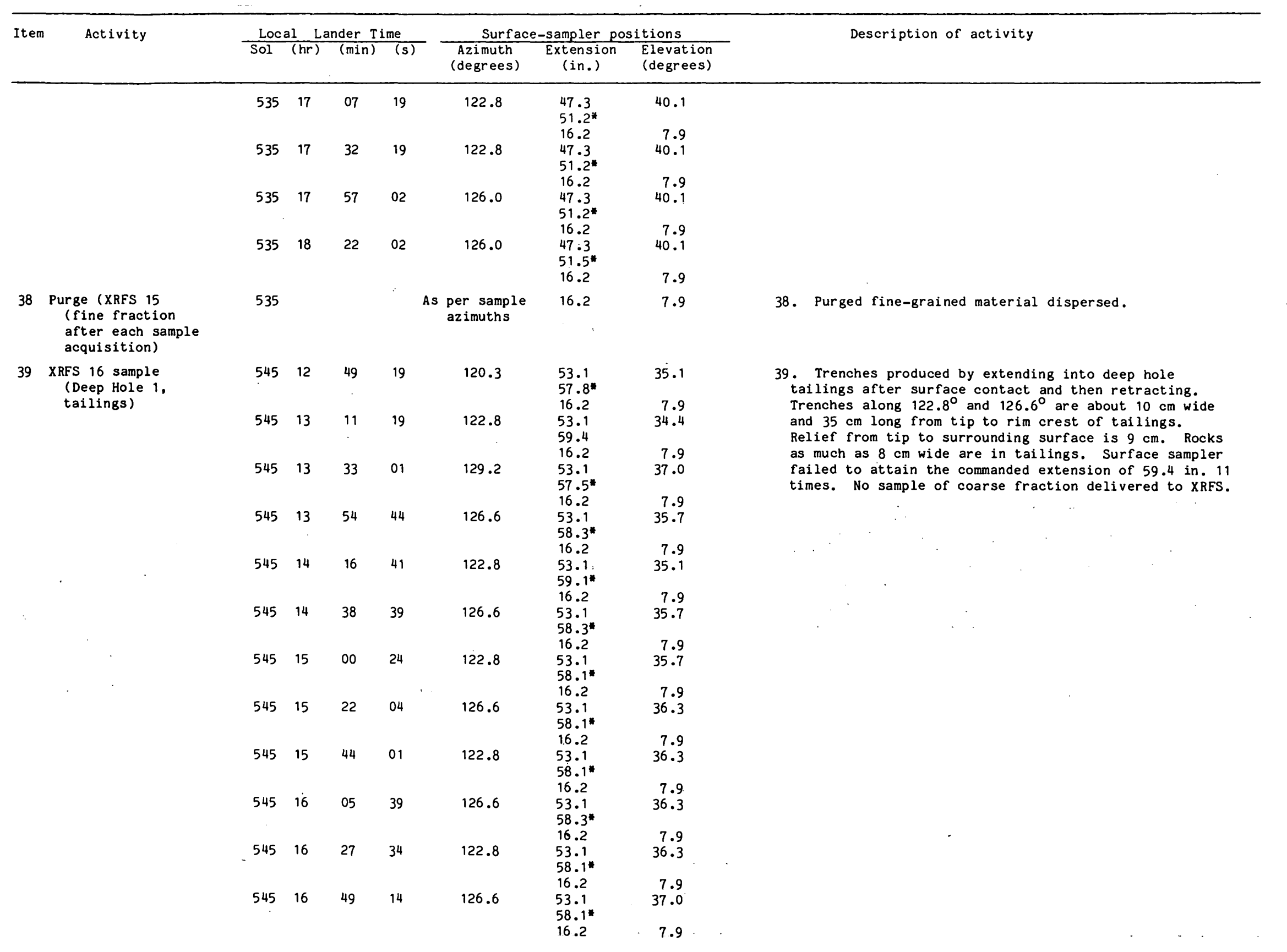


TABLE 41.-Surface-sampler activities related to the Physical Properties Investigation during the Extended Mission of Viking Lander 2-Continued

\begin{tabular}{|c|c|c|c|c|c|c|c|c|}
\hline \multirow[t]{2}{*}{ Item } & \multirow[t]{2}{*}{ Activity } & \multicolumn{4}{|c|}{ Local Lander Time } & \multicolumn{3}{|c|}{ Surface-sampler positions } \\
\hline & & Sol & $(\mathrm{hr})$ & $(\min )$ & $(s)$ & $\begin{array}{l}\text { Azimuth } \\
\text { (degrees) }\end{array}$ & $\begin{array}{l}\text { Extension } \\
\text { (in.) }\end{array}$ & $\begin{array}{l}\text { Elevation } \\
\text { (degrees) }\end{array}$ \\
\hline 40 & $\begin{array}{l}\text { Purge XRFS } 16 \\
\text { (fine fraction } \\
\text { after each sample } \\
\text { aquisition) }\end{array}$ & 545 & & & & $\begin{array}{l}\text { As per sample } \\
\text { azimuths }\end{array}$ & 16.2 & 7.9 \\
\hline 41 & $\begin{array}{l}\text { Pictures under } \\
\text { engine } 2 \text { via } \\
\text { mirror } 2\end{array}$ & $\begin{array}{l}555 \\
555 \\
555 \\
555 \\
555 \\
555 \\
555\end{array}$ & $\begin{array}{l}07 \\
07 \\
07 \\
07 \\
07 \\
07 \\
07\end{array}$ & $\begin{array}{l}05 \\
09 \\
14 \\
18 \\
23 \\
28 \\
32\end{array}$ & $\begin{array}{l}28 \\
57 \\
26 \\
55 \\
29 \\
03 \\
37\end{array}$ & $\begin{array}{l}249.1 \\
254.1 \\
259.2 \\
264.2 \\
264.2 \\
264.2 \\
264.2\end{array}$ & 8.4 & $\begin{array}{l}40.1 \\
40.1 \\
40.1 \\
40.1 \\
36.3 \\
32.5 \\
28.8\end{array}$ \\
\hline 42 & $\begin{array}{l}\text { Physical Properties } 4 \\
\text { (retraction trench) }\end{array}$ & $\begin{array}{l}559 \\
559 \\
559 \\
559 \\
559 \\
559 \\
559 \\
559 \\
559 \\
559 \\
559 \\
559 \\
559 \\
559 \\
559 \\
559\end{array}$ & $\begin{array}{l}10 \\
11 \\
11 \\
11 \\
11 \\
11 \\
11 \\
11 \\
11 \\
11 \\
11 \\
11 \\
11 \\
11 \\
11 \\
11\end{array}$ & $\begin{array}{l}56 \\
02 \\
04 \\
05 \\
07 \\
08 \\
10 \\
10 \\
13 \\
13 \\
15 \\
16 \\
18 \\
19 \\
21 \\
23\end{array}$ & $\begin{array}{l}46 \\
40 \\
55 \\
43 \\
32 \\
20 \\
09 \\
57 \\
02 \\
50 \\
54 \\
42 \\
30 \\
18 \\
06 \\
15\end{array}$ & $\begin{array}{l}149.4 \\
151.2 \\
152.5 \\
153.8 \\
152.5 \\
151.2 \\
152.5 \\
153.8\end{array}$ & $\begin{array}{l}83.3 \\
66.0 \\
69.6 \\
66.0 \\
69.6 \\
66.0 \\
69.6 \\
66.0 \\
76.2 \\
73.1 \\
83.3 \\
79.9 \\
83.3 \\
79.9 \\
83.3 \\
79.9\end{array}$ & $\begin{array}{r}26.9,28.1 \\
9.8 \\
31.9 \\
9.8 \\
31.9 \\
9.8 \\
31.9 \\
9.8 \\
29.4 \\
9.8 \\
26.9 \\
9.8 \\
26.9 \\
9.8 \\
27.5 \\
9.8\end{array}$ \\
\hline 43 & $\begin{array}{l}\text { Rock } 5 \text { chip } \\
\text { (Mole) }\end{array}$ & 559 & 11 & 31 & 47 & 191.0 & $\begin{array}{r}82.0 \\
4.1\end{array}$ & $\begin{array}{l}28.1,31.3 \\
5.3\end{array}$ \\
\hline 44 & $\begin{array}{l}\text { Physical Properties } 5 \\
\text { sample } \\
\text { (Physica Planitia) }\end{array}$ & $\begin{array}{l}595 \\
595\end{array}$ & $\begin{array}{l}08 \\
09\end{array}$ & $\begin{array}{l}09 \\
09\end{array}$ & $\begin{array}{l}45 \\
53\end{array}$ & $\begin{array}{l}238.4 \\
238.4\end{array}$ & $\begin{array}{l}76.7 \\
\text { nd } \\
74.9 \\
74.6 \\
\text { nd } \\
74.9\end{array}$ & $\begin{array}{l}\text { nd, nd } \\
\text { nd, nd }\end{array}$ \\
\hline 45 & $\begin{array}{l}\text { Conical pile } 2 \\
\quad \text { (Physica Planitia) }\end{array}$ & $\begin{array}{l}595 \\
595\end{array}$ & $\begin{array}{l}08 \\
09\end{array}$ & $\begin{array}{l}28 \\
28\end{array}$ & $\begin{array}{l}49 \\
57\end{array}$ & $\begin{array}{l}234.6 \\
234.6\end{array}$ & $\begin{array}{l}74.9 \\
74.9\end{array}$ & $\begin{array}{l}28.1 \\
28.1\end{array}$ \\
\hline 46 & Backhoe touchdown 6 & 595 & 10 & 37 & 05 & 173.3 & 75.2 & nd \\
\hline 47 & $\begin{array}{l}\text { Physical Properties } 6 \\
\quad \text { (adhesion, Alpha) }\end{array}$ & 595 & 10 & 42 & 33 & 173.3 & $\begin{array}{l}75.2 \\
\text { nd } * \\
53.9\end{array}$ & nd \\
\hline
\end{tabular}

Description of activity

40. Purged fine-grained material dispersed.

41. Mosaic of seven pictures under terminal-descent engine 2 shows three small craters that are about $6 \mathrm{~cm}$ wide and $0.5-1.0 \mathrm{~cm}$ deep. Surface material appears to be very fine grained and has some pebble-size fragments. Tip of rock 12 (Sneezy) is visible; exposed tip is $4 \times 8 \mathrm{~cm}$.

42. Trenched by retraction of backhoe after surface contact. Monoscopic estimates indicate deepest trench is 3-4 cm deep. Trench tailings composed chiefly of fine-grained material and small clods, but clods as as much as $5 \mathrm{~cm}$ wide were excavated. One rock 8-10 cm wide was excavated. retracted. No evidence for chipping or spalling of rock. Rock did not move.

44. Trenched by extending in crusty to cloddy material after surface contact and then retracting. Trench about $7-9 \mathrm{~cm}$ deep, $8.0 \mathrm{~cm}$ wide, and $40 \mathrm{~cm}$ long. Surface near tip is domed and fractured to a distance of $15 \mathrm{~cm}$ from tip of trench. Material used for conical pile. Surface sampler probably achieved commanded extension of 84.9 in. on first acquisition stroke but not on second one.

45. Monoscopic coverage does not allow good measurements of pile; slope of pile about $35^{\circ}$.

46. Backhoe penetrated crusty to cloddy material $1.35 \pm 0.25$ $\mathrm{cm}$. Collector-head tip not visible. Backhoe-touchdown site altered by subsequent trench.

47. Trenched by extending in crusty to cloddy material af ter surface contact and then retracting. Trench is $1.5-2.5 \mathrm{~cm}$ deep, $7-8 \mathrm{~cm}$ wide, and $44 \mathrm{~cm}$ long from tip to rim crest of tailings. Surface sampler probably achieved commanded extension of 81.7 in. Surfacesampler data lost. 


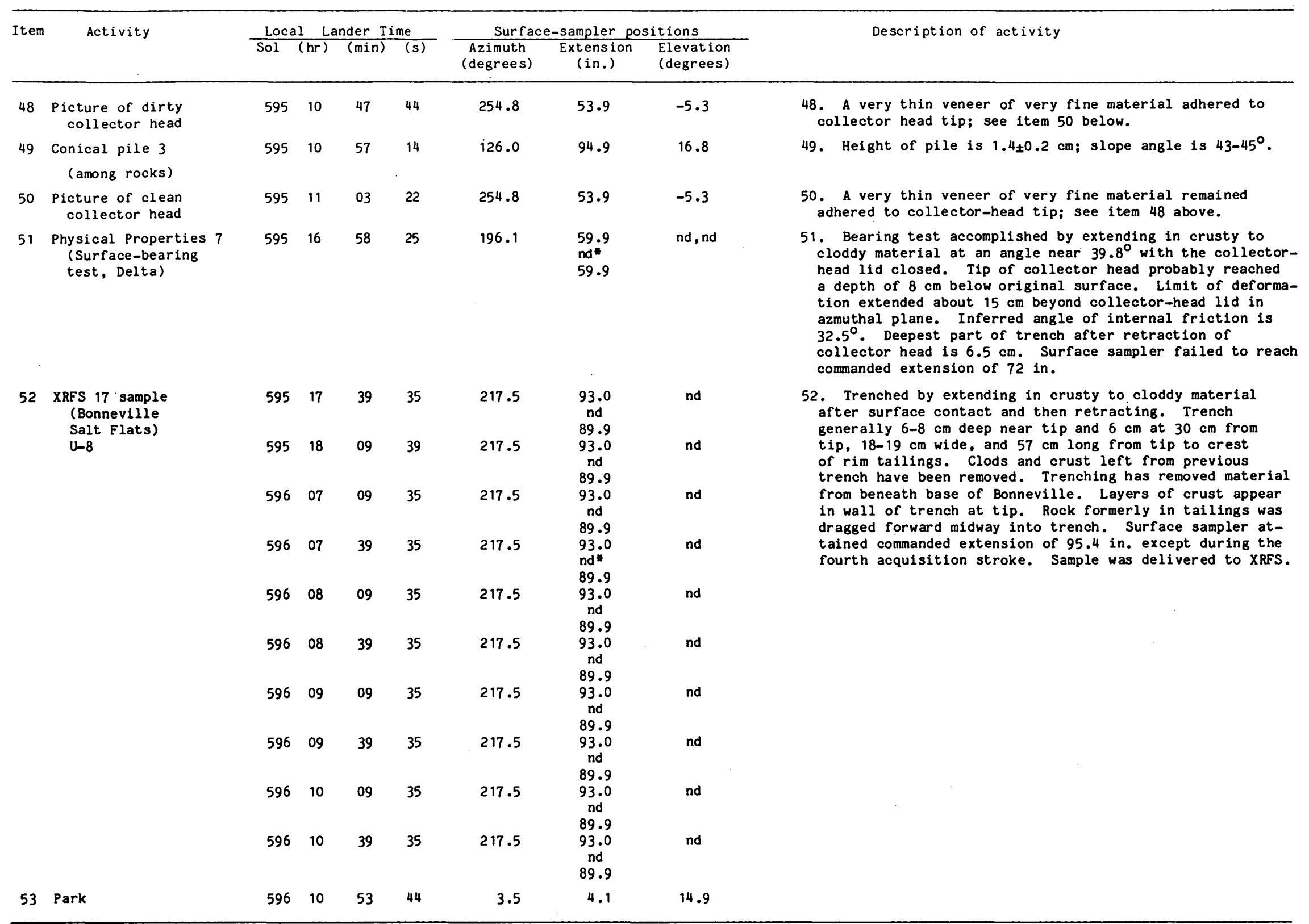




\section{OUTLINE MAPS, PROFILES, AND DATA ON TRENCHES}

This section contains outline maps and profiles of trenches excavated by the surface samplers of Landers 1 and 2 as well as other data. Figure captions include the item numbers and designations of the activities listed in the previous section (tables $38,39,40$, and 41 ) so that the activities, event times, surface-sampler achieved positions, and descriptions of the outcomes of the activities can be compared with the trenches portrayed. Figure captions also include the frame numbers of pictures used to prepare the outline maps and profiles as well as the type of coverage (that is, stereoscopic or monoscopic). The locations of the trenches in the sample fields are shown on plate 1 for Lander 1 and plate 2 for Lander 2.

In general, the outline maps and profiles were prepared using stereometric techniques, but some were, of necessity, prepared using monoscopic techniques. In a few cases no maps or outlines were prepared because pictures were not taken or were inadequate. All the outline maps and profiles are portrayed at 1:10 scale in the lander science coordinate system (LSCS); the original compilations of those that employed stereometric techniques were done at 1:5 scale. Outline maps that were made by monoscopic techniques were done at 1:10 scale; profiles were not made for trenches that were covered monoscopically. In order to aid the visualization of the geometry and true scale of the trenches, profiles include a collector head at the proper scale with the upper jaw in the closed position. Figure 92 is a map explanation for all outlines and profiles; figures 93-150 are the outline maps and profiles.

Data on the trenches include Format 5 motor-current records (or examples) if the records were used to estimate cohesions. The motor-current records were interpreted in terms of force after the base current of $0.2 \mathrm{~A}$ had been removed using a nominal $25 \mathrm{~N}$ of force for each $0.039 \mathrm{~A}$ increment of current. Cyclic currents that were measured at the lander bus and that were caused by other lander components were removed from the motor-current records. Axial forces were obtained by subtracting $50 \mathrm{~N}$ of force from the indicated forces because terrestrial tests indicate that the axial force exerted by the sampler while the collector head glided over a frictionless surface was $50 \mathrm{~N}$. In some cases, the absence of motor-current records is indicated. Complete Format 5 motor-current records may be found in L.V. Clark and others (1977) and Pike and others (1978).

\section{EXPLANATION}

TAILINGS

SLUMP

DEFORMED SURFACE

ROCK - Dashed where inferred or approximately located

CLOD-Dashed where inferred or approximately located

RIM CREST OF TRENCH-Dashed where approximately located; dotted where inferred

? CONTACT-Dashed where approximately located; dotted where inferred; queried where doubtful

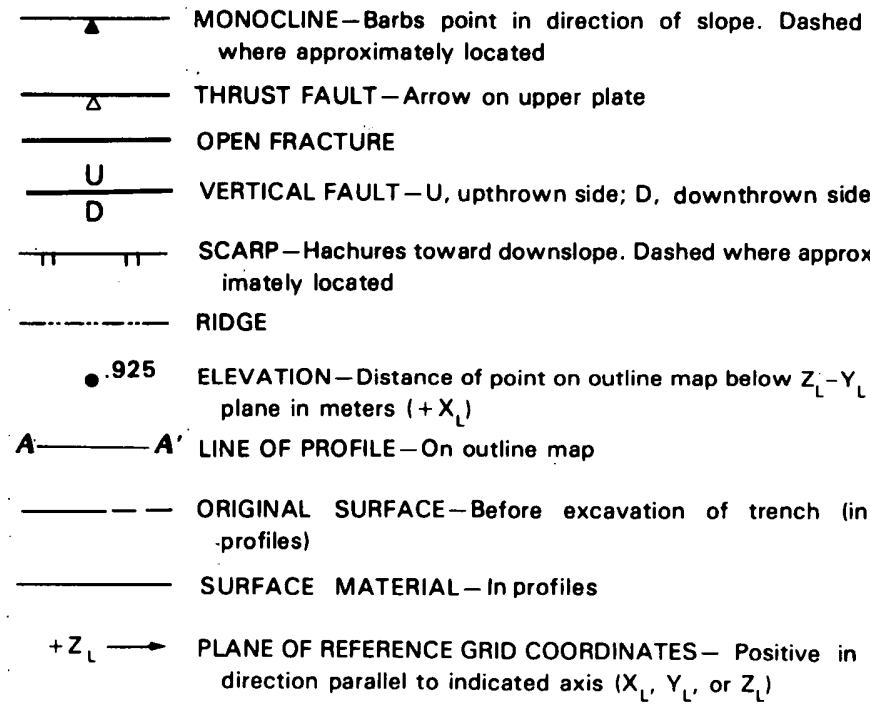

Figure 92.-Explanation for outline maps and profiles (figs. 93-150). 


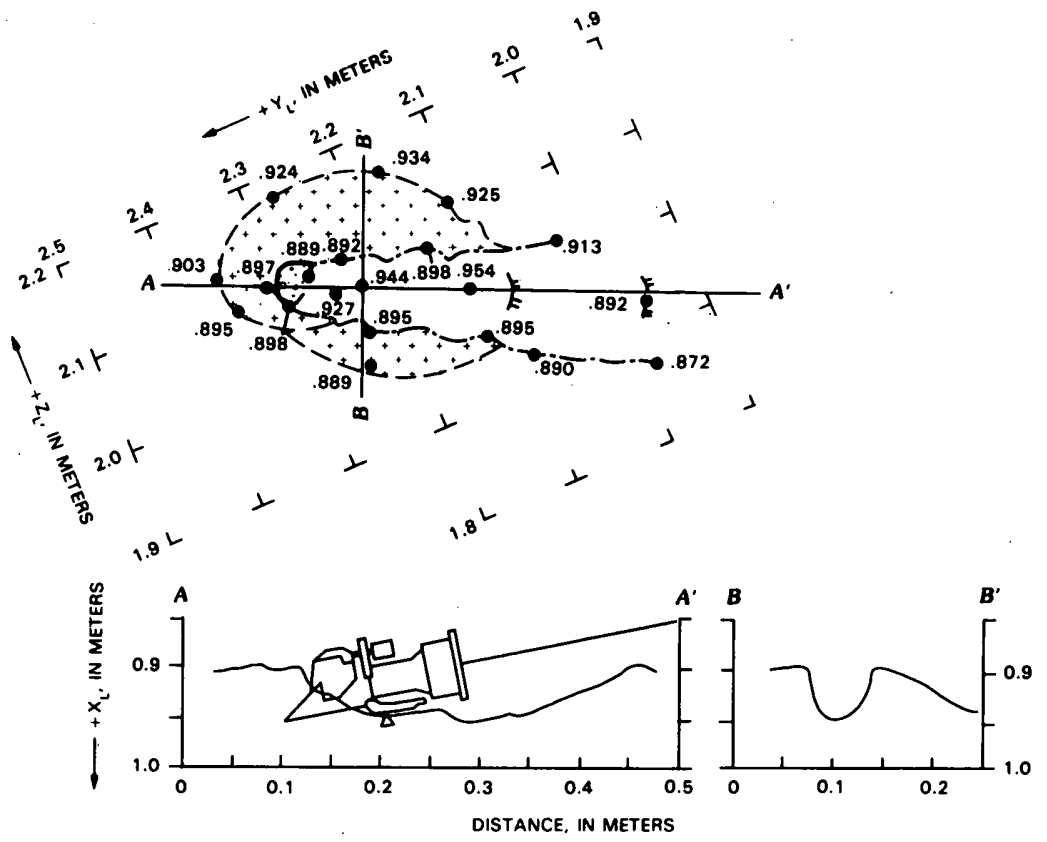

FIGURE 93.-Map and profile of first trench excavated by Lander 1 in drift material on Sol 8. No Format 5 motor-current record. (See Biology 1; table 38, item 3; stereoscopic pictures: frames $12 \mathrm{~A} 059 / 008$ and 11A058/008.)
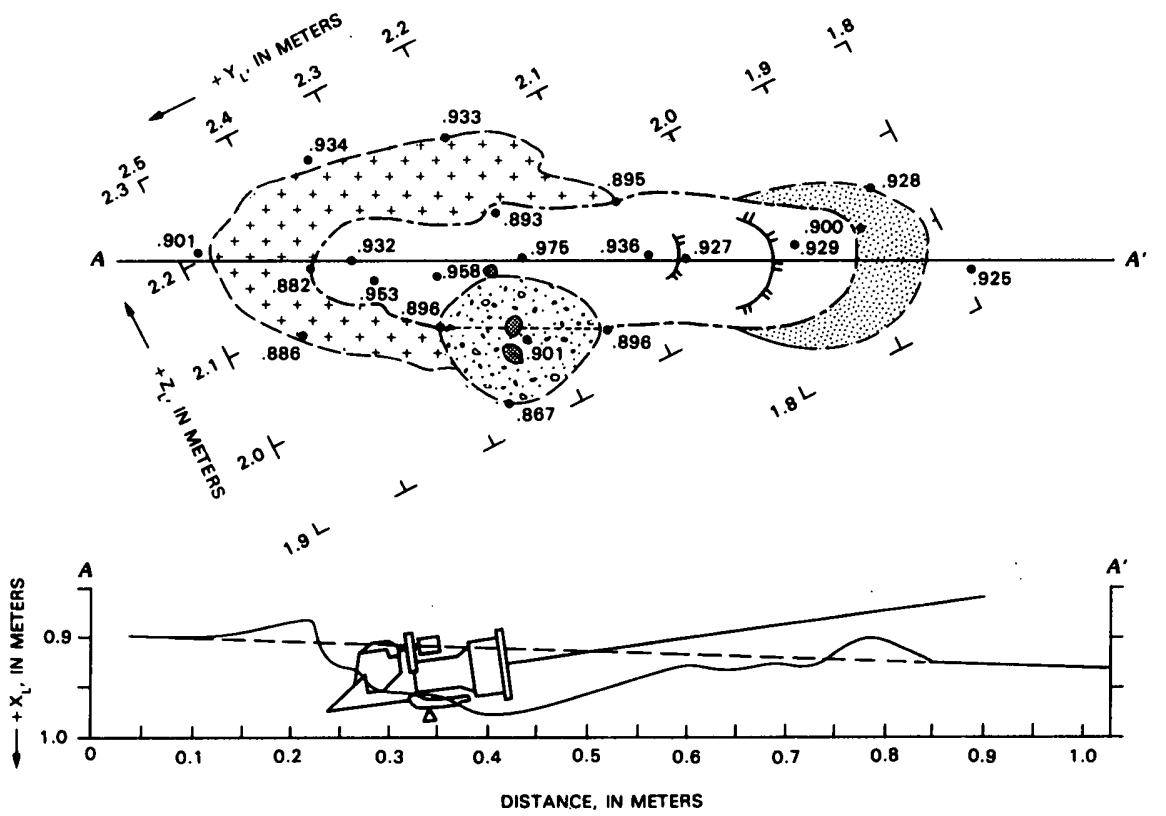

FIGURE 94.-Map and profile of trench in drift material excavated by Lander 1 on Sol 8. No Format 5 motor-current record. (See XFRS 1, sample C-1, table 38, item 7; stereoscopic pictures: frames 12A080/012 and 11A080/012.) 


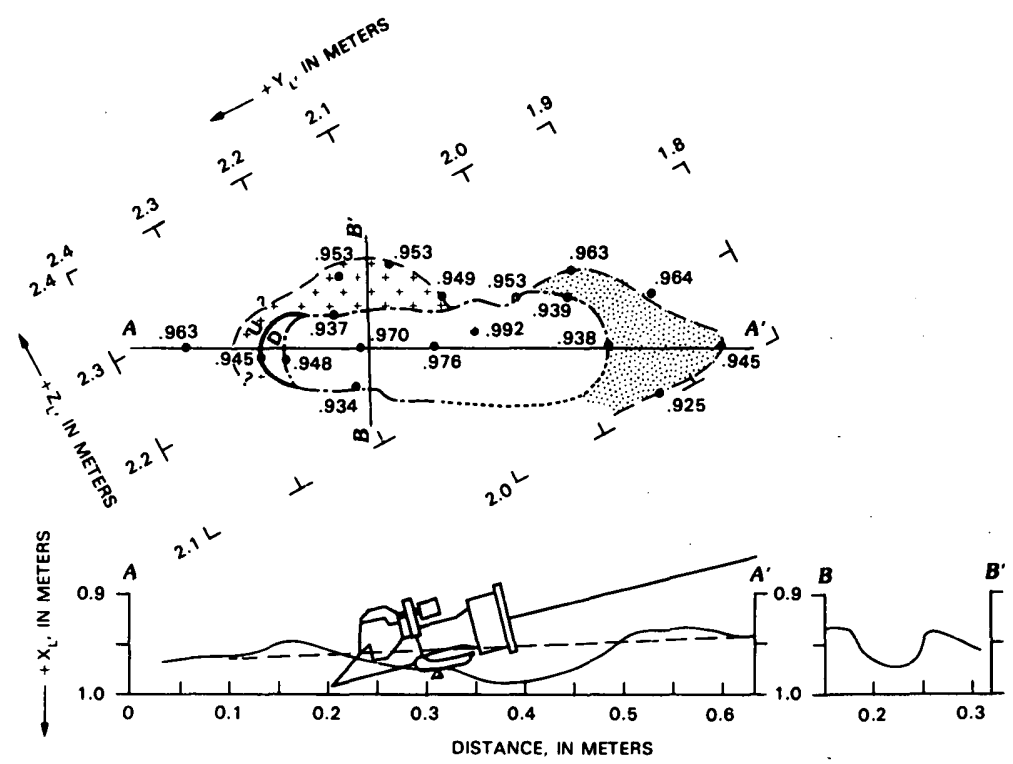

FIGURE 95.-Map and profile of trench in drift material excavated by Lander 1 on Sol 14. No Format 5 motor-current record. (See GCMS 2, table 38, item 9; stereoscopic pictures: frames 12B209/151 and 11B169/079.)
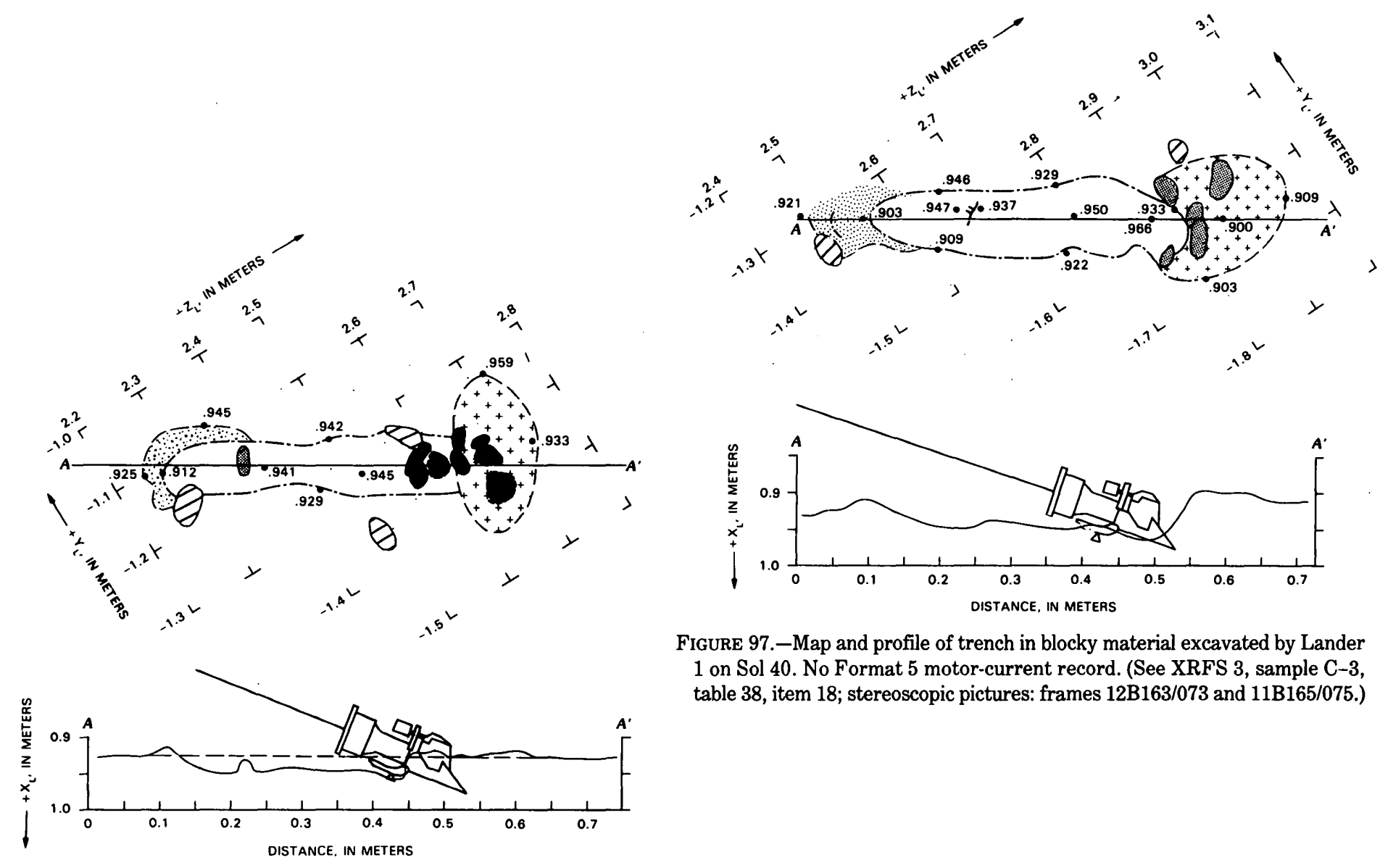

FIGURE 97.-Map and profile of trench in blocky material excavated by Lander 1 on Sol 40. No Format 5 motor-current record. (See XRFS 3, sample C-3, table 38, item 18; stereoscopic pictures: frames 12B163/073 and 11B165/075.)

FIGURE 96.-Map and profile of trench in blocky material excavated by Lander 1 on Sol 34. No Format 5 motor-current record. (See XRFS 2, sample C-2, table 38, item 13; stereoscopic pictures: frames $12 \mathrm{~B} 163 / 073$ and $11 \mathrm{~B} 165 / 075$.) 

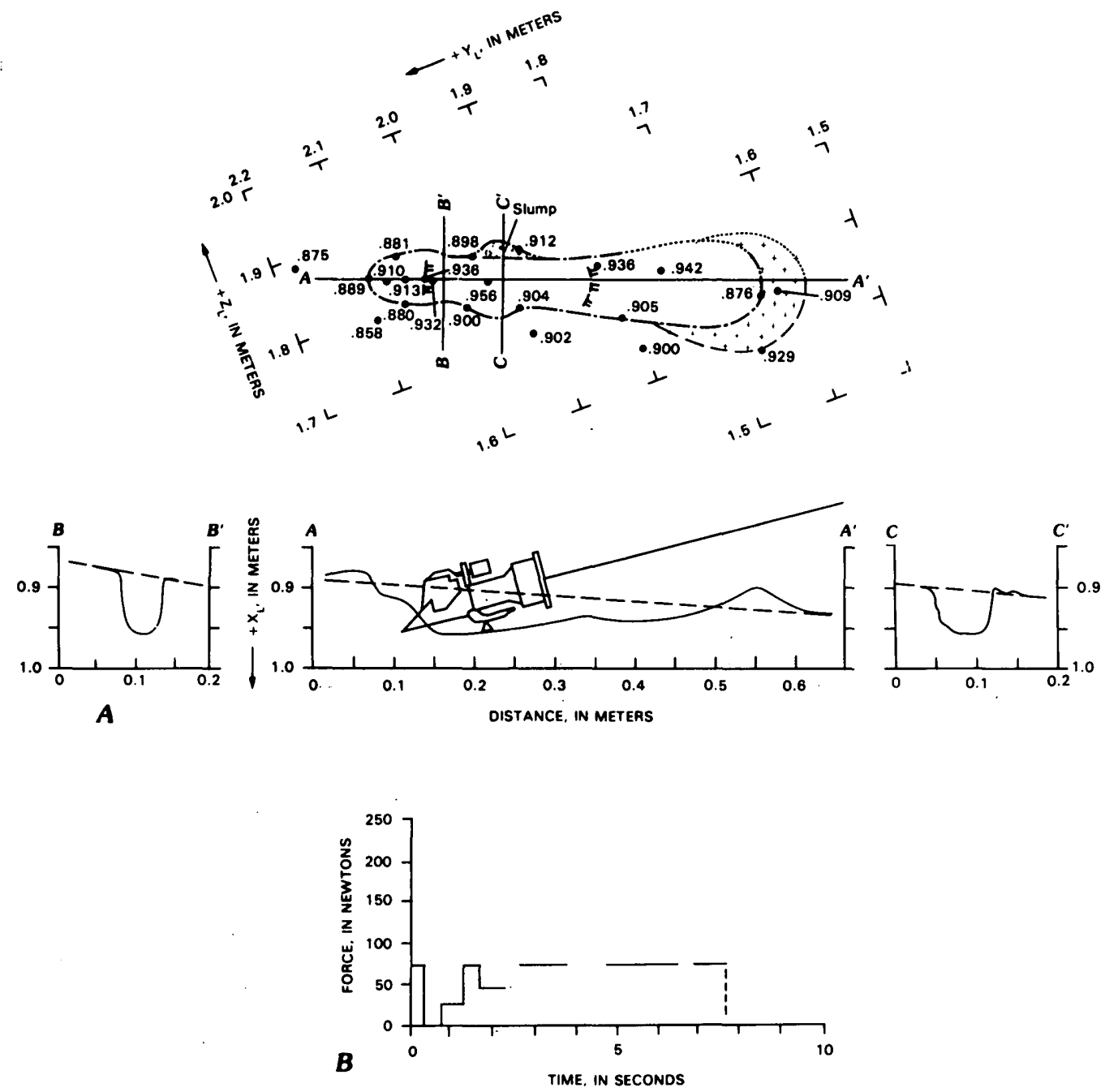

FiguRE 98.-Map, profiles, and motor-current record of trench excavated by Lander 1 in drift material on Sol 41. (See Physical Properties 1, table 38, item 22; stereoscopic pictures: frames 12B209/151 and 11B079/079.) $A$, Map and profile. $B$, Inferred forces from Format 5 motor-current record. 


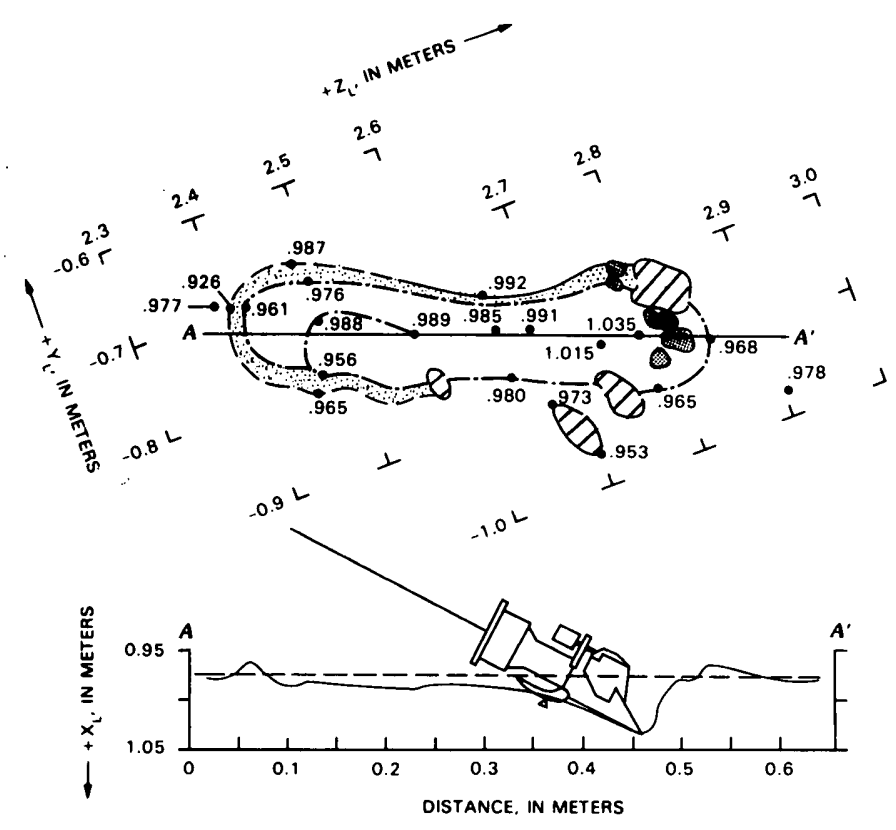

FIGURE 100.-Map and profile of trench in blocky material excavated by Lander 1 on Sols 177, 178, 179, and 180. No Format 5 motor-current record. (See XRFS 4, sample C-5, table 39, item 1; stereoscopic pictures: frames 12D186/318 and 11D185/318.)

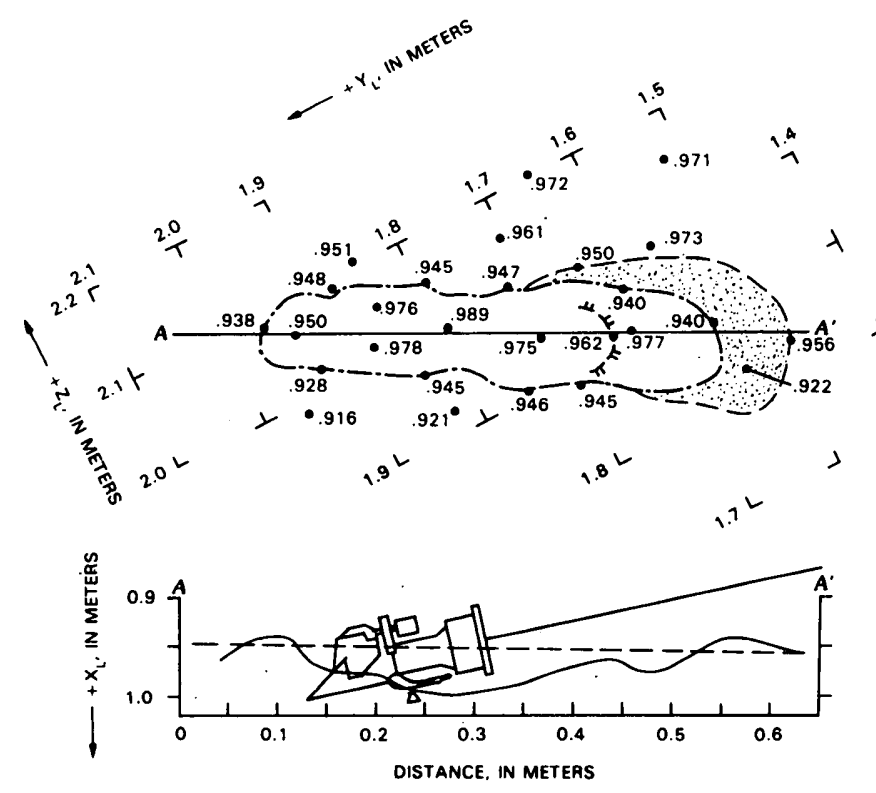

FIgURE 99.-Map and profile of trench in drift material excavated by Lander 1 on Sol 91. No Format 5 motor-current record. (See Biology 3 , table 38, item 26; stereoscopic pictures: frames 12B251/173 and 11B199/115.) A sample was also delivered to XRFS (sample C-4) because of inadvertent spillage. 

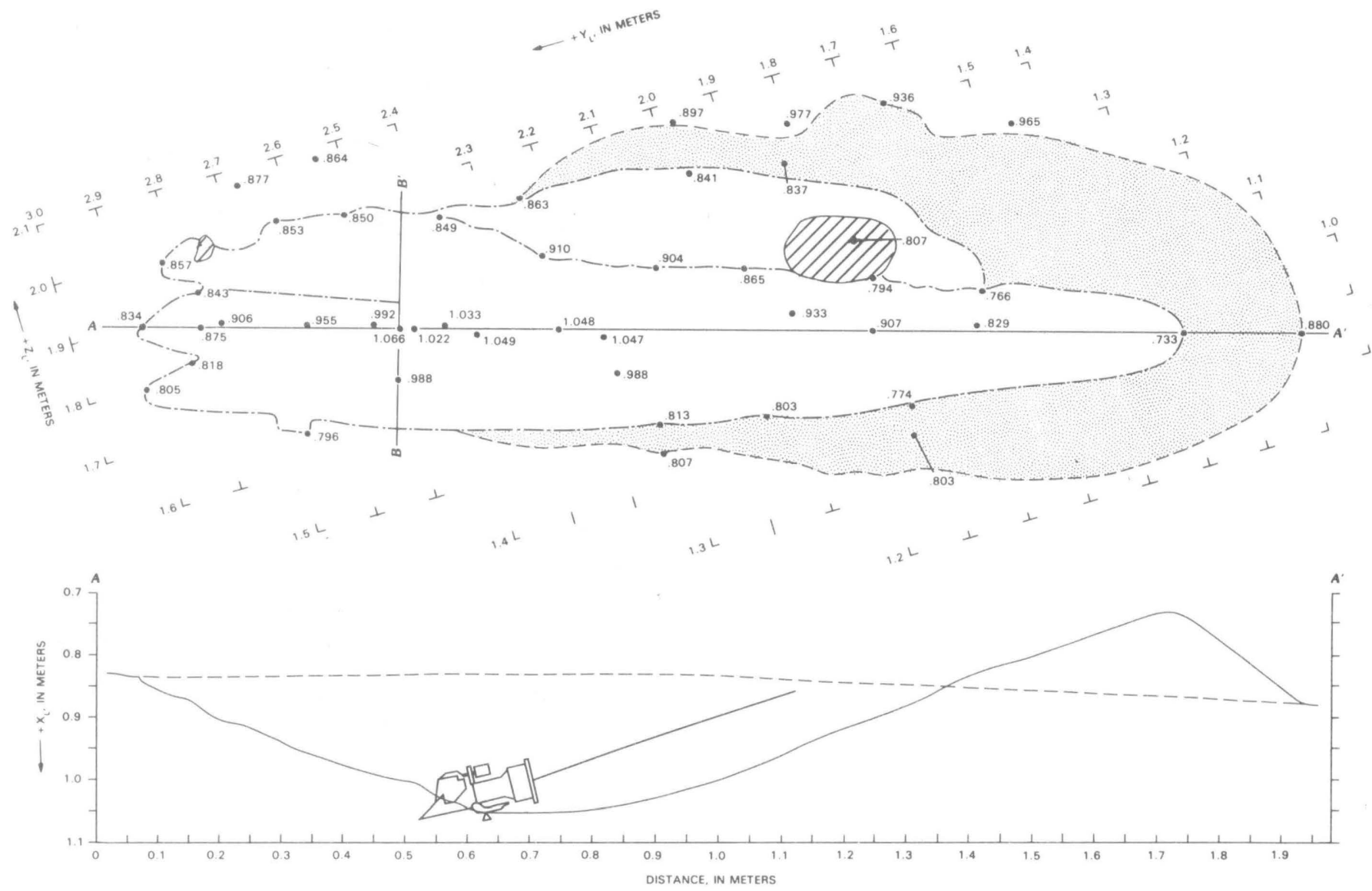

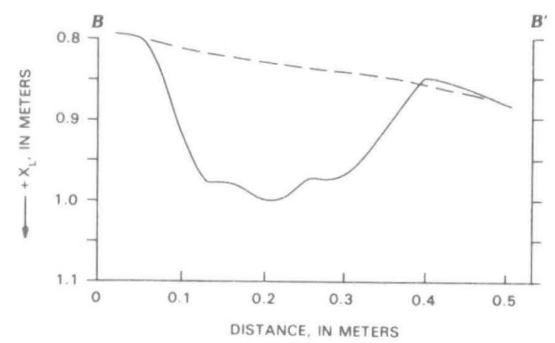

FIGURE 101.-Map and profiles of trench in Deep Hole 1 (see XRFS 5 , 6, sample C-6, table 39 , items 9, 13). Deep Hole 1 in drift material excavated by Lander 1 on Sols 202, 204, 218, and 219. For Format 5 motor-current records see Pike and others (1978). (See Deep Hole 1, table 39, items 4, 6, 7, and 8). Sample delivered to Biology on Sol 250 . (See Biology 4, table 39, item 11; stereoscopic pictures: frames $12 \mathrm{C} 079 / 204,11 \mathrm{C} 078 / 204,12 \mathrm{C} 128 / 219,11 \mathrm{C} 127 / 219,12 \mathrm{E} 003 / 330$, and 11D183/317.) 

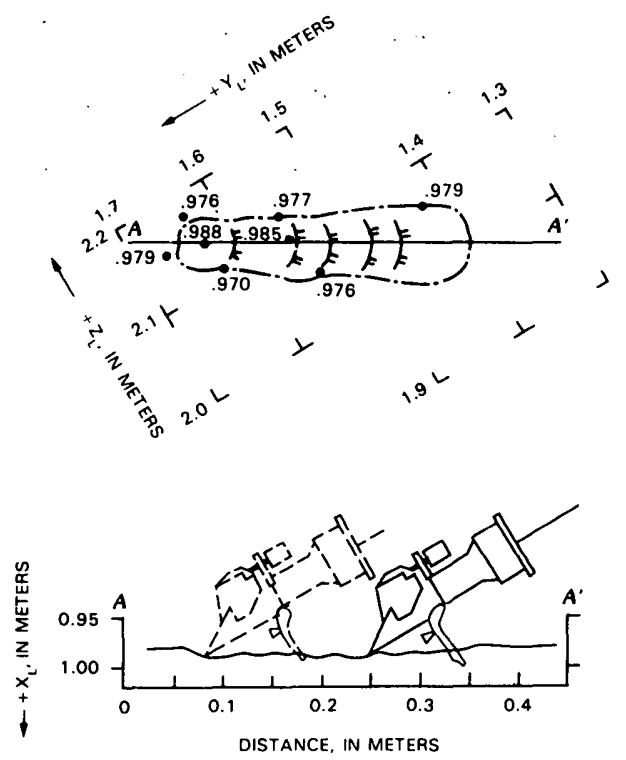

Figure 102.-Map and profile of trench in drift material excavated during magnetic mineral abundance experiment by Lander 1 on Sol 270 . No Format 5 motor-current record. (See Backhoe touchdown 2, table 39, item 15; stereoscopic pictures: $12 \mathrm{D} 139 / 303$ and 11D034/281.) Dashed outline of collector head is starting position; solid outline is final position.
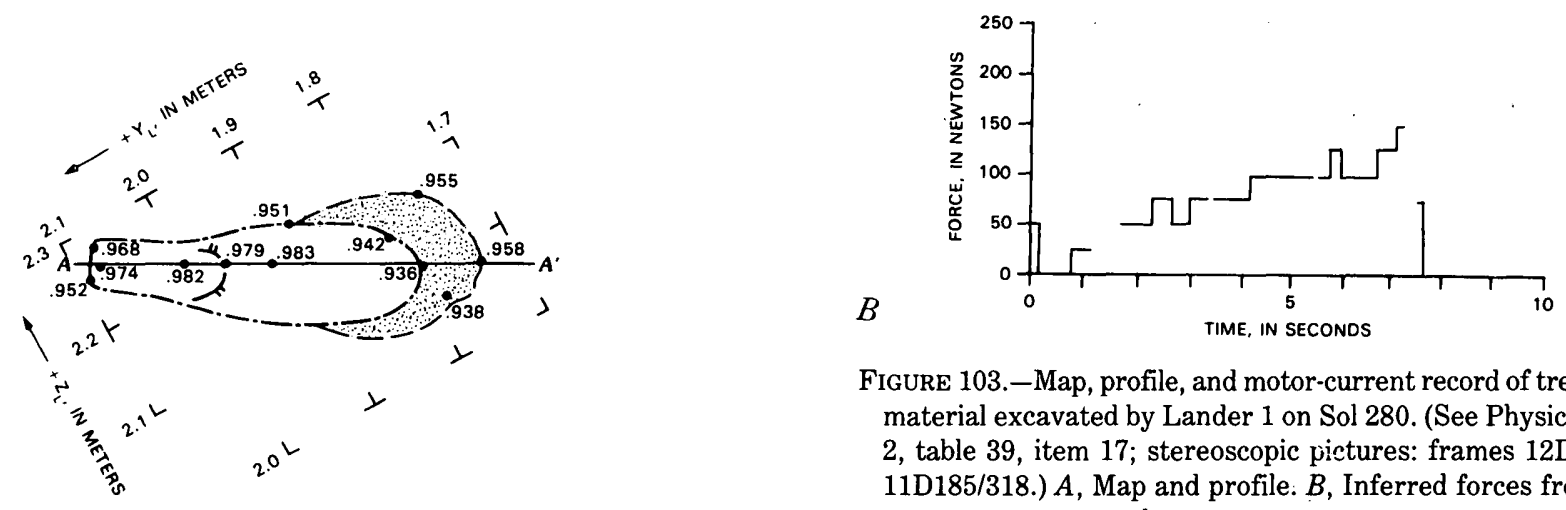

FiguRE 103.-Map, profile, and motor-current record of trench in blocky material excavated by Lander 1 on Sol 280. (See Physical Properties 2, table 39, item 17; stereoscopic pictures: frames 12D186/318 and 11D185/318.) $A$, Map and profile: $B$, Inferred forces from Format 5 motor-current record.

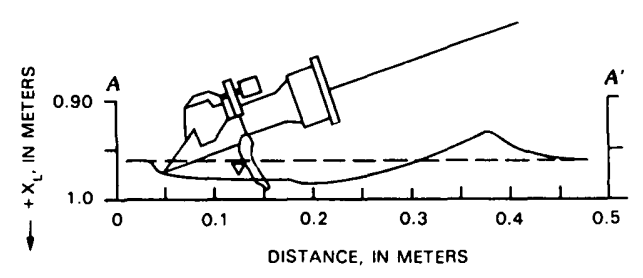

Figure 104.-Map and profile of trench in drift material excavated by Lander 1 on Sol 280 . No Format 5 motor-current record. (See Backhoe touchdown 3 , table 39 , item 20 ; stereoscopic pictures: frames 12D032/280, 12D093/296, and 11D034/281.) 

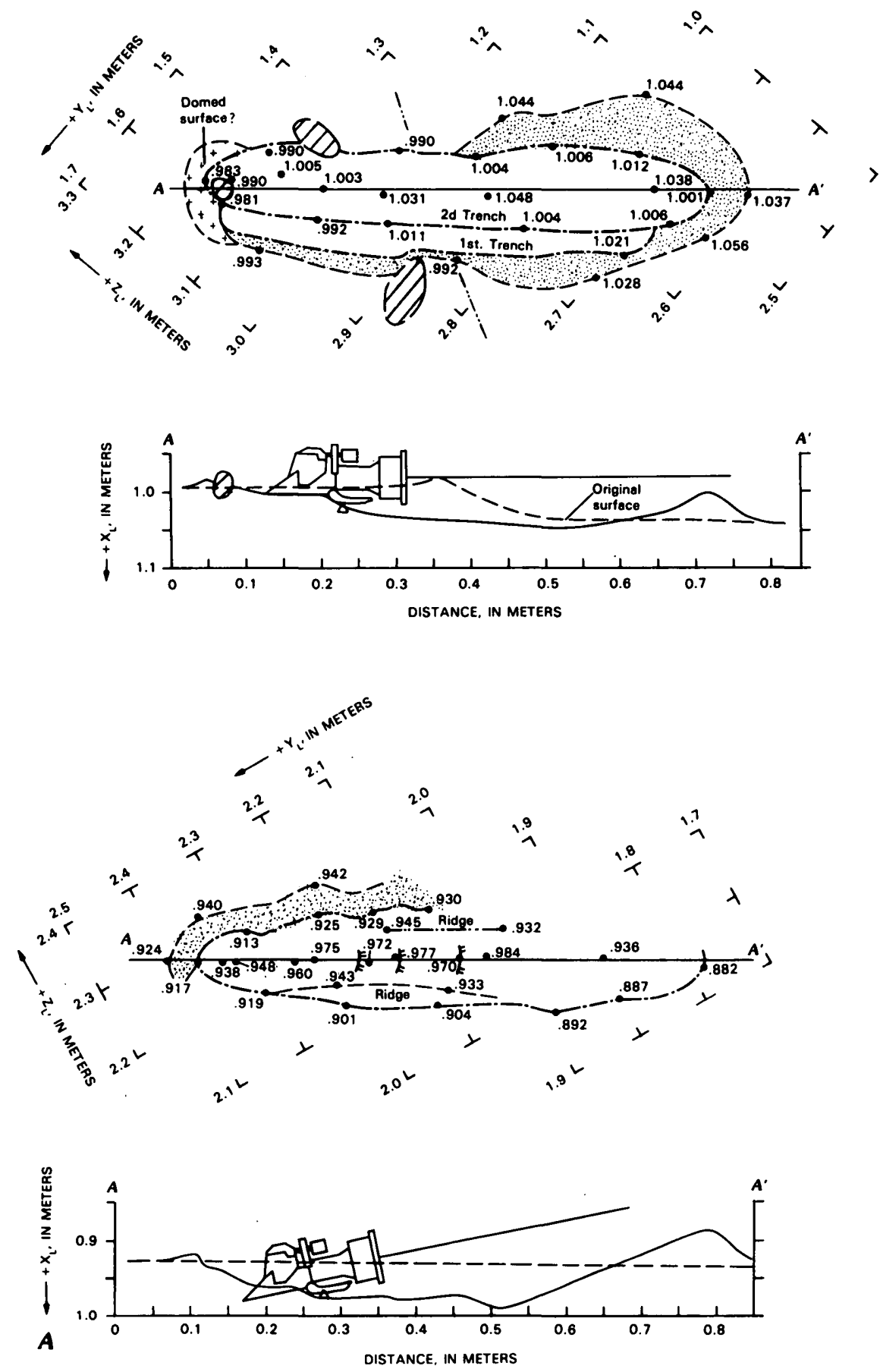

FIGURE 105.-Map and profile of trench in wind tail produced by skimming by Lander 1 on Sols 285 and 286. For Format motor-current record see Pike and others (1978). (See XRFS 7 , sample C-7, table 39 , item 21 ; stereoscopic pictures: frames 12D091/296 and 11E082/351.) 

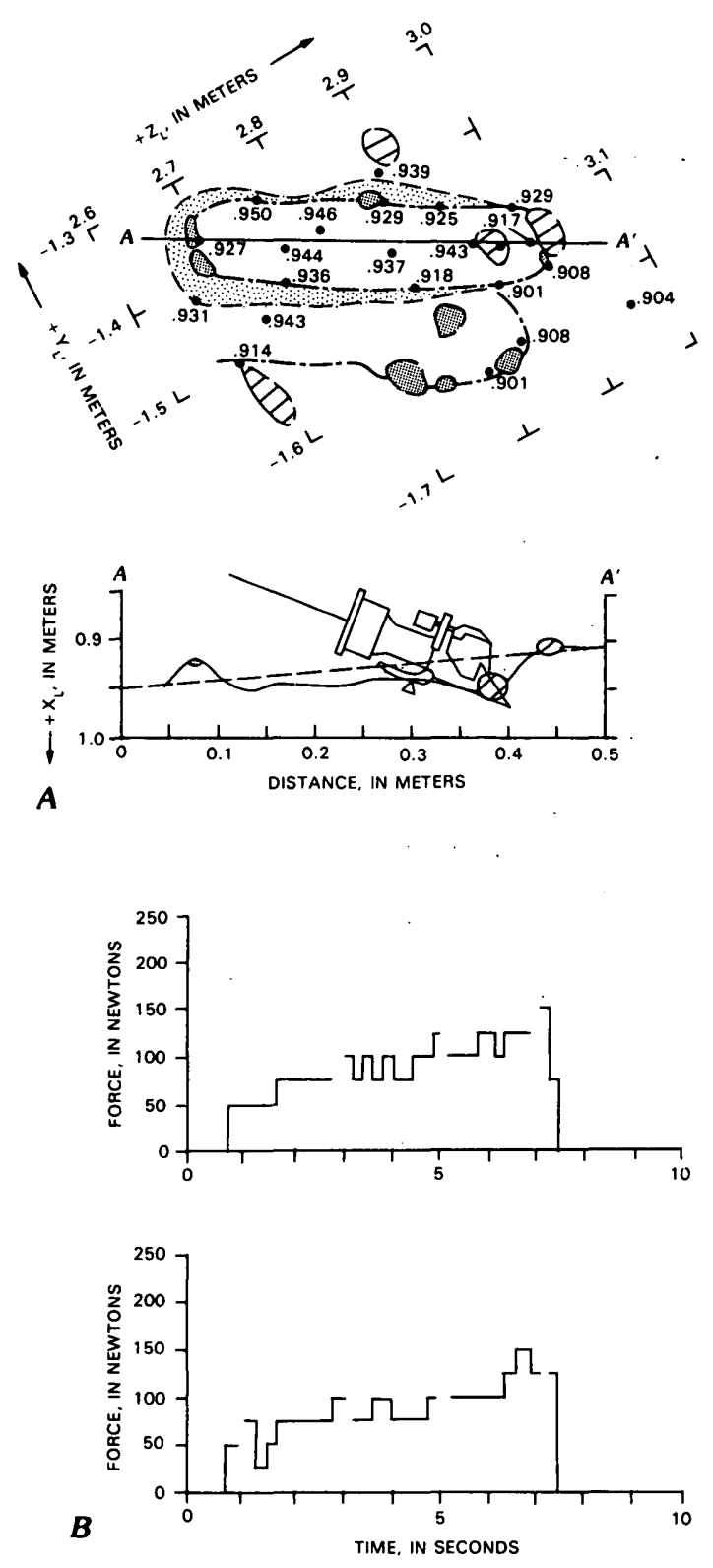

FIGURE 107.-Map, profiles, and motor-current records of trench in blocky material excavated by Lander 1 on Sols 311 and 312. (See XRFS 8, sample C-8, table 39, item 26; stereoscopic pictures: frames 12D186/318 and $11 \mathrm{D} 185 / 318$.) $A$, Map and profile. $B$, Inferred forces from Format 5 motor-current records.
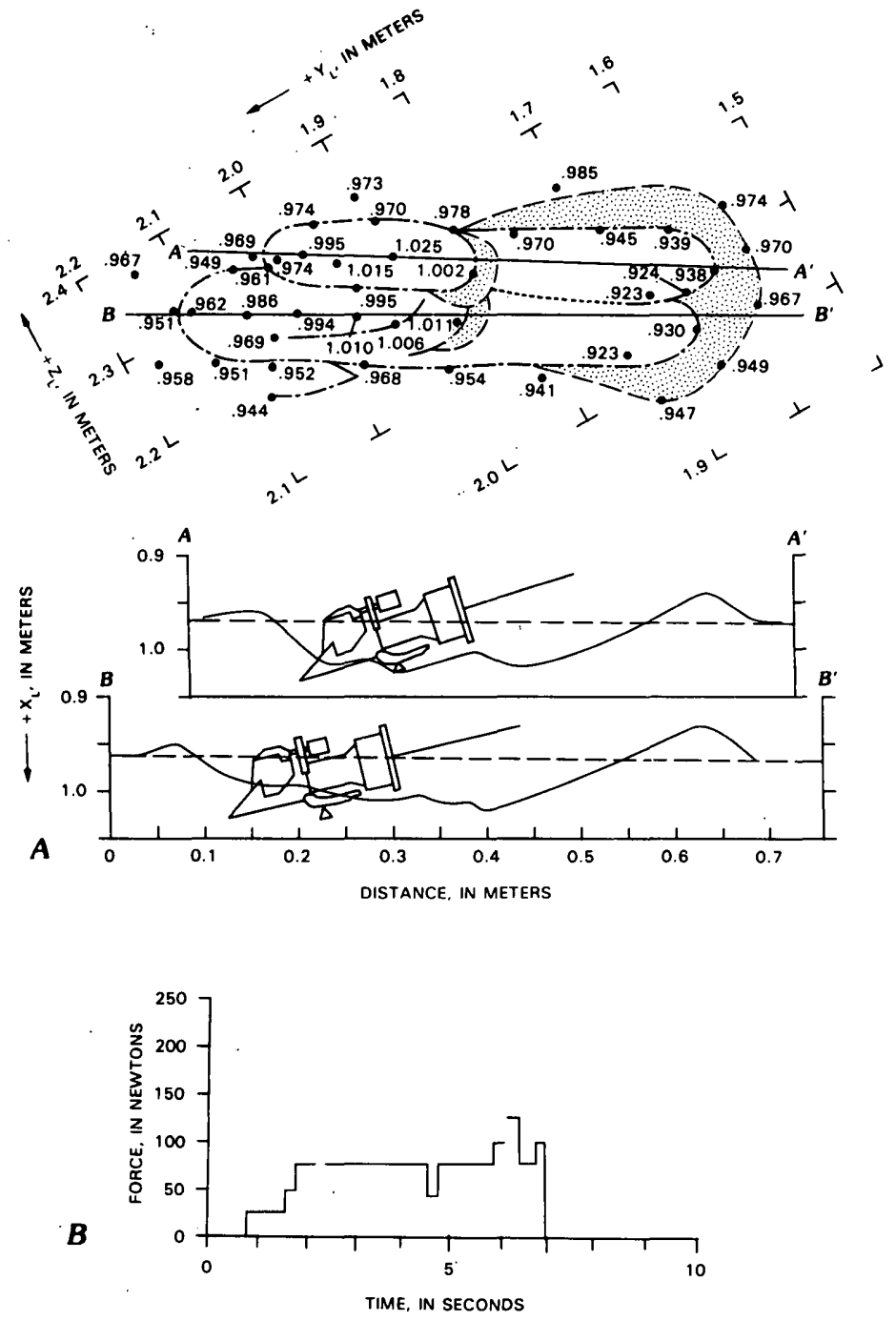

FIGURE 108.-Map, profiles, and motor-current record of trenches in drift material excavated by Lander 1 on Sol 324 . (See Physical Properties 4 and 5, table 39, items 28 and 30; stereoscopic pictures: frames 12E003/ 330 and 11D213/324.) $A$, Map and profile. $B$, Inferred forces from Format 5 motor-current record. 

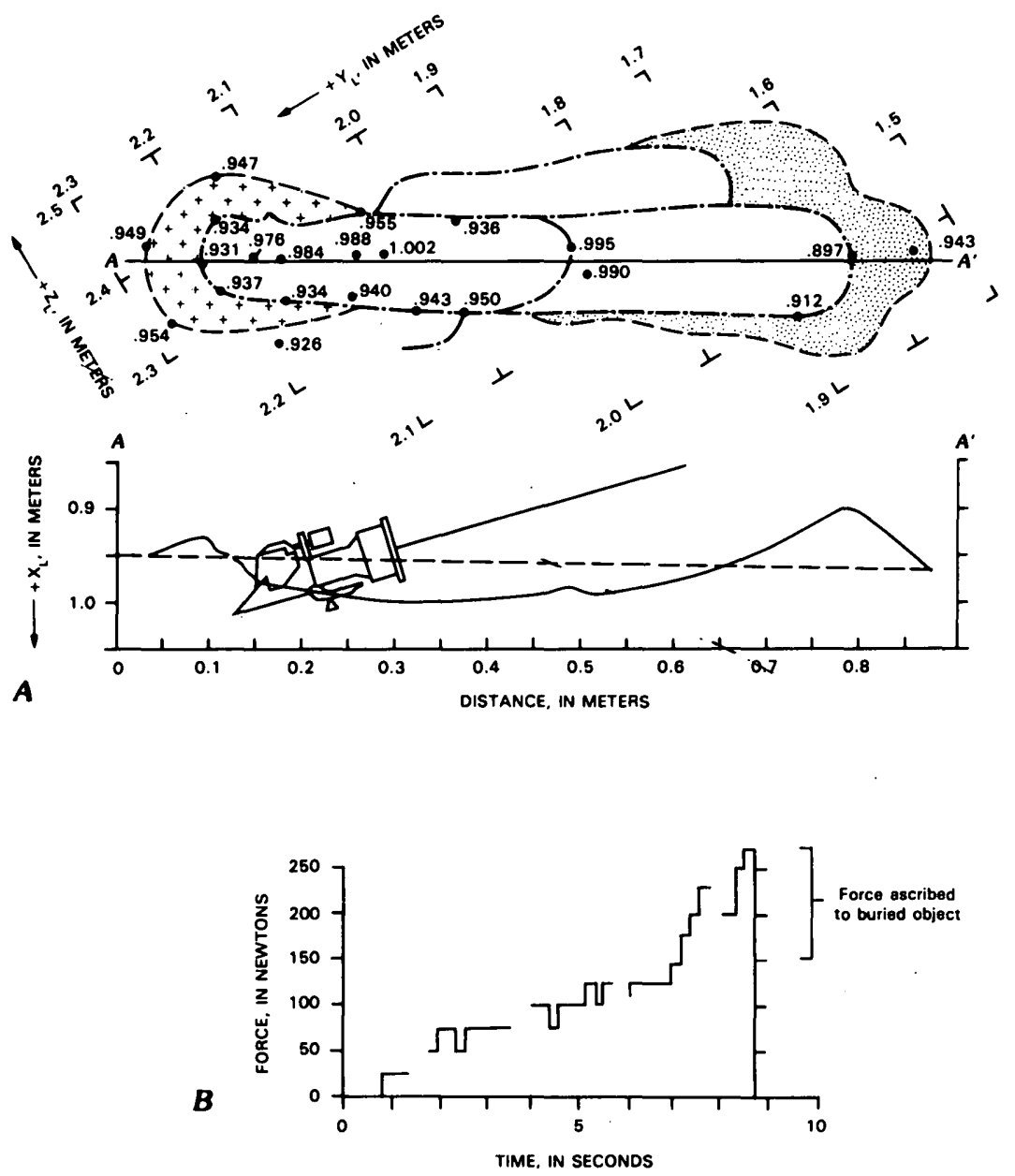

FIGURE 109.-Map, profile, and motor-current record of trench in drift material used for diurnal temperatures of Lander 1 on Sols 343 and 344. (See Physical Properties 6, table 39, item 34; stereoscopic pictures: frames 12I165/801, 11I164/801, 12F201/441, and 12I132/764.) $A$, Map and profile. $B$, Inferred forces from Format 5 motor-current record. 

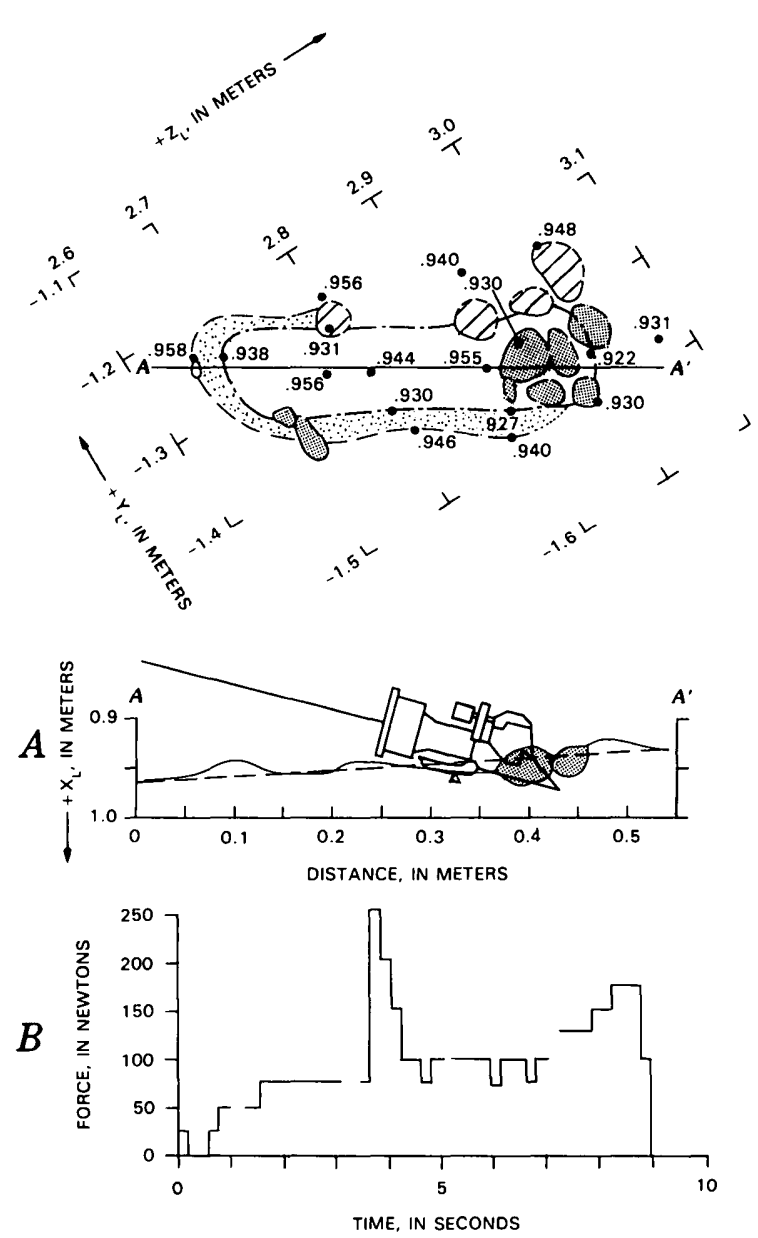

FIGURE 110.-Map, profile, and motor-current record of trench in blocky material excavated by Lander 1 on Sol 350. (See Physical Properties 7, table 39, item 36; stereoscopic pictures: frames $12 \mathrm{E} 083 / 351$ and $11 \mathrm{E} 082 / 351$.) $A$, Map and profile. $B$, Inferred forces from Format 5 motorcurrent records.

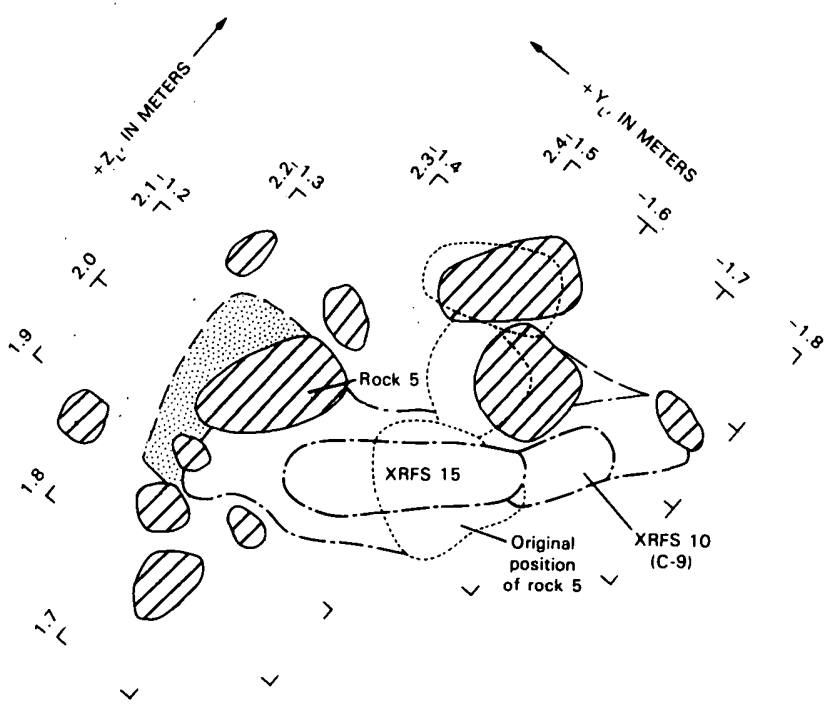

FIGURE 111.-Map of trenches near rock 5 (Bashful) excavated by Lander 1 on Sol 378 and, later, on Sol 527. No Format 5 motorcurrent record. (See XRFS 10, sample C-9, XRFS 15, table 39, items 40 and 52.) Rock pushed on Sol 369 (see table 39, item 38; monoscopic pictures: frames $12 \mathrm{E} 148 / 369,12 \mathrm{E} 208 / 384$, $12 \mathrm{G} 194 / 527$, and 12I173/804.) 


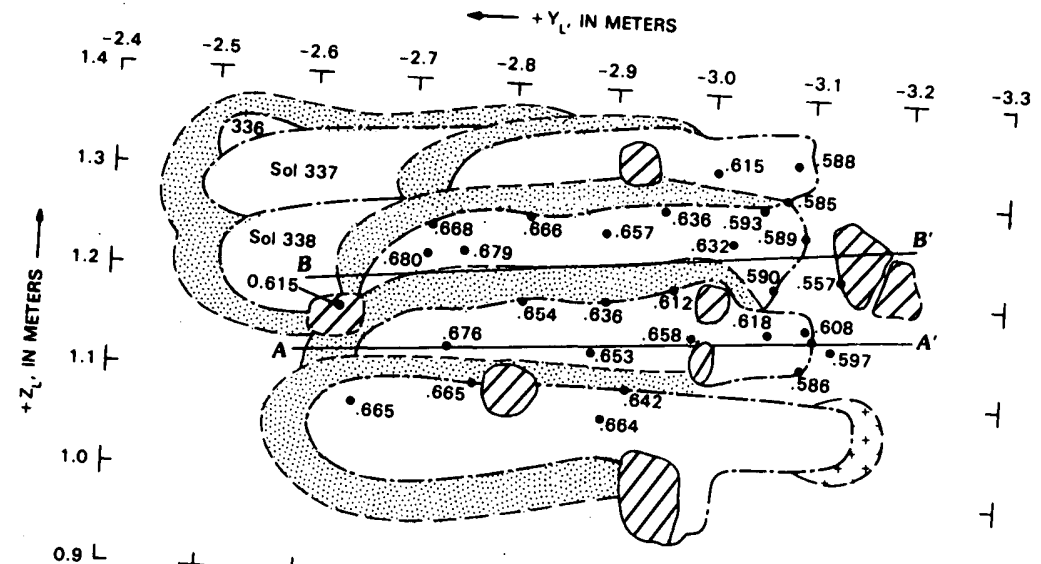

$0.9 \mathrm{~L}$

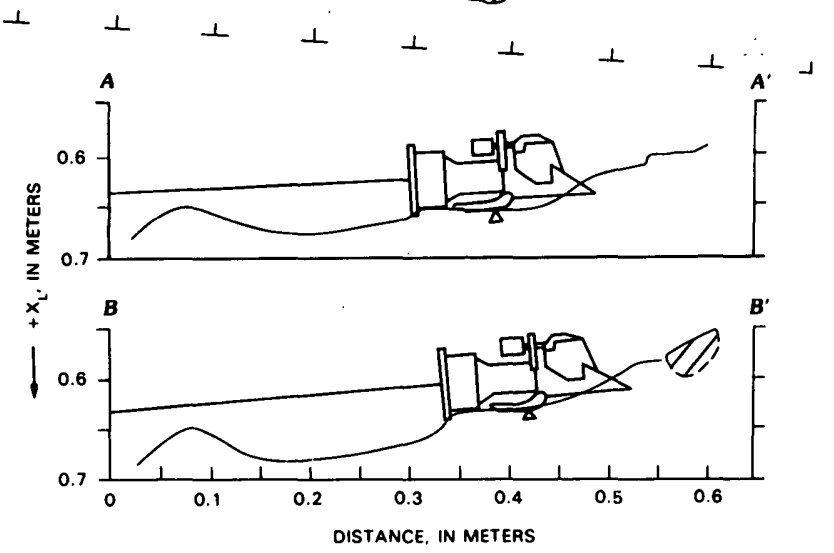

FIGURE 112.-Map and profiles of trenches near right edge of sample field excavated by Lander 1 on Sols 430 and 431. For Format 5 motor-current records see Pike and others (1978). (See XRFS 11, sample C-10, table 39, item 42.) Trenches superposed on earlier ones excavated on Sols 336,337 , and 338 (See XRFS 9, table 39, item 32; stereoscopic pictures: frames 12G241/546 and 11H086/585.)
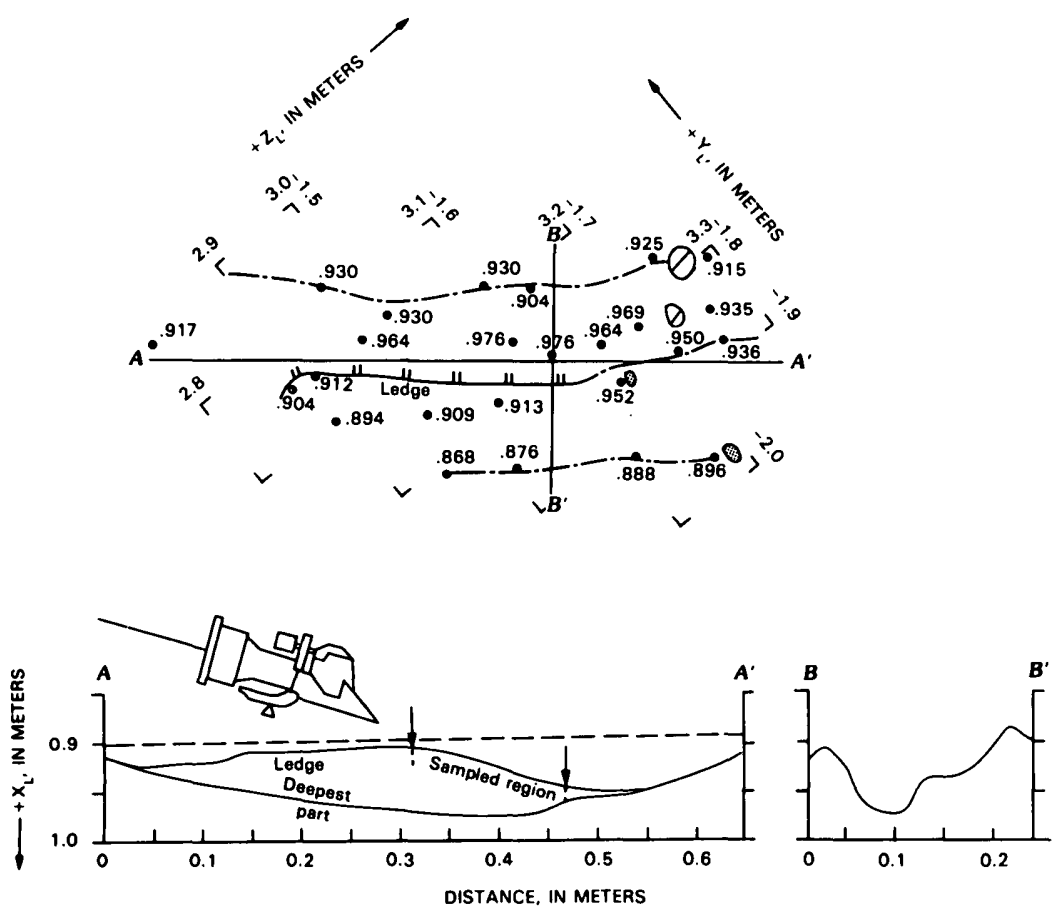

Figure 113.-Map and profiles of region of sample trench in Deep Hole 2 excavated by Lander 1 on Sol 456 prior to sampling. No Format 5 motor-current record. Sample acquired on Sol 468. (See XRFS 12, sample C-11, table 39, item 46; Deep Hole 2, table 39 , item 45; stereoscopic pictures: frames 12 F227/457 and 11 F226/457.) Arrows indicate sampled regions; there is no postsample stereoscopic coverage of sampled region. 

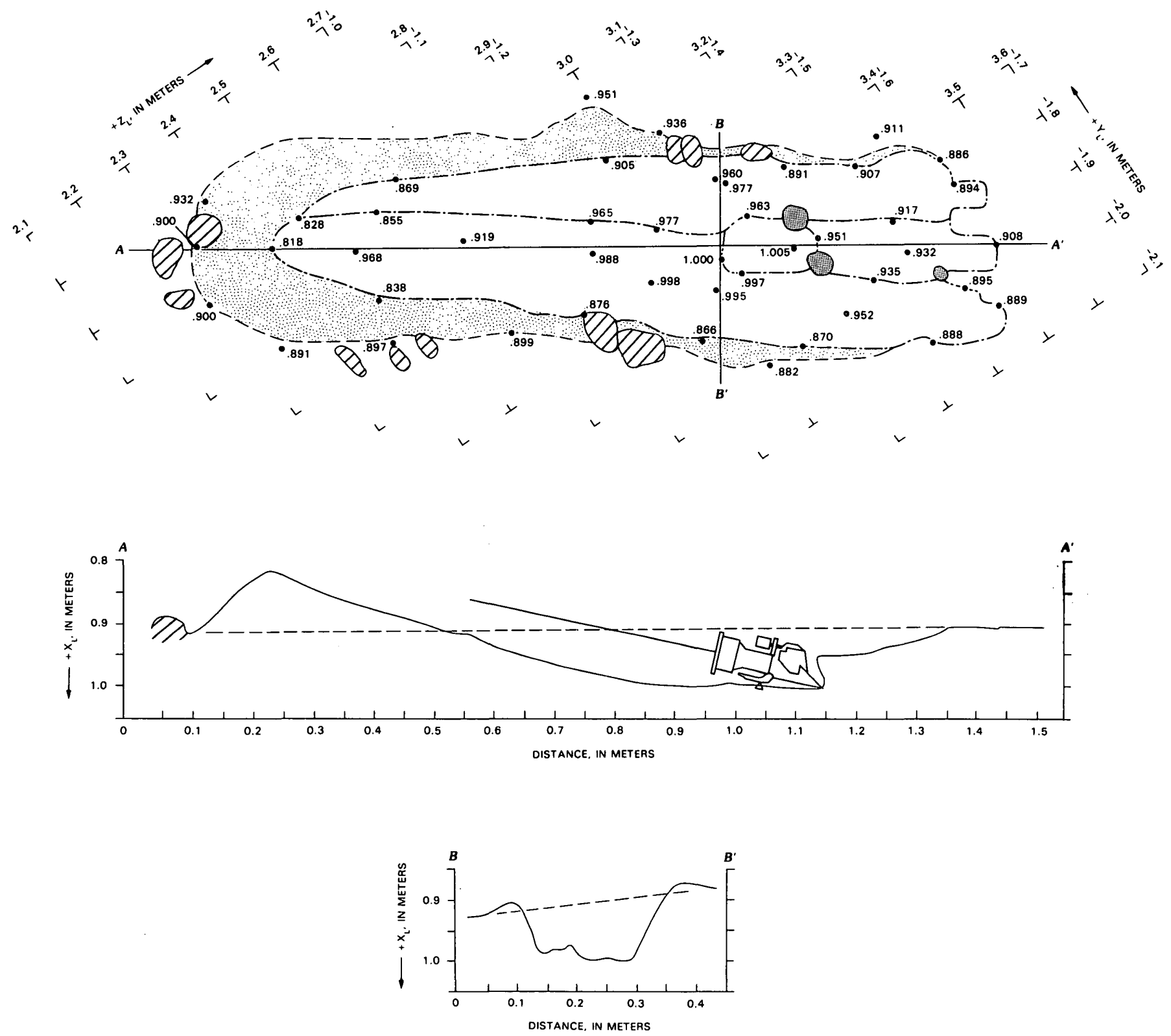

FIGURE 114.-Map and profiles of trench in Deep Hole 2 excavated by Lander 1 on Sol 502. (See XRFS 14, sample C-12, table 39, item 49; stereoscopic pictures: 12G079/488, 11G078/488, 12G100/502, and 11I159/799.) Deep Hole excavated on Sols 456 and 486 . For Format 5 motorcurrent record see Pike and others (1978). (See Deep Hole 2, table 39, items 45 and 47). 

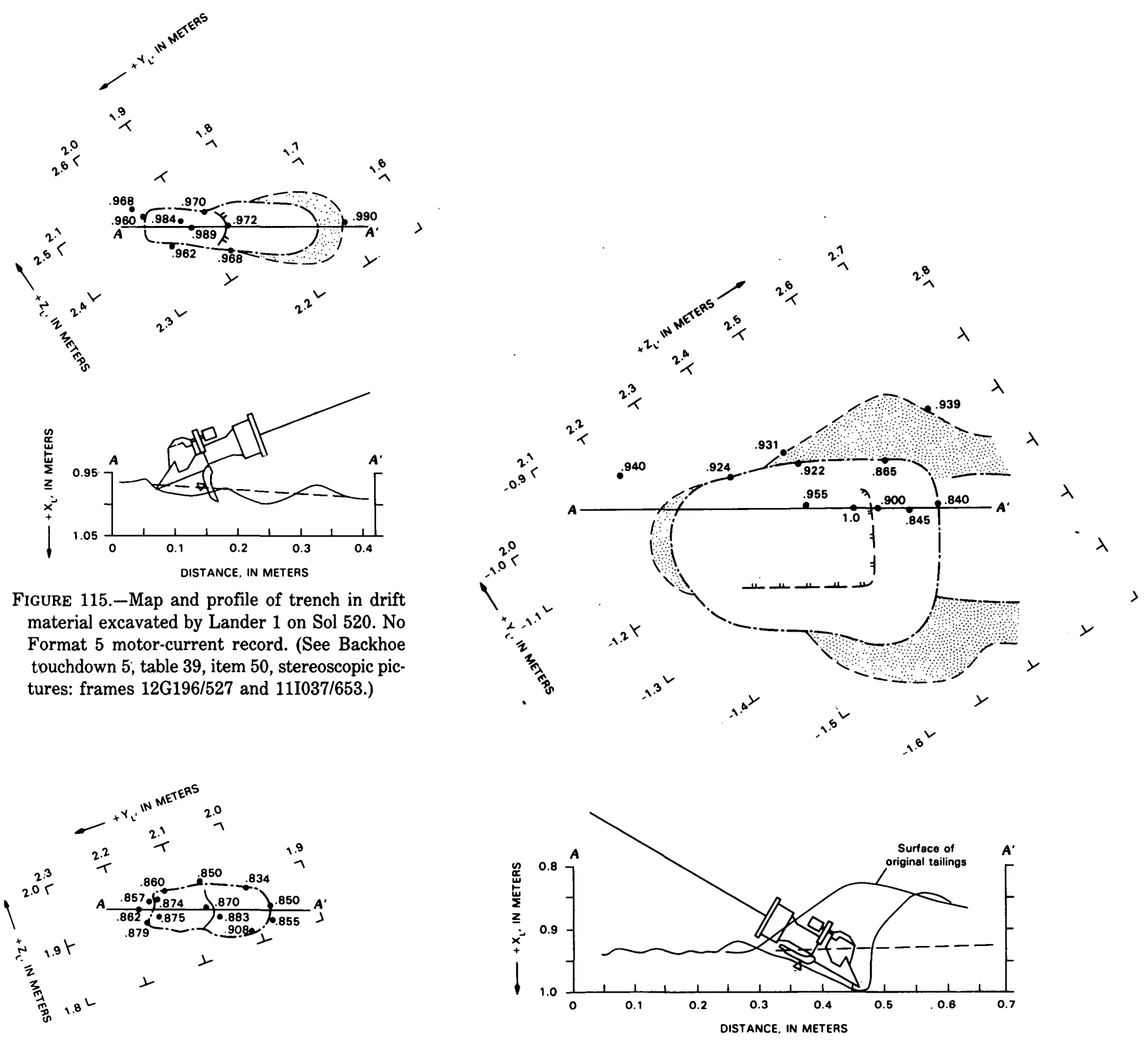

Figure 115.-Map and profile of trench in drift material excavated by Lander 1 on Sol 520 . No Format 5 motor-current record. (See Backhoe touchdown 5 , table 39 , item 50 , stereoscopic pictures: frames $12 \mathrm{G} 196 / 527$ and $11 \mathrm{I} 037 / 653$.
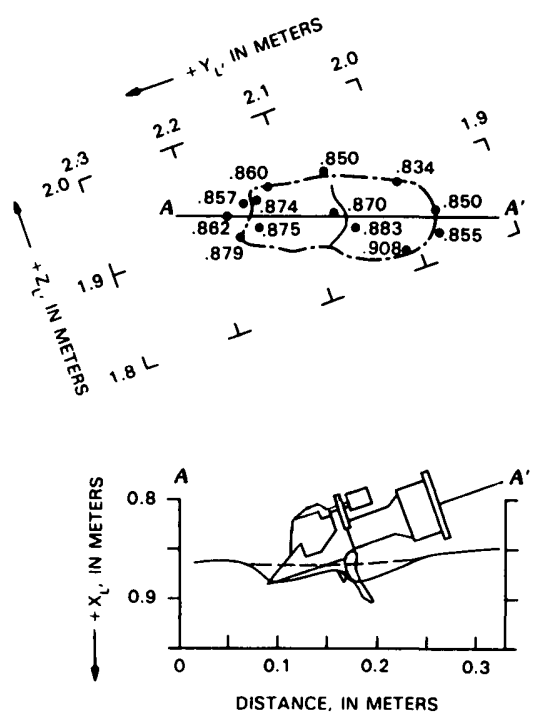

Figure 117.-Map and profile of trench in tailings of Deep Hole 2 excavated by Lander 1 on Sol 586 (and 558, 559, 560, 561, 581). For Format 5 motor-current records see Pike and others (1978). (See XRFS 16, 17, 18, sample C-13, table 39, items 58,60, and 63; stereoscopic pictures: frames 12I173/804 and 11I159/799.)

FIGURE 116.-Map and profile of trench produced in tailings of Deep Hole 1 by Lander 1 on Sol 550. No Format 5 motorcurrent record. (See Backhoe touchdown 7 , table 39 , item 56 ; stereoscopic pictures: frames $121066 / 690$ and $111065 / 690$.) 


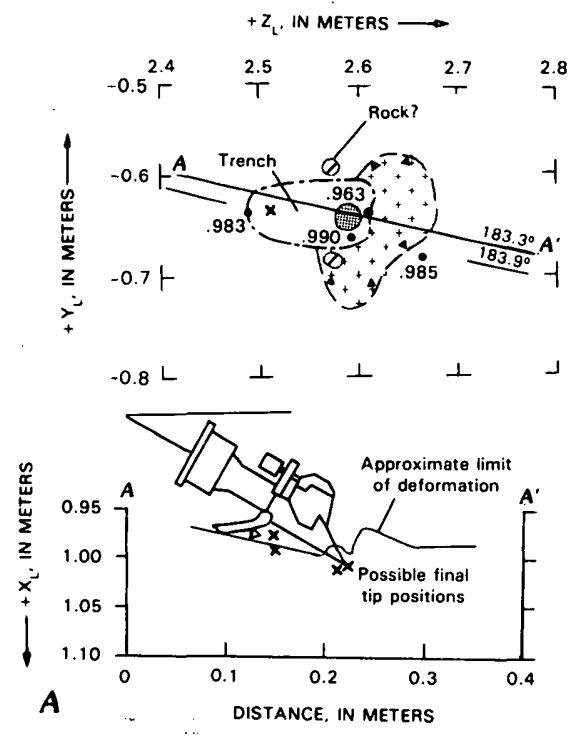

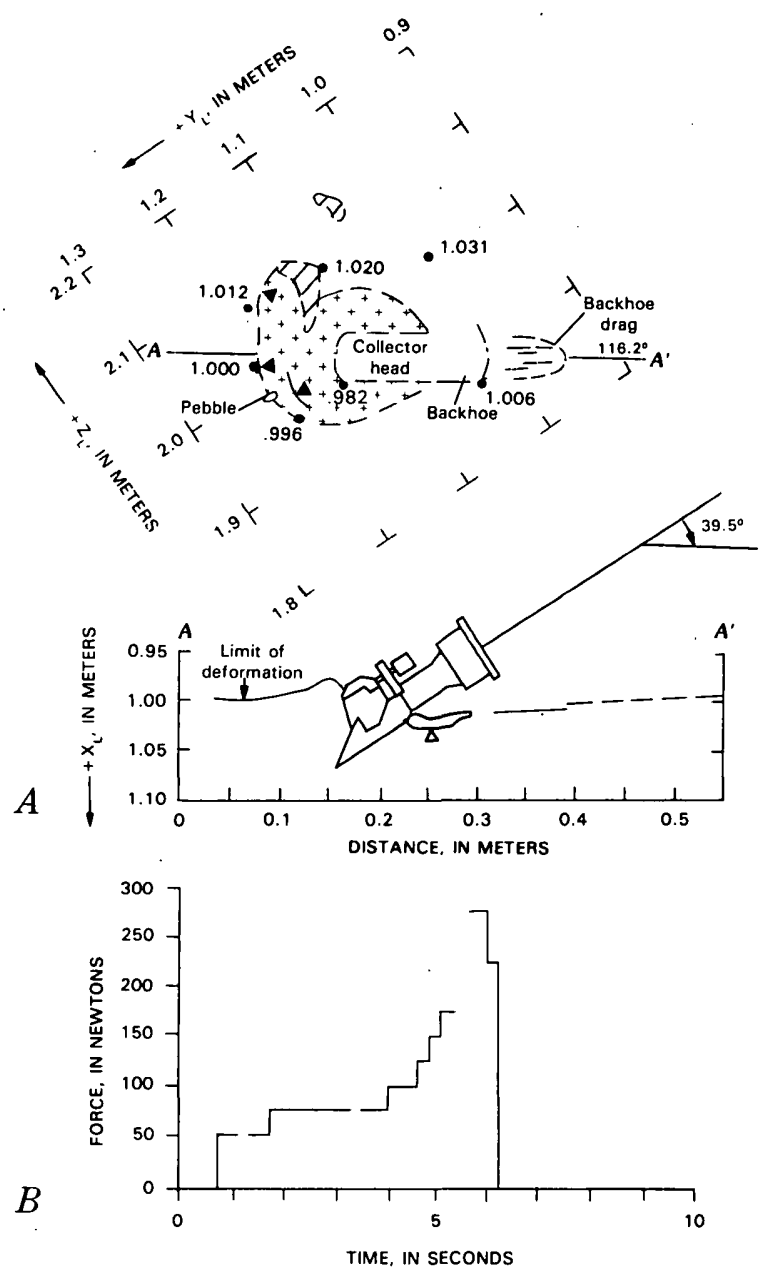

FIGURE 118.-Map, profile, and motor-current record of trench of surface-bearing test in drift material excavated by Lander 1 on Sol 612. (See Physical Properties 9, table 39, item 67; stereoscopic pictures: frames $12 \mathrm{H} 186 / 612$ and $11 \mathrm{H} 187 / 612$.) $A$, Map and profile. $B$, Inferred forces from Format 5 motor-current records. Surface-sampler elevation angle is $39.5^{\circ}$; surface-sampler azimuth is $116.2^{\circ}$.

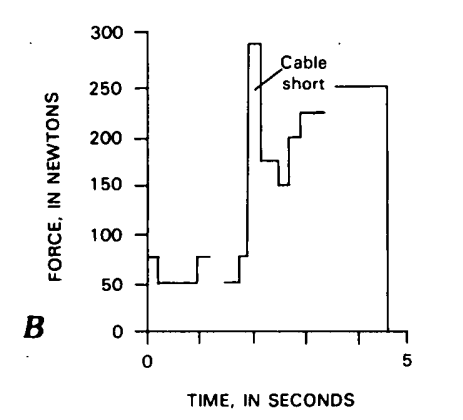

FiguRE 119.-Map, profile, and motor-current record of trench of surface-bearing test 2 in blocky material excavated by Lander 1 on Sol 612. (See Physical Properties 10, table 39, item 68; stereoscopic pictures: frames $12 \mathrm{H} 189 / 612,11 \mathrm{H} 188 / 612,12 \mathrm{H} 204 / 618$, and $11 \mathrm{H} 200 / 616$.) $A$, Map and profile. $B$, Inferred forces from Format 5 motor-current record. Surface-sampler elevation angle is $39.5^{\circ}$; surface-sampler azimuth of $183.3^{\circ}$ and $183.9^{\circ}$ are indicated by lines. Small crosses in plan view indicate locations of tip of collector head at start and end of tests; small crosses in profile indicate possible location of tip of collector head at finish of test. 

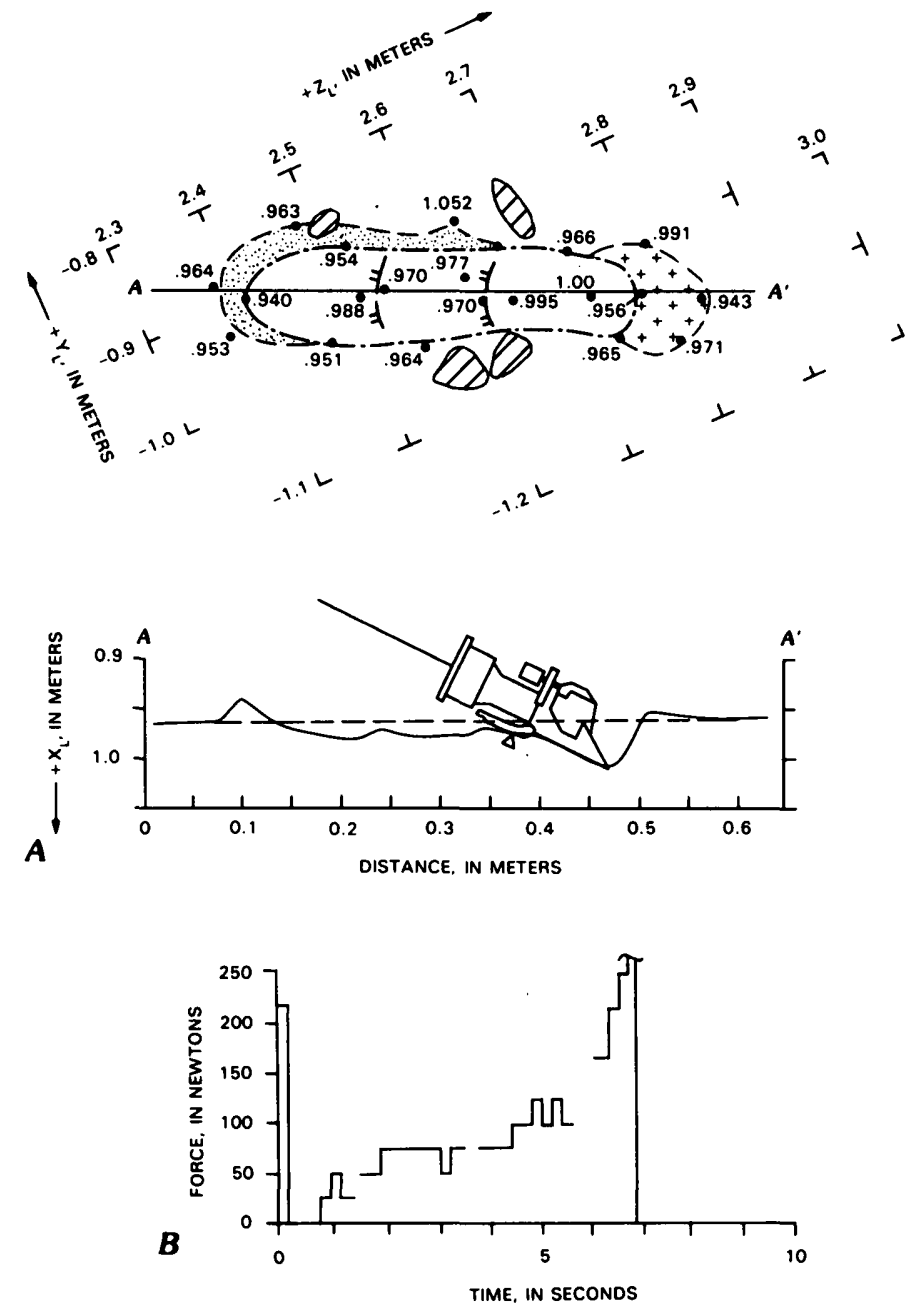

FIGURE 120.-Map, profile, and motor-current record of trench in Rocky Flats excavated in blocky material by Lander 1 on Sol 639. (See Physical Properties 11, table 39, item 70; stereoscopic pictures: frames 12I173/804 and 111159/804.) $A$, Map and profile. $B$, Inferred forces from Format 5 motor-current record.

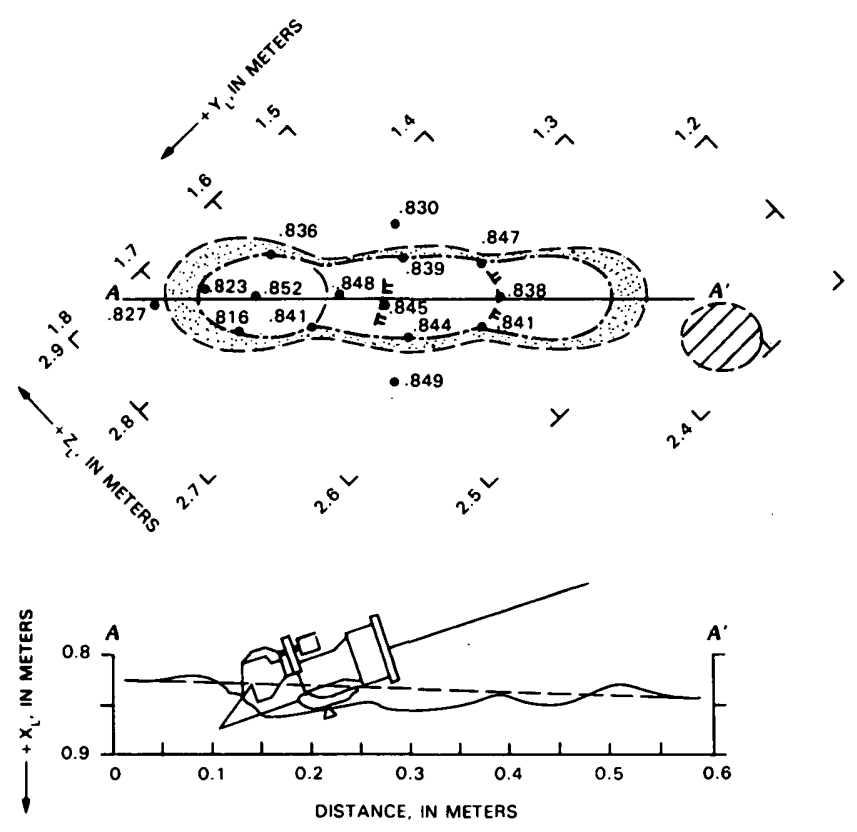

Figure 121.-Map and profile of first trench excavated by Lander 2. Trench in Beta site excavated on Sol 8. No Format 5 motor-current record. (See Biology 1, table 40, item 2; stereoscopic pictures: frames $22 \mathrm{~A} 121 / 016$ and $21 \mathrm{~A} 110 / 015$.)

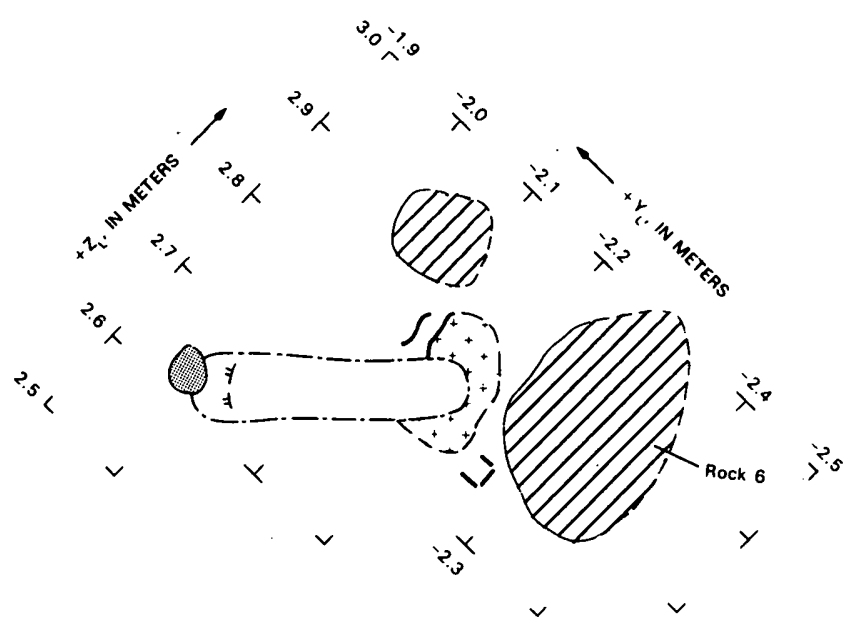

FIGURE 122.-Map of trench in Bonneville Salt Flats excavated in crusty material on Sol 21. No Format 5 motor-current record. (See GCMS 1, table 40, item 4; monoscopic picture: frame 22A162/021.) 

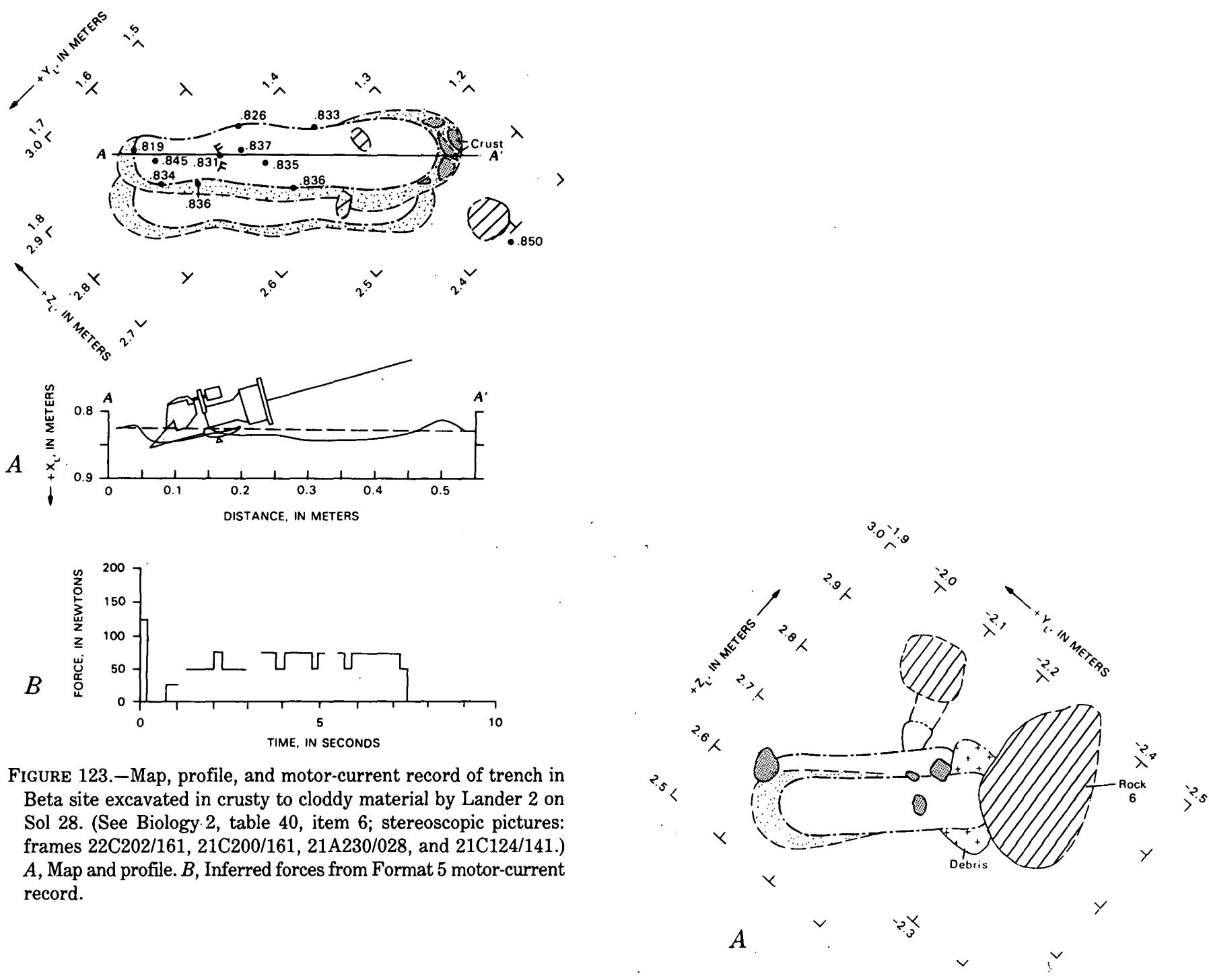

FIGURE 123.-Map, profile, and motor-current record of trench in Beta site excavated in crusty to cloddy material by Lander 2 on Sol 28. (See Biology.2, table 40, item 6; stereoscopic pictures: frames $22 \mathrm{C} 202 / 161,21 \mathrm{C} 200 / 161,21 \mathrm{~A} 230 / 028$, and $21 \mathrm{C} 124 / 141$.) $A$, Map and profile. $B$, Inferred forces from Format 5 motor-current record.

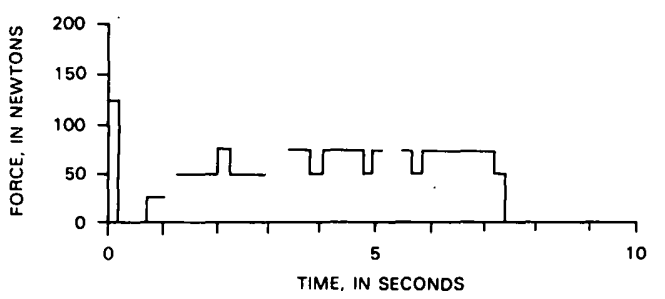



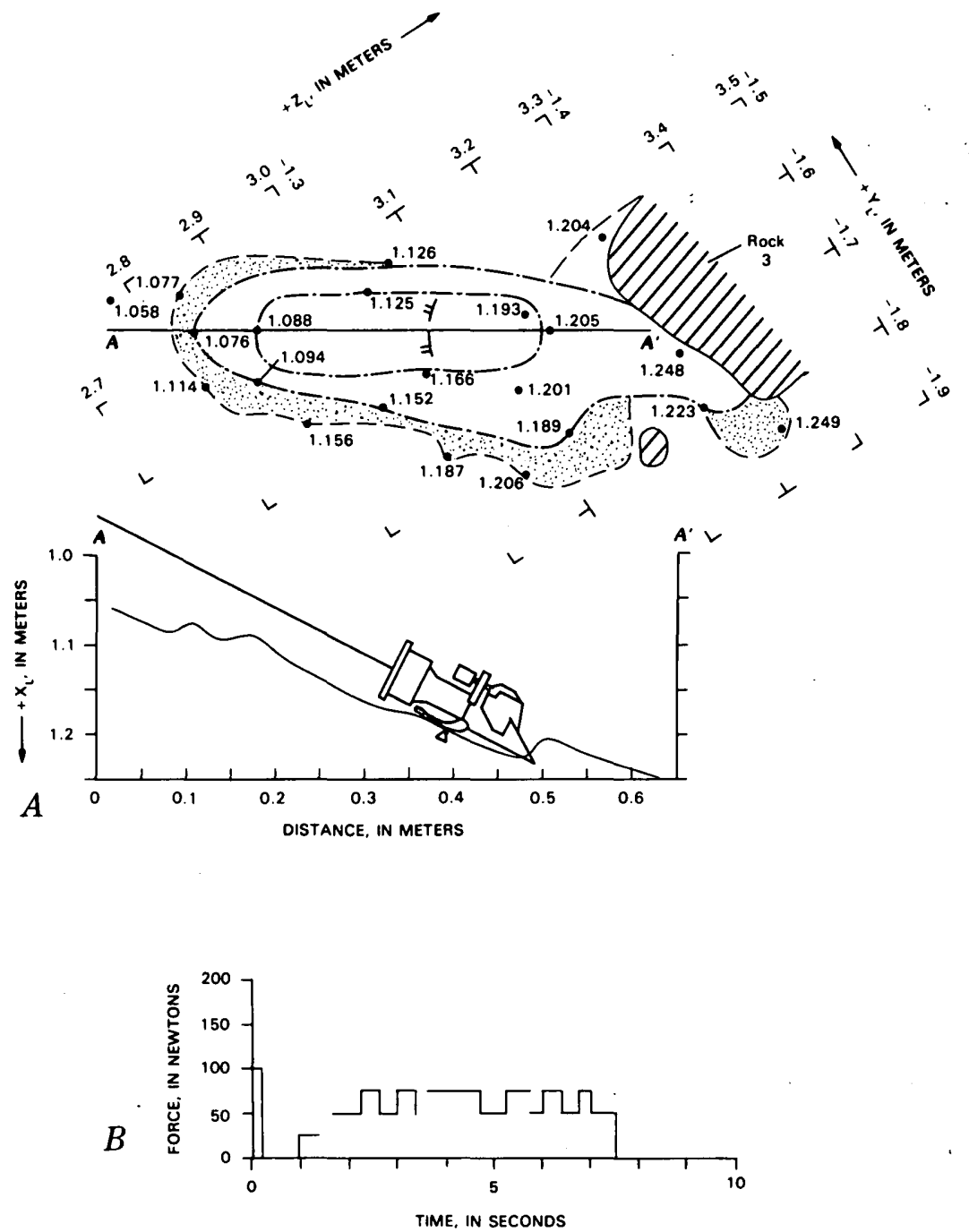

FIGURE 125.-Map, profile, and motor-current record of trench in area originally under rock 3 (Badger) excavated by Lander 2 on Sol 37. (See GCMS 2, table 40, item 13; stereoscopic pictures: frames 22C196/161 and 21C208/165.) $A$, Map and profile. $B$, Inferred forces from Format 5 motor-current record. 

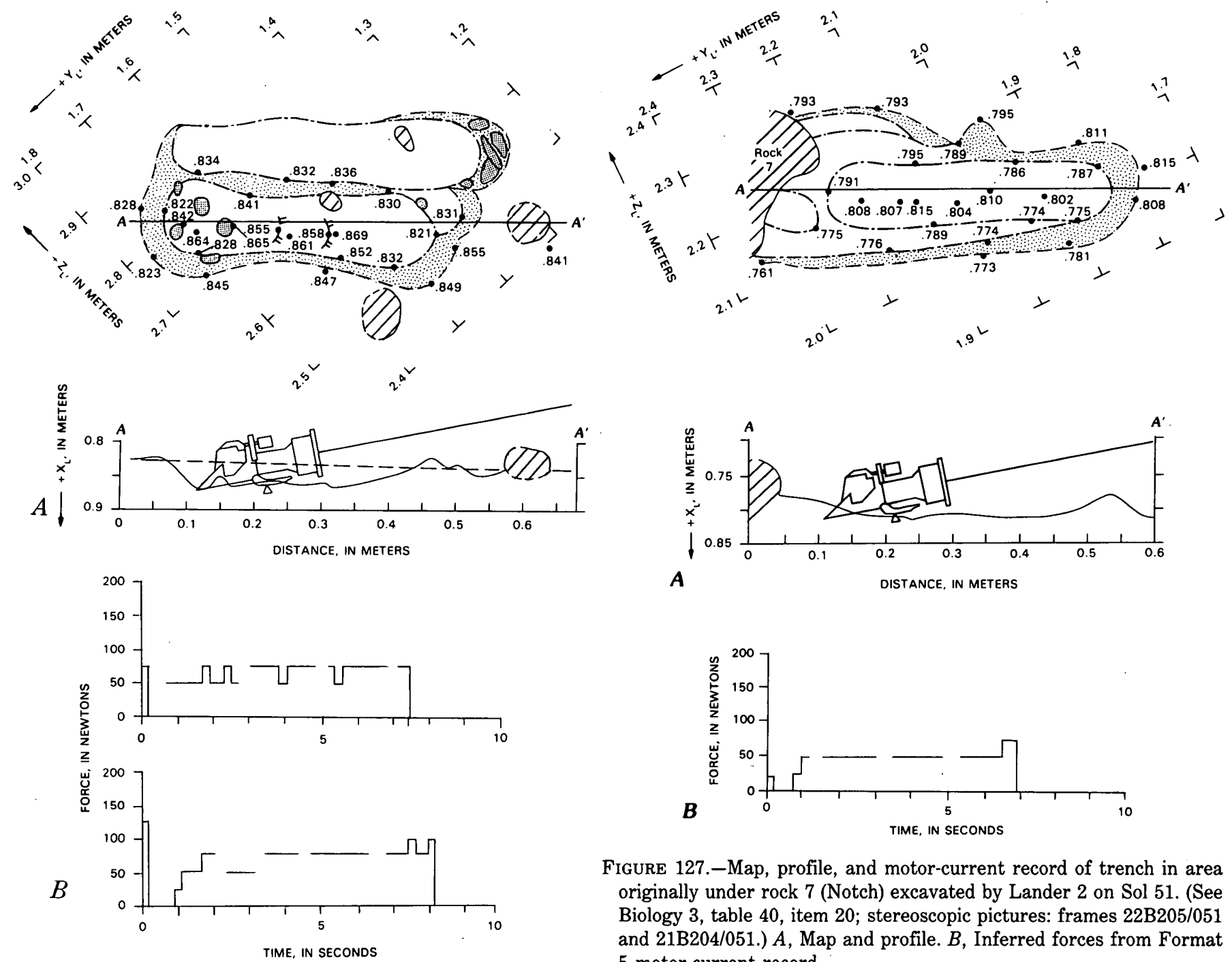

FiguRE 126.-Map, profile, and motor-current records of trenches in Beta site excavated in crusty to cloddy material by Lander 2 on Sols 46-47. (See XRFS 2, table 40, item 17; stereoscopic pictures: frames $22 \mathrm{C} 202 / 161,21 \mathrm{C} 200 / 161$, and 22C203/161.) $A$, Map and profiles. $B$, Inferred forces from Format 5 motor-current records. 


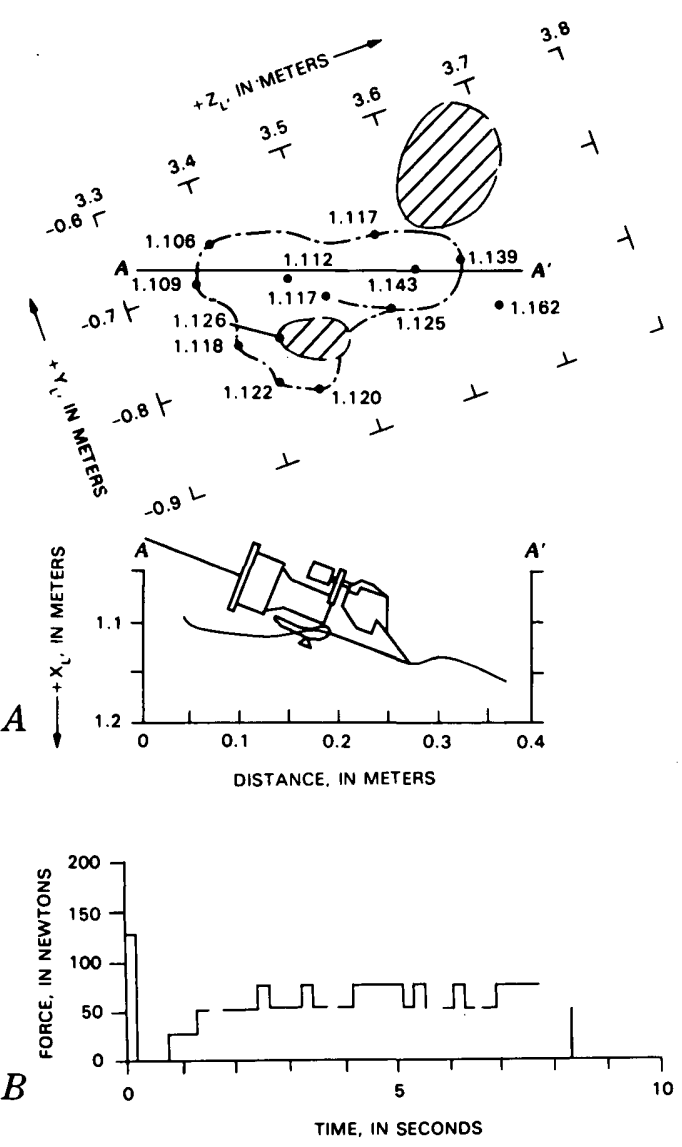

FiguRE 128.-Map, profile, and motor-current record of trench in Alpha site excavated by Lander 2 on Sol 56. (See Physical Properties 1, table 40, item 22; stereoscopic pictures: frames $22 \mathrm{H} 154 / 704$ and $21 \mathrm{H} 153 / 703$.) $A$, Map and profile. $B$, Inferred forces from Format 5 motor-current record.
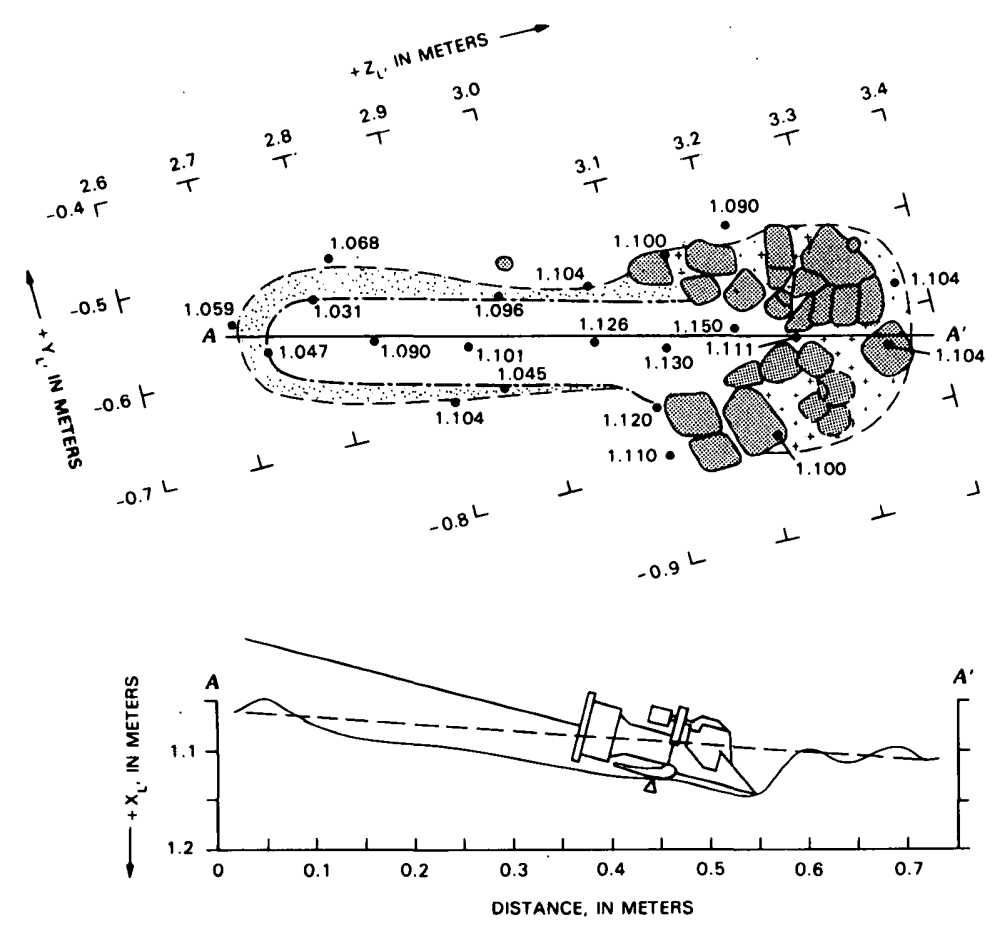

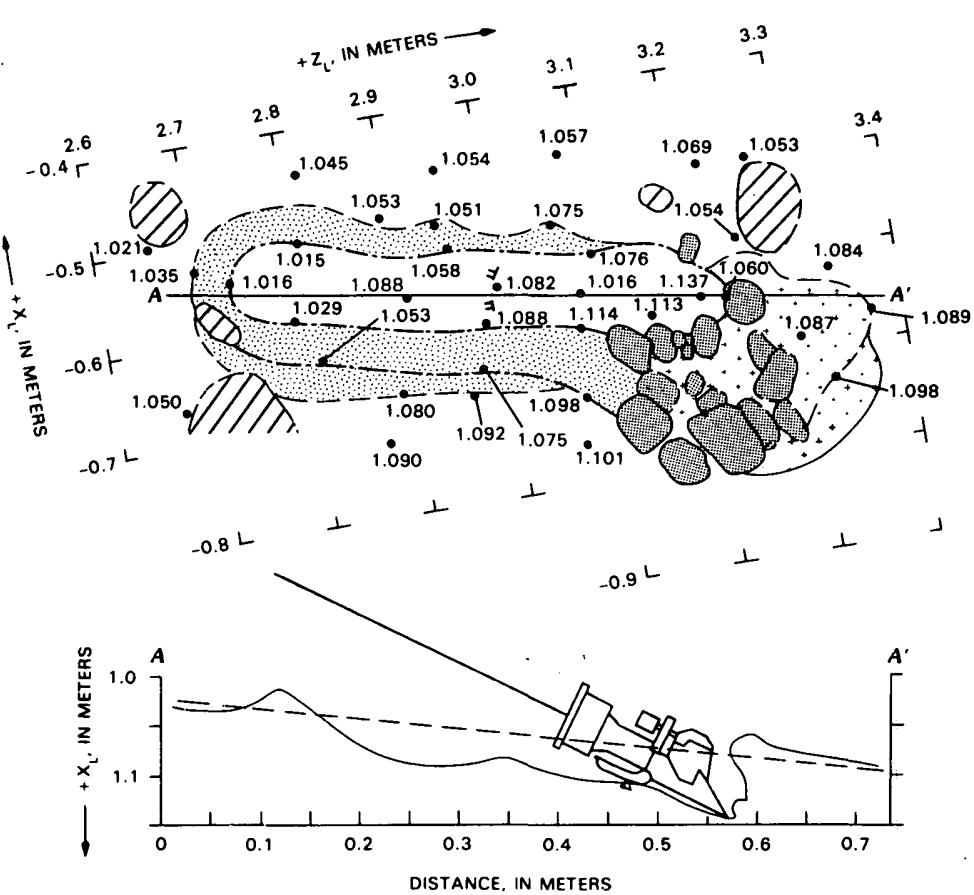

FIGURE 130.-Map and profile of trench in Alpha site excavated in crusty to cloddy material by Lander 2 on Sol 58. No Format 5 motor-current record. (See XRFS 3, table 40, item 26; stereoscopic pictures: frames 22H154/704 and $21 \mathrm{H} 153 / 704$.)
FIGURE 129.-Map and profile of trench in Alpha site excavated in crusty to cloddy material by Lander 2 on Sol 57 . No Format 5 motor-current record. (See XRFS 3, table 40, item 26; stereoscopic pictures: frames $22 \mathrm{C} 045 / 057$ and 21C044/057.) 


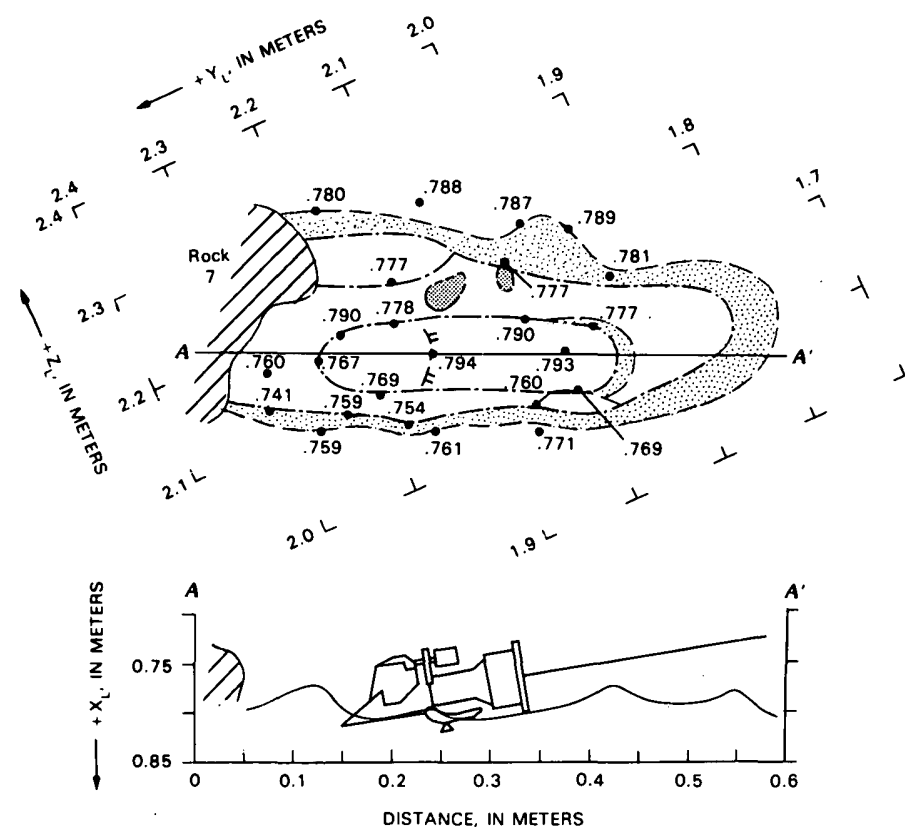

FIGURE 131.-Map and profile of trench in area originally under rock 7 (Notch) excavated by Lander 2 on Sol 131. No Format 5 motor-current record. (See XRFS 4, sample U-2, table 41, item 1; stereoscopic pictures: frames $22 \mathrm{~F} 033 / 414$ and $21 \mathrm{~F} 032 / 414$.)
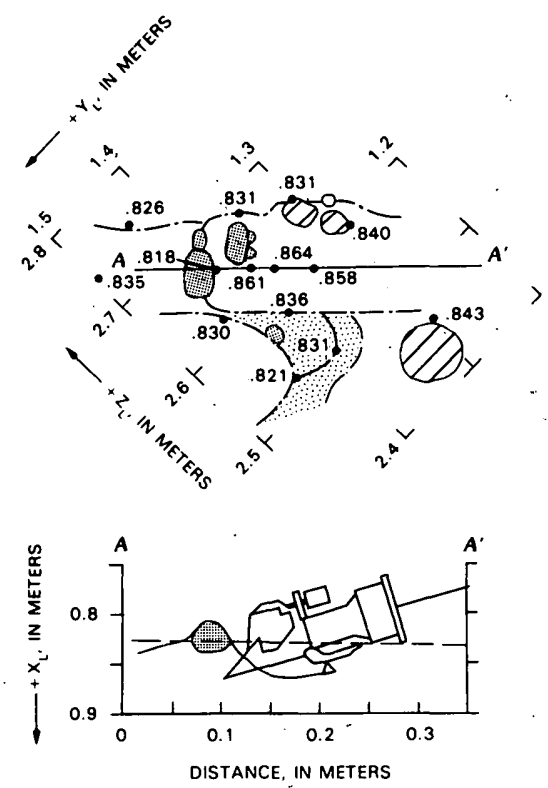

Figure 132.-Map and profile of trench in Beta site excavated in tailings of earlier trench by Lander 2 on Sol 145. No Format 5 motor-current record. (See Biology 4, table 41, item 3; stereoscopic pictures: frames $22 \mathrm{C} 202 / 161$ and $21 \mathrm{C} 200 / 161$.)

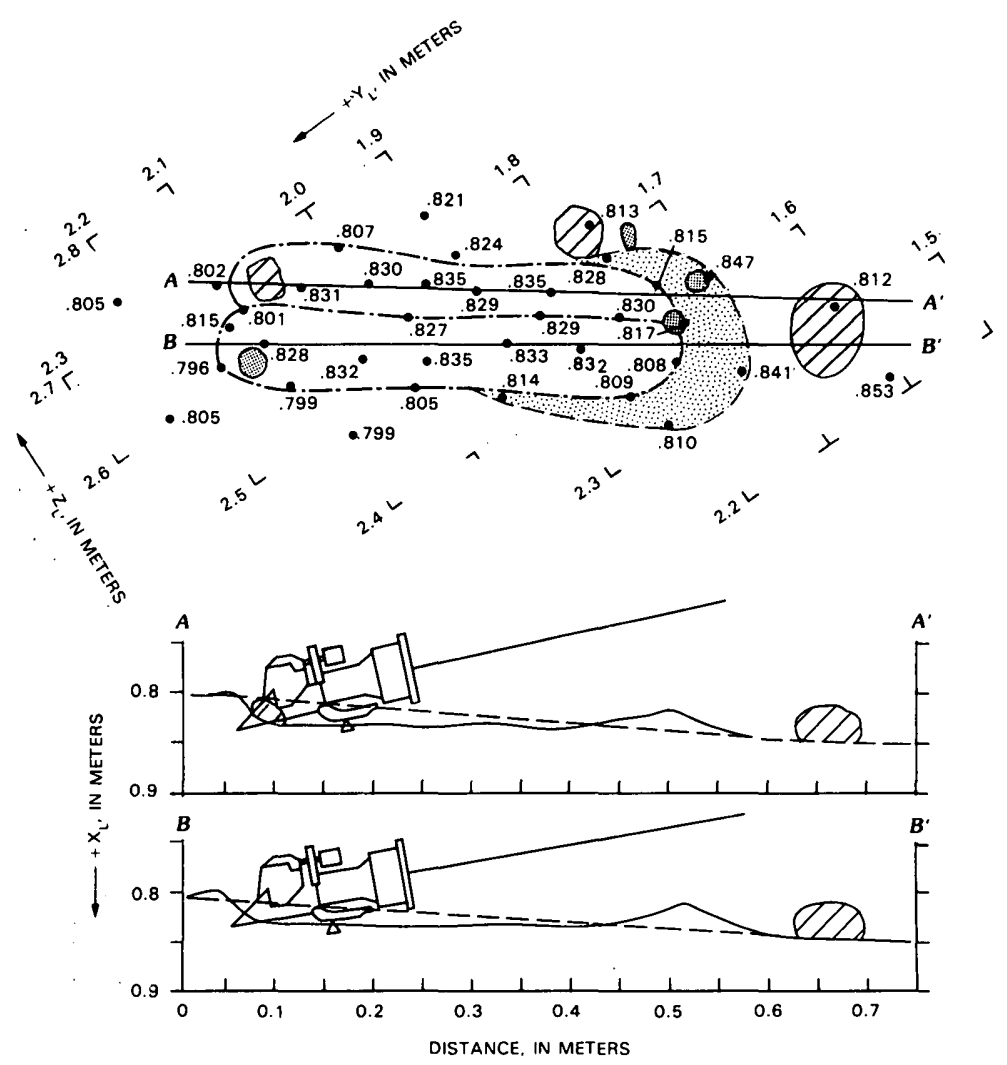

FIGURE 133.-Map and profiles of trenches in Spalling Valley (near rock 15) excavated for pile of coarse fraction by Lander 2 on Sol 145. No Format 5 motor-current record. (See XRFS 5, table 41, item 5; stereoscopic pictures: frames $22 \mathrm{C} 160 / 146,21 \mathrm{C} 159 / 146,22 \mathrm{C} 162 / 146$, and 21C161/146.) 

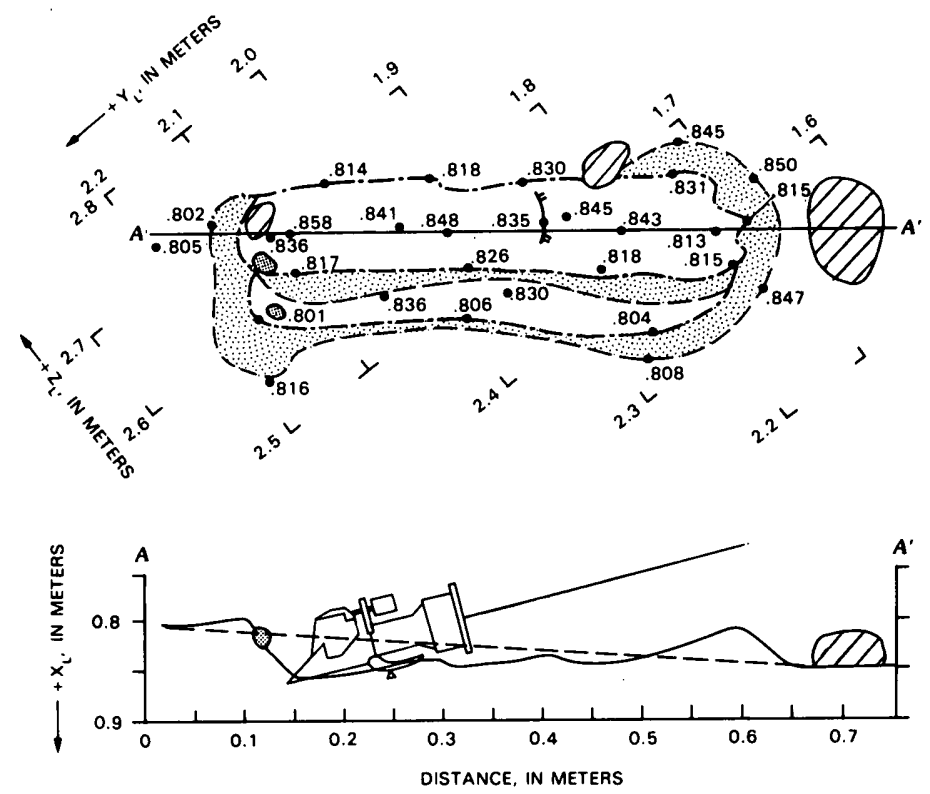

FIGURE 134.-Map and profile of trenches in Spalling Valley (near rock 15) excavated by Lander 2 on Sol 161. No Format 5 motor-current record. (See XRFS 6, sample U-3, table 41, item 9; stereoscopic pictures: frames $22 \mathrm{C} 202 / 161,21 \mathrm{C} 200 / 161,22 \mathrm{C} 203 / 161$, and 21C201/161.)

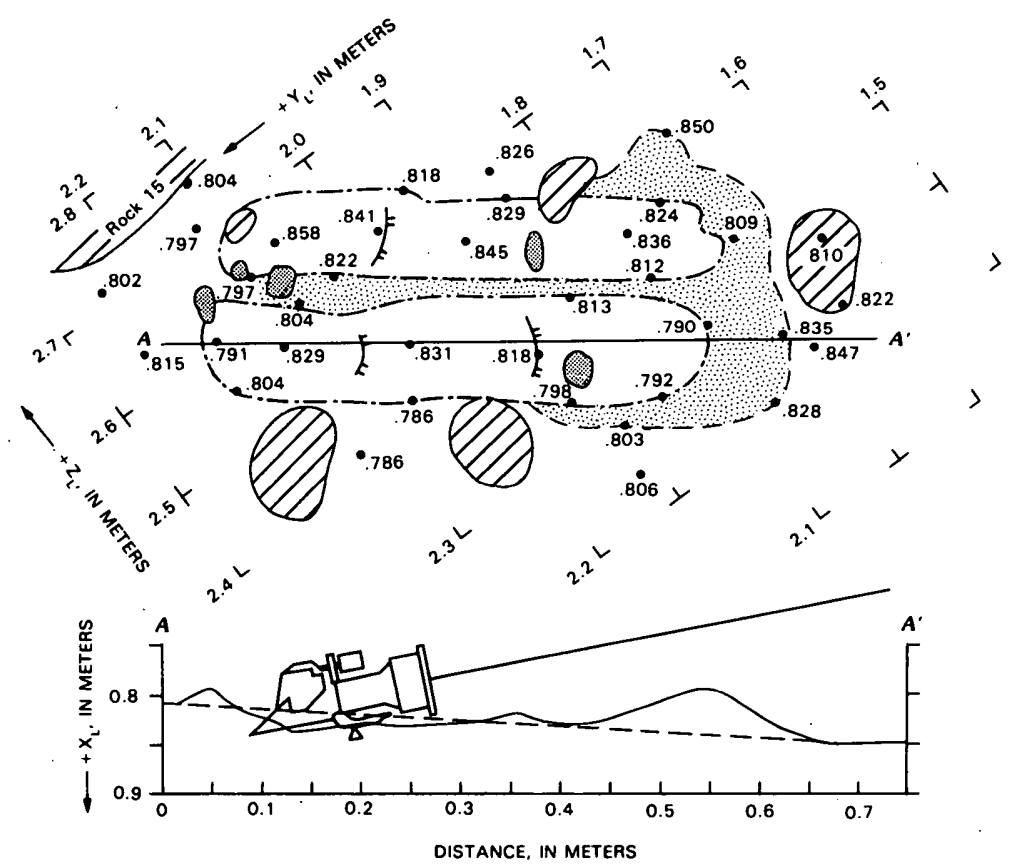

FIGURE 135.-Map and profile of trenches in Spalling Valley (near rock 15) excavated for pile of coarse fraction by Lander 2 on Sol 172. No Format 5 motor-current record. (See XRFS 7, table 41, item 11; stereoscopic pictures: frames $22 \mathrm{G} 099 / 524$ and $21 \mathrm{G} 061 / 512$.) 


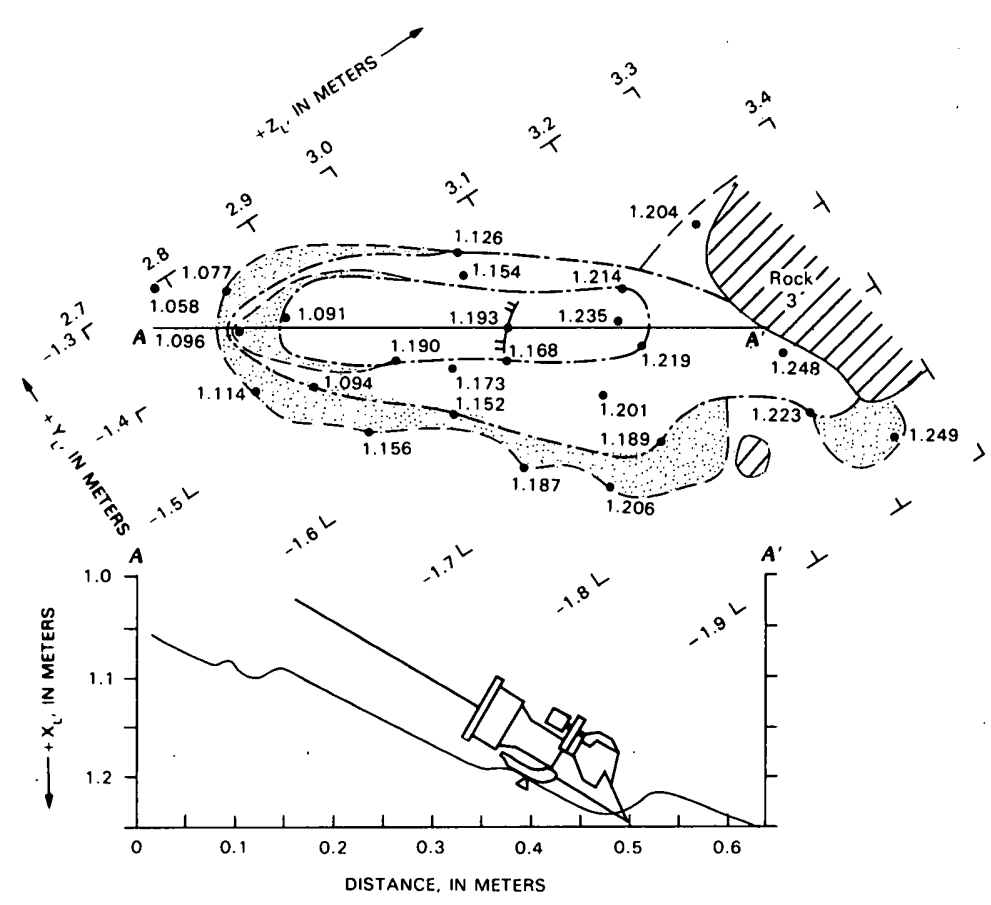

FIGURE 136.-Map and profile of trench excavated in material originally under rock 3 (Badger) by Lander 2 on Sols 185-186. No Format 5 motor-current record. (See XRFS 8, sample U-4, table 41, item 14; stereoscopic pictures: frames $22 \mathrm{~F} 125 / 446$ and $21 \mathrm{~F} 124 / 446$.)
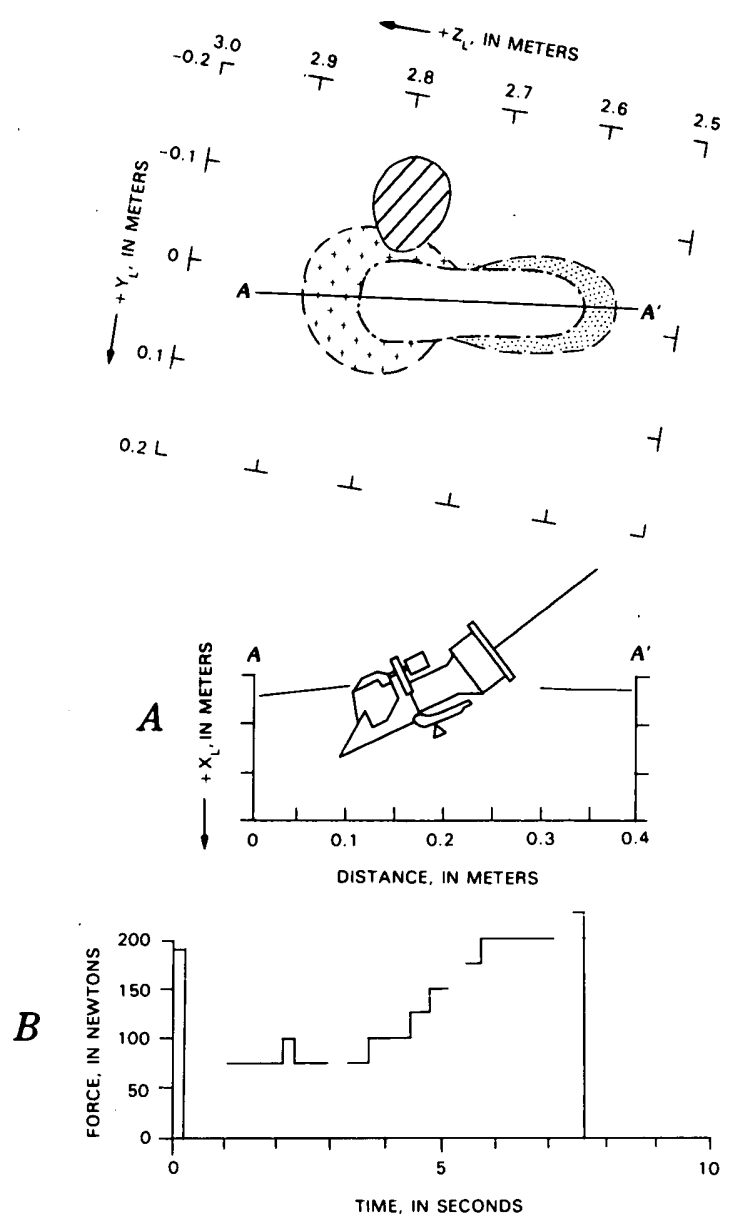

FIGURE 138.-Map, profile, and motor-current record of diurnal-temperature trench excavated by Lander 2 on Sols 405-406. (See Physical Properties 2, table 41, item 18; monoscopic pictures: frames $21 \mathrm{E} 236 / 405,21 \mathrm{E} 237 /$ 405 , and 21F023/414.) $A$, Map and profile. $B$, Inferred forces from Format 5 motor-current record.
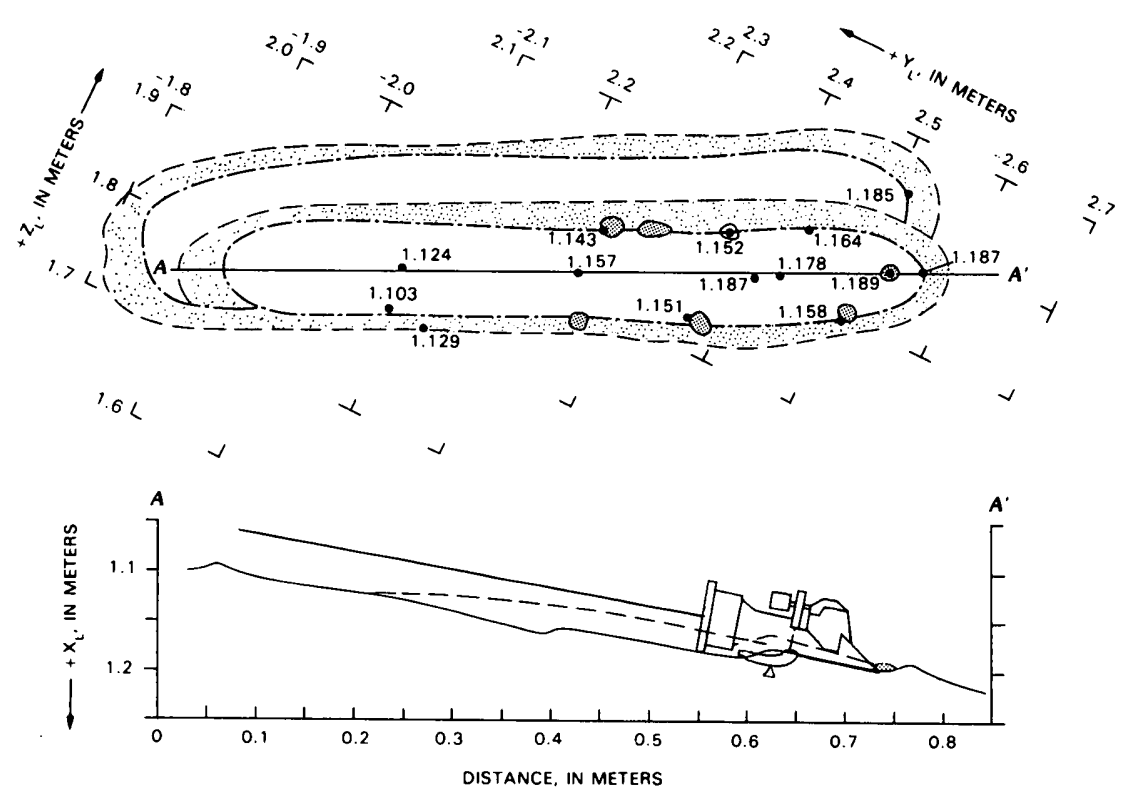

FiguRE 137.-Map and profile of trenches in Physica Planitia excavated in cloddy material with skimming motion by Lander 2 on Sol 388 . For Format 5 motor-current records see Pike and others (1978). (See XRFS 9, sample U-5, table 41, item 17; stereoscopic pictures: frames 22F213/479 and 21F209/479.) 

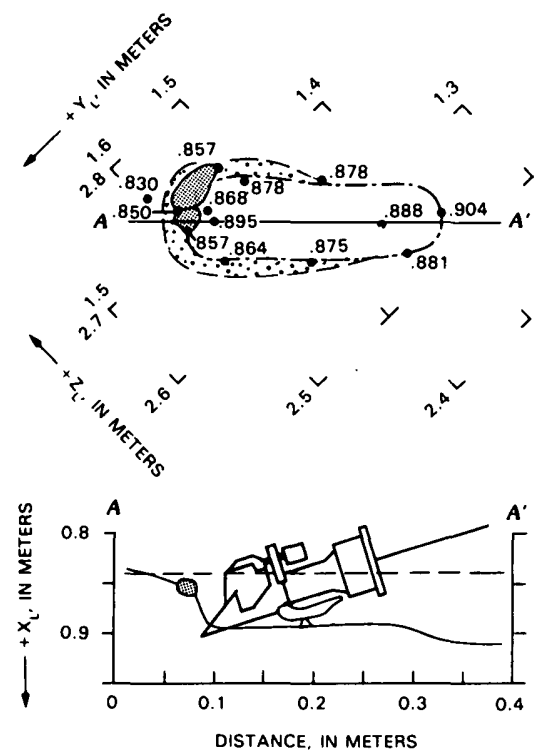

FIGURE 139.-Map and profile of trench excavated in Deep Hole by Lander 2 on Sol 417. No Format 5 motor-current record. (See XRFS 10, sample U-6, table 41, item 21; stereoscopic pictures: frames 22F049/417 and 21F048/417.)
Figure 140.-Map and profile of Deep Hole 1 excavated by Lander 2 on Sol 442 (and 417). For Format 5 motor-current records see Pike and others (1978). (See Deep Hole 1, table 41, items 20 and 22; stereoscopic pictures: frames 22G041/502, 21G040/502, 21G049/503, 21F114/443, 21G079/520, 22F049/417, 21F048/417, 22F047/417, and 21F046/417.)
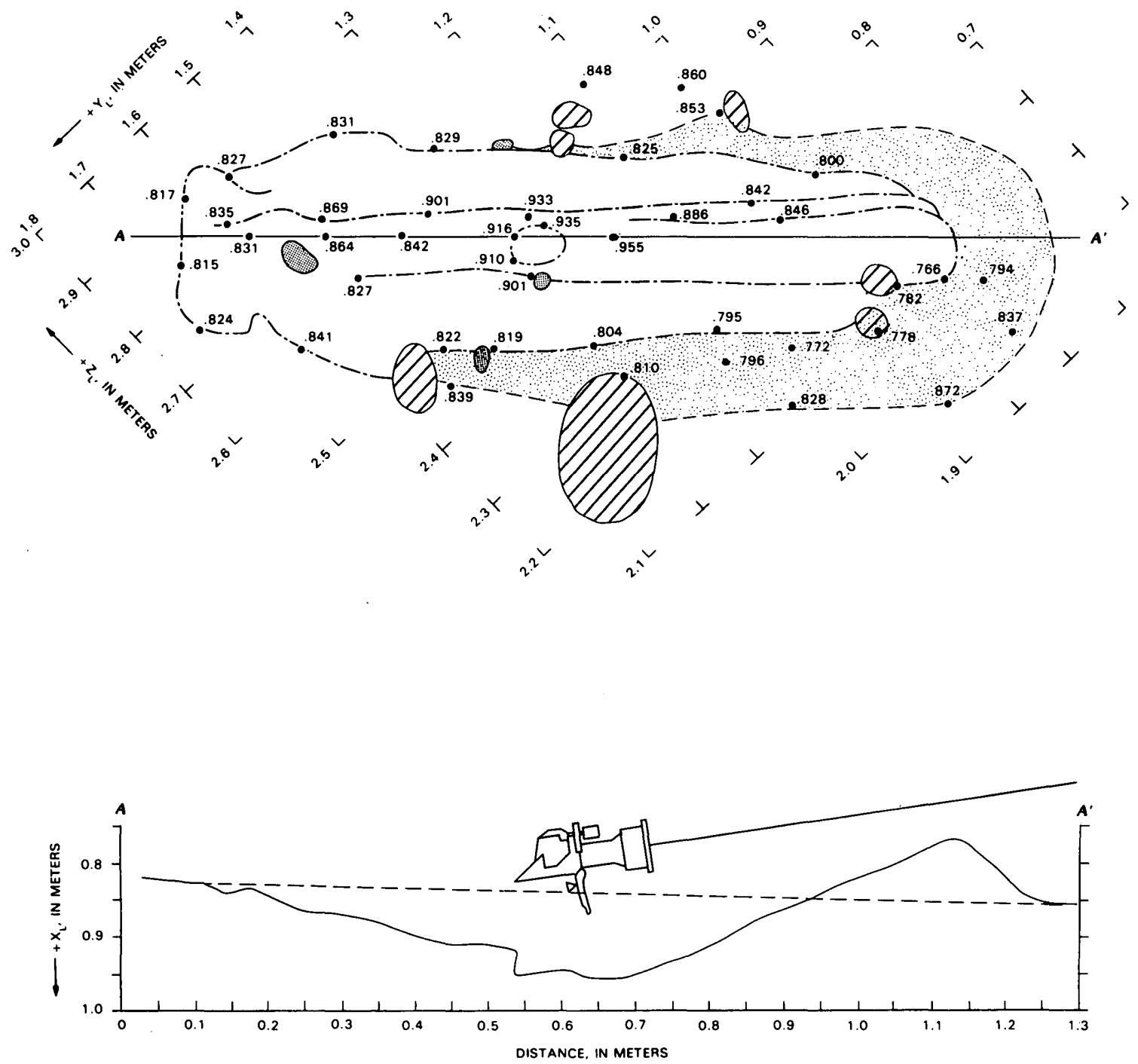


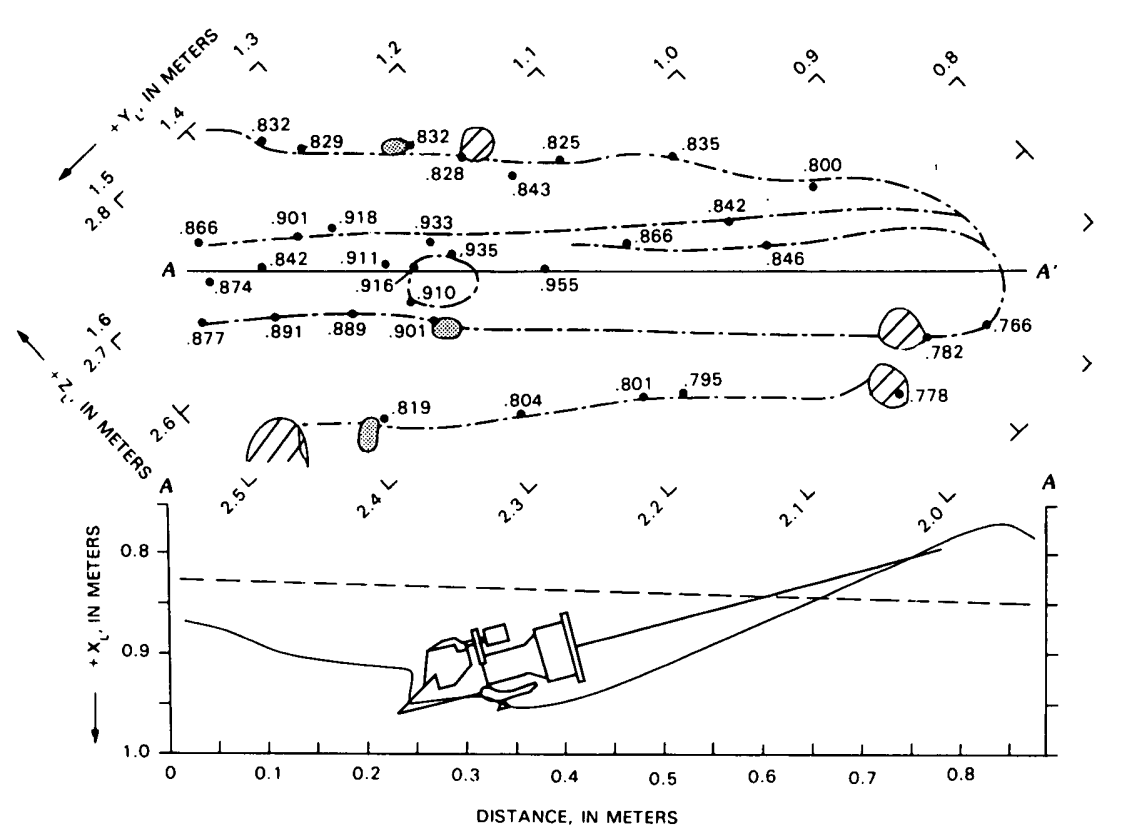

Figure 141.-Map and profile of trench in Deep Hole excavated by Lander 2 on Sol 442.। For Format 5 motor-current records see Pike and others (1978). (See XRFS 11, sample U-7, table 41, item 23; stereoscopic pictures: frames 22G041/502 and 21G049/503.)
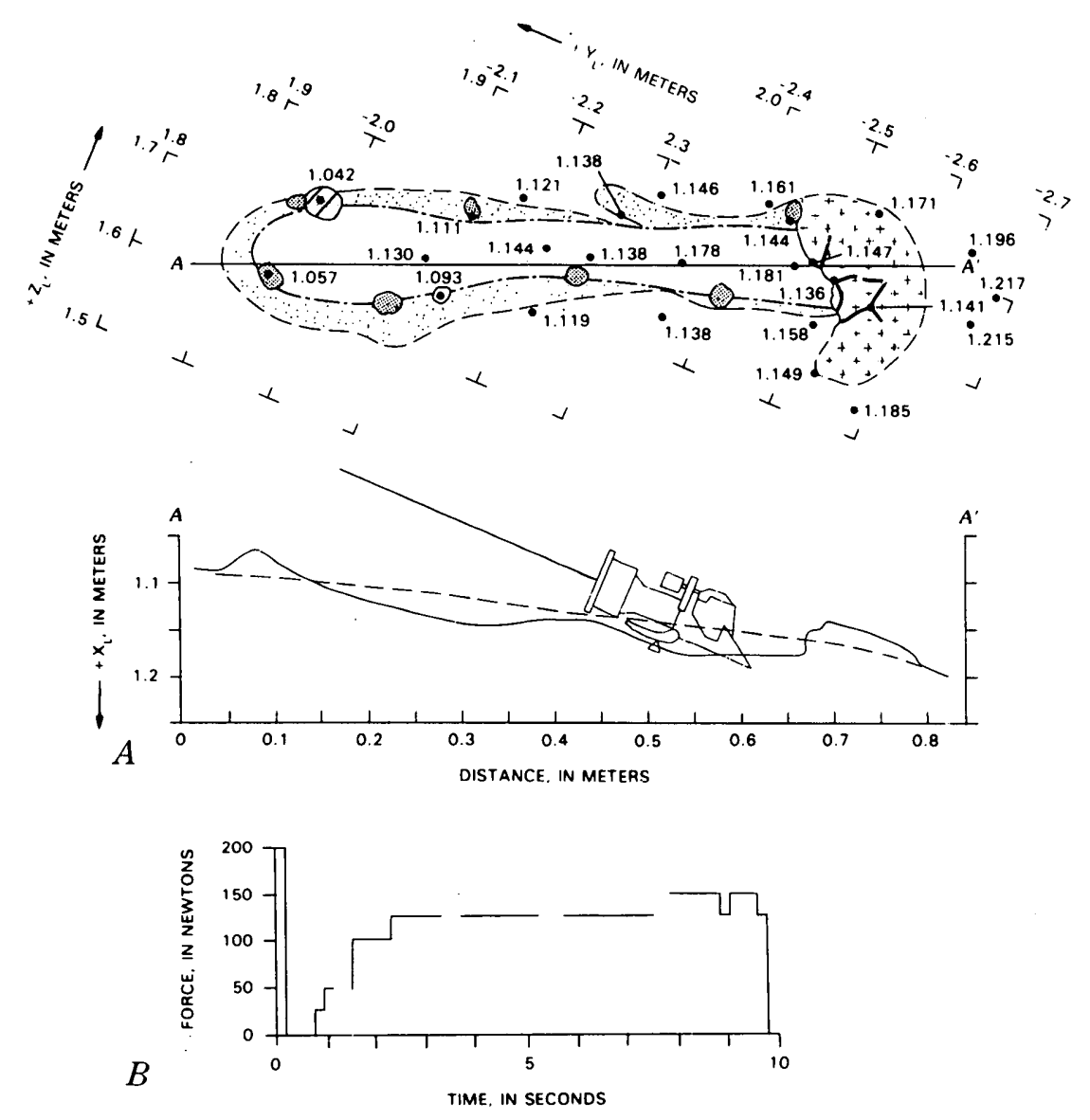

FIgURE 142.-Map, profile, and motor-current record of trench in Physica Planitia excavated in crusty to cloddy material by Lander 2 on Sol 479. (See Physical Properties 3, table 41, item 26; stereoscopic pictures: frames 22F213/479 and 21F209/479.) A, Map and profile. $B$, Inferred forces from Format 5 motor-current record.

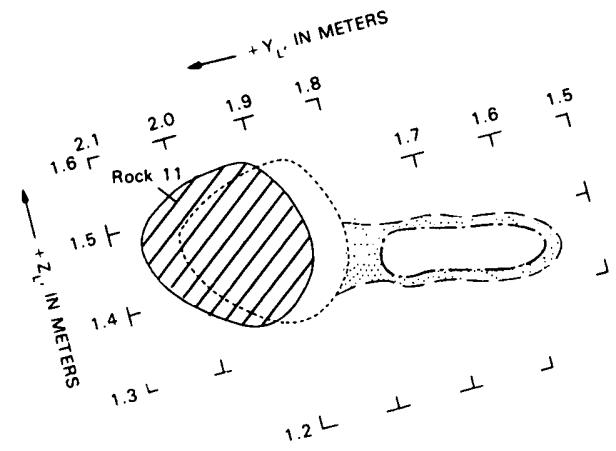

FIGURE 143.-Map of trench in front of rock 11 (Snow White) excavated by Lander 2 on Sol 483. No Format 5 motor-current record. (See XRFS 12 , table 41 , item 30 ; monoscopic pictures: frames $21 \mathrm{G} 011 / 489,21 \mathrm{~F} 182 / 471$, and 21F183/471.) 


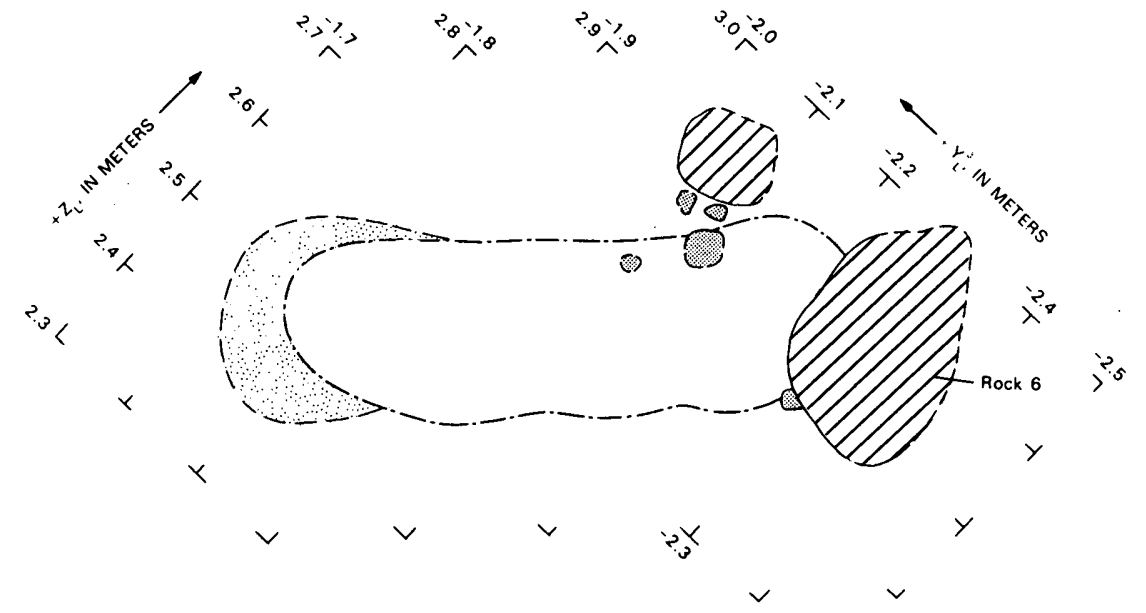

Figure 144.-Map of trench in Bonneville Salt Flats excavated in crusty to cloddy material by Lander 2 on Sol 506. For Format 5 motor-current records see Pike and others (1978). (See XRFS 13, table 41, item 32; monoscopic picture: frame 22H025/595.)
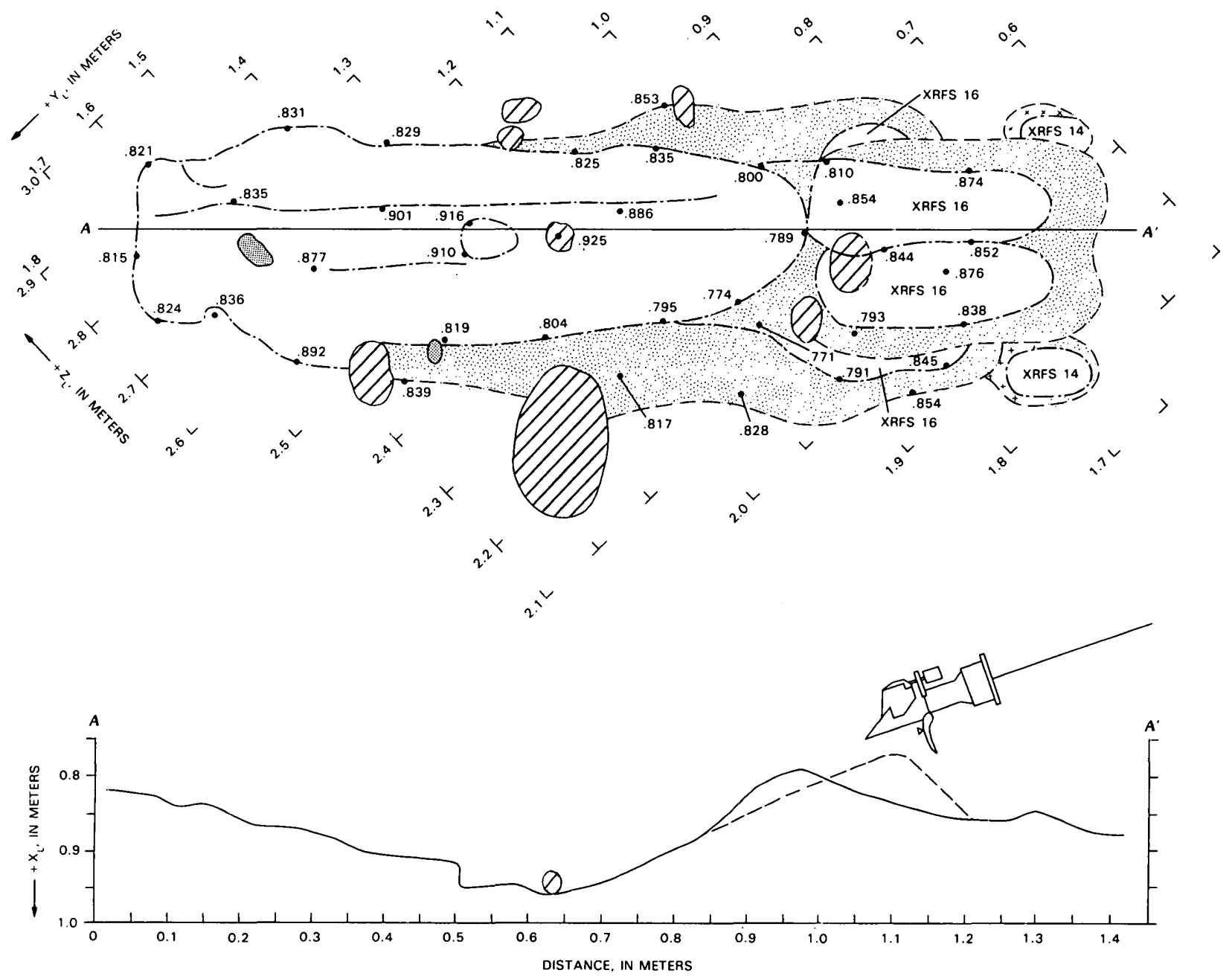

FIGURE 145.-Map and profile of trenches in Deep Hole 1 tailings, base of tailings, and deep hole. Tailings and base of tailings trenches excavated by Lander 2 on Sols 522, 523, 535, and 545. For Format 5 motor-current records see Pike and others (1978). (See XRFS 14, 15, and 16; table 41, items 34, 37, and 39; stereoscopic pictures: frames 22H029/595, 21G155/553, and 21H178/738.) Dashed line in profile shows original profile of tailings. 


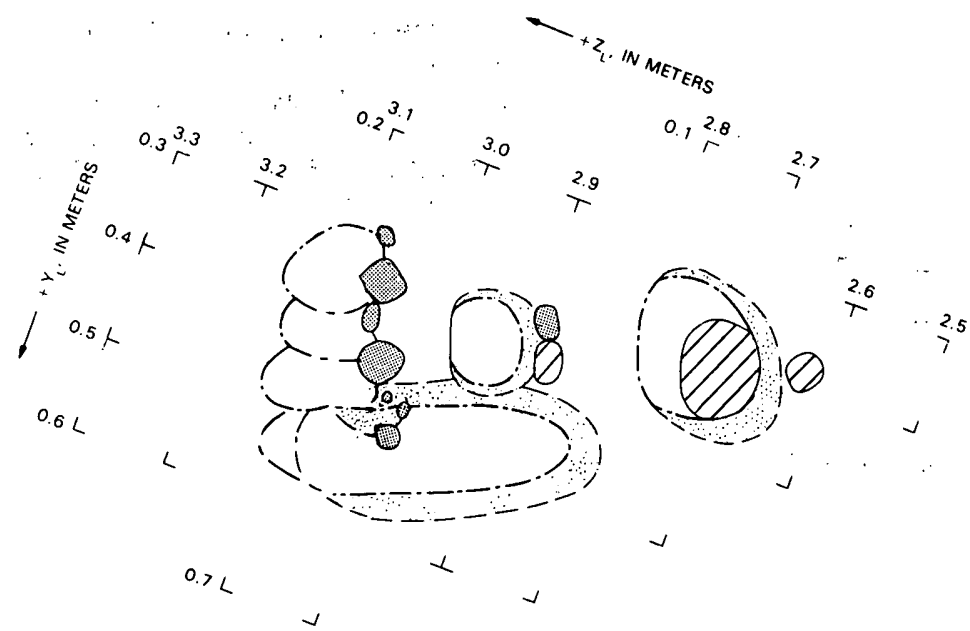

Figure 146.-Map of retraction trenches excavated by Lander 2 on Sol 559.

No Format 5 motor-current records. (See Physical Properties 4, table 41, item 42; monoscopic picture: frame $21 \mathrm{H} 178 / 738$.)

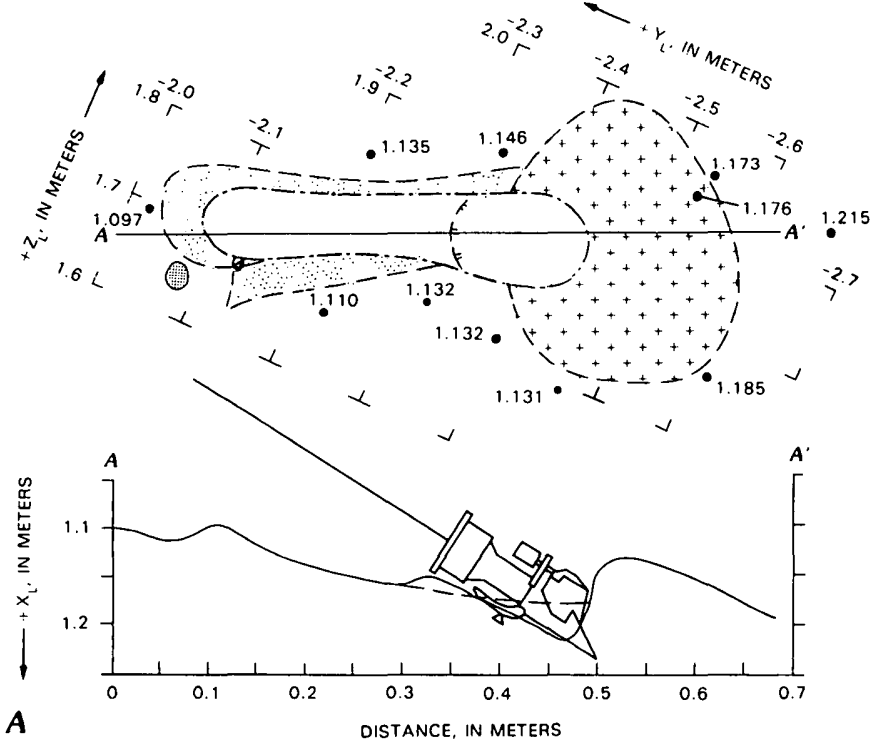

$\boldsymbol{A}$ DISTANCE, IN METERS

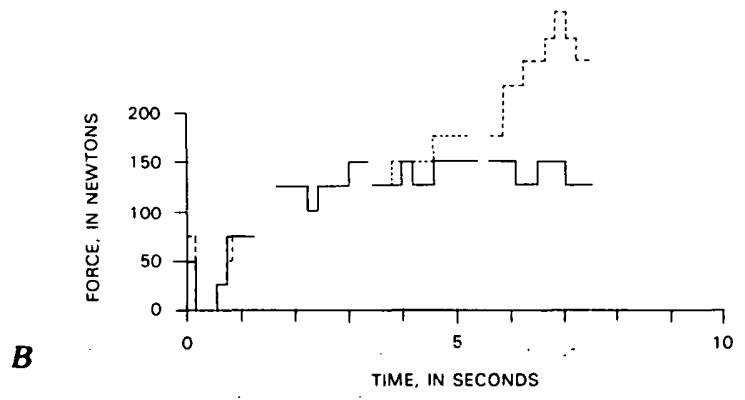

Figure 147.-Map, profile, and motor-current records of trench in Physica Planitia excavated in crusty to cloddy material by Lander 2 on Sol 595. (See Physical Properties 5, table 41, item 44; monoscopic picture: frame 22H025/595.) $A$, Map and profile. $B$, Inferred forces from Format 5 motor-current records.
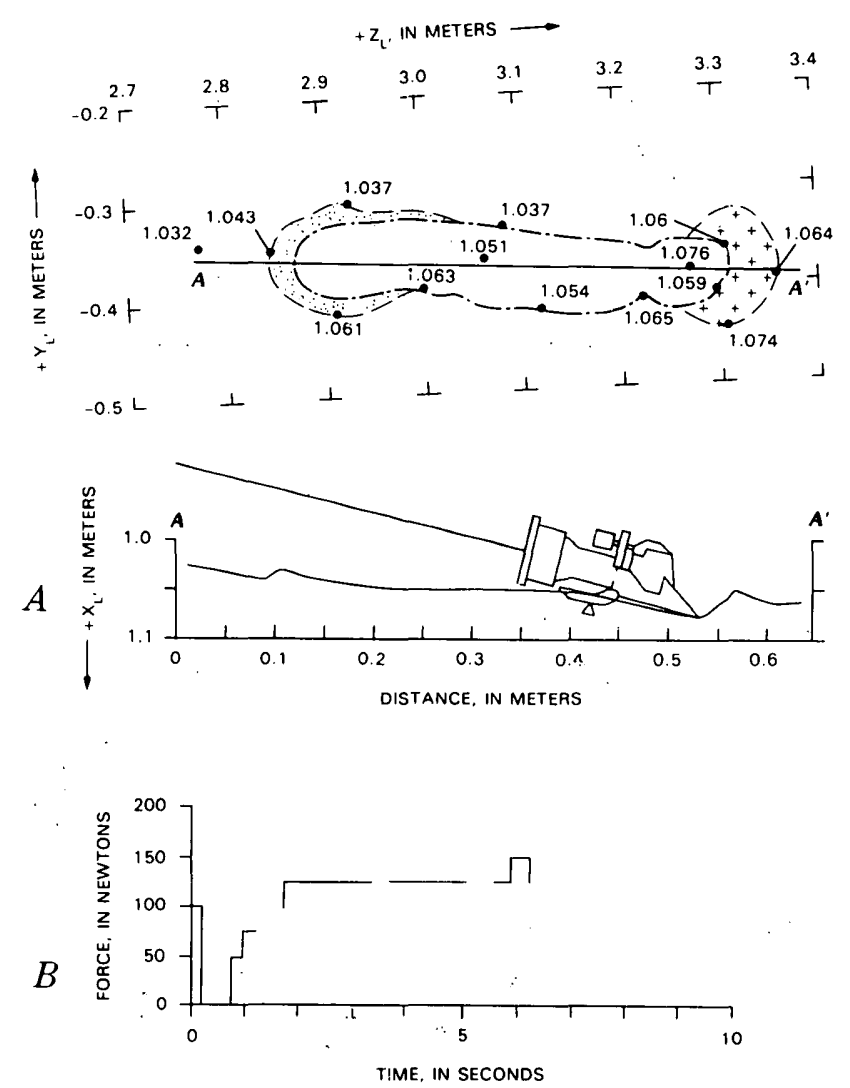

FIGURE 148.-Map, profile, and motor-current record of trench of adhesion test excavated in crusty to cloddy material by Lander 2 on Sol 595. (See Physical Properties 6, table 41, item 47; stereoscopic pictures: frames $22 \mathrm{H} 090 / 630,21 \mathrm{H} 022 / 595$, and $21 \mathrm{H} 122 / 667$.) $A$, Map and profile. $B$, Inferred forces from Format 5 motor-current record. 


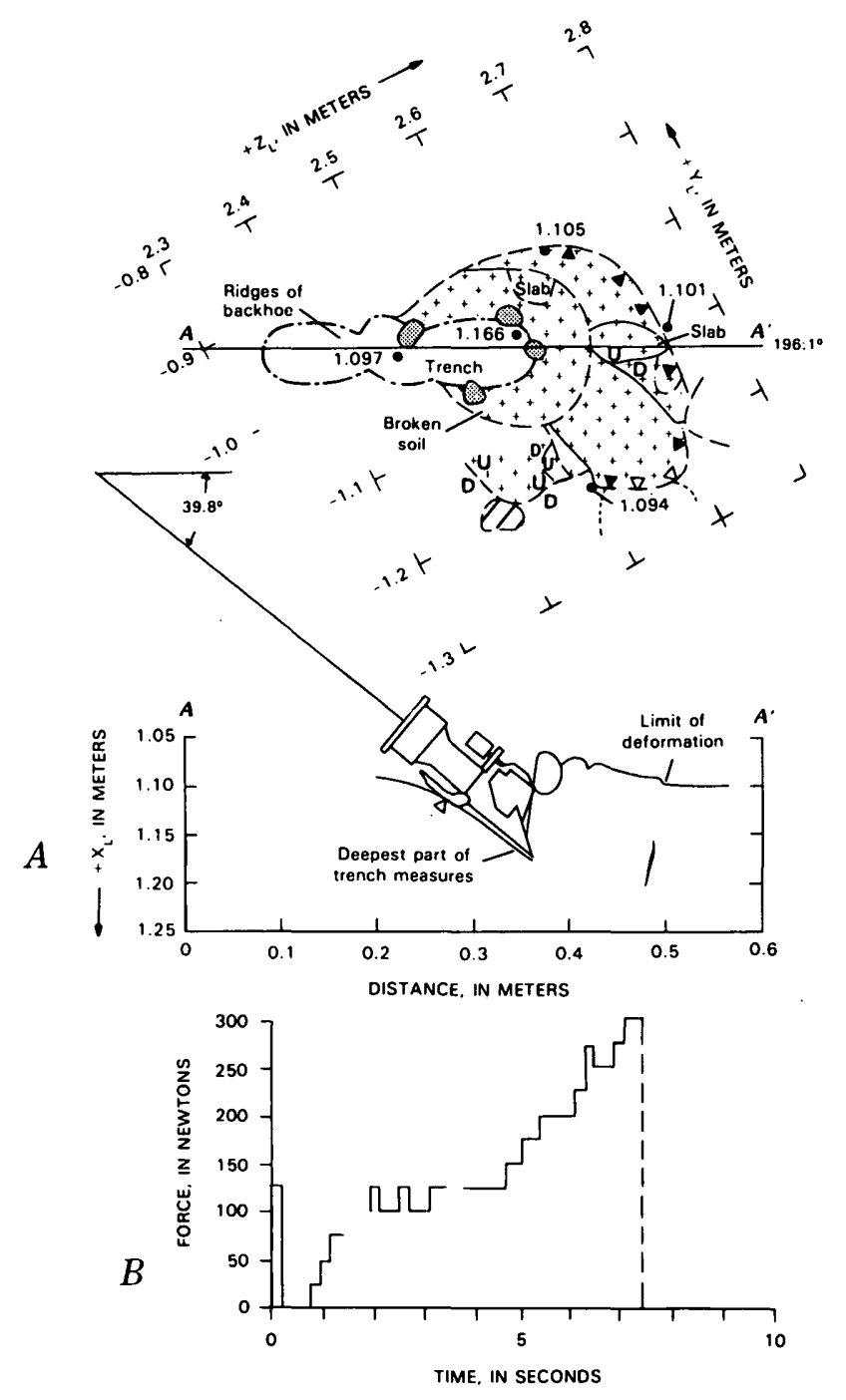

FIGURE 149.-Map, profile, and motor-current record of trench of surface-bearing test excavated in crusty to cloddy material by Lander 2 on Sol 595. (See Physical Properties 7, table 41, item 51 ; stereoscopic pictures: frames $22 \mathrm{H} 030 / 595,21 \mathrm{H} 031 / 595$, $22 \mathrm{H} 033 / 595$, and $21 \mathrm{H} 034 / 595$.) $A$, Map and profile. $B$, Inferred forces from Format 5 motor-current record.

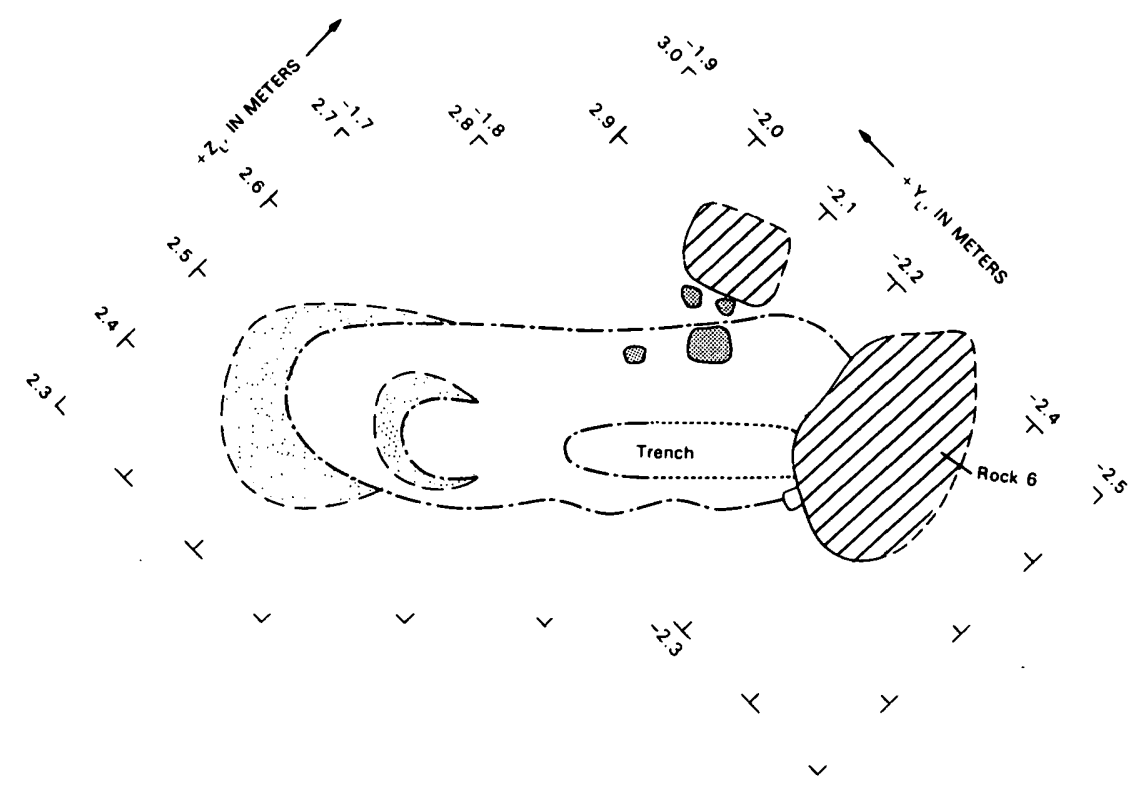

FIGURE 150.-Map of trench in Bonneville Salt Flats excavated in crusty to cloddy material by Lander 2 on Sols 595-596. For Format 5 motor-current records see Pike and others (1978). (See XRFS 17, sample U-8, table 41, item 52; monoscopic picture: frame $22 \mathrm{H} 046 /$ 604.) 


\section{ANALYSIS OF LANDING DATA}

\section{INTRODUCTION}

When any one of the three footpads touched the surface and the corresponding primary strut began to stroke, the terminal-descent engines were permanently turned off. At that time, the spacecraft had kinetic energy and potential energy which must have been lost as the lander came to rest. Kinetic energy of the spacecraft was calculated from the spacecraft mass and velocity at touchdown. Potential energy existed as a result of the higher elevation of the center of gravity of the spacecraft at initial contact with the surface than the elevation at the resting position. The initial kinetic and potential energies were converted to other forms of energy when stroking of the landing gears performed work by crushing honeycomb tube-core cartridges of the shock absorbers in the primary struts, the decaying engine exhausts performed work, and the footpads performed work as they penetrated the surface materials and slid on the surface. Additional energy might have been absorbed by load limiters. The amount of energy absorbed as work performed by the footpads penetrating the surface materials and sliding along the surface depends on the effective coefficient of friction between the footpads and the materials while that energy absorbed by the shock absorbers and decaying engine thrusts were fixed. Thus, the total work performed becomes equal to the kinetic and potential energies when a suitable coefficient of friction is used in the calculations. When a coefficient of friction that gives this equality is selected, the maximum vertical forces on the footpads can be estimated and then compared with data from terrestrial tests and other information. As will be shown below, this procedure yielded reasonable results from the data of Lander 1 . The more complex landing and footpad interactions with rocks by Lander 2 yielded equivocal results:

\section{LANDER CHARACTERISTICS}

In the analysis, the geometry of the landing gear and characteristics of the shock absorbers are major items to be considered (Pohlen and others, 1976). The load limiters also may absorb energy but there is no clear evidence that they actually.did. Each of the three landing legs consisted of a primary strut attached to a main support fitting and two secondary struts attached to load limiters at the base of the lander body. The three struts are attached to the footpad (see fig. 1). Motions of the footpads relative to the lander occurred in planes perpendicular to the upper surface of the lander body that passed: through the primary struts, footpads; and the center of the prismatic lander body. The geometry is shown and dimensions are defined in figure 151. From figure 151, horizontal translation is:

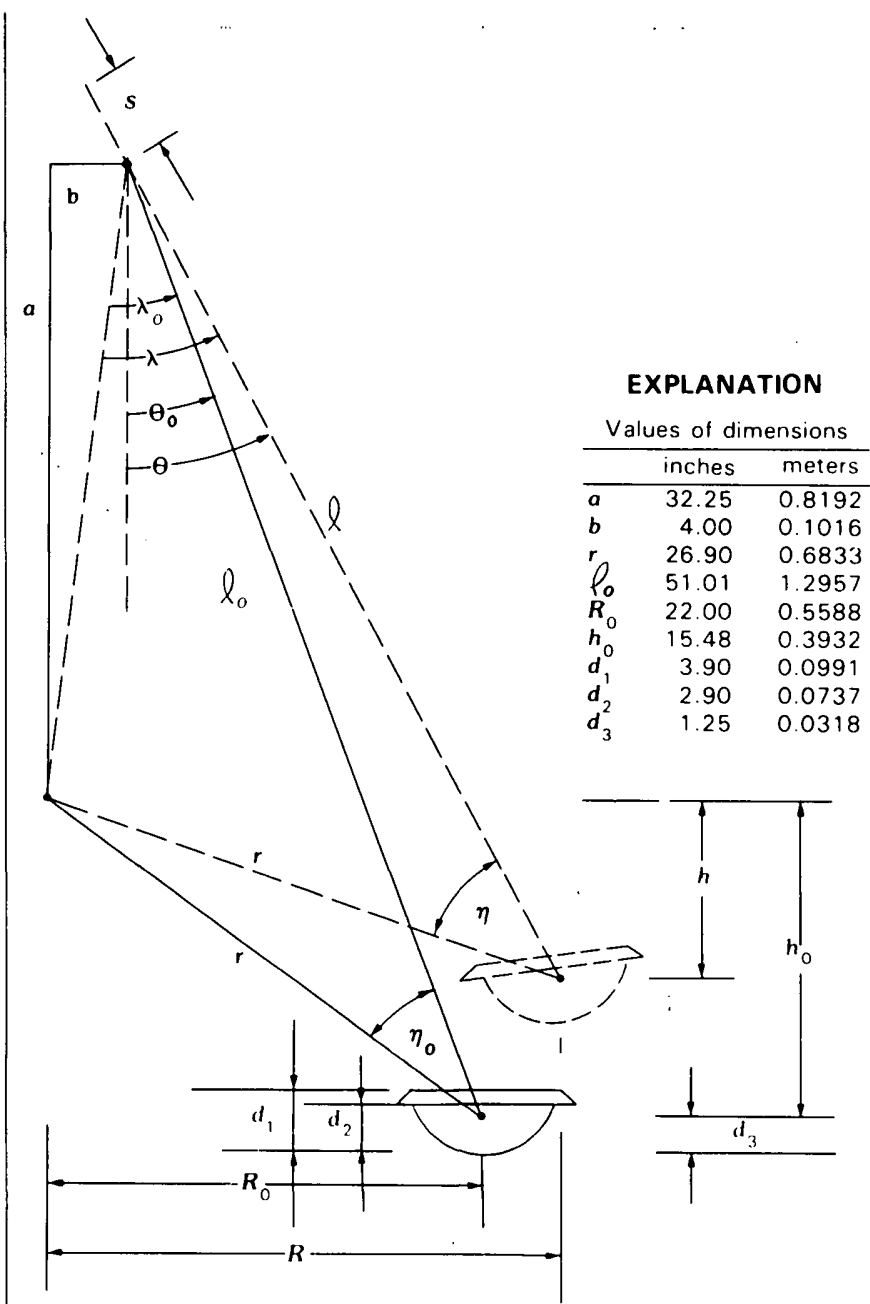

FIGURE 151.-Geometry and dimensions of landing gear of Viking Lander in plane perpendicular to upper surface of lander body $\left(Z_{L^{-}}\right.$ $Y_{L}$ plane) that includes the main strut $\left(l_{0}\right.$ and $\left.l\right)$. Leg stroke $(s)$ is equal to length of leg before stroking $\left(l_{0}\right)$ minus length of leg after stroking $(l)$.

$$
R-R_{0}=\ell \sin \left(\lambda-\tan ^{-1} \frac{b}{a}\right)+b-0.5588
$$

and vertical translation is

$$
h-h_{0}=\ell \cos \left(\lambda-\tan ^{-1} \frac{b}{a}\right)-a-0.3932
$$

and the angles are:

$$
\begin{aligned}
& \lambda=\cos ^{-1}\left(\frac{\ell^{2}+a^{2}+b^{2}-r^{2}}{2 \ell \sqrt{a^{2}+b^{2}}}\right) \\
& \because=\cos ^{-1}\left(\frac{\ell^{2}+r^{2}-a^{2}-b^{2}}{2 \ell r}\right)
\end{aligned}
$$




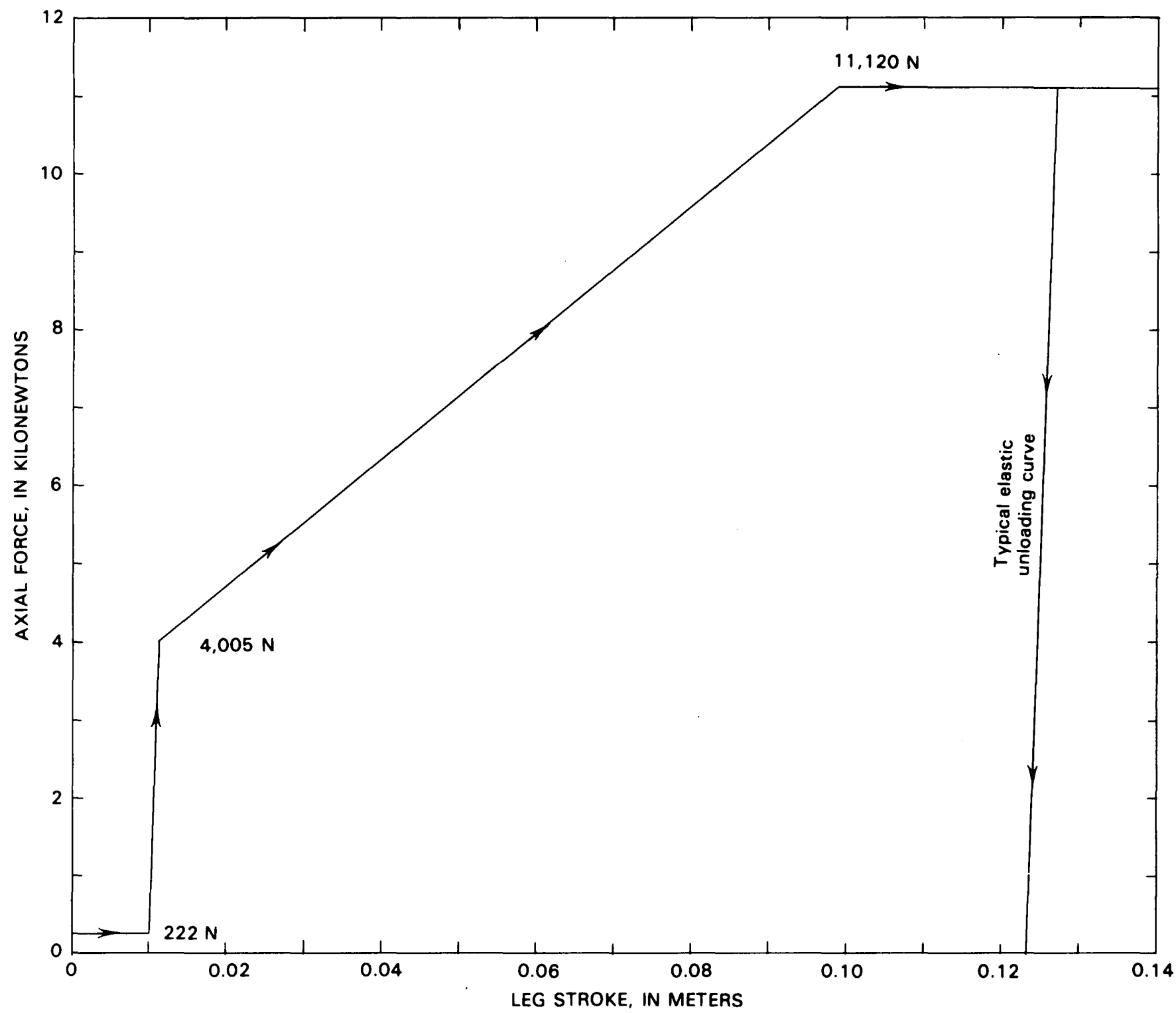

FIGURE 152.-Relation between static axial force and leg stroke for Viking Lander main strut. Initial forces during stroking result from compression of weak spring. Forces from 4,005 to $11,120 \mathrm{~N}$ result from crushing of honeycomb tube-core cartridges in primary-strut shock absorbers. Dynamic forces required to crush cartridges are variable, but they seem to average about $1 \mathrm{kN}$ higher than static forces for strokes between 0.0114 and $0.0991 \mathrm{~m}$.
These translations and angles are related to the amount of leg stroke (s) by:

$$
\ell=\ell_{0}-s
$$

where, $\ell_{o}$ is the length of the deployed primary strut before stroking and landing and $\ell$ is length of the primary strut after stroking. Leg strokes were estimated from stroke gauges and footpad travels.

The static forces that accompanied stroking of each landing gear had five segments (fig. 152; Martin Marietta Corp., 1976b; Pohlen and others, 1976). (1) Initial strok- ing required $222 \mathrm{~N}$ of force as the leg acted against a weak spring during the first $1.016 \mathrm{~cm}$ of travel. (2) This was followed by elastic compression of the shock absorber until $4,005 \mathrm{~N}$ of force was achieved and the total leg stroke was $1.143 \mathrm{~cm}$. (3) Above $4,005 \mathrm{~N}$, the force required to crush the honeycomb tube-core cartridge of the shock absorber increased linearly with stroke to $11,120 \mathrm{~N}$ at a total stroke of $9.906 \mathrm{~cm}$. (4) For leg strokes larger than $9.906 \mathrm{~cm}$, the force was constant at $11,120 \mathrm{~N}$. (5) After stroking, the shock absorber recovered elastically. Equations used in this analysis of axial forces $\left(F_{A}\right)$ on the primary strut are: 
$F_{A}=222 \mathrm{~N} \quad 0<s<0.0114 \mathrm{~m}$

$F_{A}=\left(81,194 \frac{\mathrm{N}}{\mathrm{m}}\right) s+3077 \mathrm{~N} \quad 0.0114 \mathrm{~m}<s<0.0991 \mathrm{~m}$

and elastic recovery $\left(F_{E}\right)$ is:

$$
F_{E}=\left(3,152,000 \frac{\mathrm{N}}{\mathrm{m}}\right) \Delta s
$$

where $\Delta s$ is the amount of stroke recovered elastically.

\section{ENGINE THRUST DECAY}

Nominally, the decay of the engine thrusts began about $0.02 \mathrm{~s}$ after the engine cutoff signal was initiated upon footpad contact (Martin Marietta Corp., 1976b). The thrust decays exponentially with a time constant $(\tau)$ equal to $0.02883 \mathrm{~s}$. If $T_{o}$ is taken to represent the thrust of all three engines, then the thrust is expressed by:

where

$$
T=T_{0} \mathrm{e}^{\frac{t_{1}-t}{\tau}}
$$

$T$ is the thrust at time $t$,

$T_{o}$ is the thrust at time $t_{1}$,

$\tau$ is $0.02883 \mathrm{~s}$,

$t_{1}$ is $0.020 \mathrm{~s}$,

$t$ is time, and

$t \geqslant t_{1}$.

\section{FOOTPAD PENETRATION FORCES}

Static force-penetration characteristics of a $3 / 8$-scale Viking footpad were experimentally determined using three surface-material simulants and then were scaled to a full-size footpad on Mars (Moore and others, 1977). The three simulants were dune sand, low density lunar nominal "soil", and white sand. Properties of these simulants are summarized in table 7 . In the experiments, the footpad did not penetrate more than the full-scale equivalent of $0.07 \mathrm{~m}$ so that footpad penetration was never large enough to attain a constant cross-sectional area during penetration. Analysis of the test results scaled to full-size and Mars conditions (see fig. 17) indicated that the force for penetrations less than $0.07 \mathrm{~m}$ can be expressed by:

$$
F_{p}=k P^{2} \text {, }
$$

where $F_{p}$ is the force on the footpad in the direction of penetration, $P$ is the penetration measured upward from the base, and $k$ is an experimentally determined constant for a given material.

If penetration is $0.0991 \mathrm{~m}$ and larger, the full area of the footpad $\left(0.135 \mathrm{~m}^{2}\right)$ is presented to the surface material and is constant for larger penetrations. For deep penetration, as well as for smaller penetrations, static forces on the footpad can be estimated using the staticbearing capacity equation for circular footings (Terzaghi, 1948, p. 134; Scott, 1963):

$$
F_{s}=A\left(1.3 c N_{c}+\rho g D_{p} N_{q}+0.6 \rho g r_{p} N_{\gamma}\right.
$$

where

$F_{s}$ is the static force on the footpad (footing),

$A$ is the cross-sectional area of the footpad in contact with the surface material (footing),

$c$ is the cohesion of the material,

$g$ is the acceleration of gravity $\left(3.72 \mathrm{~m} / \mathrm{s}^{2}\right)$,

$D_{p}$ is the depth of the footpad (footing),

$r_{p}$ is the effective radius of the footpad (footing), and

$N_{c}, N_{q}$, and $N_{\gamma}$ are dimensionless factors (for cohesion, surcharge, and friction, respectively) related to the angle of internal friction of the material and the roughness of the footing.

Calculations of forces using this equation for values of cohesions, densities, angles of internal friction, and lander

\begin{tabular}{|c|c|c|c|c|c|c|c|c|c|c|}
\hline \multirow{2}{*}{$\begin{array}{c}\text { Footpad } \\
\text { penetration } \\
\text { (m) }\end{array}$} & \multirow{2}{*}{$\begin{array}{c}\text { Footpad } \\
\text { area } \\
\left(\mathrm{m}^{2}\right)\end{array}$} & \multirow{2}{*}{$\begin{array}{l}\text { Assumed } \\
\text { depth of } \\
\text { footing } \\
\text { (m) }\end{array}$} & \multirow{2}{*}{$\begin{array}{l}\text { Radius } \\
\text { of } \\
\text { footing } \\
\quad(\mathrm{m})\end{array}$} & \multirow{2}{*}{$\begin{array}{c}\text { Cohesion } \\
\text { of } \\
\text { material } \\
\text { (kPa) }\end{array}$} & \multirow{2}{*}{$\begin{array}{c}\text { Angle of } \\
\text { internal } \\
\text { friction } \\
\text { (degrees) } \\
\phi\end{array}$} & \multirow{2}{*}{$\begin{array}{l}\text { Density } \\
\text { of } \\
\text { mater } \frac{1}{3} a l \\
\mathrm{~kg} / \mathrm{m}^{3}\end{array}$} & \multicolumn{3}{|c|}{$\begin{array}{c}\text { Dimensionless } \\
\text { factors }\end{array}$} & \multirow{2}{*}{$\begin{array}{c}\text { Force } \\
\text { kN }\end{array}$} \\
\hline & & & & & & & & $\stackrel{\mathrm{N}}{\mathrm{g}}$ & $\stackrel{N}{\gamma}_{\gamma}$ & \\
\hline $\begin{array}{r}0.165 \\
.099 \\
.165 \\
.099 \\
.036 \\
.036\end{array}$ & $\begin{array}{r}0.135 \\
.135 \\
.135 \\
.135 \\
.028 \\
.028\end{array}$ & $\begin{array}{c}0.066 \\
0 \\
.066 \\
0 \\
0 \\
0\end{array}$ & $\begin{array}{c}0.21 \\
.21 \\
.21 \\
.21 \\
.094 \\
.094\end{array}$ & $\begin{array}{l}1.7 \\
1.7 \\
1.0 \\
1.0 \\
7.1 \\
5.5\end{array}$ & $\begin{array}{l}20 \\
20 \\
20 \\
20 \\
30 \\
30\end{array}$ & $\begin{array}{l}1,200 \\
1,200 \\
1,200 \\
1,200 \\
1,200 \\
2,000\end{array}$ & $\begin{array}{l}17 \\
17 \\
17 \\
17 \\
34 \\
34\end{array}$ & $\begin{array}{r}7.5 \\
7.5 \\
7.5 \\
7.5 \\
21.5 \\
21.5\end{array}$ & $\begin{array}{r}2.5 \\
2.5 \\
2.5 \\
2.5 \\
20 \\
20\end{array}$ & $\begin{array}{l}5.6 \\
5.3 \\
3.5 \\
3.2 \\
9.0 \\
7.1\end{array}$ \\
\hline
\end{tabular}
data are plotted in figure 17 and listed in table 42 . The

TABLE 42.-Calculated static-bearing forces for Viking footpad from static-bearing-capacity equation for footings [Dimensionless factors obtained from Terzaghi (1948, p. 125)] 
TABLE 43.-Touchdown conditions and landing data for Landers 1 and 2

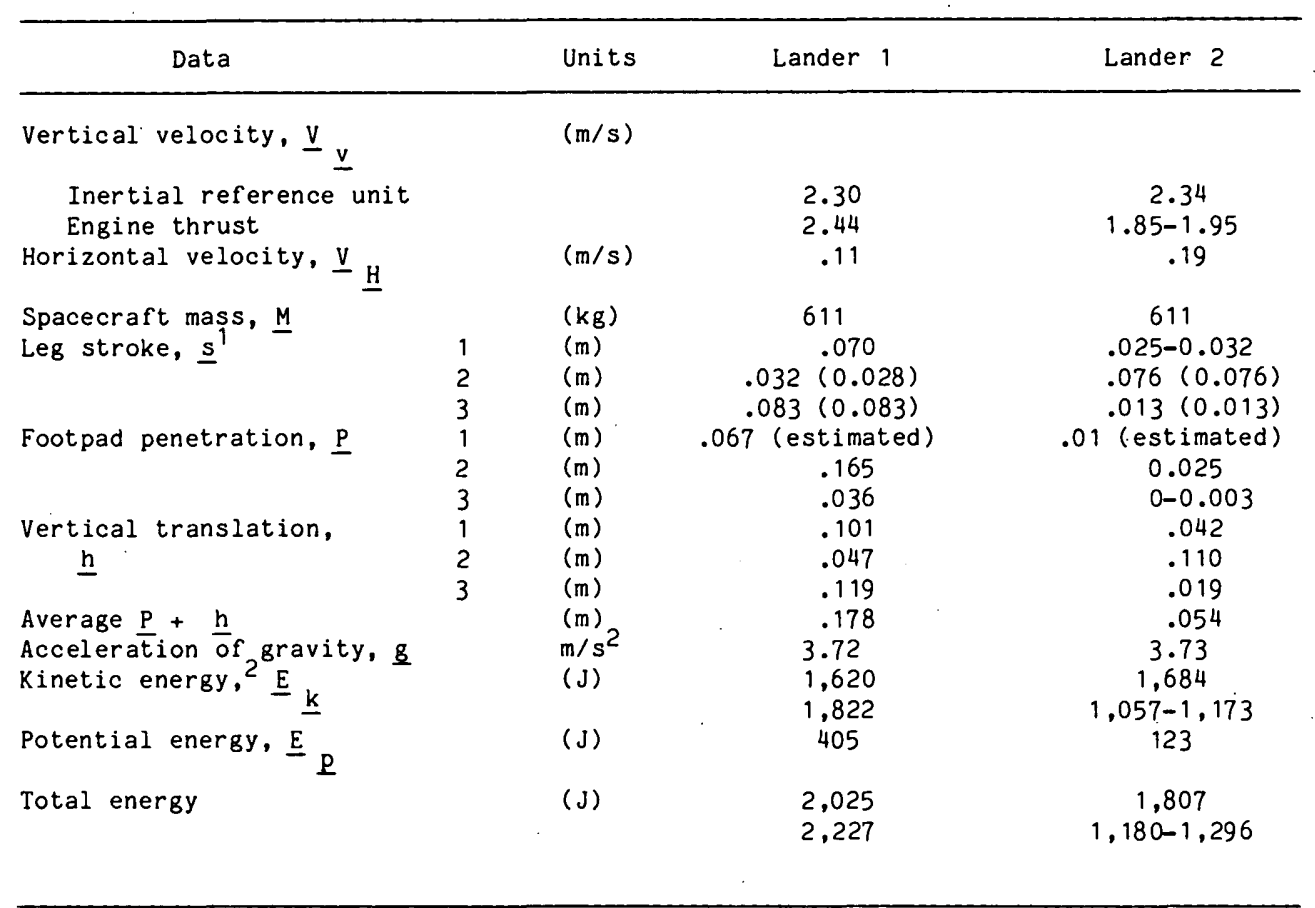

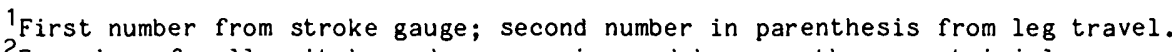

2 Energies of roll, pitch, and yaw are ignored because they are trivial.
}

surface-material parameters selected were based on estimates obtained from backhoe-touchdown experiments, surface-bearing tests, and trenching operations of the surface sampler on Mars.

\section{INITIAL CONDITIONS AT TOUCHDOWN}

The kinetic and potential energies which must be absorbed by the primary strut shock absorbers, decaying engine thrusts, and footpad work can be estimated from the known landing conditions (table 43). The kinetic energy $\left(E_{k}\right)$ is calculated from:

where

$$
E_{k}=1 / 2 M\left(V_{V}^{2}+V_{H}^{2}\right)
$$

$V_{V}$ is the vertical component of velocity at touchdown,

$V_{H}$ is the horizontal component of velocity at touchdown, and

$M$ is the mass of the spacecraft.

There is also some kinetic energy of rotation at touchdown, but because it is small compared to the kinetic energy of translation it is neglected. Potential energies $\left(E_{p}\right)$ are calculated from

$$
E_{p}=M g \Delta H
$$

where

$M$ is the mass of the spacecraft, $g$ is the acceleration of gravity, and

$\Delta H$ is the average change in height above the surface from surface contact to the final rest positions.

In these calculations, $\Delta H$ was taken as the average of the sum of the vertical translations of the footpads and their corresponding penetrations. Since footpad 1 was not visible, its penetration was estimated by assuming a linear relation between force and penetration established from the visible footpads.

For Lander 1, the kinetic and potential energy that must be absorbed ranged from 2,022 $\mathrm{J}$ to $2,227 \mathrm{~J}$. For Lander 2, the energy was difficult to establish because of the increased thrust at $0.43 \mathrm{~s}$ before touchdown. The upper limit was $1,807 \mathrm{~J}$ when the velocities at touchdown from the inertial reference unit were used but these velocities did not include the decrease to $1.85-1.95 \mathrm{~m} / \mathrm{s}$ implied by the thrust increase. Thus, the lower limit was $1,180-1,296 \mathrm{~J}$.

\section{ENERGY ABSORPTION}

Energy absorption will be outlined in order of (1) work performed by the shock absorbers during stroking of the primary strut, (2) decay of the engine thrusts, (3) work 
performed in penetrating the surface materials, and (4) work performed by footpad sliding.

Energy absorption by the primary strut or leg work $\left(W_{L}\right)$ follows directly from the force-stroke curve (fig. 152 , eq. 28,29 ) and elastic recovery curve (fig. 152, eq. 30). Here, elastic compression work is small so it is ignored. Leg work $\left(W_{L}\right)$ is the sum of the work performed on the primary shock absorber during stroking $\left(W_{s}\right)$ and the work recovered elastically $\left(W_{E}\right)$ :

where

$$
W_{L}=W_{s}+W_{E}
$$

$$
W_{s}=\int_{0}^{s} F_{A} d s
$$

and

$$
W_{E}=\int_{o}^{-\Delta s} F_{E} d(\Delta s)
$$

or

$$
W_{L}=W_{s}+W_{E}=(39,511) s^{2}+(2,998) s-39
$$

Leg work $\left(W_{L}\right)$ is listed in tables 44 and 45 for each lander.

\section{DESCENT ENGINE}

When the engine-cutoff signal was initiated upon contact, the thrust was assumed to decay as indicated in equation 31 . This decaying thrust removed energy from the system as the lander settled to its final position. The engine work, $W_{T}$, can be expressed as

$$
W_{T}=\int_{0}^{y} T d y=\int_{0}^{t_{2}} T V d t
$$

where $T$ is the thrust, $y$ is the displacement through which the thrust acts (nominally vertical), and $V$ represents the velocity along this path. Theoretical computations of landing on horizontal planes indicate that the velocity variation with time is concave downward. It is assumed here that the variation can be represented by:

$$
V=V_{o} \cos \left(\frac{\pi t}{2 t_{2}}\right)
$$

where $t_{2}$ is the time when the velocity is arrested. Upon substituting equations 41 and 31 into 40 , one obtains:

$$
\frac{W_{T}}{T_{o} V_{o}}=\int_{o}^{t_{1}} \cos \frac{\pi t}{2 t_{2}} d t+\int_{t_{1}}^{t_{2}} \mathrm{e}^{\frac{t_{1}-t}{\tau}} \cos \frac{\pi t}{2 t_{2}} d t
$$

which integrates to give:

$$
\begin{aligned}
\frac{W_{T}}{T_{o} V_{o}}= & \frac{2 t_{2}}{\pi} \sin \frac{\pi t_{1}}{2 t_{2}}+\left(\frac{1}{\frac{1}{\tau^{2}}+\frac{\pi^{2}}{4 t_{2}^{2}}}\right) \\
& \times\left(\frac{\pi}{2 t_{2}} \mathrm{e}^{\frac{t_{1}-t_{2}}{\tau}}+\frac{1}{\tau} \cos \frac{\pi t_{1}}{2 t_{2}}-\frac{\pi}{2 t_{2}} \sin \frac{\pi t_{1}}{2 t_{2}}\right)
\end{aligned}
$$

For Lander 1, a representative value of $t_{2}$ was taken to be $0.1 \mathrm{~s}$ and the values: $\tau=0.02883 \mathrm{~s}, t_{1}=0.020 \mathrm{~s}$, $V_{o}=2.44 \mathrm{~m} / \mathrm{s}$, and $T_{o}=M g=2,273 \mathrm{~N}$ were used. Substituting these values in equation 43 gave $W_{T}=221 \mathrm{~J}$. For $V_{o}=2.30 \mathrm{~m} / \mathrm{s}, W_{T}$ is equal to $208 \mathrm{~J}$. For Lander 2, a representative value of $t_{2}$ was also taken to be $0.1 \mathrm{~s}$ and the values $\tau=0.02883 \mathrm{~s}, t_{1}=0.020 \mathrm{~s}$ were used. For $V_{o}=2.34 \mathrm{~m} / \mathrm{s}, T_{o}$ is equal to $M g$ or $2,279 \mathrm{~N}$; and for $V_{o}=1.85-1.95 \mathrm{~m} / \mathrm{s}, T_{o}$ is equal to $3,678 \mathrm{~N}$ (to allow for the average increase of thrust of 62 percent). Substitution of the values in equation 43 gave $W_{T}=232 \mathrm{~J}$ to $312 \mathrm{~J}$.

\section{FOOTPAD PENETRATION WORK}

The magnitude of the forces exerted by the footpad on the surface depends on the effective coefficient of sliding friction $(\mu)$. This is expressed by:

where

$$
F_{P}=\mu F_{R}
$$

$F_{P}$ is the force in the direction of penetration of the footpad,

$F_{R}$ is the force perpendicular to the direction of penetration of the footpad, and

$\mu$ is the effective coefficient of friction.

The resultant of these two forces is the ground reaction force $\left(F_{G R}\right)$, and $F_{G R}$ is opposed by the primary and secondary strut forces $F_{A}$ and $F_{S T}$. Resolution of the forces on the struts are constrained by the geometry of the struts. Relations between these forces are shown in figure 153:

$$
\begin{gathered}
\phi=\tan ^{-1} \mu=\tan ^{-1} \frac{F_{R}}{F_{P}}, \\
F_{G R}=F_{A} \frac{\sin (180-\eta)}{\sin v}, \\
F_{P}=F_{A} \frac{\sin (180-\eta)}{\sin v} \cos \phi,
\end{gathered}
$$


TABLE 44.-Leg strokes, footpad travels, footpad penetrations, and work performed on lander shock absorbers by the engines and footpads for Lander 1

[Footpad normal forces and work are strongly dependent on assumed coefficients of friction $(\mu)$. Dashes indicate not applicable]

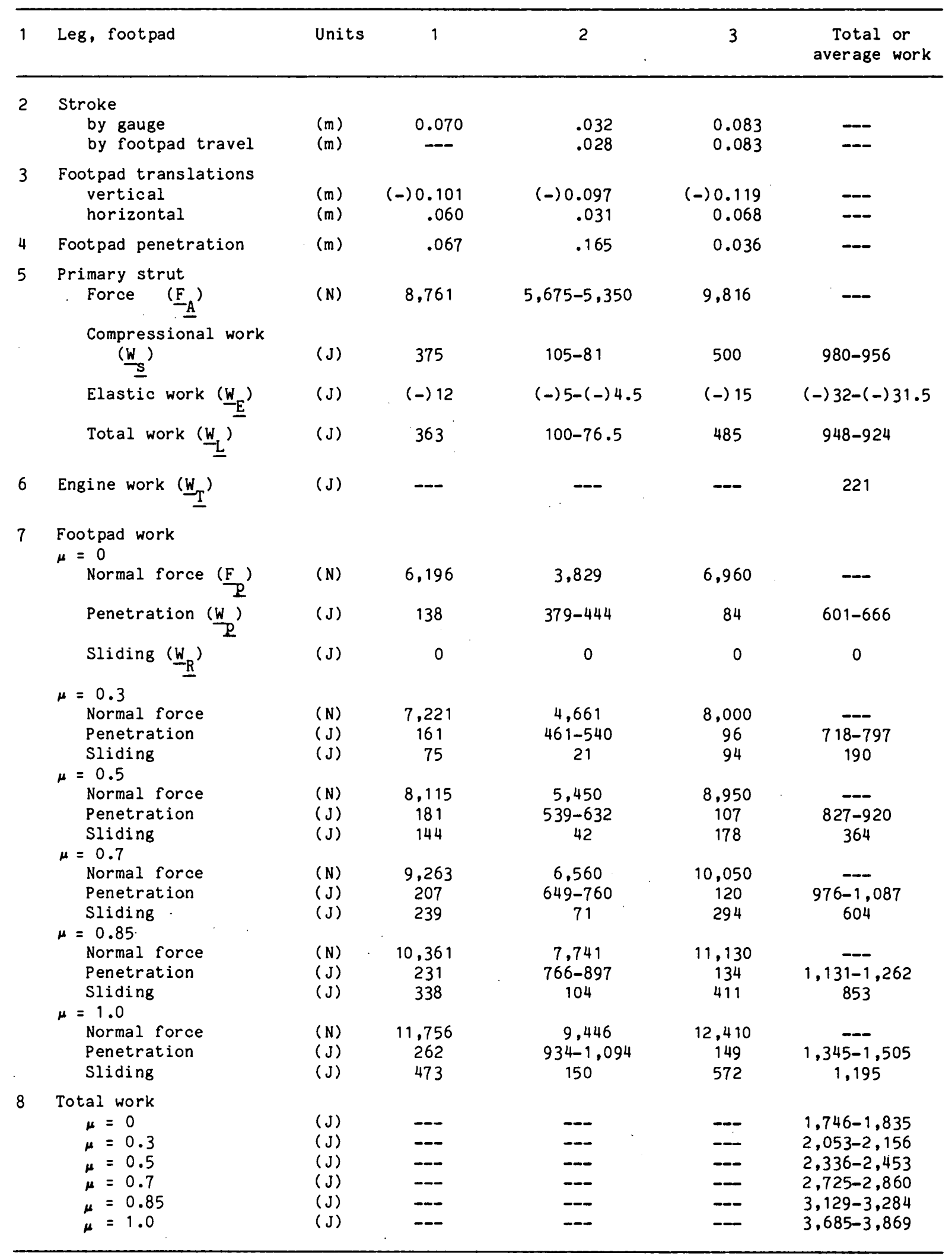


TABLE 45.-Leg strokes, footpad travels, footpad penetrations, and work performed on lander shock absorbers by the engines and footpads for Lander 2

[Footpad normal forces and work are strongly dependent on assumed coefficients of friction ( $\mu$ ). Dashes indicate not applicable]

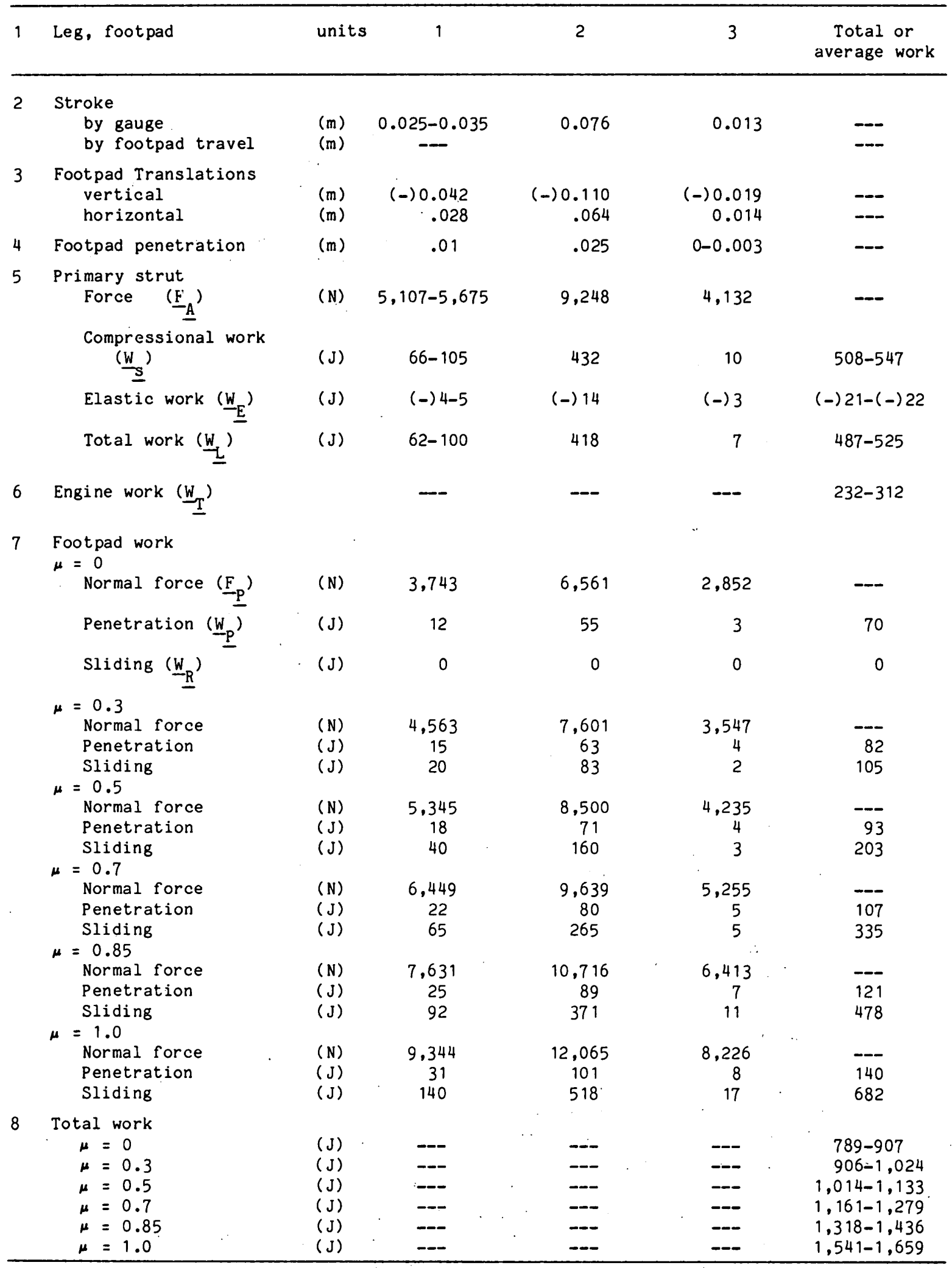




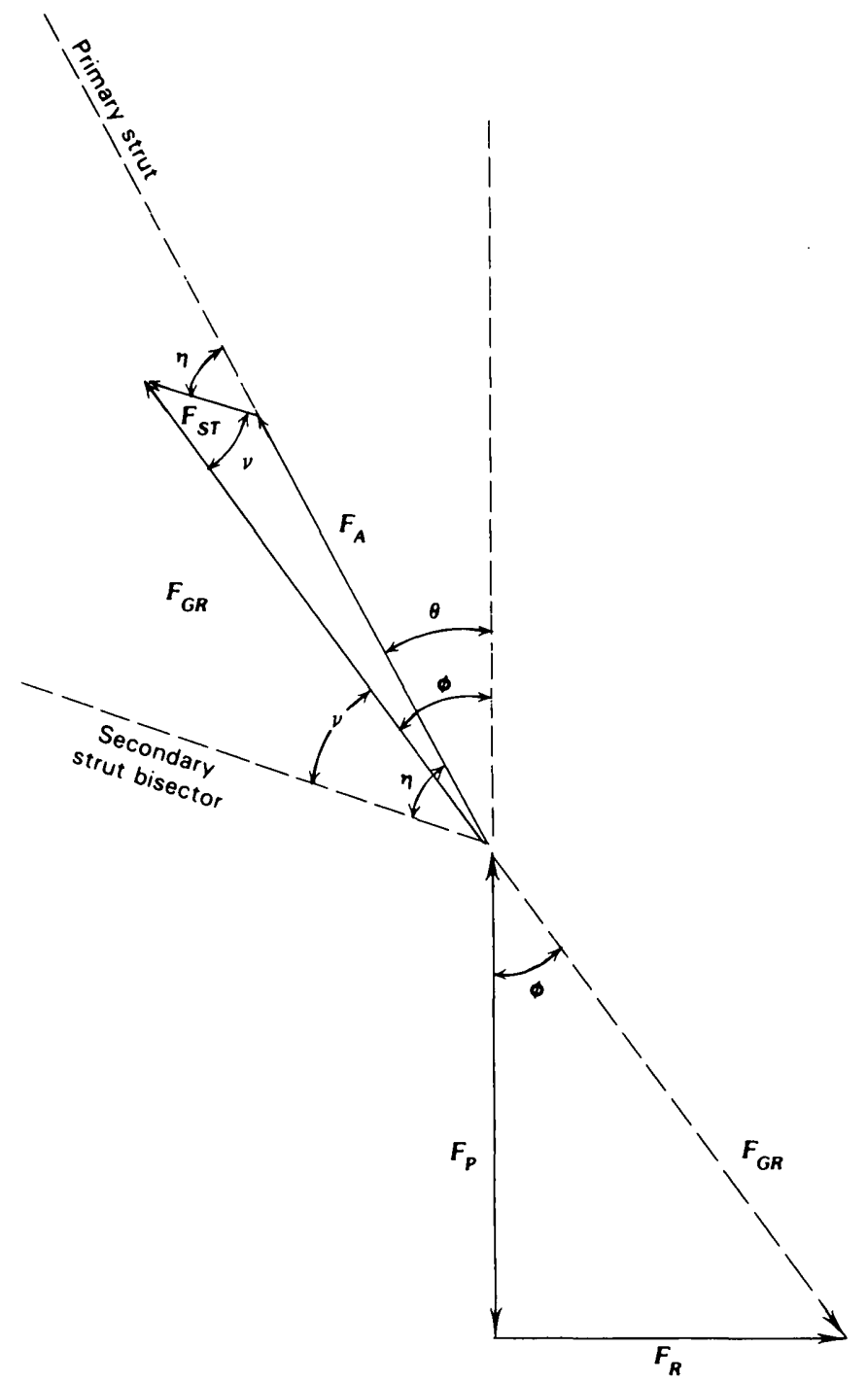

FIGURE 153.-Geometric relation between axial force on primary strut $\left(F_{A}\right)$, secondary strut force $\left(F_{S T}\right)$, ground reaction force $\left(F_{G R}\right)$, force on penetrating the footpad $\left(F_{P}\right)$ in the direction of penetration, lateral force on sliding footpad $\left(F_{R}\right)$, and effective friction angle $(\phi)$.

$$
\begin{gathered}
F_{R}=F_{A} \frac{\sin (180-\eta)}{\sin v} \sin \phi, \text { and } \\
v=\eta-\phi+\theta
\end{gathered}
$$

It should be noted that the force in the direction of penetration, $F_{P}$, is actually parallel to the $X_{L}$ direction; but it was vertical for all practical purposes because of the nearly vertical descents of the landers.

Work performed in penetrating the surface materials $\left(W_{P}\right)$ to depths as much as $0.0713 \mathrm{~m}$, which corresponds to the penetration where the skirt touches the surface material, is given by the integral of the penetration force (see eq. 32):

$$
\begin{aligned}
& W_{P}=\int_{0}^{P} F_{P} d P= \\
& k \int_{0}^{P} P^{2} d P=\left.\frac{F_{P}(\max ) P}{3}\right|_{P=0} ^{P \leqslant 0.0713}
\end{aligned}
$$

When the full area of the footpad is involved, this relation is not necessarily valid. On the basis of solutions of the static-bearing capacity equation for $c=3 \mathrm{kN}, \phi=10^{\circ}$ and $c=1.7, \phi=20^{\circ}$ (see table 7 and fig. 17), we approximated the work by assuming a constant force for penetrations $0.099 \mathrm{~m}$ and larger. The work then became:

$$
W_{P}=\frac{F_{P}(\max )}{3} 0.0991+F_{P}(\max )(P-0.0991)
$$

Because of the complex nature of the skirted footpad, we also calculated the work using:

$$
W_{P}=\frac{F_{P}(\max )}{3} 0.0713+F_{P}(\max )(P-0.0713)
$$

Here, $F_{P}(\max )$ is the maximum force in the direction of penetration. From equations $50,51,52,47$, and 48 , the force and work increased with the effective coefficient of friction. Footpad forces, $F_{P}$, are listed in tables 44 and 45 , and the relations between footpad forces and leg strokes are shown in figure 154.

\section{FOOTPAD SLIDING WORK}

Work performed by sliding is given by

$$
W_{R}=\int_{0}^{\Delta R} F_{R} d(\Delta R)
$$

We estimated sliding work by numerical integration

$$
W_{R} \approx \sum_{0}^{\Delta R} \bar{F}_{R} \Delta(\Delta R)
$$

Values of $F_{R}$ are plotted as a function of $\Delta R$ in figure 155, and sliding work, $W_{R}$, is tabulated in tables 44 and 45 .

\section{COMPARISON BETWEEN WORK AND INITIAL ENERGY}

As noted previously, the summation of work expended in the shock absorbers of the primary strut $\left(W_{L}\right)$, by the decaying engine exhausts $\left(W_{T}\right)$, in penetrating the surface materials $\left(W_{P}\right)$, and by footpad sliding $\left(W_{s}\right)$ should equal the sum of the kinetic and potential energies at the 


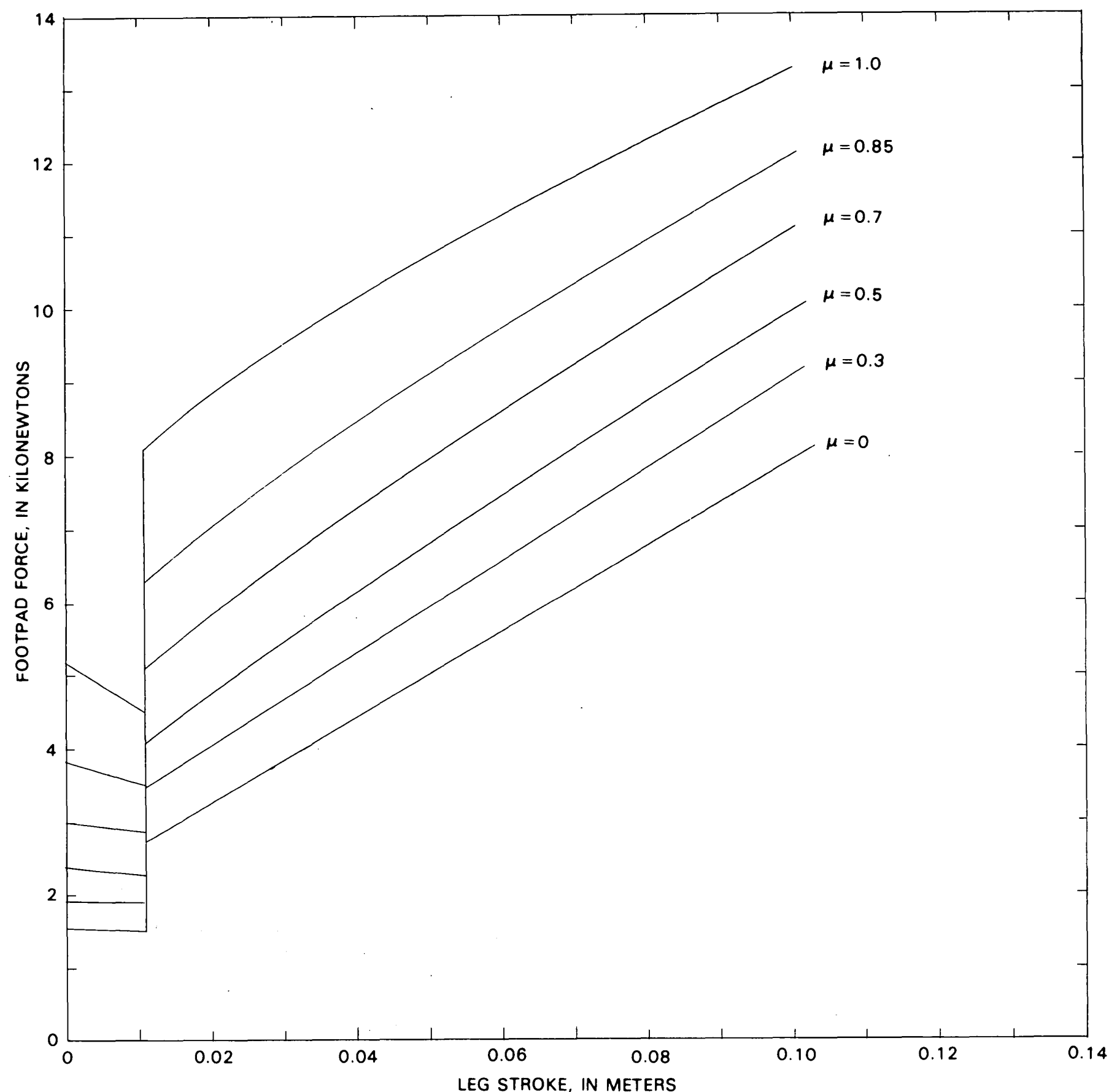

FIGURE 154.-Relation between force on footpad $\left(F_{P}\right)$ in the direction of penetration and leg stroke for six coefficients of friction $(\mu)$. Vertical line corresponds to rapid rise in axial forces from 222 to $4,005 \mathrm{~N}$ (see fig. 152).

moment of touchdown. The condition where this equality is met depends, in part, on the effective coefficient of friction. The summations of $W_{L}, W_{T}, W_{P}$, and $W_{R}$ (see tables 44 and 45) are plotted as a function of effective coefficient of friction in figure 156 for Lander 1 and figure 157 for Lander 2 and are compared with the sum of the kinetic energy and potential energy. For Lander 1 (fig. 156), this equality is achieved when the effective coefficient ranges from 0.20 to 0.43 . If the work performed during penetration is calculated using equation 50 , the effective coefficient of friction ranges from 0.43 to 0.57 and is compatible with previous calculations made by Moore and others (1977). On the basis of interpretations of cohesions and angles of internal friction from backhoe-touchdowns, surface-bearing tests, and trenching operations performed by the surface sampler, we believe that the best estimates 


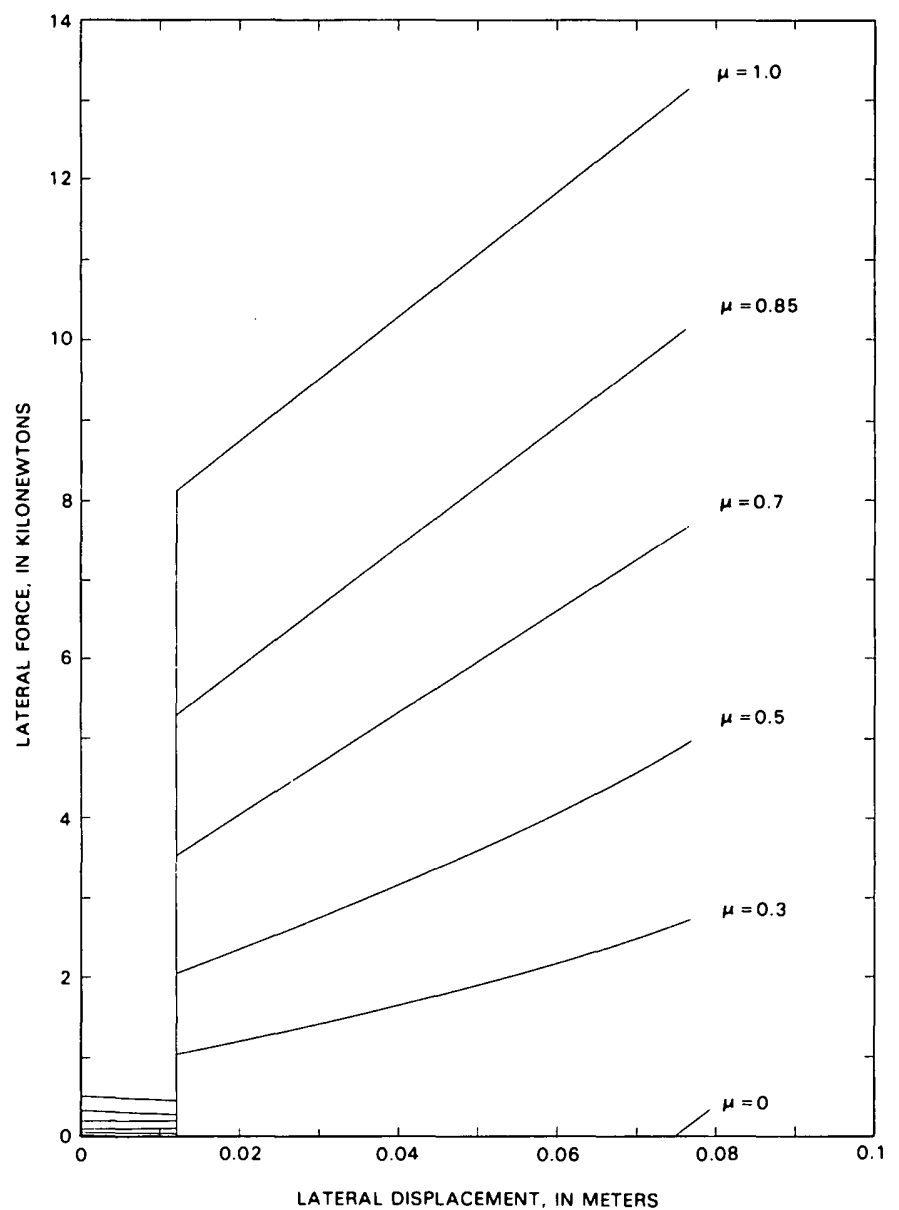

FiguRE 155.-Relation between lateral force $\left(F_{R}\right)$ on footpad and lateral translation of footpads for six coefficients of friction $(\mu)$. Vertical line corresponds to rapid rise in axial forces from 222 to 4,005 $\mathrm{N}$ (see fig. 152).

required the use of equations 50,51 , and 52 , so that the effective coefficients of friction were between 0.20 and 0.43 and the most probable range was from 0.27 to 0.43 .

In footpad forces $\left(F_{P}\right)$ in figure 17 , we used effective coefficients of sliding friction between 0 and 0.5 . For footpad 2 of Lander 1 , the forces were compatible with a surface material that had a cohesion of about $1.5 \mathrm{kPa}$, an angle of internal friction of about $20^{\circ}$, and a density of about $1,200 \mathrm{~kg} / \mathrm{m}^{3}$. For footpad 3 of Lander 1 , forces were compatible with a surface material that had a cohesion of about $6 \mathrm{kPa}$, an angle of internal friction of about $30^{\circ}$, and densities of $1,200-2,000 \mathrm{~kg} / \mathrm{m}^{3}$.

For Lander 2, we had no confidence in interpretations of the effective coefficients of sliding friction because both of the visible footpads (2 and 3 ) struck rocks upon landing. It appeared probable, however, that velocities at touchdown were, in fact, less than $2.34 \mathrm{~m} / \mathrm{s}$. The large effective coefficients of sliding friction (0.57-0.82) compared to Lander 1, suggested a complex interaction of rocks and footpads as well as a lower velocity at touchdown.

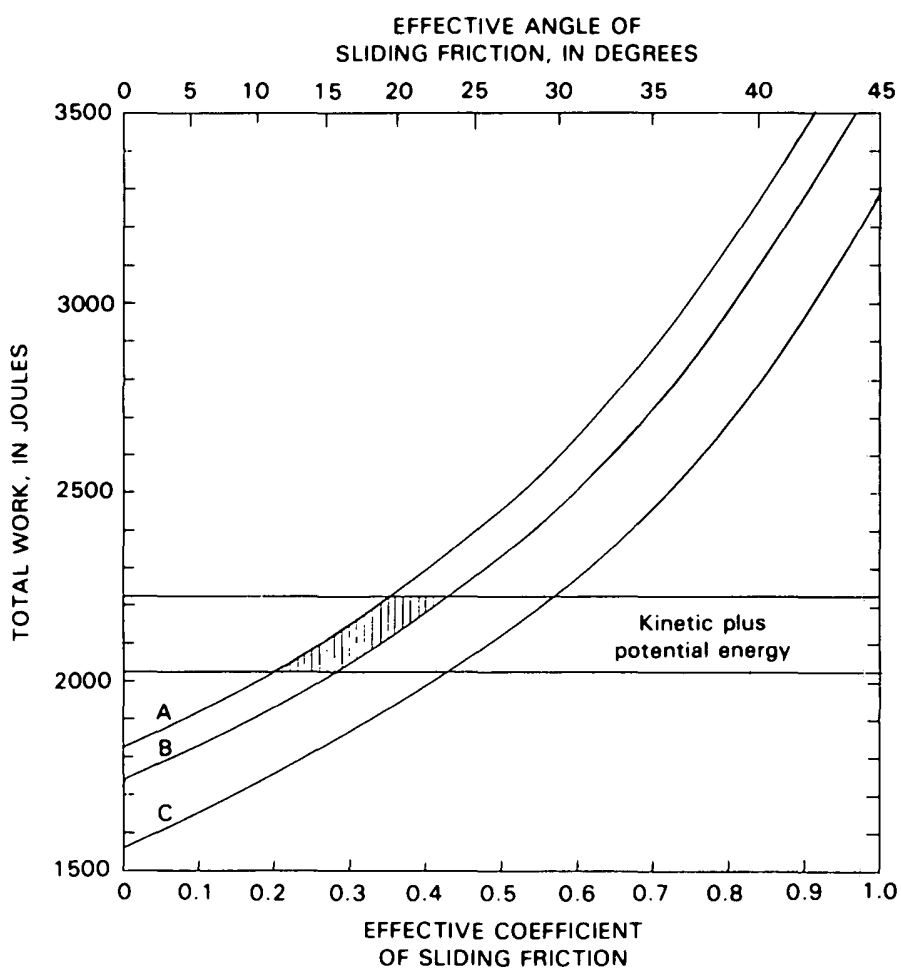

FiguRE 156.-Total work performed on primary-strut shock absorbers by decaying engine thrusts and by footpads as a function of the effective coefficient of sliding for Lander 1 . Horizontal lines represent range of energy that must be absorbed as work by these factors. Curves $\mathrm{A}$ and $B$ are best estimates of total work (see table 44). Curves A, B, and $\mathrm{C}$ show dependence of total work on force-penetration relations assumed for the penetration of footpad 2 (using equations 52,51 , and 50 , respectively). Best estimates of range of effective coefficients of sliding friction correspond to range of intersections of curves $\mathrm{A}$ and $\mathrm{B}$ with horizontal energy lines (shaded area).

\section{COMMENTS}

It should be realized that there are a number of uncertainties involved in the above analyses. First, the forces on the primary strut shock absorbers were assumed to be static loads, although they were really dynamic. Dynamic loads that crush the shock absorbers appeared to be roughly $1 \mathrm{kN}$ larger than static loads (Pohlen and others, 1976), but this difference might have been an artifact of instrumentation (J.C. Pohlen, oral commun., 1980). The effect of the larger forces, which was only about $(+) 17$ percent on the total work, tended to lower the estimates of effective coefficient of frictions, and we included this effect by using a lower value of $\mu=0$ in the force estimates. It also follows that the static forces plotted might have been $1 \mathrm{kN}$ lower than estimated, but these values are still within the range of expected forces predicted from static-bearing capacities for both footpads. In the case of footpad 2 of Lander 1 , which was submerged in drift material, plowing rather than frictional sliding seems probable. Additionally, sliding along the sur- 


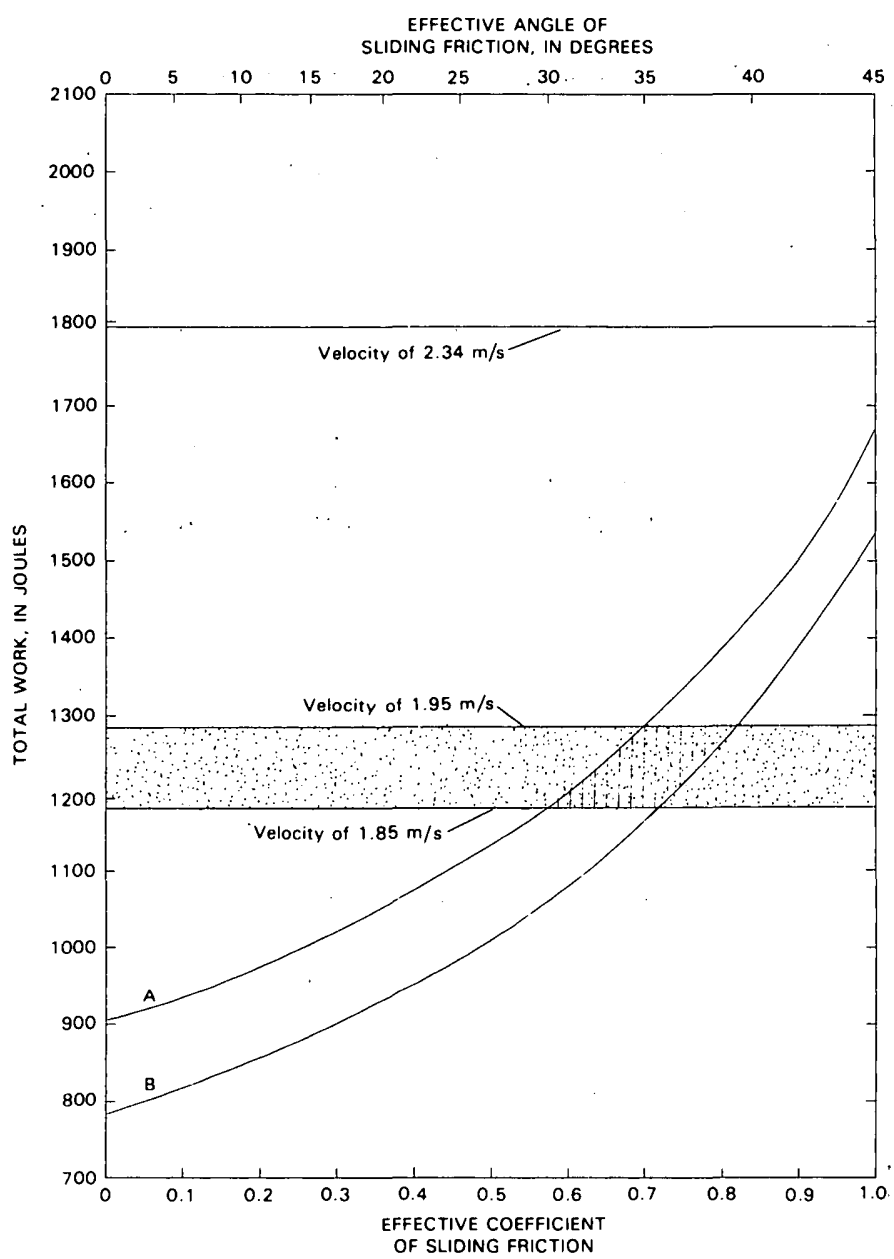

FIGURE 157.-Total work performed on primary-strut shock absorbers by decaying engine thrusts and by footpads as a function of the effective coefficient of sliding for Lander 2 . Horizontal lines indicate range of energy that must be absorbed by work. Curves A and B are best estimates of total work (see table 45). Because footpads 2 and 3 struck rocks on landing, implied effective coefficients of friction (lined area) are unreliable. Dotted area represents most probable range of kinetic and potential energy of Lander 2.

face by the entire lander also might have occurred, the effects of local topography have been ignored, and dynamic aspects of "soil" failure have been overlooked. Nevertheless, our best estimates of forces on the footpads of Lander 1 and conclusions about the surface-material properties were compatible with other data and interpretations.

For Lander 2, simple interpretations of footpad-surface material interactions were clearly invalid. It seems, however, that the velocity of Lander 2 at touchdown was less than 2.3 to $2.4 \mathrm{~m} / \mathrm{s}$.

Finally, there is no clear evidence that work was performed in deforming the load limiters. For Lander 1, forces on the load limiters should not have exceeded $3,000 \mathrm{~N}$ in any case, so that work deforming them would be very small (see Pohlen and others, 1976). For Lander 2, forces on the load limiters should not have exceeded $3,600 \mathrm{~N}$ in any case, so that work deforming them would also be small $(<<35 \mathrm{~J})$.

\section{ANALYSIS OF SAMPLE TRENCHES AND SURFACE-BEARING TESTS}

\section{INTRODUCTION}

In the analyses of the trenches and surface-bearing tests, the motion of the collector head as it moves through the surface materials was considered to be analogous to a plow with a narrow blade. Two kinds of analyses were used. The theoretical analysis, which was based on a theory for plowing by narrow blades in soil (McKyes and Ali, 1977) was used to obtain estimates of angles of internal friction and cohesions. The theoretical analyses required a complete set of data on geometrical relations for the trenches and information on forces from the motor-current records. The empirical analysis, which was based on empirical relations developed from experimental data on plowing in wet clay (Wismer and Luth, 1972) and dry sand (Luth and Wismer, 1971) and on dimensional analysis, was used to estimate cohesions of materials when the data on geometrical relations for the trenches were insufficient for the theoretical analysis to provide independent estimates of cohesion, and to calculate the forces that would be expected for sand on Mars. The two kinds of analyses are described below.

\section{THEORETICAL ANALYSES}

The theory and procedures for calculating forces on plows with narrow blades moving through soils with known properties of cohesions, densities, and angles of internal friction were developed by McKyes and Ali (1977). The procedures were similar to those used in calculations of static-bearing capacities of footings, in that three dimensionless factors for friction, cohesion, and surcharge must be determined. These dimensionless factors depended on the angle of internal friction, and the angle determined the geometry of the region of failure in front of the plow; this geometry also depended on the depth/ width ratio of the plow blade. The theory and procedures are described below.

In the model, the region of failure in front of the plow blade was made of two prisms and two mirror-image pyramidal wings (fig. 158). A lower prism was adjacent to the plow blade so that one of its sides had edges equal to the width $(w)$ of the plow and the length $(l)$ of the plow. Its lowermost edge was at a depth $(d)$ below the surface. The second, an upper prism, extended a distance $(r)$ from the plow blade to the limit of the failure along the original 

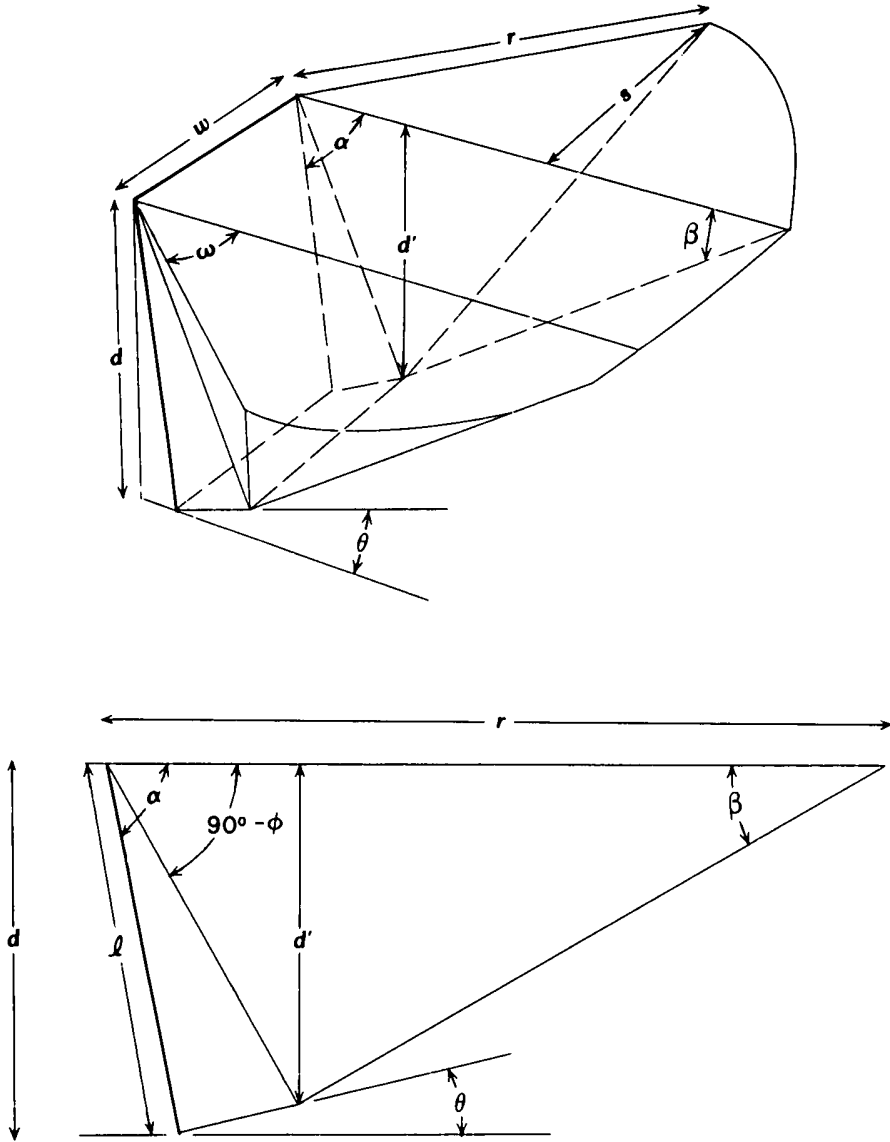

FIGURE 158.-Geometry of failure region in front of plow blade. Blade had dimensions of $w$ (width) and $l$ (length) and was inclined at an angle of $\alpha$ (rake angle). Base of blade was at a depth, $d$, below surface. Failure region was made of two prisms and pyramidal wings. Lower prism was adjacent to blade and its lower face was inclined at an angle $\theta$ (base angle) to local horizontal. Upper prism extended a distance $r$ from blade along original ground surface. Apex of upper prism was at a depth, $d^{\prime}$. Pyramidal wings extended laterally a distance, $s$, from upper prism at surface. Ratio of $s$ and $r$ defined wing angle, $\omega$. Exterior wedge angle was $\beta$.

surface. It had a width, $w$, and the apex of the upper prism was at a distance $d^{\prime}$ below the original surface. The two prisms had a common face from the upper edge of the plow blade downward to a distance of $d^{\prime}$. The pyramidal wings were bounded by the triangular face of the upper prism, the original surface included in a pie-shaped area of radius $r$ with a subtended angle of $\omega$, a conical surface, and a triangular surface. The pyramidal wings extended laterally a distance, $s$, from the upper prism so that the width of the region of failure at the surface was $2 s+w$. In profile, the angle of inclination of the plow blade from the horizontal (rake angle) was $\alpha$; the angle between the planes of the upper prism adjacent to the plow blade was $90^{\circ}-\phi$; the angle adjacent to the limit of failure was $\beta-$ here called the exterior wedge angle; and the angle between the horizontal and the base of the lower prism was $\theta$-here called the base angle.
The analyses of the forces on the prisms, pyramids, and blades given by McKyes and Ali (1977) will not be repeated here.

According to the model, the horizontal force $\left(F_{H}\right)$ on a plow blade (interface at leading edge of the collector head) is:

$$
F_{H}=\left[\rho g\left(d^{\prime}\right)^{2} N_{y H}+c d^{\prime} N_{c H}+g d^{\prime} N_{q} H\right] w
$$

where

$\rho$ is the density of the surface material,

$g$ is the acceleration of gravity,

$d^{\prime}$ is the depth of the upper prism in the region of failure,

$w$ is the width of the plow blade,

$c$ is the cohesion of the surface material, and

$q$ is the weight per unit area of the material above the prisms and pyramids (the surcharge).

$N_{\gamma H}, N_{c H}, N_{q H}$ are dimensionless factors (for friction, cohesion, and surcharge) related to the angles of internal friction $(\phi)$, metal-soil friction $(\delta)$, inclination of the plow blade $(\alpha)$, and the base angle $(\theta)$.

The dimensionless factors are given by:

$$
N_{\gamma H}=\frac{\frac{1}{2} \frac{d^{\prime}}{d}\left[\tan \phi-\cot \alpha+\frac{2 d^{\prime}}{d} N_{\gamma H}^{*} \cot (\theta+\phi)\right]}{\cot (\alpha-\delta)+\cot (\theta-\phi)}
$$

$N_{c H}=\frac{1-\frac{2 d^{\prime}}{d}-\left[\frac{2 d^{\prime}}{d} \tan \phi-\cot \alpha\right] \cot (\theta+\phi)+\frac{d^{\prime}}{d} N_{c H}^{*} \cot (\theta+\phi)}{\cot (\alpha+\delta)-\cot (\theta+\phi)}$

$$
N_{q H}=\frac{\frac{d^{\prime}}{d} N_{q H}^{*} \cot (\theta+\phi)}{\cot (\alpha+\delta)+\cot (\theta+\phi)}
$$

where

$d^{\prime}$ is the depth of the apex of the upper prism,

$d$ is the depth of the plow blade,

$\phi$ is the angle of internal friction,

$\alpha$ is the angle of inclination of the plow blade,

$\theta$ is the angle between the base of the lower prism and the horizontal,

$\delta$ is the angle of friction between the blade and surface material.

$N_{\gamma H}^{*}, N_{c H}^{*}, N_{q H}^{*}$ are dimensionless factors for blades with rake angles $(\alpha)$ equal to $90^{\circ}-\phi$ and large values of $\delta$.

From the geometry it can be shown that:

$\frac{d^{\prime}}{d}=\frac{\cos \phi}{\sin \alpha}\left[\sin (\alpha+\phi)+\cos (\alpha+\phi) \tan \left(135^{\circ}+\frac{5}{2} \phi-\alpha\right)\right]$ 
and

$$
\theta=135^{\circ}-\frac{3}{2} \phi-\alpha
$$

The dimensionless factors for equations 56,57 , and 58 are:

$$
\begin{gathered}
N_{\gamma H}^{*}=\frac{\frac{r}{2 d^{\prime}}\left[1+\frac{2 r}{3 w} \sin \omega\right]}{\cot (\alpha+\delta)+\cot (\beta+\phi)} \\
N_{c H}^{*}=\frac{1+\cot \beta \cot (\beta+\phi)\left[1+\frac{r}{w} \sin \omega\right]}{\cot (\alpha+\delta)+\cot (\beta+\phi)} \\
N_{q H}^{*}=\frac{\frac{r}{d^{\prime}}\left[1+\frac{r}{w} \sin \omega\right]}{\cot (\alpha+\delta)+\cot (\beta+\phi)}
\end{gathered}
$$

where $\alpha$ is set equal to $90^{\circ}-\phi$.

The surface material is most likely to fail under conditions in which it has the least resistance to failure, and this condition occurs when $N_{y H}^{*}$ is at a minimum (fig. 159). Minimum values of $N_{\gamma H}^{*}$ that depend only on $\phi, \beta, \delta$, and $d^{\prime} / w$ can be obtained by substitution of two relations from the model. From the geometry of the upper prism,

$$
\frac{r}{d^{\prime}}=\cot \left(90^{\circ}-\phi\right)+\cot \beta
$$

and, by definition,

$$
\cos \omega=\frac{d^{\prime}}{r} \cot \left(90^{\circ}-\phi\right)
$$

Equation 61 is now written:
Additional curves, which we called data curves, were derived from martian data (fig. 160). For the data curves, exterior wedge angles $(\beta)$ were calculated using equations 59 and 64 for several assumed values of angles of internal friction $(\phi)$. Surface-sampler and topographic data established the rake angle $(\alpha)$, the depth $(d)$ of the tip of the collector head, and the limit of the region of failure $(r)$. The depth of the upper prism $\left(d^{\prime}\right)$ was calculated with equation 59 , and then the exterior wedge angle $(\beta)$ was calculated with equation 64 .

Data curves crossed the model curves, and this fact formed the basis for our estimates of angles of internal friction. The angle of internal friction corresponding to the intersection of the data and model curves at the proper ratio of $d^{\prime} / w$ was taken to represent a good estimate of the angle of internal friction (fig. 160).

Although some of the parameters were calculated as discussed above, others were estimated. Densities of the surface materials $(\rho)$ were estimated from data supplied by the Inorganic Chemical Analysis Experiment. Acceleration of gravity $(g)$ was estimated by analyses of the inertial reference unit data from an Entry Science experiment. The surcharge, $q$, was estimated by:

$$
q=\rho g \frac{d^{\prime}}{3}
$$

This estimate was based on the assumption that the volume of the surcharge is one-fourth the volume of a spheroid with axes equal to $r, 2 s+w / 2$, and $d^{\prime} / 2$ divided by one-half the area of an ellipse with axes equal to $r$ and $2 s+w / 2$. As it turned out, terms involving $q$ were small. Additionally, variations in density from 1,200 to 2,000 $\mathrm{kg} / \mathrm{m}^{3}$ generally had little effect on the calculated cohesions. Complete sets of calculations appear in tables 46 and 47 .

$$
N_{\gamma H}^{*}=\frac{\frac{\cot \Omega+\cot \beta}{2}\left[1+\frac{2}{3} \frac{d^{\prime}}{w}(\cot \Omega+\cot \beta)\left(\sin \left\{\cos ^{-1} \frac{\cot \Omega}{\cot \Omega+\cot \beta}\right\}\right)\right]}{\cot (\Omega+\delta)+\cot (\beta+\phi)}
$$

where $\Omega=90^{\circ}-\phi$.

Curves, which we called model curves (fig. 160), were obtained by establishing exterior wedge angles $(\beta)$ and angles of internal friction $(\phi)$ for which $N_{\gamma H}^{*}$ in equation 66 were at minima (fig. 159). The two friction angles, $\phi$ and $\delta$, were equal because the interface between the region of failure and the collector head (blade) was considered to have surface materials on each side. The locations of the minima also depended on the ratio of $d^{\prime}$ and $w$.
In order to solve equation 55, frictional forces on the collector head had to be taken into account. A twodimensional limiting equilibrium model was used to estimate the horizontal force $\left(F_{T}\right)$ in the absence of adequate experimental data. In the model, three forces were considered initially (fig. 161): the axial force on the boom $\left(F_{A}\right)$, the ground reaction on the interface between the surface material and the base of the collector head $\left(f_{1}\right)$, and the ground reaction on the interface between the sur- 


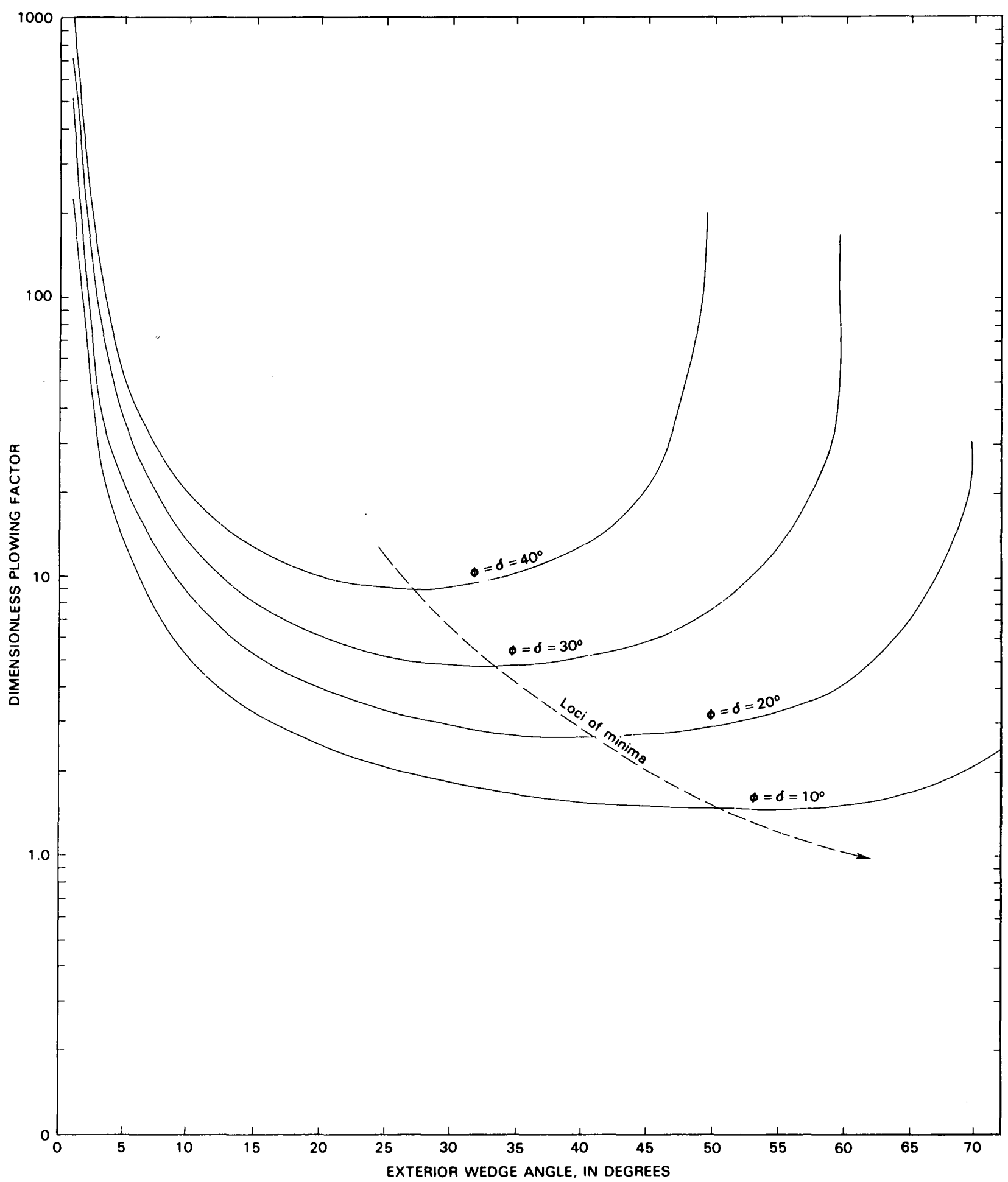

FIGURE 159.-Relation of exterior wedge angle $(\beta)$ to dimensionless plowing factor for internal friction $\left(N_{\gamma H}^{*}\right)$ for angles of internal friction $(\phi)$ of $10^{\circ}, 20^{\circ}, 30^{\circ}$, and $40^{\circ}$ with an upper prism depth-plow width ratio $\left(d^{\prime} / w\right)$ of 1 and rake angles $(\alpha)$ equal to $90^{\circ}-\phi$. For Viking data, angles of metal-surface material friction ( $\delta$ ) were taken as equal to angles of internal friction $(\phi)$ of surface materials. Line labeled loci of minima indicates where surface material has least resistance to failure. 


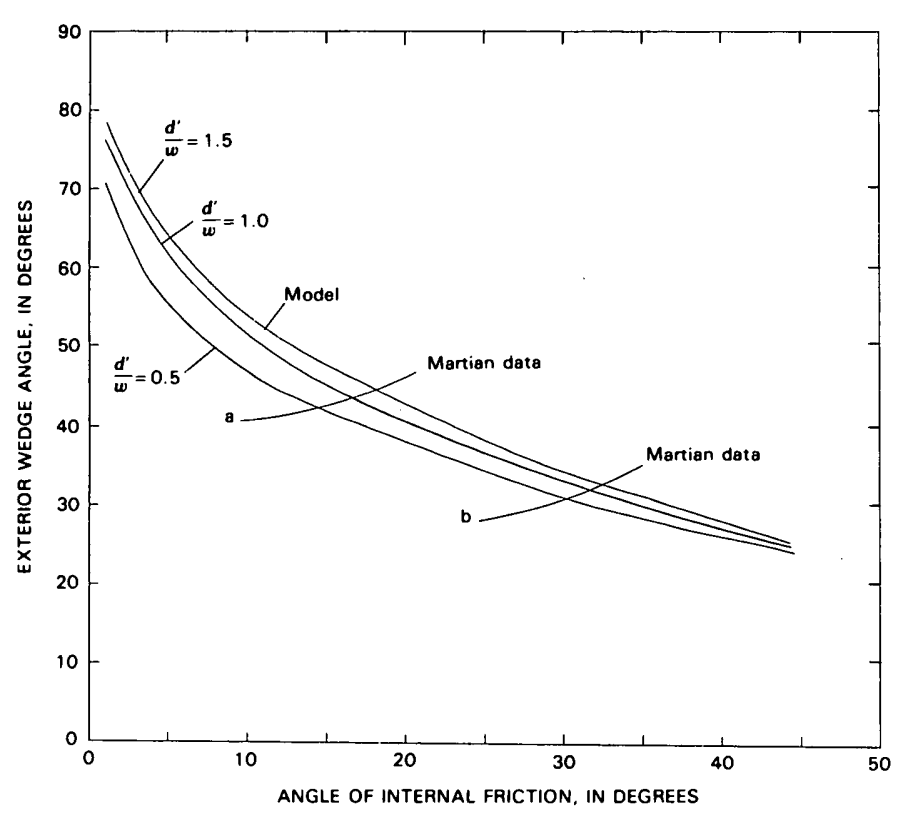

Figure 160.-Procedure for estimating angles of internal friction of martian surface materials. Exterior wedge angles $(\beta)$ as a function of angles of internal friction $(\phi)$ were calculated from model of McKyes and Ali (1977) for three depth $\left(d^{\prime}\right)$ to width $(w)$ ratios to obtain the model curves (see figure 159 and text). Exterior wedge angles and angles of internal friction were also calculated using data on martian experiments to obtain the data curves (see text). Note that model and data curves cross one another. Estimate of angle of internal friction corresponded to intersection of two sets of curves at depth-to-width ratio of martian experiment. Data curves corresponded to materials with angles of internal friction between (a) $14^{\circ}$ and $18^{\circ}$ and (b) $30^{\circ}$ and $33^{\circ}$ when depth-to-width ratios ranged from 0.5 to 1.5 .

face material and leading edge of the collector head $\left(f_{2}\right)$. Ground-reaction normal- $\left(f_{n}\right)$ and shear- $\left(f_{s}\right)$ force components for both $f_{1}$ and $f_{2}$ were fully realized in the limiting equilibrium state and were assumed to be related by:

$$
f_{s}=f_{n} \tan \delta .
$$

Thus, the angles between the normals to the interfaces and the resultant ground reaction were equal to the angle of metal-surface material friction (which was assumed to be equal to the angle of internal friction because of trapped material in the leading edge of the collector head and the rough base of the collector head). The equations of equilibrium of forces parallel and perpendicular to the boom axis respectively, are:

$$
\begin{gathered}
0=F_{A}-f_{1} \sin (\gamma+\delta)-f_{2} \cos \left(\alpha^{*}+\delta\right), \\
0=f_{1} \cos (\gamma+\delta)-f_{2} \sin \left(\alpha^{*}+\delta\right) .
\end{gathered}
$$

Eliminating force $f_{1}$ from these two equations yields:

$$
F_{A}=f_{2}\left[\sin \left(\alpha^{*}+\delta\right) \tan (\gamma+\delta)+\cos \left(\alpha^{*}+\delta\right)\right] .
$$

The angle of $\alpha^{*}$ may be related to known quantities $\gamma$ and $\theta^{*}$ by,

$$
\alpha^{*}=\frac{\pi}{2}+\gamma-\theta^{*}
$$

Substituting 72 into 71 gives:

$$
F_{A}=f_{2}\left[\cos \left(\gamma+\delta-\theta^{*}\right) \tan (\gamma+\delta)-\sin \left(\gamma+\delta-\theta^{*}\right)\right] .
$$

The horizontal component of $f_{2}$ is:

$$
F_{T}=-f_{2} \cos \left(\alpha^{*}+\delta-\varepsilon\right)=f_{2} \sin \left(\gamma+\delta-\theta^{*}-\varepsilon\right)
$$

so that

$$
\frac{F_{T}}{F_{A}}=\frac{\sin \left(\gamma+\delta-\theta^{*}-\varepsilon\right)}{\cos \left(\gamma+\delta-\theta^{*}\right) \tan (\gamma+\delta)-\sin \left(\gamma+\delta-\theta^{*}\right)}
$$

Equation 75 was used to obtain values of the horizontal force $\left(F_{T}\right)$ from the boom axial force $\left(F_{\mathrm{A}}\right)$. The ratio of these two forces varied considerably for the Viking conditions (fig. 162). The boom axial forces $\left(F_{A}\right)$ were obtained from surface-sampler data. Cohesions were estimated by iterative calculations until $F_{H}$ in equation 55 is nearly equal to $F_{T}$ in equation 75 (see tables 46 and 47 ).

We believe that our results, calculated with the models above, are reasonably correct, but it should be realized that experiments using the Viking surface sampler, its collector head, and natural soils to relate the angles of internal friction to the geometry of the region of failure and the axial forces to the horizontal forces have never been performed on Earth. There are other solutions. The estimates of angles of internal friction of blocky material and crusty to cloddy material could have been increased about $3^{\circ}$ with a concomitant reduction of about 10 percent in the cohesions by decreasing the angle between the base and leading edge of the collector head, $\theta^{*}$ (and the rake angle, $\alpha$ ) about $9^{\circ}$ from $76^{\circ}$ to $67^{\circ}$. Such manipulations, however, would not substantially affect the small angles of internal friction of drift material.

\section{EMPIRICAL ANALYSES}

Equations based on experimental data and dimensional analyses (fig. 163) provided an independent check on the magnitudes of cohesions derived from other methods, the 
TABLE 46.-Data and derived quantities used to calculate the cohesion from the surface-bearing test in blocky material of Lander 1

[Table illustrates calculations using the model of McKyes and Ali (1977)]

\begin{tabular}{|c|c|c|c|}
\hline \multirow{2}{*}{$\begin{array}{l}\text { Names of assumed, measured, and } \\
\text { calculated values of parameter"s }\end{array}$} & \multicolumn{3}{|c|}{$\begin{array}{l}\text { Values of parameters for the } \\
\text { indicated angles of internal friction }\end{array}$} \\
\hline & 25.0 & 30.0 & 35.0 \\
\hline $\begin{array}{l}\text { Assumed metal-soil friction angle, } \\
\delta \text { (degrees) }\end{array}$ & 25.0 & 30.0 & 35.0 \\
\hline $\begin{array}{l}\text { Collector head } \\
\text { width, w } \\
\text { depth, } \frac{d}{d} \\
\text { rake angle, } \alpha \text { (degrees) }\end{array}$ & $\begin{array}{c}0.048 \\
.035 \\
90.0\end{array}$ & $\begin{array}{c}0.048 \\
.035 \\
90.0\end{array}$ & $\begin{array}{l}0.048 \\
.035 \\
90.0\end{array}$ \\
\hline $\begin{array}{l}\text { Region of failure } \\
\text { limit, } \frac{r}{2} \\
\text { width, } \\
\text { exterior wedge angle, } \beta \text { (degrees) } \\
\text { base angle, } \theta \text { (degrees) } \\
\text { upper prism depth, } \underline{d}^{\prime}\end{array}$ & $\begin{aligned} 0.075 \\
.148 \\
29.0 \\
7.5 \\
.033\end{aligned}$ & $\begin{array}{c}0.075 \\
.148 \\
32.6 \\
0 \\
.035\end{array}$ & $\begin{aligned} 0.075 \\
.148 \\
38.8 \\
-7.5 \\
.039\end{aligned}$ \\
\hline $\begin{array}{l}\text { Dimensionless factors for } \\
\alpha=90-\phi \text { (degrees) } \\
\underline{N}_{c} \gamma{\frac{H^{*}}{H^{*}}}^{-}\end{array}$ & $\begin{array}{l}65.0 \\
2.652 \\
6.516\end{array}$ & $\begin{array}{l}60.0 \\
3.507 \\
7.152\end{array}$ & $\begin{array}{l}55.0 \\
5.610 \\
9.585\end{array}$ \\
\hline$\underline{N}_{g}^{-} \underline{H}^{*}$ & 6.394 & 8.456 & 13.528 \\
\hline $\begin{array}{l}\alpha \text { (degrees) } \\
\underline{N}_{c}^{N} \frac{H}{H}\end{array}$ & $\begin{array}{l}90.0 \\
3.547 \\
6.681\end{array}$ & $\begin{array}{l}90.0 \\
5.510 \\
8.130\end{array}$ & $\begin{array}{l}90.0 \\
11.037 \\
13.206\end{array}$ \\
\hline$\underline{N} q \underline{H}$ & 8.569 & 12.684 & 23.459 \\
\hline $\begin{array}{l}\text { Force calculations } \\
\text { density, } p\left(\mathrm{~kg} / \mathrm{m}^{3}\right) \\
\text { gravity, } \frac{\mathrm{g}}{\left(\mathrm{m} / \mathrm{s}^{2}\right)} \\
\text { horizontal force of surface } \\
\text { sampler (N) }\end{array}$ & $\begin{array}{c}1,200 \\
3.72 \\
210-236\end{array}$ & $\begin{aligned} 1,200 \\
3.72 \\
192-216\end{aligned}$ & $\begin{array}{c}1,200 \\
3.72 \\
173-194\end{array}$ \\
\hline $\begin{array}{l}\text { Cohesions }(\mathrm{kPa}) \\
\text { derived force }(\mathrm{N})\end{array}$ & $\begin{array}{l}19.7-22.1 \\
210-235\end{array}$ & $\begin{array}{l}13.9-15.6 \\
192-216\end{array}$ & $\begin{array}{l}6.7-7.6 \\
172-194\end{array}$ \\
\hline $\begin{array}{l}\text { Force calculations } \\
\text { density, } \rho\left(\mathrm{kg} / \mathrm{m}^{3}\right) \\
\text { Cohesions ( } \mathrm{kPa}) \\
\text { derived force (N) }\end{array}$ & $\begin{array}{c}2,000 \\
19.6-22.0 \\
210-235\end{array}$ & $\begin{array}{c}2,000 \\
13.8-15.5 \\
193-216\end{array}$ & $\begin{array}{l}2,000 \\
6.6-7.4 \\
173-193\end{array}$ \\
\hline
\end{tabular}

cohesions of materials in trenches for which there were insufficient topographic data to apply the theoretical analysis, and the estimates of forces for plowing in sand on Mars. Materials used in the experiments were wet clay (Wismer and Luth, 1972) and dry sand (Luth and Wismer, 1971). "Pure cohesion" was defined by the equations derived from the experiments with wet clay (fig. 163) because of the large cohesions $(13-26 \mathrm{kPa}$ ) and low friction angles $\left(\phi=0^{\circ}-3^{\circ}, \delta=12^{\circ}-13^{\circ}\right)$ of the test materials; densities were $1,840-1,870 \mathrm{~kg} / \mathrm{m}^{3}$. "Pure friction" was defined by equations derived from experiments with dry sand (fig. 163) because of the low cohesion $(c=0)$ and large friction angles $\left(\phi=35^{\circ}-37^{\circ} ; \delta=23^{\circ}-24^{\circ}\right)$ of the test materials; densities were $1,600-1,700 \mathrm{~kg} / \mathrm{m}^{3}$.

Values of density and acceleration of gravity that were almost the same as the experimental values (that is, $\rho=1,845 \mathrm{~kg} / \mathrm{m}^{3}$ and $g=9.8 \mathrm{~m} / \mathrm{s}^{2}$ ) were used to estimate cohesions when using the equation to calculate the horizontal force of pure cohesion ( $F_{x}$ in fig. 163). These values were used because the equation predicts that horizontal force increases if both density and acceleration of gravity decrease; whereas, the theory predicts that the 
TABLE 47.-Data and derived quantities used to calculate the cohesion from the surface-bearing test of Lander 2 [Table illustrates calculations using the model of McKyes and Ali (1977)]

\begin{tabular}{|c|c|c|c|}
\hline \multirow{2}{*}{$\begin{array}{c}\begin{array}{c}\text { Names of assumed, measured, and } \\
\text { calculated values of parameters }\end{array} \\
\begin{array}{c}\text { Assumed angle of internal friction, } \\
\phi \text { (degrees) }\end{array}\end{array}$} & \multicolumn{3}{|c|}{$\begin{array}{l}\text { Values of parameters for the } \\
\text { indicated angles of internal friction }\end{array}$} \\
\hline & 25.0 & 30.0 & 35.0 \\
\hline $\begin{array}{l}\text { Assumed metal-soil friction angle, } \\
\delta \text { (degrees) }\end{array}$ & 25.0 & 30.0 & 35.0 \\
\hline $\begin{array}{l}\text { Collector head } \\
\text { width, } \frac{w}{d} \\
\text { depth, } \frac{d}{\text { lake ange, (degrees) }}\end{array}$ & $\begin{array}{l}0.048 \\
.07 \\
84.4\end{array}$ & $\begin{array}{l}0.048 \\
.07 \\
84.4\end{array}$ & $\begin{array}{l}0.048 \\
.07 \\
84.4\end{array}$ \\
\hline $\begin{array}{l}\text { Region of failures } \\
\text { limit, } \frac{r}{2}+\underline{w} \\
\text { width, } \\
\text { exterior wedge angle, } \beta \text { (degrees) } \\
\text { base angle, } \theta \text { (degrees) } \\
\text { upper prism depth, } \underline{d}^{\prime}\end{array}$ & $\begin{aligned} & 0.15 \\
& .242 \\
& 28 . .3 \\
& 13.1 \\
& .065\end{aligned}$ & $\begin{array}{l}0.15 \\
.242 \\
31.0 \\
5.6 \\
. \quad .067\end{array}$ & $\begin{array}{l}0.15 \\
.242 \\
35.5 \\
-1.9 \\
.071\end{array}$ \\
\hline $\begin{array}{l}\text { Dimensionless factors for } \\
\alpha=90-\phi \text { (degrees) } \\
\frac{N}{N} \gamma_{c} \underline{H}^{*}\end{array}$ & $\begin{array}{l}65.0 \\
3.636 \\
9.662\end{array}$ & $\begin{array}{l}60.0 \\
4.738 \\
10.477\end{array}$ & $\begin{array}{r}55.0 \\
7.020 \\
12.779\end{array}$ \\
\hline$\underline{N}^{-} \underline{H}^{*}$ & 9.359 & 12.195 & 18.068 \\
\hline $\begin{array}{l}\alpha \text { (degrees) } \\
\frac{N}{\underline{N}} \underline{\underline{c}} \underline{\underline{H}}\end{array}$ & $\begin{array}{c}84.4 \\
4.463 \\
10.079\end{array}$ & $\begin{array}{c}84.4 \\
6.656 \\
12.087\end{array}$ & $\begin{array}{l}84.4 \\
11.860 \\
17.119\end{array}$ \\
\hline$\underline{N} q \underline{H}$ & 11.934 & 17.266 & 29.130 \\
\hline $\begin{array}{l}\text { Force calculations } \\
\text { density, } \rho\left(\mathrm{kg} / \mathrm{m}^{3}\right) \\
\text { gravity, } \mathrm{g}\left(\mathrm{m} / \mathrm{s}^{2}\right) \\
\text { horizontal force of surface } \\
\text { sampler }(\mathrm{N})\end{array}$ & $\begin{array}{c}1,200 \\
3.73 \\
210-236\end{array}$ & $\begin{array}{c}1,200 \\
3.73 \\
192-216\end{array}$ & $\begin{array}{r}1,200 \\
3.73 \\
173-194\end{array}$ \\
\hline $\begin{array}{l}\text { Cohesions }(\mathrm{kPa}) \\
\text { derived force }(\mathrm{N})\end{array}$ & $\begin{array}{l}6.4-7.3 \\
209-237\end{array}$ & $\begin{array}{l}4.7-5.2 \\
195-2.14\end{array}$ & $\begin{array}{l}2.6-2.9 \\
175-193\end{array}$ \\
\hline $\begin{array}{l}\text { Force calculations } \\
\text { density, } p\left(\mathrm{~kg} / \mathrm{m}^{3}\right)\end{array}$ & 2,000 & 2,000 & 2,000 \\
\hline $\begin{array}{l}\text { Cohesions ( } \mathrm{kPa}) \\
\text { derived force }(\mathrm{N})\end{array}$ & $\begin{array}{l}6.2-7.1 \\
208-236\end{array}$ & $\begin{array}{l}4.5-5.0 \\
195-214\end{array}$ & $\begin{array}{l}2.3-2.7 \\
173-197\end{array}$ \\
\hline
\end{tabular}

reverse will occur. Furthermore, density and acceleration of gravity should be minor factors for a material dominated by cohesion. Other parameters that were used are given in tables 11,14 , and 17 except for the width of the blade or collector head $(b=0.048 \mathrm{~m})$ and its velocity $(v=0.0254 \mathrm{~m} / \mathrm{s})$. Iterative solutions for horizontal force were calculated for a number of cohesions until the horizontal forces $\left(F_{x}\right)$ on the collector head were equal to the available horizontal forces $\left(F_{T}\right)$.

Apparent cohesions were calculated with the equation for pure cohesion and were usually larger than the cohe- sion estimates based on the plowing theoretical analysis (fig. 36). For drift material (table 11), apparent cohesions were small and within a factor of two of the cohesions obtained from theory, and this was also true for apparent cohesions of blocky material (table 14). For crusty to cloddy material (table 17), apparent cohesions, though small, were as much as five times greater than the cohesions obtained from the theoretical analyses.

Apparent cohesions were found to be linearly related to cohesions estimated with the plowing model for drift material and blocky material when the angles of internal 


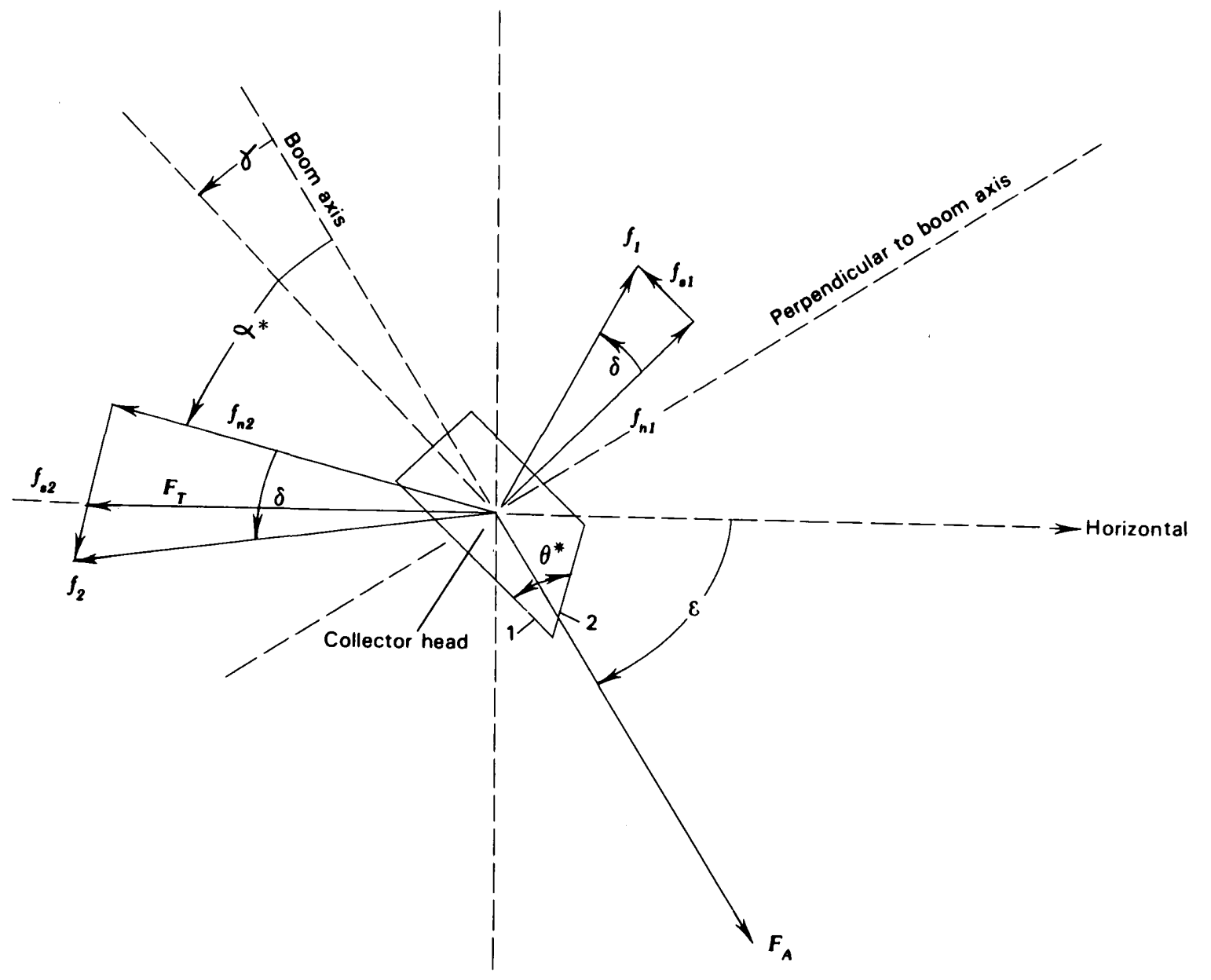

EXPLANATION

$F$ AXIAL FORCE OF BOOM

$F_{T}$ HORIZONTAL FORCE OF SURFACE MATERIAL

$f_{1}$ GROUND REACTION FORCE ON BASE OF COLLECTOR HEAD 1

$f_{.1}$ SHEARING FORCE PARALleL TO BASE OF COLLECTOR HEAD 1

$f_{n 1}$ FORCE NORMAL TO BASE OF COLLECTOR HEAD 1

$\boldsymbol{f}_{2}$ GROUND REACTION FORCE ON INTERFACE 2

$f_{\bullet 2}$ SHEAR FORCE PARALLEL TO BASE OF COLLECTOR HEAD 1

$f_{n 2}$ FORCE NORMAL TO INTERFACE

$\varepsilon$ ELEVATION ANGLE

$\gamma$ COLLECTOR-HEAD ROTATION ANGLE

$\delta$ ANGLE OF METAL SURFACE-MATERIAL FRICTION

$\theta^{*}$ aNGLE between base OF COLLECTOR HEAD 1 AND INTERFACE 2

$\alpha^{*} \quad$ ANGle between nORMAL to INTERFACE 2 AND BOOM AXIS

1 BASE OF COLLECTOR HEAD

2 INTERFACE AT LEADING EDGE OF COLLECTOR HEAD

FIGURE 161.-Two-dimensional limiting equilibrium model used to estimate horizontal force exerted by interface of leading edge of collector head on surface material $\left(F_{T}\right)$ after allowing for friction on base of collector head ( 1 in figure) and interface of leading edge of collector ( 2 in figure). 


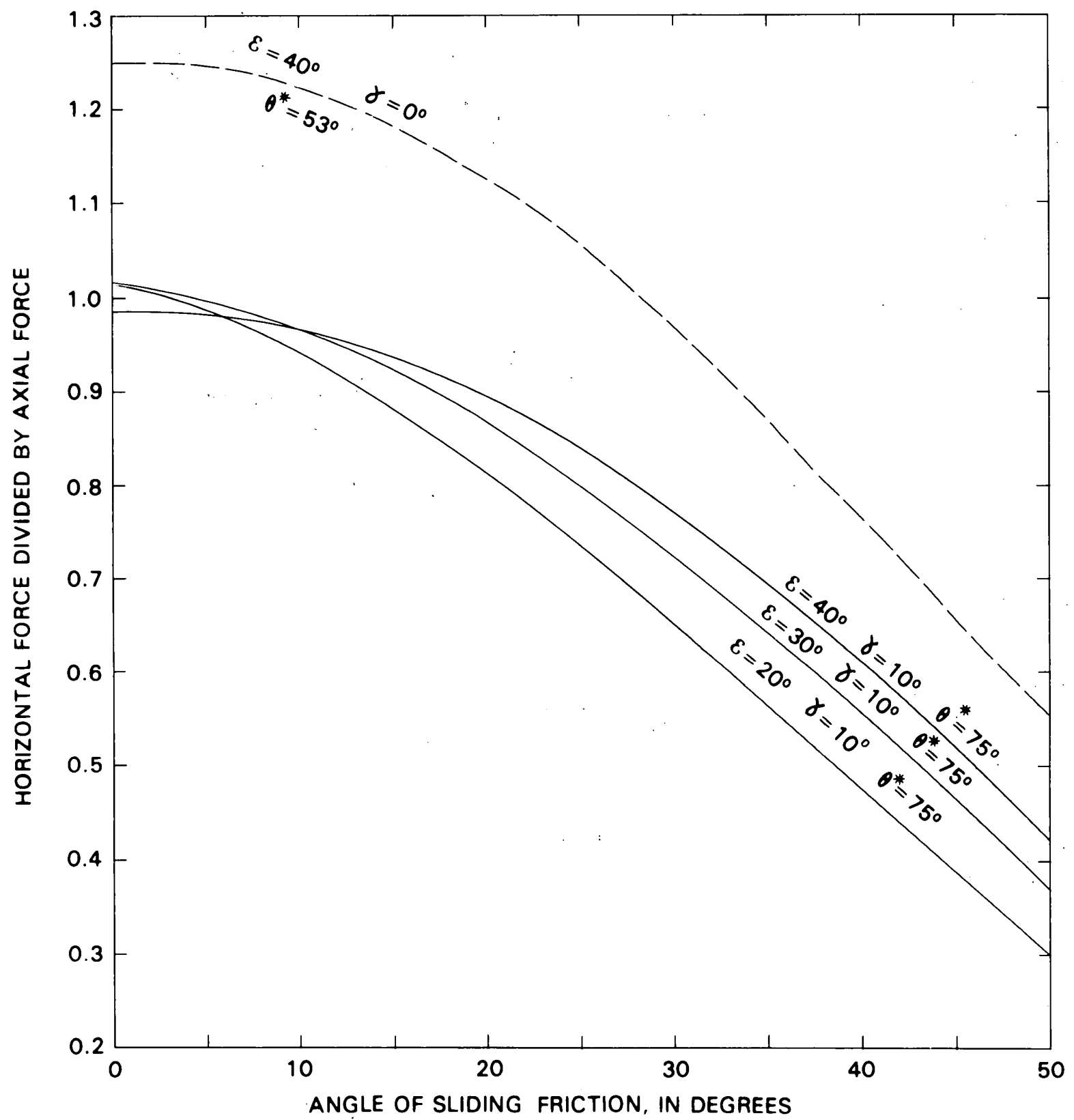

FIGURE 162.-Ratio of horizontal force on interface of leading edge of collector head and axial force of surface sampler as a function of angle of sliding friction. Elevation angles $(\varepsilon)$ of $20^{\circ}, 30^{\circ}$, and $40^{\circ}$ with a collector-head rotation $(\gamma)$ of $10^{\circ}$ include range employed on Mars. Elevation angle $(\varepsilon)$ of $40^{\circ}$ and collector-head rotation $(\gamma)$ of 0 are close to conditions for surface-bearing tests (dashed line). $\theta^{*}$, angle between base of collector head and interface. Actual values of $\theta^{*}$ used in calculations were $76^{\circ}$ for the trench analyses and $53^{\circ}$ for the surface-bearing tests.

$(\phi)$ and sliding friction $(\delta)$ were $20^{\circ}$ and $30^{\circ}$ respectively; this relation formed the basis for the estimates of probable cohesions for materials in trenches with insufficient geometrical data for theoretical analyses. A similar relation might exist for crusty to cloddy material (when $\phi=\delta=30^{\circ}$ ) (fig. 36) but the fact that results from one trench (Physical Properties 5, table 17) departed significantly from a linear relation introduces some uncertainty in the estimates of probable cohesions for the three trenches.

When using the equation for pure friction (fig. 163) to calculate horizontal forces, the acceleration of gravity of Mars $\left(g=3.71 \mathrm{~m} / \mathrm{s}^{2}\right)$ and densities from 1,200-2,000 $\mathrm{kg} / \mathrm{m}^{3}$, depending on the material, were used. Independent calculations based on the theoretical plowing model showed that these forces were reasonable. 


\section{EXPLANATION}

P DENSITY OF "SOIL"

$g$ ACCELERATION OF GRAVITY

$b$ WIDTH OF BLADE

$\ell$ HEIGHT OF BLADE

2 OPERATING DEPTH

a BLADE ANGLE (RADIANS)

$V$ VELOCITY

$C$ COHESION

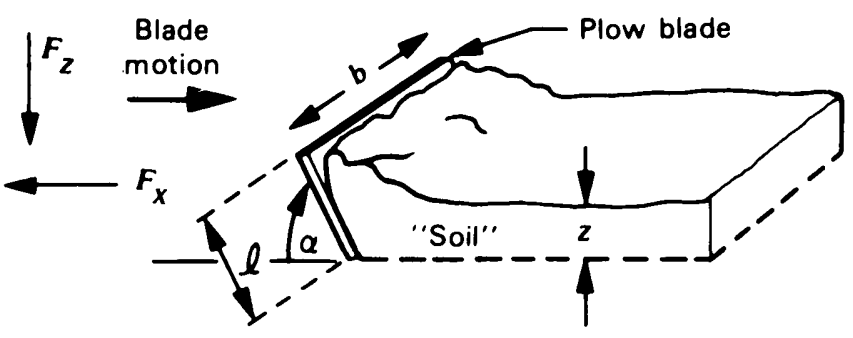

Viking sampler collector head
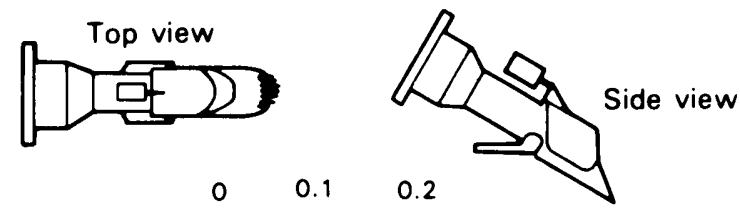

METERS

PURE FRICTION

$F_{x}=\rho g b z=l^{0.5} \alpha^{1.5}\left\{\frac{z}{l \sin \alpha}\right\}^{0.77}\left\{1.05\left(\frac{z}{b}\right)^{1.1}+1.26 \frac{V^{2}}{g l}+3.91\right\}$

$F_{z}=\rho g b z=\ell^{0.5}\left\{.5\left\{0.193-(\alpha-0.714)^{2}\right\}\left\{\frac{z}{l \sin \alpha}\right\}^{0.777}\left\{1.31\left(\frac{z}{b}\right)^{0.966}+1.43 \frac{v^{2}}{g l}+5.60\right\}\right.$

PURE COHESION

$F_{x}=\rho g b z l^{0.5} \alpha^{1.5}\left\{\frac{z}{l \sin \alpha}\right\}^{1.21}\left\{\left(\frac{11.5 C}{\rho g z}\right)^{1.21}\left(\frac{2 V}{3 b}\right)^{0.121}\left(0.055\left(\frac{z}{b}\right)^{0.78}+0.065\right)+0.64 \frac{V^{2}}{g l}\right\}$

$F_{z}=\rho g b z=l^{0.51 .5}\left\{0.48-(\alpha-0.070)^{3}\right\} \quad\left\{\frac{z}{l \sin \alpha}\right\} \quad\left\{\left(\frac{11.5 C}{\rho g z}\right)^{0.41}\left(\frac{2 V}{3 b}\right)^{0.041}\left(9.2\left(\frac{2}{b}\right)^{0.225}-5.0\right)+0.24 \frac{V^{2}}{g l}\right\}$

FIGURE 163.-Plowing model, definitions, collector head, and equations used for empirical analyses. Horizontal forces $\left(F_{x}\right)$ expected for plowing in dry sand were attained by using equation for "pure friction." "Apparent" cohesions were obtained from equation for "pure cohesion" by varying cohesion $(C)$ until horizontal forces $\left(F_{x}\right)$ were equal to available forces on collector head $\left(F_{T}\right)$. 


\section{NAMES OF AREAS IN THE SAMPLE FIELDS AND ROCKS}

The Viking explorers of Mars shared one trait with explorers on Earth: they both tended to name geographic areas and topographic features. Thus, areas and features near the landers and within their sample fields were named despite their diminutive sizes. Two purposes were served by naming the areas and rocks: (1) memorization of the geometry of the sample fields was facilitated, and (2) tensions resulting from working long, difficult hours were alleviated. A smooth rock-free area in the sample field of Lander 1 was named Sandy Flats and another area with a gravelly appearing surface and scattered rocks was called Rocky Flats (pl. 1). As it turned out, the materials at Sandy Flats were not sandy and no demonstrable pieces of rocks from Rocky Flats were ever analyzed. Two unlabeled areas in plate 1 were known as "Atlantic City" $\left(Z_{L}=2.8 \mathrm{~m} ; Y_{L}=0.9 \mathrm{~m}\right)$ for reasons unknown to the author and "Wind Tail" $\left(Z_{L}=3.0 \mathrm{~m} ; Y_{L}=1.4 \mathrm{~m}\right)$ because there was a wind tail on the lee side of rock 2 (Sponge).

An uninteresting approach to the selection of names of areas in the sample field of Lander 2 was adopted by managers within the project (also known as "hummers") who were responsible for the designations: alpha, beta, gamma, and delta (pl. 2). Some observers felt that "eenie", "meanie", "miney", and "mo" would have sufficed. Subsequent names were, perhaps, more imaginative. The name "Bonneville Salt Flats" was chosen because of a rather large exposure of martian crust, and this reminded someone on the Inorganic Chemical Analysis Team of the Bonneville Salt Flats in Utah. Physica Planitia was named in honor of the Physical Properties Investigation. Arida Fossa, which means dry valley, somehow seemed appropriate for the small rille that passed through the sample field. These last two names were patterned after those used on small-scale maps of Mars. Despite a more imaginative approach, subsequent facts did not support the selection of the name "Spalling Valley" and, sadly, no pieces of rocks or spalls were ever sampled or collected in Spalling Valley or anywhere else in the sample field of Lander 2 for that matter (pl. 2).

The reasons for the selection of names of rocks were, in part, mystifying. Rocks in the sample fields were initially named and then, at the insistence of those with excessively rigid managerial and scientific backgrounds, numbered. Some of the names deserve comment. Rock 1 of Lander 2 was named "ICL", which is an acronym for Initial Computer Load. Prior to landing, the spacecraft computers had stored commands for an automatic mission in the event that the lander could not be commanded. Had this occurred, the spacecraft would have tried to collect a sample from a point just beyond ICL, but would have failed because of ICL. A similar outcome would probably have befallen Lander 1 .
Some explorers fancied that some of the Lander 2 rocks looked like animals. Rock 3 was always known as Badger because of its shape and orientation on the surface which reminded one of an animal with a pointed nose and broad shoulders that was crouched and ready for battle. The rock proved to be worthy of the name because of its valiant struggle with the surface sampler during a rock pushing episode (Moore and others, 1978). Rock 2 (initially called "frawg") became known as Toad-a rather large one at that (15 cm long). Two rocks, originally named Middle and Right by the managers, became known as Mole and Rat in order to complete the list of principal characters in The Wind in the Willows, the well-known book by Kenneth Grahame. Of course, the area between these rocks could appropriately be called "The Willows". These rock names also stood some chance of capturing the imagination and attention of many youngsters who were an important audience. Eight rocks were named for characters in the story Snow White and the Seven Dwarfs, which was popularized by Walt Disney. Unaccountably, these names were divided between both landers; this oversight is understandable because the rocks were named during the Extended Mission when the reduced staff of explorers was very busy and all of Mars appeared to be made of red dirt and reddish rock. Somehow a rock bearing the name Prince was never selected. A host of rocks were unnamed (see figs. 11 and 12). Rock names with brief commentaries are listed as follows:

\section{Rocks in sample field of Lander 1}

Shadow. Named because the shadow of meteorology boom was cast upon it early in the mission before the first sample was acquired. Knowledge of the geometry of the spacecraft, sun azimuth, and sun elevation angle was used to locate Shadow and nearby surfaces. This independent determination of the location of Shadow was used to verify that the determination of the location of Shadow by stereometric means was correct. This knowledge, in turn, showed that the site and the surface-sampler commands selected for the very first samples of martian "soil" were valid and safe.

Sponge. Many thought that this rock looked porous, frothy and weak. Subsequent attempts to scratch it showed that it was neither frothy nor weak.

Metate 1. Looked like an American Indian mortar used to grind cereal seeds.

Grumpy. Named for one of the seven dwarfs. (We actually had a check list so as to include all.)

Bashful. Named for one of the seven dwarfs because it was hiding behind boom housing when viewed by camera 1.

Metate 2. Looked like an American Indian mortar used to grind cereal seeds. 
Dopey. Named for one of the seven dwarfs.

Phantom 309. Named for the truck driven by Big Joe in Red Solvine's ballad.

Sleepy. Named for one of the seven dwarfs; it was a "sleeper" because it was partly hidden from the view of camera 1 and could have interfered with a sample acquisition near Bashful.

Patch. Named for its patchy colorations by the Biology Team.

\section{Rocks beyond the sample field of Lander 1}

Big Joe. Originally called Big Bertha by Lander Imaging Team who later changed it to Big Joe because the name Big Bertha was thought to be chauvinistic. Big Joe is about 8 or $10 \mathrm{~m}$ from Lander $1,2 \mathrm{~m}$ wide, and $1 \mathrm{~m}$ high (figs. 11, camera 1 and $68 A, B$ ).

Muffler. Somebody on the Lander Imaging Team thought it looked like an auto muffler (M in fig. 11, camera 2).

Whale. Named by Lander Imaging Team. Rock is about $25 \mathrm{~m}$ from Lander 1 and, in some pictures, it really did look like a sperm whale; but it must be a baby because it only rose $70 \mathrm{~cm}$ above the surface and it was only $2.6 \mathrm{~m}$ long. An additional $1.3 \mathrm{~m}$ of a drift could be considered to be its tail with hidden flukes (figs. 11, camera 1 and $68 C, D$ ).

\section{Rocks in sample field of Lander 2}

ICL. Pronounced "icle". The name is an acronym for Initial Computer Load (see above and Moore and others, 1978).

Toad. Originally called "frawg" by the Surface Sampler Team but later changed to Toad. It looked like a toadwith two bulges for eyes, a "snout," a chest, and a rump. This was perhaps the most lifelike rock on Mars. The name was changed to conform with Badger.

Badger. Looked like a badger with a pointed "snout" and broad "shoulders." It put up a good fight when the sur- face sampler tried to push it. When last photographed, it was still standing on its haunches, ready for battle.

Rat. Originally called "right rock" by unimaginative managers, it was changed to Rat to conform with Toad and Badger.

Mole. Originally called "middle rock" by unimaginative managers, it was changed to Mole to conform with Toad, Badger, and Rat. Not only that, Mole was deeply buried.

Bonneville. Named for sample area.

Notch. Named because it had a notch in it that was used to push it.

Other. Named because it was the fourth of four choices of rocks that might be pushed. (The name "Another" was unaccountably never used.)

Plymouth. Named for historical reasons.

Centaur. Named for launch vehicle.

Snow White. A good friend of the seven dwarfs. The rock had a very light color. The names "Prince" and "Witch" were never used.

Sneezy. One of the seven dwarfs. Sneezy somehow seemed appropriate because it was under the terminaldecent engine.

Gibraltar. Named for the famous rock.

Happy. A fat, plump, pillowlike rock named for one of the seven dwarfs.

Doc. Named for one of the seven dwarfs.

Rocks without numbers in and near the sample field of Lander 2

Alligator. Someone thought that it looked like Albert of Pogo fame (pl. 2; $Z_{L}=4.3 \mathrm{~m}, Y_{L}=(-) 0.3 \mathrm{~m}$ ).

Guppy. Resembled a tailless guppy (pl. $2 ; Z_{L}=3.6 \mathrm{~m}$, $\left.Y_{L}=(-) 0.7 \mathrm{~m}\right)$.

Hog's Head. Someone on the Lander Imaging Team thought that it looked like one (pl. $2 ; Z_{L}=4.4 \mathrm{~m}$, $\left.Y_{L}=(-) 0.7 \mathrm{~m}\right)$.

Titan. Named for the launch vehicle (pl. $2 ; Z_{L}=1.3 \mathrm{~m}$, $\left.Y_{L}=(-) 4.0 \mathrm{~m}\right)$. 
